
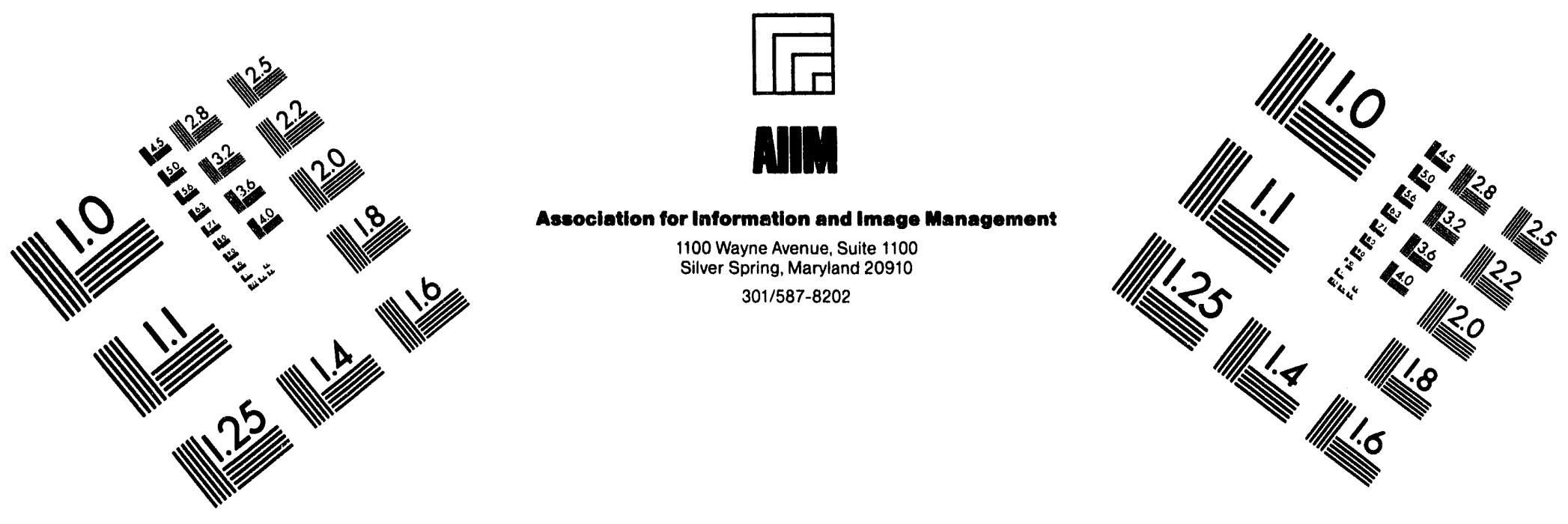

\title{
Centimeter
}

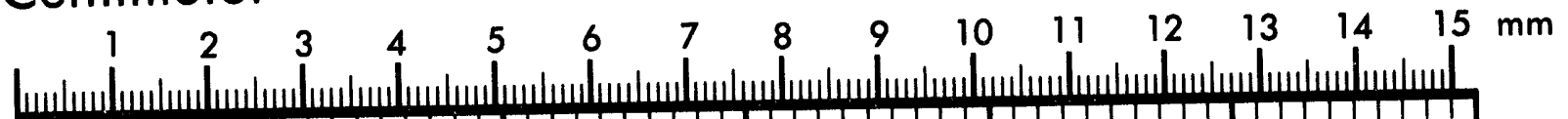

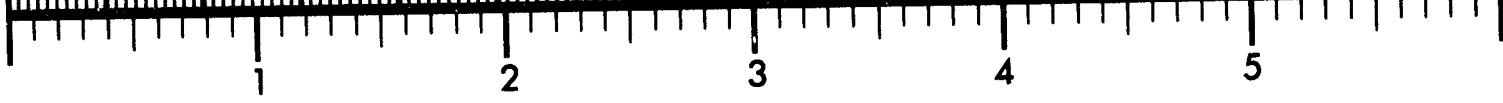
Inches
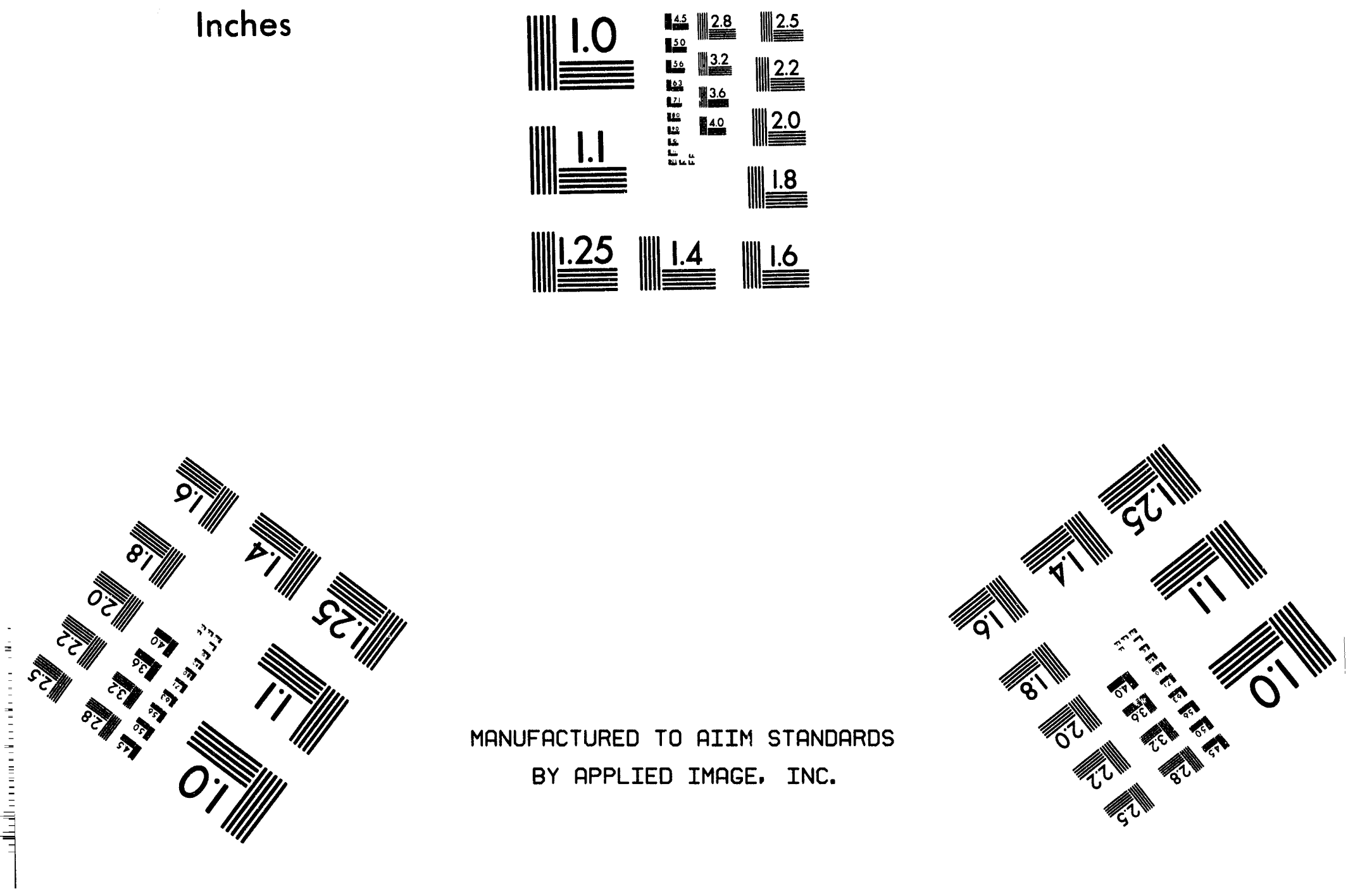

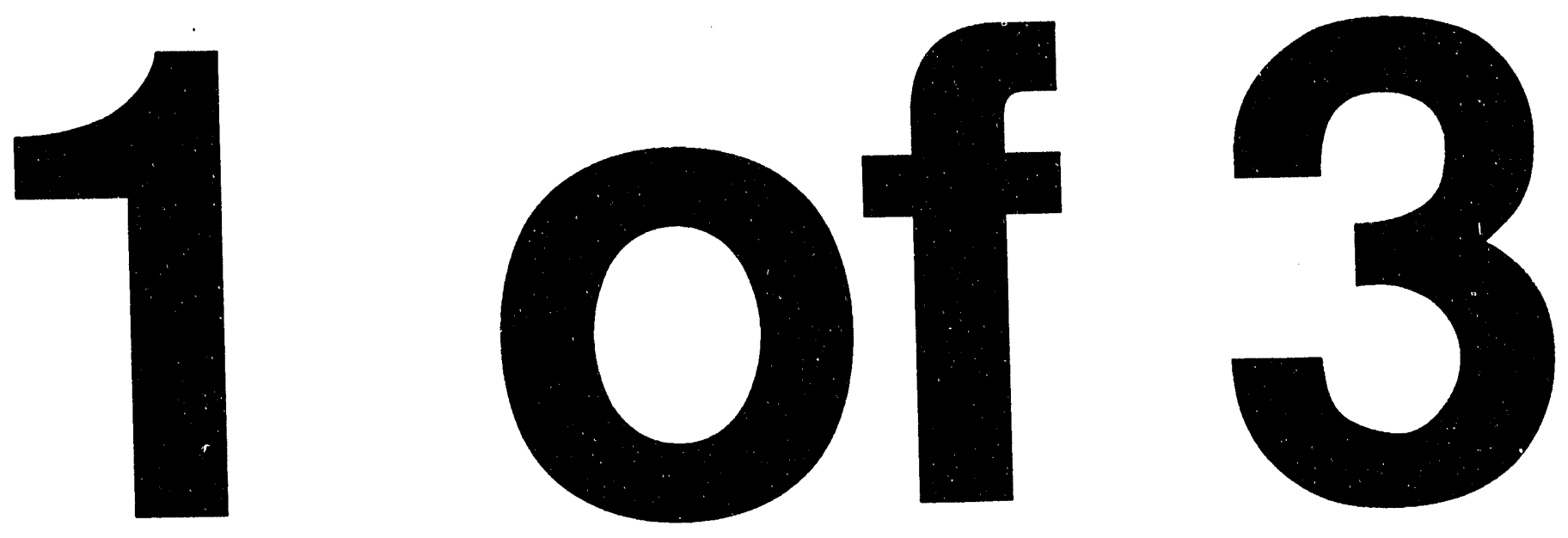
DOE/EIA-0340(93)/1

Distribution Category UC-950

\title{
Petroleum Supply Annual 1993
}

\author{
Volume 1
}

\section{June 1994}

\author{
Energy Information Administration \\ Office of Oil and Gas \\ U.S. Department of Energy \\ Washington, DC 20585
}

This report was prepared by the Energy Information Administration, the independent statistical and analytical agency within the Department of Energy. The information contained herein should not be construed as advocating or reflecting any policy position of the Department of Energy or any other organization. 


\section{Preface}

The Petroleum Supply Annual (PSA) contains information on the supply and disposition of crude oil and petroleum products. The publication reflects data that were collected from the petroleum industry during 1993 through annual and monthly surveys. The $P S A$ is divided into two volumes. This first volume contains four sections: Summary Statistics, Detailed Statistics, Refinery Capacity, and Oxygenate Capacity each with final annual data. The second volume contains final statistics for each month of 1993, and replaces data previously published in the Petroleum Supply Monthly (PSM). The tables in Volumes 1 and 2 are similarly numbered to facilitate comparison between them. Below is a description of each section in Volume 1 of the PSA.

\section{Summary Statistics}

This section contains a summary of the data presented each month in the PSM and in Volume 2 of the PSA. It includes a description of the major events in the petroleum industry during 1993 including trends in supply, consumption, and production. Graphs and tables are provided which show 13 years of data depicting the balance between supply, disposition and ending stocks for various commodities including crude oil, motor gasoline, distillate fuel oil, residual fuel oil, jet fuel, propane/propylene, and liquefied petroleum gases.

\section{Detalled Statistics}

The tables contained in this section provide 1993 detailed statistics on supply and disposition, refinery operations, imports and exports, stocks, and transportation of crude oil and petroleum products. In most cases, the statistics are presented for several geographic areas -- the United States (50 States and the District of Columbia), five Petroleum Administration for Defense (PAD) Districts, and 12 Refining Districts. At the U.S. and PAD District level, the total volume and the daily rate of activities are presented.

\section{Refinery Capacity}

This section describes major activities within the refinery industry during 1993. The tables are compiled from the Form EIA-820, "Annual Refinery Report." Of particular note are listings of facilities and associated capacities in each State. In addition, summaries of corporate refinery capacities, refinery storage facilities in selected States, and refinery shutdowns, sales and mergers during 1993 are provided.

\section{Oxygenate Capacity}

This section highlights major activities within the oxygenate industry during 1993. The tables are compiled from the Form EIA-819A, "Annual Oxygenate Capacity Report." Current production capacity by facility are provided for the following oxygenates: fuel ethanol, ethyl tertiary butyl ether (ETBE), methanol, methyl tertiary butyl ether (MTBE), tertiary amyl methyl ether (TAME), tertiary butyl alcohol (TBA) and other oxygenates. In addition, projections for the following year are provided on a PAD District basis.

\section{Appendices}

Three appendices are provided to assist in understanding and interpreting the data presented in this publication. Industry terminology and product definitions are listed alphabetically in the Glossary.

- Appendix A (District Descriptions and Maps) - Geographic aggregations of the 50 States and the District of Columbia into Refining Districts which make up the PAD Districts.

- Appendix B (Detailed Statistics Explanatory Notes) - Information describing data collection, sources, estimation methodology, data quality control procedures, modifications to reporting requirements and interpretation of tables.

- Appendix C (1992 Revised Crude Oil Production) - Updated monthly and annual crude oil production statistics received after the publication of the 1992 PSA. 


\section{Contents}

Tables

Summary Statistics

S1. Crude Oil and Petroleum Products Overview, 1981-Present ................................................................................... 2

S2. Crude Oil Supply and Disposition, 1981-Present................................................................................................. 6

S3. Crude Oil and Petroleum Product Imports, 1981-Present ......................................................................................... 8

S4. Finished Motor Gasoline Supply and Disposition, 1981-Present ........................................................................ 17

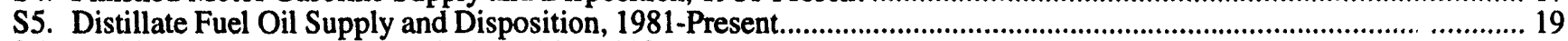

S6. Residual Fuel Oil Supply and Disposition, 1981-Present ............................................................................................ 21

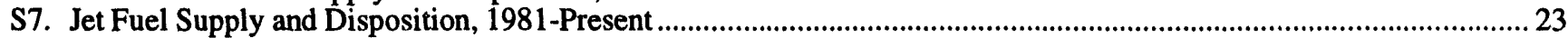

S8. Propane/Propylene Supply and Disposition, 1981-Present ……………................................................................. 25

S9. Liquefied Petroleum Gases Supply and Disposition, 1981-Present. .............................................................................. 27

S10.Other Petroleum Products Supply and Disposition, 1981-Present............................................................................. 28

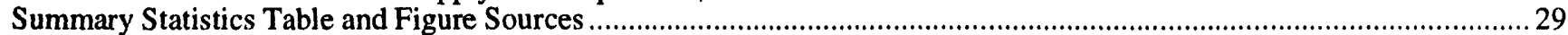

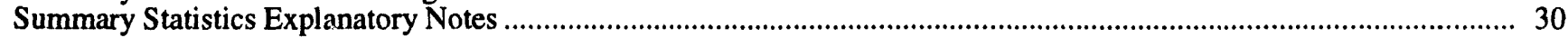

Detailed Statistics

National Statistics

1. U.S. Petroleum Balance .......................... 33

2. U.S. Supply, Disposition, and Ending Stocks of Crude Oil and Petroleum Products ……................................................ 34

3. U.S. Daily Average Supply and Disposition of Crude Oil and Petroleum Products ............................................... 35

Supply and Disposition of Crude Oil and Petroleum Products

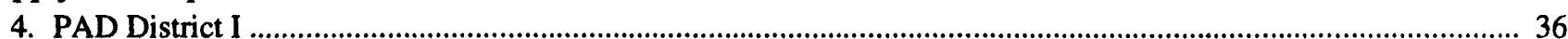

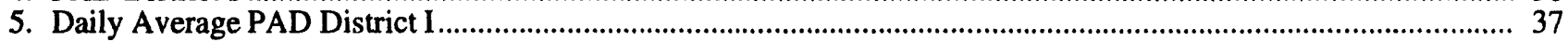

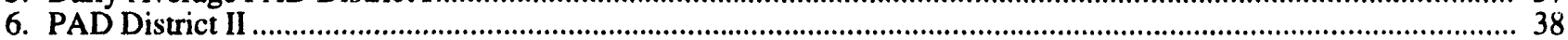

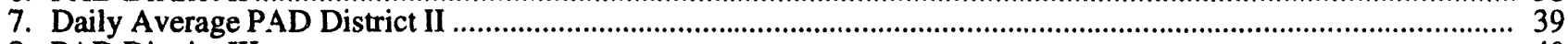

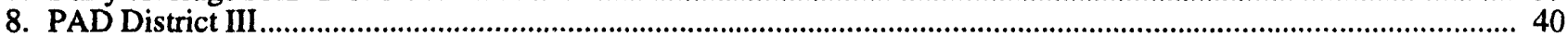

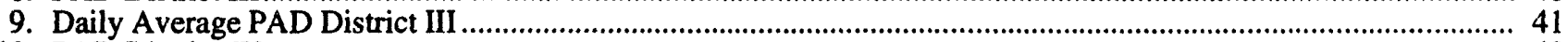

10. PAD District IV.................4. 42

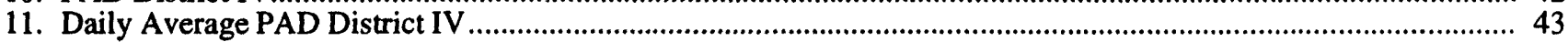

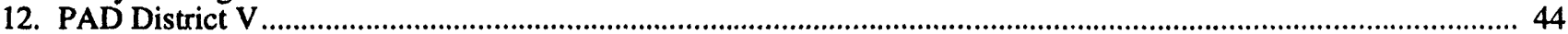

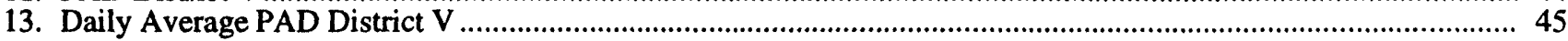

Production of Crude Oil

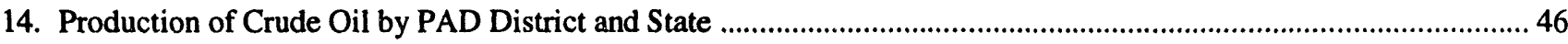

Natural Gas Processing

15. Natural Gas Plant Net Production and Stocks of Petroleum Products by PAD and Refining Districts

\section{Refinery Operations}

16. Refinery Input of Crude Oil and Petroleum Products by PAD and Refining Districts ......................................... 48

17. Refinery Net Production of Finished Petroleum Products by PAD and Refining Districts ................................... 50

18. Refinery Stocks of Crude Oil and Petroleum Products by PAD and Refining Districts .............................................. 52

19. Percent Refinery Yield of Petroleum Products by PAD and Refining Districts................................................... 54

\section{Imports of Crude Oil and Petroleum Products}

\section{PAD District}

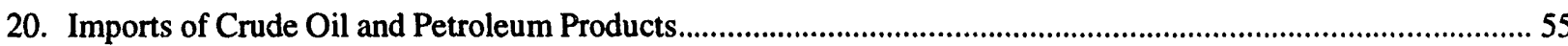

\section{Country of Origin}

21. United States

22. PAD District I.

23. PAD District II

24. PAD District III

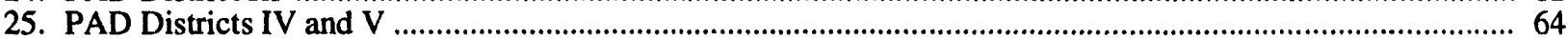

\section{State of Entry}

26. Imports of Residual Fuel Oil by Sulfur Content. 


\section{Exports of Crude Oil and Petroleum Products}

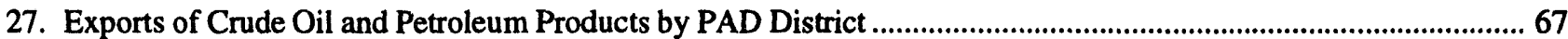

28. Exports of Crude Oil and Petroleum Products by Destination

Net Imports

29. Net Imports of Crude Oil and Petroleum Products into the United States by Country

Stocks

30. Stocks of Crude Oil and Petroleum Products by PAD District 71

31. Refinery, Bulk Terminal, and Natural Gas Plant Stocks of Selected Petroleum Products by PAD District and State

Movements of Crude Oil and Petroleum Products

32. Movements of Crude Oil and Petroleum Products by Pipeline, Tanker, and Barge Between PAD Districts

33. Movements of Crude Oil and Petroleum Products by Pipeline Between PAD Districts ......................................... 76

34. Movements of Crude Oil and Petroleum Products by Tanker and Barge Between PAD Districts

35. Net Movements of Crude Oil and Petroleum Products by Pipeline, Tanker, and Barge Between PAD Districts

\section{Refinery Capacity}

36. Number and Capacity of Operable Petroleum Refineries by PAD District and State as of January 1,1994

37. Production Capacity of Operable Petroleum Refineries by PAD District and State as of January 1, 1994

38. Capacity of Operable Petroleum Refineries by State as of January 1, 1994

39. Production Capacity of Operable Petroleum Refineries by State as of January 1, 1994

40. Refiners' Operable Atmospheric Crude Oil Distillation Capacity as of January 1, 1994

41. Operable Crude Oil and Downstream Charge Capacity of Petroleum Refineries, January 1, 1981 to January 1, 1995

42. Operable Production Capacity of Petroleum Refineries, January 1, 1981 to January 1,1995

43. Working Storage Capacity at Refineries and Gasoline Blending Plants by PAD District and State as of January 1, 1994

Shell Storage Capacity at Refineries and Gasoline Blending Plants by PAD District and State as of January 1, 1994

46. Refinery Receipts of Crude Oil by Method of Transportation by PAD District, 1993

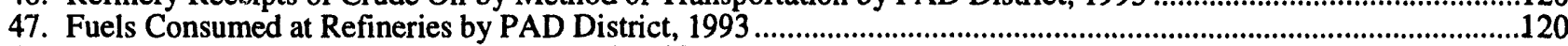

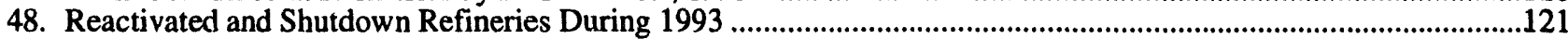

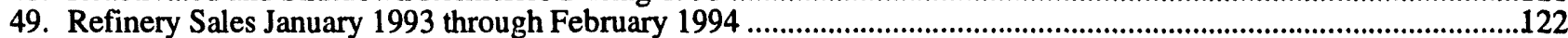

\section{Oxygenate Capacity}

50. Number and Production Capacity of Operable Oxygenate Plants by PAD

District and State as of January 1, 1994

51. Fuel Ethanol Production Capacity by PAD District as of January 1, 1994

52. Ethyl Tertiary Butyl Ether (ETBE) Production Capacity by PAD District as of January 1, 1994.

53. Methanol Production Capacity by PAD District as of January 1, 1994.

54. Tertiary Amyl Methyl Ether (TAME) Production Capacity by PAD

District as of January 1, 1994

District as of January 1, 1994

56. Tertiary Butyl Alcohol (TBA) Production Caf ucity by PAD District as of January 1, 1994.

57. Other Oxygenates Production Capacity by PAD District as of January 1, 1994

58. Production Capacity of Operable Oxygenate Plants by PAD District, January 1,1994 to January 1,1995 


\section{Illustrations}

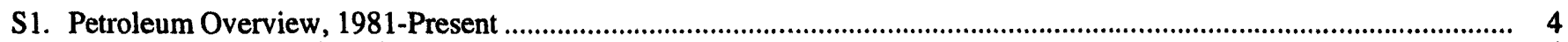

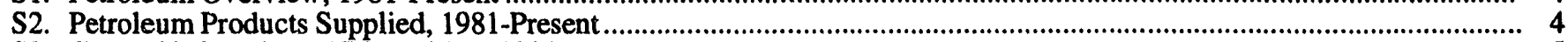

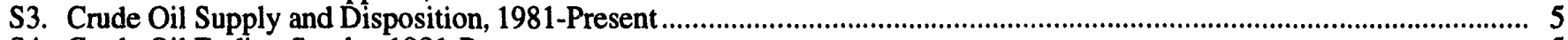

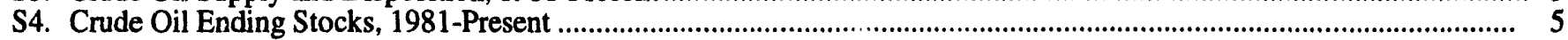

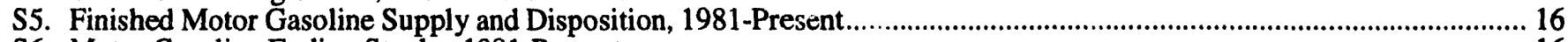

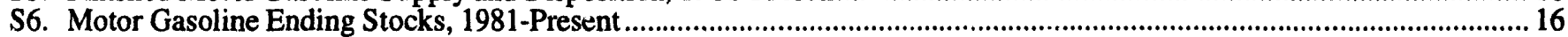

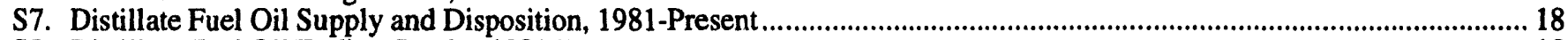

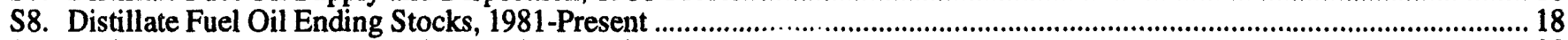

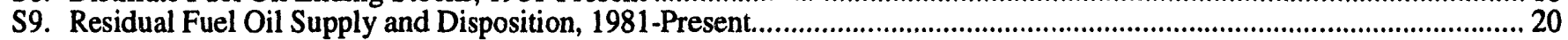

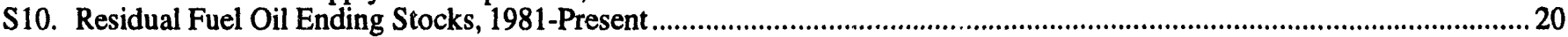

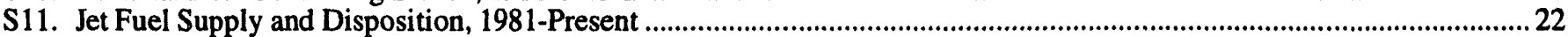

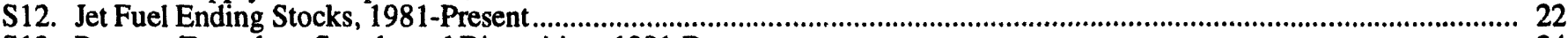

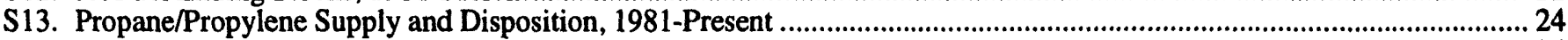

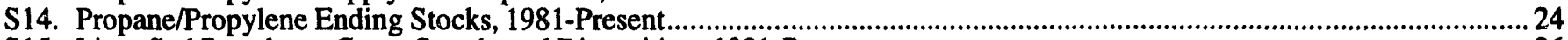

S15. Liquefied Petroleum Gases Supply and Disposition, 1981-Present ......................................................................26

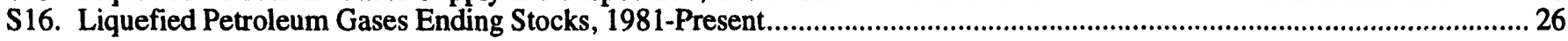

\section{Appendices}

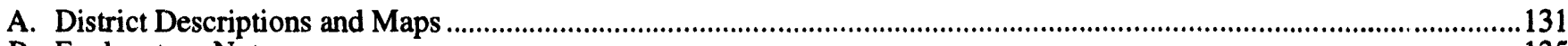

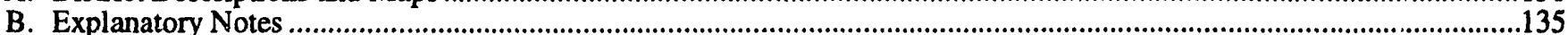

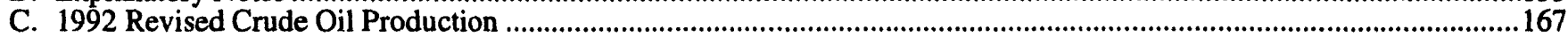

\section{Glossary}

Definitions of Petroleum Products and Other Terms 


\section{U.S. \\ Petroleum \\ Developments: 1993}

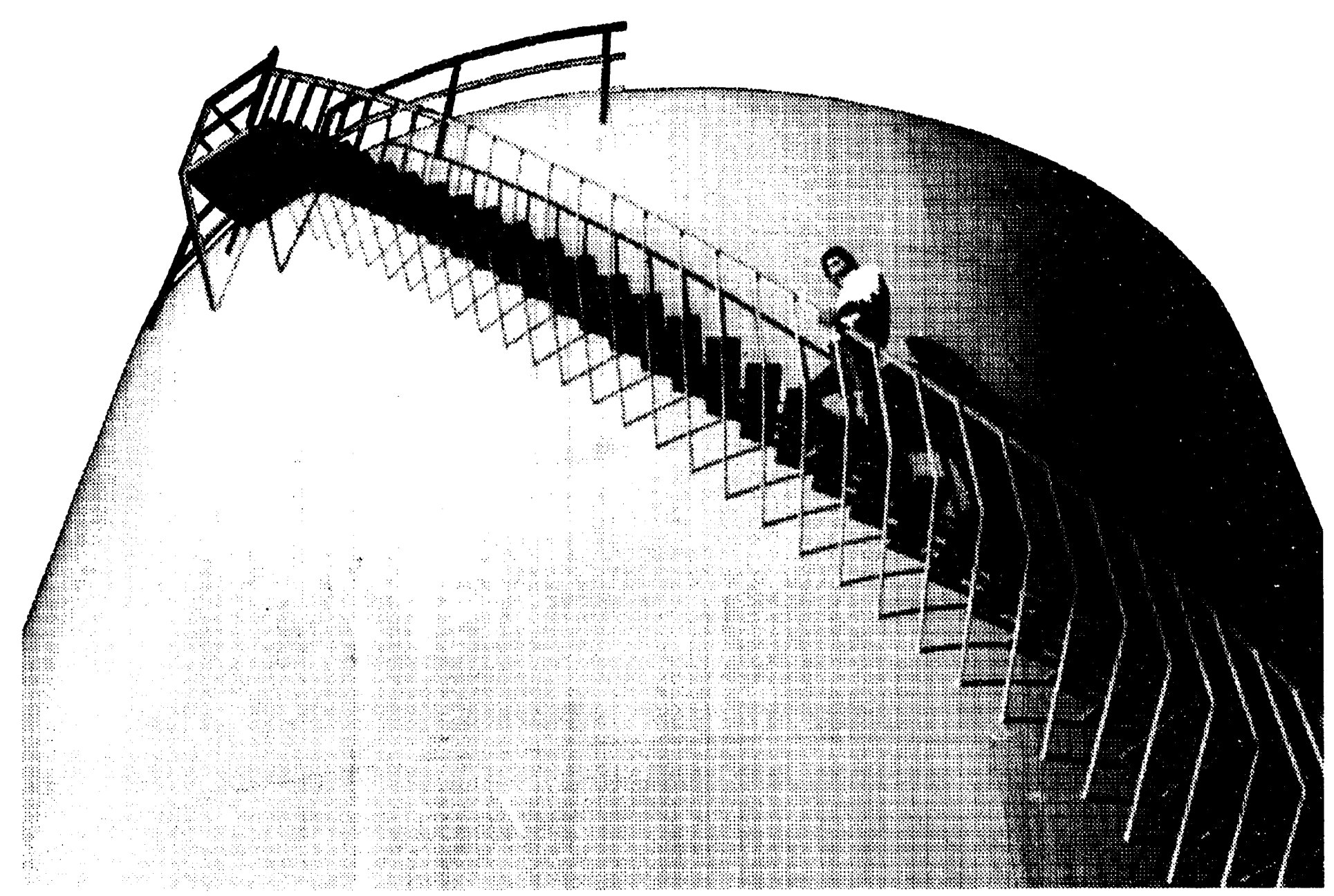




\section{U.S. Petroleum Developments: 1993}

\section{Highlights}

\section{Economic Growth Spurs Slight Increase in U.S. Petroleum Demand}

Petroleum demand (measured as product supplied) in the United States averaged 17.2 million barrels per day during 1993, an increase of 1 percent from the 1992 level. An improved economy, as measured by gross domestic product ${ }^{1}$ (GDP) and reflected in higher industrial production and real income growth by consumers, led to the slight increase in total petroleum demand during 1993.

Following a protracted period of sluggish growth and economic uncertainty that emerged after the Persian Gulf War, the U.S. economy during 1993 began to exhibit a pattern of sustained economic growth that became increasingly stronger as the year

\section{Figure FE1. Changes in U.S. Petroleum Demand by Product (1992 to 1993)}

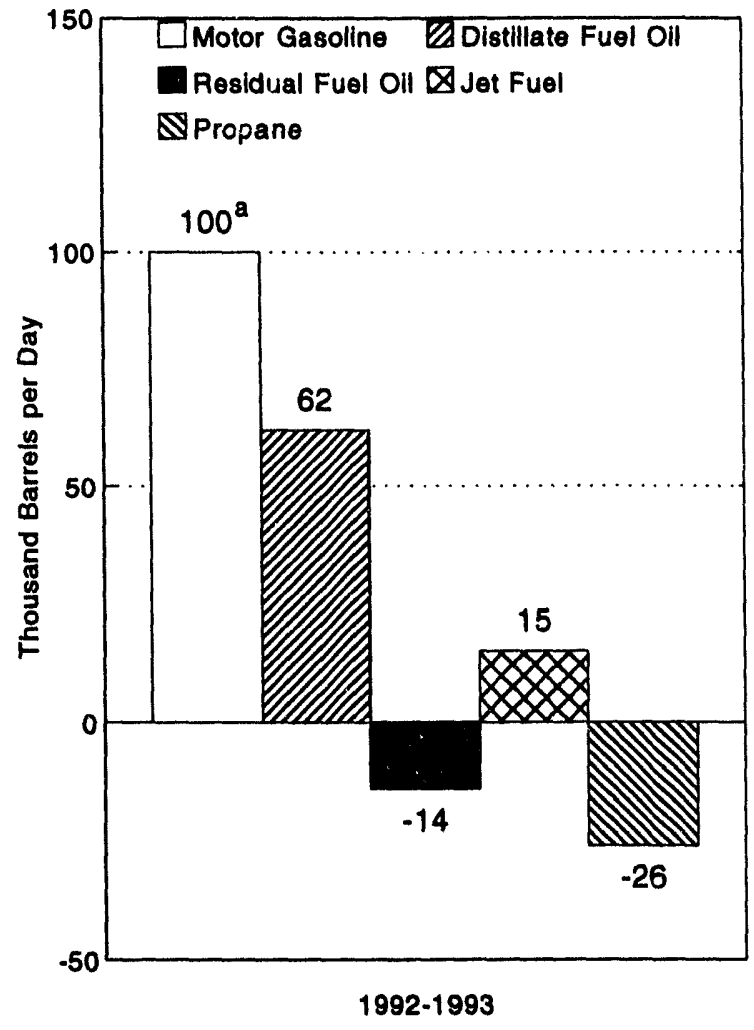

\footnotetext{
a Motor gasoline change from 1992 includes fuel ethanol and motor gasoline blending adjustments. (See Appendix B, Explanatory Note 11 Table B4.)

Source: Energy Information Administration, Petroleum Supply Annual , 1993, DOE/EIA-0304(93)/1, Volume 1, Tables S4, S5, S6, S8, and S9.
}

progressed. This growth, which marked one of the best annual performances for the economy over the past several years, spurred higher consumption for all major petroleum products, except for residual fuel oil and propane (Figure FE1).

The increase in petroleum consumption during 1993 was particularly evident within the transportation fuel sector. Motor gasoline consumption reached an all-time high during 1993, as real income growth and lower real fuel costs per mile combined to increase discretionary travel. Diesel fuel consumption increased as a result of the overall expansion of commercial and industrial activity and the subsequent rise in highway freight traffic. The rise in jet fuel consumption was brought about in part by increased commercial airline competition in the form of passenger fare discounts and incentives, which boosted passenger travel demand.

\section{Crude Oil Prices Hit 5-Year Low}

World crude oil prices ${ }^{2}$ fell to their lowest level in over 5 years during 1993. This was largely due to relatively weak world demand for petroleum, overproduction of crude oil by the Organization of Petroleum Exporting Countries (OPEC), abundant world inventories, and continued improvements in global energy efficiencies.

The overall impact of lower crude oil prices on U.S. producers, refiners, and consumers was generally mixed. While lower oil prices are viewed as being beneficial to the U.S. economy, they can adversely affect the domestic oil and gas industry if prices remain low for an extended period of time.

Lower crude oil prices historically precede lower drilling activity and accelerated declines in U.S. crude oil production volumes. Although drilling activity during 1993 did not experience the declines normally associated with falling crude oil prices, its relatively low level, compared with the past several years, was a contributing factor for the continued drop in U.S. crude oil production volumes. ${ }^{3}$ During 1993, U.S. crude oil production fell to its lowest level in 35 years.

The impact of lower crude oil prices on refiner operations was generally positive during 1993 . The refiner acquisition cost of crude oil from all sources fell by nearly $\$ 6.00$ per barrel between April and December 1993, which caused refining margins to improve for some petroleum refiners.

\footnotetext{
${ }^{1}$ Gross Domestic Product (GDP) is the measure of the sum of all domestic goods and services produced.

${ }^{2}$ Energy Information Administration, Weekly Petroleum Status Report, January 7, 1994, DOE/EIA-0208 (94-02), Figure 9, p. 19.

${ }^{3}$ Energy Information Administration, Monthly Energy Review, April 1994, DOE/EIA-0035(94/04), Table 5.1, "Rotary Rigs in Operation-Oil," p. 84.
} 
Declining crude oil costs benefitted consumers through lower product prices during 1993. All of the major petroleum products, except diesel fuel, experienced price declines during 1993. $^{4}$

\section{Federal Excise Tax Increase for Motor Fuels}

On August 10, 1993, President Clinton signed the Omnibus Budget Reconciliation Act of 1993, which raised the Federal excise tax for on-highway motor fuels by 4.3 cents per gallon starting October 1, 1993. The new tax raised the total Federal excise tax for motor gasoline and diesel fuel to 18.4 cents per gallon and 24.4 cents per gallon, respectively. Certain uses of diesel fuel, such as for farming, off-highway, and home heating, are exempt from the tax. To distinguish taxable fuel from non taxable fuel, Federal regulations required that exempt fuel be dyed while taxable fuel remain "clear."

The new Federal excise tax increase had little impact on motor gasoline retail prices, as falling crude oil prices during 1993 more than offset the effect of the tax increase on retail prices. On average, 1993 retail motor gasoline prices were about 2 cents per gallon below their 1992 average annual price.

However, the new Federal tax on diesel fuel came on top of other increased fuel costs due to new low-sulfur requirements for diesel used for transportation. These changes occurred simultaneously on October 1 and, combined with some refinery outages, the result was some localized supply shortages and steep price increases for diesel fuel in the Midwest and on the West Coast.

\section{Clean Air Act Amendments of 1990}

The quality of transportation fuels marketed in the United States during 1993 was affected by recent changes in Federal and State standards.

Noxious exhaust emissions were reduced, as the Clean Air Act Amendments of 1990 (CAAA) mandated that the maximum sulfur content of highway diesel fuel be reduced to 0.05 percent by weight. This requirement became effective October 1 , $1993^{5}$. Also effective on that date, the California Air Resources Board (CARB) reduced aromatic hydrocarbon levels to 10 percent by volume for on- and off-highway diesel fuel oil marketed in California.

As mandated by the CAAA, oxygenated motor gasoline was marketed during the winter months generally in specified carbon monoxide nonattainment areas. These cleaner burning fuels contain high levels of oxygen that reduce carbon monoxide emissions from automobiles. The first oxygenated gasoline season was carried out in the designated areas mainly from November 1992 through February $1993,{ }^{6}$ and the second season was between October 1993 and March 1994 in most regions. In New York and northern New Jersey, the oxygenated season continued until the end of April 1994.

Oxygenates are comprised of alcohols and ethers and are used by petroleum refiners and marketers as octane boosters for motor gasolines as well as blending materials to improve motor gasoline specifications under the mandate of the CAAA. The second oxygenated fuel season resulted in reduced carbon monoxide emissions in naany areas. ${ }^{7}$ However, while most oxygenated motor gasoline operations were carried out smoothly and without complaints, numerous low-sulfur diesel fuel oil cold-weather handling and performance problems were reported. These problems occurred mainly because of jelling and solidifying at temperatures considerably higher than in similar fuels with higher sulfur levels, and consumers and marketers blended increased quantities of kerosene with the diesel fuel oil to alleviate the cold-temperature flow problems.

The petroleum industry invested in new and modified storage, distribution, and transportation systems to accommodate these new fuel standards. Many petroleum refiners and distributors constructed separate storage tanks to segregate oxygenate materials, finished motor gasoline, and low-sulfur diesel fuel oil; modifications were also made to highway, waterway, and pipeline transportation equipment and systems to handle these particular petroleum products.

CAAA and State requirements imposed significant changes and upgrading in petroleum refinery fluid catalytic cracking and catalytic reforming units to raise the oxygen content of automotive fuel. U.S. refiners increased the output of oxygenated finished motor gasoline, produced mainly with methyl tertiary butyl ether (MTBE) and fuel ethanol, and they invested significantly in additional hydrotreaters and desulfurization units necessary to satisfy the need for the cleaner burning distillate fuel oils.

\section{Propane Inventories Rebound from Record Low Level}

Rebounding from an all-time low reached at the end of March 1993, U.S. stocks of propane were replenished to normal levels by the start of the 1993-1994 heating season. The concern among industry observers prior to the buildup was whether the industry could rebuild stocks to a comfortable level in time for the start of the winter heating season. However, by the end of September 1993, the Nation's supply of propane reached over

\footnotetext{
${ }^{4}$ Energy Information Administration, Short-Term Energy Outlook, DOE/EIA-0202(94/1Q), Figure 13, "U.S. Petroleum Prices," p. 14.

5"Distillate Fuel Oil Outlook for Winter 1993-94," Petroleum Supply Monthly, DOE/EIA-0109(93/10), October 1993, p. xiii-xxiv.

6"The Economics of the Clean Air Act Amendments of 1990: Review of the 1992-1993 Oxygenated Motor Gasoline Season," Petroleum Supply Monthly, DOE/EIA-0109(93/07), July 1993, pp. xiii-xxv.

7"1993-94 Oxygenated Fuel Seasons Come to Quiet Conclusion," Oxy-Fuel News, March 7, 1994, pp. 1, 8, and 9.
} 
61 million barrels, a level that many industry observers regard as adequate to meet normal winter demand (Figure FE2).

The dramatic stockbuild was accomplished in part by a high volume of waterborne imports into the East Coast and Gulf Coast regions during spring and summer months. Lower demand for propane in European and Asian markets and relatively attractive propane prices in the United States combined to attract supplemental imports of propane to the United States. Other factors attributable to propane's stockbuild were sustained high production levels and lower demand. Moreover, a repeat of the near-record corn harvest and associated crop drying demand was precluded, due in part to heavy flooding in the Midwest during the summer of 1993.

\section{Product Demand}

\section{Motor Gasoline}

Demand for motor gasoline averaged a record 7.5 million barrels per day during 1993, breaking the long-standing record of 7.4 million barrels per day set in 1978 .

Falling crude oil prices, especially in the second half of 1993 , resulted in high refinery runs and high motor gasoline stock

Figure FE2. Propane Stocks-March 31 and September 30, 1989-1993

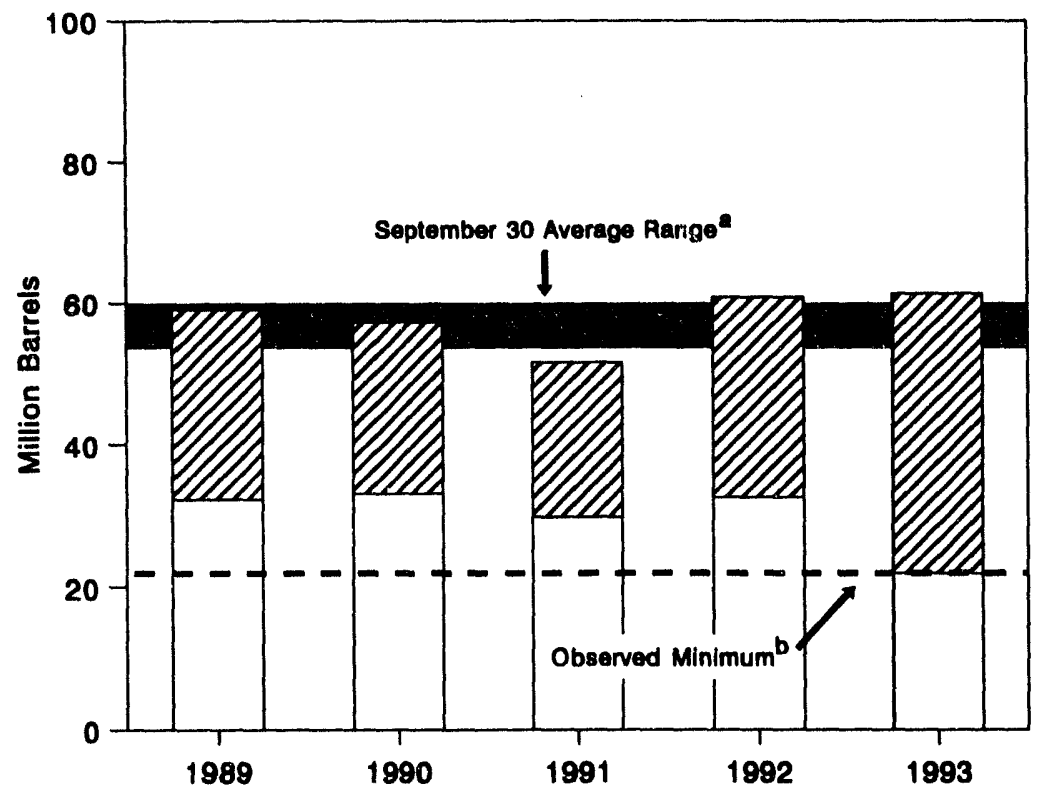

March 31 Build as of Sept. 30

\footnotetext{
${ }^{a}$ Average level and width of average range based on 3 years of monthly data: January 1991 December 1993.

${ }^{b}$ The Observed Mininum for propane stocks is based on final monthly data for the last 36-month period and was 21.8 million barrels, occurring in March 1993.

Source: Energy information Administration (EIA), Petroleum Supply Annual, 1989-1993, DOE/EIA-0340(89/93)/1, Volume 1, Table 2.
}

levels. This in turn led to lower gasoline prices which helped to stimulate gasoline demand.

Imports of motor gasoline have declined in recent years while exports have risen. Net imports (gross imports minus exports) of motor gasoline averaged over 4 percent of product supplied annually between 1986 and 1990, dropping to under 3 percent during 1991 and 1992, and to 1.9 percent in 1993.

Stock patterns during 1993 were influenced by motor gasoline composition changes, crude oil prices, weather, and driving patterns. February 1993 inventories were at a 3-year high, which provided a strong supply for the beginning of the summer driving season. Stocks of motor gasoline fell in August as the low-Reid Vapor Pressure (RVP) ${ }^{8}$ summer gasolines were replaced by new supplies of oxygenated gasolines to meet the winter requirements. August inventories of motor gasoline were the lowest monthly level for both 1992 and 1993, standing at 201 million barrels and 202 million barrels, respectively.

The demand for oxygenates and oxygenated gasoline was slightly less during 1993 than for 1992's initial oxygenated gasoline season. One of the causes of the decline was that the Hartford, Connecticut; Syracuse, New York; Cleveland, Ohio areas, and areas in Alaska where oxygenated fuels were required last year, did not participate in the oxgenated gasoline program this year. Their nonparticipation resulted in a decline of about 145,000 barrels per day of required oxygenated gasoline.

\section{Distillate Fuel Oil}

Adaptation to meet the new requirements of the 1990 CAAA was the major industry event for producers, transporters and suppliers of distillate fuel oil during 1993. Those requirements included distillate standards for fuels designated for on-highway use of a maximum sulfur content of 0.05 percent by weight and a minimum cetane level of 40 . Further, these fuels must also be colorless to clearly designate them for use on-highway. Other diesel or distillate will be dyed blue. The restrictions were designed to combat emissions of sulfur oxides, and to assure the ignition performance of the diesel meets the American

\footnotetext{
${ }^{8}$ Reid Vapor Pressure (RVP). The measure of volatility. The more volatile a gasoline is, the more readily it evaporates and causes vapor
} lock. 
Society for Testing and Materials standards for combustion. The 1990 CAAA standard took effect October 1, 1993, and affects about 46 percent of the total domestic demand for distillate fuel oil, or about 8 percent of total U.S. petroleum demand, according to estimates based on preliminary data from the 1993 Fuel Oil and Kerosene Sales Report.

Because of the difficulties in maintaining separate storage facilities for the on-highway (clear) and off-highway (blue) diesel used in Alaska, the State petitioned the Environmental Protection Agency (EPA) for exemption from the low-sulfur ruling until 1996 for those areas served by the Federal Highway System, and for a permanent exemption for the remainder of the State. The exemption was granted in March 1994. In the interim between October 1, 1993, and the final decision in March 1994 by EPA, the Agency used its enforcement discretion under the terms of the Regulation.

In California, even stricter diesel regulations were set by the California Air Resources Board (CARB). In addition to the 0.05 percent or less sulfur content, aromatic hydrocarbons were limited to 10 percent by volume, which implies a minimum cetane level of 55. Furthermore, all diesel fuel used in California, except that used by railroad engines and marine vessels, must meet the standards. This contrasts to the Federal regulation that permits some government and charitable organization diesel vehicles, and all off-highway agricultural and construction vehicles to use higher sulfur diesel fuel.

Implementation of the low-sulfur diesel rules brought reports of shortages and price spikes in parts of the Midwest, Nevada, and particularly in California. Because of the expense and number of modifications necessary for refiners to produce the CARB standard fuel beyond those modifications necessitated by the Federal mandate, a number of refiners who traditionally marketed diesel in California elected not to do so. Instead, their diesel production was sold wherever the Federal regulations required. Spot prices rose by as much as 30 cents per gallon in parts of California by early October 1993. Truckers refueled across State borders, putting extraordinary stress on terminals in States bordering California. Although reports of shortages were short-lived, a strike called by California truckers brought a 6 week moratorium on regulations in California markets that allowed Federal low-sulfur diesel to be sold for off-highway use. This respite allowed refiners to increase supplies of California-specification fuel, and helped stabilize prices.

Demand for distillate fuel oil of 3.0 million barrels per day was 2 percent higher in 1993 than in 1992. Distillate refinery production during 1993 of 3.1 million barrels per day was 5 percent higher than in 1992. Inventories remained within or above normal ranges throughout the year, with low-sulfur stocks constituting 45 percent of total distillate stocks by the end of the year.
Imports were 184,000 barrels per day during 1993, down 15 percent from the 1992 average of 216,000 barrels per day. This included 41,000 barrels per day of low-sulfur distillate from Canada, Venezuela, and the Virgin Islands. For the third consecutive year, exports of distillate exceeded imports. Exports were 90,000 barrels per day greater than imports in 1993, compared with a 3,000-barrels-per-day difference in 1992.

\section{Residual Fuel Oil}

The steady decline in the demand for residual fuel oil that began in the late 1970's moderated during 1993. The slowdown in the decline in demand was partly the result of falling prices that caused residual fuel oil to be competitive with natural gas at electric utilities.

During 1993, residual fuel oil demand averaged 1.1 million barrels per day, a decline of 1.3 percent from 1992. Dorsestic production, imports, and exports were below comparable 1992 levels, while stocks remained within normal levels for most of the year.

\section{Propane}

Because of an atypical combination of demand factors, specifically high and late crop-drying demand during the fall of 1992, and unseasonably cold weather during the first quarter of 1993, U.S. inventories of propane fell to record low levels by the end of the 1992-1993 heating season (March 31, 1993). With less than 22 million barrels remaining in storage, industry focused on rebuilding stocks to adequate levels by the start of the 1993-1994 heating season. Between March and September 1993, U.S. inventories of propane were replenished at a record pace to more than 61 million barrels, a level that industry observers regarded as adequate to meet normal winter demand. The record stockbuild ' $w$ as the result of sustained high levels of refinery and natural gas plant production, lower demand, particularly petrochemical feedstock demand for propane, and higher waterborne iniports compared with the previous year. Overall demand during 1993 declined by about 3 percent from the 1992 level.

\section{Jet Fuel}

Although total jet fuel demand increased a modest 1 percent during 1993, demand for kerosene-type jet fuel (the largest component of total jet fuel) recorded nearly a 4-percent gain during this same period. The recovery in air travel demand that began in early 1993 was a major factor for the increase.

Contributing to the increase in demand for kerosene-type jet fuel was marketer and end-user blending of kerosene-type jet fuel into heating oil and diesel fuel. Refiners blended kerosene-type jet fuel into those fuels during the winter months to alleviate viscosity, handling, and performance problems associated with cold weather. Refiners also began blending

xiv 
kerosene-type jet fuel with on-highway diesel fuel in order to meet new Federal requirements for lower sulfur fuels.

Another factor for the increase in demand for kerosene-type jet fuel was the October 1, 1993, start of the U.S. military's conversion from naphtha-type jet fuel to kerosene-type jet fuel. The military changeover, which only affected West Coast bases during 1993, is expected to be completed by 1995 .

Despite slightly higher production and import volumes, jet fuel inventories remained below average levels for most of 1993 , particularly during the fourth quarter when the new Federal regulations for lower sulfur fuels took effect.

\section{Crude Oll}

In 1993 U.S. crude oil production reached its lowest level since 1958 as declining world crude oil prices, costly environmental regulations, and extensive foreign oil exploration opportunities contributed to the ongoing decline in U.S. oil reserves. Low crude oil prices throughout 1993 forced companies to temporarily abandon marginally profitable wells, pushing production even lower.

Crude oil production from Alaskan fields declined nearly 8 percent from 1992 as warm-weather oil field problems affected production during the spring, and field and pipeline maintenance projects affected summer production. Unseasonably warm temperatures affected the efficiency of gas-fired turbines, and ongoing work on gas-handling projects at Prudhoe Bay field hindered production during the second and third quarters. As a result, Alaskan crude oil output was at its lowest level in 14 years.

The large 1993 decline in crude oil production occurred despite the long-delayed expansion of production from the Point Arguello field off the coast of California. This field in the Pacific Federal Offshore Continental Shelf (OCS) started producing during the second quarter of 1991 , and initially it produced at lower than expected levels because of problems in transporting the produced oil to refineries. During the end of the second quarter, the field was producing about 60,000 barrels per day. By the end of the third quarter of 1993 , West Coast tankering operations began moving crude oil from the Point Arguello field to nearby petroleum refineries. Consequently, oil

\footnotetext{
${ }^{9}$ As U.S. Oil Output Falls, Reliance on Imports Grows," The Christian Science Monitor, January 29, 1994, p. 2.
}

production increased to 80,000 barrels per day by year-end 1993.

Along with declining domestic crude oil priduction, falling crude oil prices combined with high refiner, runs during much of 1993 resulted in high volumes of imported oil, pushing net petroleum imports' share of the U.S. product supplied to 44 percent during 1993, its highest level since 1977 (Figure FE3). Weak world oil demand and over production by OPEC depressed world oil prices throughout 1993, prompting record levels of foreign purchases of crude oil during June, July, October, and November. Crude oil imports filled the gap created by a nearly 5-percent drop in U.S. crude oil production, averaging a record 6.8 million barrels per day, eclipsing the previous record of 6.6 million barrels per day set in 1977 .

Worldwide sources of crude oil over the past several years have become more diversified, and therefore more secure. From the North Sea to Nigeria, new supplies are coming on stream. This has removed some of the leverage of oil-rich Mideast nations. Further adding to U.S. energy security, three of America's top five sources of foreign oil: Venezuela (2nd), Canada (3rd), and Mexico (4th), are friendly neighbors. Venezuelan crude oil shipments to the U.S. grew by 0.2 million barrels per day, or 22 percent, to 1.0 million barrels per day in 1993, as several companies stepped up purchases of these South American crude oils. In addition, Colombia raised its exports of crude oil by 38 percent, despite problems with guerrillas who repeatedly attacked its export pipeline. Although Saudi Arabia continued to lead the list of foreign petroleum sources for the United
Figure FE3. United States Dependence on Net Petroleum Imports, 1984-1993

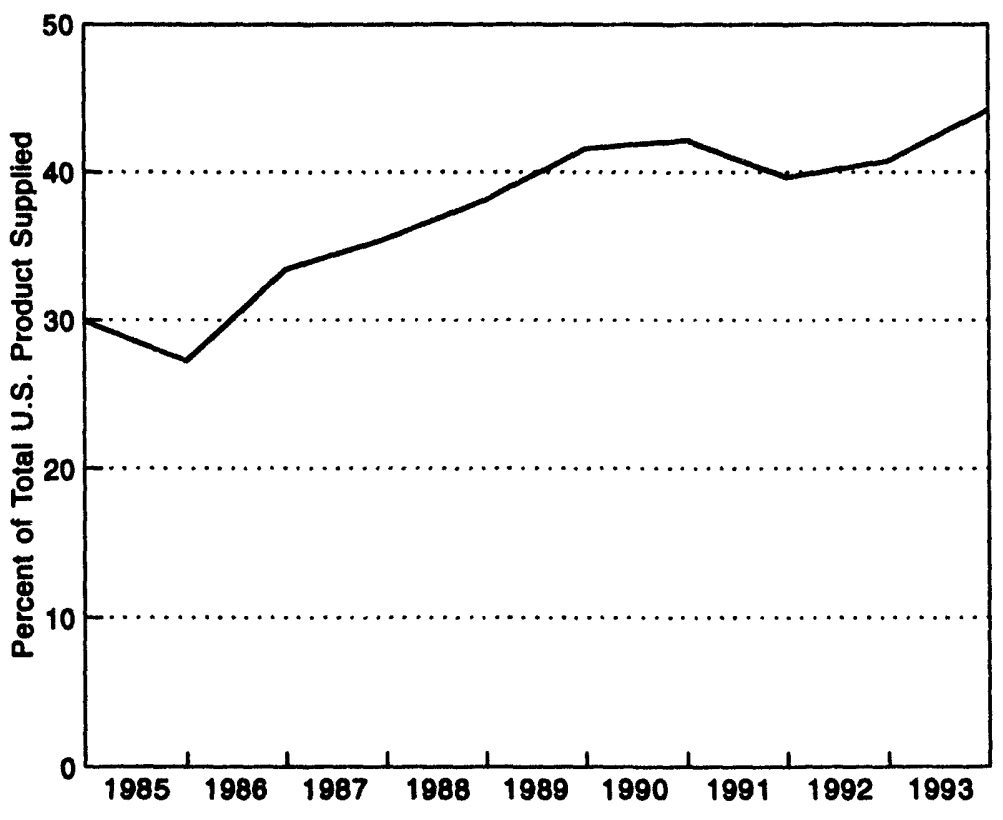

Source: Energy Information Administration, Petroloum Supply Annual 1993, DOE/EIA-0340 (93)/1, Volume 1, Table 3, and predecessor reports. 
States during 1993, its share of the U.S. crude oil import market fell below 20 percent for the first time in 5 years. Conversely, countries supplying crude oil from the Western Hemisphere claimed a greater percentage of total U.S. imports (Table FE1).

During 1993, crude oil imports from Saudi Arabia averaged 1.3 million barrels per day, down 20 percent from a year earlier. Some industry analysts reported that the slump in U.S. purchases of Saudi crude oil was due in part to tying crude oil term contract prices to Alaskan North Slope spot prices at the U.S. Gulf Coast. Indications were that the declining North Slope production, coupled with strong demand for this crude oil on the U.S. West Coast, frequently inflated the prices of North Slope and other crude oils tied to crude oil term contract prices. Compensating for Saudi Arabia's loss of market share of imports to the United States, Kuwait boosted its exports nearly eight-fold as crude oil imports from the emirate eclipsed previous levels between July and December 1993, averaging a record 344,000 barrels per day for 1993.

Despite the gains by Kuwait and Venezuela, the share of total U.S. crude oil imports from OPEC declined to 54 percent from 56 percent a year earlier. Among non-OPEC suppliers, the increased shares from the United Kingdom and Canada contributed to the ongoing decline in OPEC crude oil imports. Continuing a trend observed in recent years, the United States imported a smaller proportion of their crude oil supply from countries belonging to the Arab OPEC (Figure FE4).

Falling crude oil prices throughout 1993, combined with a shift in emphasis by major oil companies toward oil exploration in less mature, lower cost producing regions overseas, resulted in oil well completions in the United States dropping for the ninth straight year. Since most of the world's largest undeveloped crude oil reserves are located outside of the United States, many companies, continuing a recent trend, choose to boost spending on exploration outside of the United States. ${ }^{10}$

Although drilling activity did not experience the decline normally associated with falling crude oil prices, its relatively low level in 1993 was a contributing factor for the continued drop in U.S. crude oil production. Furthermore, due in part to tax incentives for unconventional gas drilling, for the first time in U.S. history, oil well completions fell below the number of wells drilled for gas. Oil well completions totaled 7,994 by the close of 1993, 6 percent below last year's level.
Ten Leading Sources of Crude Oil Imports, 1992 and 1993 (Thousand Barrels per Day, Except Where Noted)

\begin{tabular}{clrrrr}
\hline Rank & & Country & 1992 & 1993 & \% Difference \\
\hline 1 & Saudi Arabia & 1,597 & 1,282 & -19.7 \\
2 & Venezuela & 826 & 1,010 & 22.3 \\
3 & Canada & 797 & 900 & 12.9 \\
4 & Mexico & 787 & 863 & 9.7 \\
5 & Nigeria & 665 & 722 & 8.6 \\
6 & Kuwait & 39 & 344 & 782.1 \\
7 & Angola & 336 & 336 & 0.0 \\
8 & United Kingdom & 200 & 312 & 56.0 \\
9 & Gabon & 123 & 151 & 22.8 \\
10 & Colombia & 102 & 141 & 38.2 \\
& Total & 5,472 & 6,061 & 10.8 \\
& Percent of Total Imports & 90.0 & 89.3 &.- \\
& Other Countries & 611 & 726 & 18.8 \\
& Total U.S. Imports & 6,083 & 6,787 & 11.6 \\
\hline
\end{tabular}

Note: Totals may not equal sum of components due to independent rounding.

Scurce: Energy Information Administration, Petroleum Supply Annual 1993, DOE/EIA$0340(93) / 1$ Volume 1, Table 21, and predecessor reports.

For much of 1993, low crude oil prices and strong petroleum demand encouraged brisk refining activity, prompting U.S. refiners to maintain higher crude oil inventories than had been observed in recent years. The substantial stock overhang was largely a result of improved refining margins, attributed to low world oil prices brought on by overproduction by OPEC. As the world crude oil price dipped below $\$ 16$ per barrel during June, refiners padded inventories from record imports, lifting stocks, for the first time since 1990, above their average range in June and July. Crude oil inventories ended the year at 335 million barrels, their highest level since 1989.

Strategic Petroleum Reserve (SPR) inventories rose in 1993. During April 1993, imports to the SPR reached their highest level in nearly 8 years, causing the largest month-to-month stock increase for that period since 1984. By the end of 1993, the SPR reached 587 million barrels, more than 12 million barrels higher than year-end 1992.

\section{Refinery Activity}

As of January 1, 1994, operable crude oil distillation capacity was about 15.0 million barrels per day, less than 1 percent below the level a year earlier. There were 179 operable refineries on January 1,1994, compared with 187 a year earlier. The result of the net loss of eight refineries, combined with some capacity upgrades during the year, was a decline of about 87,000 barrels per day capacity. This is the second consecutive year that crude oil distillation capacity declined, continuing the trend to shut down uneconomical refineries.

\footnotetext{
${ }^{10}$ "Five U.S. Majors Expect to Boost Spending on Oil Exploration in 1994," Oil Buyers' Guide, November 15, 1993, p. 1.
} 
Figure FE4. Shares of the U.S. Crude OII Import Market, 1992-1993

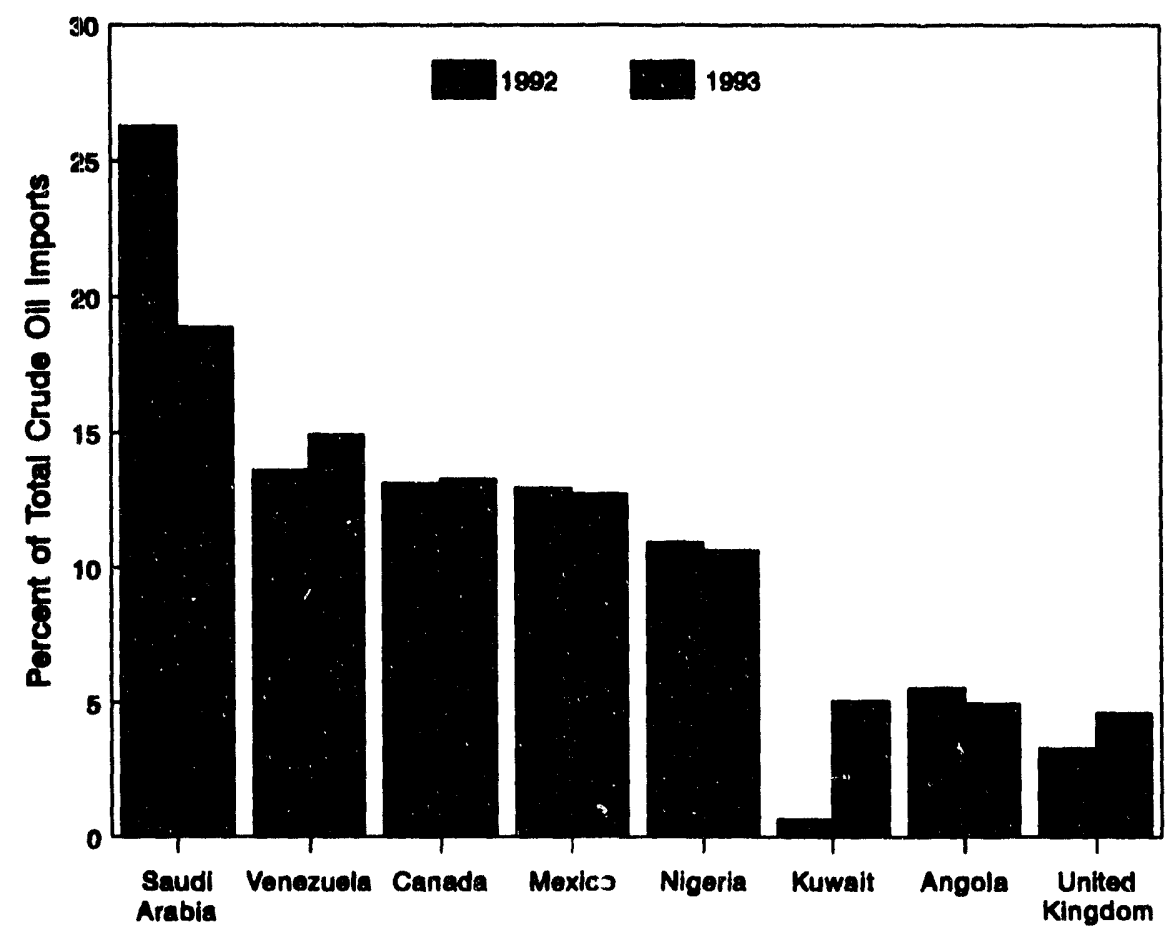

Source: Energy Information Administration, Petroleum Supply Annual 1993, DOE/EIA-0340(93)/1, Volume 1, Table 21, and predecessor reports.

Ten U.S. refineries with total crude oil capacity of about 166,000 barrels per day were shut down in 1993 , while 22,000 barrels per day were gained through two refinery reactivations. The largest single refinery to cease operations during 1993 was Marathon's 50,000-barrel-a-day Indianapolis, Indiana refinery. In addition, Coastal Refining and Marketing ceased refinery operations at their Augusta and Wichita, Kansas, facilities and operated both as bulk terminals. A third Coastal refinery at El Dorado, Kansas, is now operating as an asphalt plant. See a complete list of refinery shutdowns and reactivations beginning on page 121 of this publication.

Cyril Petrochemical in Cyril, Oklahoma, reactivated their 7,400-barrel-per-day refinery in late 1993, and Petroleum Fuel and Terminal (formerly Edgington Oil) in California reactivated their 15,000-barrel-a-day refinery. In addition, El Paso Refining in El Paso, Texas, which was shut down in 1992, was partially reactivated by Chevron. Chevron merged this refinery with its own El Paso facility into one operation. Concurrently, modernization of existing refineries through the upgrading of downstream intermediate processes continued throughout the industry, as refiners met the challenges of deteriorating crude oil quality, stringent requirements for oxygenated and reformulated gasoline, and reductions in allowable sulfur content of diesel fuels.

From January 1, 1993, to January 1, 1994, distillate hydrotreating (desulfurization) capacity increased 29 percent; other residual hydrotreating capacity increased by 11 percent (Table FE2). While the capacities of all cracking processes combined experienced a slight decline from the previous year, coking capacity actually increased 4 percent. Catalytic hydrocracking capacity and catalytic cracking capacity experienced declines of 1.5 percent and 0.1 percent, respectively from the previous year. Capacities for the three octane enhancement processes combined experienced a 3percent increase by January 1994, primarily due to a 4-percent increase in catalytic reforming capacity. In addition, hydrogen capacity, essential to the production of reformulated motor gasoline, increased 10 percent during 1993. See capacity tables beginning on page 81 of this publication.

During 1993, total crude oil received at refineries averaged 14.0 million barrels per day, 2 percent above the 1992 level. Receipts of foreign crude oil averaged 7.0 million barrels per day in 1993, a 10-percent increase from the previous year. Domestic receipts declined to 7.1 million barrels per day, 4 percent lower than the 1992 level. Domestic receipts constituted half of the total crude oil received at refineries: about 50 percent of the total in 1993, compared with 54 percent in 1992. Alaskan receipts at U.S. refineries dropped 6 percent from the 1992 level; however, Alaskan receipts still constituted about 21 percent of all domestic crude oil received at refineries. See new Table 45 in refinery capacity section of this publication for a summary of inputs, capacities, and utilization rates of downstream processing units. 
Table FE2. Number and Operable Capacity of Select Processing Units at U.S. Petroleum Refineries, 1989-1994

\begin{tabular}{|c|c|c|c|c|c|c|c|c|}
\hline \multirow{3}{*}{$\begin{array}{c}\text { As of } \\
\text { January } 1 \text { of } \\
\text { Year }\end{array}$} & \multirow[b]{3}{*}{ Number } & \multirow{3}{*}{$\begin{array}{c}\text { Crude } \\
\text { Distillation } \\
\text { (thousand } \\
\text { barrels per } \\
\text { calendar day) }\end{array}$} & \multirow[b]{2}{*}{ Cracking } & \multirow[b]{2}{*}{$\begin{array}{c}\text { Octane } \\
\text { Enhancements }\end{array}$} & \multicolumn{4}{|c|}{ Desulfurization } \\
\hline & & & & & Diatillate & $\begin{array}{l}\text { Naphthal } \\
\text { Roformor } \\
\text { Feads }\end{array}$ & $\begin{array}{c}\text { Hoayy Gas } \\
\text { Oil }\end{array}$ & $\begin{array}{l}\text { Other } \\
\text { Residual }\end{array}$ \\
\hline & & & \multicolumn{6}{|c|}{ (Thoueand Barrole per Stream Day) } \\
\hline 1989 & 204 & 15,655 & 8,961 & 5,395 & 2,746 & 3,993 & 1,685 & 1,016 \\
\hline 1990 & 205 & 15,572 & 9,145 & 5,382 & 2,780 & 4,047 & 1,749 & 961 \\
\hline 1991 & 202 & 15,676 & 9,328 & 5,493 & 2,866 & 4,072 & 1,833 & 906 \\
\hline 1992 & 199 & 15,696 & 9,351 & 5,496 & 2,784 & 4,090 & 1,867 & 904 \\
\hline 1993 & 187 & 15,121 & 9,263 & 5,310 & 2,849 & 4,034 & 1,891 & 904 \\
\hline 1994 & 179 & 15,034 & 9,259 & 5,459 & 3,662 & 4,067 & 1,888 & 999 \\
\hline
\end{tabular}

Includes catalytic cracking, hydrocracking, and thermal cracking.

'Includes catalytic reforming, isomerization, and alkylation.

Source: Energy Information Administration (EIA), Petroleum Supply Annual, DOE/EIA-0340(88-93)/1 Volume 1, Table 38 and 39.

\section{Prices}

Lower worldwide demand for petroleum, abundant worldwide inventories, increasing global energy efficiency, and overproduction by OPEC and non-OPEC countries caused world oil prices to fall to their lowest level since the latter part of 1988.

World oil prices followed a modest upward trend during the early months of 1993 , reaching a yearly high of $\$ 17.60$ per barrel by early March. By May, world oil prices began a steady decline that continued through the end of the year. By the end of December 1993, world oil prices declined to $\$ 12.44$ per barrel, about $\$ 4.00$ per barrel below the year-end 1992 level.

The refiner acquisition cost of crude oil from all sources drifted upward from January and reached a yearly high of $\$ 18.40$ per barrel by the end of April 1993. However, following the drop in world oil prices, the refiner acquisition cost began a steady decline and by December 1993, equalled $\$ 12.51$ per barrel, a drop of nearly $\$ 5.00$ per barrel compared with December 1992 (Table FE3).

U.S. annual motor gasoline prices during 1993 averaged about 2 cents per gallon below comparable 1992 average prices, as falling crude oil prices more than offset the 4.3-cents-per-gallon motor fuel tax.

Gasoline inventories remained unseasonably high during the first quarter of 1993, which contributed to lower gasoline prices during this period. Beginning in April 1993, gasoline prices began to rise slightly, and by June 1993, average retail motor gasoline prices reached a peak driving season high of $\$ 1.19$ per gallon. Retail gasoline prices trended downward until October 1993, when imposition of the motor fuel tax caused prices to increase to $\$ 1.19$ per gallon. However, falling crude oil prices during the fourth quarter 1993 caused motor gasoline prices to fall to $\$ 1.14$ per gallon by the end of December 1993 , the lowest level of the year.

On average, residential heating oil prices for 1993 were nearly 3 cents per gallon less than comparable 1992 average prices. Increased heating oil demand due to colder temperatures pushed heating oil prices up to a yearly high of 95 cents per gallon by the end of the first quarter of 1993 . However, relatively low heating oil demand during the second quarter of 1993 caused prices to fall and by August 1993 were at a yearly low of 84 cents per gallon. As of the end of December 1993, residential fuel oil prices stood at about 87 cents per gallon, about 8 cents per gallon lower than the December 1992 price.

Table FE3. Year-End U.S. Petroloum Prices"

\begin{tabular}{|c|c|c|c|c|}
\hline & 1990 & 1891 & 1992 & 1993 \\
\hline & \multicolumn{4}{|c|}{ Dollars per Barrel } \\
\hline \multicolumn{5}{|l|}{$\begin{array}{l}\text { Refiner Acquisition Cost } \\
\text { of Crude Oil }\end{array}$} \\
\hline Domestic & 26.46 & 17.84 & 17.85 & 12.45 \\
\hline Imported & 25.56 & 17.17 & 16.94 & 12.56 \\
\hline \multirow[t]{2}{*}{ Composite } & 26.09 & 17.56 & 17.43 & 12.51 \\
\hline & \multicolumn{4}{|c|}{ Cents per Gallon } \\
\hline $\begin{array}{l}\text { Motor Gasoline } \\
\text { All Types, Retail }\end{array}$ & 141.0 & 118.2 & 120.1 & $\$ 13.6$ \\
\hline No. 2 Heating Oll Retail ${ }^{b}$ & 119.7 & 95.9 & 94.6 & 86.7 \\
\hline
\end{tabular}

Price as of December 31 of the year indicated, except for Refiner Acquisition Cost, which is based on an average December price.

${ }^{b}$ Residential heating oil prices exclude taxes.

Source: Energy Information Administration (EIA), Petroleum Marketing Annual 1992, DOE/EIA-0487(92), Tables 1 and 16; Petroleum Marketing Monthly, March 1994, DOE/EIA-0380 (93/03), Tables 1 and 16; Motor Gasoline Prices; Bureau of Labor Statistics. 


\section{Summary Statistics}
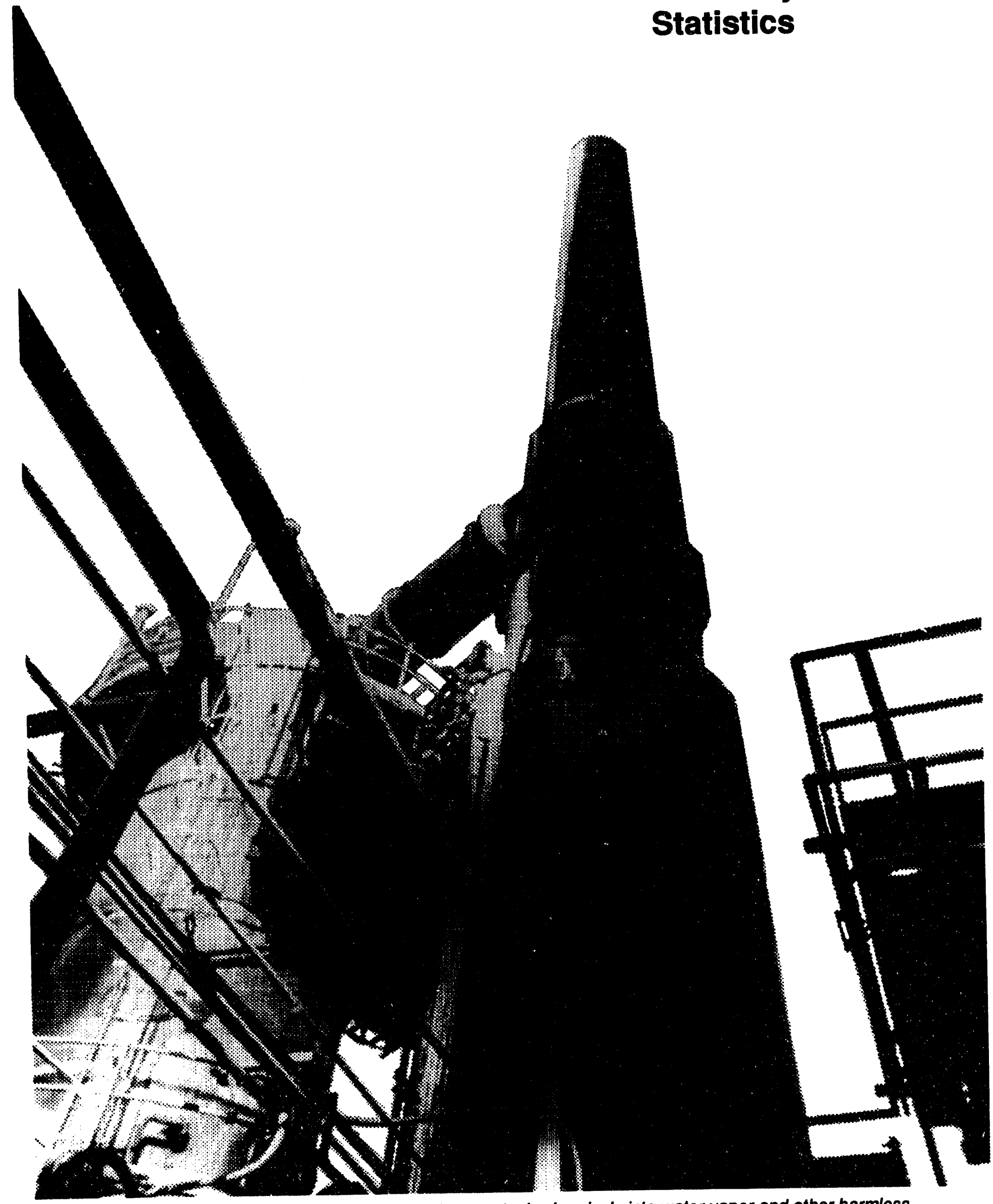

Incinerators such as this one at a chemical installation turn toxic chemicals into water vapor and other harmless elements. 
Table S1. Crude Oil and Petroleum Products Overview, 1981 - Present (Thousand Barrels per Day, Except Where Noted)

\begin{tabular}{|c|c|c|c|c|c|c|c|c|}
\hline \multirow{2}{*}{\multicolumn{2}{|c|}{ YearMonth }} & \multicolumn{3}{|c|}{ Fiald Production } & \multicolumn{2}{|c|}{ Stock Change" } & \multirow[b]{2}{*}{$\begin{array}{l}\text { Petroloum } \\
\text { Products } \\
\text { Supplled }\end{array}$} & \multirow{2}{*}{\begin{tabular}{|c}
$\begin{array}{c}\text { Ending } \\
\text { Stooks } \\
\text { (Million Bamela) }\end{array}$ \\
Crude Oil and \\
Petroleum \\
Products
\end{tabular}} \\
\hline & & $\begin{array}{c}\text { Total } \\
\text { Domestlec }\end{array}$ & $\begin{array}{c}\text { Crude } \\
\text { OII }\end{array}$ & $\begin{array}{l}\text { Natural } \\
\text { Cas Plant } \\
\text { Llquids }\end{array}$ & $\begin{array}{c}\text { Cruge } \\
\text { oll }^{-1}\end{array}$ & $\begin{array}{l}\text { Petrolaum } \\
\text { Products }\end{array}$ & & \\
\hline $\begin{array}{l}1981 \\
1982 \\
1983 \\
1984 \\
1985 \\
1988 \\
1987 \\
1988 \\
1989 \\
1990\end{array}$ & $\begin{array}{l}\text { Average ................ } \\
\text { Average ............... } \\
\text { Average ................ } \\
\text { Average ............... } \\
\text { Average ................ } \\
\text { Average } \\
\text { Average }\end{array}$ & $\begin{array}{r}10,230 \\
10,252 \\
10,299 \\
10,554 \\
10,636 \\
10,289 \\
10,008 \\
9,818 \\
9,219 \\
8,994\end{array}$ & $\begin{array}{l}\mathbf{8 , 5 7 2} \\
\mathbf{8 , 6 4 9} \\
\mathbf{8 , 6 8 8} \\
\mathbf{8 , 8 7 9} \\
8,971 \\
8,680 \\
\mathbf{8 , 3 4 0} \\
\mathbf{8 , 1 4 0} \\
\mathbf{7 , 6 1 3} \\
\mathbf{7 , 3 5 5}\end{array}$ & $\begin{array}{l}1,609 \\
1,550 \\
1,559 \\
1,630 \\
1,609 \\
1,551 \\
1,595 \\
1,625 \\
1,548 \\
1,659\end{array}$ & $\begin{array}{r}9_{290} \\
138 \\
9214 \\
199 \\
50 \\
78 \\
128 \\
1 \\
86 \\
-35\end{array}$ & $\begin{array}{r}9-130 \\
-283 \\
0-234 \\
81 \\
-153 \\
124 \\
-87 \\
-29 \\
-129 \\
142\end{array}$ & $\begin{array}{l}16,058 \\
15,296 \\
15,231 \\
15,726 \\
15,728 \\
16,281 \\
16,685 \\
17,283 \\
17,325 \\
16,988\end{array}$ & $\begin{array}{r}1,484 \\
91,430 \\
1,454 \\
1,556 \\
1,519 \\
1,598 \\
1,607 \\
1,597 \\
1,581 \\
1,621\end{array}$ \\
\hline \multicolumn{2}{|c|}{ 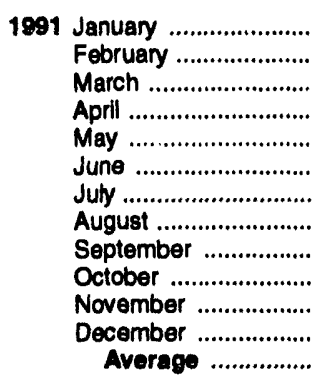 } & $\begin{array}{l}9,255 \\
9,424 \\
9,301 \\
9,262 \\
9,157 \\
9,032 \\
9,056 \\
9,027 \\
9,088 \\
9,212 \\
9,129 \\
9,089 \\
9,168\end{array}$ & $\begin{array}{l}7,500 \\
7,637 \\
7,546 \\
7,509 \\
7,409 \\
7,320 \\
7,347 \\
7,316 \\
7,368 \\
7,437 \\
7,328 \\
7,299 \\
7,417\end{array}$ & $\begin{array}{l}1,647 \\
1,695 \\
1,683 \\
1,665 \\
1,657 \\
1,627 \\
1,622 \\
1,627 \\
1,623 \\
1,686 \\
1,697 \\
1,686 \\
1,659\end{array}$ & $\begin{array}{r}-71 \\
231 \\
-239 \\
50 \\
566 \\
-299 \\
-153 \\
103 \\
-156 \\
51 \\
43 \\
-611 \\
-42\end{array}$ & $\begin{array}{r}-1,027 \\
-704 \\
-268 \\
628 \\
888 \\
546 \\
199 \\
316 \\
653 \\
-659 \\
62 \\
-365 \\
32\end{array}$ & $\begin{array}{l}16,893 \\
16,339 \\
16,212 \\
16,139 \\
16,189 \\
16,878 \\
16,971 \\
17,183 \\
16,848 \\
16,996 \\
16,730 \\
17,145 \\
16,714\end{array}$ & $\begin{array}{r}1,587 \\
1,573 \\
1,558 \\
1,578 \\
1,626 \\
1,634 \\
1,635 \\
1,648 \\
1,663 \\
1,644 \\
1,647 \\
1,617 \\
\end{array}$ \\
\hline \multicolumn{2}{|c|}{ 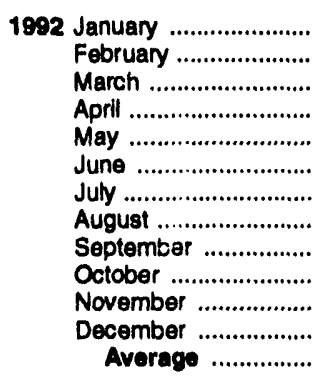 } & $\begin{array}{l}9,176 \\
9,175 \\
9,123 \\
9,072 \\
8,949 \\
8,968 \\
8,961 \\
8,678 \\
8,843 \\
9,025 \\
8,975 \\
9,019 \\
8,996\end{array}$ & $\begin{array}{l}7,361 \\
7,389 \\
7,348 \\
7,293 \\
7,169 \\
7,167 \\
7,131 \\
6,922 \\
7,030 \\
7,126 \\
7,024 \\
7,103 \\
7,171\end{array}$ & $\begin{array}{l}1,688 \\
1,696 \\
1,694 \\
1,693 \\
1,695 \\
1,701 \\
1,683 \\
1,638 \\
1,660 \\
1,722 \\
1,754 \\
1,744 \\
1,697\end{array}$ & $\begin{array}{r}540 \\
171 \\
-250 \\
315 \\
-144 \\
-581 \\
244 \\
-124 \\
-160 \\
411 \\
-227 \\
-212 \\
-1\end{array}$ & $\begin{array}{r}-757 \\
-951 \\
-291 \\
92 \\
770 \\
6 C \\
290 \\
161 \\
653 \\
-258 \\
77 \\
-1,203 \\
-68\end{array}$ & $\begin{array}{l}17,012 \\
16,893 \\
16,825 \\
16,764 \\
16,485 \\
16,978 \\
17,143 \\
16,929 \\
16,876 \\
17,448 \\
17,091 \\
17,928 \\
17,033\end{array}$ & $\begin{array}{r}1,610 \\
1,588 \\
1,571 \\
1,583 \\
1,602 \\
1,603 \\
1,620 \\
1,621 \\
1,636 \\
1,640 \\
1,636 \\
91,592 \\
-\end{array}$ \\
\hline \multicolumn{2}{|c|}{ 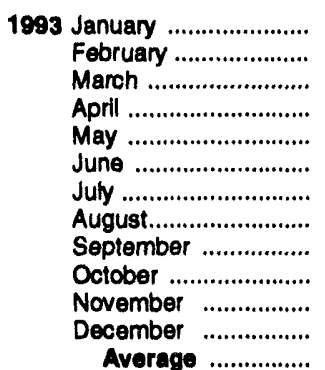 } & $\begin{array}{l}9,254 \\
8,907 \\
8,987 \\
8,897 \\
8,800 \\
8,747 \\
8,657 \\
8,720 \\
8,652 \\
8,893 \\
8,847 \\
8,668 \\
8,836\end{array}$ & $\begin{array}{l}6,961 \\
6,943 \\
6,974 \\
6,881 \\
6,847 \\
6,795 \\
6,688 \\
6,758 \\
6,712 \\
6,839 \\
6,912 \\
6,858 \\
6,847\end{array}$ & $\begin{array}{l}1,737 \\
1,777 \\
1,793 \\
1,802 \\
1,732 \\
1,753 \\
1,741 \\
1,747 \\
1,732 \\
1,768 \\
1,670 \\
1,579 \\
1,738\end{array}$ & $\begin{array}{r}295 \\
219 \\
212 \\
523 \\
147 \\
2 \\
6 \\
-505 \\
-439 \\
328 \\
251 \\
-53 \\
81\end{array}$ & $\begin{array}{r}9560 \\
-796 \\
-602 \\
356 \\
915 \\
573 \\
497 \\
299 \\
86 \\
403 \\
-320 \\
-1,199 \\
70\end{array}$ & $\begin{array}{l}16,173 \\
17,334 \\
17,575 \\
16,781 \\
16,508 \\
17,096 \\
17,357 \\
17,332 \\
17,650 \\
17,323 \\
17,780 \\
17,953 \\
17,237\end{array}$ & $\begin{array}{r}1,618 \\
1,602 \\
1,590 \\
1,617 \\
1,650 \\
1,667 \\
1,682 \\
1,676 \\
1,665 \\
1,688 \\
1,686 \\
1,647 \\
-\end{array}$ \\
\hline
\end{tabular}

a negative number indicates a decrease in stocks and a positive number indicates an increase.

b Stocks are totals as of end of period.

c Includes crude oll, natural gas plant llquids, and other llquids. Beginning in 1993, fuel ethanol blended into finished motor gasoline and oxygenate production from merchant MTBE plants are also included.

Includes stocks located in the Strategic Petroleum Reserve.

- Includes crude oll for storage in the Strategic Petroleum Reserve.

1 Net Imports equal Imports minus Exports.

9 In January 1981 and 1983 , numerous respondents were added to surveys affecting stocks reported and stock change calculations. Stock changes are calculated using new basis stock levels. Bulk terminal and plpeline stocks of oxygenates were added beginning in January 1993 . See Summary Statistics Explanatory Note 4.

Footnotes continued on following page. 
Table S1. Crude Oll and Petroleum Products Overview, 1981 - Present (Continued) (Thousand Barrels per Day, Except Where Noted)

\begin{tabular}{|c|c|c|c|c|c|c|c|}
\hline \multirow[b]{2}{*}{ Yoarmionth } & \multicolumn{3}{|c|}{ Imports } & \multicolumn{3}{|c|}{ Exports } & \multirow[b]{2}{*}{$\begin{array}{c}\text { Net } \\
\text { Importe? }\end{array}$} \\
\hline & Total & Crude & $\begin{array}{l}\text { Petroleum } \\
\text { Producte }\end{array}$ & Total & $\begin{array}{c}\text { Crud } \\
\text { Oll }\end{array}$ & $\begin{array}{l}\text { Petroloum } \\
\text { Products }\end{array}$ & \\
\hline 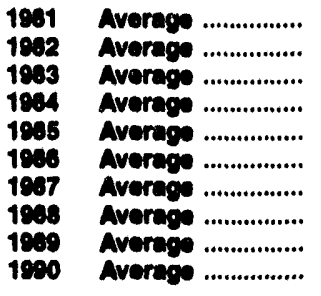 & $\begin{array}{l}5,996 \\
5,113 \\
5,051 \\
5,437 \\
5,007 \\
6,201 \\
6,078 \\
7,402 \\
8,031 \\
8,018\end{array}$ & $\begin{array}{l}4,390 \\
3,486 \\
3,320 \\
3,420 \\
3,201 \\
4,178 \\
4,674 \\
5,107 \\
5,843 \\
8,004\end{array}$ & $\begin{array}{l}1,690 \\
1,625 \\
1,722 \\
2,011 \\
1,806 \\
2,045 \\
2,004 \\
2,205 \\
2,217 \\
2,123\end{array}$ & $\begin{array}{l}595 \\
815 \\
739 \\
720 \\
781 \\
785 \\
784 \\
815 \\
850 \\
857\end{array}$ & $\begin{array}{l}228 \\
283 \\
164 \\
181 \\
204 \\
154 \\
151 \\
155 \\
142 \\
100\end{array}$ & $\begin{array}{l}387 \\
579 \\
675 \\
641 \\
577 \\
681 \\
613 \\
601 \\
717 \\
748\end{array}$ & $\begin{array}{l}5,401 \\
4,298 \\
4,312 \\
4,715 \\
4,200 \\
5,439 \\
5,914 \\
6,587 \\
7,202 \\
7,161\end{array}$ \\
\hline 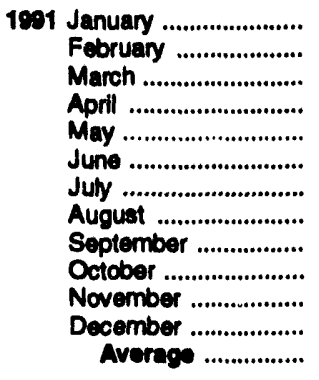 & $\begin{array}{l}7,103 \\
6,866 \\
6,646 \\
7,418 \\
8,518 \\
8,246 \\
7,755 \\
8,670 \\
7,826 \\
7,467 \\
7,615 \\
7,337 \\
7,627\end{array}$ & $\begin{array}{l}\mathbf{5 , 2 9 6} \\
\mathbf{5 , 4 8 5} \\
\mathbf{5 , 1 6 6} \\
\mathbf{5 , 6 2 9} \\
\mathbf{6 , 3 6 3} \\
\mathbf{6 , 3 3 4} \\
\mathbf{5 , 9 5 5} \\
\mathbf{6 , 6 4 5} \\
\mathbf{5 , 8 1 2} \\
\mathbf{5 , 6 8 3} \\
\mathbf{5 , 6 2 8} \\
\mathbf{5 , 5 6 5} \\
\mathbf{5 , 7 0 2}\end{array}$ & $\begin{array}{l}1,808 \\
1,380 \\
1,480 \\
1,888 \\
2,165 \\
1,911 \\
1,801 \\
2,025 \\
2,015 \\
1,784 \\
2,087 \\
1,772 \\
1,844\end{array}$ & $\begin{array}{r}1,199 \\
1,441 \\
944 \\
737 \\
1,149 \\
921 \\
903 \\
837 \\
785 \\
918 \\
926 \\
1,213 \\
1,001\end{array}$ & $\begin{array}{r}50 \\
152 \\
137 \\
162 \\
165 \\
78 \\
139 \\
55 \\
109 \\
92 \\
126 \\
133 \\
118\end{array}$ & $\begin{array}{r}1,149 \\
1,288 \\
807 \\
575 \\
984 \\
843 \\
824 \\
783 \\
676 \\
826 \\
800 \\
1,081 \\
886\end{array}$ & $\begin{array}{l}5,904 \\
5,424 \\
5,702 \\
6,690 \\
7,369 \\
7,323 \\
6,793 \\
7,832 \\
7,042 \\
6,550 \\
6,690 \\
6,124 \\
6,628\end{array}$ \\
\hline 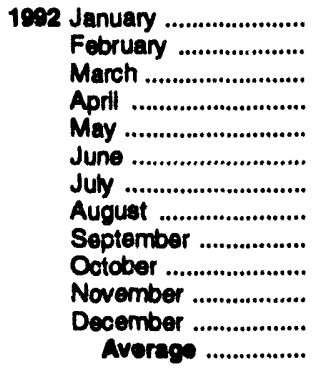 & $\begin{array}{l}7,712 \\
6,827 \\
7,068 \\
8,092 \\
7,823 \\
7,946 \\
8,479 \\
8,260 \\
8,178 \\
8,505 \\
7,872 \\
7,839 \\
7,606\end{array}$ & $\begin{array}{l}5,956 \\
5,079 \\
5,321 \\
6,127 \\
6,060 \\
6.171 \\
6,796 \\
6,457 \\
6,218 \\
6,696 \\
6,121 \\
5,937 \\
6,003\end{array}$ & $\begin{array}{l}1,756 \\
1,748 \\
1,747 \\
1,966 \\
1,763 \\
1,775 \\
1,683 \\
1,803 \\
1,960 \\
1,810 \\
1,751 \\
1,901 \\
1,805\end{array}$ & $\begin{array}{r}1,144 \\
632 \\
912 \\
937 \\
885 \\
957 \\
929 \\
789 \\
848 \\
902 \\
995 \\
1,237 \\
950\end{array}$ & $\begin{array}{r}118 \\
22 \\
105 \\
23 \\
23 \\
106 \\
107 \\
53 \\
133 \\
68 \\
106 \\
111 \\
107 \\
8\end{array}$ & $\begin{array}{r}1,026 \\
829 \\
807 \\
914 \\
779 \\
850 \\
876 \\
657 \\
780 \\
796 \\
885 \\
1,130 \\
8081\end{array}$ & $\begin{array}{l}6,568 \\
5,975 \\
6,156 \\
7,155 \\
6,939 \\
6,989 \\
7,550 \\
7,470 \\
7,330 \\
7,603 \\
6,877 \\
6,602 \\
6,938\end{array}$ \\
\hline 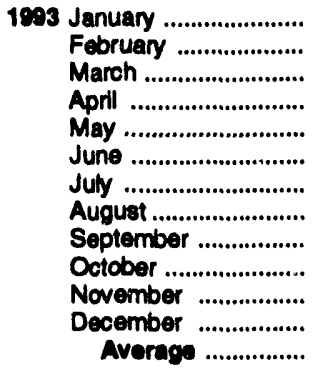 & $\begin{array}{l}8,004 \\
7,948 \\
8,285 \\
8,768 \\
8,663 \\
8,805 \\
9,219 \\
8,429 \\
8,531 \\
9,197 \\
8,903 \\
8,645 \\
8,620\end{array}$ & $\begin{array}{l}6,292 \\
6,156 \\
6,488 \\
6,928 \\
6,809 \\
7,201 \\
7,289 \\
6,641 \\
6,581 \\
7,181 \\
6,997 \\
6,838 \\
6,787\end{array}$ & $\begin{array}{l}1,712 \\
1,792 \\
1,797 \\
1,840 \\
1,854 \\
1,604 \\
1,930 \\
1,789 \\
1,950 \\
2,015 \\
1,906 \\
1,807 \\
1,833\end{array}$ & $\begin{array}{r}1,135 \\
1,033 \\
970 \\
1,067 \\
1,082 \\
900 \\
1,001 \\
829 \\
902 \\
881 \\
980 \\
1,250 \\
1,003\end{array}$ & $\begin{array}{r}129 \\
166 \\
139 \\
73 \\
112 \\
150 \\
62 \\
55 \\
107 \\
62 \\
67 \\
63 \\
98\end{array}$ & $\begin{array}{r}1,006 \\
867 \\
831 \\
994 \\
970 \\
750 \\
938 \\
774 \\
795 \\
819 \\
913 \\
1,188 \\
804\end{array}$ & $\begin{array}{l}6,869 \\
6,915 \\
7,315 \\
7,701 \\
7,581 \\
7,905 \\
8,218 \\
7,600 \\
7,629 \\
8,316 \\
7,923 \\
7,394 \\
7,618\end{array}$ \\
\hline
\end{tabular}

Footnotes continued.

Notes: - Crude oll includes lease condensate. - Geographic coverage is the 50 States and the District of Columbia. - Totals may not equal sum of components due to independent rounding.

Source: See Summary Statistics Table and Figure Sources. 
Figure S1. Petroleum Overvlew, 1981 - Present

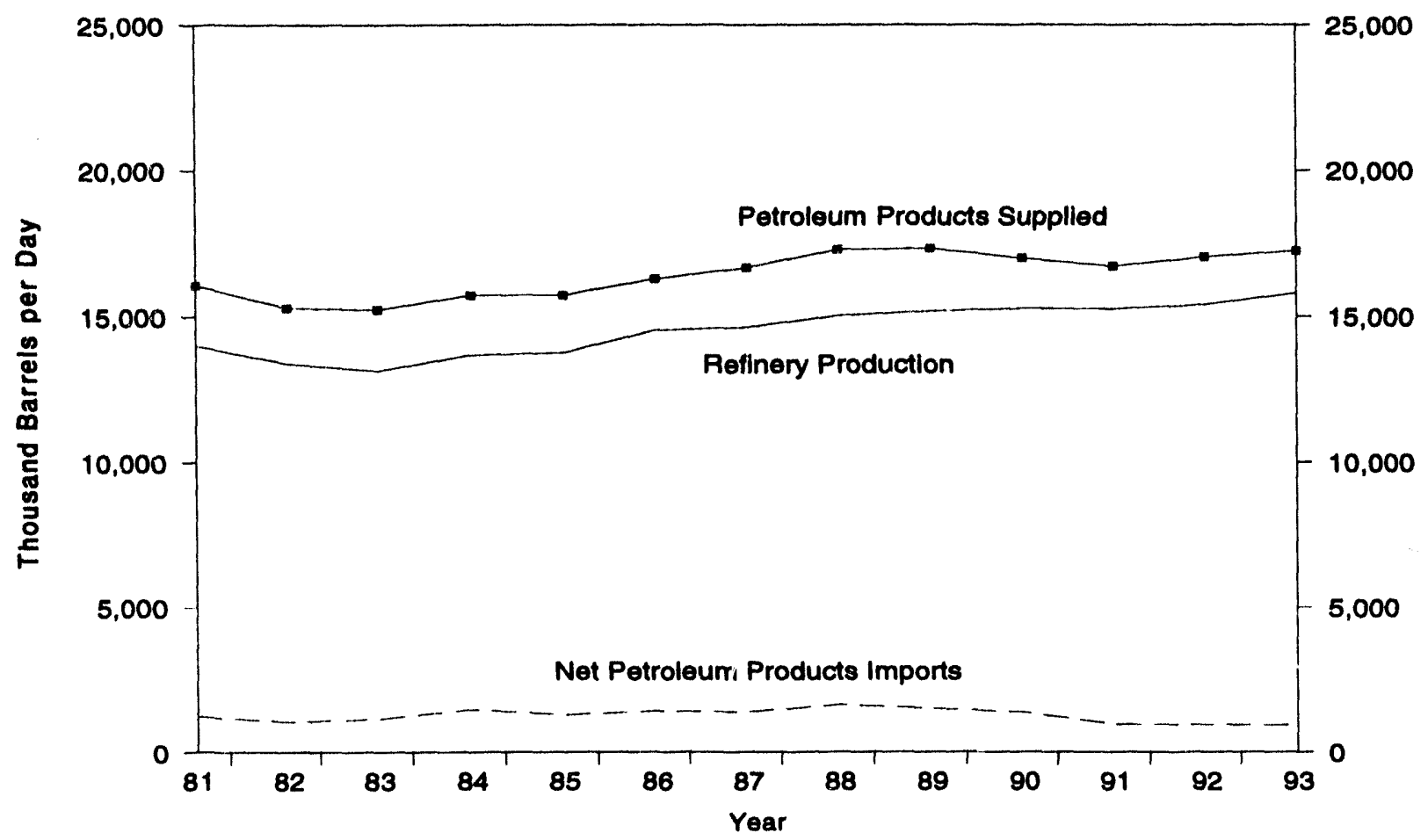

Source: Energy Information Administration. Petroleum Supply Annual, Table S1. See Summary Statistics Table and Figure Sources.

Figure S2. Petroleum Products Supplied, 1981 - Present

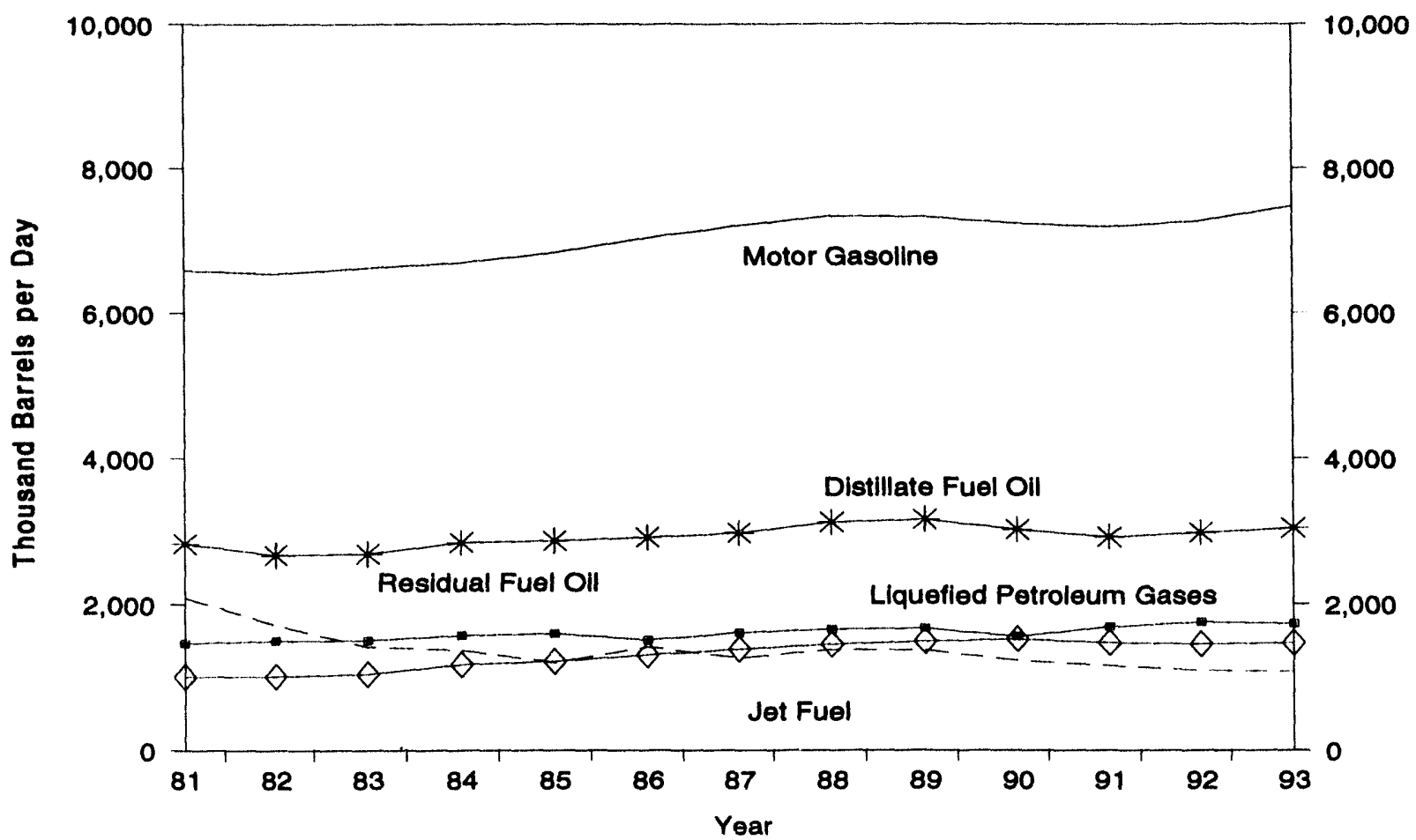

Source: Energy Information Administration, Petroleum Supply Annual, Tables S4 - S8. See Summary Statistics Table and Figure Sources. 
Figure S3. Crude OII Supply and Dlsposition, 1981 - Present

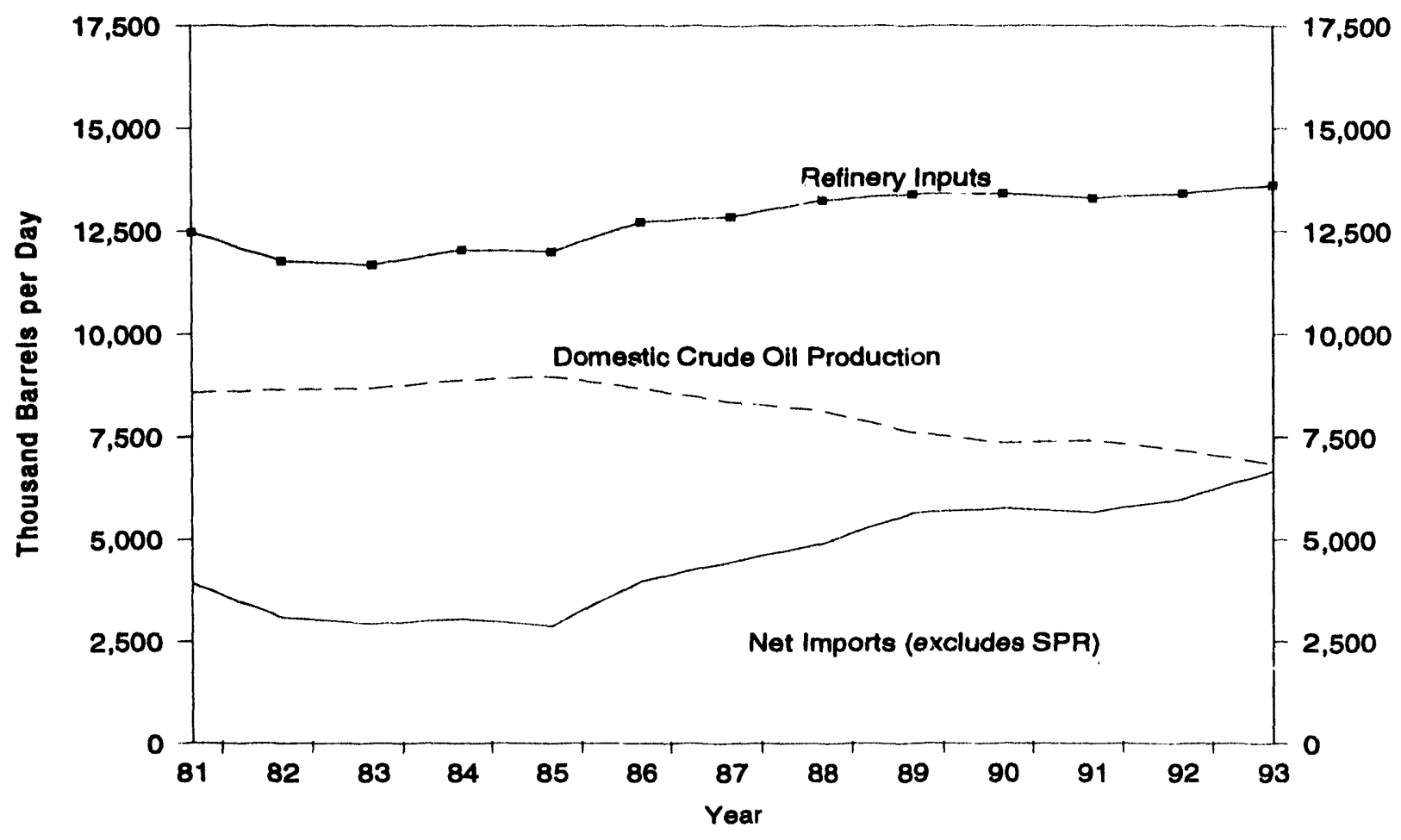

Source: Energy Information Administration, Petroleum Supply Annual, Table S2. See Summary Statistics Table and Figure Sources.

Figure S4. Crude Oll Ending Stocks', 1981 - Present

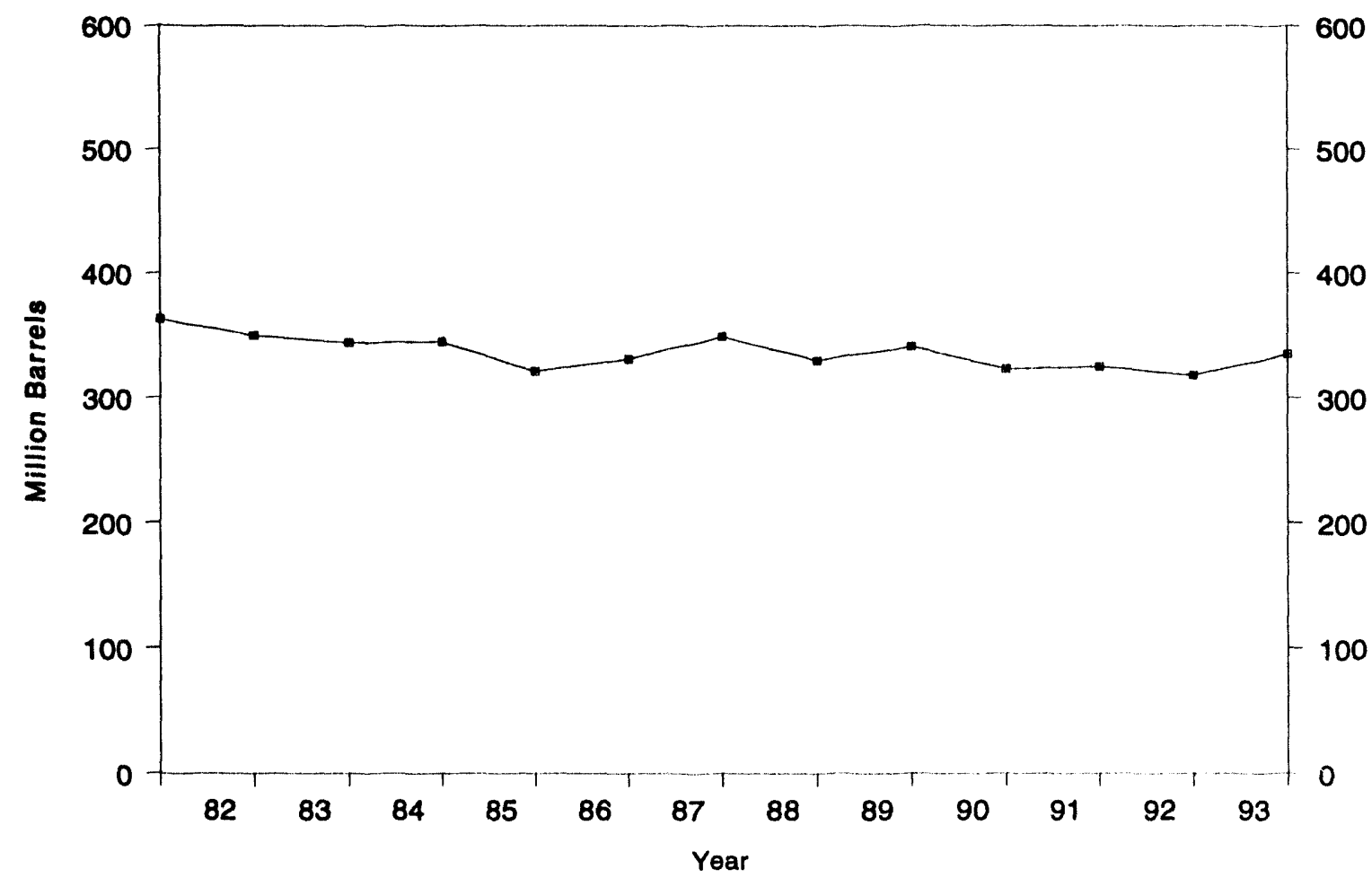

'Excludes stocks held in the Strategic Petroleum Reserve (SPR).

Source: Energy Information Administration, Petroleum Supply Annual, Table S2. See Summary Statistics Table and Figure Sources. 
Table 52. Crude Oll Supply and Disposttion, 1981 - Present (Thousand Barrels per Day, Except Where Noted)

\begin{tabular}{|c|c|c|c|c|c|c|c|c|}
\hline \multirow{3}{*}{\multicolumn{2}{|c|}{ Yoamionth }} & \multicolumn{6}{|c|}{ supply } & \multirow{3}{*}{$\begin{array}{c}\text { Dlepostion } \\
\text { Crude } \\
\text { Losews }\end{array}$} \\
\hline & & \multicolumn{2}{|c|}{ Field Production } & \multicolumn{3}{|c|}{ Imports } & \multirow{2}{*}{$\begin{array}{c}\text { Uneccounted } \\
\text { for Crude } \\
\text { Olle }\end{array}$} & \\
\hline & & $\begin{array}{c}\text { Total } \\
\text { Domentio }\end{array}$ & Alabkan & Total & 8PR & Othor & & \\
\hline $\begin{array}{l}1981 \\
1902 \\
1983 \\
1984 \\
1985 \\
1989 \\
1987 \\
1980 \\
1989 \\
1900\end{array}$ & 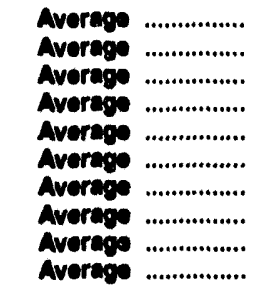 & $\begin{array}{l}8,672 \\
8,649 \\
8,689 \\
8,879 \\
0,071 \\
0,680 \\
0,340 \\
0,140 \\
7,613 \\
7,365\end{array}$ & $\begin{array}{l}1,009 \\
1,000 \\
1,714 \\
1,702 \\
1,025 \\
1,807 \\
1,002 \\
2,017 \\
1,874 \\
1,773\end{array}$ & $\begin{array}{l}4,393 \\
3,409 \\
3,329 \\
3,490 \\
3,201 \\
4,178 \\
4,674 \\
5,107 \\
5,843 \\
5,094\end{array}$ & $\begin{array}{r}258 \\
108 \\
284 \\
197 \\
118 \\
48 \\
73 \\
51 \\
86 \\
27\end{array}$ & $\begin{array}{l}4,141 \\
3,323 \\
3,009 \\
3,209 \\
3,083 \\
4,130 \\
4,601 \\
5,068 \\
5,787 \\
5,867\end{array}$ & $\begin{array}{r}83 \\
71 \\
114 \\
185 \\
145 \\
139 \\
145 \\
196 \\
200 \\
258\end{array}$ & $\begin{array}{l}5 \\
3 \\
2 \\
2 \\
1 \\
(a) \\
(0) \\
(8) \\
(0) \\
(0)\end{array}$ \\
\hline 1001 & 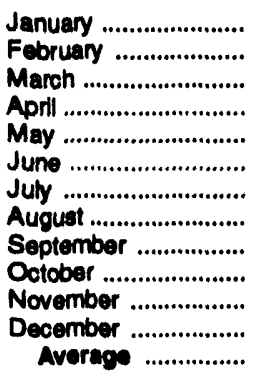 & $\begin{array}{l}7,500 \\
7,637 \\
7,546 \\
7,509 \\
7,409 \\
7,320 \\
7,347 \\
7,316 \\
7,368 \\
7,437 \\
7,328 \\
7,299 \\
7,417\end{array}$ & $\begin{array}{l}1,848 \\
1,908 \\
1,887 \\
1,798 \\
1,771 \\
1,757 \\
1,775 \\
1,731 \\
1,787 \\
1,843 \\
1,765 \\
1,718 \\
1,790\end{array}$ & $\begin{array}{l}5,298 \\
5,485 \\
5,166 \\
5,529 \\
6,363 \\
6,334 \\
5,965 \\
6,645 \\
5,812 \\
5,683 \\
5,528 \\
\mathbf{5 , 5 6 5} \\
\mathbf{5 , 7 8 2}\end{array}$ & $\begin{array}{l}0 \\
0 \\
0 \\
0 \\
0 \\
0 \\
0 \\
0 \\
0 \\
0 \\
0 \\
0 \\
0\end{array}$ & $\begin{array}{l}\mathbf{5 , 2 9 6} \\
\mathbf{5 , 4 8 5} \\
5,166 \\
5,529 \\
\mathbf{6 , 3 6 3} \\
\mathbf{6 , 3 3 4} \\
\mathbf{5 , 9 5 5} \\
\mathbf{6 , 6 4 5} \\
\mathbf{5 , 8 1 2} \\
\mathbf{5 , 6 8 3} \\
\mathbf{5 , 5 2 8} \\
\mathbf{5 , 5 6 5} \\
\mathbf{5 , 7 8 2}\end{array}$ & $\begin{array}{r}-59 \\
324 \\
43 \\
236 \\
513 \\
59 \\
403 \\
11 \\
484 \\
-59 \\
263 \\
146 \\
195\end{array}$ & $\begin{array}{r}0 \\
0 \\
(8) \\
(8) \\
(8) \\
(8) \\
0 \\
0 \\
0 \\
(8) \\
(8) \\
0 \\
(8)\end{array}$ \\
\hline 1992 & 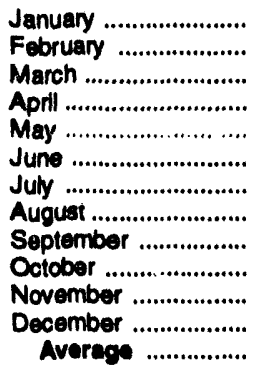 & $\begin{array}{l}7,361 \\
7,389 \\
7,348 \\
7,293 \\
7,169 \\
7,167 \\
7,131 \\
6,922 \\
7,030 \\
7,126 \\
7,024 \\
7,103 \\
7,171\end{array}$ & $\begin{array}{l}1,789 \\
1,608 \\
1,785 \\
1,741 \\
1,682 \\
1,703 \\
1,655 \\
1,635 \\
1,700 \\
1,696 \\
1,674 \\
1,705 \\
1,714\end{array}$ & $\begin{array}{l}5,966 \\
5,079 \\
5,321 \\
6,127 \\
6,080 \\
6,171 \\
6,796 \\
6,457 \\
6,218 \\
6,686 \\
6,121 \\
5,937 \\
6,083\end{array}$ & $\begin{array}{r}0 \\
0 \\
0 \\
0 \\
0 \\
34 \\
0 \\
18 \\
16 \\
49 \\
0 \\
0 \\
10\end{array}$ & $\begin{array}{l}\mathbf{5 , 9 5 6} \\
\mathbf{5 , 0 7 9} \\
\mathbf{5 , 3 2 1} \\
\mathbf{6 , 1 2 7} \\
\mathbf{6 , 0 6 0} \\
\mathbf{6 , 1 3 8} \\
\mathbf{6 , 7 8 6} \\
\mathbf{6 , 4 3 9} \\
\mathbf{6 , 2 0 2} \\
\mathbf{6 , 6 4 7} \\
\mathbf{6 , 1 2 1} \\
\mathbf{5 , 9 3 7} \\
\mathbf{6 , 0 7 3}\end{array}$ & $\begin{array}{r}290 \\
229 \\
287 \\
189 \\
421 \\
259 \\
332 \\
65 \\
385 \\
290 \\
296 \\
61 \\
258\end{array}$ & $\begin{array}{r}0 \\
(8) \\
(8) \\
0 \\
0 \\
(8) \\
0 \\
(8) \\
0 \\
(8) \\
(8) \\
(8) \\
(8)\end{array}$ \\
\hline 1993 & 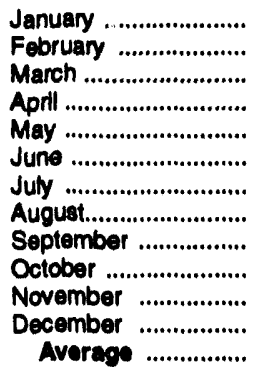 & $\begin{array}{l}6,961 \\
6,943 \\
6,974 \\
6,881 \\
6,847 \\
6,795 \\
6,688 \\
6,758 \\
6,712 \\
6,839 \\
6,912 \\
6,858 \\
6,847\end{array}$ & $\begin{array}{l}1,654 \\
1,628 \\
1,689 \\
1,587 \\
1,568 \\
1,520 \\
1,441 \\
1,528 \\
1,471 \\
1,610 \\
1,670 \\
1,671 \\
1,520\end{array}$ & $\begin{array}{l}6,292 \\
6,156 \\
6,488 \\
6,928 \\
6,809 \\
7,201 \\
7,289 \\
6,641 \\
6,581 \\
7,181 \\
6,997 \\
6,838 \\
6,787\end{array}$ & $\begin{array}{r}0 \\
0 \\
32 \\
112 \\
0 \\
0 \\
0 \\
0 \\
34 \\
0 \\
0 \\
0 \\
15\end{array}$ & $\begin{array}{l}6,292 \\
6,156 \\
6,455 \\
6,817 \\
6,809 \\
7,201 \\
7,289 \\
6,641 \\
6,547 \\
7,181 \\
6,997 \\
6,838 \\
6,772\end{array}$ & $\begin{array}{r}118 \\
162 \\
101 \\
333 \\
443 \\
293 \\
236 \\
3 \\
224 \\
109 \\
106 \\
.98 \\
168\end{array}$ & $\begin{array}{r}(8) \\
(8) \\
0 \\
(8) \\
0 \\
0 \\
0 \\
0 \\
(8) \\
0 \\
0 \\
0 \\
(8)\end{array}$ \\
\hline
\end{tabular}

- Stocks are totals as of end of period.

A negative number indicates a decrease in stocks and a positive number indicates an increase.

c Unaccounted for crude oll represents the difierence between the supply and disposition of crude oll.

d Previously published as crude used directly.

Stock changes are caloulated uaing new basis slock levels. See Summary Statistlcs Explanatory Note 4.

Footnotes continued on following page. 
Table S2. Crude Oll Supply and Disposition, 1981 - Present (Continued) (Thousand Barrels per Day, Except Where Noted)

\begin{tabular}{|c|c|c|c|c|c|c|c|c|c|}
\hline & \multirow[b]{3}{*}{ Yearkionth } & \multicolumn{5}{|c|}{ Diopostilon } & \multirow{2}{*}{\multicolumn{3}{|c|}{$\begin{array}{l}\text { Ending stooks } \\
\text { (Million Bamble) }\end{array}$}} \\
\hline & & \multicolumn{2}{|c|}{ Stock Changeb } & \multirow[b]{2}{*}{$\begin{array}{l}\text { Rofinery } \\
\text { Inpute }\end{array}$} & \multirow[b]{2}{*}{ Exports } & \multirow[b]{2}{*}{$\begin{array}{l}\text { Product } \\
\text { supplled }\end{array}$} & & & \\
\hline & & 8PR & Orhor & & & & Total & SPR & $\begin{array}{l}\text { Other } \\
\text { Primary }\end{array}$ \\
\hline $\begin{array}{l}1901 \\
1982 \\
1993 \\
1994 \\
1905 \\
1898 \\
1997 \\
1998 \\
1998 \\
1990\end{array}$ & $\begin{array}{l}\text { Average ............. } \\
\text { Averags ............ } \\
\text { Averags ............ } \\
\text { Averags ............ } \\
\text { Averags ............ } \\
\text { Averags ............. } \\
\text { Averags ........... } \\
\text { Averegs ............ } \\
\text { Averags ............ } \\
\text { Averags ............ }\end{array}$ & $\begin{array}{r}338 \\
174 \\
204 \\
195 \\
117 \\
50 \\
80 \\
52 \\
68 \\
16\end{array}$ & $\begin{array}{r}-48 \\
-38 \\
-20 \\
4 \\
-67 \\
28 \\
40 \\
-51 \\
30 \\
-51\end{array}$ & $\begin{array}{l}12,470 \\
11,774 \\
11,085 \\
12,044 \\
12,002 \\
12,716 \\
12,854 \\
13,240 \\
13,401 \\
13,400\end{array}$ & $\begin{array}{l}220 \\
286 \\
184 \\
181 \\
204 \\
184 \\
151 \\
185 \\
142 \\
109\end{array}$ & $\begin{array}{l}d 98 \\
d y 9 \\
68 \\
64 \\
60 \\
49 \\
44 \\
40 \\
24 \\
24\end{array}$ & $\begin{array}{l}004 \\
944 \\
723 \\
700 \\
614 \\
843 \\
000 \\
00 \\
001 \\
000\end{array}$ & $\begin{array}{l}230 \\
294 \\
370 \\
481 \\
493 \\
512 \\
841 \\
800 \\
800 \\
880\end{array}$ & $\begin{array}{r}383 \\
\mathbf{3 5 0} \\
\mathbf{3 4 4} \\
\mathbf{3 4 5} \\
\mathbf{3 2 1} \\
\mathbf{3 3 1} \\
\mathbf{3 4 0} \\
\mathbf{3 3 0} \\
\mathbf{3 4 1} \\
\mathbf{3 2 3}\end{array}$ \\
\hline 1891 & 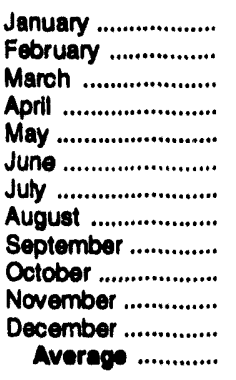 & $\begin{array}{r}0 \\
-147 \\
-422 \\
0 \\
0 \\
(8) \\
(8) \\
(8) \\
0 \\
(8) \\
(8) \\
(8) \\
47\end{array}$ & $\begin{array}{r}-71 \\
379 \\
183 \\
50 \\
566 \\
-299 \\
-153 \\
103 \\
-156 \\
51 \\
43 \\
-611 \\
5\end{array}$ & $\begin{array}{l}12,735 \\
13,046 \\
12,839 \\
13,042 \\
13,539 \\
13,918 \\
13,703 \\
13,800 \\
13,694 \\
12,896 \\
12,929 \\
13,465 \\
13,301\end{array}$ & $\begin{array}{r}50 \\
152 \\
137 \\
162 \\
165 \\
78 \\
139 \\
55 \\
55 \\
109 \\
92 \\
126 \\
133 \\
110\end{array}$ & $\begin{array}{l}23 \\
17 \\
18 \\
21 \\
15 \\
16 \\
15 \\
13 \\
16 \\
22 \\
22 \\
23 \\
18\end{array}$ & $\begin{array}{r}908 \\
913 \\
905 \\
907 \\
924 \\
915 \\
911 \\
914 \\
909 \\
911 \\
912 \\
893 \\
-\end{array}$ & $\begin{array}{r}588 \\
582 \\
568 \\
568 \\
568 \\
568 \\
569 \\
569 \\
569 \\
569 \\
569 \\
569 \\
-\end{array}$ & $\begin{array}{r}320 \\
33 i \\
337 \\
338 \\
356 \\
347 \\
342 \\
345 \\
341 \\
342 \\
344 \\
325 \\
-\end{array}$ \\
\hline 1992 & 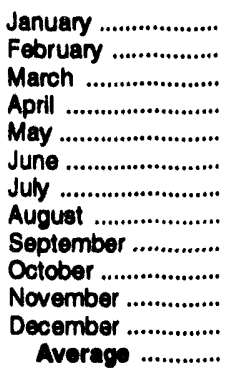 & $\begin{array}{l}(8) \\
0 \\
(8) \\
0 \\
(8) \\
34 \\
(8) \\
20 \\
43 \\
69 \\
15 \\
22 \\
17\end{array}$ & $\begin{array}{r}540 \\
171 \\
-250 \\
315 \\
-145 \\
-615 \\
244 \\
-144 \\
-204 \\
342 \\
-243 \\
-234 \\
-18\end{array}$ & $\begin{array}{l}12,923 \\
12,486 \\
13,083 \\
13,260 \\
13,679 \\
14,059 \\
13,953 \\
13,426 \\
13,714 \\
13,584 \\
13,547 \\
13,194 \\
13,411\end{array}$ & $\begin{array}{r}118 \\
22 \\
105 \\
23 \\
106 \\
107 \\
53 \\
133 \\
68 \\
108 \\
111 \\
107 \\
8\end{array}$ & $\begin{array}{r}26 \\
17 \\
18 \\
11 \\
10 \\
12 \\
9 \\
8 \\
11 \\
10 \\
10 \\
12 \\
13\end{array}$ & $\begin{array}{r}910 \\
915 \\
807 \\
917 \\
912 \\
895 \\
902 \\
898 \\
893 \\
908 \\
899 \\
893 \\
-\end{array}$ & $\begin{array}{r}\mathbf{5 6 9} \\
\mathbf{5 6 9} \\
\mathbf{5 6 9} \\
\mathbf{5 6 9} \\
\mathbf{5 6 9} \\
\mathbf{5 7 0} \\
\mathbf{5 7 0} \\
\mathbf{5 7 0} \\
\mathbf{5 7 1} \\
\mathbf{5 7 4} \\
\mathbf{5 7 4} \\
\mathbf{5 7 5} \\
-\end{array}$ & $\begin{array}{r}341 \\
346 \\
339 \\
348 \\
344 \\
325 \\
333 \\
328 \\
322 \\
333 \\
325 \\
318 \\
-\end{array}$ \\
\hline 1993 & 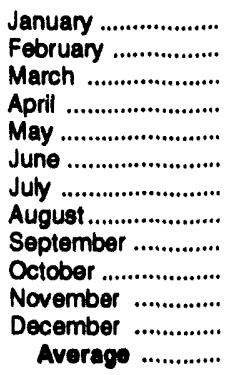 & $\begin{array}{r}19 \\
18 \\
58 \\
136 \\
13 \\
21 \\
19 \\
24 \\
52 \\
19 \\
18 \\
9 \\
34\end{array}$ & $\begin{array}{l}276 \\
201 \\
154 \\
387 \\
134 \\
-20 \\
-13 \\
-529 \\
-491 \\
309 \\
233 \\
-62 \\
47\end{array}$ & $\begin{array}{l}12,938 \\
12,865 \\
13,200 \\
13,538 \\
13,829 \\
14,129 \\
14,136 \\
13,844 \\
13,841 \\
13,729 \\
13,686 \\
13,571 \\
13,613\end{array}$ & $\begin{array}{r}129 \\
166 \\
139 \\
73 \\
112 \\
150 \\
62 \\
55 \\
107 \\
62 \\
67 \\
63 \\
98\end{array}$ & $\begin{array}{r}10 \\
10 \\
11 \\
9 \\
10 \\
8 \\
9 \\
8 \\
8 \\
10 \\
10 \\
16 \\
10\end{array}$ & $\begin{array}{r}902 \\
908 \\
915 \\
930 \\
935 \\
935 \\
935 \\
920 \\
908 \\
917 \\
924 \\
922 \\
-\end{array}$ & $\begin{array}{r}575 \\
576 \\
578 \\
582 \\
582 \\
583 \\
583 \\
584 \\
586 \\
586 \\
587 \\
587 \\
-\end{array}$ & $\begin{array}{r}327 \\
332 \\
337 \\
349 \\
353 \\
352 \\
352 \\
335 \\
321 \\
330 \\
337 \\
335 \\
-\end{array}$ \\
\hline
\end{tabular}

Footnoles continued.

SPR = Strateglc Petroleum Reserve.

(8)=Less than 500 barrels per day.

Notes: - Crude oll includes lease condensate. - Geographle coverage is the 50 States and the District of Columbla. - Totals may not equal sum of components due to independent rounding.

Source: See Summary S!atistics Table and Figure Sources. 
Table 53. Crude OIl and Petroleum Product Imports, 1981 - Present (Thousand Barrels per Day)

\begin{tabular}{|c|c|c|c|c|c|c|c|c|c|}
\hline & \multirow{3}{*}{ Yearmonth } & \multicolumn{8}{|c|}{ Imports from Arab-OPEC Sourese } \\
\hline & & \multicolumn{2}{|c|}{ Algeria } & \multicolumn{2}{|c|}{ Iraq } & \multicolumn{2}{|c|}{ Kunvalt } & \multicolumn{2}{|c|}{ Lubye } \\
\hline & & Total & Crude OII & Total & Crude Oll & Total & Crude Oll & Total & Crude Oll \\
\hline $\begin{array}{l}1981 \\
1982 \\
1983 \\
1984 \\
1985 \\
1988 \\
1987 \\
1988 \\
1989 \\
1900\end{array}$ & 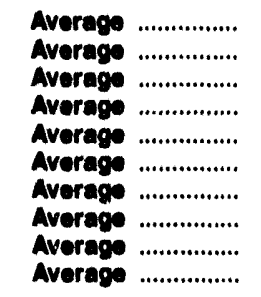 & $\begin{array}{l}311 \\
170 \\
240 \\
323 \\
187 \\
271 \\
295 \\
300 \\
200 \\
280\end{array}$ & $\begin{array}{r}281 \\
80 \\
176 \\
184 \\
84 \\
78 \\
115 \\
88 \\
60 \\
63\end{array}$ & $\begin{array}{r}(b) \\
3 \\
10 \\
12 \\
48 \\
81 \\
83 \\
345 \\
449 \\
818\end{array}$ & $\begin{array}{r}0 \\
3 \\
10 \\
12 \\
46 \\
81 \\
82 \\
343 \\
441 \\
514\end{array}$ & $\begin{array}{r}0 \\
5 \\
14 \\
38 \\
21 \\
68 \\
84 \\
02 \\
157 \\
88\end{array}$ & $\begin{array}{r}0 \\
2 \\
7 \\
24 \\
4 \\
28 \\
70 \\
80 \\
155 \\
79\end{array}$ & $\begin{array}{r}310 \\
20 \\
0 \\
1 \\
4 \\
0 \\
0 \\
0 \\
0 \\
0\end{array}$ & $\begin{array}{r}317 \\
23 \\
0 \\
0 \\
0 \\
0 \\
0 \\
0 \\
0 \\
0\end{array}$ \\
\hline 1991 & 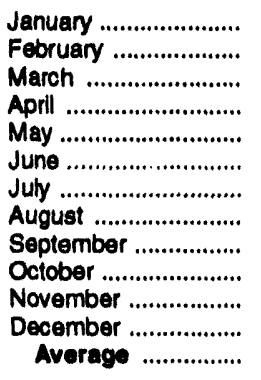 & $\begin{array}{l}327 \\
246 \\
222 \\
282 \\
308 \\
304 \\
202 \\
182 \\
205 \\
235 \\
278 \\
247 \\
253\end{array}$ & $\begin{array}{l}48 \\
20 \\
45 \\
74 \\
72 \\
37 \\
28 \\
16 \\
19 \\
53 \\
58 \\
54 \\
44\end{array}$ & $\begin{array}{l}0 \\
0 \\
0 \\
0 \\
0 \\
0 \\
0 \\
0 \\
0 \\
0 \\
0 \\
0 \\
0\end{array}$ & $\begin{array}{l}0 \\
0 \\
0 \\
0 \\
0 \\
0 \\
0 \\
0 \\
0 \\
0 \\
0 \\
0 \\
0\end{array}$ & $\begin{array}{r}0 \\
0 \\
0 \\
0 \\
0 \\
0 \\
0 \\
0 \\
34 \\
33 \\
0 \\
0 \\
6\end{array}$ & $\begin{array}{l}0 \\
0 \\
0 \\
0 \\
0 \\
0 \\
0 \\
0 \\
34 \\
33 \\
0 \\
0 \\
6\end{array}$ & $\begin{array}{l}0 \\
0 \\
0 \\
0 \\
0 \\
0 \\
0 \\
0 \\
0 \\
0 \\
0 \\
0 \\
0\end{array}$ & $\begin{array}{l}0 \\
0 \\
0 \\
0 \\
0 \\
0 \\
0 \\
0 \\
0 \\
0 \\
0 \\
0 \\
0\end{array}$ \\
\hline 1992 & 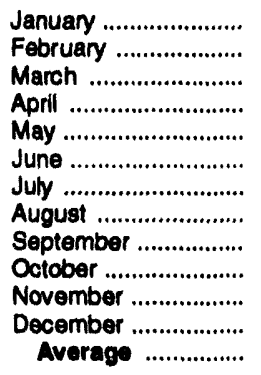 & $\begin{array}{l}206 \\
218 \\
215 \\
182 \\
202 \\
144 \\
179 \\
261 \\
184 \\
186 \\
171 \\
203 \\
196\end{array}$ & $\begin{array}{r}37 \\
57 \\
37 \\
19 \\
7 \\
72 \\
37 \\
45 \\
19 \\
8 \\
0 \\
9 \\
24\end{array}$ & $\begin{array}{l}0 \\
0 \\
0 \\
0 \\
0 \\
0 \\
0 \\
0 \\
0 \\
0 \\
0 \\
0 \\
0\end{array}$ & $\begin{array}{l}0 \\
0 \\
0 \\
0 \\
0 \\
0 \\
0 \\
0 \\
0 \\
0 \\
0 \\
0 \\
0\end{array}$ & $\begin{array}{r}0 \\
0 \\
0 \\
0 \\
0 \\
0 \\
58 \\
66 \\
70 \\
137 \\
117 \\
165 \\
51\end{array}$ & $\begin{array}{r}0 \\
0 \\
0 \\
0 \\
0 \\
0 \\
23 \\
33 \\
33 \\
109 \\
117 \\
149 \\
39\end{array}$ & $\begin{array}{l}0 \\
0 \\
0 \\
0 \\
0 \\
0 \\
0 \\
0 \\
0 \\
0 \\
0 \\
0 \\
0\end{array}$ & $\begin{array}{l}0 \\
0 \\
0 \\
0 \\
0 \\
0 \\
0 \\
0 \\
0 \\
0 \\
0 \\
0 \\
0\end{array}$ \\
\hline 1903 & 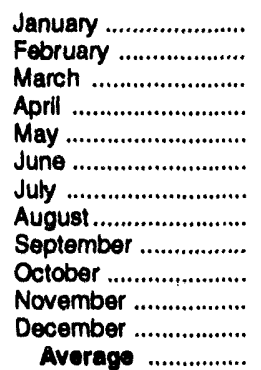 & $\begin{array}{l}153 \\
256 \\
185 \\
258 \\
228 \\
169 \\
246 \\
241 \\
192 \\
317 \\
222 \\
169 \\
220\end{array}$ & $\begin{array}{r}28 \\
0 \\
7 \\
26 \\
3 \\
32 \\
6 \\
28 \\
0 \\
80 \\
52 \\
25 \\
24\end{array}$ & $\begin{array}{l}0 \\
0 \\
0 \\
0 \\
0 \\
0 \\
0 \\
0 \\
0 \\
0 \\
0 \\
0 \\
0\end{array}$ & $\begin{array}{l}0 \\
0 \\
0 \\
0 \\
0 \\
0 \\
0 \\
0 \\
0 \\
0 \\
0 \\
0 \\
0\end{array}$ & $\begin{array}{l}144 \\
251 \\
316 \\
279 \\
222 \\
235 \\
368 \\
467 \\
445 \\
530 \\
486 \\
484 \\
353\end{array}$ & $\begin{array}{l}129 \\
229 \\
300 \\
279 \\
222 \\
235 \\
362 \\
451 \\
431 \\
526 \\
470 \\
484 \\
344\end{array}$ & $\begin{array}{l}0 \\
0 \\
0 \\
0 \\
0 \\
0 \\
0 \\
0 \\
0 \\
0 \\
0 \\
0 \\
0\end{array}$ & $\begin{array}{l}0 \\
0 \\
0 \\
0 \\
0 \\
0 \\
0 \\
0 \\
0 \\
0 \\
0 \\
0 \\
0\end{array}$ \\
\hline
\end{tabular}

See footnotes at end of table. 
Table S3. Crude Oll and Petroleum Product Imports, 1981 - Present (Continued) (Thousand Barrels per Day)

\begin{tabular}{|c|c|c|c|c|c|c|c|c|c|}
\hline & \multirow{3}{*}{ Yoarnionth } & \multicolumn{6}{|c|}{ Imports from Arab-OPEC sources } & \multirow{2}{*}{\multicolumn{2}{|c|}{$\begin{array}{l}\text { Total } \\
\text { Arab } \\
\text { OPEC }\end{array}$}} \\
\hline & & \multicolumn{2}{|c|}{ onthr } & \multicolumn{2}{|c|}{$\begin{array}{l}\text { saudl } \\
\text { Anpinb }\end{array}$} & \multicolumn{2}{|c|}{$\begin{array}{c}\text { United } \\
\text { Arab } \\
\text { Emirethe }\end{array}$} & & \\
\hline & & Total & Crude Oll & Totel & Crude OII & Total & Crude OII & Total & Crude Oil \\
\hline $\begin{array}{l}1981 \\
1902 \\
1803 \\
1984 \\
1905 \\
1000 \\
1987 \\
1989 \\
1909 \\
1900\end{array}$ & $\begin{array}{l}\text { Average ............ } \\
\text { Avernge ............ } \\
\text { Average ............ } \\
\text { Averege ............ } \\
\text { Average ............ } \\
\text { Avernge ............ } \\
\text { Average ............ } \\
\text { Average ............ } \\
\text { Average ............ } \\
\text { Average ............ }\end{array}$ & $\begin{array}{r}7 \\
7 \\
(5) \\
5 \\
(5) \\
13 \\
0 \\
0 \\
2 \\
4\end{array}$ & $\begin{array}{r}7 \\
7 \\
0 \\
4 \\
0 \\
12 \\
0 \\
0 \\
2 \\
4\end{array}$ & $\begin{array}{r}1,129 \\
582 \\
337 \\
325 \\
168 \\
685 \\
751 \\
1,073 \\
1,224 \\
1,330\end{array}$ & $\begin{array}{r}1,112 \\
530 \\
321 \\
300 \\
132 \\
618 \\
842 \\
011 \\
1,116 \\
1,198\end{array}$ & $\begin{array}{r}81 \\
92 \\
30 \\
117 \\
45 \\
44 \\
61 \\
20 \\
28 \\
17\end{array}$ & $\begin{array}{l}77 \\
81 \\
18 \\
90 \\
35 \\
38 \\
56 \\
23 \\
21 \\
9\end{array}$ & $\begin{array}{r}1,848 \\
854 \\
632 \\
819 \\
472 \\
1,162 \\
1,274 \\
1,839 \\
2,130 \\
2,244 \\
\end{array}$ & $\begin{array}{r}1,774 \\
736 \\
533 \\
634 \\
300 \\
854 \\
965 \\
1,415 \\
1,794 \\
1,864\end{array}$ \\
\hline 1902 & 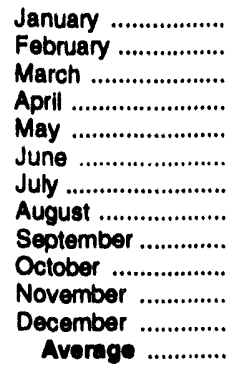 & $\begin{array}{l}0 \\
0 \\
0 \\
0 \\
0 \\
0 \\
8 \\
0 \\
0 \\
0 \\
0 \\
0 \\
1\end{array}$ & $\begin{array}{l}0 \\
0 \\
0 \\
0 \\
0 \\
0 \\
0 \\
0 \\
0 \\
0 \\
0 \\
0 \\
0\end{array}$ & $\begin{array}{l}2,017 \\
1,776 \\
1,707 \\
1,734 \\
1,764 \\
1,744 \\
1,713 \\
1,594 \\
1,593 \\
1,593 \\
1,608 \\
1,793 \\
1,720\end{array}$ & $\begin{array}{l}1,900 \\
1,687 \\
1,568 \\
1,524 \\
1,584 \\
1,610 \\
1,599 \\
1,473 \\
1,477 \\
1,482 \\
1,540 \\
1,725 \\
1,597\end{array}$ & $\begin{array}{r}18 \\
0 \\
0 \\
0 \\
0 \\
0 \\
0 \\
7 \\
0 \\
4 \\
17 \\
28 \\
6\end{array}$ & $\begin{array}{l}0 \\
0 \\
0 \\
0 \\
0 \\
0 \\
0 \\
0 \\
0 \\
0 \\
0 \\
0 \\
0\end{array}$ & $\begin{array}{l}2,241 \\
1,995 \\
1,922 \\
1,916 \\
1,966 \\
1,888 \\
1,958 \\
1,929 \\
1,847 \\
1,920 \\
1,913 \\
2,188 \\
1,974\end{array}$ & $\begin{array}{l}1,937 \\
1,745 \\
1,605 \\
1,543 \\
1,591 \\
1,621 \\
1,659 \\
1,651 \\
1,529 \\
1,599 \\
1,657 \\
1,882 \\
1,660\end{array}$ \\
\hline 1903 & 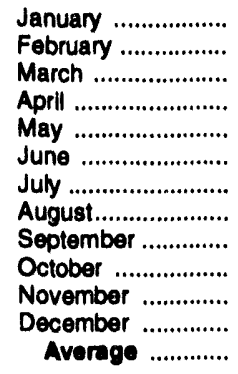 & $\begin{array}{l}0 \\
0 \\
6 \\
0 \\
0 \\
0 \\
0 \\
0 \\
0 \\
0 \\
0 \\
0 \\
1\end{array}$ & $\begin{array}{l}0 \\
0 \\
0 \\
0 \\
0 \\
0 \\
0 \\
0 \\
0 \\
0 \\
0 \\
0 \\
0\end{array}$ & $\begin{array}{l}1,688 \\
1,626 \\
1,479 \\
1,644 \\
1,524 \\
1,540 \\
1,283 \\
1,151 \\
1,329 \\
1,115 \\
1,281 \\
1,330 \\
1,414\end{array}$ & $\begin{array}{r}1,571 \\
1,480 \\
1,349 \\
1,515 \\
1,361 \\
1,413 \\
1,171 \\
1,036 \\
1,181 \\
969 \\
1,152 \\
1,205 \\
1,282 \\
\end{array}$ & $\begin{array}{r}0 \\
0 \\
0 \\
17 \\
59 \\
66 \\
19 \\
0 \\
0 \\
0 \\
1 \\
0 \\
14\end{array}$ & $\begin{array}{r}0 \\
0 \\
0 \\
17 \\
59 \\
66 \\
0 \\
0 \\
0 \\
0 \\
0 \\
0 \\
12\end{array}$ & $\begin{array}{l}1,984 \\
2,133 \\
1,987 \\
2,198 \\
2,034 \\
2,010 \\
1,917 \\
1,859 \\
1,966 \\
1,961 \\
1,989 \\
1,983 \\
2,000\end{array}$ & $\begin{array}{l}1,728 \\
1,709 \\
1,655 \\
1,837 \\
1,646 \\
1,746 \\
1,538 \\
1,515 \\
1,612 \\
1,574 \\
1,673 \\
1,713 \\
1,661\end{array}$ \\
\hline
\end{tabular}

See footnotes at end of table. 
Table S3. Crude Oll and Petroleum Product Imports, 1981 - Presont (Continued) (Thousand Barrels per Day)

\begin{tabular}{|c|c|c|c|c|c|c|c|c|c|}
\hline & \multirow{3}{*}{ Yoarmonth } & \multicolumn{8}{|c|}{ Imports from Othor-OPEC sources } \\
\hline & & \multicolumn{2}{|c|}{ Eouador } & \multicolumn{2}{|c|}{ Cmbon } & \multicolumn{2}{|c|}{ Indonesia } & \multicolumn{2}{|c|}{ Iran } \\
\hline & & Total & Crude OII & Totel & Crude Oll & Total & Crude Oll & Total & Crude OII \\
\hline $\begin{array}{l}1991 \\
1982 \\
1983 \\
1984 \\
1985 \\
1989 \\
1987 \\
1998 \\
1999 \\
1980\end{array}$ & 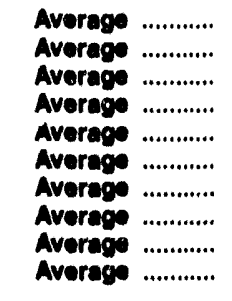 & $\begin{array}{l}49 \\
42 \\
61 \\
58 \\
67 \\
77 \\
29 \\
47 \\
89 \\
49\end{array}$ & $\begin{array}{l}38 \\
32 \\
58 \\
47 \\
58 \\
64 \\
23 \\
33 \\
80 \\
38\end{array}$ & $\begin{array}{l}36 \\
40 \\
59 \\
58 \\
52 \\
20 \\
35 \\
16 \\
50 \\
64\end{array}$ & $\begin{array}{l}35 \\
40 \\
59 \\
57 \\
51 \\
25 \\
35 \\
15 \\
49 \\
64\end{array}$ & $\begin{array}{l}308 \\
248 \\
338 \\
343 \\
314 \\
318 \\
206 \\
208 \\
103 \\
114\end{array}$ & $\begin{array}{r}318 \\
228 \\
315 \\
304 \\
292 \\
297 \\
202 \\
180 \\
188 \\
98\end{array}$ & $\begin{array}{r}0 \\
35 \\
48 \\
10 \\
27 \\
19 \\
98 \\
01 \\
0 \\
0\end{array}$ & $\begin{array}{r}0 \\
38 \\
48 \\
10 \\
27 \\
19 \\
98 \\
0 \\
0 \\
0\end{array}$ \\
\hline 1901 & 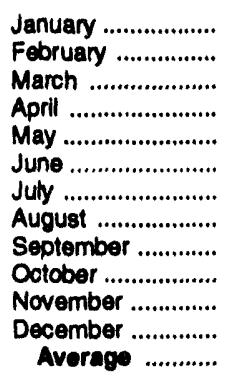 & $\begin{array}{r}18 \\
68 \\
67 \\
35 \\
109 \\
129 \\
62 \\
112 \\
31 \\
30 \\
55 \\
41 \\
63\end{array}$ & $\begin{array}{r}6 \\
55 \\
58 \\
24 \\
103 \\
126 \\
47 \\
93 \\
25 \\
24 \\
48 \\
23 \\
53\end{array}$ & $\begin{array}{r}41 \\
95 \\
29 \\
72 \\
96 \\
70 \\
137 \\
56 \\
91 \\
137 \\
91 \\
91 \\
84\end{array}$ & $\begin{array}{r}41 \\
95 \\
29 \\
72 \\
96 \\
70 \\
137 \\
56 \\
91 \\
137 \\
91 \\
91 \\
84\end{array}$ & $\begin{array}{r}70 \\
162 \\
93 \\
69 \\
97 \\
187 \\
88 \\
93 \\
83 \\
118 \\
120 \\
163 \\
111\end{array}$ & $\begin{array}{r}70 \\
153 \\
93 \\
69 \\
97 \\
187 \\
88 \\
87 \\
64 \\
91 \\
96 \\
134 \\
102\end{array}$ & $\begin{array}{r}0 \\
0 \\
0 \\
0 \\
0 \\
0 \\
81 \\
48 \\
152 \\
43 \\
64 \\
0 \\
32\end{array}$ & $\begin{array}{r}0 \\
0 \\
0 \\
0 \\
0 \\
0 \\
81 \\
48 \\
152 \\
43 \\
64 \\
0 \\
32\end{array}$ \\
\hline 1992 & 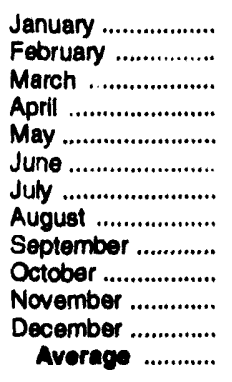 & $\begin{array}{r}56 \\
61 \\
26 \\
53 \\
51 \\
105 \\
111 \\
99 \\
97 \\
42 \\
53 \\
24 \\
85\end{array}$ & $\begin{array}{r}58 \\
48 \\
26 \\
46 \\
51 \\
101 \\
111 \\
93 \\
97 \\
36 \\
53 \\
24 \\
62\end{array}$ & $\begin{array}{r}91 \\
105 \\
25 \\
186 \\
135 \\
129 \\
143 \\
108 \\
165 \\
167 \\
114 \\
120 \\
124\end{array}$ & $\begin{array}{r}91 \\
105 \\
25 \\
186 \\
135 \\
129 \\
143 \\
109 \\
158 \\
167 \\
114 \\
120 \\
123\end{array}$ & $\begin{array}{r}125 \\
39 \\
85 \\
54 \\
155 \\
109 \\
65 \\
91 \\
57 \\
54 \\
36 \\
60 \\
78\end{array}$ & $\begin{array}{r}117 \\
39 \\
83 \\
49 \\
133 \\
102 \\
65 \\
85 \\
38 \\
43 \\
23 \\
60 \\
70\end{array}$ & $\begin{array}{l}0 \\
0 \\
0 \\
0 \\
0 \\
0 \\
0 \\
0 \\
0 \\
0 \\
0 \\
0 \\
0\end{array}$ & $\begin{array}{l}0 \\
0 \\
0 \\
0 \\
0 \\
0 \\
0 \\
0 \\
0 \\
0 \\
0 \\
0 \\
0\end{array}$ \\
\hline 1993 & 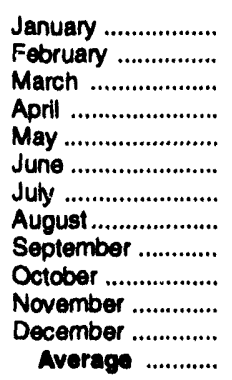 & $\begin{array}{r}76 \\
14 \\
59 \\
74 \\
56 \\
75 \\
96 \\
121 \\
49 \\
146 \\
115 \\
84 \\
81\end{array}$ & $\begin{array}{r}70 \\
14 \\
59 \\
62 \\
56 \\
75 \\
96 \\
121 \\
49 \\
135 \\
108 \\
84 \\
78\end{array}$ & $\begin{array}{r}90 \\
88 \\
126 \\
127 \\
169 \\
107 \\
168 \\
152 \\
211 \\
242 \\
143 \\
191 \\
152\end{array}$ & $\begin{array}{r}89 \\
88 \\
123 \\
127 \\
169 \\
107 \\
166 \\
152 \\
211 \\
242 \\
136 \\
191 \\
151\end{array}$ & $\begin{array}{r}37 \\
52 \\
67 \\
76 \\
82 \\
97 \\
55 \\
95 \\
51 \\
131 \\
74 \\
156 \\
81\end{array}$ & $\begin{array}{r}37 \\
51 \\
64 \\
76 \\
82 \\
67 \\
55 \\
80 \\
40 \\
82 \\
34 \\
114 \\
65\end{array}$ & $\begin{array}{l}0 \\
0 \\
0 \\
0 \\
0 \\
0 \\
0 \\
0 \\
0 \\
0 \\
0 \\
0 \\
0\end{array}$ & $\begin{array}{l}0 \\
0 \\
0 \\
0 \\
0 \\
0 \\
0 \\
0 \\
0 \\
0 \\
0 \\
0 \\
0\end{array}$ \\
\hline
\end{tabular}

See footnotes at end of table. 
Table S3. Crude Oll and Petroleum Product Imports, 1981 - Present (Continued) (Thousand Barrels per Day)

\begin{tabular}{|c|c|c|c|c|c|c|c|c|c|}
\hline & \multirow{3}{*}{ Yoarmonth } & \multicolumn{6}{|c|}{ importe from Other-OPEC sources } & \multirow{2}{*}{\multicolumn{2}{|c|}{$\begin{array}{l}\text { Total } \\
\text { OPECe }\end{array}$}} \\
\hline & & \multicolumn{2}{|c|}{ Meprin } & \multicolumn{2}{|c|}{ Monmuln } & \multicolumn{2}{|c|}{$\begin{array}{l}\text { Totel } \\
\text { Oxhor } \\
\text { opze }\end{array}$} & & \\
\hline & & Total & Crude oll & Total & Crude OII & Total & Crude Oll & Total & Crude Oil \\
\hline $\begin{array}{l}1891 \\
1892 \\
1893 \\
1894 \\
1885 \\
1898 \\
1987 \\
1988 \\
1809 \\
1900\end{array}$ & $\begin{array}{l}\text { Average ............. } \\
\text { Average ............ } \\
\text { Average ............. } \\
\text { Average ............ } \\
\text { Average ............. } \\
\text { Averags ............ } \\
\text { Average …......... } \\
\text { Average ............ } \\
\text { Average ............ } \\
\text { Average ............. }\end{array}$ & $\begin{array}{l}620 \\
554 \\
302 \\
216 \\
203 \\
440 \\
535 \\
618 \\
815 \\
800\end{array}$ & $\begin{array}{l}611 \\
510 \\
301 \\
207 \\
200 \\
437 \\
509 \\
607 \\
600 \\
784\end{array}$ & $\begin{array}{l}408 \\
412 \\
402 \\
540 \\
605 \\
703 \\
804 \\
704 \\
673 \\
1,025\end{array}$ & $\begin{array}{l}147 \\
156 \\
164 \\
203 \\
308 \\
416 \\
488 \\
489 \\
408 \\
608\end{array}$ & $\begin{array}{l}1,476 \\
1,291 \\
1,231 \\
1,230 \\
1,358 \\
1,074 \\
1,787 \\
1,601 \\
2,010 \\
2,052\end{array}$ & $\begin{array}{r}1,149 \\
998 \\
844 \\
878 \\
1,012 \\
1,259 \\
1,435 \\
1,281 \\
1,582 \\
1,650\end{array}$ & $\begin{array}{l}3,323 \\
2,148 \\
1,832 \\
2,049 \\
1,830 \\
2,837 \\
3,060 \\
3,520 \\
4,140 \\
4,240\end{array}$ & $\begin{array}{l}2,922 \\
1,734 \\
1,477 \\
1,512 \\
1,312 \\
2,113 \\
2,400 \\
2,683 \\
3,376 \\
3,514\end{array}$ \\
\hline 1801 & 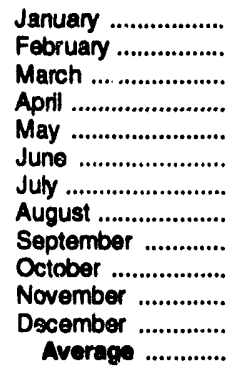 & $\begin{array}{r}504 \\
721 \\
531 \\
677 \\
860 \\
832 \\
833 \\
1,016 \\
489 \\
651 \\
704 \\
617 \\
703 \\
\end{array}$ & $\begin{array}{l}481 \\
717 \\
531 \\
649 \\
838 \\
827 \\
817 \\
883 \\
467 \\
623 \\
674 \\
593 \\
683\end{array}$ & $\begin{array}{r}1,005 \\
959 \\
998 \\
845 \\
997 \\
1,135 \\
1,102 \\
1,070 \\
1,163 \\
1,087 \\
1,065 \\
987 \\
1,085\end{array}$ & $\begin{array}{l}673 \\
686 \\
631 \\
470 \\
581 \\
705 \\
683 \\
701 \\
790 \\
777 \\
671 \\
655 \\
668\end{array}$ & $\begin{array}{l}1,637 \\
2,003 \\
1,718 \\
1,698 \\
2,158 \\
2,354 \\
2,304 \\
2,394 \\
2,009 \\
2,067 \\
2,099 \\
1,899 \\
2,028\end{array}$ & $\begin{array}{l}1,271 \\
1,705 \\
1,342 \\
1,283 \\
1,715 \\
1,915 \\
1,855 \\
1,966 \\
1,589 \\
1,694 \\
1,644 \\
1,496 \\
1,622\end{array}$ & $\begin{array}{l}3,898 \\
3,815 \\
3,623 \\
3,744 \\
4,724 \\
4,498 \\
4,232 \\
4,602 \\
3,956 \\
4,023 \\
4,171 \\
3,791 \\
4,092\end{array}$ & $\begin{array}{l}3,101 \\
3,264 \\
3,033 \\
3,059 \\
3,839 \\
3,747 \\
3,525 \\
3,946 \\
3,204 \\
3,343 \\
3,328 \\
3,116 \\
3,377\end{array}$ \\
\hline 1892 & 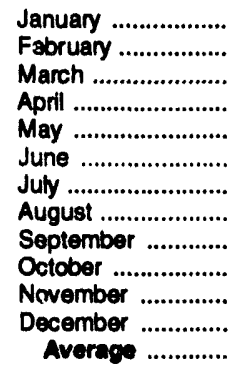 & $\begin{array}{l}693 \\
322 \\
441 \\
798 \\
773 \\
740 \\
900 \\
815 \\
774 \\
827 \\
626 \\
549 \\
681\end{array}$ & $\begin{array}{l}566 \\
303 \\
409 \\
788 \\
773 \\
740 \\
883 \\
795 \\
754 \\
813 \\
608 \\
532 \\
685\end{array}$ & $\begin{array}{l}1,119 \\
1,028 \\
1,106 \\
1,079 \\
1,038 \\
1,059 \\
1,163 \\
1,102 \\
1,333 \\
1,497 \\
1,343 \\
1,164 \\
1,170\end{array}$ & $\begin{array}{r}787 \\
655 \\
793 \\
722 \\
745 \\
738 \\
912 \\
841 \\
953 \\
1,073 \\
921 \\
763 \\
828\end{array}$ & $\begin{array}{l}1,984 \\
1,555 \\
1,684 \\
2,169 \\
2,152 \\
2,141 \\
2,382 \\
2,215 \\
2,426 \\
2,587 \\
2,173 \\
1,917 \\
2,117\end{array}$ & $\begin{array}{l}1,617 \\
1,150 \\
1,336 \\
1,791 \\
1,837 \\
1,809 \\
2,114 \\
1,922 \\
2,001 \\
2,133 \\
1,719 \\
1,499 \\
1,746\end{array}$ & $\begin{array}{l}4,224 \\
3,549 \\
3,606 \\
4,085 \\
4,118 \\
4,029 \\
4,339 \\
4,144 \\
4,274 \\
4,507 \\
4,086 \\
4,105 \\
4,092\end{array}$ & $\begin{array}{l}3,554 \\
2,895 \\
2,941 \\
3,334 \\
3,428 \\
3,430 \\
3,772 \\
3,473 \\
3,531 \\
3,732 \\
3,376 \\
3,381 \\
3,403\end{array}$ \\
\hline 1993 & 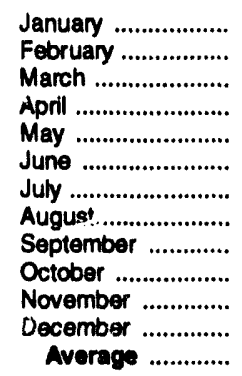 & $\begin{array}{l}729 \\
927 \\
928 \\
892 \\
760 \\
848 \\
893 \\
562 \\
514 \\
603 \\
636 \\
598 \\
740\end{array}$ & $\begin{array}{l}729 \\
913 \\
892 \\
871 \\
741 \\
827 \\
888 \\
549 \\
496 \\
593 \\
612 \\
569 \\
722 \\
\end{array}$ & $\begin{array}{l}1,397 \\
1,296 \\
1,173 \\
1,314 \\
1,264 \\
1,292 \\
1,384 \\
1,383 \\
1,273 \\
1,276 \\
1,322 \\
1,230 \\
1,300\end{array}$ & $\begin{array}{r}1,038 \\
925 \\
835 \\
1,023 \\
992 \\
999 \\
1,068 \\
1,135 \\
1,050 \\
993 \\
1,108 \\
952 \\
1,010\end{array}$ & $\begin{array}{l}2,330 \\
2,377 \\
2,354 \\
2,483 \\
2,331 \\
2,418 \\
2,596 \\
2,313 \\
2,097 \\
2,398 \\
2, \% 90 \\
2,260 \\
2,354\end{array}$ & $\begin{array}{l}1,962 \\
1,990 \\
1,973 \\
2,158 \\
2,040 \\
2,075 \\
2,273 \\
2,037 \\
1,845 \\
2,045 \\
1,997 \\
1,910 \\
2,028\end{array}$ & $\begin{array}{l}4,314 \\
4,510 \\
4,341 \\
4,682 \\
4,365 \\
4,428 \\
4,513 \\
4,172 \\
4,063 \\
4,359 \\
4,279 \\
4,242 \\
4,354\end{array}$ & $\begin{array}{l}3,650 \\
3,699 \\
3,628 \\
3,995 \\
3,686 \\
3,821 \\
3,811 \\
3,552 \\
3,457 \\
3,619 \\
3,670 \\
3,624 \\
3,687\end{array}$ \\
\hline
\end{tabular}

See footnotes at end of table. 
Table S3. Cruds OII and Petroleum Product Imports, 1981 - Present (Continued) (Thousand Barrels per Day)

\begin{tabular}{|c|c|c|c|c|c|c|c|c|c|c|c|c|c|}
\hline & \multirow{3}{*}{ YcarkMonth } & \multicolumn{12}{|c|}{ Imports from Non-OpEC sources" } \\
\hline & & \multicolumn{2}{|c|}{ 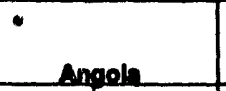 } & \multicolumn{2}{|c|}{ Auntrilln } & \multicolumn{2}{|c|}{$\begin{array}{l}\text { Bahama } \\
\text { infynde }\end{array}$} & \multicolumn{2}{|c|}{ Arall } & \multicolumn{2}{|c|}{ capin } & \multicolumn{2}{|c|}{$\begin{array}{c}\text { China } \\
\text { Puople's } \\
\text { Pepulblic }\end{array}$} \\
\hline & & Total & Crude OII & Tolet & Crude Oll & Totel & Crude on & Tocal & Crude oll & Total & Crude oil & Total & Crude a \\
\hline $\begin{array}{l}1981 \\
1982 \\
1983 \\
1984 \\
1985 \\
1996 \\
1907 \\
1999 \\
1909 \\
1900\end{array}$ & 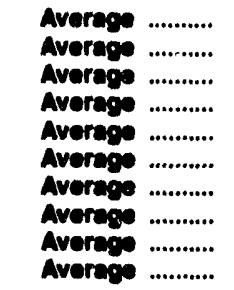 & $\begin{array}{r}49 \\
44 \\
78 \\
90 \\
110 \\
112 \\
192 \\
212 \\
204 \\
207\end{array}$ & $\begin{array}{r}45 \\
42 \\
71 \\
85 \\
104 \\
102 \\
180 \\
203 \\
270 \\
208\end{array}$ & $\begin{array}{l}5 \\
5 \\
4 \\
34 \\
37 \\
41 \\
6 \\
64 \\
38 \\
53\end{array}$ & $\begin{array}{r}0 \\
(0) \\
0 \\
25 \\
21 \\
30 \\
40 \\
50 \\
31 \\
47\end{array}$ & $\begin{array}{r}74 \\
65 \\
125 \\
88 \\
40 \\
37 \\
37 \\
32 \\
34 \\
37\end{array}$ & $\begin{array}{l}0 \\
0 \\
0 \\
0 \\
0 \\
0 \\
0 \\
0 \\
0 \\
0\end{array}$ & $\begin{array}{l}23 \\
47 \\
41 \\
60 \\
61 \\
50 \\
94 \\
98 \\
62 \\
40\end{array}$ & $\begin{array}{r}14 \\
10 \\
2 \\
(0) \\
0 \\
0 \\
0 \\
0 \\
0 \\
0\end{array}$ & $\begin{array}{l}497 \\
492 \\
647 \\
630 \\
770 \\
607 \\
948 \\
900 \\
931 \\
934\end{array}$ & $\begin{array}{l}164 \\
214 \\
274 \\
341 \\
498 \\
570 \\
603 \\
601 \\
690 \\
643\end{array}$ & $\begin{array}{l}18 \\
40 \\
34 \\
40 \\
60 \\
80 \\
62 \\
8 \\
80 \\
80\end{array}$ & $\begin{array}{r}0 \\
6 \\
6 \\
15 \\
38 \\
63 \\
63 \\
62 \\
76 \\
77\end{array}$ \\
\hline 1991 & 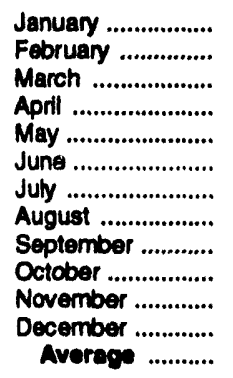 & $\begin{array}{l}232 \\
202 \\
186 \\
337 \\
220 \\
205 \\
264 \\
298 \\
230 \\
300 \\
213 \\
359 \\
254\end{array}$ & $\begin{array}{l}232 \\
202 \\
186 \\
337 \\
220 \\
205 \\
264 \\
298 \\
230 \\
300 \\
213 \\
359 \\
264\end{array}$ & $\begin{array}{r}21 \\
0 \\
0 \\
55 \\
64 \\
43 \\
20 \\
37 \\
24 \\
13 \\
25 \\
13 \\
20\end{array}$ & $\begin{array}{r}21 \\
0 \\
0 \\
55 \\
57 \\
31 \\
20 \\
22 \\
24 \\
0 \\
13 \\
13 \\
21\end{array}$ & $\begin{array}{r}25 \\
14 \\
0 \\
35 \\
42 \\
30 \\
19 \\
78 \\
23 \\
51 \\
46 \\
53 \\
35\end{array}$ & $\begin{array}{l}0 \\
0 \\
0 \\
0 \\
0 \\
0 \\
0 \\
0 \\
0 \\
0 \\
0 \\
0 \\
0\end{array}$ & $\begin{array}{r}31 \\
13 \\
0 \\
17 \\
31 \\
41 \\
21 \\
27 \\
19 \\
16 \\
45 \\
6 \\
22\end{array}$ & $\begin{array}{l}0 \\
0 \\
0 \\
0 \\
0 \\
0 \\
0 \\
0 \\
0 \\
0 \\
0 \\
0 \\
0\end{array}$ & $\begin{array}{r}978 \\
1,135 \\
1,058 \\
1,103 \\
1,027 \\
986 \\
948 \\
1,011 \\
1,137 \\
938 \\
1,107 \\
1,083 \\
1,033\end{array}$ & $\begin{array}{l}718 \\
881 \\
764 \\
768 \\
752 \\
705 \\
615 \\
694 \\
849 \\
639 \\
796 \\
759 \\
743\end{array}$ & $\begin{array}{r}68 \\
102 \\
86 \\
113 \\
119 \\
144 \\
88 \\
85 \\
91 \\
29 \\
98 \\
65 \\
91\end{array}$ & $\begin{array}{r}63 \\
96 \\
96 \\
113 \\
113 \\
139 \\
88 \\
75 \\
86 \\
24 \\
96 \\
65 \\
87\end{array}$ \\
\hline 1992 & 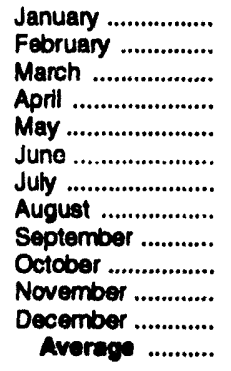 & $\begin{array}{l}360 \\
246 \\
339 \\
381 \\
264 \\
286 \\
443 \\
335 \\
248 \\
395 \\
458 \\
279 \\
385\end{array}$ & $\begin{array}{l}380 \\
246 \\
339 \\
381 \\
264 \\
286 \\
443 \\
323 \\
248 \\
395 \\
458 \\
279 \\
389\end{array}$ & $\begin{array}{r}11 \\
10 \\
0 \\
39 \\
0 \\
21 \\
20 \\
21 \\
0 \\
11 \\
53 \\
38 \\
19\end{array}$ & $\begin{array}{r}11 \\
10 \\
0 \\
22 \\
0 \\
21 \\
20 \\
21 \\
0 \\
11 \\
49 \\
38 \\
17\end{array}$ & $\begin{array}{r}63 \\
47 \\
76 \\
67 \\
46 \\
57 \\
22 \\
8 \\
8 \\
1 \\
20 \\
19 \\
38\end{array}$ & $\begin{array}{l}0 \\
0 \\
0 \\
0 \\
0 \\
0 \\
0 \\
0 \\
0 \\
0 \\
0 \\
0 \\
0\end{array}$ & $\begin{array}{l}18 \\
12 \\
(8) \\
17 \\
18 \\
28 \\
25 \\
10 \\
21 \\
10 \\
32 \\
50 \\
20\end{array}$ & $\begin{array}{l}0 \\
0 \\
0 \\
0 \\
0 \\
0 \\
0 \\
0 \\
0 \\
0 \\
0 \\
0 \\
0\end{array}$ & $\begin{array}{r}1,045 \\
1,147 \\
1,100 \\
1,121 \\
1,013 \\
970 \\
1,044 \\
1,038 \\
1,131 \\
1,063 \\
1,037 \\
1,122 \\
1,000\end{array}$ & $\begin{array}{l}786 \\
834 \\
832 \\
835 \\
779 \\
738 \\
798 \\
762 \\
839 \\
761 \\
784 \\
816 \\
797\end{array}$ & $\begin{array}{r}144 \\
80 \\
75 \\
86 \\
129 \\
110 \\
68 \\
66 \\
80 \\
61 \\
86 \\
97 \\
90\end{array}$ & $\begin{array}{r}144 \\
69 \\
75 \\
69 \\
114 \\
95 \\
64 \\
66 \\
75 \\
61 \\
86 \\
90 \\
84\end{array}$ \\
\hline 1803 & 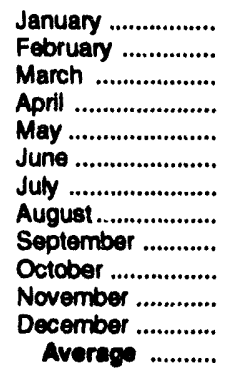 & $\begin{array}{l}354 \\
348 \\
408 \\
344 \\
299 \\
209 \\
402 \\
258 \\
282 \\
440 \\
307 \\
379 \\
336\end{array}$ & $\begin{array}{l}354 \\
348 \\
408 \\
344 \\
299 \\
209 \\
402 \\
258 \\
282 \\
440 \\
307 \\
379 \\
336\end{array}$ & $\begin{array}{r}(8) \\
0 \\
0 \\
0 \\
13 \\
34 \\
40 \\
33 \\
0 \\
53 \\
0 \\
53 \\
19\end{array}$ & $\begin{array}{r}0 \\
0 \\
0 \\
0 \\
13 \\
34 \\
40 \\
27 \\
0 \\
47 \\
0 \\
53 \\
10\end{array}$ & $\begin{array}{r}18 \\
26 \\
38 \\
16 \\
8 \\
7 \\
31 \\
41 \\
37 \\
53 \\
29 \\
30 \\
28\end{array}$ & $\begin{array}{l}0 \\
0 \\
0 \\
0 \\
0 \\
0 \\
0 \\
0 \\
0 \\
0 \\
0 \\
0 \\
0\end{array}$ & $\begin{array}{l}3 \\
22 \\
27 \\
56 \\
41 \\
19 \\
48 \\
32 \\
59 \\
15 \\
61 \\
10 \\
33\end{array}$ & $\begin{array}{l}0 \\
0 \\
0 \\
0 \\
0 \\
0 \\
0 \\
0 \\
0 \\
0 \\
0 \\
0 \\
0\end{array}$ & $\begin{array}{l}1,052 \\
1,095 \\
1,033 \\
1,052 \\
1,128 \\
1,117 \\
1,264 \\
1,247 \\
1,319 \\
1,370 \\
1,236 \\
1,255 \\
1,181\end{array}$ & $\begin{array}{r}778 \\
782 \\
770 \\
783 \\
874 \\
911 \\
991 \\
968 \\
1,023 \\
1,030 \\
917 \\
964 \\
900\end{array}$ & $\begin{array}{r}60 \\
44 \\
79 \\
0 \\
40 \\
48 \\
24 \\
38 \\
91 \\
61 \\
68 \\
61 \\
51\end{array}$ & $\begin{array}{r}60 \\
44 \\
73 \\
0 \\
40 \\
46 \\
24 \\
38 \\
89 \\
61 \\
68 \\
61 \\
50\end{array}$ \\
\hline
\end{tabular}

See footnotes at end of table. 
Table S3. Crude OII and Petroloum Product Imports, 1981 - Present (Continued) (Thousand Barrels per Day)

\begin{tabular}{|c|c|c|c|c|c|c|c|c|c|c|c|}
\hline & \multirow{3}{*}{ Yearmionth } & \multicolumn{10}{|c|}{ Imports from Non-OPEC sources" } \\
\hline & & \multicolumn{2}{|c|}{ Colombla } & \multicolumn{2}{|c|}{ Raty } & \multicolumn{2}{|c|}{ Maleysia } & \multicolumn{2}{|c|}{ Moxico } & \multicolumn{2}{|c|}{ Netherlands } \\
\hline & & Total & Crude oll & Total & Cruds Oll & Total & Cruds OIl & Total & Crude Oll & Total & Crude Oll \\
\hline 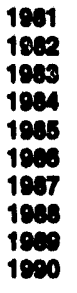 & 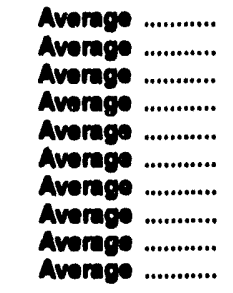 & $\begin{array}{r}1 \\
5 \\
10 \\
8 \\
28 \\
97 \\
148 \\
134 \\
172 \\
182\end{array}$ & $\begin{array}{r}0 \\
0 \\
0 \\
0 \\
0 \\
07 \\
115 \\
100 \\
138 \\
140\end{array}$ & $\begin{array}{l}11 \\
18 \\
18 \\
45 \\
60 \\
76 \\
84 \\
68 \\
34 \\
68\end{array}$ & $\begin{array}{l}0 \\
(0) \\
(0) \\
(0) \\
(0) \\
0 \\
1 \\
8 \\
3 \\
2\end{array}$ & $\begin{array}{r}30 \\
20 \\
4 \\
1 \\
3 \\
12 \\
13 \\
19 \\
39 \\
41\end{array}$ & $\begin{array}{r}33 \\
18 \\
3 \\
0 \\
1 \\
11 \\
12 \\
10 \\
30 \\
40\end{array}$ & $\begin{array}{l}622 \\
608 \\
824 \\
746 \\
816 \\
609 \\
885 \\
747 \\
707 \\
785\end{array}$ & $\begin{array}{l}460 \\
645 \\
785 \\
689 \\
716 \\
821 \\
602 \\
674 \\
716 \\
880\end{array}$ & $\begin{array}{l}30 \\
35 \\
65 \\
65 \\
58 \\
54 \\
60 \\
61 \\
49 \\
85\end{array}$ & $\begin{array}{l}(0) \\
(0) \\
3 \\
3 \\
0 \\
0 \\
0 \\
0 \\
0 \\
0\end{array}$ \\
\hline 1901 & 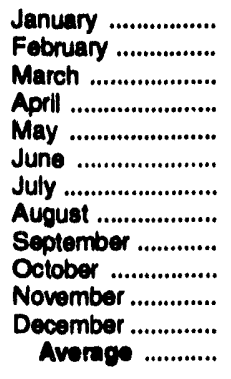 & $\begin{array}{l}194 \\
151 \\
157 \\
163 \\
163 \\
169 \\
163 \\
219 \\
168 \\
128 \\
145 \\
138 \\
163\end{array}$ & $\begin{array}{r}174 \\
88 \\
127 \\
131 \\
112 \\
124 \\
111 \\
162 \\
103 \\
80 \\
135 \\
117 \\
123\end{array}$ & $\begin{array}{l}25 \\
42 \\
29 \\
41 \\
60 \\
46 \\
54 \\
57 \\
89 \\
41 \\
16 \\
61 \\
47\end{array}$ & $\begin{array}{r}0 \\
13 \\
0 \\
12 \\
0 \\
0 \\
0 \\
11 \\
0 \\
0 \\
0 \\
0 \\
3\end{array}$ & $\begin{array}{r}0 \\
9 \\
21 \\
0 \\
68 \\
63 \\
9 \\
14 \\
10 \\
64 \\
10 \\
14 \\
24\end{array}$ & $\begin{array}{r}0 \\
9 \\
21 \\
0 \\
68 \\
63 \\
9 \\
14 \\
10 \\
64 \\
10 \\
14 \\
24\end{array}$ & $\begin{array}{l}788 \\
742 \\
795 \\
891 \\
757 \\
919 \\
835 \\
878 \\
805 \\
811 \\
716 \\
732 \\
807\end{array}$ & $\begin{array}{l}778 \\
693 \\
772 \\
819 \\
736 \\
872 \\
748 \\
797 \\
768 \\
754 \\
656 \\
708 \\
759\end{array}$ & $\begin{array}{r}6 \\
17 \\
33 \\
35 \\
45 \\
49 \\
47 \\
30 \\
44 \\
16 \\
24 \\
4 \\
29\end{array}$ & $\begin{array}{l}0 \\
0 \\
0 \\
0 \\
0 \\
0 \\
0 \\
0 \\
0 \\
0 \\
0 \\
0 \\
0\end{array}$ \\
\hline 1002 & 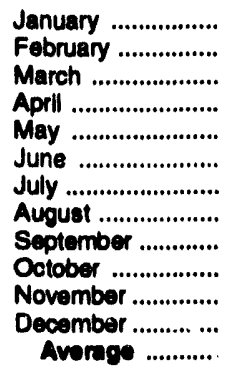 & $\begin{array}{r}158 \\
114 \\
101 \\
150 \\
57 \\
135 \\
103 \\
168 \\
180 \\
153 \\
127 \\
66 \\
128\end{array}$ & $\begin{array}{r}111 \\
92 \\
74 \\
129 \\
46 \\
114 \\
93 \\
142 \\
179 \\
132 \\
84 \\
34 \\
102\end{array}$ & $\begin{array}{l}51 \\
48 \\
44 \\
75 \\
57 \\
69 \\
36 \\
84 \\
81 \\
37 \\
33 \\
37 \\
85\end{array}$ & $\begin{array}{l}0 \\
0 \\
0 \\
0 \\
0 \\
0 \\
0 \\
0 \\
0 \\
0 \\
0 \\
0 \\
0\end{array}$ & $\begin{array}{r}0 \\
0 \\
0 \\
0 \\
5 \\
8 \\
40 \\
22 \\
17 \\
17 \\
8 \\
4 \\
10\end{array}$ & $\begin{array}{r}0 \\
0 \\
0 \\
0 \\
5 \\
8 \\
40 \\
22 \\
17 \\
17 \\
8 \\
4 \\
10\end{array}$ & $\begin{array}{l}764 \\
838 \\
846 \\
857 \\
788 \\
905 \\
830 \\
857 \\
755 \\
829 \\
762 \\
930 \\
830\end{array}$ & $\begin{array}{l}721 \\
807 \\
809 \\
795 \\
764 \\
883 \\
788 \\
790 \\
720 \\
783 \\
700 \\
888 \\
787\end{array}$ & $\begin{array}{r}31 \\
9 \\
34 \\
8 \\
27 \\
25 \\
21 \\
45 \\
39 \\
18 \\
26 \\
33 \\
28\end{array}$ & $\begin{array}{l}0 \\
0 \\
0 \\
0 \\
0 \\
0 \\
0 \\
0 \\
0 \\
0 \\
0 \\
0 \\
0\end{array}$ \\
\hline 1908 & 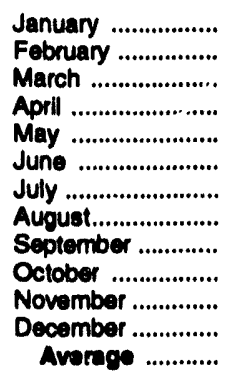 & $\begin{array}{l}188 \\
148 \\
161 \\
178 \\
147 \\
176 \\
204 \\
131 \\
224 \\
192 \\
164 \\
134 \\
171\end{array}$ & $\begin{array}{r}167 \\
137 \\
129 \\
165 \\
90 \\
143 \\
184 \\
101 \\
170 \\
182 \\
143 \\
85 \\
141\end{array}$ & $\begin{array}{r}56 \\
34 \\
43 \\
14 \\
26 \\
25 \\
25 \\
50 \\
32 \\
40 \\
30 \\
0 \\
31\end{array}$ & $\begin{array}{l}0 \\
0 \\
0 \\
0 \\
0 \\
0 \\
0 \\
0 \\
0 \\
0 \\
0 \\
0 \\
0\end{array}$ & $\begin{array}{r}0 \\
0 \\
11 \\
8 \\
21 \\
0 \\
11 \\
14 \\
28 \\
14 \\
0 \\
28 \\
11\end{array}$ & $\begin{array}{r}0 \\
0 \\
10 \\
8 \\
10 \\
0 \\
11 \\
14 \\
28 \\
10 \\
0 \\
28 \\
10\end{array}$ & $\begin{array}{r}858 \\
807 \\
844 \\
832 \\
917 \\
987 \\
943 \\
862 \\
929 \\
1,013 \\
1,116 \\
909 \\
919\end{array}$ & $\begin{array}{r}820 \\
748 \\
798 \\
796 \\
846 \\
959 \\
878 \\
809 \\
867 \\
951 \\
1,041 \\
837 \\
883\end{array}$ & $\begin{array}{r}11 \\
18 \\
10 \\
0 \\
10 \\
10 \\
21 \\
17 \\
22 \\
0 \\
(8) \\
6 \\
10\end{array}$ & $\begin{array}{l}0 \\
0 \\
0 \\
0 \\
0 \\
0 \\
0 \\
0 \\
0 \\
0 \\
0 \\
0 \\
0\end{array}$ \\
\hline
\end{tabular}

See footnotes at end of table. 
Table 53. Crude Oil and Petroleum Product Imports, 1981 - Present (Continued) (Thousand Barrels per Day)

\begin{tabular}{|c|c|c|c|c|c|c|c|c|c|c|c|c|c|}
\hline & \multirow{3}{*}{ Ycarnmonth } & \multicolumn{12}{|c|}{ Imports from Non-OPEC sources" } \\
\hline & & \multicolumn{2}{|c|}{$\begin{array}{c}\text { Nethorlands } \\
\text { Antilli: }\end{array}$} & \multicolumn{2}{|c|}{ Noring } & \multicolumn{2}{|c|}{$\begin{array}{l}\text { Puerto } \\
\text { Plese }\end{array}$} & \multicolumn{2}{|c|}{ Bungled } & \multicolumn{2}{|c|}{ sping } & \multicolumn{2}{|c|}{$\begin{array}{c}\text { Trinided } \\
\text { and } \\
\text { Topoes }\end{array}$} \\
\hline & & Total & Crude oll & Total & Crude OII & Total & Cruds oll & Tot: & Crude ofl & Total & Crude oll & Total & Crude oll \\
\hline $\begin{array}{l}1891 \\
1802 \\
1908 \\
1984 \\
1005 \\
1090 \\
1907 \\
1008 \\
1000 \\
1900\end{array}$ & 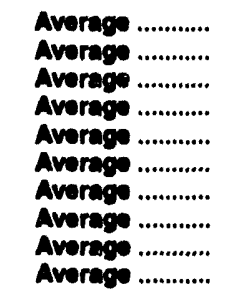 & $\begin{array}{r}197 \\
175 \\
180 \\
189 \\
40 \\
25 \\
20 \\
30 \\
42 \\
31\end{array}$ & $\begin{array}{l}0 \\
0 \\
0 \\
0 \\
0 \\
0 \\
0 \\
0 \\
0 \\
0\end{array}$ & $\begin{array}{r}110 \\
102 \\
68 \\
114 \\
30 \\
60 \\
80 \\
67 \\
139 \\
102\end{array}$ & $\begin{array}{r}114 \\
102 \\
85 \\
112 \\
31 \\
83 \\
70 \\
02 \\
127 \\
8\end{array}$ & $\begin{array}{l}62 \\
50 \\
40 \\
42 \\
28 \\
21 \\
21 \\
22 \\
32 \\
32\end{array}$ & $\begin{array}{l}0 \\
0 \\
0 \\
0 \\
0 \\
0 \\
0 \\
0 \\
0 \\
0\end{array}$ & $\begin{array}{r}5 \\
1 \\
1 \\
13 \\
8 \\
18 \\
11 \\
28 \\
48 \\
45\end{array}$ & $\begin{array}{l}(0) \\
0 \\
(0) \\
(0) \\
(0) \\
(0) \\
0 \\
0 \\
0 \\
1\end{array}$ & $\begin{array}{l}1 \\
3 \\
2 \\
11 \\
29 \\
83 \\
85 \\
69 \\
67 \\
47\end{array}$ & $\begin{array}{l}(0) \\
(0) \\
(0) \\
0 \\
1 \\
0 \\
0 \\
0 \\
0 \\
0\end{array}$ & $\begin{array}{r}133 \\
112 \\
83 \\
84 \\
113 \\
123 \\
105 \\
97 \\
84 \\
86\end{array}$ & $\begin{array}{r}102 \\
92 \\
83 \\
97 \\
98 \\
98 \\
75 \\
71 \\
73 \\
78\end{array}$ \\
\hline 1901 & 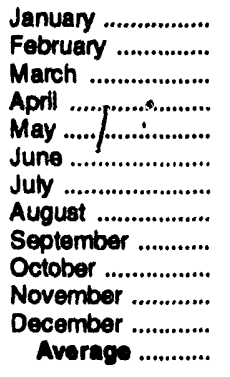 & $\begin{array}{r}103 \\
23 \\
56 \\
61 \\
110 \\
84 \\
86 \\
100 \\
67 \\
80 \\
100 \\
88 \\
81\end{array}$ & $\begin{array}{l}0 \\
0 \\
0 \\
0 \\
0 \\
0 \\
0 \\
0 \\
0 \\
0 \\
0 \\
0 \\
0\end{array}$ & $\begin{array}{r}45 \\
37 \\
25 \\
51 \\
165 \\
99 \\
69 \\
142 \\
79 \\
89 \\
73 \\
94 \\
82\end{array}$ & $\begin{array}{r}34 \\
37 \\
16 \\
35 \\
156 \\
84 \\
63 \\
138 \\
72 \\
98 \\
65 \\
88 \\
74\end{array}$ & $\begin{array}{l}22 \\
20 \\
14 \\
23 \\
42 \\
19 \\
25 \\
42 \\
34 \\
12 \\
35 \\
36 \\
27\end{array}$ & $\begin{array}{l}0 \\
0 \\
0 \\
0 \\
0 \\
0 \\
0 \\
0 \\
0 \\
0 \\
0 \\
0 \\
0\end{array}$ & $\begin{array}{r}28 \\
17 \\
13 \\
39 \\
42 \\
0 \\
58 \\
80 \\
23 \\
13 \\
16 \\
16 \\
20\end{array}$ & $\begin{array}{r}0 \\
0 \\
0 \\
0 \\
0 \\
0 \\
0 \\
11 \\
0 \\
0 \\
0 \\
0 \\
1\end{array}$ & $\begin{array}{l}26 \\
18 \\
13 \\
66 \\
53 \\
41 \\
22 \\
48 \\
42 \\
24 \\
19 \\
26 \\
33\end{array}$ & $\begin{array}{l}0 \\
0 \\
0 \\
0 \\
0 \\
0 \\
0 \\
0 \\
0 \\
0 \\
0 \\
0 \\
0\end{array}$ & $\begin{array}{r}75 \\
76 \\
86 \\
84 \\
61 \\
118 \\
91 \\
91 \\
119 \\
88 \\
77 \\
87 \\
8\end{array}$ & $\begin{array}{r}64 \\
76 \\
73 \\
64 \\
61 \\
104 \\
72 \\
66 \\
75 \\
76 \\
69 \\
71 \\
72\end{array}$ \\
\hline 1892 & 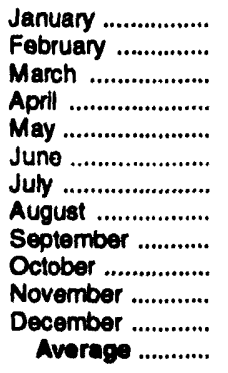 & $\begin{array}{l}40 \\
82 \\
49 \\
73 \\
59 \\
83 \\
49 \\
65 \\
60 \\
90 \\
56 \\
80 \\
65\end{array}$ & $\begin{array}{l}0 \\
0 \\
0 \\
0 \\
0 \\
0 \\
0 \\
0 \\
0 \\
0 \\
0 \\
0 \\
0\end{array}$ & $\begin{array}{r}25 \\
11 \\
11 \\
155 \\
210 \\
234 \\
186 \\
142 \\
103 \\
180 \\
111 \\
140 \\
127\end{array}$ & $\begin{array}{r}17 \\
0 \\
0 \\
147 \\
200 \\
225 \\
179 \\
134 \\
102 \\
177 \\
104 \\
133 \\
119\end{array}$ & $\begin{array}{l}32 \\
23 \\
18 \\
14 \\
22 \\
36 \\
11 \\
38 \\
37 \\
29 \\
26 \\
28 \\
26\end{array}$ & $\begin{array}{l}0 \\
0 \\
0 \\
0 \\
0 \\
0 \\
0 \\
0 \\
0 \\
0 \\
0 \\
0 \\
0\end{array}$ & $\begin{array}{r}17 \\
3 \\
0 \\
0 \\
0 \\
0 \\
72 \\
62 \\
53 \\
9 \\
0 \\
0 \\
18\end{array}$ & $\begin{array}{r}0 \\
0 \\
0 \\
0 \\
0 \\
0 \\
32 \\
31 \\
0 \\
0 \\
0 \\
0 \\
5\end{array}$ & $\begin{array}{l}35 \\
16 \\
37 \\
35 \\
30 \\
46 \\
18 \\
29 \\
56 \\
32 \\
36 \\
17 \\
32\end{array}$ & $\begin{array}{l}0 \\
0 \\
0 \\
0 \\
0 \\
0 \\
0 \\
0 \\
0 \\
0 \\
0 \\
0 \\
0\end{array}$ & $\begin{array}{r}108 \\
109 \\
105 \\
79 \\
69 \\
94 \\
103 \\
108 \\
84 \\
108 \\
85 \\
91 \\
95\end{array}$ & $\begin{array}{l}78 \\
76 \\
85 \\
75 \\
54 \\
74 \\
78 \\
54 \\
56 \\
71 \\
62 \\
71 \\
70\end{array}$ \\
\hline 1003 & 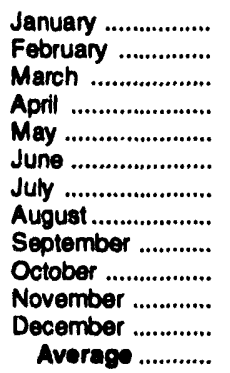 & $\begin{array}{r}73 \\
80 \\
61 \\
97 \\
81 \\
55 \\
52 \\
56 \\
101 \\
122 \\
90 \\
118 \\
62\end{array}$ & $\begin{array}{l}0 \\
0 \\
0 \\
0 \\
0 \\
0 \\
0 \\
0 \\
0 \\
0 \\
0 \\
0 \\
0\end{array}$ & $\begin{array}{r}70 \\
62 \\
122 \\
170 \\
222 \\
160 \\
215 \\
180 \\
113 \\
115 \\
162 \\
108 \\
142\end{array}$ & $\begin{array}{r}70 \\
61 \\
115 \\
170 \\
222 \\
160 \\
215 \\
161 \\
113 \\
93 \\
155 \\
101 \\
137\end{array}$ & $\begin{array}{l}37 \\
21 \\
26 \\
18 \\
38 \\
29 \\
49 \\
30 \\
28 \\
30 \\
23 \\
14 \\
29\end{array}$ & $\begin{array}{l}0 \\
0 \\
0 \\
0 \\
0 \\
0 \\
0 \\
0 \\
0 \\
0 \\
0 \\
0 \\
0\end{array}$ & $\begin{array}{r}0 \\
0 \\
0 \\
32 \\
32 \\
77 \\
157 \\
26 \\
57 \\
176 \\
56 \\
38 \\
55\end{array}$ & $\begin{array}{r}0 \\
0 \\
0 \\
32 \\
32 \\
51 \\
134 \\
0 \\
29 \\
123 \\
32 \\
0 \\
30\end{array}$ & $\begin{array}{l}44 \\
19 \\
21 \\
61 \\
42 \\
20 \\
41 \\
37 \\
54 \\
33 \\
30 \\
42 \\
37\end{array}$ & $\begin{array}{l}0 \\
0 \\
0 \\
0 \\
0 \\
0 \\
0 \\
0 \\
0 \\
0 \\
0 \\
0 \\
0\end{array}$ & $\begin{array}{r}59 \\
72 \\
92 \\
78 \\
68 \\
77 \\
62 \\
50 \\
70 \\
69 \\
68 \\
103 \\
74\end{array}$ & $\begin{array}{l}\mathbf{4 8} \\
\mathbf{5 8} \\
\mathbf{7 1} \\
55 \\
51 \\
55 \\
53 \\
37 \\
55 \\
54 \\
55 \\
71 \\
55\end{array}$ \\
\hline
\end{tabular}

See footnotes at end of table. 
Table S3. Crude OIl and Petroleum Product Imports, 1981 - Present (Continued) (Thnusand Barrels per Day)

\begin{tabular}{|c|c|c|c|c|c|c|c|c|c|c|c|}
\hline & \multirow{3}{*}{ Yoarmonth } & \multicolumn{6}{|c|}{ Imports trom Non-OPEC sources" } & \multirow{2}{*}{\multicolumn{2}{|c|}{$\begin{array}{l}\text { Total } \\
\text { Non- } \\
\text { Opge }\end{array}$}} & \multirow{2}{*}{\multicolumn{2}{|c|}{$\begin{array}{c}\text { Toul } \\
\text { Inoent: }\end{array}$}} \\
\hline & & \multicolumn{2}{|c|}{$\begin{array}{l}\text { United } \\
\text { Kinodem }\end{array}$} & \multicolumn{2}{|c|}{$\begin{array}{l}\text { Vroin } \\
\text { Inlends }\end{array}$} & \multicolumn{2}{|c|}{$\begin{array}{l}\text { Other } \\
\text { Non: } \\
\text { Opac }\end{array}$} & & & & \\
\hline & & Total & Crude oll & Total & Crude OII & Total & Crudo oll & Total & Crude oll & Total & Crude oll \\
\hline 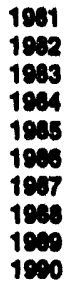 & 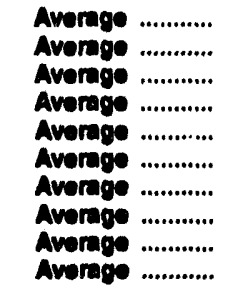 & $\begin{array}{l}375 \\
458 \\
382 \\
402 \\
310 \\
380 \\
382 \\
315 \\
215 \\
189\end{array}$ & $\begin{array}{l}369 \\
441 \\
365 \\
378 \\
278 \\
317 \\
304 \\
254 \\
160 \\
165\end{array}$ & $\begin{array}{l}327 \\
316 \\
282 \\
294 \\
247 \\
244 \\
272 \\
242 \\
321 \\
282\end{array}$ & $\begin{array}{l}0 \\
0 \\
0 \\
0 \\
0 \\
0 \\
0 \\
0 \\
0 \\
0\end{array}$ & $\begin{array}{l}238 \\
308 \\
378 \\
411 \\
394 \\
428 \\
450 \\
407 \\
457 \\
417\end{array}$ & $\begin{array}{l}163 \\
174 \\
215 \\
210 \\
137 \\
144 \\
168 \\
183 \\
197 \\
180\end{array}$ & $\begin{array}{l}2,672 \\
2,968 \\
3,180 \\
3,380 \\
3,237 \\
3,387 \\
3,617 \\
3,802 \\
3,921 \\
3,721\end{array}$ & $\begin{array}{l}1,474 \\
1,754 \\
1,858 \\
1,914 \\
1,085 \\
2,008 \\
2,274 \\
2,411 \\
2,407 \\
2,391\end{array}$ & $\begin{array}{l}8,908 \\
5,113 \\
8,081 \\
8,487 \\
8,097 \\
8,204 \\
6,078 \\
7,402 \\
8,001 \\
0,018\end{array}$ & $\begin{array}{l}4,303 \\
3,406 \\
3,320 \\
3,420 \\
3,201 \\
4,178 \\
4,674 \\
5,107 \\
5,849 \\
5,004\end{array}$ \\
\hline 1091 & 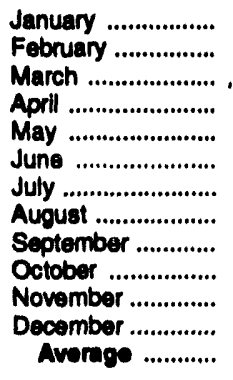 & $\begin{array}{r}32 \\
34 \\
48 \\
81 \\
222 \\
105 \\
228 \\
254 \\
218 \\
201 \\
84 \\
154 \\
138\end{array}$ & $\begin{array}{r}19 \\
21 \\
19 \\
37 \\
188 \\
70 \\
164 \\
217 \\
194 \\
168 \\
18 \\
151 \\
108\end{array}$ & $\begin{array}{l}261 \\
222 \\
214 \\
245 \\
264 \\
234 \\
191 \\
208 \\
269 \\
262 \\
264 \\
286 \\
243\end{array}$ & $\begin{array}{l}0 \\
0 \\
0 \\
0 \\
0 \\
0 \\
0 \\
0 \\
0 \\
0 \\
0 \\
0 \\
0\end{array}$ & $\begin{array}{l}235 \\
180 \\
179 \\
256 \\
239 \\
349 \\
384 \\
369 \\
374 \\
252 \\
335 \\
229 \\
282\end{array}$ & $\begin{array}{r}91 \\
96 \\
60 \\
99 \\
63 \\
189 \\
275 \\
197 \\
197 \\
139 \\
130 \\
104 \\
137\end{array}$ & $\begin{array}{l}3,205 \\
3,051 \\
3,023 \\
3,674 \\
3,794 \\
3,747 \\
3,524 \\
4,067 \\
3,871 \\
3,444 \\
3,444 \\
3,546 \\
3,536\end{array}$ & $\begin{array}{l}2,195 \\
2,221 \\
2,133 \\
2,470 \\
2,524 \\
2,587 \\
2,430 \\
2,699 \\
2,608 \\
2,340 \\
2,200 \\
2,448 \\
2,405\end{array}$ & $\begin{array}{l}7,103 \\
6,865 \\
6,646 \\
7,418 \\
8,518 \\
8,245 \\
7,755 \\
8,670 \\
7,826 \\
7,467 \\
7,615 \\
7,307 \\
7,627\end{array}$ & $\begin{array}{l}\mathbf{5 , 2 9 0} \\
\mathbf{5 , 4 8 5} \\
\mathbf{5 , 1 6 8} \\
\mathbf{5 , 6 2 9} \\
\mathbf{6 , 3 6 3} \\
\mathbf{6 , 3 3 4} \\
\mathbf{5 , 9 5 5} \\
\mathbf{6 , 6 4 5} \\
\mathbf{5 , 6 1 2} \\
\mathbf{5 , 6 8 3} \\
\mathbf{5 , 5 2 8} \\
\mathbf{5 , 6 6 5} \\
\mathbf{8 , 7 0 2}\end{array}$ \\
\hline 1902 & 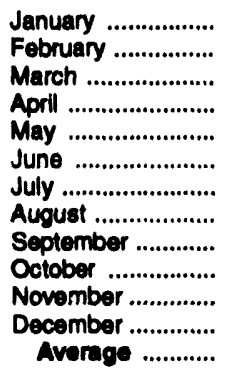 & $\begin{array}{r}129 \\
63 \\
79 \\
157 \\
198 \\
248 \\
354 \\
295 \\
341 \\
411 \\
336 \\
148 \\
230\end{array}$ & $\begin{array}{r}115 \\
0 \\
52 \\
128 \\
180 \\
206 \\
337 \\
282 \\
291 \\
411 \\
285 \\
110 \\
200\end{array}$ & $\begin{array}{l}250 \\
222 \\
202 \\
234 \\
246 \\
266 \\
260 \\
263 \\
217 \\
254 \\
274 \\
273 \\
240\end{array}$ & $\begin{array}{l}0 \\
0 \\
0 \\
0 \\
0 \\
0 \\
0 \\
0 \\
0 \\
0 \\
0 \\
0 \\
0\end{array}$ & $\begin{array}{l}208 \\
196 \\
345 \\
458 \\
467 \\
297 \\
415 \\
464 \\
382 \\
279 \\
219 \\
283 \\
335\end{array}$ & $\begin{array}{r}59 \\
50 \\
114 \\
212 \\
225 \\
95 \\
152 \\
357 \\
160 \\
144 \\
124 \\
92 \\
149\end{array}$ & $\begin{array}{l}3,488 \\
3,278 \\
3,462 \\
4,007 \\
3,705 \\
3,917 \\
4,140 \\
4,116 \\
3,904 \\
3,998 \\
3,786 \\
3,734 \\
3,786\end{array}$ & $\begin{array}{l}2,402 \\
2,184 \\
2,380 \\
2,793 \\
2,633 \\
2,711 \\
3,024 \\
2,984 \\
2,687 \\
2,964 \\
2,745 \\
2,556 \\
2,676\end{array}$ & $\begin{array}{l}7,712 \\
6,827 \\
7,068 \\
8,092 \\
7,823 \\
7,946 \\
8,479 \\
8,260 \\
4,178 \\
8,505 \\
7,872 \\
7,839 \\
7,898\end{array}$ & $\begin{array}{l}\mathbf{5 , 9 6 8} \\
\mathbf{5 , 0 7 9} \\
\mathbf{5 , 3 2 1} \\
6,127 \\
\mathbf{6 , 0 8 0} \\
\mathbf{6 , 1 7 1} \\
6,796 \\
\mathbf{6 , 4 5 7} \\
\mathbf{6 , 2 1 8} \\
\mathbf{6 , 6 9 8} \\
\mathbf{6 , 1 2 1} \\
\mathbf{5 , 9 3 7} \\
\mathbf{6 , 0 0 3}\end{array}$ \\
\hline $1 \cos$ & 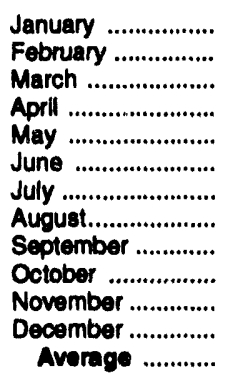 & $\begin{array}{l}229 \\
173 \\
332 \\
413 \\
522 \\
458 \\
292 \\
343 \\
286 \\
353 \\
351 \\
432 \\
350\end{array}$ & $\begin{array}{l}201 \\
127 \\
298 \\
337 \\
495 \\
408 \\
247 \\
323 \\
217 \\
338 \\
340 \\
403 \\
312\end{array}$ & $\begin{array}{l}252 \\
244 \\
244 \\
245 \\
279 \\
290 \\
202 \\
256 \\
184 \\
236 \\
330 \\
288 \\
254\end{array}$ & $\begin{array}{l}0 \\
0 \\
0 \\
0 \\
0 \\
0 \\
0 \\
0 \\
0 \\
0 \\
0 \\
0 \\
0\end{array}$ & $\begin{array}{l}325 \\
223 \\
393 \\
472 \\
363 \\
581 \\
600 \\
556 \\
552 \\
453 \\
503 \\
394 \\
452\end{array}$ & $\begin{array}{l}104 \\
151 \\
186 \\
243 \\
152 \\
405 \\
299 \\
356 \\
251 \\
233 \\
270 \\
231 \\
240\end{array}$ & $\begin{array}{l}3,690 \\
3,438 \\
3,944 \\
4,087 \\
4,298 \\
4,377 \\
4,705 \\
4,257 \\
4,468 \\
4,838 \\
4,624 \\
4,402 \\
4,268\end{array}$ & $\begin{array}{l}2,602 \\
2,457 \\
2,859 \\
2,933 \\
3,123 \\
3,380 \\
3,477 \\
3,088 \\
3,124 \\
3,562 \\
3,327 \\
3,214 \\
3,100\end{array}$ & $\begin{array}{l}8,004 \\
7,948 \\
8,285 \\
8,768 \\
8,663 \\
8,805 \\
9,219 \\
8,429 \\
8,531 \\
9,197 \\
8,903 \\
8,645 \\
8,620\end{array}$ & $\begin{array}{l}6,292 \\
6,156 \\
6,486 \\
6,928 \\
6,809 \\
7,201 \\
7,289 \\
6,641 \\
6,681 \\
7,181 \\
6,997 \\
6,838 \\
6,787\end{array}$ \\
\hline
\end{tabular}

Includes petroleum imported into the United States indirectly from members of the Organization of Petroleum Exporting Countries (OPEC) primarily from Carlbbean and West European areas as petroleum products that were refined from crude oil produced by OPEC.

IImports trom the Neutral Zone between Kuwait and Saudl Arabla are included in imports from Saudi Arabia.

Excludes petroleum imported into the United States indirectly from members of the Organization of Petroleum Exporting Countrles (OPEC), primarliy from Caribbean and West European areas, as petroleum products that were refined from crude oll produced by OPEC.

Imports from other States in the former U.S.S.R. may be included in imports from Russla for the years 1981 through 1992.

- A small amount of iranian crude oll entered the United States in January 1988 from the Virgin Isiands. This oll originated in Iran and was exported to the Virgin lalands prior to the signing of Executive Order 12613 on October 29, 1987.

(s) $=$ Less than 500 barrels per day.

Notes: - Geographic coverage is the 50 States and the District of Columbia. - Totals may not equal sum of components due to independent rounding. Source: See Summary Statistice Table and Figure Sources. 
Figure S5. Finished Motor Gasoline Supply and Disposition, 1981 - Present

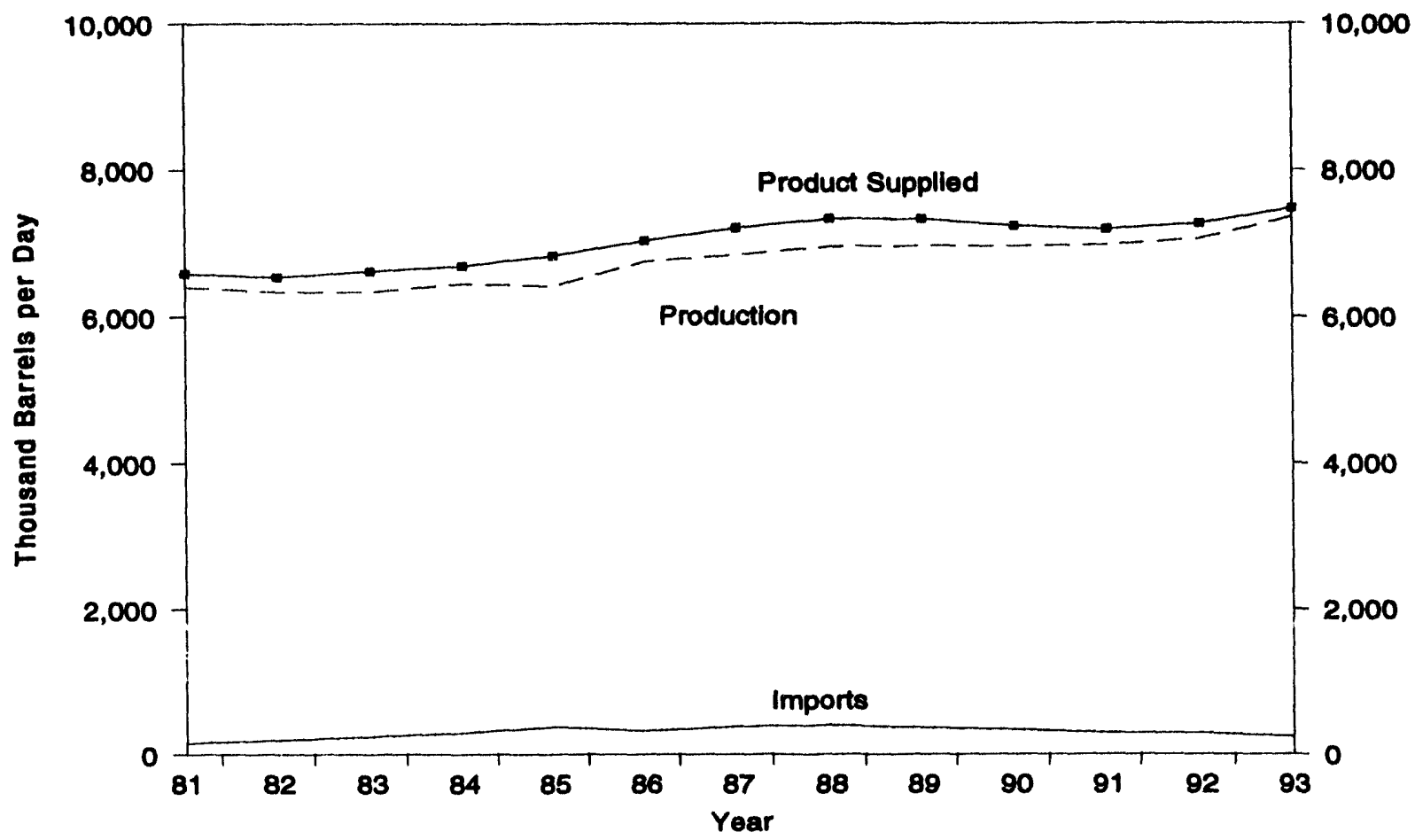

Source: Energy Information Administration, Petroleum Supply Annual, Table S4. See Summary Statistics Table and Figure Sources.

Flgure S6. Motor Gasoline Ending Stocks, 1981 - Present

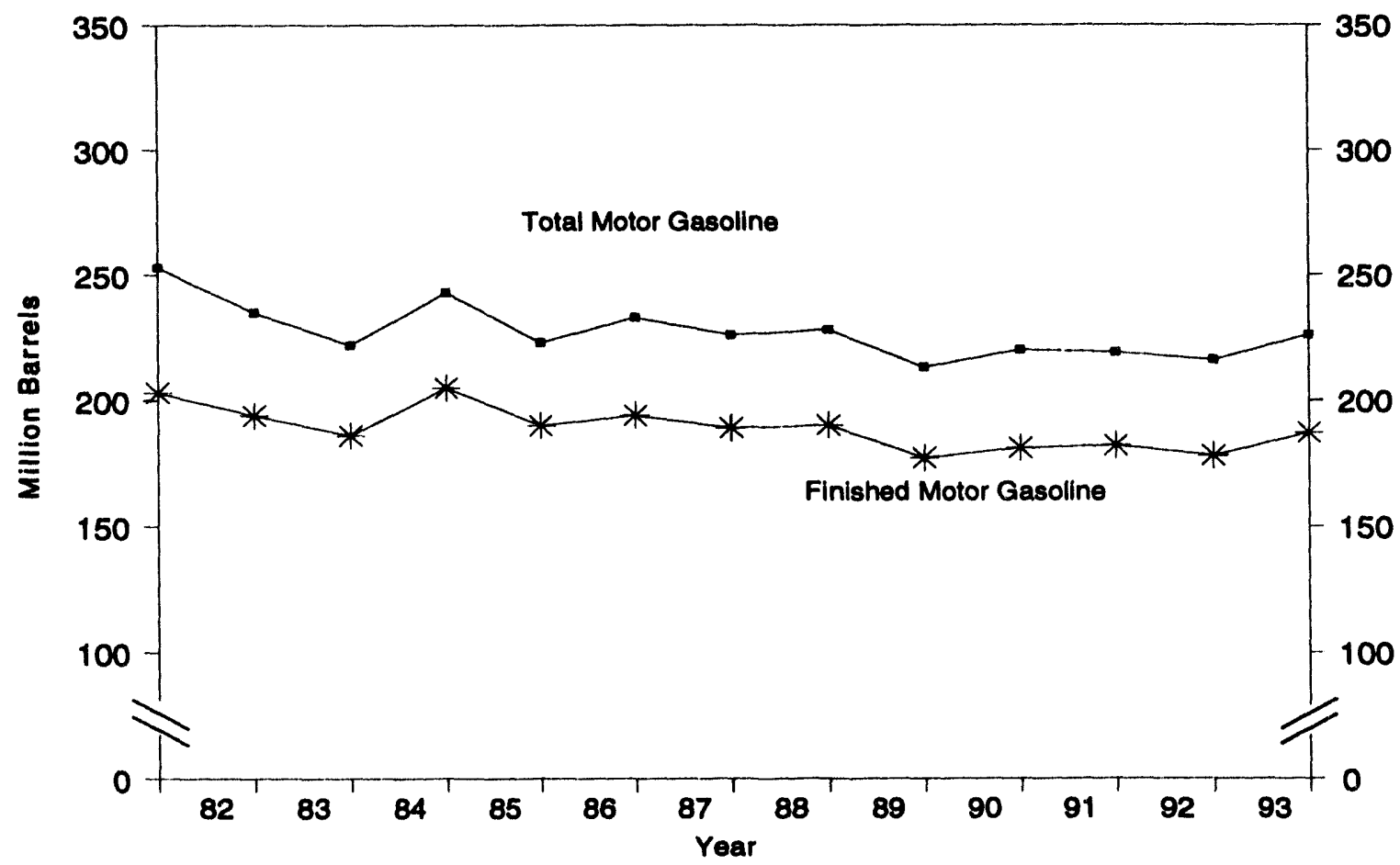

Note: Total motor gasoline includes motor gasoline blending components and finished motor gasoline.

Source: Energy Information Administration, Petroleum Supply Annual, Table S4. See Summary Statistics Table and Figure Sources. 
Table S4. Finished Motor Gasoline Supply and Disposition, 1981 - Present (Thousand Barrels per Day, Except Where Noted)

\begin{tabular}{|c|c|c|c|c|c|c|c|c|c|}
\hline & \multirow{3}{*}{ Yearmonth } & \multicolumn{2}{|c|}{ Supply } & \multicolumn{3}{|c|}{ Diepostiton } & \multirow{2}{*}{\multicolumn{2}{|c|}{$\begin{array}{l}\begin{array}{l}\text { Ending stooks } \\
\text { (Million Barrols) }\end{array} \\
\text { Motor Cesolin }\end{array}$}} & \multirow{3}{*}{$\begin{array}{l}\text { Ending Etocks } \\
\text { (Million Barrole) } \\
\\
\text { Oxyounates }\end{array}$} \\
\hline & & \multirow{2}{*}{$\begin{array}{l}\text { Total } \\
\text { Production }\end{array}$} & \multirow[b]{2}{*}{ Imports" } & \multirow{2}{*}{$\begin{array}{l}\text { Btook } \\
\text { Change,d }\end{array}$} & \multirow[b]{2}{*}{ Exports } & \multirow{2}{*}{$\begin{array}{l}\text { Product } \\
\text { supplled }\end{array}$} & & & \\
\hline & & & & & & & Totap & Finlohod & \\
\hline $\begin{array}{l}1091 \\
1082 \\
1983 \\
1984 \\
1985 \\
1989 \\
1907 \\
1009 \\
1009 \\
1000\end{array}$ & 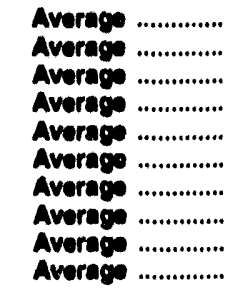 & $\begin{array}{l}6,408 \\
6,308 \\
6,340 \\
6,485 \\
6,410 \\
6,762 \\
6,841 \\
6,988 \\
6,983 \\
6,969\end{array}$ & $\begin{array}{l}187 \\
197 \\
247 \\
290 \\
391 \\
396 \\
324 \\
408 \\
309 \\
342\end{array}$ & $\begin{array}{r}1.28 \\
-25 \\
-45 \\
54 \\
41 \\
11 \\
-15 \\
3 \\
-35 \\
10\end{array}$ & $\begin{array}{r}2 \\
20 \\
10 \\
6 \\
10 \\
33 \\
35 \\
22 \\
39 \\
85\end{array}$ & $\begin{array}{l}6,608 \\
6,639 \\
6,622 \\
6,693 \\
6,831 \\
7,034 \\
7,200 \\
7,330 \\
7,328 \\
7,238\end{array}$ & $\begin{array}{l}283 \\
1235 \\
222 \\
243 \\
223 \\
223 \\
220 \\
228 \\
213 \\
220\end{array}$ & $\begin{array}{l}203 \\
194 \\
180 \\
205 \\
180 \\
184 \\
189 \\
190 \\
177 \\
181\end{array}$ & $\begin{array}{l}- \\
= \\
= \\
= \\
= \\
-\end{array}$ \\
\hline 1901 & 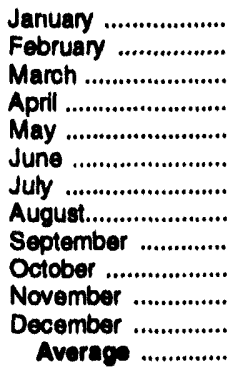 & $\begin{array}{l}6,629 \\
6,573 \\
6,643 \\
6,742 \\
7,063 \\
7,351 \\
7,274 \\
7,247 \\
7,030 \\
6,749 \\
7,018 \\
7,354 \\
6,975\end{array}$ & $\begin{array}{l}228 \\
115 \\
235 \\
381 \\
528 \\
384 \\
232 \\
385 \\
312 \\
236 \\
322 \\
216 \\
297\end{array}$ & $\begin{array}{r}162 \\
-252 \\
-236 \\
-67 \\
95 \\
160 \\
-177 \\
7 \\
195 \\
-354 \\
228 \\
267 \\
3\end{array}$ & $\begin{array}{r}60 \\
102 \\
97 \\
53 \\
59 \\
99 \\
122 \\
98 \\
63 \\
58 \\
104 \\
79 \\
82\end{array}$ & $\begin{array}{l}8,645 \\
6,838 \\
7,017 \\
7,137 \\
7,437 \\
7,456 \\
7,561 \\
7,528 \\
7,083 \\
7,281 \\
7,008 \\
7,224 \\
7,188\end{array}$ & $\begin{array}{r}225 \\
219 \\
210 \\
205 \\
209 \\
214 \\
208 \\
209 \\
216 \\
203 \\
209 \\
219 \\
-\end{array}$ & $\begin{array}{r}186 \\
179 \\
171 \\
169 \\
172 \\
177 \\
172 \\
172 \\
178 \\
167 \\
173 \\
182 \\
-\end{array}$ & $\begin{array}{l}- \\
\ddot{-} \\
\ddot{.} \\
\ddot{-} \\
\ddot{-} \\
\ddot{-} \\
- \\
-\end{array}$ \\
\hline 1092 & 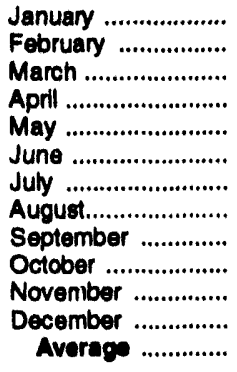 & $\begin{array}{l}7,013 \\
6,726 \\
6,683 \\
6,954 \\
7,092 \\
7,198 \\
7,195 \\
6,817 \\
7,071 \\
7,198 \\
7,323 \\
7,411 \\
7,058\end{array}$ & $\begin{array}{l}246 \\
275 \\
247 \\
428 \\
392 \\
424 \\
303 \\
240 \\
418 \\
193 \\
170 \\
202 \\
294\end{array}$ & $\begin{array}{r}304 \\
-22 \\
-278 \\
54 \\
74 \\
76 \\
-249 \\
-446 \\
60 \\
-41 \\
318 \\
32 \\
-11\end{array}$ & $\begin{array}{r}87 \\
59 \\
71 \\
90 \\
82 \\
86 \\
108 \\
123 \\
85 \\
94 \\
74 \\
184 \\
80\end{array}$ & $\begin{array}{l}6,869 \\
6,963 \\
7,137 \\
7,238 \\
7,328 \\
7,460 \\
7,639 \\
7,380 \\
7,344 \\
7,338 \\
7,102 \\
7,396 \\
7,268\end{array}$ & $\begin{array}{l}229 \\
230 \\
220 \\
218 \\
220 \\
225 \\
215 \\
201 \\
206 \\
204 \\
214 \\
216 \\
-\end{array}$ & $\begin{array}{l}191 \\
191 \\
182 \\
183 \\
186 \\
188 \\
180 \\
167 \\
168 \\
167 \\
177 \\
178 \\
-\end{array}$ & $\begin{array}{l}\ddot{-} \\
\overline{-} \\
= \\
\ddot{-} \\
= \\
\overline{-} \\
\overline{-} \\
-\end{array}$ \\
\hline 1093 & 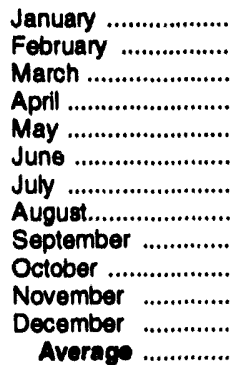 & $\begin{array}{l}7,228 \\
7,144 \\
6,904 \\
7,126 \\
7,446 \\
7,442 \\
7,337 \\
7,335 \\
7,573 \\
7,394 \\
7,652 \\
7,725 \\
7,360\end{array}$ & $\begin{array}{l}204 \\
216 \\
177 \\
253 \\
323 \\
251 \\
300 \\
283 \\
267 \\
210 \\
252 \\
231 \\
247\end{array}$ & $\begin{array}{r}652 \\
149 \\
-417 \\
-168 \\
93 \\
-88 \\
-240 \\
-323 \\
148 \\
142 \\
245 \\
132 \\
28\end{array}$ & $\begin{array}{r}142 \\
99 \\
109 \\
111 \\
90 \\
81 \\
92 \\
77 \\
85 \\
80 \\
126 \\
162 \\
105\end{array}$ & $\begin{array}{l}6,639 \\
7,112 \\
7,389 \\
7,435 \\
7,585 \\
7,700 \\
7,785 \\
7,864 \\
7,607 \\
7,382 \\
7,533 \\
7,661 \\
7,476\end{array}$ & $\begin{array}{r}240 \\
245 \\
230 \\
225 \\
225 \\
221 \\
215 \\
202 \\
208 \\
212 \\
222 \\
226 \\
-\end{array}$ & $\begin{array}{r}198 \\
202 \\
189 \\
184 \\
187 \\
184 \\
177 \\
167 \\
171 \\
176 \\
183 \\
187 \\
-\end{array}$ & $\begin{array}{l}15 \\
14 \\
15 \\
15 \\
17 \\
18 \\
20 \\
21 \\
19 \\
18 \\
16 \\
13 \\
-\end{array}$ \\
\hline
\end{tabular}

Stocks are totals as of end of period.

b Beginning in 1993, motor gasoline production and product supplied includes blending of fuel ethanol and an adjustment to correct for the imbalance of motor gasoline blending components. Reler to Appendix B. Explanatory Note 11 for 1992 new basis product supplled.

c Beginning in 1981, excludes blending components.

- A negative number indlcates a decrease in stocks and a positive number indicates an increase.

- includes motor gasoline blending components but excludes stocks of oxygenates.

1 In January 1981 and 1983, numerous respondents were added to surveys affecting stocks reported and stock change calculations. Stock changes are calculated using new basis stock levels. See Summary Statistics Explanatory Note 4.

Notes: - Geographic coverage is the 50 States and the District of Columbia. - Totals may not equal sum of components due to independent rounding

Source: See Summary Statistics Table and Figure Sources. 
Figure 87. Distillate Fuol Oll 8upply and Dieposition, 1981 - Present

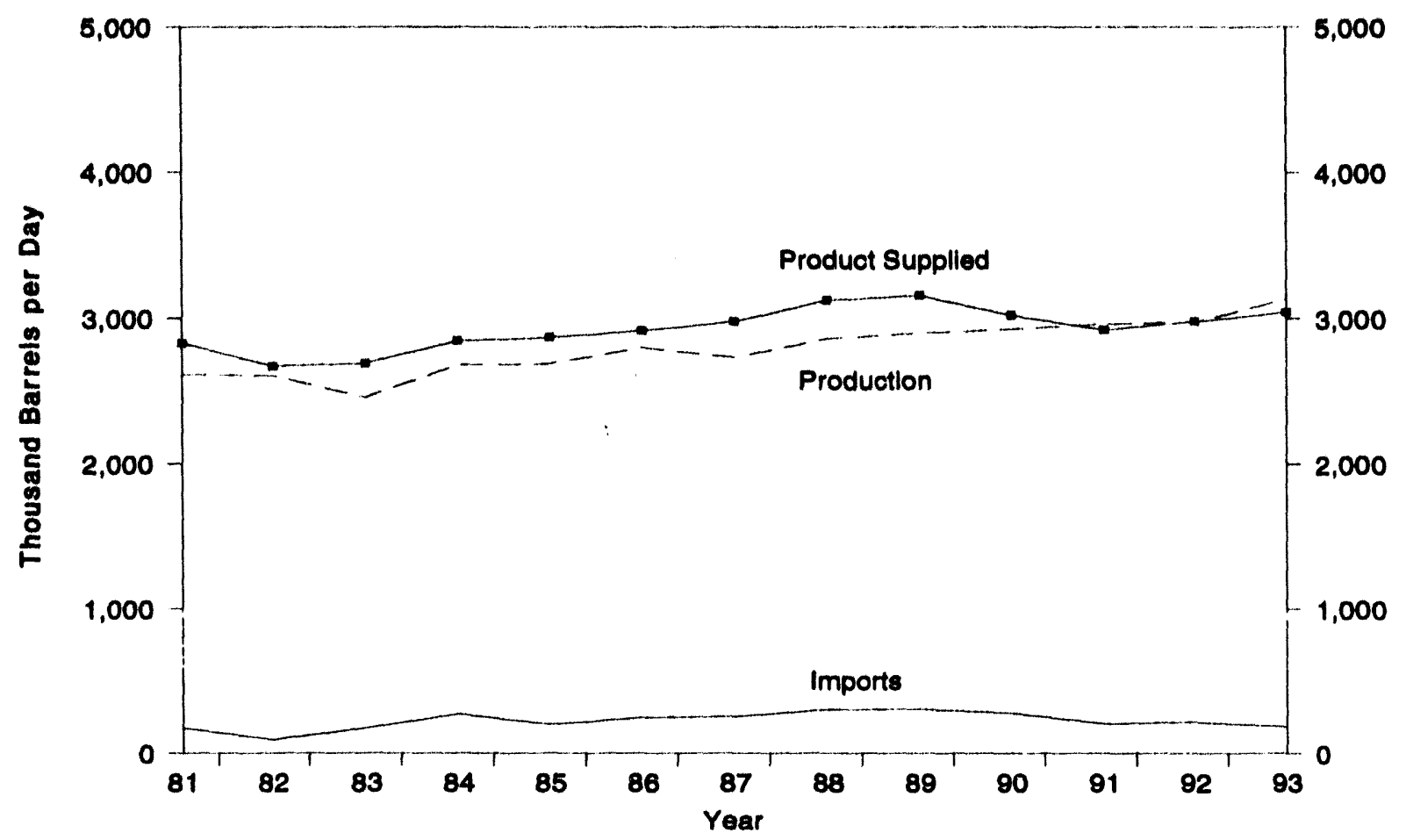

Source: Energy Information Administration, Petroleum Supply Annual, Table S5. See Summary Statistice Table and Figure Sources.

Figure S8. Distlliate Fuel Oll Ending Stocks, 1981 - Present

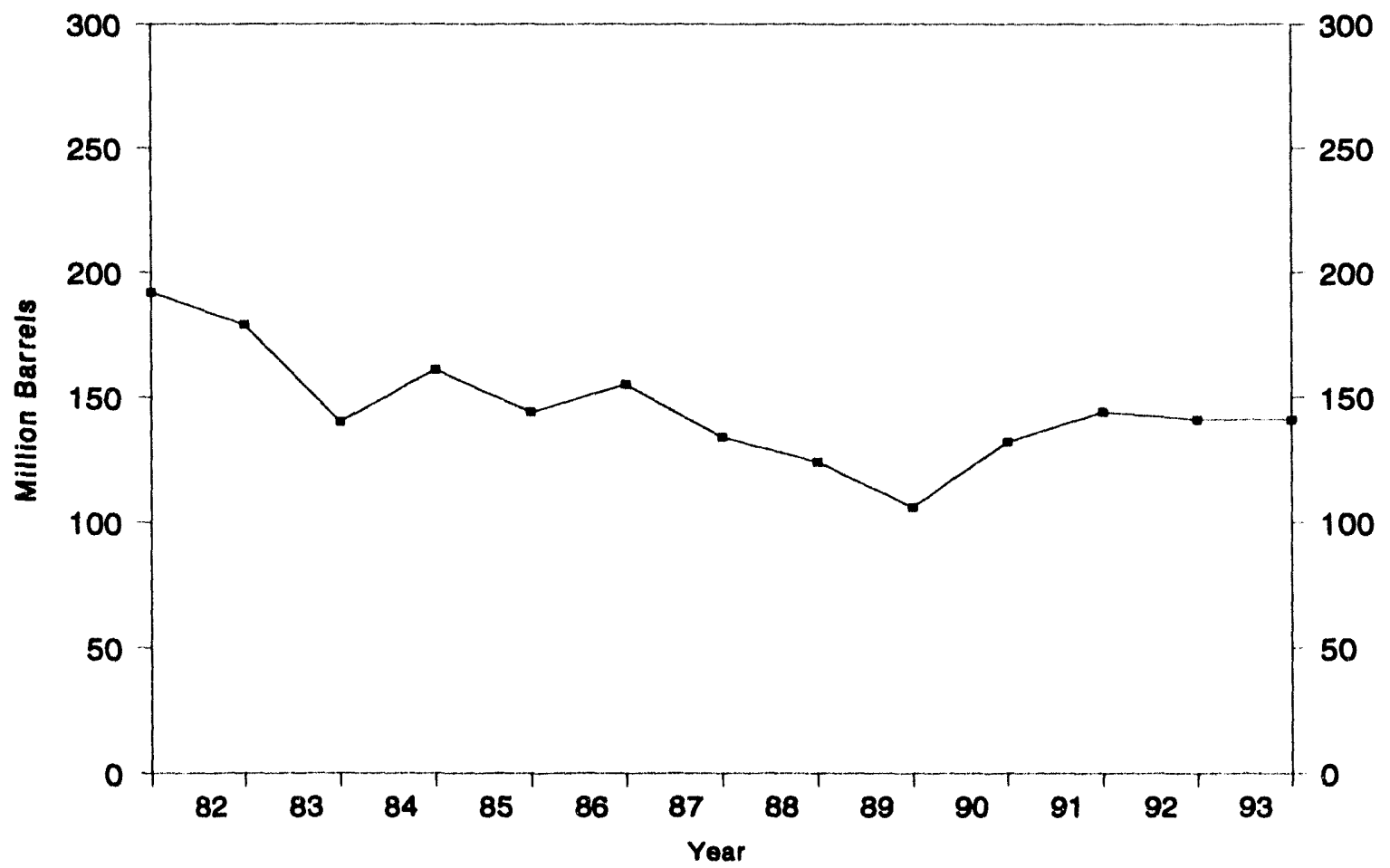

Source: Energy information Administration, Petroleum Supply Annual, Table S5. See Summary Statistics Table and Figure Sources. 
Table 85. Dietillate Fuel Oll Supply and Dispoaltion, 1881 - Precent (Thousand Barrels per Day, Except Where Noted)

\begin{tabular}{|c|c|c|c|c|c|c|c|c|c|}
\hline \multirow{3}{*}{\multicolumn{2}{|c|}{ Youmbonth }} & \multicolumn{2}{|c|}{ supply } & \multicolumn{3}{|c|}{ Dieporition } & \multirow{2}{*}{\multicolumn{3}{|c|}{$\begin{array}{l}\text { Ending stooke" } \\
\text { (Million Barrote) }\end{array}$}} \\
\hline & & \multirow[b]{2}{*}{$\begin{array}{c}\text { Totel } \\
\text { Produetion }\end{array}$} & \multirow[b]{2}{*}{ Importe } & \multirow[b]{2}{*}{$\begin{array}{l}\text { Crook } \\
\text { Chenge }\end{array}$} & \multirow[b]{2}{*}{ suports } & \multirow[b]{2}{*}{$\begin{array}{l}\text { roduct } \\
\text { upplled }\end{array}$} & & & \\
\hline & & & & & & & Total & $\begin{array}{l}\text { 0.05\% guflur } \\
\text { and Under }\end{array}$ & $\begin{array}{l}\text { Ornewer then } \\
\text { o.cos sulfur }\end{array}$ \\
\hline & 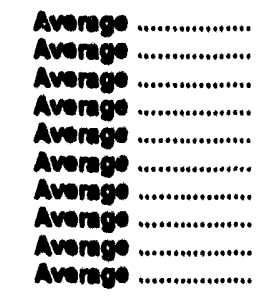 & $\begin{array}{l}2,013 \\
2,600 \\
2,400 \\
2,001 \\
2,097 \\
2,700 \\
2,791 \\
2,090 \\
2,000 \\
2,900\end{array}$ & $\begin{array}{r}173 \\
93 \\
174 \\
278 \\
200 \\
247 \\
248 \\
300 \\
305 \\
270\end{array}$ & $\begin{array}{r}-80 \\
-10 \\
70\end{array}$ & $\begin{array}{r}8 \\
74 \\
64 \\
81 \\
67 \\
100 \\
68 \\
67 \\
100\end{array}$ & $\begin{array}{l}2,000 \\
2,071 \\
2,090 \\
2,40 \\
2,000 \\
2,914 \\
2,97 \\
3,192 \\
3,197 \\
3,021\end{array}$ & $\begin{array}{l}198 \\
d 170 \\
140 \\
181 \\
144 \\
185 \\
134 \\
184 \\
103 \\
182\end{array}$ & $\begin{array}{l}\overline{-} \\
= \\
= \\
= \\
=\end{array}$ & $\begin{array}{l}\bar{z} \\
\bar{z} \\
\overline{ } \\
\overline{-}\end{array}$ \\
\hline teer & 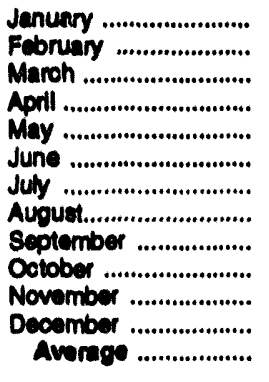 & $\begin{array}{l}2,845 \\
2,870 \\
2,806 \\
2,819 \\
2,929 \\
2,941 \\
2,998 \\
2,901 \\
3,065 \\
3,040 \\
3,103 \\
3,107 \\
2,902\end{array}$ & $\begin{array}{l}192 \\
139 \\
208 \\
288 \\
188 \\
200 \\
186 \\
168 \\
237 \\
207 \\
249 \\
252 \\
208\end{array}$ & $\begin{array}{r}-602 \\
-350 \\
-112 \\
150 \\
132 \\
225 \\
358 \\
214 \\
291 \\
-59 \\
208 \\
-30 \\
31\end{array}$ & $\begin{array}{r}332 \\
393 \\
198 \\
81 \\
218 \\
150 \\
149 \\
144 \\
138 \\
269 \\
224 \\
302 \\
218\end{array}$ & $\begin{array}{l}3,387 \\
2,076 \\
2,084 \\
2,839 \\
2,768 \\
2,775 \\
2,848 \\
2,770 \\
2,868 \\
3,047 \\
2,021 \\
3,087 \\
2,921\end{array}$ & $\begin{array}{r}112 \\
102 \\
90 \\
103 \\
107 \\
114 \\
128 \\
131 \\
140 \\
138 \\
144 \\
144 \\
-\end{array}$ & $\begin{array}{l}= \\
= \\
= \\
= \\
= \\
= \\
=\end{array}$ & $\begin{array}{l}= \\
= \\
= \\
= \\
= \\
=\end{array}$ \\
\hline 1092 & 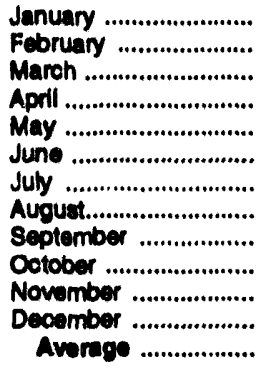 & $\begin{array}{l}2,818 \\
2,681 \\
2,749 \\
2,980 \\
2,933 \\
2,995 \\
3,087 \\
2,865 \\
2,983 \\
3,251 \\
3,240 \\
3,179 \\
2,974\end{array}$ & $\begin{array}{l}232 \\
217 \\
238 \\
202 \\
179 \\
157 \\
172 \\
229 \\
237 \\
283 \\
238 \\
229 \\
216\end{array}$ & $\begin{array}{r}-541 \\
-610 \\
-358 \\
-185 \\
139 \\
268 \\
328 \\
262 \\
168 \\
280 \\
316 \\
-183 \\
-8\end{array}$ & $\begin{array}{l}360 \\
278 \\
138 \\
278 \\
222 \\
205 \\
201 \\
127 \\
145 \\
169 \\
230 \\
276 \\
210\end{array}$ & $\begin{array}{l}3,231 \\
3,210 \\
3,207 \\
3,039 \\
2,768 \\
2,679 \\
2,710 \\
2,705 \\
2,908 \\
3,058 \\
2,929 \\
3,316 \\
2,970\end{array}$ & $\begin{array}{r}127 \\
109 \\
98 \\
92 \\
98 \\
104 \\
116 \\
123 \\
128 \\
137 \\
146 \\
141 \\
-\end{array}$ & $\begin{array}{l}- \\
= \\
= \\
= \\
= \\
= \\
=\end{array}$ & $\begin{array}{l}\overline{-} \\
\overline{-} \\
\overline{-} \\
= \\
= \\
= \\
=\end{array}$ \\
\hline 1000 & 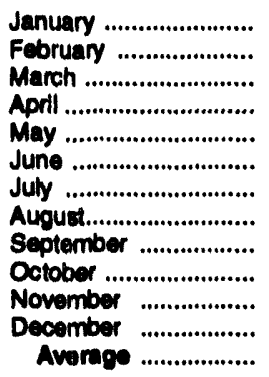 & $\begin{array}{l}2,914 \\
2,815 \\
2,919 \\
3,047 \\
2,994 \\
3,093 \\
3,188 \\
3,100 \\
3,205 \\
3,432 \\
3,474 \\
3,382 \\
3,132 \\
\end{array}$ & $\begin{array}{l}182 \\
224 \\
236 \\
209 \\
153 \\
168 \\
130 \\
169 \\
137 \\
242 \\
214 \\
160 \\
184\end{array}$ & $\begin{array}{r}-318 \\
-727 \\
-420 \\
71 \\
108 \\
241 \\
346 \\
243 \\
102 \\
453 \\
127 \\
-267 \\
1\end{array}$ & $\begin{array}{l}287 \\
301 \\
154 \\
241 \\
355 \\
158 \\
298 \\
198 \\
287 \\
237 \\
342 \\
453 \\
274\end{array}$ & $\begin{array}{l}3,128 \\
3,465 \\
3,420 \\
2,943 \\
2,685 \\
2,860 \\
2,674 \\
2,820 \\
2,973 \\
2,983 \\
3,218 \\
3,357 \\
3,041\end{array}$ & $\begin{array}{r}131 \\
110 \\
97 \\
99 \\
103 \\
110 \\
121 \\
128 \\
131 \\
145 \\
149 \\
141 \\
-\end{array}$ & $\begin{array}{l}15 \\
12 \\
11 \\
12 \\
12 \\
15 \\
21 \\
44 \\
48 \\
55 \\
64 \\
64 \\
-\end{array}$ & $\begin{array}{r}115 \\
89 \\
87 \\
88 \\
91 \\
95 \\
100 \\
84 \\
84 \\
90 \\
85 \\
77 \\
-\end{array}$ \\
\hline
\end{tabular}

axcludes 10,000 barrols per day in 1981 and 1982 prevloudy published as crude used directly.

b Stocks are totals as of end of period.

- A negative number indicates a decrease in stocks and a postive number indicates an increase.

- In January 1881 and 1983 , numerous reepondents were added to surveys afiecting stocks reported and stock change caloulatlons. Stock changes are calculated using new stock basis stock levels. See Summary Statistios Explanatory Note 4.

Notes: - Geographic coverage is the 60 Stites and the District of Columbla. - Totab may not equal sum of components due to independent rounding. Source: See summary Statistice Table and Floure Sources. 
Figure 89. Resldual Fuel Oll Bupply and Dispostion, 1981 - Present

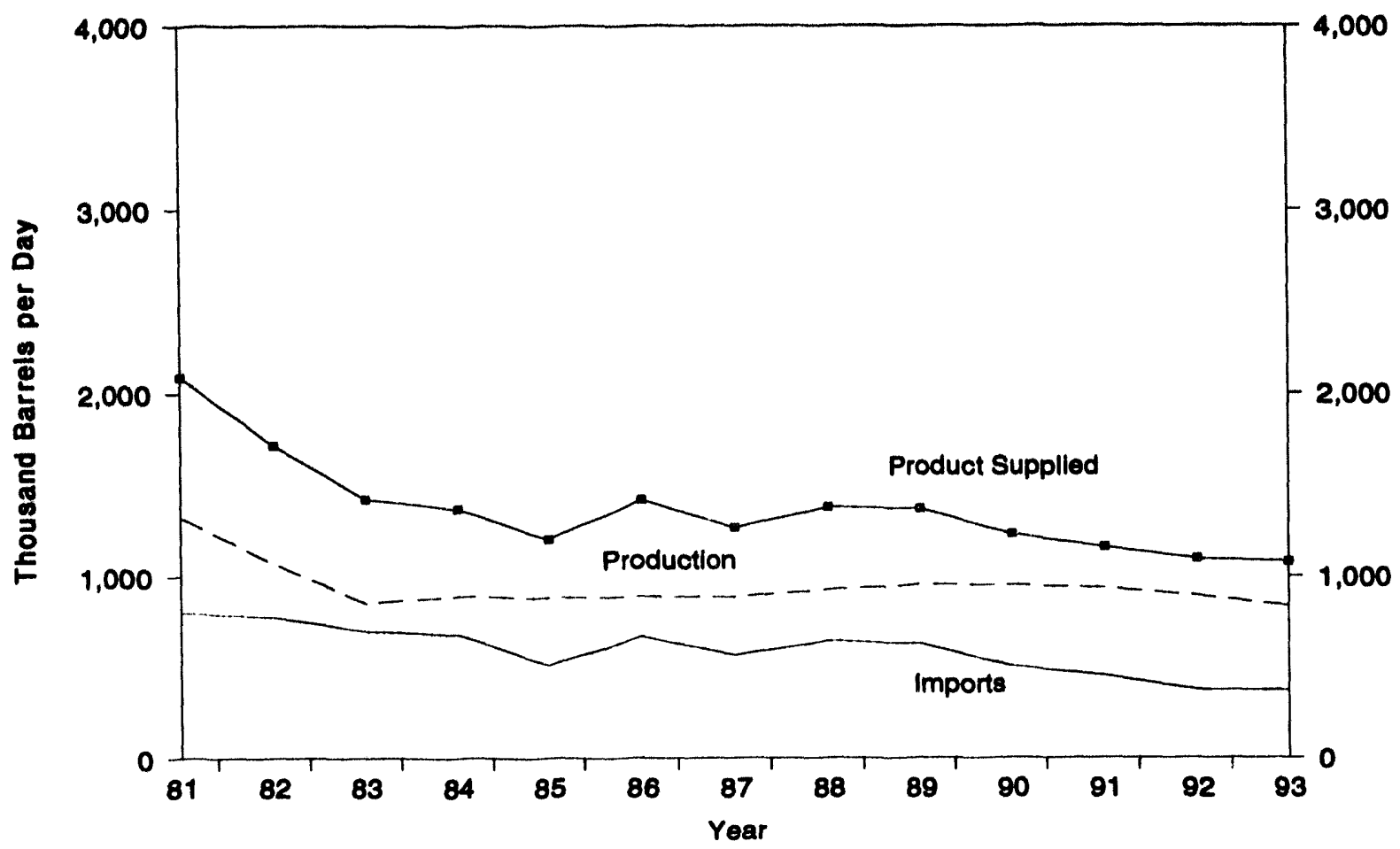

Source: Energy Information Administration, Petroleum Supply Annual, Table S6. See Summary Statistice Table and Figure Sources.

Figure S10. Residual Fuel Oll Ending Stocks, 1981 - Present

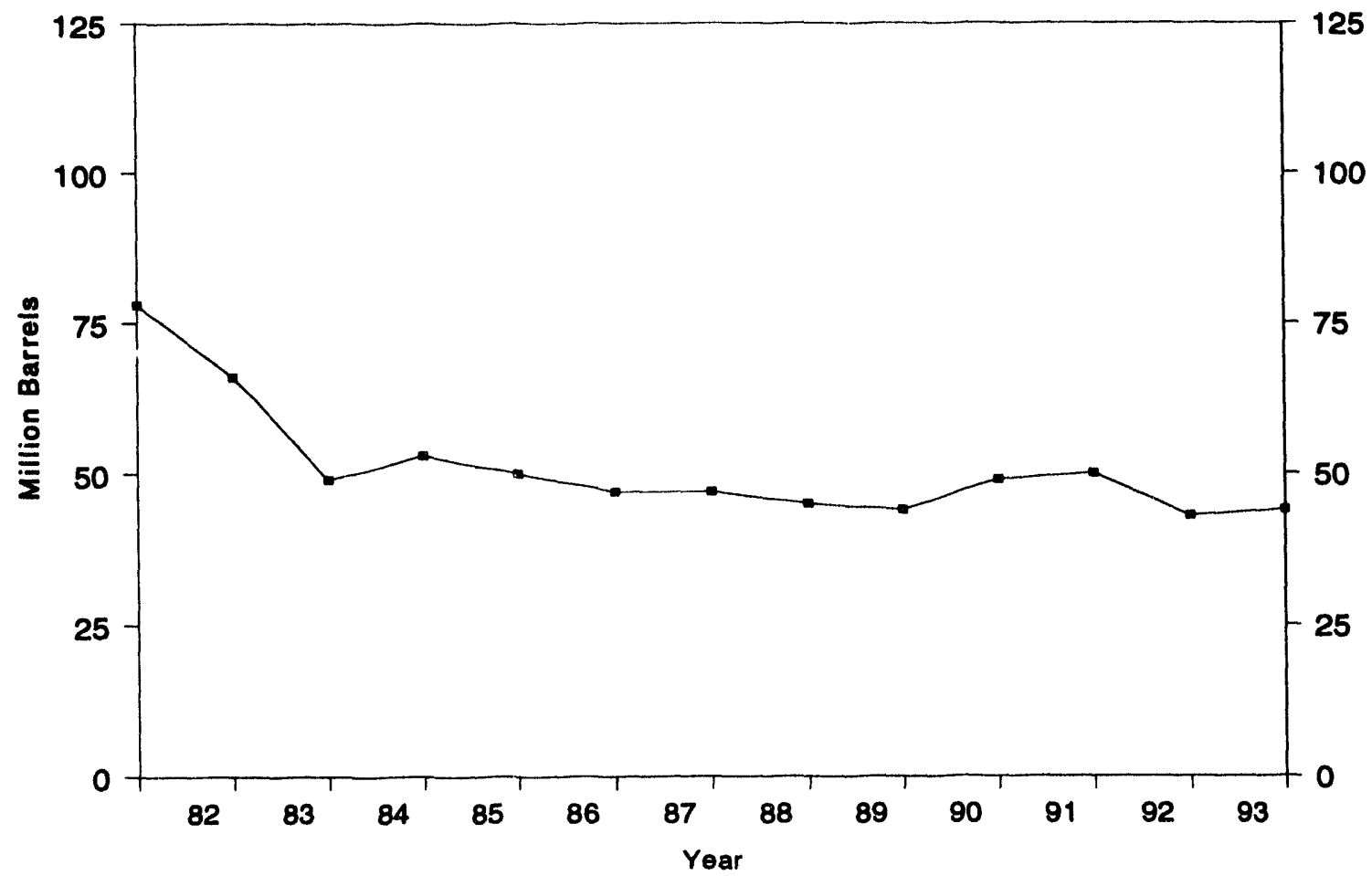

Source: Energy Information Administration, Petroleum Supply Annual, Table S6. See Summary Statistics Table and Figure Sources. 
Table S6. Realdual Fuel Oll Supply and Dlaposition, 1981 - Present (Thousand Barrels per Day, Except Where Noted)

\begin{tabular}{|c|c|c|c|c|c|c|c|}
\hline \multirow{2}{*}{\multicolumn{2}{|c|}{ YearfMonth }} & \multicolumn{2}{|c|}{ supphy } & \multicolumn{3}{|c|}{ Diepestilon } & \multirow{2}{*}{ 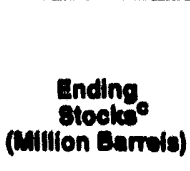 } \\
\hline & & $\begin{array}{c}\text { Total } \\
\text { Production }\end{array}$ & Importe & $\begin{array}{l}\text { stook } \\
\text { Change }\end{array}$ & Exports & $\begin{array}{l}\text { Product } \\
\text { Suppliad }\end{array}$ & \\
\hline $\begin{array}{l}1901 \\
1802 \\
1803 \\
1904 \\
1905 \\
1900 \\
1901 \\
1909 \\
1800 \\
1800\end{array}$ & 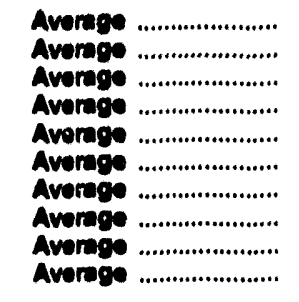 & $\begin{array}{r}1,321 \\
1,070 \\
892 \\
891 \\
092 \\
080 \\
085 \\
928 \\
984 \\
980\end{array}$ & $\begin{array}{l}800 \\
770 \\
690 \\
681 \\
510 \\
800 \\
808 \\
644 \\
620 \\
804\end{array}$ & $\begin{array}{r}d-37 \\
-32 \\
-35 \\
12 \\
-7 \\
-8 \\
(0) \\
-8 \\
-2 \\
13\end{array}$ & $\begin{array}{l}118 \\
200 \\
188 \\
180 \\
197 \\
147 \\
189 \\
200 \\
215 \\
211\end{array}$ & $\begin{array}{l}2,009 \\
1,710 \\
1,421 \\
1,390 \\
1,202 \\
1,418 \\
1,294 \\
1,370 \\
1,370 \\
1,220\end{array}$ & $\begin{array}{r}76 \\
\mathrm{~d} \\
49 \\
83 \\
80 \\
47 \\
47 \\
40 \\
44 \\
40\end{array}$ \\
\hline 1991 & 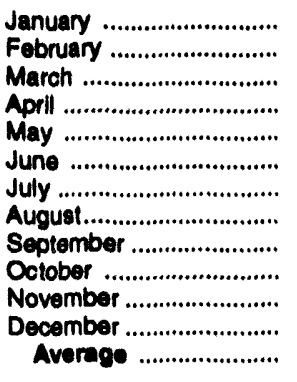 & $\begin{array}{r}1,001 \\
1,050 \\
905 \\
916 \\
929 \\
933 \\
871 \\
925 \\
838 \\
814 \\
896 \\
1,051 \\
934\end{array}$ & $\begin{array}{l}425 \\
384 \\
332 \\
416 \\
425 \\
512 \\
420 \\
599 \\
481 \\
438 \\
455 \\
547 \\
453\end{array}$ & $\begin{array}{r}-19 \\
-76 \\
-85 \\
68 \\
60 \\
-103 \\
-1 \\
68 \\
78 \\
6 \\
24 \\
28 \\
4\end{array}$ & $\begin{array}{l}320 \\
299 \\
178 \\
145 \\
300 \\
245 \\
176 \\
216 \\
168 \\
217 \\
189 \\
264 \\
224\end{array}$ & $\begin{array}{l}1,124 \\
1,211 \\
1,234 \\
1,119 \\
1,003 \\
1,303 \\
1,117 \\
1,240 \\
1,074 \\
1,029 \\
1,139 \\
1,307 \\
1,189\end{array}$ & $\begin{array}{l}48 \\
46 \\
43 \\
45 \\
47 \\
44 \\
44 \\
46 \\
48 \\
48 \\
49 \\
50 \\
-\end{array}$ \\
\hline 1992 & 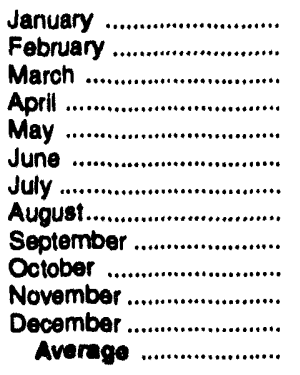 & $\begin{array}{l}965 \\
957 \\
990 \\
900 \\
964 \\
894 \\
838 \\
815 \\
810 \\
818 \\
895 \\
862 \\
892\end{array}$ & $\begin{array}{l}364 \\
498 \\
397 \\
342 \\
328 \\
334 \\
280 \\
347 \\
349 \\
376 \\
411 \\
481 \\
375\end{array}$ & $\begin{array}{r}-144 \\
-55 \\
-77 \\
-78 \\
67 \\
-11 \\
-37 \\
125 \\
123 \\
-72 \\
49 \\
-127 \\
-20\end{array}$ & $\begin{array}{r}184 \\
176 \\
310 \\
265 \\
207 \\
230 \\
169 \\
86 \\
149 \\
156 \\
216 \\
158 \\
193\end{array}$ & $\begin{array}{r}1,289 \\
1,334 \\
1,154 \\
1,055 \\
1,019 \\
1,009 \\
986 \\
941 \\
887 \\
1,110 \\
1,041 \\
1,312 \\
1,004\end{array}$ & $\begin{array}{l}45 \\
44 \\
41 \\
39 \\
41 \\
41 \\
40 \\
44 \\
47 \\
45 \\
47 \\
43\end{array}$ \\
\hline 1993 & 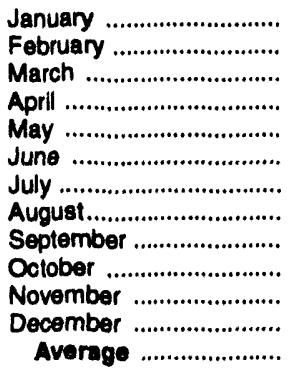 & $\begin{array}{l}820 \\
840 \\
818 \\
896 \\
908 \\
795 \\
762 \\
752 \\
822 \\
841 \\
899 \\
869 \\
835\end{array}$ & $\begin{array}{l}385 \\
332 \\
360 \\
377 \\
316 \\
308 \\
337 \\
387 \\
430 \\
412 \\
361 \\
467 \\
373\end{array}$ & $\begin{array}{r}44 \\
-74 \\
-47 \\
32 \\
54 \\
87 \\
-102 \\
64 \\
-31 \\
103 \\
48 \\
-129 \\
4\end{array}$ & $\begin{array}{r}133 \\
113 \\
152 \\
169 \\
137 \\
147 \\
122 \\
120 \\
110 \\
94 \\
86 \\
98 \\
123\end{array}$ & $\begin{array}{r}1,028 \\
1,132 \\
1,073 \\
1,071 \\
1,033 \\
870 \\
1,079 \\
956 \\
1,173 \\
1,057 \\
1,126 \\
1,367 \\
1,080\end{array}$ & $\begin{array}{l}44 \\
42 \\
40 \\
41 \\
43 \\
46 \\
43 \\
44 \\
44 \\
47 \\
48 \\
44 \\
-\end{array}$ \\
\hline
\end{tabular}

a Excludes 48,000 barrels per day in 1981 and 1982 prevlously published as crude used directly.

b A negative number indicates a decrease in stocks and a positive number indicates an increase.

c Stocks are totals as of end of period.

d In January 1981 and 1983 , numerous respondents were added to surveys affecting stocks reported and stock change calculations. Stock changee are calculated using new basis stock levels. See Summary Statistics Explanatory Note 4.

(8) $=$ Less than 500 barrels per day.

Notes: - Geographic coverage is the 50 States and the District of Columbla. - Totals may not equal sum of components due to independent rounding.

Source: See Summary Statistics Table and Figure Sources. 
Fisure 811. Jot Fuel Supply and Diaposttion, 1981 - Prosent

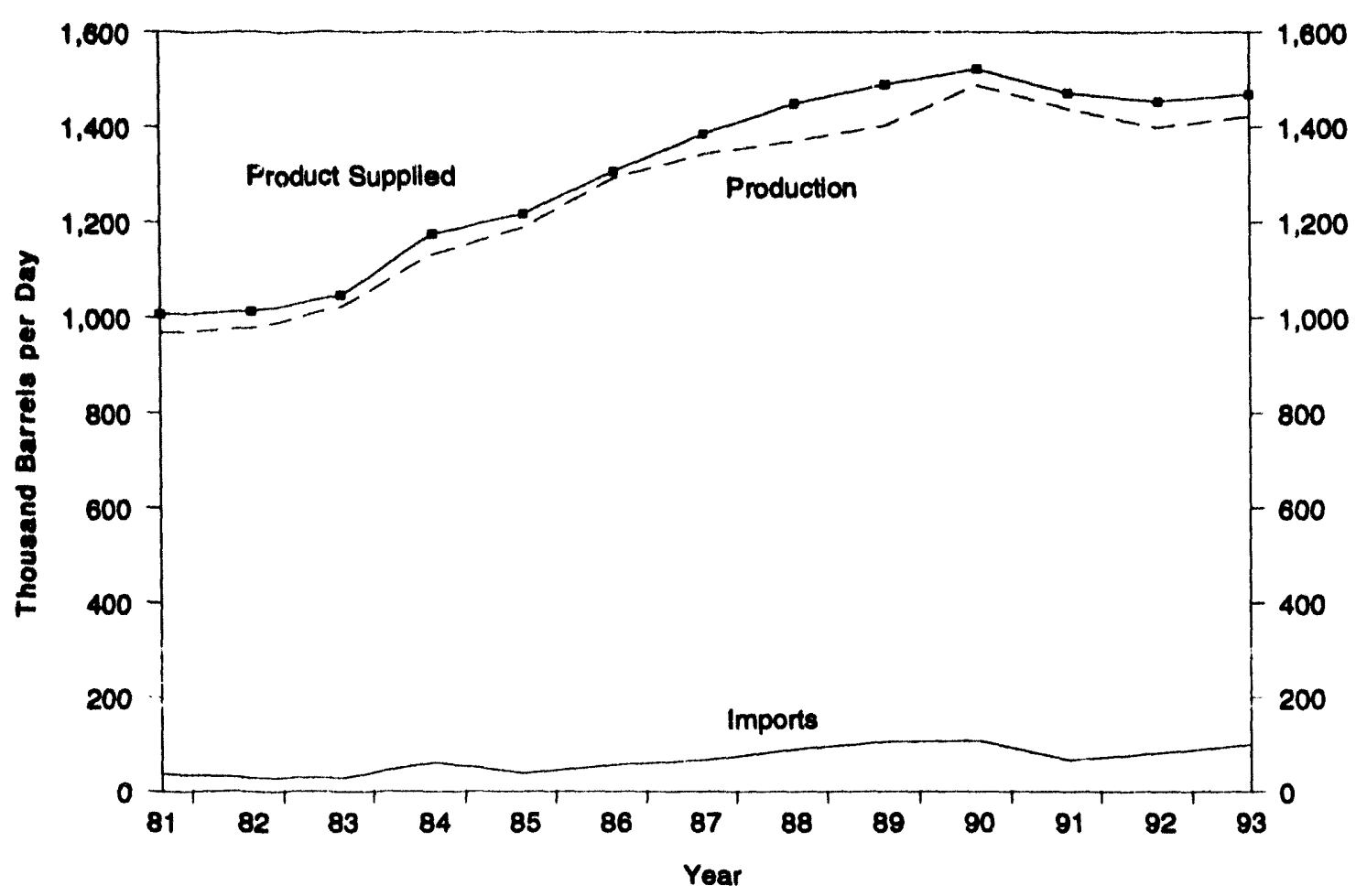

Source: Energy Information Administration, Petroleum Supply Annual, Table S7. See Summary Statiotice Table and Figure Sources.

\section{Figure 812. Jot Fuol Ending Stocks, 1981 - Presont}

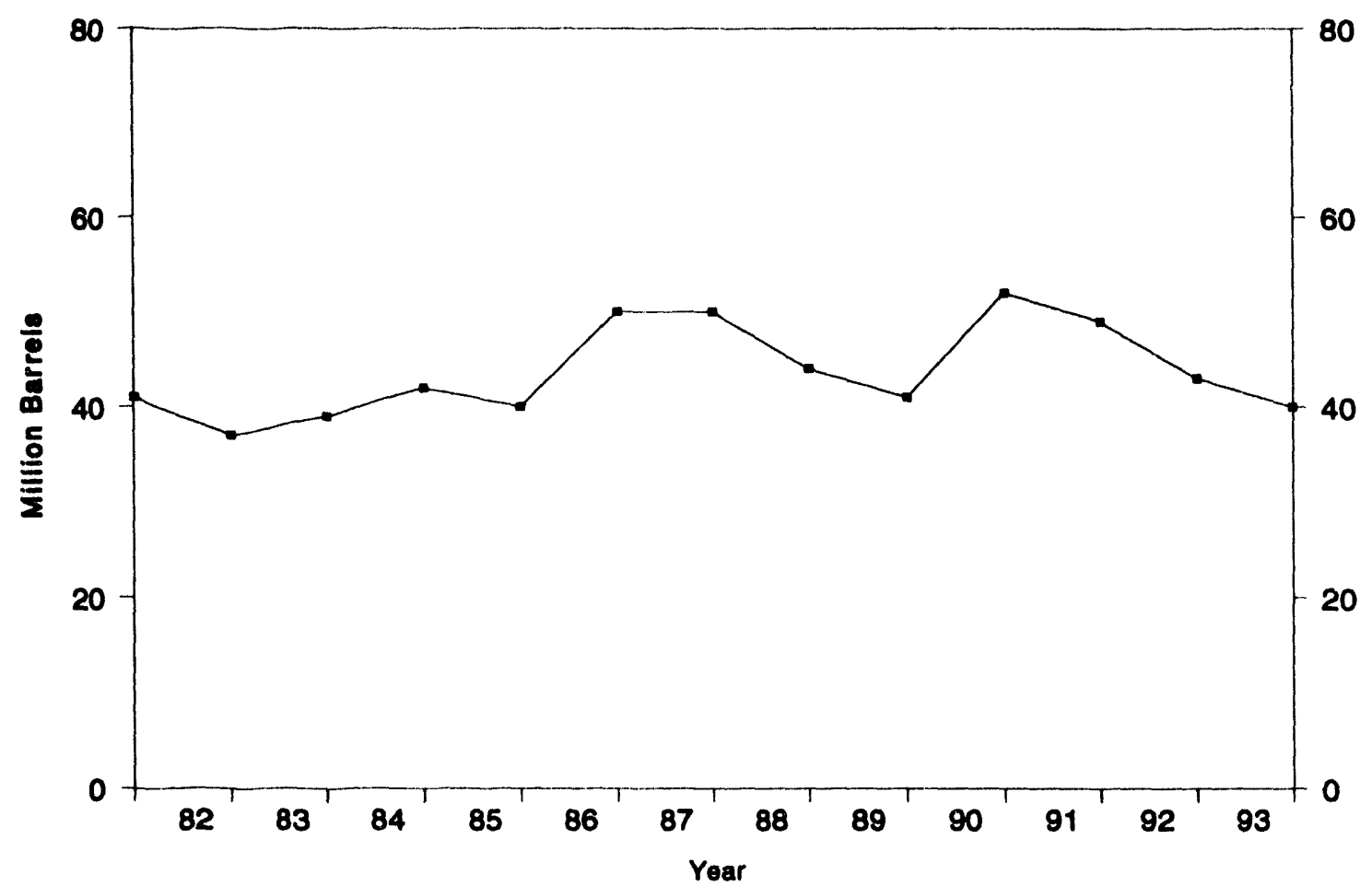

Sourco: Energy Information Adminstration, Petroleum Supply Annual, Table S7. Seo Summary Statistice Table and Figure Sources. 
Tablo 87. Jot Fuel Supply and Dlsposition, 1981 - Prosent (Thousand Barrels per Day, Except Where Noted)

\begin{tabular}{|c|c|c|c|c|c|c|c|c|c|c|}
\hline & \multirow{3}{*}{ Yearnionth } & \multicolumn{3}{|c|}{ Eupply } & \multicolumn{4}{|c|}{ Dleposition } & \multirow{2}{*}{\multicolumn{2}{|c|}{$\begin{array}{l}\text { Ending stoekn } \\
\text { (Million Eamblo) }\end{array}$}} \\
\hline & & \multicolumn{2}{|c|}{ Production } & \multirow[b]{2}{*}{ Imports } & \multirow{2}{*}{$\begin{array}{l}\text { stook } \\
\text { Changob }\end{array}$} & \multirow[b]{2}{*}{ Exports } & \multicolumn{2}{|c|}{ Product supplied } & & \\
\hline & & Total & Kerosene-Type & & & & Total & Korosene-Type & Total & Roroesm \\
\hline 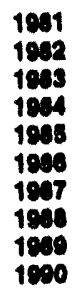 & 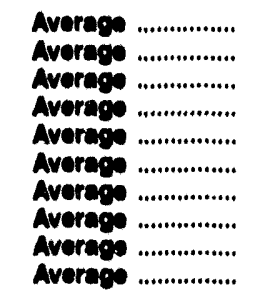 & $\begin{array}{r}909 \\
070 \\
1,000 \\
1,120 \\
1,100 \\
1,203 \\
1,345 \\
1,370 \\
1,400 \\
1,400\end{array}$ & $\begin{array}{r}773 \\
779 \\
017 \\
910 \\
919 \\
1,097 \\
1,138 \\
1,194 \\
1,197 \\
1,311\end{array}$ & $\begin{array}{r}30 \\
20 \\
29 \\
62 \\
39 \\
57 \\
67 \\
09 \\
100 \\
108\end{array}$ & $\begin{array}{c}0.4 \\
-12 \\
0(0) \\
0 \\
-4 \\
25 \\
(0) \\
-17 \\
0 \\
31\end{array}$ & $\begin{array}{r}2 \\
8 \\
8 \\
9 \\
13 \\
18 \\
24 \\
20 \\
27 \\
43\end{array}$ & $\begin{array}{l}1,007 \\
1,013 \\
1,040 \\
1,176 \\
1,216 \\
1,307 \\
1,389 \\
1,440 \\
1,409 \\
1,820\end{array}$ & $\begin{array}{r}600 \\
804 \\
890 \\
903 \\
1,008 \\
1,108 \\
1,181 \\
1,283 \\
1,294 \\
1,340\end{array}$ & $\begin{array}{r}41 \\
037 \\
30 \\
40 \\
40 \\
60 \\
60 \\
41 \\
41 \\
62\end{array}$ & $\begin{array}{l}3 \\
0 \\
3 \\
25 \\
4 \\
4 \\
4 \\
4 \\
4\end{array}$ \\
\hline 1901 & 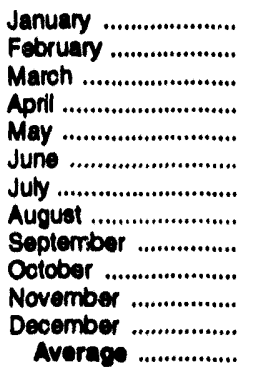 & $\begin{array}{l}1,600 \\
1,648 \\
1,200 \\
1,280 \\
1,367 \\
1,473 \\
1,428 \\
1,486 \\
1,405 \\
1,415 \\
1,433 \\
1,530 \\
1,438\end{array}$ & $\begin{array}{l}1,364 \\
1,384 \\
1,157 \\
1,136 \\
1,191 \\
1,300 \\
1,265 \\
1,316 \\
1,322 \\
1,253 \\
1,276 \\
1,357 \\
1,274\end{array}$ & $\begin{array}{l}67 \\
44 \\
65 \\
73 \\
87 \\
64 \\
67 \\
88 \\
92 \\
59 \\
56 \\
42 \\
67\end{array}$ & $\begin{array}{r}-56 \\
-108 \\
-99 \\
-8 \\
85 \\
58 \\
-47 \\
21 \\
71 \\
-68 \\
15 \\
22 \\
-9\end{array}$ & $\begin{array}{r}73 \\
159 \\
40 \\
38 \\
35 \\
13 \\
31 \\
11 \\
10 \\
50 \\
5 \\
59 \\
43\end{array}$ & $\begin{array}{l}1,569 \\
1,641 \\
1,423 \\
1,329 \\
1,334 \\
1,465 \\
1,509 \\
1,543 \\
1,506 \\
1,489 \\
1,469 \\
1,492 \\
1,471\end{array}$ & $\begin{array}{l}1,378 \\
1,360 \\
1,270 \\
1,173 \\
1,143 \\
1,280 \\
1,343 \\
1,343 \\
1,321 \\
1,319 \\
1,282 \\
1,338 \\
1,298\end{array}$ & $\begin{array}{l}60 \\
47 \\
44 \\
44 \\
47 \\
48 \\
47 \\
48 \\
60 \\
48 \\
48 \\
49 \\
-\end{array}$ & $\begin{array}{l}44 \\
41 \\
38 \\
38 \\
41 \\
43 \\
41 \\
42 \\
46 \\
43 \\
44 \\
44 \\
-\end{array}$ \\
\hline 1002 & 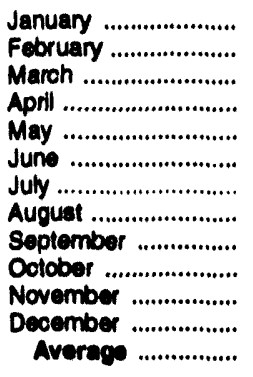 & $\begin{array}{l}1,362 \\
1,311 \\
1,347 \\
1,286 \\
1,393 \\
1,374 \\
1,473 \\
1,471 \\
1,448 \\
1,408 \\
1,466 \\
1,462 \\
1,390\end{array}$ & $\begin{array}{l}1,200 \\
1,164 \\
1,215 \\
1,131 \\
1,214 \\
1,234 \\
1,328 \\
1,339 \\
1,286 \\
1,265 \\
1,310 \\
1,388 \\
1,264\end{array}$ & $\begin{array}{r}39 \\
56 \\
68 \\
74 \\
93 \\
86 \\
81 \\
111 \\
83 \\
105 \\
80 \\
102 \\
62\end{array}$ & $\begin{array}{r}-127 \\
-73 \\
31 \\
-68 \\
114 \\
-21 \\
59 \\
-32 \\
78 \\
-12 \\
-41 \\
-101 \\
-16\end{array}$ & $\begin{array}{r}44 \\
42 \\
7 \\
18 \\
26 \\
45 \\
62 \\
28 \\
20 \\
44 \\
59 \\
112 \\
43\end{array}$ & $\begin{array}{l}1,473 \\
1,398 \\
1,365 \\
1,409 \\
1,346 \\
1,436 \\
1,433 \\
1,585 \\
1,442 \\
1,480 \\
1,528 \\
1,553 \\
1,454\end{array}$ & $\begin{array}{l}1,314 \\
1,250 \\
1,218 \\
1,262 \\
1,189 \\
1,308 \\
1,280 \\
1,438 \\
1,313 \\
1,315 \\
1,411 \\
1,410 \\
1,310\end{array}$ & $\begin{array}{l}45 \\
43 \\
44 \\
42 \\
45 \\
45 \\
46 \\
45 \\
46 \\
47 \\
46 \\
43 \\
-\end{array}$ & $\begin{array}{l}40 \\
38 \\
30 \\
37 \\
40 \\
39 \\
42 \\
41 \\
43 \\
43 \\
41 \\
30 \\
-\end{array}$ \\
\hline 1003 & 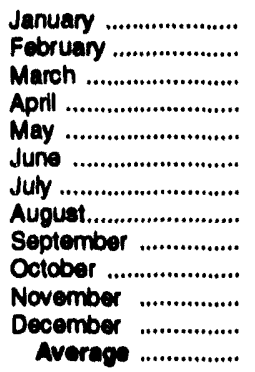 & $\begin{array}{l}1,437 \\
1,440 \\
1,463 \\
1,391 \\
1,427 \\
1,547 \\
1,485 \\
1,368 \\
1,338 \\
1,329 \\
1,386 \\
1,469 \\
1,492\end{array}$ & $\begin{array}{l}1,308 \\
1,316 \\
1,332 \\
1,268 \\
1,302 \\
1,407 \\
1,359 \\
1,257 \\
1,241 \\
1,242 \\
1,301 \\
1,382 \\
1,300\end{array}$ & $\begin{array}{r}89 \\
110 \\
76 \\
88 \\
75 \\
111 \\
94 \\
100 \\
108 \\
143 \\
105 \\
105 \\
100\end{array}$ & $\begin{array}{r}-64 \\
53 \\
-15 \\
-23 \\
42 \\
83 \\
42 \\
-98 \\
-69 \\
-27 \\
8 \\
-13 \\
-7\end{array}$ & $\begin{array}{r}134 \\
17 \\
101 \\
88 \\
60 \\
45 \\
71 \\
42 \\
16 \\
20 \\
29 \\
85 \\
80\end{array}$ & $\begin{array}{l}1,456 \\
1,480 \\
1,453 \\
1,413 \\
1,401 \\
1,530 \\
1,466 \\
1,514 \\
1,497 \\
1,479 \\
1,453 \\
1,493 \\
1,469\end{array}$ & $\begin{array}{l}1,369 \\
1,337 \\
1,335 \\
1,299 \\
1,288 \\
1,362 \\
1,338 \\
1,413 \\
1,357 \\
1,389 \\
1,357 \\
1,441 \\
1,367\end{array}$ & $\begin{array}{l}41 \\
43 \\
42 \\
41 \\
43 \\
45 \\
47 \\
43 \\
41 \\
41 \\
41 \\
40 \\
-\end{array}$ & $\begin{array}{l}36 \\
38 \\
38 \\
37 \\
38 \\
41 \\
43 \\
40 \\
38 \\
37 \\
38 \\
38 \\
-\end{array}$ \\
\hline
\end{tabular}

- Stocks are totals as of end of period.

- A negative number indicates a decrease in stocks and a postive number indicates an increase.

- In January 1881, 1893, and 1884, a new took basla was cotabllshed affecting stocks reported and stock change calculations. Stock changes are calculated ueing now basis stock lovels. See Summary Statiatics Explanatory Note 4.

(8) = Leas than 500 barrels per day.

Notes: - Geogrephle coverage bs the 50 States and the District of Columbia. - Totals may not equal sum of components due to independent rounding.

Source: See Summary Statiotice Table and Floure Sources. 
Figure 813. Propane/Propylene Supply and Disposition, 1981 - Present

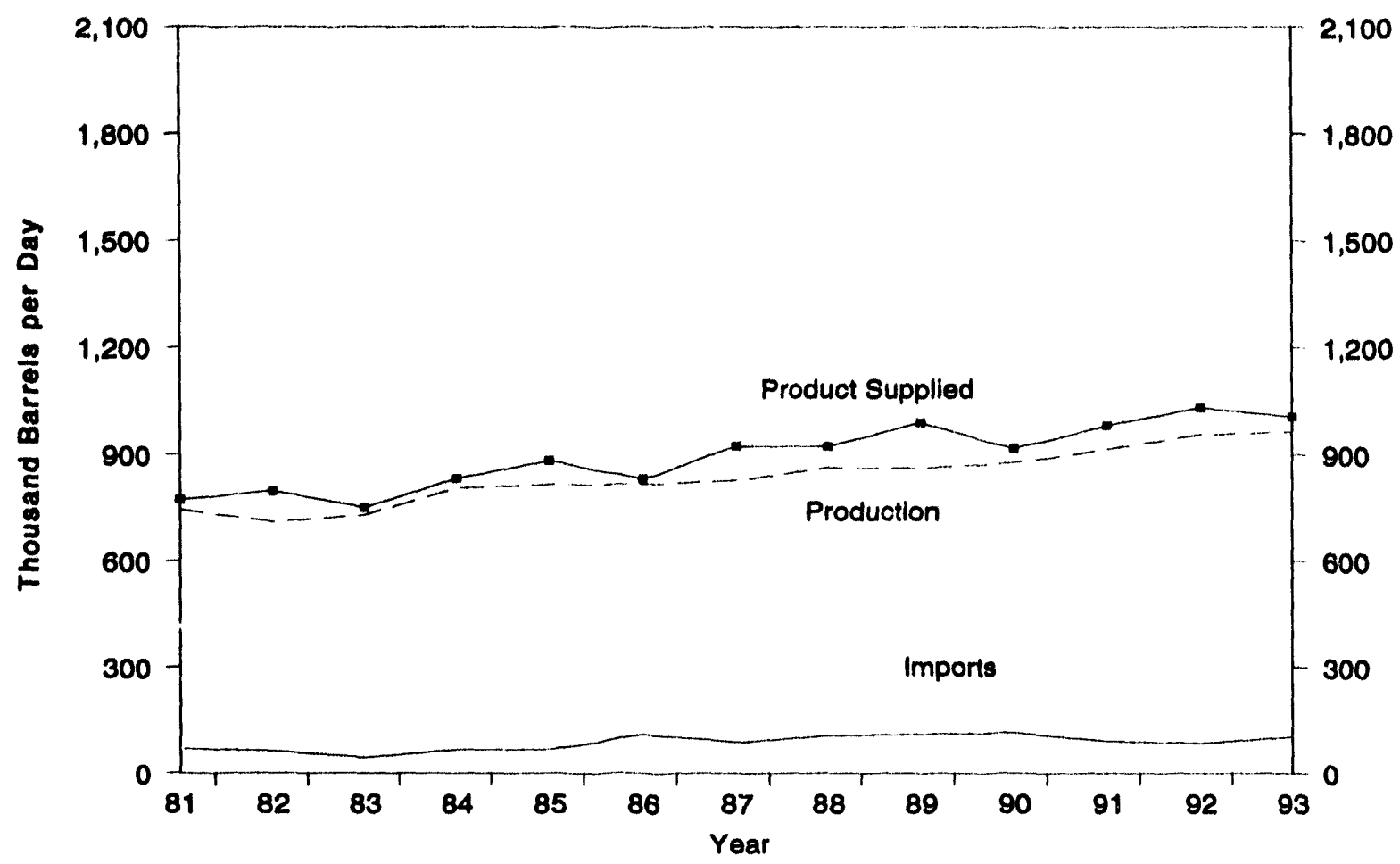

Source: Energy Information Administration, Petroleum Supply Annual, Table S8. See Summary Statistics Table and Figure Sources.

Figure S14. Propane/Propylene Ending Stocks, 1981 - Present

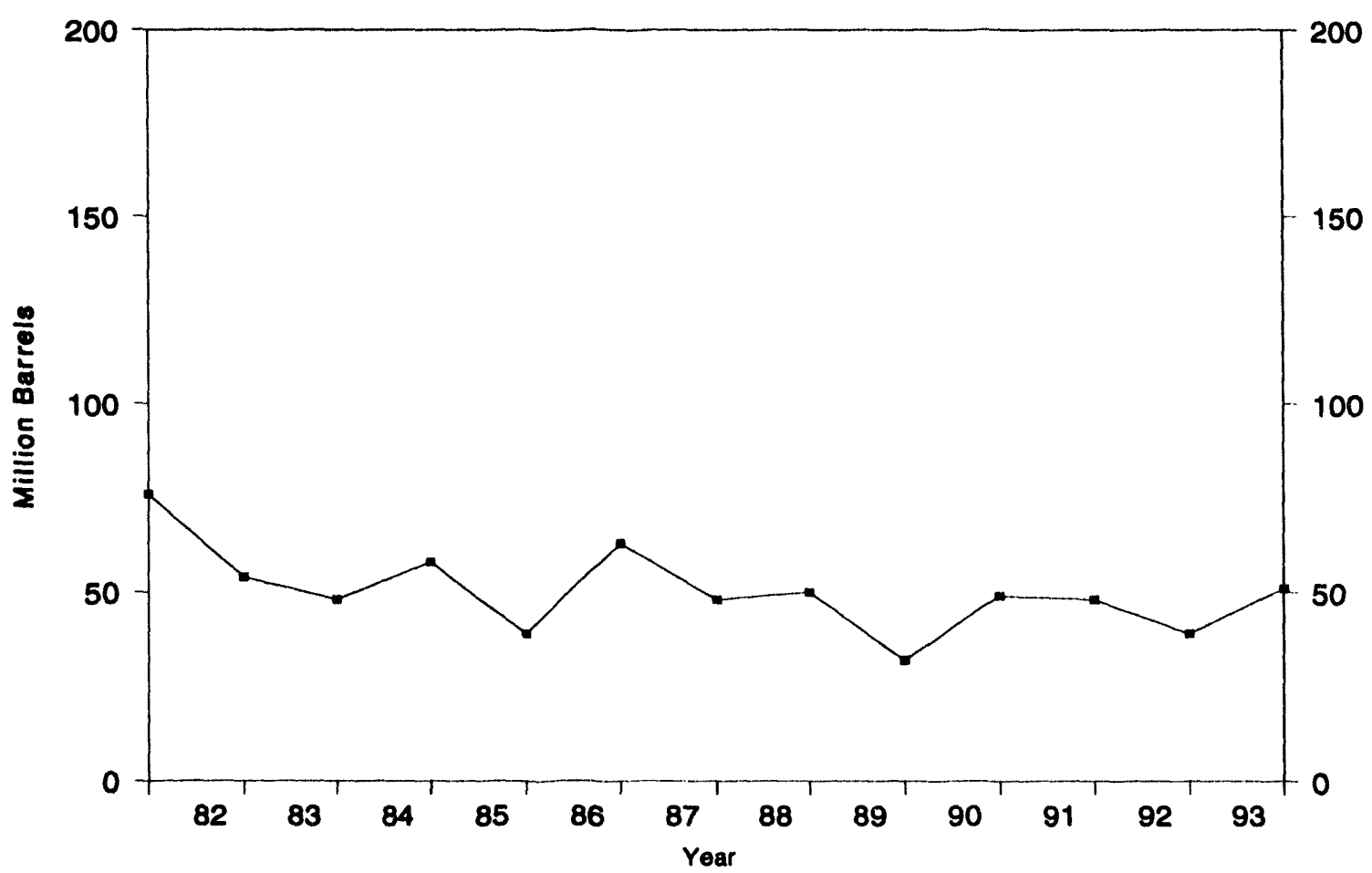

Source: Energy Information Administration, Petroleum Supply Annual, Table S8. See Summary Statistics Table and Figure Sources. 
Table S8. Propane/Propylene Supply and Disposition, 1981 - Present (Thousand Barrels per Day, Except Where Noted)

\begin{tabular}{|c|c|c|c|c|c|c|c|c|}
\hline \multirow{2}{*}{\multicolumn{2}{|c|}{ Ycaminomth }} & \multicolumn{2}{|c|}{ Eupphy } & \multicolumn{4}{|c|}{ Dispoction } & \multirow{2}{*}{ 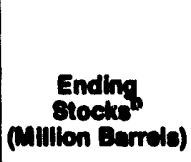 } \\
\hline & & $\begin{array}{c}\text { Tetal } \\
\text { Production }\end{array}$ & inports & $\begin{array}{l}\text { Etook } \\
\text { Chance }\end{array}$ & $\begin{array}{l}\text { Pofinery } \\
\text { Inpute }\end{array}$ & Exports & $\begin{array}{l}\text { Product } \\
\text { supplied }\end{array}$ & \\
\hline $\begin{array}{l}1600 \\
1900\end{array}$ & 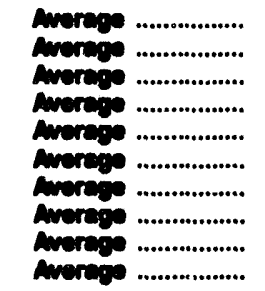 & 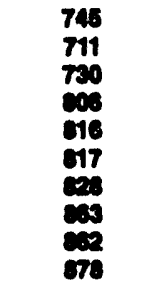 & $\begin{array}{r}70 \\
63 \\
44 \\
67 \\
67 \\
110 \\
09 \\
108 \\
111 \\
118\end{array}$ & $\begin{array}{r}18 \\
-60 \\
0-24 \\
67 \\
-60 \\
64 \\
41 \\
7 \\
-4 \\
4\end{array}$ & $\begin{array}{c}5 \\
4 \\
4 \\
4 \\
3 \\
6 \\
6 \\
11 \\
\text { (b) }\end{array}$ & $\begin{array}{l}16 \\
31 \\
43 \\
30 \\
4 \\
2 \\
24 \\
31 \\
24 \\
24\end{array}$ & $\begin{array}{l}773 \\
703 \\
761 \\
203 \\
003 \\
031 \\
91 \\
923 \\
\infty 00 \\
917\end{array}$ & $\begin{array}{l}76 \\
64 \\
640 \\
56 \\
30 \\
63 \\
40 \\
80 \\
30 \\
40\end{array}$ \\
\hline 1901 & 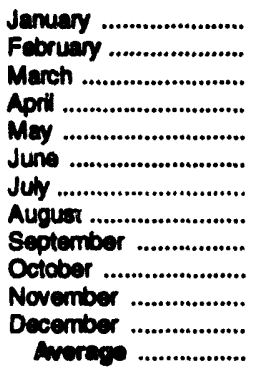 & $\begin{array}{l}920 \\
923 \\
912 \\
900 \\
922 \\
908 \\
901 \\
891 \\
905 \\
902 \\
980 \\
984 \\
915\end{array}$ & $\begin{array}{r}105 \\
90 \\
56 \\
101 \\
90 \\
81 \\
91 \\
73 \\
92 \\
146 \\
82 \\
86 \\
91\end{array}$ & $\begin{array}{r}-449 \\
-174 \\
-10 \\
179 \\
214 \\
223 \\
81 \\
40 \\
-22 \\
35 \\
-37 \\
-128 \\
-3\end{array}$ & $\begin{array}{l}0 \\
0 \\
0 \\
0 \\
0 \\
0 \\
0 \\
0 \\
0 \\
0 \\
0 \\
(8) \\
(0)\end{array}$ & $\begin{array}{l}51 \\
40 \\
45 \\
25 \\
31 \\
22 \\
15 \\
13 \\
14 \\
18 \\
20 \\
38 \\
28\end{array}$ & $\begin{array}{r}1,422 \\
1,147 \\
938 \\
788 \\
767 \\
741 \\
895 \\
910 \\
1,006 \\
995 \\
1,030 \\
1,139 \\
892\end{array}$ & $\begin{array}{l}35 \\
30 \\
30 \\
35 \\
42 \\
49 \\
51 \\
52 \\
52 \\
53 \\
52 \\
48 \\
-\end{array}$ \\
\hline 1902 & 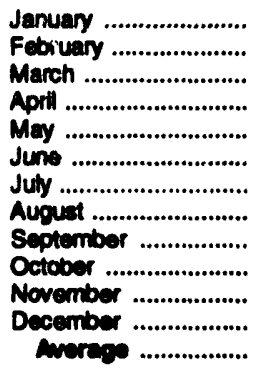 & $\begin{array}{l}949 \\
955 \\
940 \\
981 \\
977 \\
978 \\
894 \\
946 \\
831 \\
938 \\
884 \\
977 \\
893\end{array}$ & $\begin{array}{r}90 \\
86 \\
69 \\
60 \\
72 \\
66 \\
68 \\
85 \\
71 \\
104 \\
99 \\
131 \\
85\end{array}$ & $\begin{array}{r}-282 \\
-200 \\
-15 \\
120 \\
253 \\
206 \\
176 \\
117 \\
51 \\
-88 \\
-243 \\
-385 \\
-24\end{array}$ & $\begin{array}{r}(8) \\
(8) \\
(8) \\
0 \\
(8) \\
(8) \\
(8) \\
(8) \\
(8) \\
(8) \\
0 \\
0 \\
(8)\end{array}$ & $\begin{array}{l}72 \\
27 \\
26 \\
24 \\
23 \\
27 \\
35 \\
25 \\
25 \\
30 \\
33 \\
45 \\
33\end{array}$ & $\begin{array}{r}1,249 \\
1,214 \\
997 \\
896 \\
773 \\
811 \\
821 \\
889 \\
927 \\
1,095 \\
1,273 \\
1,448 \\
1,082 \\
\end{array}$ & $\begin{array}{l}30 \\
33 \\
33 \\
38 \\
44 \\
50 \\
56 \\
59 \\
61 \\
58 \\
51 \\
39 \\
-\end{array}$ \\
\hline 1903 & 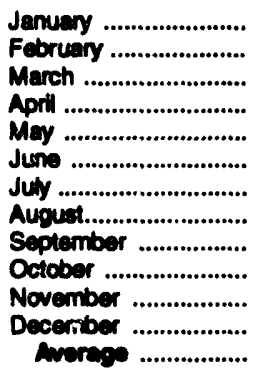 & $\begin{array}{l}968 \\
864 \\
966 \\
960 \\
951 \\
967 \\
963 \\
980 \\
969 \\
964 \\
963 \\
853 \\
963\end{array}$ & $\begin{array}{r}79 \\
82 \\
85 \\
108 \\
96 \\
75 \\
118 \\
116 \\
132 \\
107 \\
138 \\
102 \\
103\end{array}$ & $\begin{array}{r}-212 \\
-255 \\
-109 \\
238 \\
266 \\
265 \\
256 \\
178 \\
92 \\
-11 \\
-126 \\
-195 \\
34\end{array}$ & $\begin{array}{r}1 \\
(8) \\
(8) \\
(8) \\
0 \\
0 \\
0 \\
0 \\
0 \\
0 \\
0 \\
0 \\
(s)\end{array}$ & $\begin{array}{l}31 \\
37 \\
32 \\
40 \\
30 \\
23 \\
26 \\
27 \\
17 \\
13 \\
17 \\
25 \\
28\end{array}$ & $\begin{array}{r}1,227 \\
1,264 \\
1,129 \\
809 \\
750 \\
754 \\
800 \\
871 \\
992 \\
1,059 \\
1,209 \\
1,225 \\
1,006\end{array}$ & $\begin{array}{l}32 \\
25 \\
22 \\
29 \\
37 \\
45 \\
53 \\
59 \\
61 \\
61 \\
57 \\
51 \\
-\end{array}$ \\
\hline
\end{tabular}

\footnotetext{
a A negative mumber indicates a decrease in stocls and a positive number indicates an increase.

b Stocks are totals as of end of period.

c In January 1981, 1983, and 1984, a new stock basis was established affecting stocks reported and stock change calculations. Stock changes are calculated uing new bacis stcck lovels. See Summary Statistios Explanatory Note 4.

(8) = Leas than 500 berrele per day.

Notes: - Geogrephic coverage is the 50 States and the District of Columbia. - Totals may not equal sum of components due to independent rounding.

Sourca: See Summary Suntiatics Table and Foure Sources.
} 
Figure S15. Liquefied Petroleum Gases Supply and Disposttion, 1981 - Present

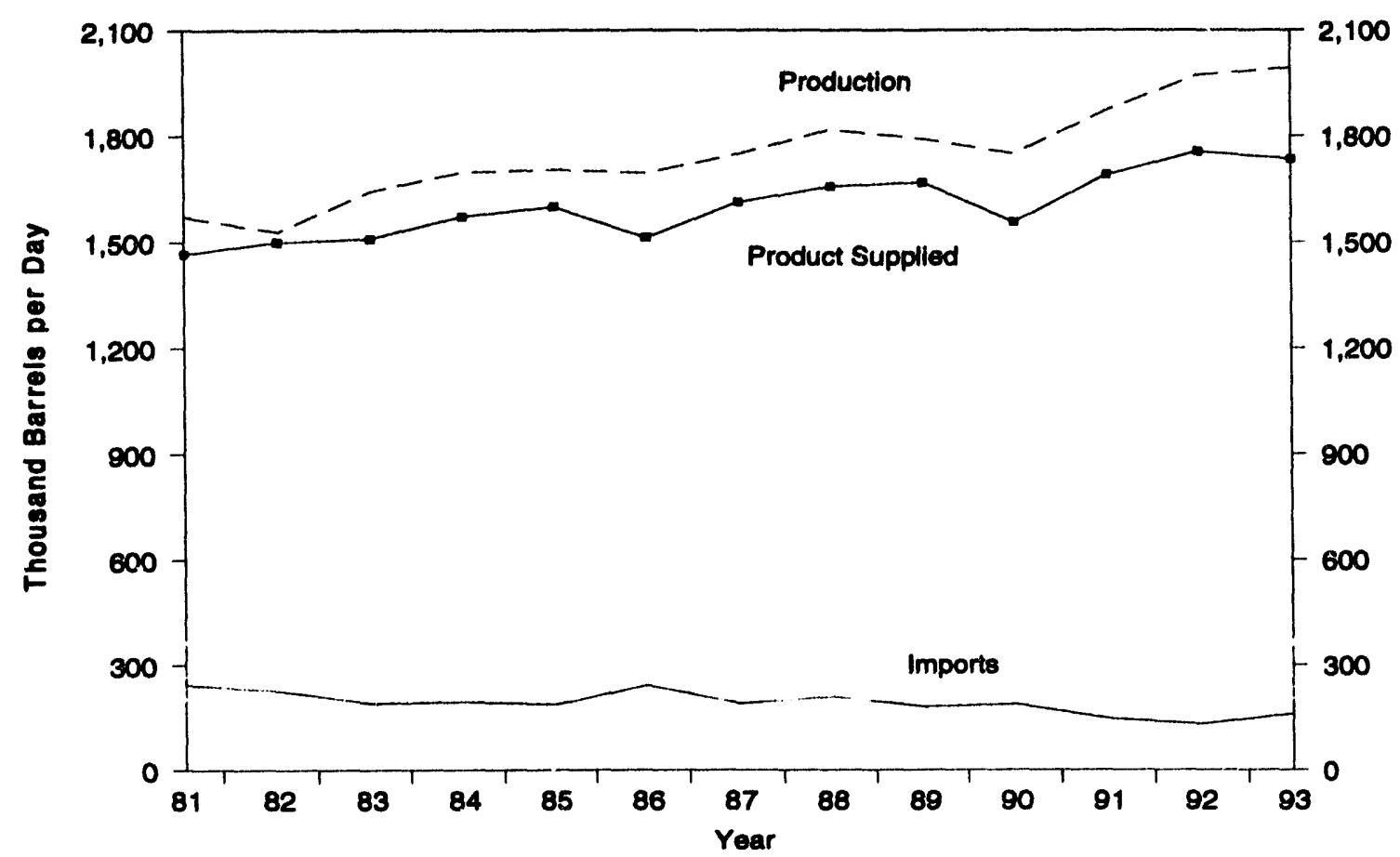

Source: Energy Information Administration, Petroleum Supply Annual, Table S9. See Summary Statistics Table and Figure Sources.

Figure S16. Liquefied Petroleum Gases Ending Stocks, 1981 - Present

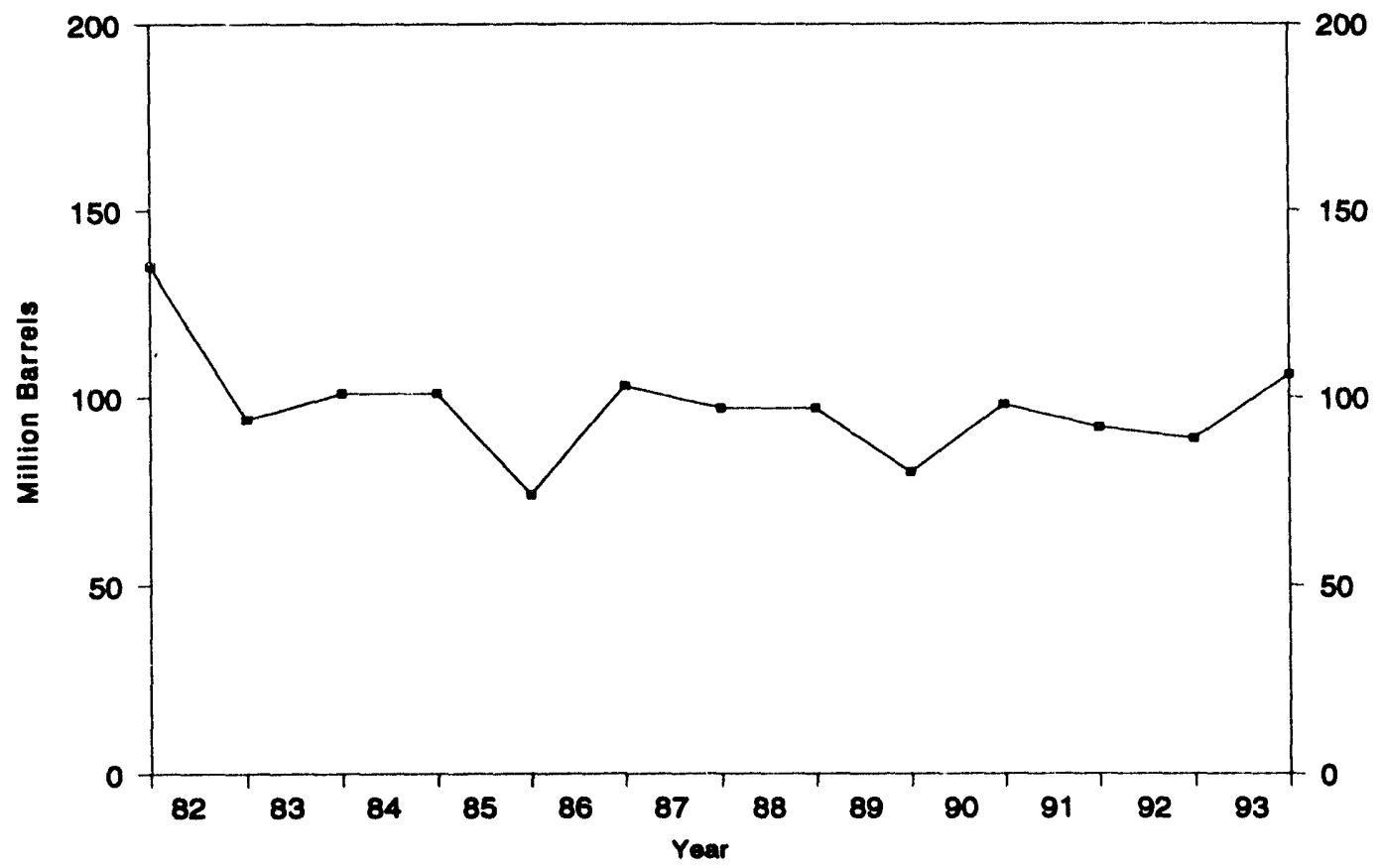

Source: Energy Information Administration, Petroloum Supply Annual, Table S9. See Summary Statistics Table and Figure Sources. 
Table S9. Liquofied Petroleum Gases Supply and Dlspoattion, 1981 - Present (Thousand Barrels per Day, Except Where Noted)

\begin{tabular}{|c|c|c|c|c|c|c|c|c|}
\hline & \multirow[b]{2}{*}{ Yearndonth } & \multicolumn{2}{|c|}{ supply } & \multicolumn{4}{|c|}{ Diepostion } & \multirow[b]{2}{*}{$\begin{array}{c}\text { Ending } \\
\text { Stoeks } \\
\text { (Muliton Barrola) }\end{array}$} \\
\hline & & $\begin{array}{l}\text { Total } \\
\text { Production }\end{array}$ & Imports & $\begin{array}{l}\text { Etook } \\
\text { Change }\end{array}$ & $\begin{array}{l}\text { Refinery } \\
\text { Inputs }\end{array}$ & Exports & $\begin{array}{l}\text { Product } \\
\text { supplied }\end{array}$ & \\
\hline $\begin{array}{l}1801 \\
1802 \\
1893 \\
1984 \\
1805 \\
1808 \\
1807 \\
1808 \\
1000 \\
1800\end{array}$ & 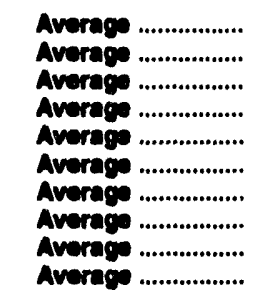 & $\begin{array}{l}1,571 \\
1,598 \\
1,042 \\
1,097 \\
1,704 \\
1,095 \\
1,748 \\
1,017 \\
1,701 \\
1,749\end{array}$ & $\begin{array}{l}244 \\
220 \\
190 \\
195 \\
197 \\
242 \\
190 \\
200 \\
181 \\
180\end{array}$ & $\begin{array}{r}c 18 \\
-111 \\
0-4 \\
0-19 \\
-75 \\
80 \\
-15 \\
1 \\
-47 \\
48\end{array}$ & $\begin{array}{l}280 \\
300 \\
253 \\
201 \\
304 \\
302 \\
304 \\
321 \\
315 \\
203\end{array}$ & $\begin{array}{l}42 \\
65 \\
73 \\
46 \\
62 \\
42 \\
38 \\
49 \\
35 \\
40\end{array}$ & $\begin{array}{l}1,408 \\
1,409 \\
1,509 \\
1,572 \\
1,500 \\
1,512 \\
1,612 \\
1,080 \\
1,608 \\
1,560\end{array}$ & $\begin{array}{r}135 \\
c 94 \\
c 101 \\
101 \\
74 \\
103 \\
97 \\
97 \\
80 \\
80\end{array}$ \\
\hline 1901 & 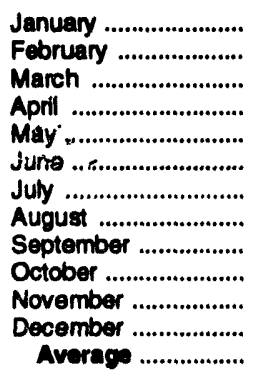 & $\begin{array}{l}1,753 \\
1,865 \\
1,842 \\
1,837 \\
1,889 \\
1,949 \\
1,913 \\
1,899 \\
1,806 \\
1,805 \\
1,789 \\
1,810 \\
1,871\end{array}$ & $\begin{array}{r}148 \\
126 \\
91 \\
154 \\
129 \\
148 \\
151 \\
143 \\
147 \\
233 \\
156 \\
139 \\
147\end{array}$ & $\begin{array}{r}-658 \\
-271 \\
113 \\
346 \\
428 \\
328 \\
211 \\
175 \\
-84 \\
33 \\
-330 \\
-488 \\
-15\end{array}$ & $\begin{array}{l}384 \\
322 \\
249 \\
237 \\
239 \\
245 \\
253 \\
255 \\
288 \\
345 \\
413 \\
437 \\
304\end{array}$ & $\begin{array}{l}56 \\
60 \\
56 \\
31 \\
45 \\
32 \\
24 \\
18 \\
31 \\
31 \\
40 \\
73 \\
41\end{array}$ & $\begin{array}{l}2,139 \\
1,880 \\
1,615 \\
1,477 \\
1,407 \\
1,492 \\
1,575 \\
1,594 \\
1,718 \\
1,629 \\
1,821 \\
1,927 \\
1,609\end{array}$ & $\begin{array}{r}78 \\
70 \\
73 \\
84 \\
97 \\
107 \\
113 \\
119 \\
116 \\
117 \\
107 \\
92 \\
-\end{array}$ \\
\hline 1992 & 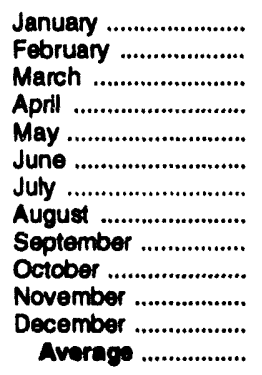 & $\begin{array}{l}1,820 \\
1,917 \\
2,033 \\
2,102 \\
2,106 \\
2,102 \\
2,090 \\
2,016 \\
1,886 \\
1,892 \\
1,854 \\
1,849 \\
1,972\end{array}$ & $\begin{array}{r}142 \\
126 \\
97 \\
127 \\
106 \\
104 \\
106 \\
148 \\
114 \\
171 \\
148 \\
176 \\
131\end{array}$ & $\begin{array}{r}-452 \\
-365 \\
153 \\
401 \\
489 \\
334 \\
345 \\
369 \\
37 \\
-242 \\
-541 \\
-660 \\
-10\end{array}$ & $\begin{array}{l}384 \\
326 \\
247 \\
233 \\
245 \\
257 \\
255 \\
233 \\
299 \\
389 \\
403 \\
453 \\
309\end{array}$ & $\begin{array}{l}80 \\
33 \\
43 \\
45 \\
44 \\
59 \\
52 \\
55 \\
45 \\
39 \\
43 \\
49 \\
49\end{array}$ & $\begin{array}{l}1,950 \\
2,051 \\
1,687 \\
1,549 \\
1,433 \\
1,556 \\
1,544 \\
1,507 \\
1,620 \\
1,898 \\
2,097 \\
2,184 \\
1,755\end{array}$ & $\begin{array}{r}78 \\
68 \\
72 \\
84 \\
100 \\
110 \\
120 \\
132 \\
133 \\
125 \\
109 \\
89 \\
-\end{array}$ \\
\hline 1993 & 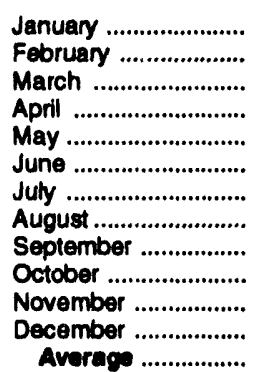 & $\begin{array}{l}1,845 \\
1,929 \\
2,103 \\
2,172 \\
2,116 \\
2,141 \\
2,125 \\
2,105 \\
1,984 \\
1,899 \\
1,789 \\
1,710 \\
1,993\end{array}$ & $\begin{array}{l}126 \\
138 \\
124 \\
161 \\
153 \\
111 \\
175 \\
168 \\
210 \\
200 \\
181 \\
166 \\
160\end{array}$ & $\begin{array}{r}-492 \\
-309 \\
53 \\
472 \\
540 \\
489 \\
391 \\
442 \\
204 \\
-154 \\
-527 \\
-545 \\
49\end{array}$ & $\begin{array}{l}444 \\
363 \\
256 \\
250 \\
254 \\
247 \\
246 \\
269 \\
312 \\
381 \\
469 \\
440 \\
327\end{array}$ & $\begin{array}{l}39 \\
55 \\
47 \\
69 \\
50 \\
41 \\
54 \\
45 \\
35 \\
21 \\
21 \\
40 \\
43\end{array}$ & $\begin{array}{l}1,980 \\
1,958 \\
1,871 \\
1,542 \\
1,425 \\
1,476 \\
1,609 \\
1,517 \\
1,644 \\
1,851 \\
2,007 \\
1,942 \\
1,734\end{array}$ & $\begin{array}{r}73 \\
65 \\
66 \\
81 \\
97 \\
112 \\
124 \\
138 \\
144 \\
139 \\
123 \\
106 \\
-\end{array}$ \\
\hline
\end{tabular}

- A negative number indicates a decrease in stocks and a positive number indicates an increase.

b Stocks are totals as of end of perlod.

c In January 1981, 1983, and 1984, a new stock basis was established affecting stocks reported and stock change calculations. Stock changes are calculated using new basis stock levels. See Summary Statistics Explanatory Note 4.

Notes: - Llquefied petroleum gases includes ethane/ethylene, propane/propylene, normal butane/butylene, and isobutane/isobutylene. Beginning in January 1984, unfrectionated stream, is reported by individual product. - Geographic coverage is the 50 States and the District of Columbla. • Totals may not equal sum of components due to independent rounding.

Source: See Summary Statistics Table and Figure Sources. 
Table 510. Other Petroleum Products Supply and Disposition, 1981 - Precent (Thousand Barrels per Day, Except Where Noted)

\begin{tabular}{|c|c|c|c|c|c|c|c|c|}
\hline \multirow{2}{*}{\multicolumn{2}{|c|}{ Yoarkmonth }} & \multicolumn{2}{|c|}{ 8upply } & \multicolumn{4}{|c|}{ Diepostion } & \multirow{2}{*}{$\begin{array}{l}\text { Ending } \\
\text { Btocks } \\
\text { (Militon Eamble) }\end{array}$} \\
\hline & & $\begin{array}{c}\text { Total } \\
\text { Production }\end{array}$ & Imports & $\begin{array}{l}\text { Stook } \\
\text { Change- }\end{array}$ & $\begin{array}{l}\text { Refinery } \\
\text { Inputs }\end{array}$ & Exports & $\begin{array}{l}\text { Producte } \\
\text { supplied }\end{array}$ & \\
\hline 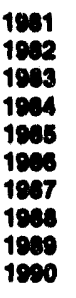 & 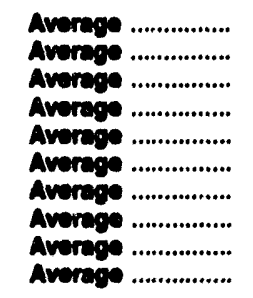 & $\begin{array}{l}2,771 \\
2,475 \\
2,437 \\
2,500 \\
2,892 \\
2,704 \\
2,737 \\
2,773 \\
2,771 \\
2,40\end{array}$ & $\begin{array}{l}189 \\
305 \\
302 \\
603 \\
850 \\
604 \\
543 \\
645 \\
627 \\
705\end{array}$ & $\begin{array}{r}c-42 \\
-88 \\
c-8 \\
c-32 \\
22 \\
-15 \\
-1 \\
22 \\
12 \\
-32\end{array}$ & $\begin{array}{l}723 \\
787 \\
712 \\
791 \\
808 \\
898 \\
609 \\
790 \\
797 \\
807\end{array}$ & $\begin{array}{l}197 \\
208 \\
220 \\
220 \\
227 \\
201 \\
204 \\
204 \\
305 \\
200\end{array}$ & $\begin{array}{l}2,001 \\
1,088 \\
1,077 \\
2,007 \\
1,047 \\
2,048 \\
2,187 \\
2,303 \\
2,208 \\
2,402\end{array}$ & $\begin{array}{l}241 \\
\text { c } 218 \\
217 \\
198 \\
200 \\
201 \\
200 \\
208 \\
213 \\
201\end{array}$ \\
\hline 1001 & 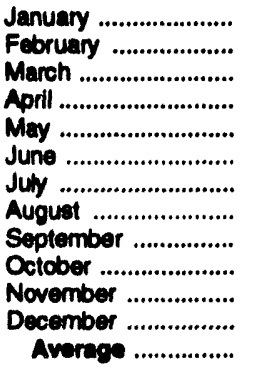 & $\begin{array}{l}2,653 \\
2,688 \\
2,576 \\
2,724 \\
2,853 \\
3,030 \\
3,029 \\
2,993 \\
3,010 \\
2,824 \\
2,750 \\
2,797 \\
2,828\end{array}$ & $\begin{array}{l}748 \\
575 \\
551 \\
607 \\
800 \\
615 \\
776 \\
642 \\
746 \\
611 \\
850 \\
577 \\
675\end{array}$ & $\begin{array}{r}204 \\
363 \\
151 \\
133 \\
198 \\
-123 \\
-143 \\
-169 \\
101 \\
-218 \\
-81 \\
-163 \\
18\end{array}$ & $\begin{array}{r}844 \\
726 \\
819 \\
753 \\
900 \\
1,092 \\
1,081 \\
1,013 \\
802 \\
944 \\
1,093 \\
1,147 \\
986\end{array}$ & $\begin{array}{l}317 \\
275 \\
239 \\
228 \\
327 \\
304 \\
321 \\
296 \\
267 \\
211 \\
238 \\
304 \\
277\end{array}$ & $\begin{array}{l}2,036 \\
1,876 \\
1,919 \\
2,217 \\
2,228 \\
2,372 \\
2,545 \\
2,496 \\
2,586 \\
2,498 \\
2,349 \\
2,085 \\
2,200\end{array}$ & $\begin{array}{r}207 \\
217 \\
222 \\
226 \\
232 \\
228 \\
224 \\
219 \\
222 \\
215 \\
213 \\
208 \\
-\end{array}$ \\
\hline 1902 & 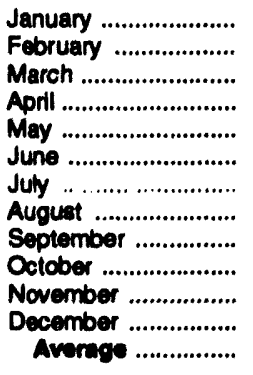 & $\begin{array}{l}2,702 \\
2,642 \\
2,752 \\
2,900 \\
2,929 \\
3,126 \\
3,207 \\
3,068 \\
3,114 \\
2,923 \\
2,915 \\
2,853 \\
2,928\end{array}$ & $\begin{array}{l}734 \\
575 \\
713 \\
793 \\
665 \\
669 \\
740 \\
729 \\
748 \\
701 \\
697 \\
711 \\
707\end{array}$ & $\begin{array}{r}203 \\
183 \\
238 \\
-31 \\
-113 \\
-42 \\
-156 \\
-116 \\
188 \\
-182 \\
-24 \\
-165 \\
-3\end{array}$ & $\begin{array}{r}787 \\
883 \\
730 \\
1,043 \\
910 \\
787 \\
996 \\
884 \\
675 \\
954 \\
989 \\
1,223 \\
906\end{array}$ & $\begin{array}{l}272 \\
240 \\
239 \\
217 \\
199 \\
225 \\
284 \\
227 \\
336 \\
295 \\
264 \\
352 \\
233\end{array}$ & $\begin{array}{l}2,175 \\
1,911 \\
2,258 \\
2,464 \\
2,598 \\
2,826 \\
2,822 \\
2,802 \\
2,683 \\
2,557 \\
2,383 \\
2,164 \\
2,470\end{array}$ & $\begin{array}{r}214 \\
219 \\
227 \\
226 \\
222 \\
221 \\
216 \\
212 \\
218 \\
212 \\
212 \\
207 \\
-\end{array}$ \\
\hline 1093 & 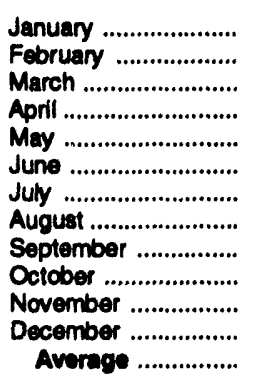 & $\begin{array}{l}3,147 \\
2,853 \\
2,887 \\
2,935 \\
2,941 \\
3,099 \\
3,213 \\
3,167 \\
3,087 \\
3,195 \\
3,080 \\
2,816 \\
3,035\end{array}$ & $\begin{array}{l}726 \\
773 \\
826 \\
753 \\
834 \\
654 \\
894 \\
693 \\
600 \\
810 \\
795 \\
678 \\
770\end{array}$ & $\begin{array}{r}739 \\
111 \\
245 \\
-29 \\
80 \\
-239 \\
61 \\
-28 \\
-268 \\
-114 \\
-222 \\
-376 \\
-2\end{array}$ & $\begin{array}{r}929 \\
1,057 \\
843 \\
1,033 \\
1,048 \\
1,064 \\
1,008 \\
940 \\
1,104 \\
1,189 \\
1,355 \\
1,403 \\
1,081\end{array}$ & $\begin{array}{l}271 \\
282 \\
269 \\
315 \\
278 \\
278 \\
303 \\
294 \\
282 \\
369 \\
309 \\
349 \\
300\end{array}$ & $\begin{array}{l}1,933 \\
2,176 \\
2,356 \\
2,368 \\
2,368 \\
2,650 \\
2,735 \\
2,654 \\
2,749 \\
2,561 \\
2,433 \\
2,117 \\
2,426\end{array}$ & $\begin{array}{r}229 \\
233 \\
240 \\
239 \\
242 \\
235 \\
237 \\
236 \\
228 \\
224 \\
217 \\
206 \\
-\end{array}$ \\
\hline
\end{tabular}

a A negative number indicates a decrease in stocks and a positive number indicates an increase.

Stocks are totals as of end of period.

c In January 1981, 1983, and 1984, a new stock basis was established affecting stocks reported and stock chance calculations. Stock changes are calculated using new basis stock levels. Bulk terminal and pipeline stocks of oxygenates were added beginning in January 1993 . See Summary Statistics Explanatory Note 4.

Notes: - Other petroleum products includes pentanes plus, other hydrocarbons and oxygenates, untinished olls, gasoline blending components and all finished petroleum products except finished motor gasoline, distillate fuel oil, residual fuel oll, jet fuel, and liquefied petroleum gases. • Geographic coverage is the 50 States and the District of Columbla. - Totals may not equal sum of components due to independent rounding.

Source: See Summary Statistics Table and Figure Sources. 


\section{Summary Statistics Table and Figure Sources}

Information about petroleum supply and disposition at the National level are presented in the Summary Statistics tables. Industry terminology and product definitions are listed alphabetically in the Glossary.

The data presented in these tables are from several sources and represent different levels of timeliness and data finality.

- U.S. Department of Energy, Energy Information Administration (EIA), Petroleum Supply Annual (1981 through 1993).

- Data on crude oil production are reported to the EIA by State government agencies. Data on crude oil production for Federal offshore areas are reported to the
EIA by the Minerals Management Service of the U.S. Department of the Interior and the Conservation Committee of California Oil Producers. Crude oil production data for 1993 reflect data received as of April 1994. Data for 1993 received after April will be published as an appendix in the following year's Petroleum Supply Annual.

- Data on exports of crude oil and petroleum products are received from the U.S. Bureau of the Census. Export statistics reflect exports of domestic and foreign merchandise from the United States (the 50 States and the District of Columbia) to foreign countries and U.S. possessions. 


\section{Summary Statistics Explanatory Notes}

The following notes are provided to assist in understanding and interpreting the data presented in the Summary Statistics section of this publication.

\section{Note 1. Domestic Crude Oll Production}

The Energy Information Administration (EIA) collects monthly crude oil production data on an ongoing basis. Data on crude oil production for States are reported to the EIA by State govemment agencies. Data on crude oil production for Federal offshore areas are reported to the EIA by the Minerals Management Service of the U.S. Department of the Interior and the Conservation Committee of California Oil and Gas Producers.

Currently, all except four crude oil producing States (Michigan, New York, Ohio, and Pennsylvania) report production on a monthly basis. These four States report crude oil on an annual basis. Estimates of monthly crude oil production for these four States are made by the EIA using data reported on Form ElA-182, "Domestic Crude Oil First Purchase Report."

After the end of each calendar year, the monthly crude oil production estimates are updated using annual reports from various State agencies, the Mineral Management Service, and the Conservation Committee of California Oil and Gas Producers. The EIA incorporates production data into its Crude Oil Production System (COPS) as the data are received from the reporting agencies. Tables S1 and S2 present the 1993 crude oil production data received by the EIA as of April 1994. Crude oil production data for 1993 received after April 1994 will be published later as an appendix in the following year's Petroleum Supply Annual (PSA). Table C1 of this publication presents the 1992 crude oil production a year after it was published in the PSA 1992.

\section{Note 2. Frames Maintenance}

In January 1981 and 1983, numerous respondents were added to bulk terminal and pipeline surveys affecting subsequent stocks reported and stock change calculations. Using the expanded coverage (new basis), the end-of-year stocks, in million barrels, would have been as listed below.

- Crude Oil: 1982-645 (Total) and 351 (Other Primary).

- Crude Oil and Petroleum Products: 1980-1,425; and 1982-1,461.

- Motor Gasoline: 1980-263 (Total) and 214 (Finished); 1982-244 (Total) and 202 (Finished).

- Distillate Fuel Oil: 1980-205; and 1982-186.
Residual Fuel Oil: 1980-91; and 1982-69.

- Jet Fuel: 1980-42 (Total) and 36 (Kerosene-type); and 1982-39 (Total) and 32 (Kerosene-type).

- Propane/Propylene: 1980-69; and 1982-57.

- Liquefied Petroleum Gases: 1980-128; and 1982-102.

- Other Petroleum Products: 1980-207; and 1982-219.

Stock change calculations beginning in 1981 and 1983 were made using new basis stock levels.

Stocks of Alaskan crude oil in-transit were included for the first time in January 1981. The major impact of this change is on the reporting of stock change calculations. Using the expanded coverage (new basis), 1980 end-of-year crude oil stocks would have been $\mathbf{4 8 8}$ million barrels (Total) and $\mathbf{3 8 0}$ million barrels (Other Primary).

Beginning with January 1984, natural gas liquids supply and disposition data were collected on a component basis rather than a product basis. This change affected stocks reported and stock change calculations. Under the new basis, end-of-year 1983 stocks would have been:

- Propane/Propylene: 1983-55.

- Liquefied Petroleum Gases: 1983-108.

- Other Petroleum Products: 1983-210.

In response to changes in the Clean Air Act Amendments of 1990 requiring that all gasoline sold in carbon monoxide nonattainment areas have an oxygen content of 2.7 percent (by weight) during winter months, the Energy Information Administration (EIA) conducted a frame identifier survey in 1991 of companies that produce, blend, store, or import oxygenates. The purpose of this survey was to (1) identify all U.S. producers, blenders, storers, and importers of oxygenates; and (2) collect supply and blending data for 1990 and end of 1990 inventory data on those oxygenates blended into motor gasoline. A summary of the results from the identification survey were published in the Weekly Petroleum Status Report dated February 12, 1992 and in the February 1992 issue of the Petroleum Supply Monthly.

In order to continue to provide relevant information about U.S. and regional gasoline supply, the EIA conducted a second frame identifier survey of these companies during 1992. As a result, a number of respondents were added to the monthly surveys effective in January 1993: 19 blenders, 25 stock holders, and 8 importers. This change did not affect stocks reported and therefore did not cause a new basis stock level to be calculated. 


\section{Detalled Statistics}

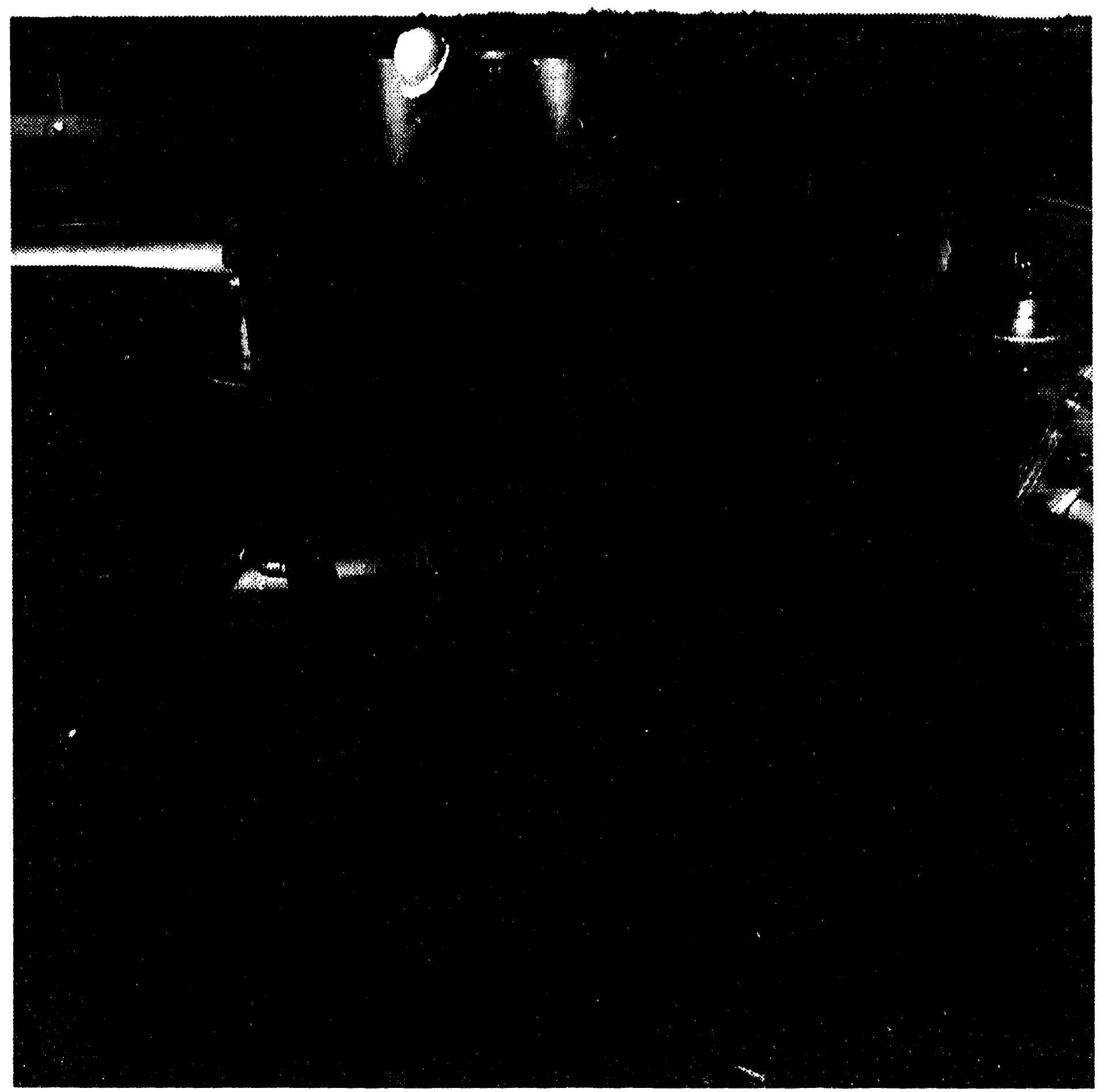

At some locations, oil skimmers and knockout tanks (in background) are used to remove waste water from the crude oil. The crude oil is then put into storage tanks and gauged. 


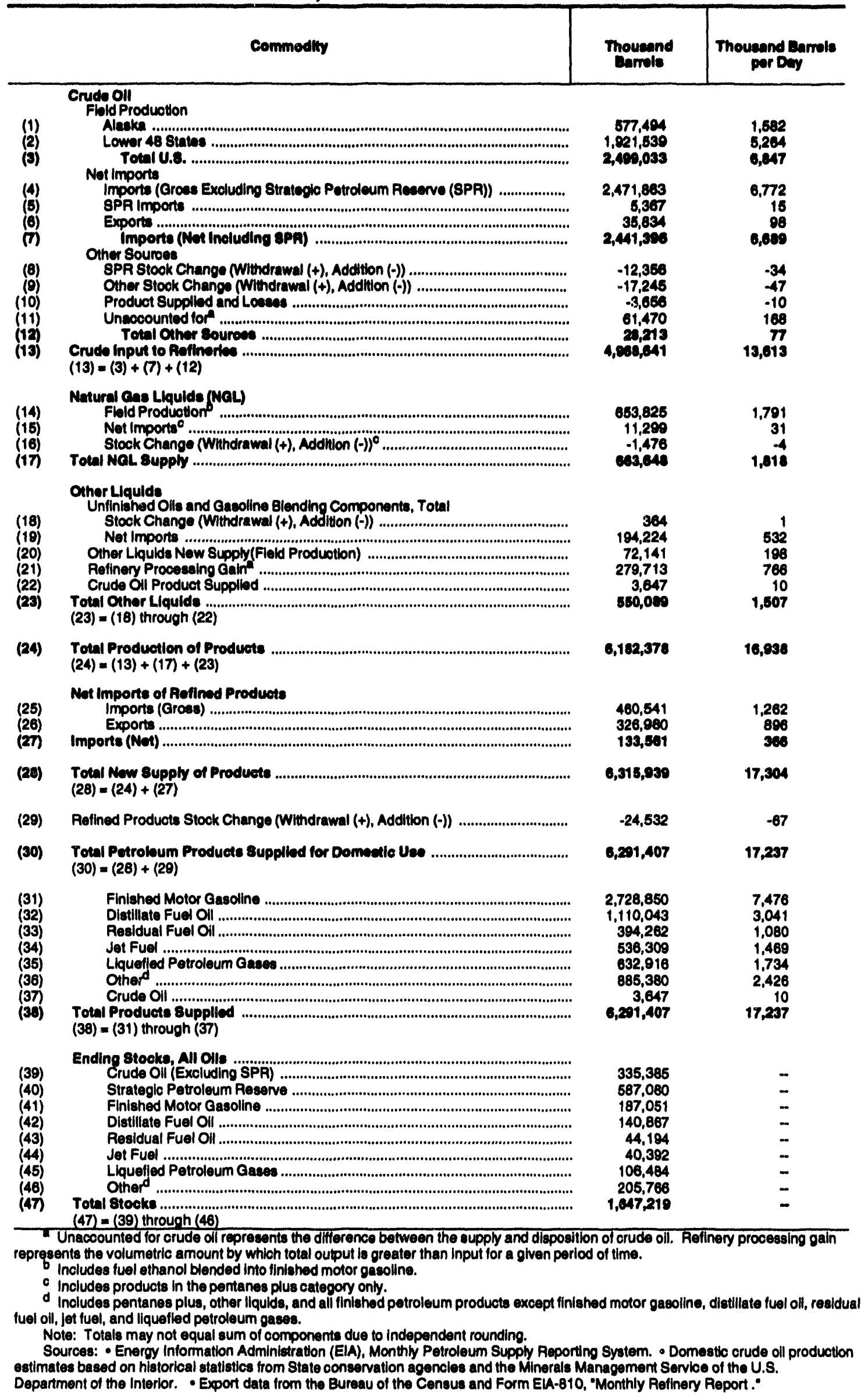


Table 2. U.S. 8upply, Disposition, and Ending Stooks of Cruds Oll and Potroloum Products, 1993 (Thousand Barrels)

\begin{tabular}{|c|c|c|c|c|c|c|c|c|c|c|}
\hline \multirow[b]{2}{*}{ Commodity } & \multicolumn{4}{|c|}{ expply } & \multicolumn{5}{|c|}{ Dleposttion } & \multirow[b]{2}{*}{$\begin{array}{l}\text { Ending } \\
\text { Stooks }\end{array}$} \\
\hline & $\begin{array}{c}\text { Flold } \\
\text { Production }\end{array}$ & Predunory & Imports & $\begin{array}{c}\text { Uneoeounted } \\
\text { For Cruds } \\
\text { olf }\end{array}$ & $\begin{array}{l}\text { Stook } \\
\text { Changeb }\end{array}$ & $\begin{array}{l}\text { Crude } \\
\text { Loneses }\end{array}$ & $\begin{array}{l}\text { Refinery } \\
\text { Inpute }\end{array}$ & Exports & $\begin{array}{l}\text { Products } \\
\text { Suppliod }\end{array}$ & \\
\hline 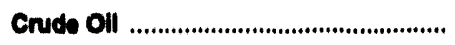 & $2,400,003$ & - & $2,477,200$ & 01,470 & 20,001 & 9 & $4,938,041$ & 38,084 & 3,047 & $\bullet 22,408$ \\
\hline $\begin{array}{l}\text { Netural Cas Liqulde and LACs ............ } \\
\text { Pentanes Plus ...................................... } \\
\text { Liquefled Petroleum Gases ............... } \\
\text { Ethane/Ethylene ............................... } \\
\text { Propane/Propyene ........................ } \\
\text { Normal Butane/Butylene ................ } \\
\text { Isobutane/leobutylene ................... }\end{array}$ & $\begin{array}{r}\mathbf{8 3 3 , 6 1 8} \\
121,852 \\
511,686 \\
202,782 \\
187,168 \\
\mathbf{5 1 , 6 8 1} \\
70,057\end{array}$ & $\begin{array}{r}218,907 \\
215,007 \\
10,104 \\
104,324 \\
38,026 \\
4,868\end{array}$ & $\begin{array}{r}70,090 \\
11,842 \\
88,227 \\
6,268 \\
37,714 \\
10,709 \\
4,490\end{array}$ & 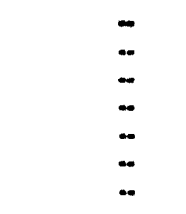 & $\begin{array}{r}10,200 \\
1,476 \\
17,763 \\
1,606 \\
12,321 \\
4,480 \\
.644\end{array}$ & $\begin{array}{l}\ddot{-} \\
\ddot{-} \\
\ddot{-} \\
\ddot{-}\end{array}$ & $\begin{array}{r}179,213 \\
59,762 \\
119,461 \\
0 \\
29 \\
57,652 \\
61,880\end{array}$ & $\begin{array}{r}18,203 \\
543 \\
15,660 \\
0 \\
9,648 \\
6,011 \\
0\end{array}$ & $\begin{array}{r}704,030 \\
71,923 \\
632,916 \\
216,668 \\
367,208 \\
31,342 \\
17,703\end{array}$ & $\begin{array}{r}117,036 \\
10,581 \\
106,484 \\
20,230 \\
51,205 \\
24,812 \\
10,237\end{array}$ \\
\hline $\begin{array}{l}\text { Othor Llquide .................................... } \\
\text { Other Hydrocarbona/Onygenates ...... } \\
\text { Unfiniahed Olls ................................... } \\
\text { Motor Gasoline Blend. Comp. ............. } \\
\text { Aviation Gasollne Biand. Comp. ......... }\end{array}$ & $\begin{array}{r}72,141 \\
68,384 \\
3,747 \\
=\end{array}$ & $\ddot{-}$ & $\begin{array}{r}193,011 \\
7,680 \\
179,301 \\
0,984 \\
0\end{array}$ & $\ddot{-}$ & $\begin{array}{r}\mathbf{2 0 4} \\
5,797 \\
6,870 \\
690 \\
10\end{array}$ & $\ddot{-}$ & $\begin{array}{r}394,044 \\
70,153 \\
254,166 \\
10,424 \\
-48\end{array}$ & $\begin{array}{r}2,617 \\
0 \\
0 \\
2,617 \\
0\end{array}$ & $\begin{array}{r}-67,985 \\
0 \\
-67,984 \\
0 \\
29\end{array}$ & $\begin{array}{r}140,677 \\
12,673 \\
88,420 \\
39,406 \\
78\end{array}$ \\
\hline 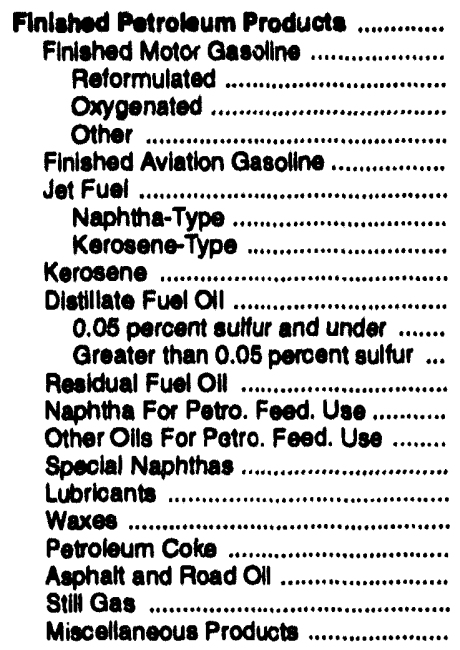 & $\begin{array}{r}20,307 \\
20,307 \\
240,540 \\
-220,233 \\
- \\
- \\
- \\
- \\
- \\
- \\
- \\
- \\
- \\
- \\
- \\
- \\
- \\
- \\
- \\
- \\
- \\
- \\
- \\
-\end{array}$ & $\begin{array}{r}8,846,344 \\
2,686,980 \\
0 \\
329,213 \\
2,336,767 \\
7,782 \\
518,881 \\
40,900 \\
477,881 \\
17,438 \\
1,143,076 \\
327,834 \\
815,241 \\
304,780 \\
53,680 \\
104,045 \\
19,827 \\
68,288 \\
7,328 \\
226,016 \\
164,714 \\
238,365 \\
16,169\end{array}$ & $\begin{array}{r}402,314 \\
80,242 \\
0 \\
421 \\
89,821 \\
78 \\
36,483 \\
3,690 \\
32,790 \\
307 \\
67,184 \\
27,067 \\
40,127 \\
136,151 \\
13,286 \\
40,343 \\
1,790 \\
2,496 \\
646 \\
680 \\
11,771 \\
0 \\
048\end{array}$ & 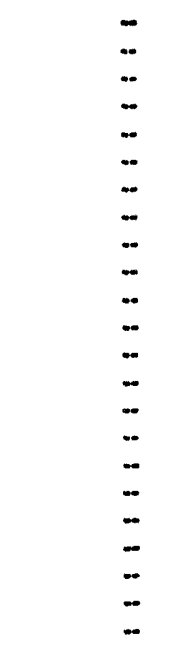 & $\begin{array}{r}0,709 \\
9,488 \\
N A \\
N A \\
N A \\
254 \\
-2,675 \\
-2,077 \\
-588 \\
-1,658 \\
280 \\
N A \\
N A \\
1,605 \\
170 \\
-518 \\
380 \\
-1,487 \\
33 \\
-1,114 \\
1,476 \\
0 \\
557\end{array}$ & 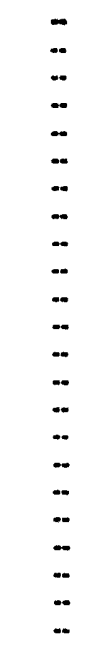 & 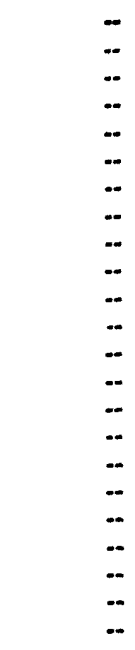 & $\begin{array}{r}311,320 \\
38,183 \\
N A \\
N A \\
N A \\
0 \\
21,700 \\
5,916 \\
15,784 \\
1,289 \\
99,957 \\
N A \\
N A \\
45,064 \\
0 \\
0 \\
1,309 \\
6,782 \\
712 \\
94,336 \\
1,860 \\
0 \\
118\end{array}$ & $\begin{array}{r}5,850,876 \\
2,728,850 \\
N A \\
N A \\
N A \\
7,606 \\
536,309 \\
40,841 \\
495,468 \\
18,114 \\
1,110,043 \\
N A \\
N A \\
394,262 \\
66,805 \\
144,906 \\
19,928 \\
55,486 \\
7,229 \\
133,483 \\
173,149 \\
238,365 \\
16,342\end{array}$ & $\begin{array}{r}467,112 \\
187,051 \\
0 \\
33,648 \\
153,503 \\
1,811 \\
40,392 \\
2,348 \\
38,044 \\
4,069 \\
140,867 \\
63,690 \\
77,177 \\
44,194 \\
1,865 \\
1,375 \\
2,516 \\
11,862 \\
854 \\
8,511 \\
19,192 \\
0 \\
2,553\end{array}$ \\
\hline , & $3,224,000$ & $5,702,281$ & $3,140,454$ & 81,470 & 85,245 & ? & $8,482,638$ & 385,974 & $6,291,407$ & $1,647,210$ \\
\hline
\end{tabular}

- Unaccounted for crude ofl represents the difference between the supply and dieposition of crude oll.

- A negative number indicates a decrease in atocks and a poeltive number indloates an increase in stocks.

- Products supplied is equal to tield production, plus refinery production, plus imports, plus unaccounted for crude oll, minus stock ohange, minus crude losses, minus refinery inputs, minus exports.

(8) = Less than 500 barrels.

LRG a Llquelled Refinery Gas.

NA $=$ Not avallable.

Note: Totals may not equal sum of components dus to independent rounding.

Sources: - Energy Information Administration (EIA) Forms EIA-810, "Monthly Refinery Report," EIA-81 1, "Monthly Bulk Terminal Report," EIA-812, "Monthly Product Pipeline Report," EIA-813, "Monthly Crude OII Report," EIA-814, "Monthly Imports Report," ElA-816, "Monthly Natural Gas Llquids Report," EIA-817, "Monthly Tanker and Barge Movement Repont, and ElA-818M, "Monthly Oxygenate Telephone Report" - Domeatic crude oll production from State conservation agencies and the Minerals Management Service of the U.S. Department of the Interior. - Export data from the Bureau of the Census and Form ElA-810, "Monthly Refinen Report." 
Table 3. U.S. Dally Average Supply and Dlaposttion of Crude Oll and Potroloum Products, 1993 (Thousand Barrels per Day)

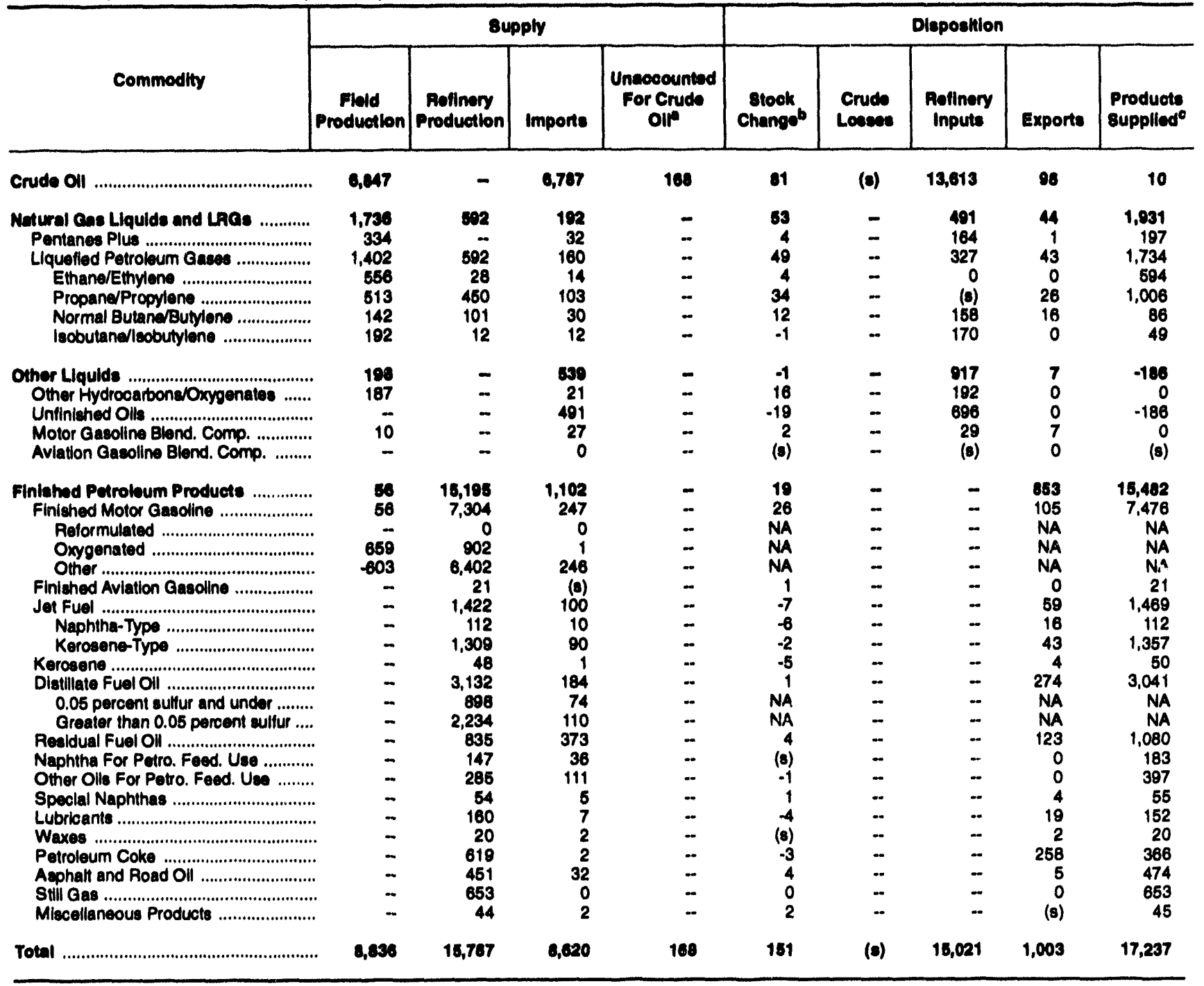

- Unaccounted for crude oll represants the difference between the supply and disposition of crude oll.

b A negative number indloates a decrease in stocks and a positive number indicates an increase in stocks.

c Products aupplled is equal to field production, plus refinery produotion, plus importa, plus unaccounted for crude oll, minus stock change, minus crude losses, minus refinery inpute, minus exports.

(s) = Less than 600 barrels per day.

LRG = Llquefled Refinery Gas.

NA $=$ Not avallable.

Note: Totals may not equal sum of components due to independent rounding.

Sources: - Energy Intormation Administration (EIA) Forms EIA-810. "Monthly Refinery Report. EIA-811, "Monthly Bulk Terminal Report," EIA-812. "Monthly Product Pipeline Report," ElA-813, "Monthly Crude Ol Report," ElA-814, "Monthly Imports Report," ElA-816, "Monthly Natural Gas Llquids Report," EIA-817, "Monthly Tanker and Barge Movement Report," and ElA-819M, "Monthly Oxygenate Telephone Report". - Domestic crude ofl production from State conservation agencles and the Minerals Management Service of the U.S. Department of the Interior. • Export data from the Bureau of the Census and Form ElA-810, "Monthly Refinery Report." 
Table 4. PAD District ⺊Supply, Disposhtion, and Ending Stocks of Crude Oll and Petrobum Producte, 1993 (Thousand Barrels)

\begin{tabular}{|c|c|c|c|c|c|c|c|c|c|c|c|}
\hline \multirow[b]{2}{*}{ Commodity } & \multicolumn{5}{|c|}{ 8upply } & \multicolumn{5}{|c|}{ Diepostiton } & \multirow[b]{2}{*}{$\begin{array}{l}\text { Ending } \\
\text { gleolne }\end{array}$} \\
\hline & $\begin{array}{c}\text { Fiold } \\
\text { Preduction }\end{array}$ & $\begin{array}{c}\text { Refinery } \\
\text { Productlon }\end{array}$ & $\begin{array}{c}\text { Importe by } \\
\text { PAD } \\
\text { Diatrlot } \\
\text { of Entre }\end{array}$ & $\begin{array}{l}\text { Uneo- } \\
\text { coumbed } \\
\text { For } \\
\text { crude olp }\end{array}$ & $\begin{array}{c}\text { Net } \\
\text { Breadoth }\end{array}$ & $\begin{array}{c}\text { Stock } \\
\text { Chmnen }\end{array}$ & $\begin{array}{l}\text { Cruds } \\
\text { Leane: }\end{array}$ & $\begin{array}{c}\text { Rofinery } \\
\text { Inpite }\end{array} \mid$ & Expontel & $\begin{array}{l}\text { Producte } \\
\text { Supollind }\end{array}$ & \\
\hline 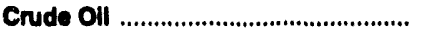 & 10,032 & - & 498,003 & $-3,077$ & 270 & 298 & $\mathbf{0}$ & 408,241 & 2 & $\mathbf{0}$ & 14,320 \\
\hline $\begin{array}{l}\text { Netural Cas Llquide and LRGs ........ } \\
\text { Pentanes Plus ............................... } \\
\text { Llquefled Petroleum Gases ........... } \\
\text { Ethane/Ethylene ......................... } \\
\text { Propane/Propylene .................. } \\
\text { Normal Butane/Butylene ............ } \\
\text { Isobutane/lsobutylene ............... }\end{array}$ & $\begin{array}{r}10,071 \\
1,293 \\
8,778 \\
3,04 \\
3,821 \\
1,426 \\
491\end{array}$ & $\begin{array}{r}16,784 \\
- \\
16,754 \\
0 \\
16,503 \\
793 \\
-542\end{array}$ & $\begin{array}{r}0,002 \\
1,512 \\
7,150 \\
1 \\
5,977 \\
1,086 \\
108\end{array}$ & $\begin{array}{l}- \\
- \\
- \\
- \\
-\end{array}$ & $\begin{array}{r}39,712 \\
0 \\
39,712 \\
0 \\
37,679 \\
1,650 \\
383\end{array}$ & $\begin{array}{r}518 \\
239 \\
279 \\
0 \\
-43 \\
342 \\
-20\end{array}$ & 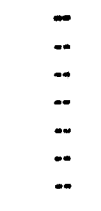 & $\begin{array}{r}5,009 \\
942 \\
4,067 \\
0 \\
0 \\
2,727 \\
1,340\end{array}$ & $\begin{array}{r}681 \\
20 \\
661 \\
0 \\
363 \\
298 \\
0\end{array}$ & $\begin{array}{r}68,091 \\
1,604 \\
67,387 \\
3,041 \\
63,680 \\
1,568 \\
-882\end{array}$ & $\begin{array}{r}5,492 \\
288 \\
5,204 \\
0 \\
3,650 \\
1,444 \\
110\end{array}$ \\
\hline 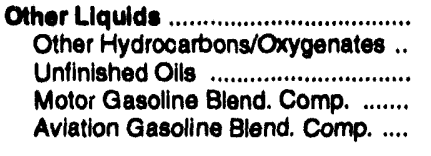 & $\begin{array}{r}16,908 \\
11,939 \\
\ldots \\
4,964 \\
\ldots\end{array}$ & $\begin{array}{l}- \\
\cdots \\
\cdots\end{array}$ & $\begin{array}{r}56,353 \\
2,398 \\
45,612 \\
7,343 \\
0\end{array}$ & $\begin{array}{l}\ddot{-} \\
\ddot{-} \\
\ddot{-}\end{array}$ & $\begin{array}{r}8,175 \\
0 \\
3,623 \\
1,652 \\
0\end{array}$ & $\begin{array}{r}-2,74 \\
348 \\
-2,147 \\
-1,037 \\
42\end{array}$ & 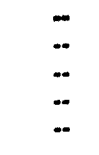 & $\begin{array}{r}84,815 \\
13,899 \\
55,615 \\
14,959 \\
-48\end{array}$ & $\begin{array}{r}37 \\
0 \\
0 \\
37 \\
0\end{array}$ & $\begin{array}{r}-4,327 \\
0 \\
-4,333 \\
0 \\
6\end{array}$ & $\begin{array}{r}16,114 \\
1,560 \\
8,937 \\
5,575 \\
42\end{array}$ \\
\hline 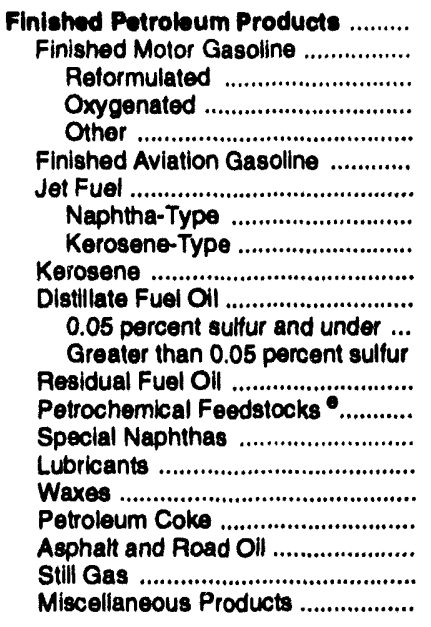 & $\begin{array}{r}-3,762 \\
-3,762 \\
\ldots \\
12,027 \\
-15,789 \\
\ldots \\
\ldots \\
\ldots \\
\ldots \\
\ldots \\
\ldots \\
\ldots \\
\ldots \\
\ldots \\
\ldots \\
\ldots \\
\ldots \\
\ldots \\
\ldots \\
.-\end{array}$ & $\begin{array}{r}593,003 \\
285,612 \\
0 \\
64,793 \\
220,819 \\
199 \\
31,591 \\
2,825 \\
28,768 \\
1,195 \\
145,951 \\
35,762 \\
110,189 \\
44,424 \\
5,939 \\
485 \\
6,234 \\
1,480 \\
17,887 \\
29,096 \\
22,103 \\
797\end{array}$ & $\begin{array}{r}300,860 \\
84,432 \\
0 \\
421 \\
84,011 \\
0 \\
29,937 \\
2,046 \\
27,891 \\
239 \\
59,237 \\
22,128 \\
37,109 \\
110,481 \\
1,808 \\
188 \\
2,156 \\
413 \\
0 \\
10,960 \\
0 \\
701\end{array}$ & 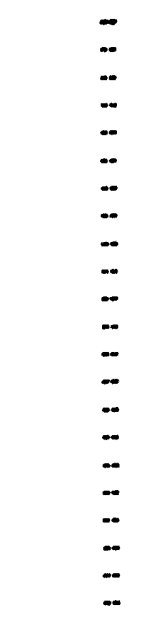 & $\begin{array}{r}892,903 \\
543,191 \\
0 \\
68,595 \\
474,596 \\
1,208 \\
120,323 \\
1,990 \\
118,333 \\
6,680 \\
207,595 \\
28,968 \\
178,627 \\
3,084 \\
-528 \\
877 \\
6,000 \\
26 \\
0 \\
3,698 \\
0 \\
751\end{array}$ & $\begin{array}{r}61 \\
4,891 \\
N A \\
N A \\
N A \\
215 \\
-700 \\
-16 \\
-684 \\
-657 \\
-2,541 \\
N A \\
N A \\
-779 \\
54 \\
-27 \\
-649 \\
81 \\
-80 \\
319 \\
0 \\
-56\end{array}$ & 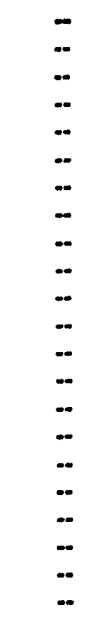 & 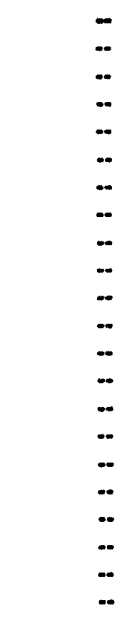 & $\begin{array}{r}10,979 \\
1,532 \\
N A \\
N A \\
N A \\
0 \\
334 \\
18 \\
316 \\
27 \\
1,684 \\
N A \\
N A \\
3,552 \\
0 \\
112 \\
1,405 \\
150 \\
1,894 \\
112 \\
0 \\
76\end{array}$ & $\begin{array}{r}1,71,745 \\
903,050 \\
N A \\
N A \\
N A \\
1,190 \\
182,217 \\
6,859 \\
175,358 \\
8,744 \\
413,640 \\
N A \\
N A \\
155,216 \\
7,163 \\
1,465 \\
13,634 \\
1,688 \\
16,083 \\
43,323 \\
22,103 \\
2,229\end{array}$ & $\begin{array}{r}157,610 \\
69,378 \\
0 \\
15,014 \\
44,364 \\
613 \\
8,922 \\
271 \\
8,651 \\
1,640 \\
62,539 \\
19,928 \\
42,611 \\
16,288 \\
275 \\
2222 \\
2,695 \\
163 \\
911 \\
3,504 \\
0 \\
460\end{array}$ \\
\hline Total & 33,244 & 600,047 & $\mathbf{8 5 3 , 4 0 9}$ & 2,677 & 938,009 & $-1,022$ & $\mathbf{0}$ & 604,785 & 11,690 & $1,836,400$ & 193,553 \\
\hline
\end{tabular}

a Represents the PAD District in which the material entered the United Stabes and not necessarly where the crude oll or product is processed andror consumed.

b Unaccounted for crude oll represents the difference between the supply and dieposition of crude oll.

c A negative number indlcates a decrease in stocks and a positve number indloates an increase in stocks.

d Products supplied is equal to fleld production, plus refinery production, plus imports, plus unacoounted for crude oll, plus net recelpts, minus stock change, minus crude losses, minus refinery inputs, minus exports.

Includes naphtha less than $401^{\circ} \mathrm{F}$ endpoint and other olls equal to or greater than $401^{\circ} \mathrm{F}$ endpoint.

(s) = Less than 500 barrels.

LRG a Llquefled Refinery Gas.

NA $=$ Not available.

Note: Totals may not equal sum of components due to independent rounding.

Sources: - Energy Information Administration (EIA) Forms EIA-810, "Monthly Refinery Report," ElA-811, "Monthly Bulk Terminal Report," EIA-812, "Monthly Product Pipeline Report," ElA-813, "Monthly Crude OIl Report," EIA-814, "Monthly Import Report," ElA-816, "Monthly Natural Gas Liquids Report," ElA-817, "Monthly Tanker and Barge Movement Report," and EIA-819M, "Monthly Oxygenate Telephone Report". - Domestio crude oll production from State conservation agencies and the Minerals Management Service of the U.S. Department of the Interior. - Export data from the Bursau of the Census and Form EIA-810, "Monthly Refinery Report." 
Table 5. PAD Distriet 1 Dally Average Supply and Dlaposition of Cruds Oll and Potroloum Producte, 1993 (Thousand Barrels per Day)

\begin{tabular}{|c|c|c|c|c|c|c|c|c|c|c|}
\hline \multirow[b]{2}{*}{ Commodisy } & \multicolumn{5}{|c|}{ aupply } & \multicolumn{5}{|c|}{ Dlepostion } \\
\hline & $\begin{array}{l}\text { Fibld } \\
\text { Enoduction }\end{array}$ & $\begin{array}{l}\text { Rofinery } \\
\text { Prodpelten }\end{array}$ & $\begin{array}{c}\text { Imports by } \\
\text { PAD } \\
\text { Dietrict } \\
\text { a Entine }\end{array}$ & $\begin{array}{l}\text { Unao } \\
\text { counted } \\
\text { For } \\
\text { Crund oub }\end{array}$ & $\begin{array}{c}\text { Net } \\
\text { Incoilot }\end{array}$ & $\begin{array}{l}\text { Stook } \\
\text { Chrnese }\end{array}$ & $\begin{array}{l}\text { Crude } \\
\text { Learen }\end{array}$ & $\begin{array}{c}\text { Refinory } \\
\text { Inpuin }\end{array}$ & Expenth & $\begin{array}{l}\text { Producte } \\
\text { Pupollad }\end{array}$ \\
\hline 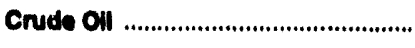 & 27 & 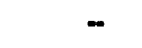 & 1,390 & -10 & 1 & 1 & $\mathbf{0}$ & 1,367 & (c) & $\mathbf{0}$ \\
\hline 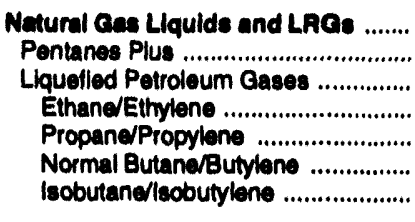 & $\begin{array}{r}24 \\
4 \\
24 \\
8 \\
10 \\
4 \\
1\end{array}$ & $\begin{array}{r}46 \\
-. \\
46 \\
0 \\
46 \\
2 \\
.1\end{array}$ & $\begin{array}{r}24 \\
4 \\
20 \\
(8) \\
16 \\
3 \\
(8)\end{array}$ & $\begin{array}{l}\ddot{-} \\
\ddot{-} \\
\ddot{*} \\
\cdots \\
\cdots\end{array}$ & $\begin{array}{r}100 \\
0 \\
109 \\
0 \\
103 \\
5 \\
1\end{array}$ & $\begin{array}{r}1 \\
1 \\
1 \\
0 \\
(0) \\
1 \\
(8)\end{array}$ & $\begin{array}{l}- \\
\ddot{-} \\
\ddot{*} \\
\ddot{\cdots} \\
\ddot{-}\end{array}$ & $\begin{array}{r}14 \\
3 \\
11 \\
0 \\
0 \\
7 \\
4\end{array}$ & $\begin{array}{r}2 \\
(8) \\
2 \\
0 \\
1 \\
1 \\
0\end{array}$ & $\begin{array}{r}100 \\
4 \\
185 \\
8 \\
174 \\
4 \\
-2\end{array}$ \\
\hline 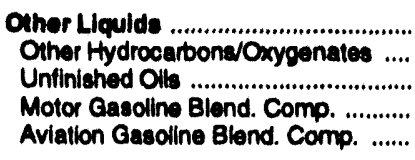 & $\begin{array}{c}48 \\
33 \\
\ddot{14} \\
\cdots\end{array}$ & $\begin{array}{l}- \\
\ddot{-} \\
\ddot{-}\end{array}$ & $\begin{array}{r}182 \\
7 \\
125 \\
20 \\
0\end{array}$ & $\begin{array}{l}\ddot{ } \\
\ddot{-} \\
\ddot{*} \\
\cdots\end{array}$ & $\begin{array}{r}14 \\
0 \\
10 \\
5 \\
0\end{array}$ & $\begin{array}{r}-8 \\
1 \\
-6 \\
-3 \\
(8)\end{array}$ & $\begin{array}{l}- \\
\cdots \\
\cdots \\
\cdots\end{array}$ & $\begin{array}{r}232 \\
38 \\
152 \\
41 \\
(8)\end{array}$ & $\begin{array}{r}(0) \\
0 \\
0 \\
(8) \\
0\end{array}$ & $\begin{array}{r}-12 \\
0 \\
-12 \\
0 \\
(8)\end{array}$ \\
\hline 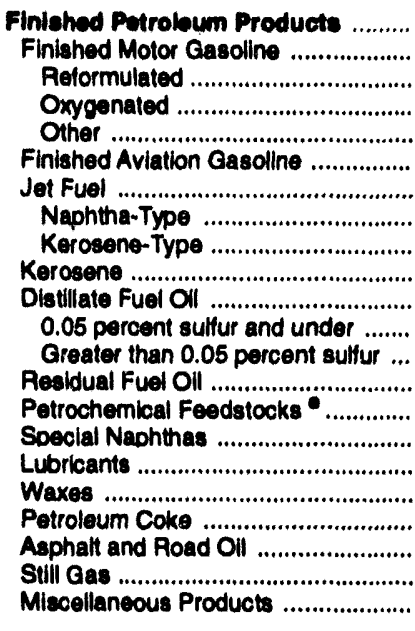 & 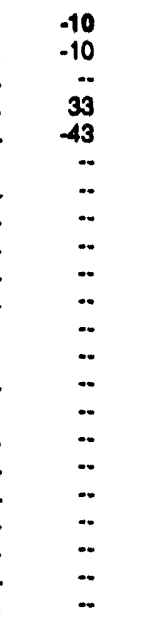 & $\begin{array}{r}1,628 \\
782 \\
0 \\
178 \\
605 \\
1 \\
87 \\
8 \\
79 \\
3 \\
300 \\
8 \\
80 \\
302 \\
122 \\
16 \\
1 \\
17 \\
4 \\
49 \\
80 \\
61 \\
2\end{array}$ & $\begin{array}{r}23 \\
231 \\
0 \\
1 \\
230 \\
0 \\
82 \\
6 \\
76 \\
1 \\
162 \\
61 \\
102 \\
303 \\
5 \\
1 \\
6 \\
1 \\
0 \\
30 \\
0 \\
2\end{array}$ & 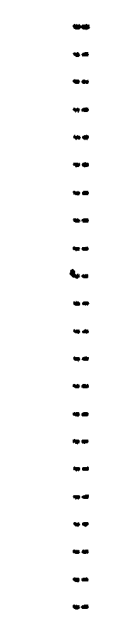 & $\begin{array}{r}2,449 \\
1,488 \\
0 \\
188 \\
1,300 \\
3 \\
330 \\
5 \\
324 \\
18 \\
569 \\
79 \\
489 \\
8 \\
-1 \\
2 \\
16 \\
(8) \\
0 \\
10 \\
0 \\
2\end{array}$ & $\begin{array}{r}(0) \\
13 \\
N A \\
N A \\
N A \\
1 \\
-2 \\
(8) \\
-2 \\
-2 \\
-7 \\
N A \\
N A \\
-2 \\
(8) \\
(8) \\
-2 \\
(8) \\
(8) \\
1 \\
0 \\
(8)\end{array}$ & 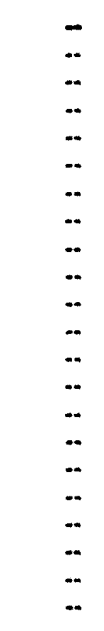 & 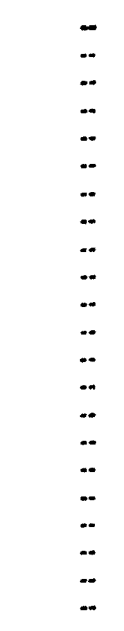 & $\begin{array}{r}30 \\
4 \\
N A \\
N A \\
N A \\
0 \\
1 \\
(8) \\
1 \\
(8) \\
5 \\
N A \\
N A \\
10 \\
0 \\
(8) \\
4 \\
(8) \\
5 \\
(8) \\
0 \\
(8)\end{array}$ & $\begin{array}{r}4,084 \\
2,474 \\
N A \\
N A \\
N A \\
3 \\
499 \\
19 \\
480 \\
24 \\
1,133 \\
N A \\
N A \\
425 \\
20 \\
4 \\
37 \\
5 \\
44 \\
119 \\
61 \\
6\end{array}$ \\
\hline Total & 91 & 1,671 & 2,330 & -10 & 2,570 & -5 & 0 & 1,602 & 32 & 5,031 \\
\hline
\end{tabular}

- Represente the PAD District in which the material entered the United States and not necessarlly where the crude oil or product ls processed and/or consumed.

b Unaccounted for crude oll represents the difference between the supply and disposition of crude oll.

c A negative number indicates a decrease in stocks and a positive number indicates an increase in stocks.

d Products supplied is equal to field production, plus refinery production, plus imports, plus unaccounted for crude oll, plus net receipts, minus stock change, minus crude losses, minus refinery inputs, minus exports.

- Includes naphtha less than $401^{\circ} \mathrm{F}$ endpoint and other olls equal to or greater than $401^{\circ} \mathrm{F}$ endpoint.

(8) = Less than 500 barrels per day.

LRG = Llquefied Retinery Gas.

NA $=$ Not available.

Note: Totals may not equal sum of components due to independent rounding

Sources: - Energy Information Adminiatration (EIA) Forms ElA-810, "Monthly Refinery Report," ElA-811, "Monthly Bulk Terminal Report," ElA-812, "Monthly Product Plpeline Report," ElA-813, "Monthly Crude Oll Report," ElA-814, "Monthly Imports Report," ElA-816, "Monthly Natural Gas Llquids Repont," EIA-817, "Monthty Tanker and Barge Movement Report, and EIA-819M, "Monthly Oxygenate Telephone Report". - Domestic crude oil production from State

conservation agencies and the Minerals Management Service of the U.S. Department of the Interior. - Export data from the Bureau of the Census and Form EIA-810, "Monthly Refinery Report." 
Table 6. PAD District I-Supply, Dispostition, and Ending Stooks of Crude Oll and Potroloum Producte, 1993 (Thousand Barrels)

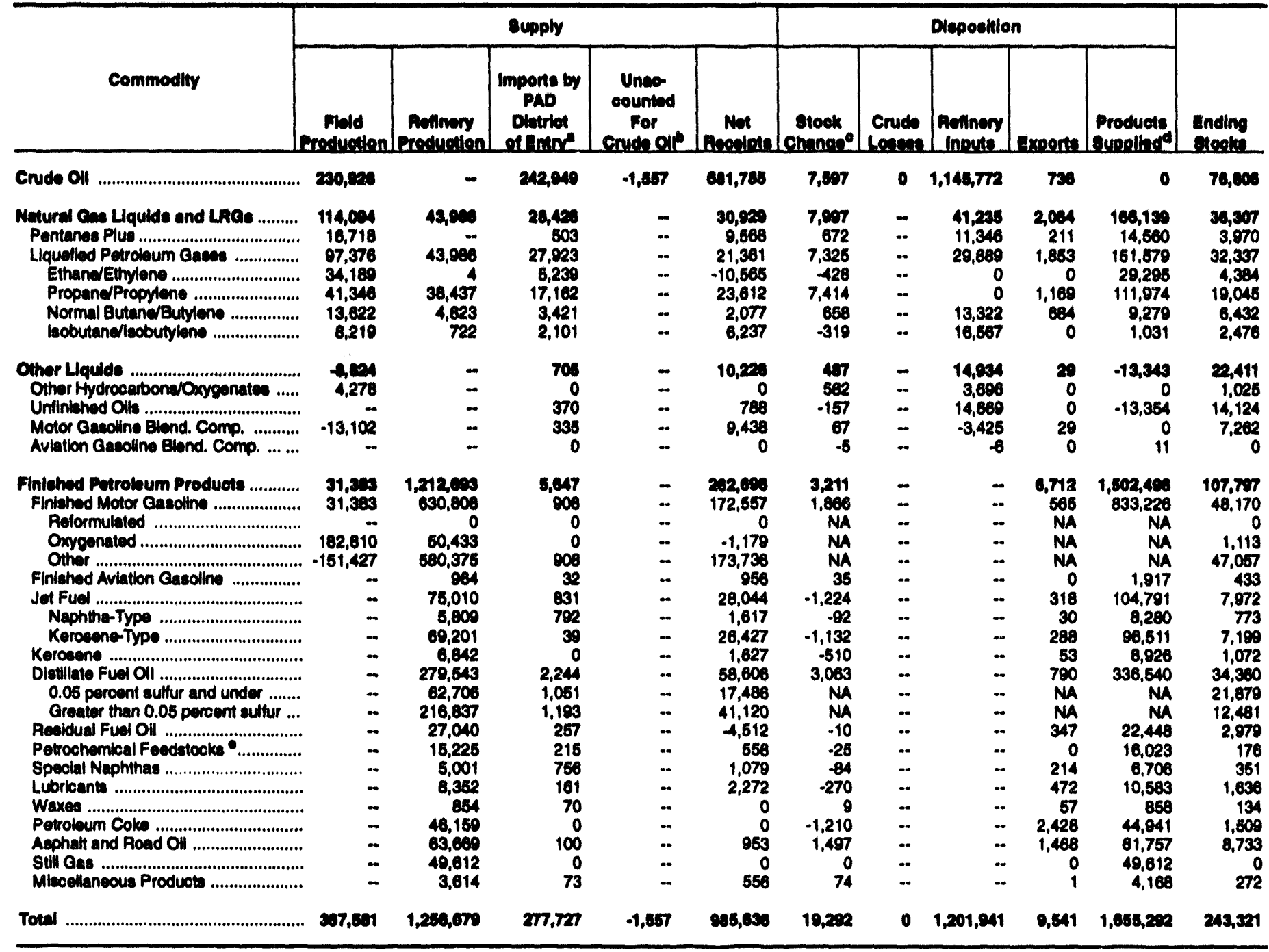

- Represents the PAD Distribt in which the material entered the United States and not necessarlly where the crude oll or product is proceses and/or consumed.

- Unaccounted for crude oll represents the difference between the supply and disposition of crude oll.

- Anegative number indicates a decrease in stocks and a positive number indicates an increase in stocks.

d Products supplied is equal to field production, plus refinery production, plus imports, plus unaccounted for crude oll, plus net recelpts, minus stock change, minus crude losses, minus refinery inputs, minus exports.

- Includes naphtha lees than $401^{\circ} \mathrm{F}$ endpoint and other olls equal to or greater than $401^{\circ} \mathrm{F}$ endpoint.

(8) - Less than 500 barrels.

LRG = Llquefied Refinery Gas.

NA = Not avallable.

Note: Totals may not equal sum of components due to independent rounding.

Sources: - Eneroy Intormation Adminiatration (EIA) Forms EL-810, "Monthly Rolinery Report," ElA-811, "Monthly Bulk Terminal Report," ElA-812, "Monthly Product Ppeline Report," EIA-813, "Monthly Crude OII Report," EIA-814, "Monthly Imports Report, "EIA-816, "Monthly Natural Gas LIquids Report," EIA-817, "Monthly Tenker and Barge Movement Repon," and EIA-819M, "Monthy Oxygenate Telephone Report". - Domeatic crude oll production from State coneervation agencies and the Minerals Management Service of the U.S. Department of the Interior. - Export data from the Bureau of the Census and Form EIA-810, "Monthly Refinery Report." 
Table 7. PAD Diatrict If-Dally Average supply and Dieposition of Cruds Oll and Potroloum Producte, 1903 (Thousand Barrels per Day)

\begin{tabular}{|c|c|c|c|c|c|c|c|c|c|c|}
\hline \multirow[b]{2}{*}{ Commodlyy } & \multicolumn{5}{|c|}{ Supply } & \multicolumn{5}{|c|}{ Bleposillon } \\
\hline & $\begin{array}{l}\text { Fibld } \\
\text { Prodicetion }\end{array}$ & $\begin{array}{l}\text { Rofinery } \\
\text { Prodicilon }\end{array}$ & $\begin{array}{c}\text { Imports by } \\
\text { Pat } \\
\text { Dietrier } \\
\text { of Initur }\end{array}$ & $\begin{array}{l}\text { Unae- } \\
\text { counted } \\
\text { Por } \\
\text { Cruce oub }\end{array}$ & Net & $\begin{array}{l}\text { Stook } \\
\text { then }\end{array}$ & Cructs & $\begin{array}{l}\text { Refiney } \\
\text { hopith }\end{array}$ & moort & $\begin{array}{l}\text { Producte } \\
\text { guoplladt }\end{array}$ \\
\hline 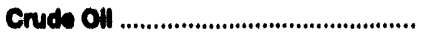 & 033 & - & 693 & 4 & 1,690 & 21 & 0 & 3,130 & 2 & $\mathbf{0}$ \\
\hline 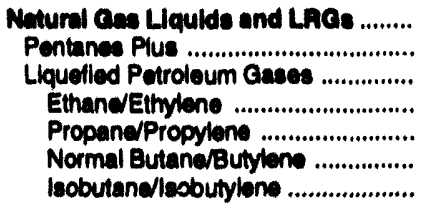 & $\begin{array}{r}313 \\
16 \\
287 \\
04 \\
113 \\
37 \\
23\end{array}$ & $\begin{array}{r}121 \\
121 \\
(8) \\
105 \\
13 \\
2\end{array}$ & $\begin{array}{r}79 \\
1 \\
77 \\
14 \\
47 \\
9 \\
6\end{array}$ & $\begin{array}{l}- \\
\cdots \\
\cdots \\
\cdots \\
\cdots\end{array}$ & $\begin{array}{r}80 \\
26 \\
89 \\
-29 \\
66 \\
6 \\
17\end{array}$ & $\begin{array}{r}22 \\
2 \\
20 \\
-1 \\
20 \\
2 \\
-1\end{array}$ & $\begin{array}{l}\ddot{-} \\
\ddot{-} \\
\ddot{-} \\
\ddot{-}\end{array}$ & $\begin{array}{r}113 \\
31 \\
82 \\
0 \\
0 \\
38 \\
46\end{array}$ & $\begin{array}{l}0 \\
1 \\
5 \\
0 \\
3 \\
2 \\
0\end{array}$ & $\begin{array}{r}485 \\
40 \\
416 \\
80 \\
307 \\
26 \\
3\end{array}$ \\
\hline 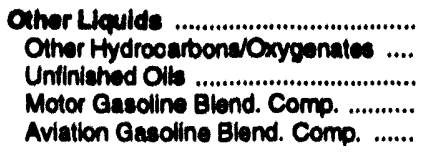 & $\begin{array}{r}24 \\
12 \\
-38 \\
-.\end{array}$ & $\begin{array}{l}- \\
\cdots \\
\cdots\end{array}$ & $\begin{array}{l}2 \\
0 \\
1 \\
1 \\
0\end{array}$ & $\begin{array}{l}- \\
\cdots \\
\cdots\end{array}$ & $\begin{array}{r}2 \\
0 \\
2 \\
28 \\
0\end{array}$ & $\begin{array}{l}1 \\
2 \\
(0) \\
(0)\end{array}$ & $\begin{array}{l}- \\
- \\
-\end{array}$ & $\begin{array}{l}41 \\
10 \\
40 \\
-9 \\
(8)\end{array}$ & $\begin{array}{r}(0) \\
0 \\
0 \\
(0) \\
0\end{array}$ & $\begin{array}{r}-37 \\
0 \\
-37 \\
0 \\
(0)\end{array}$ \\
\hline 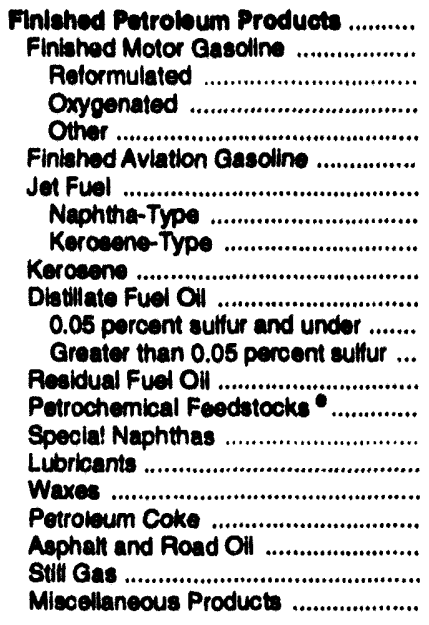 & 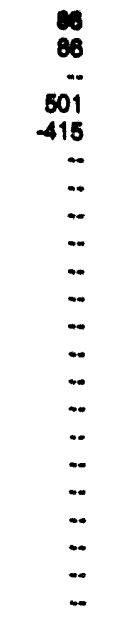 & $\begin{array}{r}3,302 \\
1,728 \\
0 \\
138 \\
1,690 \\
3 \\
208 \\
16 \\
180 \\
19 \\
768 \\
172 \\
504 \\
74 \\
42 \\
14 \\
23 \\
2 \\
126 \\
174 \\
136 \\
10\end{array}$ & $\begin{array}{r}18 \\
2 \\
0 \\
0 \\
2 \\
(8) \\
2 \\
2 \\
(8) \\
0 \\
6 \\
3 \\
3 \\
1 \\
1 \\
2 \\
(8) \\
(8) \\
0 \\
(8) \\
0 \\
(8)\end{array}$ & 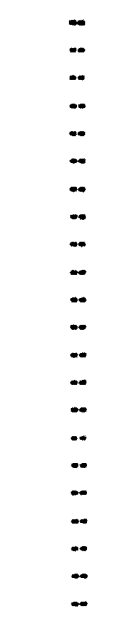 & $\begin{array}{r}720 \\
473 \\
0 \\
-3 \\
478 \\
3 \\
77 \\
4 \\
72 \\
4 \\
161 \\
48 \\
113 \\
-12 \\
2 \\
3 \\
6 \\
0 \\
0 \\
3 \\
0 \\
2\end{array}$ & $\begin{array}{c}9 \\
N \\
N A \\
N A \\
(0) \\
-3 \\
(8) \\
-3 \\
-1 \\
8 \\
N A \\
N A \\
(0) \\
(0) \\
(8) \\
-1 \\
(3) \\
-3 \\
4 \\
0 \\
(0)\end{array}$ & 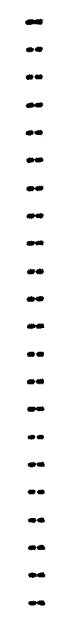 & 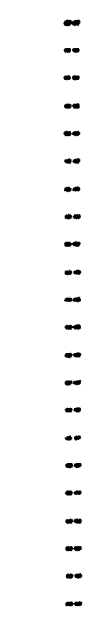 & $\begin{array}{r}18 \\
2 \\
N A \\
N A \\
N A \\
0 \\
1 \\
(8) \\
1 \\
(8) \\
2 \\
N A \\
N A \\
1 \\
0 \\
1 \\
1 \\
(8) \\
7 \\
4 \\
0 \\
(8)\end{array}$ & $\begin{array}{r}4,118 \\
2,283 \\
N A \\
N A \\
N A \\
5 \\
287 \\
23 \\
284 \\
24 \\
922 \\
N A \\
N A \\
82 \\
44 \\
18 \\
29 \\
2 \\
123 \\
169 \\
138 \\
11\end{array}$ \\
\hline 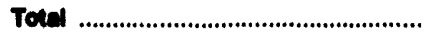 & 1,007 & 3,443 & 781 & 4 & 2,700 & 83 & $\mathbf{0}$ & 3,209 & 28 & 4,838 \\
\hline
\end{tabular}

- Represents the PAD District in which the material entered the United States and not necesaarly where the crude oll or product be procesesed andior consumed.

b Unaccounted for crude oll represents the difference between the supply and dispoeltion of orude oll.

c A negative number indicates a decrease in stocks and a positive number indicates an increase in stocks.

d Products supplied ls equal to field production, plus refinery production, plus imports, plus unaccounted for crude oll, plus net receipte, minus stock change, minus crude losses, minus refinery inputs, minus exports.

- Includes naphtha leas than $401^{\circ} \mathrm{F}$ endpoint and other ols equal to or greater than $401^{\circ} \mathrm{F}$ encpoint.

(8) = Less than 500 barrels per day.

LRG = Liquefled Refinery Gas.

NA = Not available.

Note: Totals may not equal sum of components due to independent rounding.

Sources: - Energy Information Administration (EIA) Forms ElA-810, "Monthly Refinery Repont," ElA-811, "Monthly Bulk Terminal Report," EIA-812, "Monthly Product Pipeline Report," ElA-813, "Monthly Crude Oil Report," ELA-814, "Monthly imports Report," ElA-816, "Monthly Natural Ges Liquide Report," ElA-817, "Monthly Tanker and Barge Movement Report," and EIA-819M, "Monthly Oxygenate Telephone Report". - Domestic orude oll production from State conservation agencies and the Minerals Management Service of the U.S. Department of the Interior. - Export data from the Bureau of the Census and Form EIA-810, "Monthly Refinery Report." 
Table 8. PAD District III-Supply, Disposition, and Ending Stocks of Crude OIl and Petroleum Products, 1998 (Thousand Barrels)

\begin{tabular}{|c|c|c|c|c|c|c|c|c|c|c|c|}
\hline \multirow[b]{2}{*}{ Commodity } & \multicolumn{5}{|c|}{ 8upply } & \multicolumn{5}{|c|}{ Diepostion } & \multirow[b]{2}{*}{$\begin{array}{l}\text { Ending } \\
\text { Exesis: }\end{array}$} \\
\hline & $\begin{array}{c}\text { Fivid } \\
\text { Production }\end{array}$ & $\begin{array}{c}\text { Retinery } \\
\text { Production }\end{array}$ & $\begin{array}{c}\text { Importe by } \\
\text { PAD } \\
\text { Dietriet } \\
\text { of Entin }\end{array}$ & $\begin{array}{l}\text { Unee- } \\
\text { countied } \\
\text { For } \\
\text { Crude ore }\end{array}$ & 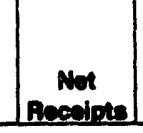 & $\begin{array}{c}\text { Stock } \\
\text { Chnnose }\end{array}$ & $\begin{array}{l}\text { Crude } \\
\text { Loesese } \\
\end{array}$ & $\begin{array}{c}\text { Refinary } \\
\text { Ingute }\end{array}$ & Exports & $\begin{array}{l}\text { Products } \\
\text { Supollade }\end{array}$ & \\
\hline 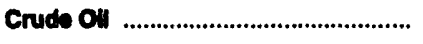 & $1,178,587$ & - & $1,612,135$ & 47,307 & $-570,123$ & 26,028 & 3 & $2,241,351$ & 1 & 0 & 752,220 \\
\hline $\begin{array}{l}\text { Metural Ces Liquide and LRGs ......... } \\
\text { Pentanes Plus .............................. } \\
\text { Liquefied Petroleum Gases ............... } \\
\text { Ethane/Ethylene ............................ } \\
\text { Propane/Propylene ..................... } \\
\text { Normal Butane/Butylene ................ } \\
\text { leobutane/lsobutylene ................... }\end{array}$ & $\begin{array}{r}423,953 \\
73,415 \\
350,538 \\
153,523 \\
122,821 \\
21,801 \\
52,393\end{array}$ & $\begin{array}{r}121,643 \\
121,643 \\
10,100 \\
91,549 \\
16,532 \\
3,462\end{array}$ & $\begin{array}{r}27,919 \\
7,159 \\
20,460 \\
46 \\
12,994 \\
5,429 \\
1,991\end{array}$ & $\begin{array}{l}- \\
- \\
- \\
- \\
-\end{array}$ & $\begin{array}{r}-41,051 \\
-5,846 \\
-36,005 \\
21,950 \\
-53,796 \\
75 \\
-4,234\end{array}$ & $\begin{array}{r}10,190 \\
548 \\
9,611 \\
1,936 \\
4,826 \\
3,394 \\
-545\end{array}$ & $\begin{array}{l}- \\
- \\
- \\
- \\
-\end{array}$ & $\begin{array}{r}98,090 \\
31,004 \\
62,885 \\
0 \\
3 \\
27,258 \\
35,604\end{array}$ & $\begin{array}{r}8,970 \\
293 \\
8,676 \\
0 \\
6,580 \\
2,097 \\
0\end{array}$ & $\begin{array}{r}418,308 \\
42,883 \\
375,484 \\
183,683 \\
162,159 \\
11,088 \\
18,553\end{array}$ & $\begin{array}{r}70,202 \\
6,074 \\
64,218 \\
15,670 \\
26,950 \\
14,608 \\
6,790\end{array}$ \\
\hline $\begin{array}{l}\text { Other Liquids ........................ } \\
\text { Other Hydrocaroons/Oxygenates ..... } \\
\text { Unitinished Olls ................................ } \\
\text { Motor Gasoline Biend. Comp. .......... } \\
\text { Aviation Gasoline Blend. Comp. ....... }\end{array}$ & $\begin{array}{r}47,200 \\
31,431 \\
15,769 \\
-\end{array}$ & $\begin{array}{l}- \\
- \\
- \\
-\end{array}$ & $\begin{array}{r}130,633 \\
262 \\
128,504 \\
1,867 \\
0\end{array}$ & $\ddot{-}$ & $\begin{array}{r}-17,124 \\
0 \\
-4,985 \\
-12,139 \\
0\end{array}$ & $\begin{array}{r}-1,710 \\
3,473 \\
-4,882 \\
-177 \\
-24\end{array}$ & $\begin{array}{l}- \\
\ddot{m} \\
\ddot{-}\end{array}$ & $\begin{array}{r}210,894 \\
28,220 \\
177,795 \\
4,857 \\
12\end{array}$ & $\begin{array}{r}817 \\
0 \\
0 \\
817 \\
0\end{array}$ & $\begin{array}{r}49,292 \\
0 \\
49,294 \\
0 \\
12\end{array}$ & $\begin{array}{r}63,192 \\
6,013 \\
41,449 \\
15,703 \\
27\end{array}$ \\
\hline 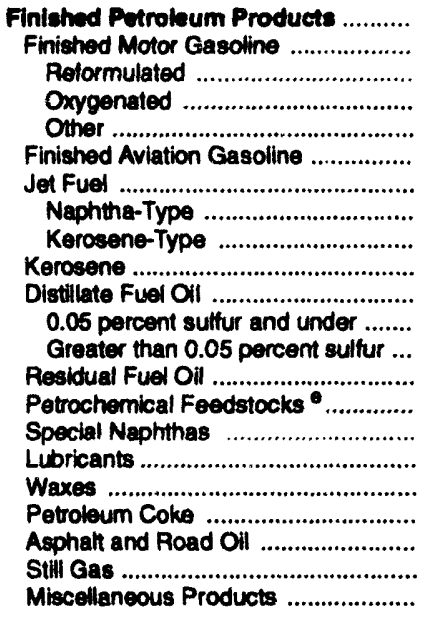 & $\begin{array}{r}-13,044 \\
-13,844 \\
. . \\
19,243 \\
-33,088 \\
. . \\
- \\
.- \\
- \\
. \\
- \\
- \\
- \\
- \\
- \\
- \\
- \\
- \\
- \\
. \\
. \\
-\end{array}$ & $\begin{array}{r}2,580,150 \\
1,208,397 \\
0 \\
91,524 \\
1,116,873 \\
4,399 \\
254,062 \\
22,740 \\
231,322 \\
7,795 \\
510,539 \\
142,762 \\
367,777 \\
128,789 \\
134,402 \\
13,617 \\
36,119 \\
3,907 \\
101,507 \\
41,879 \\
106,565 \\
8,173\end{array}$ & $\begin{array}{r}77,651 \\
1,363 \\
0 \\
0 \\
1,363 \\
0 \\
2,445 \\
584 \\
1,861 \\
47 \\
2,399 \\
2,399 \\
0 \\
18,195 \\
51,353 \\
796 \\
148 \\
105 \\
512 \\
220 \\
0 \\
68\end{array}$ & $\begin{array}{l}- \\
-. \\
-- \\
-- \\
-- \\
-. \\
-. \\
-. \\
-- \\
-- \\
- \\
-- \\
-- \\
-- \\
--\end{array}$ & $\begin{array}{r}-1,199,202 \\
-741,918 \\
0 \\
0 \\
-67,515 \\
-674,403 \\
-2,293 \\
-159,010 \\
-3,713 \\
-155,297 \\
-8,111 \\
-272,646 \\
-51,179 \\
-221,467 \\
1,428 \\
-30 \\
-1,956 \\
-8,782 \\
-26 \\
0 \\
-4,651 \\
0 \\
-1,287\end{array}$ & $\begin{array}{r}63 \\
1,471 \\
N A \\
N A \\
N A \\
-17 \\
-1,449 \\
-989 \\
-460 \\
-507 \\
-1,764 \\
N A \\
N A \\
2,431 \\
-465 \\
504 \\
-246 \\
4 \\
-384 \\
0 \\
0 \\
359\end{array}$ & 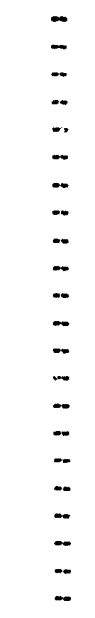 & $\begin{array}{l}- \\
-. \\
- \\
-. \\
-- \\
-- \\
- \\
- \\
- \\
-- \\
-- \\
-- \\
-- \\
-- \\
-- \\
-- \\
-\end{array}$ & $\begin{array}{r}184,994 \\
27,355 \\
N A \\
N A \\
N A \\
0 \\
9,304 \\
1,577 \\
7,727 \\
1,187 \\
53,198 \\
N A \\
N A \\
20,517 \\
0 \\
689 \\
3,559 \\
333 \\
48,734 \\
78 \\
0 \\
30\end{array}$ & $\begin{array}{r}1,269,754 \\
425,171 \\
N A \\
N A \\
N A \\
2,123 \\
89,642 \\
19,023 \\
70,619 \\
-949 \\
188,858 \\
N A \\
N A \\
125,464 \\
186,190 \\
11,264 \\
24,172 \\
3,649 \\
53,669 \\
37,370 \\
106,565 \\
6,565\end{array}$ & $\begin{array}{r}129,517 \\
49,481 \\
0 \\
6,642 \\
42,839 \\
323 \\
13,838 \\
755 \\
13,083 \\
1,179 \\
29,017 \\
12,181 \\
16,836 \\
17,610 \\
2,557 \\
1,894 \\
5,835 \\
449 \\
2,191 \\
3,615 \\
0 \\
1,528\end{array}$ \\
\hline 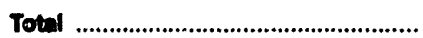 & $1,035,875$ & $2,681,793$ & $1,848,038$ & 47,307 & $-1,028,343$ & 34,914 & 3 & $2,546,104$ & 174,71 & $1,028,038$ & $1,015,200$ \\
\hline
\end{tabular}

aepresents the PAD District in which the material entered the United States and not necessarily where the crude oil or product is processed and/or consumed.

b Unaccounted for crude oll represents the difference between the supply and dispostion of crude oll.

c A negative number indicates a decrease in stocks and a positive number indicates an increase in stocks.

d Products supplied is equal to field production, plus refinery production, plus imports, plus unaccounted for crude oll, plus nel receipts, minus stock change, minus crude losses, minus refinery inputs, minus exports.

Includes naphtha less than $401^{\circ} \mathrm{F}$ endpoint and other oils equal to or greater than $401^{\circ} \mathrm{F}$ endpoint

(s) = Less than 500 barrels.

LRG $=$ Liquefied Refinery Gas.

NA $=$ Not available.

Note: Totals may not equal sum of components due to independent rounding.

Sources: - Energy Information Administration (EIA) Forms ElA-810, "Monthly Refinery Report," EIA-811, "Monthly Bulk Terminal Report," EIA-812, "Monthly Product Pipeline Report, ElA-813, "Monthly Crude Oil Report," ElA-814, "Monthly Imports Report," ElA-816, "Monthly Natural Gas Liquids Report," ElA-817, "Monthly Tanker and Barge Movement Report," and ElA-819M, "Monthly Oxygenate Telephone Report". - Domestic crude oll production from State conservation agencies and the Minerals Management Service of the U.S. Department of the interior. - Export data from the Bureau of the Census and Form ElA-810, "Monthly Refinery Report." 
Table 9. PAD District II-Dally Average Supply and Dieposition of Crude Oil and Petroleum Products, 1993 (Thousand Barrels per Day)

\begin{tabular}{|c|c|c|c|c|c|c|c|c|c|c|}
\hline \multirow[b]{2}{*}{ Commodity } & \multicolumn{5}{|c|}{ Supply } & \multicolumn{5}{|c|}{ Diepostion } \\
\hline & $\begin{array}{l}\text { Field } \\
\text { Produsion }\end{array}$ & $\begin{array}{c}\text { Rofinury } \\
\text { Produaten }\end{array}$ & $\begin{array}{c}\text { importo by } \\
\text { PAD } \\
\text { Dietriat } \\
\text { dening }\end{array}$ & $\begin{array}{l}\text { Unae- } \\
\text { counted } \\
\text { For } \\
\text { Crudiente }\end{array}$ & $\begin{array}{c}\text { Met } \\
\text { Resiphes }\end{array}$ & $\begin{array}{c}\text { 8tock } \\
\text { Chen:e" }\end{array}$ & $\begin{array}{l}\text { Crude } \\
\text { Lenees }\end{array}$ & $\begin{array}{c}\text { Refinery } \\
\text { Inpute }\end{array}$ & Exponts & $\begin{array}{l}\text { Products } \\
\text { suppliadd }\end{array}$ \\
\hline 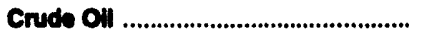 & 3,290 & - & 4,417 & 130 & $-1,522$ & 73 & (e) & 6,141 & (c) & 0 \\
\hline $\begin{array}{l}\text { Meturd Ces Llquids and LRCe ........ } \\
\text { Pentanes Plus .................................. } \\
\text { Liquefied Petroleum Gases .............. } \\
\text { Ethane/Ethylene .............................. } \\
\text { Propane/Propylene .................... } \\
\text { Normal Butane/Butylene ............... } \\
\text { Isobutane/lsobutylene ................... }\end{array}$ & $\begin{array}{r}1,162 \\
201 \\
960 \\
421 \\
336 \\
60 \\
144\end{array}$ & $\begin{array}{r}353 \\
333 \\
28 \\
251 \\
45 \\
9\end{array}$ & $\begin{array}{r}70 \\
20 \\
56 \\
(8) \\
36 \\
15 \\
5\end{array}$ & $\begin{array}{l}- \\
- \\
- \\
- \\
-\end{array}$ & $\begin{array}{r}-115 \\
-16 \\
-99 \\
60 \\
-147 \\
(8) \\
-12\end{array}$ & $\begin{array}{r}20 \\
2 \\
26 \\
5 \\
13 \\
9 \\
-1\end{array}$ & $\begin{array}{l}- \\
\ddot{-} \\
\ddot{-} \\
-- \\
-\end{array}$ & $\begin{array}{r}257 \\
85 \\
172 \\
0 \\
(8) \\
75 \\
98\end{array}$ & $\begin{array}{r}25 \\
1 \\
24 \\
0 \\
18 \\
6 \\
0\end{array}$ & $\begin{array}{r}1,146 \\
117 \\
1,029 \\
503 \\
444 \\
30 \\
51\end{array}$ \\
\hline $\begin{array}{l}\text { Other Llquids .................................... } \\
\text { Other Hydrocarbons/Oxygenates .... } \\
\text { Unfinished Olls ................................. } \\
\text { Motor Gasoline Blend. Comp. ......... } \\
\text { Aviation Gasoline Blend. Comp. ....... }\end{array}$ & $\begin{array}{r}129 \\
86 \\
0 \\
0\end{array}$ & $\begin{array}{l}- \\
\ddot{-} \\
-\end{array}$ & $\begin{array}{r}383 \\
1 \\
352 \\
5 \\
0\end{array}$ & $\begin{array}{l}- \\
- \\
-\end{array}$ & $\begin{array}{r}-47 \\
0 \\
-14 \\
-33 \\
0\end{array}$ & $\begin{array}{r}-6 \\
10 \\
-14 \\
(8) \\
(8)\end{array}$ & $\begin{array}{l}- \\
- \\
- \\
-\end{array}$ & $\begin{array}{r}578 \\
77 \\
487 \\
13 \\
(8)\end{array}$ & $\begin{array}{l}2 \\
0 \\
0 \\
2 \\
0\end{array}$ & $\begin{array}{r}-135 \\
0 \\
-135 \\
0 \\
(8)\end{array}$ \\
\hline 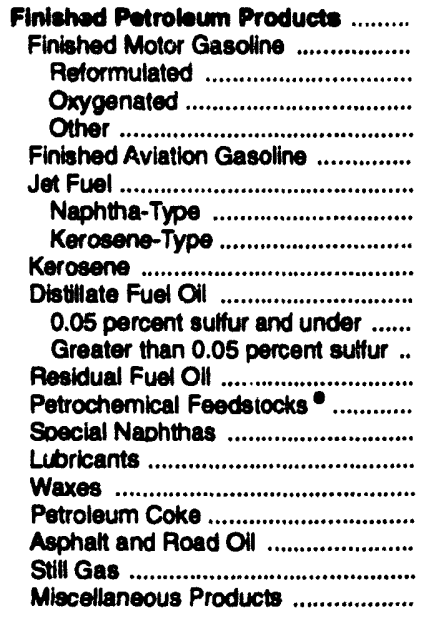 & 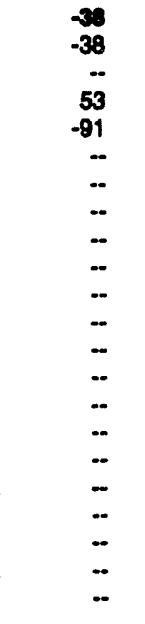 & $\begin{array}{r}7,014 \\
3,311 \\
0 \\
251 \\
3,060 \\
12 \\
696 \\
62 \\
634 \\
21 \\
1,399 \\
391 \\
1,008 \\
353 \\
368 \\
37 \\
99 \\
11 \\
278 \\
115 \\
292 \\
22\end{array}$ & $\begin{array}{r}213 \\
4 \\
0 \\
0 \\
4 \\
0 \\
7 \\
2 \\
5 \\
(8) \\
7 \\
7 \\
0 \\
50 \\
141 \\
2 \\
(8) \\
(8) \\
1 \\
1 \\
0 \\
(8)\end{array}$ & $\begin{array}{l}- \\
\ddot{-} \\
= \\
- \\
-- \\
- \\
- \\
- \\
- \\
- \\
- \\
- \\
- \\
- \\
- \\
-\end{array}$ & $\begin{array}{r}3,223 \\
-2,033 \\
0 \\
-185 \\
-1,848 \\
-6 \\
-436 \\
-10 \\
-425 \\
-22 \\
-747 \\
-140 \\
-607 \\
4 \\
(8) \\
-5 \\
-24 \\
(8) \\
0 \\
-13 \\
0 \\
-4\end{array}$ & $\begin{array}{r}\text { (s) } \\
4 \\
N A \\
N A \\
N A \\
(8) \\
-4 \\
-3 \\
-1 \\
-1 \\
-5 \\
N A \\
N A \\
7 \\
-1 \\
1 \\
-1 \\
(8) \\
-1 \\
0 \\
0 \\
1\end{array}$ & 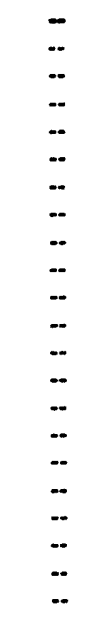 & 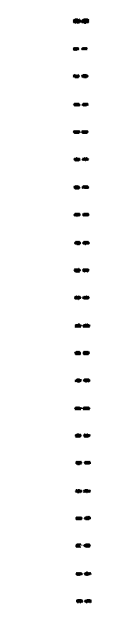 & $\begin{array}{r}452 \\
75 \\
N A \\
N A \\
N A \\
0 \\
25 \\
4 \\
21 \\
3 \\
146 \\
N A \\
N A \\
56 \\
0 \\
2 \\
10 \\
1 \\
134 \\
(s) \\
0 \\
(s)\end{array}$ & $\begin{array}{r}3,451 \\
1,165 \\
N A \\
N A \\
N A \\
6 \\
246 \\
52 \\
193 \\
-3 \\
517 \\
\text { NA } \\
\text { NA } \\
344 \\
510 \\
31 \\
66 \\
10 \\
147 \\
102 \\
292 \\
18\end{array}$ \\
\hline 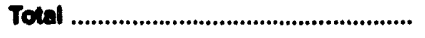 & 4,492 & 7,347 & $8,0.83$ & 130 & $-6,000$ & $\boldsymbol{8}$ & (s) & 6,978 & 479 & 4,463 \\
\hline
\end{tabular}

aepresents the PAD District in which the material entered the United States and not necessarily where the crude oll or product is processed and/or consumed.

b Unaccounted for crude cil represents the difference between the supply and dieposition of crude oll.

c A negative number indicates a decrease in stocks and a positive number indicates an increase in stocks.

d Products supplied is equal to field production, phus refinery production, plus imports, phus unaccounted for crude oil, plus net receipts, minus stock change, minus crude losses, minus refinery inputs, minus exports.

- Includes naphtha less than $401^{\circ} \mathrm{F}$ endpoint and other olls equal to or greater than $401^{\circ} \mathrm{F}$ endpoint.

(s) = Less than 500 barrels per day.

LRG = Liquefied Refinery Gas.

$N A=$ Not avaliable.

Note: Totals may not equal sum of components due to independent rounding.

Sources: - Energy Information Administration (EIA) Forms ElA-810, "Monthly Refinery Report," ELA-811, "Monthly Bulk Terminal Report," EIA-812, "Monthly Product Pipellne Report," EIA-813, "Monthly Crude Oll Report," ELA-814, "Monthly Imports Report," ELA-816, "Monthly Natural Gas Liquids Report," EIA-817, "Monthly Tanker and Barge Movement Report," and EIA-819M, Monthly Oxygenate Telephone Report". - Domestic crude oil production from State conservation agencies and the Minerals Management Senvice of the U.S. Department of the Interior. - Export data from the Bureau of the Census and Form EIA-810, "Monthly Refinery Report." 
Table 10. PAD District IV-Supply, Disposition, and Ending Stocks of Crude Oll and Petroloum Products, 1993 (Thousand Barrels)

\begin{tabular}{|c|c|c|c|c|c|c|c|c|c|c|c|}
\hline \multirow[b]{2}{*}{ Commodity } & \multicolumn{5}{|c|}{ 8upply } & \multicolumn{5}{|c|}{ Diepostion } & \multirow[b]{2}{*}{$\begin{array}{l}\text { Ending } \\
\text { sioeln }\end{array}$} \\
\hline & $\begin{array}{l}\text { Fildd } \\
\text { Production }\end{array}$ & $\begin{array}{l}\text { Refinery } \\
\text { Prodinetlon }\end{array}$ & $\begin{array}{c}\text { Importe by } \\
\text { PAD } \\
\text { Dietrict } \\
\text { of Entre }\end{array}$ & $\begin{array}{l}\text { Uneo- } \\
\text { counted } \\
\text { For } \\
\text { Candecele }\end{array}$ & 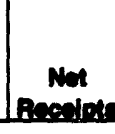 & $\begin{array}{c}\text { Block } \\
\text { Ch:nese }\end{array}$ & $\begin{array}{l}\text { Crude } \\
\text { leme }\end{array}$ & {$\left[\begin{array}{c}\text { Refinery } \\
\text { Inouth }\end{array}\right.$} & Expon: & $\begin{array}{l}\text { Producte } \\
\text { Elypoll det }\end{array}$ & \\
\hline Crude Oll & . 158,351 & - & 32,971 & 13,670 & 40,101 & 271 & 0 & 183,252 & $\mathbf{0}$ & 0 & 11,133 \\
\hline 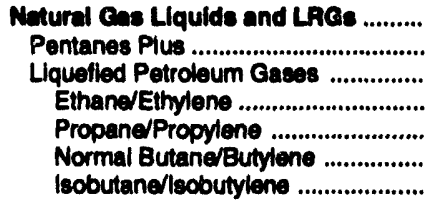 & $\begin{array}{r}\mathbf{4}, 494 \\
11,451 \\
37,013 \\
12,011 \\
15,628 \\
5,689 \\
3,687\end{array}$ & $\begin{array}{r}2,403 \\
- \\
2,563 \\
0 \\
2,877 \\
-153 \\
-161\end{array}$ & $\begin{array}{r}5,130 \\
2,688 \\
2,462 \\
0 \\
1,469 \\
845 \\
148\end{array}$ & $\begin{array}{l}\ddot{-} \\
\ddot{-} \\
\ddot{-} \\
\ddot{-}\end{array}$ & $\begin{array}{r}-20,700 \\
-3,722 \\
-25,068 \\
-11,385 \\
-7,495 \\
-3,802 \\
-2,386\end{array}$ & $\begin{array}{r}61 \\
31 \\
50 \\
-2 \\
-11 \\
28 \\
37\end{array}$ & $\begin{array}{l}- \\
- \\
- \\
-\end{array}$ & $\begin{array}{r}0,320 \\
2,012 \\
4,316 \\
0 \\
0 \\
3,015 \\
1,301\end{array}$ & $\begin{array}{r}100 \\
19 \\
60 \\
0 \\
2 \\
78 \\
0\end{array}$ & $\begin{array}{r}20,858 \\
8,335 \\
12,524 \\
628 \\
12,488 \\
-540 \\
-50\end{array}$ & $\begin{array}{r}1,390 \\
183 \\
1,176 \\
178 \\
480 \\
311 \\
200\end{array}$ \\
\hline $\begin{array}{l}\text { Other Lquids ................................... } \\
\text { Oiher Hydrocarbona/Oxygenates ..... } \\
\text { Unfinished Olls ..................................... } \\
\text { Motor Gasolline Blend. Comp. .......... } \\
\text { Aviation Gasoline Blend. Comp. ........ }\end{array}$ & $\begin{array}{r}1,402 \\
1,209 \\
253 \\
\cdots\end{array}$ & $\begin{array}{l}- \\
- \\
-\end{array}$ & $\begin{array}{l}0 \\
0 \\
0 \\
0 \\
0\end{array}$ & $\ddot{-}$ & $\begin{array}{l}0 \\
0 \\
0 \\
0 \\
0\end{array}$ & $\begin{array}{r}090 \\
143 \\
328 \\
218 \\
0\end{array}$ & $\begin{array}{l}\ddot{m} \\
\ddot{m}\end{array}$ & $\begin{array}{r}214 \\
1,088 \\
-887 \\
35 \\
0\end{array}$ & $\begin{array}{l}0 \\
0 \\
0 \\
0 \\
0\end{array}$ & $\begin{array}{r}569 \\
0 \\
559 \\
0 \\
0\end{array}$ & $\begin{array}{r}5,034 \\
245 \\
2,727 \\
2,082 \\
0\end{array}$ \\
\hline 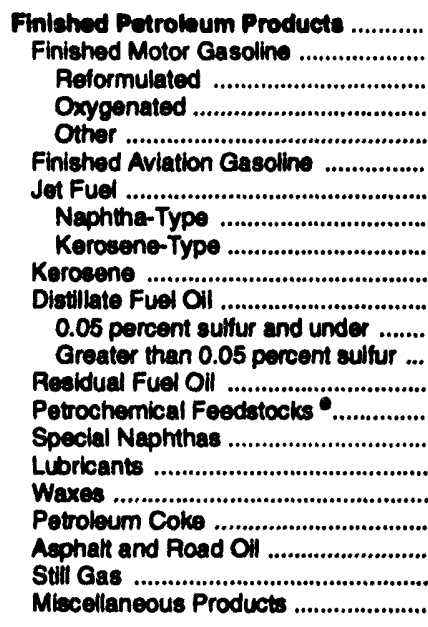 & $\begin{array}{r}220 \\
228 \\
. . \\
4,811 \\
-4,583 \\
-. \\
-. \\
-. \\
-. \\
-. \\
-. \\
- \\
- \\
- \\
- \\
-. \\
- \\
- \\
- \\
-\end{array}$ & $\begin{array}{r}17,670 \\
84,465 \\
0 \\
4,107 \\
80,358 \\
272 \\
12,231 \\
3,775 \\
8,466 \\
607 \\
45,189 \\
12,460 \\
32,729 \\
3,857 \\
255 \\
0 \\
0 \\
381 \\
5,079 \\
12,225 \\
7,428 \\
807\end{array}$ & $\begin{array}{r}1,310 \\
120 \\
0 \\
0 \\
120 \\
0 \\
0 \\
0 \\
0 \\
0 \\
1,138 \\
475 \\
663 \\
18 \\
0 \\
0 \\
0 \\
34 \\
0 \\
0 \\
0 \\
0\end{array}$ & $\begin{array}{l}= \\
= \\
= \\
= \\
= \\
= \\
= \\
= \\
= \\
= \\
= \\
= \\
= \\
- \\
-\end{array}$ & $\begin{array}{r}11,600 \\
5,551 \\
0 \\
89 \\
5,452 \\
131 \\
5,860 \\
-1,812 \\
7,672 \\
-196 \\
463 \\
3,329 \\
-2,868 \\
0 \\
0 \\
0 \\
0 \\
0 \\
0 \\
0 \\
0 \\
0\end{array}$ & $\begin{array}{r}-63 \\
-66 \\
N A \\
N A \\
N A \\
2 \\
-122 \\
-46 \\
-78 \\
20 \\
162 \\
N A \\
N A \\
45 \\
0 \\
0 \\
0 \\
-14 \\
24 \\
-415 \\
0 \\
20\end{array}$ & $\begin{array}{l}= \\
= \\
= \\
= \\
= \\
= \\
= \\
= \\
= \\
= \\
= \\
= \\
= \\
-\end{array}$ & $\begin{array}{l}\ddot{0} \\
= \\
= \\
= \\
= \\
= \\
- \\
- \\
- \\
- \\
- \\
- \\
-\end{array}$ & $\begin{array}{r}117 \\
3 \\
N A \\
N A \\
N A \\
0 \\
0 \\
0 \\
0 \\
0 \\
12 \\
N A \\
N A \\
0 \\
0 \\
3 \\
49 \\
1 \\
39 \\
9 \\
0 \\
(8)\end{array}$ & $\begin{array}{r}186,490 \\
90,417 \\
N A \\
N A \\
N A \\
401 \\
18,213 \\
2,009 \\
16,204 \\
460 \\
46,616 \\
N A \\
N A \\
3,830 \\
255 \\
-3 \\
-49 \\
408 \\
5,016 \\
12,631 \\
7,428 \\
887\end{array}$ & $\begin{array}{r}10,420 \\
4,532 \\
0 \\
655 \\
3,877 \\
47 \\
713 \\
251 \\
462 \\
60 \\
2,794 \\
1,731 \\
1,080 \\
402 \\
2 \\
1 \\
0 \\
30 \\
257 \\
1,668 \\
0 \\
24\end{array}$ \\
\hline 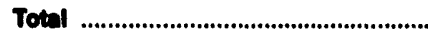 & $200,4 \times$ & 175,430 & 30,411 & 13,070 & $-67,172$ & 108 & 0 & 160,794 & 217 & 207,917 & 27,977 \\
\hline
\end{tabular}

- Represents the PAD District in which the material entered the United States and not nececearly where the crude oll or product be proceseed and/or consumed.

b Unaccounted for crude oil represents the difference between the supply and dieposition of crude oll.

c A negative number indicates a decrease in stocks and a poedtive number indicates an increase in stocks.

d Products supplled is equal to fleld production, plus refinery production, plus imports, plus unaccounted for crude oll, phus net recelpta, minus stock change, minus crude losses, minus refinery inputs, minus exports.

- Includes naphtha less than $401^{\circ} \mathrm{F}$ endpoint and other olls equal to or greater than $401^{\circ} \mathrm{F}$ endpoint.

(8) = Less than 500 barrols.

LRG = Liquefied Refinery Gas.

NA $=$ Not available.

Note: Totals may not equal sum of components due to independent rounding.

Sources: - Energy information Administration (EIA) Forms EIA-810, "Monthly Refinery Report," ELA-811, "Monthly Bulk Terminal Report," ElA-812, "Monthly Product Pipeline Report," ElA-813, "Monthly Crude Oll Report," EIA-B14, "Monthly Imports Report," ElA-816, "Monthly Natural Cas Liquide Report" ElA-817, "Monthly Tanker and Barce Movement Report" and EIA-819M, "Monthly Oxyoenate Telephone Report". Domestic crude oll production from State coneervation agencies and the Minerale Management Service of the U.S. Department of the Interior. - Export data from the Bureau of the Census and Form EL-810, "Monthly Refinery Report." 
Table 11. PAD District IV-Dally Average Supply and Disposition of Crude Oll and Petroleum Products, 1993 (Thousand Barrels per Day)

\begin{tabular}{|c|c|c|c|c|c|c|c|c|c|c|}
\hline \multirow[b]{2}{*}{ Commodity } & \multicolumn{5}{|c|}{ 8upply } & \multicolumn{5}{|c|}{ Diepostion } \\
\hline & $\begin{array}{l}\text { Fieid } \\
\text { Produetion }\end{array}$ & $\begin{array}{l}\text { Rofinery } \\
\text { Produetion }\end{array}$ & $\begin{array}{c}\text { Importe by } \\
\text { PAD } \\
\text { Dietriet } \\
\text { of Enite }\end{array}$ & $\begin{array}{l}\text { Unne- } \\
\text { counted } \\
\text { For } \\
\text { encilo: }\end{array}$ & $\begin{array}{c}\text { Not } \\
\text { Propilots }\end{array}$ & $\begin{array}{c}\text { Stock } \\
\text { Chnne. }\end{array}$ & $\begin{array}{l}\text { Cruds } \\
\text { Lonen }\end{array}$ & $\begin{array}{l}\text { Rollinery } \\
\text { Inpule }\end{array}$ & Expont: & $\begin{array}{l}\text { Products } \\
\text { Sypolliked }\end{array}$ \\
\hline 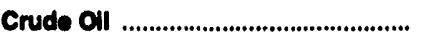 & 420 & - & $\boldsymbol{0}$ & 30 & -110 & -1 & $\mathbf{0}$ & 447 & $\mathbf{0}$ & $\mathbf{0}$ \\
\hline 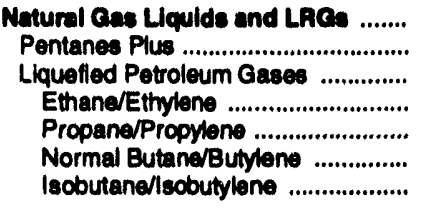 & $\begin{array}{r}133 \\
31 \\
101 \\
33 \\
43 \\
16 \\
10\end{array}$ & $\begin{array}{l}7 \\
77 \\
0 \\
8 \\
\text { (b) } \\
\text { (d) }\end{array}$ & $\begin{array}{r}14 \\
7 \\
7 \\
0 \\
4 \\
2 \\
(0)\end{array}$ & $\begin{array}{l}\ddot{-} \\
\ddot{z} \\
\ddot{-}\end{array}$ & $\begin{array}{l}-70 \\
-10 \\
-69 \\
-31 \\
-21 \\
-10 \\
-7\end{array}$ & $\begin{array}{l}\text { (o) } \\
\text { (f) } \\
\text { (b) } \\
\text { (s) } \\
\text { (s) } \\
\text { (s) } \\
\text { (s) }\end{array}$ & $\begin{array}{l}\check{-} \\
\ddot{-} \\
\ddot{-}\end{array}$ & $\begin{array}{r}17 \\
6 \\
12 \\
0 \\
0 \\
8 \\
4\end{array}$ & $\begin{array}{l}\text { (g) } \\
(8) \\
(8) \\
0 \\
(8) \\
(8) \\
0\end{array}$ & $\begin{array}{l}57 \\
23 \\
34 \\
2 \\
34 \\
-1 \\
(8)\end{array}$ \\
\hline 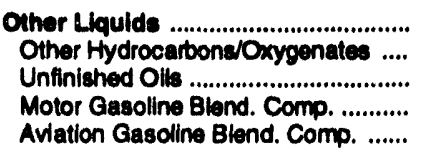 & $\frac{4}{3}$ & $\begin{array}{l}- \\
-\end{array}$ & $\begin{array}{l}0 \\
0 \\
0 \\
0 \\
0\end{array}$ & $\bar{z}$ & $\begin{array}{l}0 \\
0 \\
0 \\
0 \\
0\end{array}$ & $\begin{array}{r}2 \\
(0) \\
1 \\
1 \\
0\end{array}$ & $\bar{~}$ & $\begin{array}{r}1 \\
3 \\
-2 \\
(8) \\
0\end{array}$ & $\begin{array}{l}0 \\
0 \\
0 \\
0 \\
0\end{array}$ & $\begin{array}{l}2 \\
0 \\
2 \\
0 \\
0\end{array}$ \\
\hline 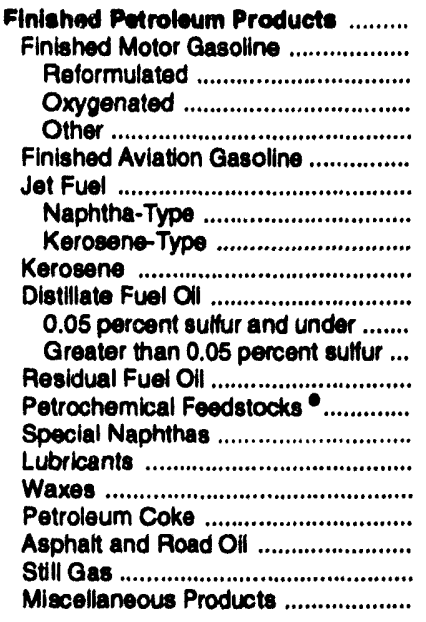 & $\begin{array}{r}1 \\
1 \\
13 \\
-13 \\
= \\
= \\
= \\
= \\
= \\
= \\
= \\
= \\
= \\
=\end{array}$ & $\begin{array}{r}274 \\
231 \\
0 \\
11 \\
220 \\
1 \\
34 \\
10 \\
23 \\
2 \\
124 \\
34 \\
80 \\
11 \\
1 \\
0 \\
0 \\
1 \\
14 \\
33 \\
20 \\
2\end{array}$ & $\begin{array}{r}1 \\
(8) \\
0 \\
0 \\
(8) \\
0 \\
0 \\
0 \\
0 \\
0 \\
3 \\
1 \\
2 \\
(0) \\
0 \\
0 \\
0 \\
(8) \\
0 \\
0 \\
0 \\
0\end{array}$ & $\begin{array}{l}= \\
= \\
= \\
= \\
= \\
= \\
= \\
= \\
= \\
=\end{array}$ & $\begin{array}{c}32 \\
15 \\
0 \\
0 \\
(0) \\
16 \\
(0) \\
16 \\
-5 \\
21 \\
-1 \\
1 \\
0 \\
0 \\
0 \\
0 \\
0 \\
0 \\
0 \\
0 \\
0 \\
0 \\
0 \\
0\end{array}$ & $\begin{array}{c}-1 \\
(8) \\
N A \\
N A \\
N A \\
(8) \\
(8) \\
(8) \\
(8) \\
(8) \\
(8) \\
N A \\
N A \\
(8) \\
0 \\
0 \\
0 \\
(8) \\
(8) \\
-1 \\
0 \\
(0)\end{array}$ & $\begin{array}{l}= \\
= \\
= \\
= \\
= \\
= \\
= \\
= \\
= \\
= \\
= \\
= \\
=\end{array}$ & 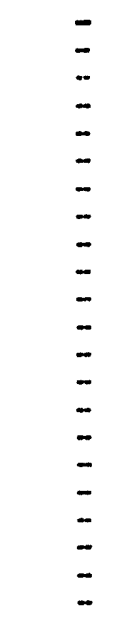 & $\begin{array}{r}(8) \\
(s) \\
N A \\
N A \\
N A \\
0 \\
0 \\
0 \\
0 \\
0 \\
(8) \\
N A \\
N A \\
0 \\
0 \\
(8) \\
(8) \\
(8) \\
(8) \\
(8) \\
0 \\
(8)\end{array}$ & $\begin{array}{r}511 \\
248 \\
\text { NA } \\
\text { NA } \\
\text { NA } \\
1 \\
50 \\
6 \\
44 \\
1 \\
128 \\
\text { NA } \\
\text { NA } \\
10 \\
1 \\
18) \\
(8) \\
1 \\
14 \\
35 \\
20 \\
2\end{array}$ \\
\hline 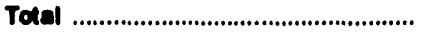 & 868 & 481 & 100 & 30 & -157 & (o) & 0 & 468 & 1 & 570 \\
\hline
\end{tabular}

- Represents the PAD District in which the material entered the United Staice and not necsecarly where the crude oll or product is proceseed andlor consumed.

b Unaccounted for crude oll represents the difference between the eupply and diepceition of crude oll.

c A negattve number indicates a decrease in stocks and a posithe number indioates an inoruase in stocks.

d Products supplled is equal to fleld production, plus relinery production, plus imports, plus unsccounted for crude oll, plus net receipts, minus stock ohange, minus crude losses, minus refinery inputs, minus exports.

- Includes naphtha less than $401^{\circ} \mathrm{F}$ endpoint and other oils equal to or greater than $401^{\circ} \mathrm{F}$ encpoint.

(s) = Less than 500 barrels per day.

LRG $=$ Liquefied Refinery Gas.

NA = Not available.

Note: Totals may not equal sum of components due to independemt rounding.

Sources: - Energy Intormation Administration (EIA) Forms EIA-810, "Monthly Rofinery Report," ELA-811, "Monthly Bulk Terminal Report," EIA-812, "Monthly Product Pipeline Report," ElA-813, "Monthly Crude Oll Report," ElA-814, "Monthly Importe Report," ElA-816, "Monthly Natural Gas Llquids Report," ElA-817. "Monthly Tanker and Barge Movement Report," and ElA-818M. "Monthy Oxycenate Telephone Report". - Domestic crude oll productlon from State conservation agencles and the Minerals Management Service of the U.S. Department of the Interior. - Export data from the Bureau of the Ceneus and Form EIA-810, "Monthly Refinery Report." 
Tablo 12. PAD District V-Supply, Diaposition, and Ending Stocks of Crude Oll and Potroleium Products, 1993 (Thousand Barrels)

\begin{tabular}{|c|c|c|c|c|c|c|c|c|c|c|c|}
\hline \multirow[b]{2}{*}{ Commodity } & \multicolumn{5}{|c|}{ supply } & \multicolumn{5}{|c|}{ Dieposillon } & \multirow[b]{2}{*}{$\begin{array}{l}\text { Ending } \\
\text { Enolas }\end{array}$} \\
\hline & $\begin{array}{l}\text { Fleld } \\
\text { explupiton }\end{array}$ & Rofinery & $\begin{array}{c}\text { importe by } \\
\text { pas } \\
\text { Distriot } \\
\text { of Enine }\end{array}$ & $\begin{array}{l}\text { Uneo- } \\
\text { oounied } \\
\text { For } \\
\text { crudegie }\end{array}$ & $\begin{array}{c}\text { Not } \\
\text { exsidinte }\end{array}$ & $\begin{array}{c}\text { Bloek } \\
\text { Chnn'es }\end{array}$ & $\begin{array}{l}\text { Cnded } \\
\text { Loneil }\end{array}$ & $\begin{array}{c}\text { Ronnery } \\
\text { inputh }\end{array}$ & Expont: & $\begin{array}{l}\text { Producte } \\
\text { Bupollad }\end{array}$ & \\
\hline 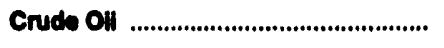 & 924,175 & $\sim$ & 100,212 & 8,827 & $-71,747$ & 4,048 & 6 & 923,025 & 38,000 & 3,647 & 07,976 \\
\hline 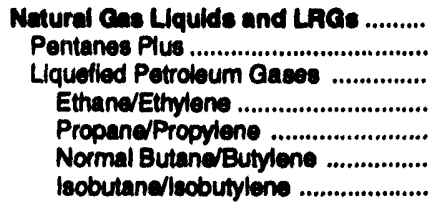 & $\begin{array}{r}3,923 \\
18,975 \\
17,861 \\
10 \\
3,652 \\
9,123 \\
5,267\end{array}$ & $\begin{array}{r}30,961 \\
30,861 \\
0 \\
14,858 \\
14,931 \\
1,072\end{array}$ & $\begin{array}{r}232 \\
0 \\
232 \\
0 \\
112 \\
37 \\
83\end{array}$ & 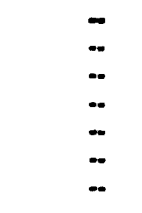 & $\begin{array}{l}0 \\
0 \\
0 \\
0 \\
0 \\
0 \\
0\end{array}$ & $\begin{array}{r}494 \\
-14 \\
498 \\
0 \\
136 \\
60 \\
303\end{array}$ & $\begin{array}{l}\ddot{-} \\
\ddot{-} \\
\ddot{-} \\
\ddot{-}\end{array}$ & $\begin{array}{r}32,772 \\
14,448 \\
18,324 \\
0 \\
26 \\
11,230 \\
7,088\end{array}$ & $\begin{array}{r}4,309 \\
(8) \\
4,389 \\
0 \\
1,534 \\
2,854 \\
0\end{array}$ & $\begin{array}{r}30,494 \\
4,641 \\
25,943 \\
19 \\
16,927 \\
9,947 \\
-949\end{array}$ & $\begin{array}{r}3,615 \\
68 \\
3,549 \\
0 \\
1,080 \\
1,817 \\
652\end{array}$ \\
\hline $\begin{array}{l}\text { Other Lquide .................................... } \\
\text { Other Hydrocarbona/Oxygenates ..... } \\
\text { Untinithed Olts .................................... } \\
\text { Motor Gasoline Blend. Comp. .......... } \\
\text { Avlation Gasollne Blend. Comp. ........ }\end{array}$ & $\begin{array}{r}18,400 \\
19,5077 \\
\ldots \\
4,137 \\
\cdots\end{array}$ & $\begin{array}{l}- \\
- \\
-\end{array}$ & $\begin{array}{r}10,160 \\
4,896 \\
4,815 \\
439 \\
0\end{array}$ & $\begin{array}{l}- \\
\ddot{-} \\
\ddot{-}\end{array}$ & $\begin{array}{r}1,720 \\
0 \\
674 \\
1,049 \\
0\end{array}$ & $\begin{array}{r}2,924 \\
1,261 \\
88 \\
1,619 \\
6\end{array}$ & $\begin{array}{l}- \\
- \\
-\end{array}$ & $\begin{array}{r}24,137 \\
23,182 \\
6,903 \\
-6,002 \\
-6\end{array}$ & $\begin{array}{r}1,724 \\
0 \\
0 \\
1,734 \\
0\end{array}$ & $\begin{array}{r}-1,892 \\
0 \\
-1,582 \\
0 \\
0\end{array}$ & $\begin{array}{r}33,603 \\
3,830 \\
21,183 \\
8,784 \\
9\end{array}$ \\
\hline 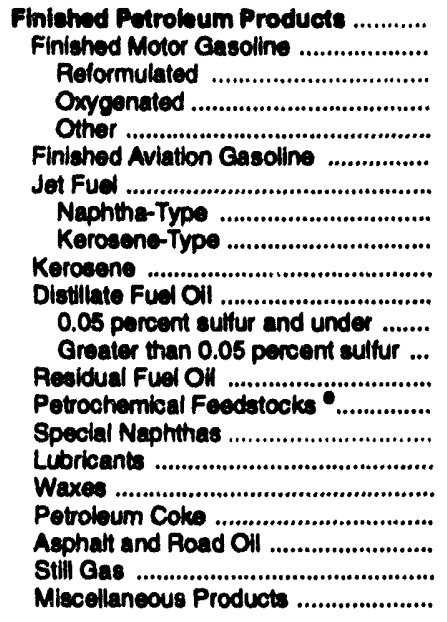 & $\begin{array}{r}6,302 \\
6,302 \\
21,649 \\
-15,347 \\
. . \\
. . \\
. . \\
- \\
- \\
- \\
- \\
- \\
- \\
- \\
- \\
- \\
-\end{array}$ & $\begin{array}{r}1,007,862 \\
456,688 \\
0 \\
118,368 \\
338,342 \\
1,848 \\
145,957 \\
5,841 \\
140,116 \\
9999 \\
161,853 \\
74,144 \\
87,709 \\
100,670 \\
1,913 \\
724 \\
7,680 \\
726 \\
55,284 \\
17,845 \\
52,657 \\
2,678\end{array}$ & $\begin{array}{r}17,168 \\
3,419 \\
0 \\
0 \\
3,419 \\
46 \\
3,270 \\
268 \\
3,002 \\
21 \\
2,163 \\
1,004 \\
1,162 \\
7,200 \\
255 \\
50 \\
31 \\
24 \\
177 \\
491 \\
0 \\
6\end{array}$ & $\begin{array}{l}- \\
- \\
= \\
= \\
= \\
= \\
- \\
- \\
- \\
- \\
- \\
- \\
- \\
-\end{array}$ & $\begin{array}{r}31,874 \\
20,619 \\
0 \\
0 \\
20,619 \\
0 \\
4,783 \\
1,918 \\
2,806 \\
0 \\
5,982 \\
1,396 \\
4,608 \\
0 \\
0 \\
0 \\
510 \\
0 \\
0 \\
0 \\
0 \\
-20\end{array}$ & $\begin{array}{r}3,933 \\
1,314 \\
N A \\
N A \\
N A \\
10 \\
820 \\
-834 \\
1,754 \\
55 \\
1,340 \\
N A \\
N A \\
-82 \\
88 \\
-13 \\
-322 \\
47 \\
546 \\
75 \\
0 \\
180\end{array}$ & $\begin{array}{l}- \\
- \\
- \\
- \\
= \\
- \\
- \\
- \\
- \\
- \\
- \\
- \\
- \\
- \\
- \\
-\end{array}$ & 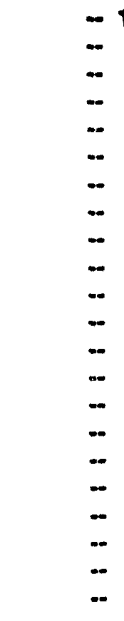 & $\begin{array}{r}128,629 \\
8,737 \\
N A \\
N A \\
N A \\
0 \\
11,745 \\
4,291 \\
7,454 \\
22 \\
44,272 \\
N A \\
N A \\
20,647 \\
0 \\
291 \\
1,297 \\
171 \\
41,142 \\
193 \\
0 \\
12\end{array}$ & $\begin{array}{r}930,392 \\
476,986 \\
N A \\
N A \\
N A \\
1,975 \\
141,445 \\
4,670 \\
136,775 \\
943 \\
124,389 \\
N A \\
N A \\
67,305 \\
2,080 \\
498 \\
7,146 \\
626 \\
13,773 \\
18,088 \\
52,657 \\
2,492\end{array}$ & $\begin{array}{r}r 1,790 \\
25,490 \\
0 \\
10,124 \\
15,383 \\
395 \\
8,947 \\
298 \\
8,649 \\
118 \\
12,157 \\
7,971 \\
4,188 \\
6,915 \\
280 \\
48 \\
1,698 \\
78 \\
3,643 \\
1,774 \\
0 \\
289\end{array}$ \\
\hline 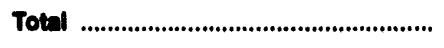 & 891,013 & $1,028,493$ & 127,010 & 6.827 & 28,180 & 2,055 & 6 & 970,934 & 160,747 & ex2,est & 167,183 \\
\hline
\end{tabular}

- Represents the PAD District in which the material entered the United States and not necesearly where the crude oll or product ls processed andlor consumed.

b Uneccoumted for crude oll represents the difterence between the supply and dieposition of crude oll.

c A negative number indicates a decrease in stocks and a poeltwe number indicates an increase in stocks.

d Products supplied is equal to field production, plus refinery production, plus imports, plus uneccounted for crude oll, plus net receipts, minus stock change, minus crude losses, minus refinery inputs, minus exports.

- Includes naphtha lees than $401^{\circ} \mathrm{F}$ endpoint and other olls equal to or greater than $401^{\circ} \mathrm{F}$ endpoint.

(8) = Less than 500 barrels.

LRG = Liquefled Retinery Gas.

NA = Not avallable.

Note: Totals may not equal sum of components dua to independent rounding.

Sources: - Energy Intormation Administration (EIA) Forms EIA-810, "Monthly Refinery Report," ELA-811, "Monthly Bulk Terminal Report," EIA-812, "Monthly Product Pipeline Report," ElA-813, "Monthly Crude Oll Report," ElA-814, "Monthly Imports Report," EIA-816, "Monthly Natural Gas Liquids Report," EIA-817, "Monthly Tanker and Barge Movement Report, and EIA-81OM, "Monthly Oxygenate Telephone Report". " Domeatic crude oll production from State conservation agencles and the Minerals Management Service of the U.S. Department of the Interlor. - Export data from the Bureau of the Census and Form ElA-810, "Monthly Refinery Report." 
Table 13. PAD Dietrict V - Dally Average Bupply and Dispoakion of Cruds Oll and Petroloum Producta, 1993 (Thousand Barrels per Day)

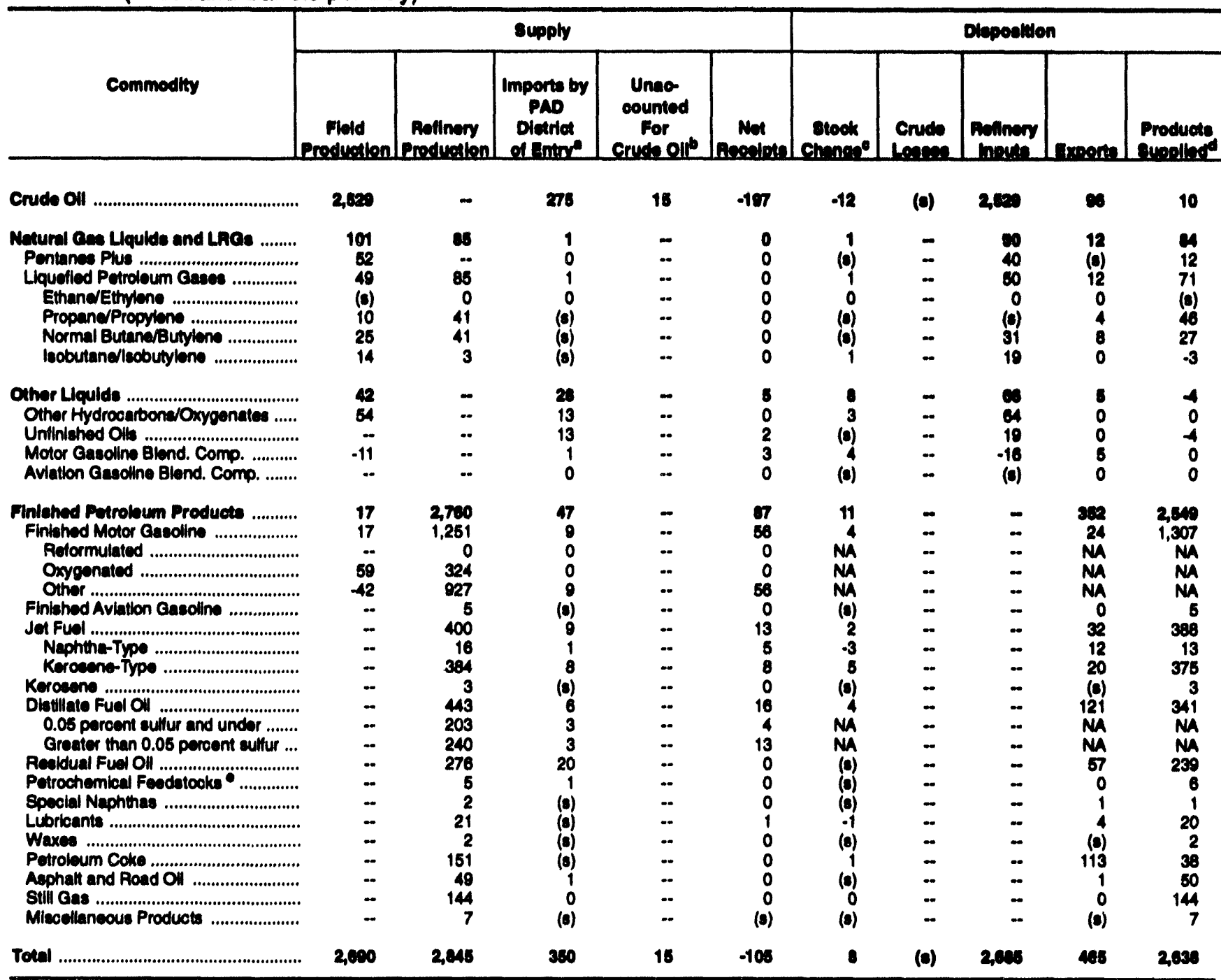

- Represents the PAD Divetrict in whioh the material entered the United States and not necesearily where the orude oll or product is proceseed and/or conaumed.

b Unaccounted for crude oll represents the diflerence between the supply and disposition of crude oll.

- A negative number indicates a decrease in stocks and a positive number indlcates an incresse in stocks.

d Producte supplied le equal to field production, plus refinery production, plus imports, plus unacoounted for cnude oll, plus not recelpte, minus atcok change, minus crude losese, minus retinery inputs, minus exports.

Includes naphtha leas than $401^{\circ} \mathrm{F}$ endpoint and other olls equal to or greater than $401^{\circ} \mathrm{F}$ endpoint.

(8) = Lees than 500 barrele per day.

LRG = Llquefled Refinery Gas.

NA $=$ Not avallable.

Note: Totals may not equal sum of components due to independent rounding.

Sources: - Energy Information Administration (EIA) Forms EIA-810, "Monthly Reflnery Report," EIA-811, "Monthly Bulk Terminal Report," ElA-812, "Monthly Product Plpeline Report," EIA-813, "Monthly Crude Oll Report," EIA-814, "Monthly Imports Report," EIA-816, "Monthly Natural Gae Liquide Report," ElA-817, "Monthly Tanker and Barge Movement Report," and EIA-818M, "Monthly Oxygenate Telephone Report". - Domestio crude oil production from Stato concervation agencies and the Minerals Management Service of the U.S. Department of the Interior. - Export data from the Bureau of the Census and Form ElA-810, Monthly Retinery Report." 
Table 14. Production of Crude OII by PAD District and Stato, 1993 (Thousand Barrels)

\begin{tabular}{|c|c|c|}
\hline PAD Dletrict and Btate & Total & $\begin{array}{c}\text { Dally } \\
\text { Avernges }\end{array}$ \\
\hline 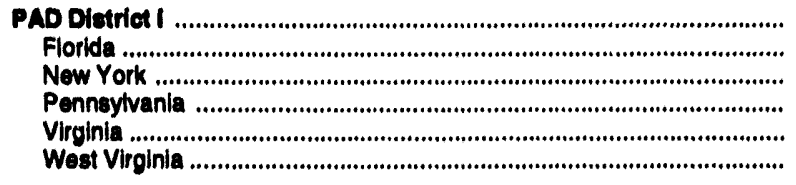 & $\begin{array}{r}10,032 \\
5,604 \\
331 \\
2,036 \\
12 \\
2,048\end{array}$ & $\begin{array}{r}27 \\
15 \\
1 \\
6 \\
(8) \\
6\end{array}$ \\
\hline $\begin{array}{l}\text { PD Diatrict II } \\
\text { Illinols } \\
\text { Indiana . } \\
\text { Kansas ... } \\
\text { Kentucky } \\
\text { Michigan } \\
\text { Missourl } \\
\text { Nebraska } \\
\text { North Dakota } \\
\text { Ohlo } \\
\text { Oklahoma } \\
\text { South Dakota } \\
\text { Tennessee }\end{array}$ & $\begin{array}{r}230,926 \\
17,406 \\
2,761 \\
49,625 \\
4,693 \\
13,799 \\
135 \\
4,868 \\
30,917 \\
8,282 \\
96,624 \\
1,500 \\
419\end{array}$ & $\begin{array}{r}683 \\
48 \\
8 \\
136 \\
13 \\
38 \\
(8) \\
13 \\
85 \\
23 \\
265 \\
4 \\
1\end{array}$ \\
\hline 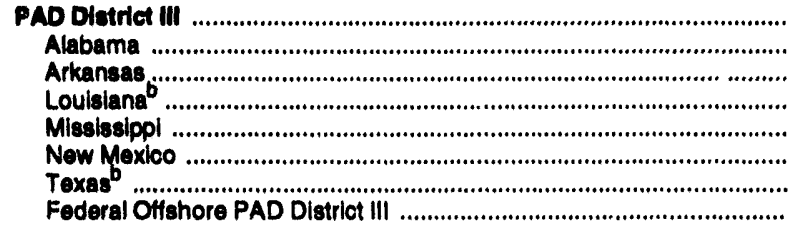 & $\begin{array}{r}1,178,607 \\
18,677 \\
9,974 \\
138,673 \\
22,615 \\
68,422 \\
619,088 \\
301,117\end{array}$ & $\begin{array}{r}3,229 \\
51 \\
27 \\
380 \\
62 \\
187 \\
1,686 \\
825\end{array}$ \\
\hline 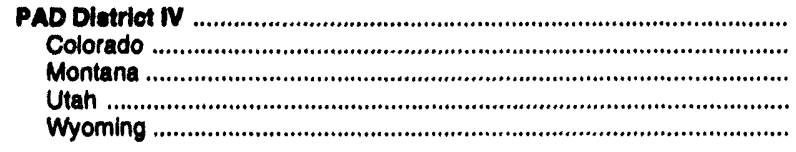 & $\begin{array}{r}168,331 \\
29,398 \\
17,448 \\
21,819 \\
87,667\end{array}$ & $\begin{array}{r}428 \\
81 \\
48 \\
60 \\
240\end{array}$ \\
\hline $\begin{array}{l}\text { PAD District V } \\
\text { Alaska } \\
\text { South Alaska } \\
\text { North Slope } \\
\text { Arlzona } \\
\text { Callforniab } \\
\text { Nevada } \\
\text { Federal Ofishore PAD District V }\end{array}$ & $\begin{array}{r}923,175 \\
577,494 \\
13,848 \\
563,646 \\
73 \\
293,089 \\
1,880 \\
50,639\end{array}$ & $\begin{array}{r}2,529 \\
1,582 \\
38 \\
1,544 \\
(8) \\
803 \\
5 \\
139\end{array}$ \\
\hline U.8. Total ${ }^{b}$ & $2,499,033$ & 6,847 \\
\hline
\end{tabular}

ancludes the following oftshore production (thousand barrels): Alaska: State - 58,735; California: State - 20,900 Loulsiana: State - 23,081; Texas: State -1,935; U.S. Total, Including Federal offshore - $456,408$.

$(s)=$ Less than 500 barrels or less than 500 barrels per day.

Note: Crude oil production data for Indlana, and New Mexico, are unchanged from those reported in the Petroleum Supply Monthly during 1993. - Annual crude oll production data for Pennsylvania were estimated based on first purchaser monthly crude oll volumes collected on Form EIA-182, "Domestic Crude Oll First Purchase Report." - A tinal revision to the State data for 1993 will appear in the 1994 Petroleum Supply Annual. - Totals may not equal sum of components due to independent rounding.

Sources: State government agencies, U.S. Department of the Interior, Minerals Management Service and the Conservation Committee of Callifornia Oll and Gas Producers.

Revised 1992 crude oll production statistics are avallable in Appendix C. 
Tablo 15. Netural Gas Plant Not Production and Stcoke of Potroloum Producte by PAD and Roinning Diatricta, 1993

(Thousand Barrels)

\begin{tabular}{|c|c|c|c|c|c|c|c|}
\hline \multirow[b]{2}{*}{ Commodihy } & \multicolumn{3}{|c|}{ PAD Dictriat I } & \multicolumn{4}{|c|}{ PAD Dictritot II } \\
\hline & $\begin{array}{l}\text { Gent } \\
\text { cont }\end{array}$ & $\begin{array}{c}\text { Appalachlen } \\
\text { No. } 1\end{array}$ & Told & Ind., In., Ky. & Ylow & Owla, Kenn, & Total \\
\hline \multirow{3}{*}{ 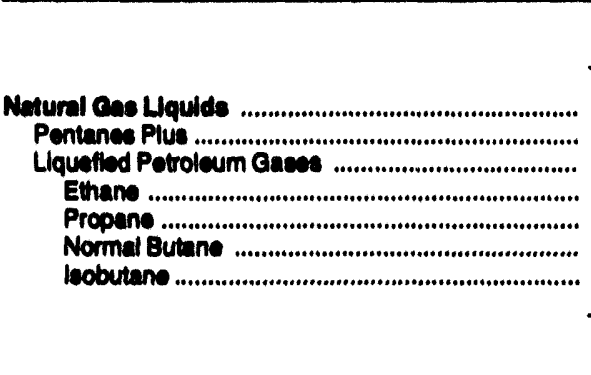 } & \multicolumn{7}{|c|}{ Net Production } \\
\hline & $\begin{array}{r}2,372 \\
388 \\
1,084 \\
602 \\
773 \\
473 \\
76\end{array}$ & $\begin{array}{r}7,000 \\
805 \\
6,704 \\
2,378 \\
3,048 \\
853 \\
415\end{array}$ & $\begin{array}{r}10,071 \\
1,283 \\
8,778 \\
3,040 \\
3,821 \\
1,428 \\
491\end{array}$ & $\begin{array}{r}6,800 \\
1,238 \\
5,342 \\
703 \\
2,688 \\
090 \\
808\end{array}$ & $\begin{array}{r}4,840 \\
1,106 \\
3,360 \\
10 \\
2,000 \\
1,270 \\
2\end{array}$ & $\begin{array}{r}102,09 \\
14,288 \\
88,084 \\
38,300 \\
38,000 \\
11,348 \\
7,360\end{array}$ & $\begin{array}{r}114,094 \\
16,718 \\
97,370 \\
34,180 \\
41,346 \\
13,622 \\
8,210\end{array}$ \\
\hline & \multicolumn{7}{|c|}{ Stooks } \\
\hline 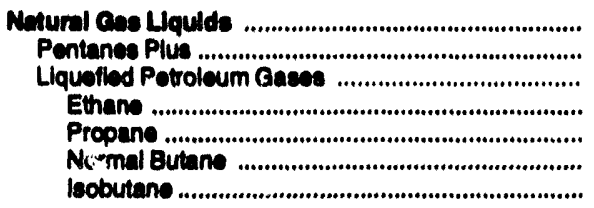 & $\begin{array}{r}22 \\
1 \\
21 \\
0 \\
18 \\
3 \\
0\end{array}$ & $\begin{array}{r}30 \\
6 \\
30 \\
0 \\
26 \\
2 \\
3\end{array}$ & $\begin{array}{r}86 \\
7 \\
61 \\
0 \\
43 \\
5 \\
3\end{array}$ & $\begin{array}{r}111 \\
22 \\
80 \\
11 \\
39 \\
23 \\
18\end{array}$ & $\begin{array}{r}7 \\
28 \\
29 \\
0 \\
18 \\
11 \\
0\end{array}$ & $\begin{array}{r}3,100 \\
303 \\
2,712 \\
739 \\
1,246 \\
842 \\
186\end{array}$ & $\begin{array}{r}2,279 \\
449 \\
2,830 \\
750 \\
1,302 \\
678 \\
202\end{array}$ \\
\hline
\end{tabular}

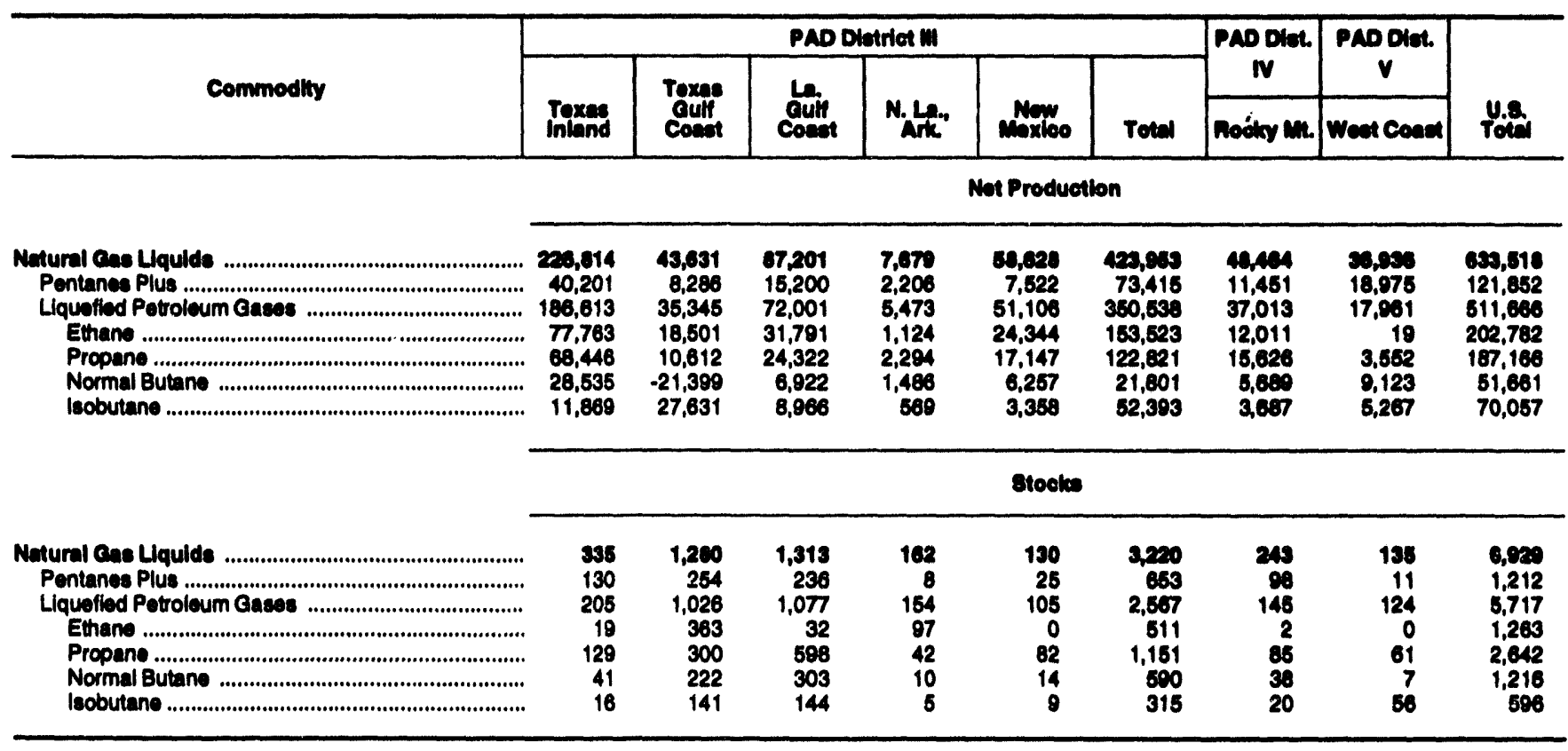

Note: - Slocks are reported as of the end of December. $\bullet$ Reter to Appendlx A for Refining Distriot descriptions.

Source: Energy Information Administration (EIA) Form EIA-816, "Monthly Naturai Gas Liquids Report." 
Table 16. Rofinery Input of Crude OII and Petroleum Producte by PAD and Rofining Dictriots, 1993 (Thousand Barrels, Except Where Noted)

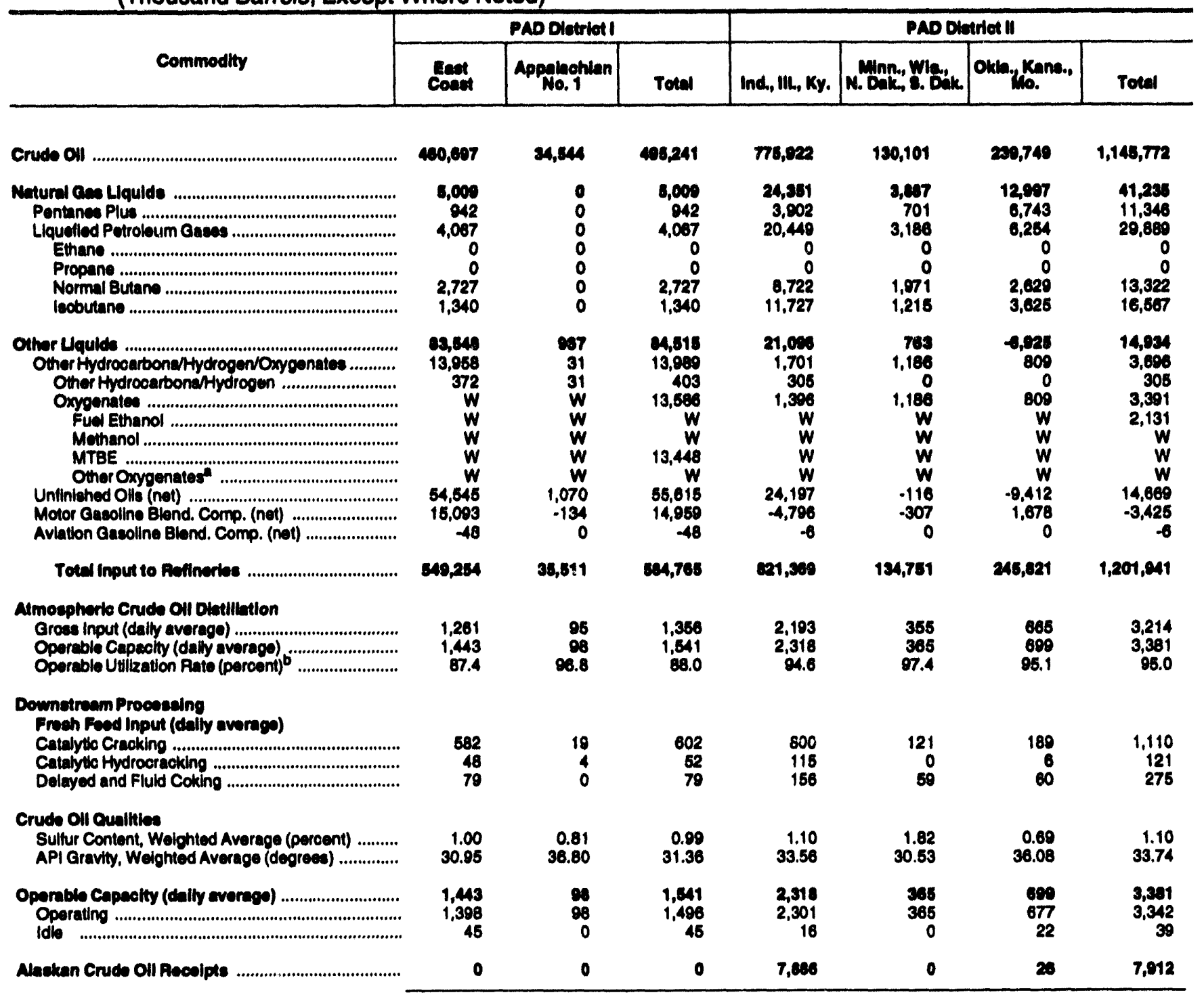

See footnotes at end of table. 
Table 16. Rofinery Input of Crude Oll and Potroloum Products by PAD and Rofining Districte, 1993 (Continued)

(Thousand Barrels, Except Where Noted)

\begin{tabular}{|c|c|c|c|c|c|c|c|c|c|}
\hline \multirow{3}{*}{ Commodity } & \multicolumn{6}{|c|}{ PAD Dictriot III } & \multirow{3}{*}{$\begin{array}{c}\begin{array}{c}\text { PAD DICR. } \\
\text { IV }\end{array} \\
\text { Rooky m. }\end{array}$} & \multirow{3}{*}{\begin{tabular}{|c|}
$\begin{array}{c}\text { PAD Diat. } \\
V\end{array}$ \\
Weat Conet \\
\end{tabular}} & \multirow{3}{*}{ Yotil } \\
\hline & \multirow{2}{*}{$\begin{array}{l}\text { Torres } \\
\text { Intand }\end{array}$} & \multirow{2}{*}{$\begin{array}{l}\text { Tougs } \\
\text { oult } \\
\text { Coatt }\end{array}$} & \multirow{2}{*}{ cont } & \multirow{2}{*}{ N.Len." } & \multirow{2}{*}{ Moxico } & \multirow[b]{2}{*}{ Total } & & & \\
\hline & & & & & & & & & \\
\hline Crude Oll & 190,460 & $1,060,460$ & 607,048 & 71,201 & 22,130 & $2,241,341$ & 169,202 & 023,028 & $4,638,641$ \\
\hline 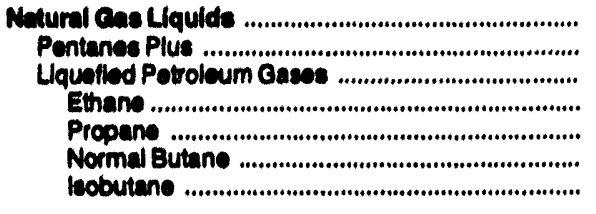 & $\begin{array}{r}13,041 \\
6,240 \\
6,801 \\
0 \\
0 \\
5,213 \\
1,888\end{array}$ & $\begin{array}{r}43,923 \\
16,753 \\
27,170 \\
0 \\
3 \\
6,487 \\
18,710\end{array}$ & $\begin{array}{r}31,239 \\
4,723 \\
26,616 \\
0 \\
0 \\
13,328 \\
13,191\end{array}$ & $\begin{array}{r}2,908 \\
1,731 \\
1,175 \\
0 \\
0 \\
227 \\
948\end{array}$ & $\begin{array}{r}2,760 \\
1,867 \\
1,203 \\
0 \\
0 \\
30 \\
1,167\end{array}$ & $\begin{array}{r}93,690 \\
31,004 \\
62,806 \\
0 \\
3 \\
27,258 \\
35,604\end{array}$ & $\begin{array}{r}0,393 \\
2,012 \\
4,316 \\
0 \\
0 \\
3,018 \\
1,301\end{array}$ & $\begin{array}{r}32,772 \\
14,448 \\
18,324 \\
0 \\
28 \\
11,230 \\
7,068\end{array}$ & $\begin{array}{r}170,213 \\
69,762 \\
119,401 \\
0 \\
29 \\
67,682 \\
61,880\end{array}$ \\
\hline 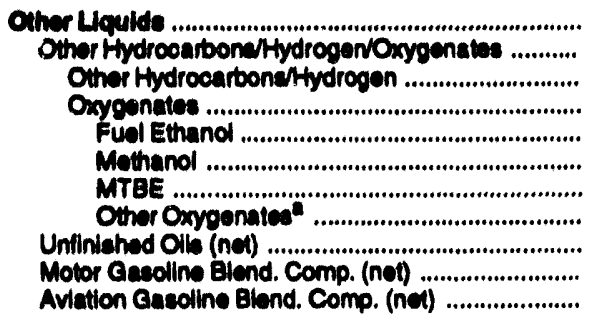 & $\begin{array}{r}1,847 \\
1,340 \\
607 \\
w \\
w \\
w \\
w \\
-1,468 \\
-988 \\
0\end{array}$ & $\begin{array}{r}162,007 \\
18,421 \\
1,770 \\
13,642 \\
w \\
w \\
12,803 \\
W \\
139,490 \\
7,909 \\
-12\end{array}$ & $\begin{array}{r}16,164 \\
10,716 \\
5,065 \\
5,651 \\
W \\
W \\
W \\
W \\
37,547 \\
-2,103 \\
24\end{array}$ & $\begin{array}{r}1,049 \\
39 \\
0 \\
w \\
w \\
w \\
w \\
w \\
w \\
2,334 \\
-630 \\
0\end{array}$ & $\begin{array}{r}197 \\
0 \\
w \\
w \\
w \\
w \\
w \\
-127 \\
476 \\
0\end{array}$ & $\begin{array}{r}210,194 \\
28,220 \\
8,184 \\
20,038 \\
W \\
W \\
18,046 \\
W \\
17,708 \\
4,857 \\
12\end{array}$ & $\begin{array}{r}214 \\
1,060 \\
0 \\
1,060 \\
w \\
w \\
w \\
w \\
w \\
-887 \\
36 \\
0\end{array}$ & $\begin{array}{r}24,197 \\
23,182 \\
6,061 \\
16,031 \\
W \\
W \\
16,265 \\
W \\
6,063 \\
6,002 \\
-6\end{array}$ & $\begin{array}{r}394,044 \\
70,163 \\
15,, 643 \\
54,010 \\
3,361 \\
782 \\
40,303 \\
1,084 \\
254,158 \\
10,424 \\
48\end{array}$ \\
\hline Tocal Inpert to Rofthorice ....................................... & 202,911 & $1,207,260$ & 894,471 & 78,000 & 38,442 & $2,646,104$ & 100,794 & 979,094 & $5,412,538$ \\
\hline 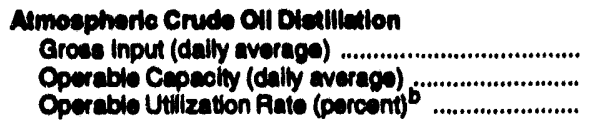 & $\begin{array}{l}526 \\
567 \\
94.4\end{array}$ & $\begin{array}{r}2,961 \\
3,223 \\
91.9\end{array}$ & $\begin{array}{r}2,481 \\
2,683 \\
93.2\end{array}$ & $\begin{array}{l}198 \\
240 \\
78.4\end{array}$ & $\begin{array}{r}89 \\
97 \\
\infty 0.4\end{array}$ & $\begin{array}{r}6,251 \\
6,780 \\
02.1\end{array}$ & $\begin{array}{l}463 \\
618 \\
87.4\end{array}$ & $\begin{array}{r}2,578 \\
2,914 \\
88.6\end{array}$ & $\begin{array}{r}13,881 \\
16,143 \\
81.6\end{array}$ \\
\hline 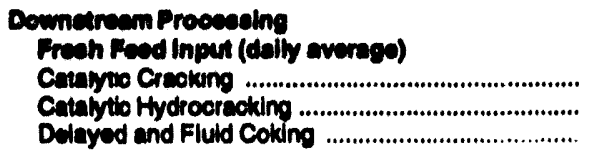 & $\begin{array}{r}172 \\
22 \\
5\end{array}$ & $\begin{array}{r}1,261 \\
213 \\
288\end{array}$ & $\begin{array}{l}870 \\
199 \\
347\end{array}$ & $\begin{array}{r}27 \\
0 \\
13\end{array}$ & $\begin{array}{r}29 \\
0 \\
0\end{array}$ & $\begin{array}{r}2,360 \\
434 \\
663\end{array}$ & $\begin{array}{r}148 \\
34\end{array}$ & $\begin{array}{l}706 \\
460 \\
474\end{array}$ & $\begin{array}{l}4,026 \\
1,084 \\
1,514\end{array}$ \\
\hline 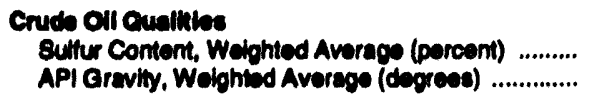 & $\begin{array}{r}0.91 \\
38.47\end{array}$ & $\begin{array}{r}1.14 \\
32.13\end{array}$ & $\begin{array}{r}1.32 \\
31.05\end{array}$ & $\begin{array}{r}1.44 \\
32.33\end{array}$ & $\begin{array}{r}1.03 \\
37.41\end{array}$ & $\begin{array}{r}1.20 \\
32.32\end{array}$ & $\begin{array}{r}1.18 \\
34.51\end{array}$ & $\begin{array}{r}1.16 \\
25.39\end{array}$ & $\begin{array}{r}1.16 \\
31.30\end{array}$ \\
\hline 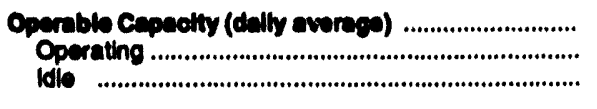 & $\begin{array}{r}557 \\
557 \\
0\end{array}$ & $\begin{array}{r}3,2223 \\
3,196 \\
27\end{array}$ & $\begin{array}{r}2,000 \\
2,662 \\
1\end{array}$ & $\begin{array}{r}240 \\
244 \\
5\end{array}$ & $\begin{array}{l}97 \\
97 \\
0\end{array}$ & $\begin{array}{r}6,700 \\
6,768 \\
33\end{array}$ & $\begin{array}{l}810 \\
607 \\
11\end{array}$ & $\begin{array}{r}2,914 \\
2,860 \\
54\end{array}$ & $\begin{array}{r}18,143 \\
14,061 \\
182\end{array}$ \\
\hline 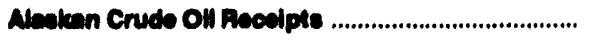 & 238 & 6,257 & 8,008 & 327 & $\mathbf{0}$ & 11,907 & 0 & 800,600 & 523,700 \\
\hline
\end{tabular}

- Includes ethyl brtary butyl ther (ETBE), tortiary armyl methyl ether (TAME), tertiary butyl alcohol (TBA), and other allphatio alcohols and ethers intended lor mobor gaesolino blending (0.g., bopropyl other (IPE) or n-propanol).

- Represente grose input divided by operable capacity.

$W$ - With held to evold disclocure of indwidual company date.

Noto: - Totals may not equal sum of components dus to independent rounding. - Reter to Appendix A for Refining Diatriot descriptions.

Source: Energy Information Adminiatration (EIA) Form ELA-810, "Monthly Refinery Report." 
Table 17. Refinery Not Produotion of Finlahed Potroloum Producte by PAD and Rofining Distrlote, 1993

(Thousand Barrels)

\begin{tabular}{|c|c|c|c|c|c|c|c|}
\hline \multirow{2}{*}{ Commodty } & \multicolumn{3}{|c|}{ PAD Dietriet I } & \multicolumn{4}{|c|}{ PAD Dietritot II } \\
\hline & Cont & Appolechlan & Total & Ind, Ill., Ky. & & Okla phe & Totat \\
\hline 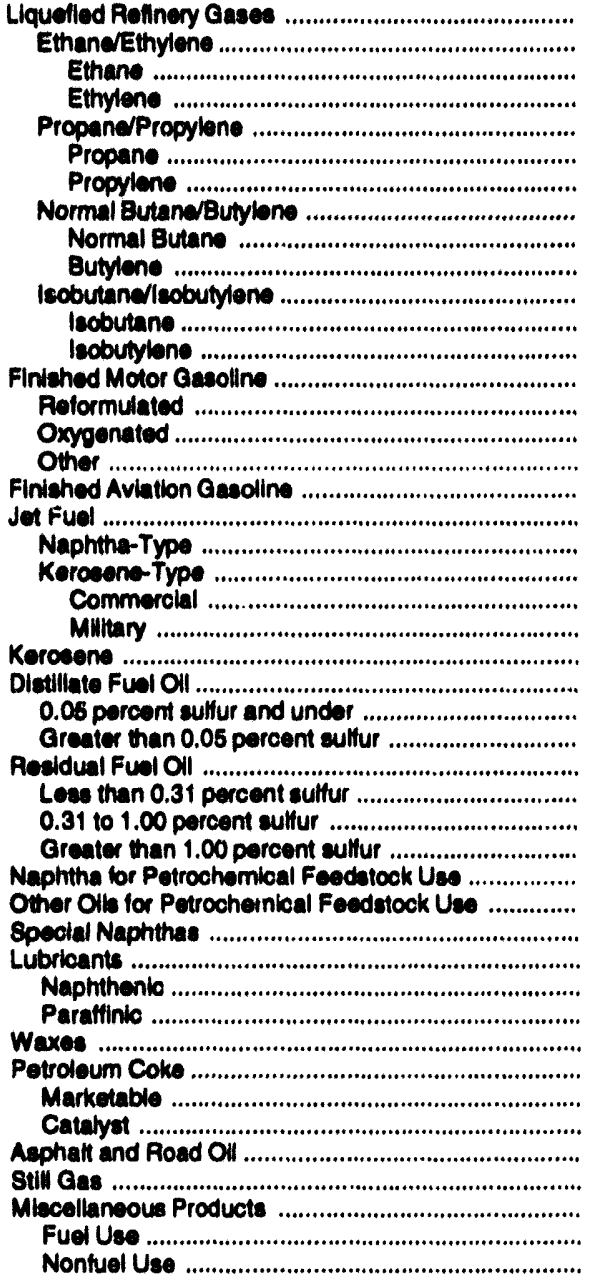 & $\begin{array}{r}16,679 \\
0 \\
W \\
W \\
16,146 \\
W \\
W \\
903 \\
W \\
W \\
-469 \\
W \\
W \\
271,977 \\
0 \\
64,793 \\
207,184 \\
109 \\
31,591 \\
2,825 \\
28,766 \\
28,431 \\
22,335 \\
472 \\
136,348 \\
32,277 \\
104,071 \\
43,455 \\
5,168 \\
34,714 \\
3,573 \\
5,807 \\
132 \\
138 \\
3,025 \\
0 \\
3,025 \\
0 \\
17,696 \\
6,690 \\
11,006 \\
25,823 \\
20,450 \\
304 \\
0 \\
304\end{array}$ & $\begin{array}{r}175 \\
0 \\
W \\
W \\
368 \\
W \\
W \\
.110 \\
W \\
W \\
-73 \\
W \\
W \\
13,636 \\
0 \\
0 \\
13,636 \\
0 \\
0 \\
0 \\
0 \\
0 \\
0 \\
723 \\
723 \\
9,603 \\
3,485 \\
6,118 \\
089 \\
361 \\
608 \\
0 \\
0 \\
0 \\
347 \\
3,209 \\
0 \\
3,209 \\
1,480 \\
291 \\
0 \\
291 \\
3,273 \\
1,653 \\
493 \\
0 \\
493\end{array}$ & $\begin{array}{r}16,764 \\
0 \\
W \\
W \\
16,603 \\
W \\
W \\
793 \\
W \\
W \\
-842 \\
W \\
W \\
285,612 \\
0 \\
24,793 \\
220,819 \\
190 \\
31,691 \\
2,825 \\
28,768 \\
26,431 \\
2,335 \\
1,195 \\
145,951 \\
35,762 \\
110,189 \\
44,424 \\
5,529 \\
35,322 \\
3,573 \\
6,607 \\
132 \\
485 \\
6,234 \\
0 \\
6,234 \\
1,460 \\
17,987 \\
6,600 \\
11,297 \\
29,098 \\
22,103 \\
797 \\
0 \\
797\end{array}$ & $\begin{array}{r}32,126 \\
0 \\
W \\
W \\
27,828 \\
W \\
W \\
3,268 \\
W \\
W \\
1,040 \\
W \\
W \\
432,903 \\
0 \\
14,433 \\
418,470 \\
521 \\
50,192 \\
2,849 \\
47,343 \\
46,152 \\
2,191 \\
5,318 \\
179,832 \\
46,605 \\
134,227 \\
22,051 \\
0 \\
3,919 \\
18,132 \\
7,108 \\
7,232 \\
3,823 \\
5,573 \\
0 \\
5,573 \\
528 \\
30,030 \\
16,485 \\
13,646 \\
46,316 \\
34,140 \\
2,446 \\
0 \\
2,446\end{array}$ & $\begin{array}{r}3,871 \\
0 \\
W \\
W \\
3,218 \\
W \\
W \\
602 \\
W \\
W \\
51 \\
W \\
W \\
71,268 \\
0 \\
12,814 \\
68,464 \\
328 \\
8,364 \\
180 \\
8,174 \\
8,174 \\
0 \\
965 \\
34,062 \\
4,172 \\
29,890 \\
2,939 \\
0 \\
0 \\
2,939 \\
0 \\
0 \\
0 \\
0 \\
0\end{array}$ & $\begin{array}{r}7,089 \\
4 \\
W \\
W \\
7,391 \\
W \\
W \\
963 \\
W \\
W \\
-389 \\
W \\
W \\
126,637 \\
0 \\
23,186 \\
103,461 \\
115 \\
16,464 \\
2,780 \\
13,684 \\
13,666 \\
18 \\
589 \\
65,649 \\
12,929 \\
52,720 \\
2,050 \\
320 \\
468 \\
1,282 \\
192 \\
695 \\
1,178 \\
2,779 \\
0 \\
2,779 \\
336 \\
8,786 \\
6,075 \\
2,711 \\
9,573 \\
11,024 \\
590 \\
0 \\
590\end{array}$ & $\begin{array}{r}43,986 \\
4 \\
W \\
W \\
38,437 \\
W \\
W \\
4,823 \\
W \\
W \\
722 \\
W \\
W \\
630,808 \\
0 \\
60,433 \\
680,375 \\
864 \\
75,010 \\
5,809 \\
69,201 \\
66,992 \\
2,209 \\
6,842 \\
279,543 \\
62,706 \\
216,837 \\
27,040 \\
320 \\
4,387 \\
22,333 \\
7,298 \\
7,927 \\
5,001 \\
8,362 \\
0 \\
8,352 \\
864 \\
46,159 \\
27,987 \\
18,172 \\
63,669 \\
49,612 \\
3,614 \\
0 \\
3,614\end{array}$ \\
\hline Total & $\mathbf{5 7 3}, \boldsymbol{\oplus 9}$ & 38,081 & 600,947 & 00,143 & 141,020 & 284,618 & $1,263,670$ \\
\hline 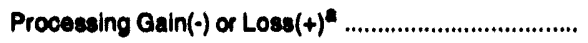 & $-24,742$ & .340 & $-25,082$ & $-38,774$ & $-7,169$ & $-8,795$ & $-54,738$ \\
\hline
\end{tabular}

See footnotes at end of table. 
Table 17. Rofinery Not Production of Finiched Petroloum Producte by PAD and Rofining Dietriots, 1903 (Continued)

(Thousand Barrels)

\begin{tabular}{|c|c|c|c|c|c|c|c|c|c|}
\hline \multirow{2}{*}{ Comnnedlyy } & \multicolumn{6}{|c|}{ PAD Dieniriot II } & \multirow{2}{*}{\begin{tabular}{|c|}
$\begin{array}{c}\text { Pab oich. } \\
\text { N }\end{array}$ \\
pooly im.
\end{tabular}} & \multirow{2}{*}{$\begin{array}{c}\begin{array}{c}\text { Pab bict. } \\
\text { v }\end{array} \\
\text { weat conet }\end{array}$} & \multirow[b]{2}{*}{$\begin{array}{l}\text { Y.s. } \\
\text { Totel }\end{array}$} \\
\hline & & & & & & Toind & & & \\
\hline 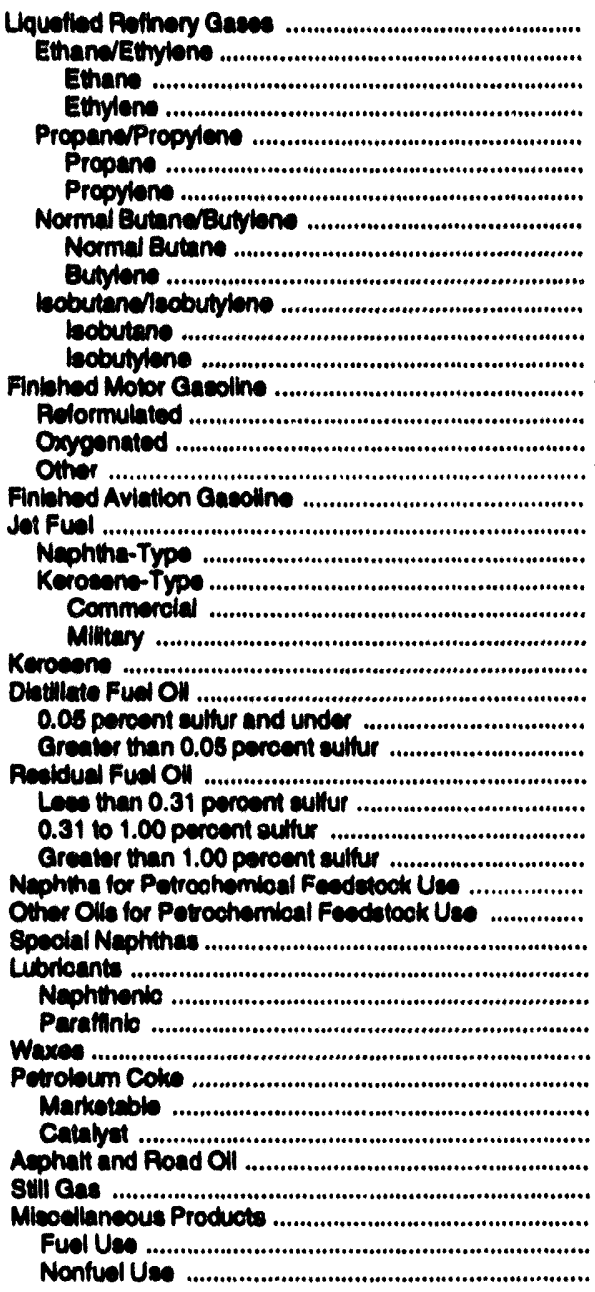 & 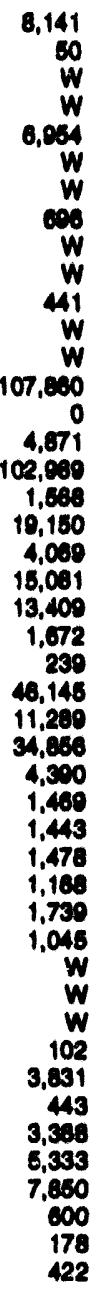 & 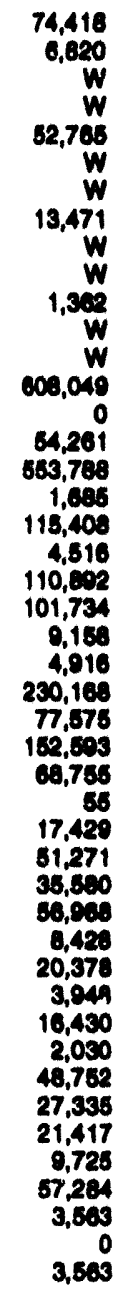 & 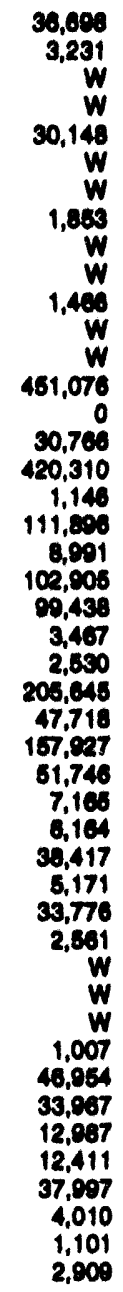 & 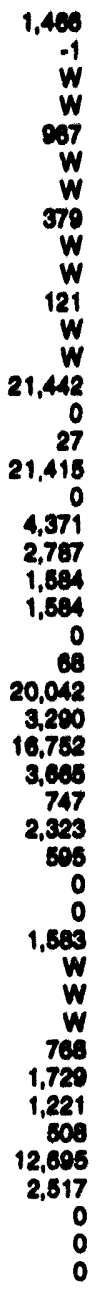 & $\begin{array}{r}920 \\
0 \\
W \\
W \\
718 \\
W \\
W \\
139 \\
W \\
W \\
72 \\
W \\
W \\
10,970 \\
0 \\
1,800 \\
18,371 \\
0 \\
3,237 \\
2,377 \\
8.0 \\
107 \\
753 \\
42 \\
8,850 \\
2,090 \\
6,040 \\
283 \\
19 \\
214 \\
0 \\
0 \\
0 \\
0 \\
W \\
W \\
W \\
0 \\
241 \\
0 \\
241 \\
1,716 \\
917 \\
0 \\
0 \\
0\end{array}$ & 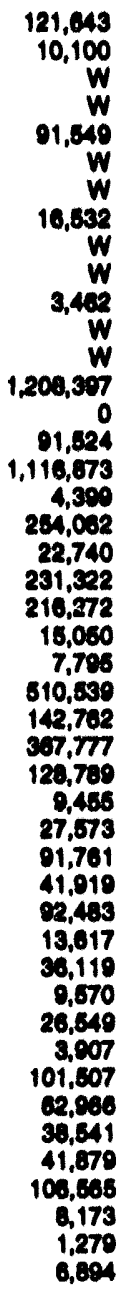 & 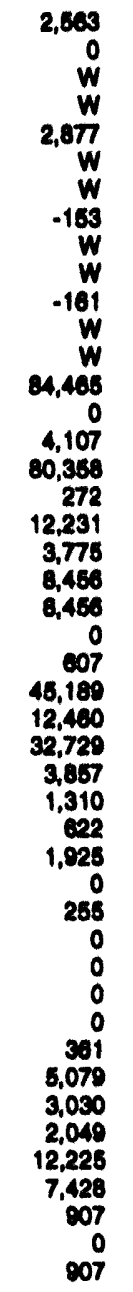 & 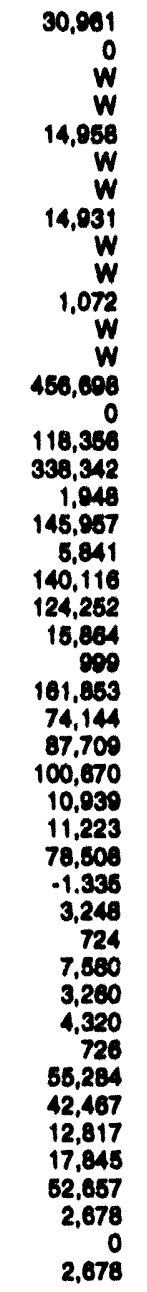 & 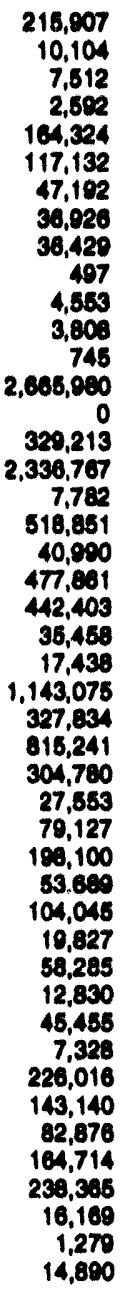 \\
\hline (1) & 0 & 34,101 & . & $\pi, 004$ & 38,014 & $2,10,703$ & 178,450 & $1,084,493$ & $0,792,291$ \\
\hline Ing Cain $(-)$ or Loas $(+)^{a}$ & $-6,685$ & $-78,827$ & $-46,801$ & $-1,004$ & -372 & $-136,689$ & $-6,646$ & $-58,569$ & $-279,713$ \\
\hline
\end{tabular}

- Represents the arthmatic difterence between input and produotion.

$W=$ Withheld to avoid dicolosure of individual company data.

Note: Roter to Apoendtx A for refining Ditriot decortptione.

Source: Energy Information Adminibtration (ELA) Form ElA-810, "Monthly Rofinery Report." 
Table 18. Rofinery Btocke of Cruds Oll and Potroloum Producte by PAD and Rofining Dictricte, 1983 (Thousand Barrels)

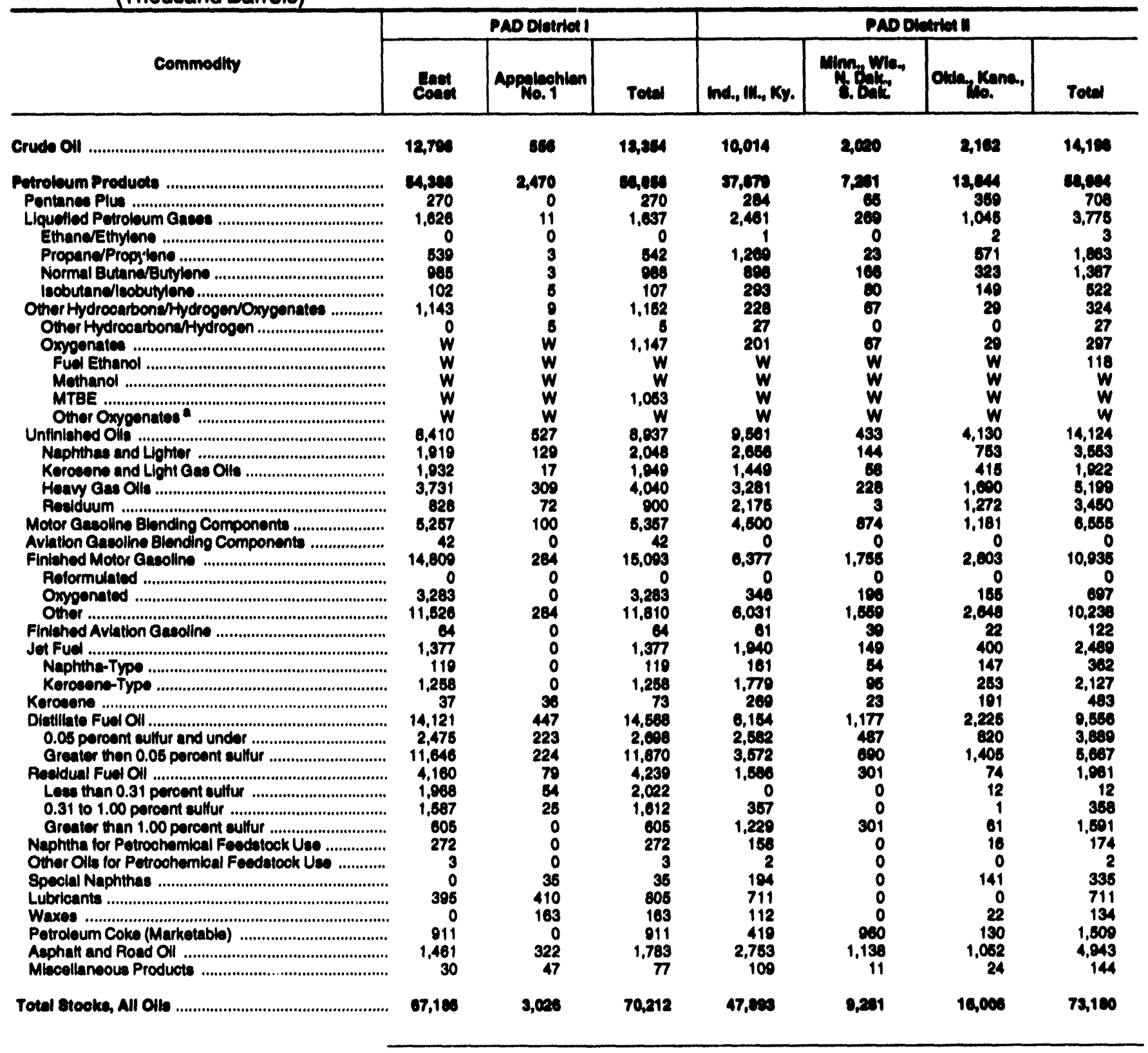

See footnotes at end of table. 
Tablo 18. Rofinery Stooks of Cruds Oll and Potroloum Produots by PAD and Aolining Diatriote, 1993 (Continued)

(Thousand Barrels)

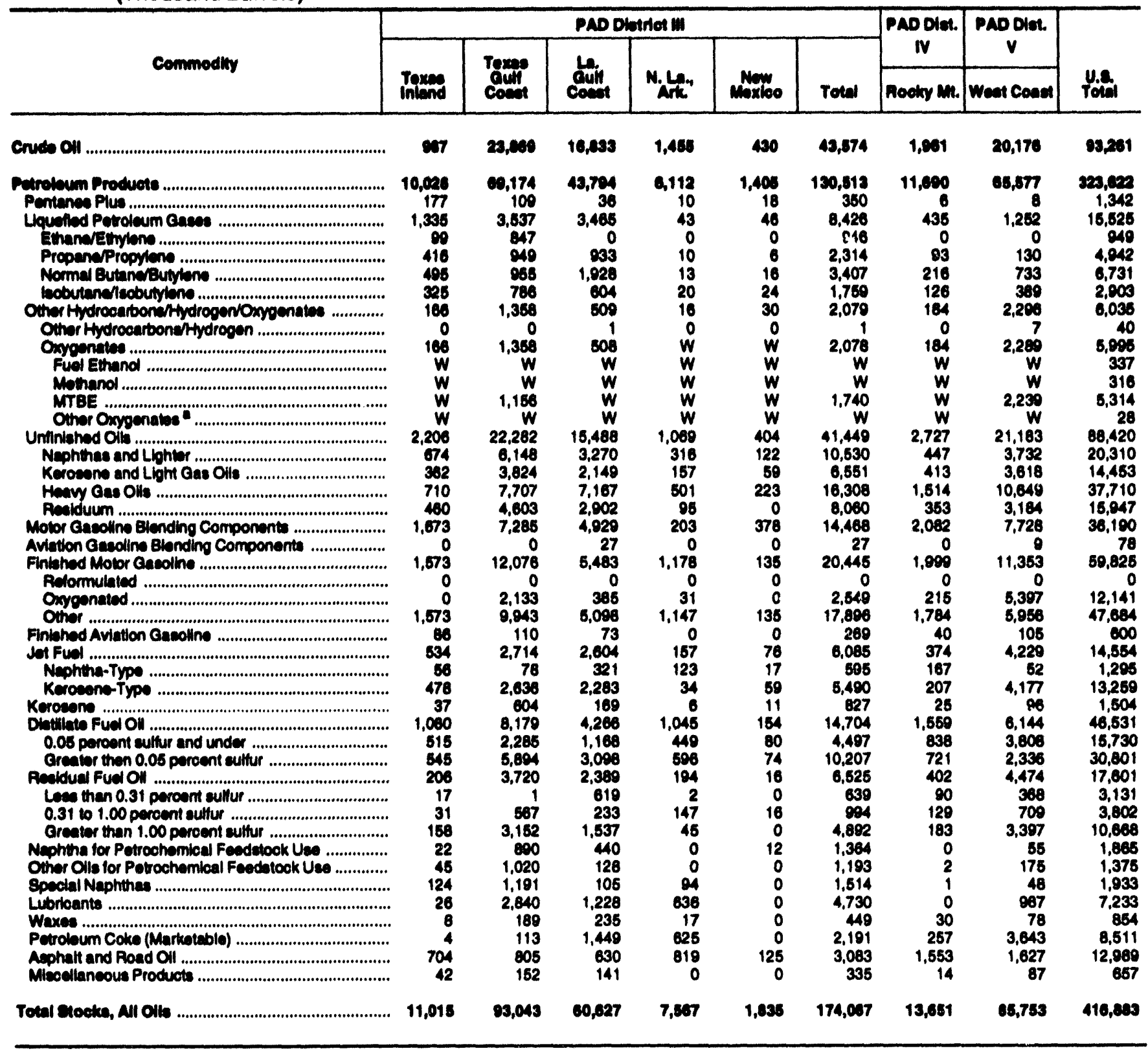

- Includes ethyl tertiary butyl ether (ETBE), tertiary amyl mothyl ether (TAME), tertiary butyl alcohol (TBA), and other aliphatic alcohols and ethers intended for motor gasoline blending (e.g. bopropyl ther (IPE) or n-propanol).

$W$ - Witheld to avold disolosure of individual company data

Notex. B Stocks are reporied as of the end of December. - Reler to Appendix A for Refining Diatrict descriptions.

Source: Energy Information Administration (EIA) Form ELA-810, "Monthly Refinery Report." 
Table 19. Percent Refinery Yild of Petroloum Products by PAD and Rofining Districte," 1993

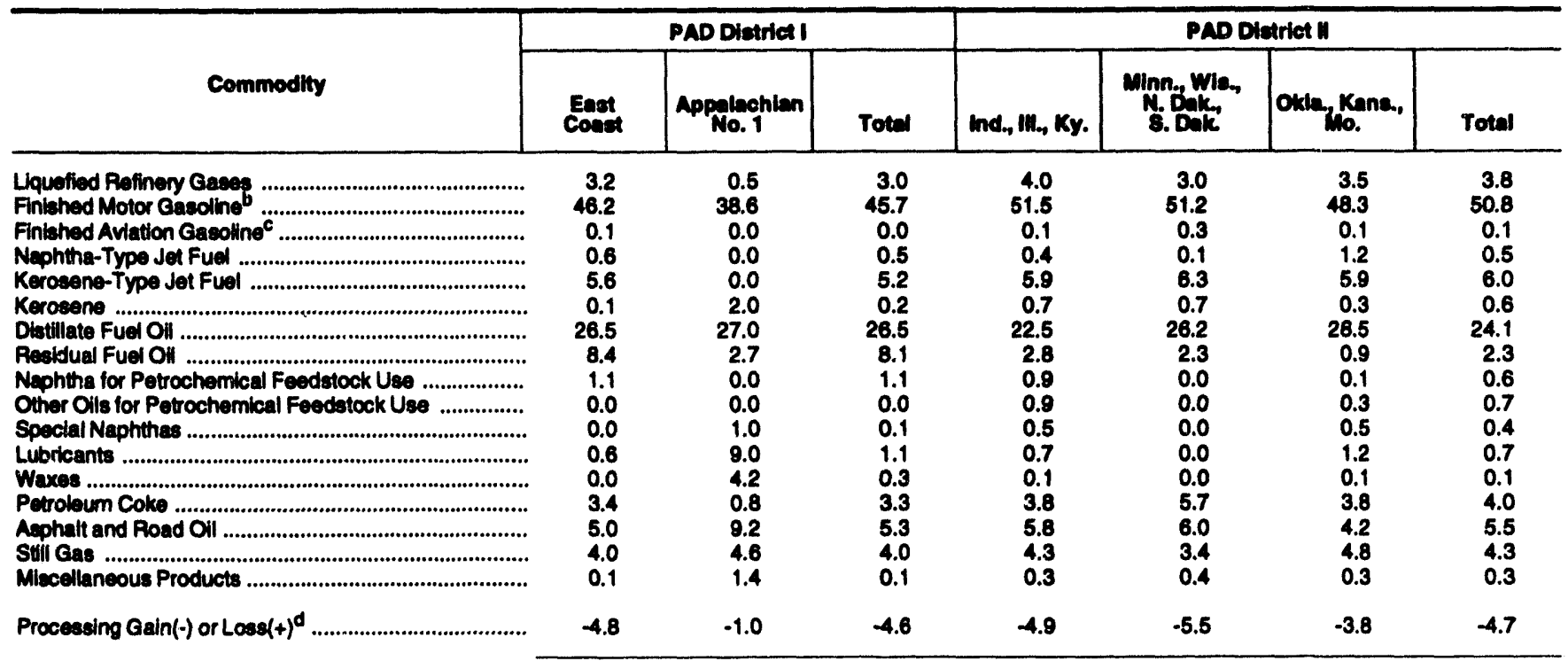

\begin{tabular}{|c|c|c|c|c|c|c|c|c|c|}
\hline \multirow[b]{2}{*}{ Commodity } & \multicolumn{6}{|c|}{ PAD Dicariat II } & \multirow{2}{*}{$\begin{array}{c}\text { PAD Diot. } \\
\mathbf{N}\end{array} \mid$} & \multirow{2}{*}{$\begin{array}{c}\begin{array}{c}\text { PAD Dist. } \\
V\end{array} \\
\text { west Conet }\end{array}$} & \multirow[b]{2}{*}{$\begin{array}{l}\text { U.S. } \\
\text { Tota: }\end{array}$} \\
\hline & $\begin{array}{l}\text { Toxes } \\
\text { Intand }\end{array}$ & $\begin{array}{l}\text { Toxas } \\
\text { Culf } \\
\text { Cosest }\end{array}$ & $\begin{array}{c}\text { La. } \\
\text { Cout }\end{array}$ & N.Lse, & Moxico & Total & & & \\
\hline 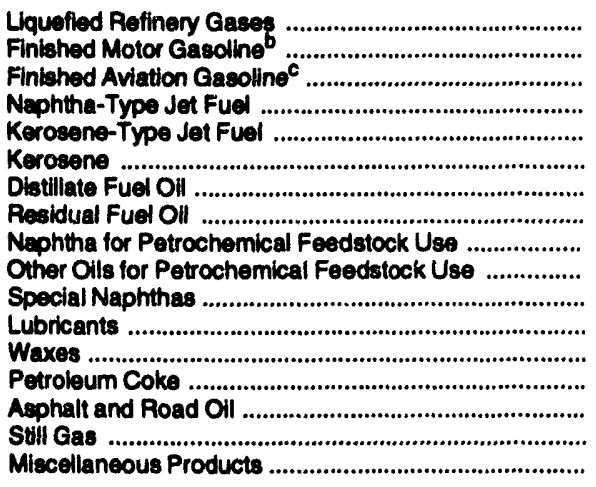 & $\begin{array}{r}4.3 \\
49.7 \\
0.8 \\
2.2 \\
8.0 \\
0.1 \\
24.4 \\
2.3 \\
0.6 \\
0.9 \\
0.6 \\
0.2 \\
0.1 \\
2.0 \\
2.8 \\
4.2 \\
0.3\end{array}$ & $\begin{array}{r}6.2 \\
45.1 \\
0.1 \\
0.4 \\
9.2 \\
0.4 \\
19.2 \\
5.7 \\
3.0 \\
4.8 \\
0.7 \\
1.7 \\
0.2 \\
4.1 \\
0.8 \\
4.8 \\
0.3\end{array}$ & $\begin{array}{r}4.0 \\
44.5 \\
0.1 \\
1.0 \\
11.1 \\
0.3 \\
22.2 \\
5.6 \\
0.6 \\
3.7 \\
0.3 \\
0.9 \\
0.1 \\
5.1 \\
1.3 \\
4.1 \\
0.4\end{array}$ & $\begin{array}{r}2.0 \\
25.9 \\
0.0 \\
3.8 \\
2.2 \\
0.1 \\
27.2 \\
5.0 \\
0.0 \\
0.0 \\
2.2 \\
9.1 \\
1.0 \\
2.4 \\
17.3 \\
3.4 \\
0.0\end{array}$ & $\begin{array}{r}2.9 \\
51.7 \\
0.0 \\
7.4 \\
2.7 \\
0.1 \\
26.7 \\
0.7 \\
0.0 \\
0.0 \\
0.0 \\
0.0 \\
0.0 \\
0.8 \\
5.4 \\
2.9 \\
0.0\end{array}$ & $\begin{array}{r}5.0 \\
44.7 \\
0.2 \\
0.9 \\
9.6 \\
0.3 \\
21.1 \\
5.3 \\
1.7 \\
3.8 \\
0.6 \\
1.5 \\
0.2 \\
4.2 \\
1.7 \\
4.4 \\
0.3\end{array}$ & $\begin{array}{r}1.6 \\
47.5 \\
0.2 \\
2.3 \\
5.2 \\
0.4 \\
27.8 \\
2.4 \\
0.0 \\
0.2 \\
0.0 \\
0.0 \\
0.2 \\
3.1 \\
7.5 \\
4.6 \\
0.6\end{array}$ & $\begin{array}{r}3.3 \\
43.7 \\
0.2 \\
0.6 \\
15.1 \\
0.1 \\
17.4 \\
10.8 \\
-0.1 \\
0.4 \\
0.1 \\
0.8 \\
0.1 \\
5.9 \\
1.9 \\
5.7 \\
0.3\end{array}$ & $\begin{array}{r}4.1 \\
46.1 \\
0.2 \\
0.8 \\
9.2 \\
0.3 \\
21.9 \\
5.8 \\
1.0 \\
2.0 \\
0.4 \\
1.1 \\
0.1 \\
4.3 \\
3.2 \\
4.6 \\
0.3\end{array}$ \\
\hline Processing Gain(-) or Loss $(+)^{d}$ & -3.5 & -6.6 & -5.3 & -1.4 & -1.2 & -5.6 & -3.5 & -6.3 & -5.4 \\
\hline
\end{tabular}

- Based on crude oil input and net reruns of unfinished olls.

b Based on total finished motor gasoline output minus net input of motor gasoline blending components, minus input of natural gas plant llquids, other hydrocarbons and oxygenates.

c Based on finished aviation gasoline output minus net input of aviation gasoline blending components.

d Represents the difference between input and production.

Notes: - Totals may not equal sum of components due to independent rounding. - Refer to Appendix A for Refining District descriptions.

Sources: Calculated from data on Tables 17 and 18. 
Table 20. Imports of Crude Oll and Petroleum Products by PAD District, 1993 (Thousand Barrels)

\begin{tabular}{|c|c|c|c|c|c|c|c|}
\hline \multirow{2}{*}{ Commodliy } & \multicolumn{6}{|c|}{ Petroleum Adminiatration for Dofones Distriets } & \multirow[b]{2}{*}{$\begin{array}{c}\text { Dalfy } \\
\text { Average }\end{array}$} \\
\hline & $\mathbf{I}$ & $\mathbf{n}$ & m & IV & $\mathbf{v}$ & $\begin{array}{l}\text { U.8. } \\
\text { Total }\end{array}$ & \\
\hline 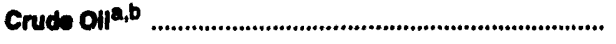 & 492,908 & 491,674 & $1,303,540$ & 32,941 & 100,272 & $2,477,230$ & 6,787 \\
\hline 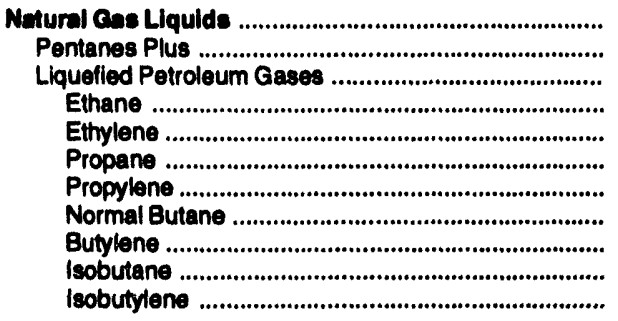 & $\begin{array}{r}8,062 \\
1,512 \\
7,150 \\
1 \\
0 \\
5,977 \\
0 \\
1,086 \\
0 \\
106 \\
0\end{array}$ & $\begin{array}{r}28,428 \\
503 \\
27,923 \\
5,239 \\
0 \\
15,683 \\
1,499 \\
3,421 \\
0 \\
2,101 \\
0\end{array}$ & $\begin{array}{r}27,619 \\
7,159 \\
20,460 \\
0 \\
46 \\
12,994 \\
0 \\
5,326 \\
103 \\
1,975 \\
16\end{array}$ & $\begin{array}{r}5,130 \\
2,668 \\
2,462 \\
0 \\
0 \\
1,469 \\
0 \\
845 \\
0 \\
148 \\
0\end{array}$ & $\begin{array}{r}232 \\
0 \\
232 \\
0 \\
0 \\
112 \\
0 \\
37 \\
0 \\
83 \\
0\end{array}$ & $\begin{array}{r}70,069 \\
11,842 \\
58,227 \\
5,240 \\
46 \\
36,215 \\
1,499 \\
10,695 \\
103 \\
4,413 \\
16\end{array}$ & $\begin{array}{r}192 \\
32 \\
160 \\
14 \\
(8) \\
99 \\
4 \\
29 \\
(8) \\
12 \\
(8)\end{array}$ \\
\hline 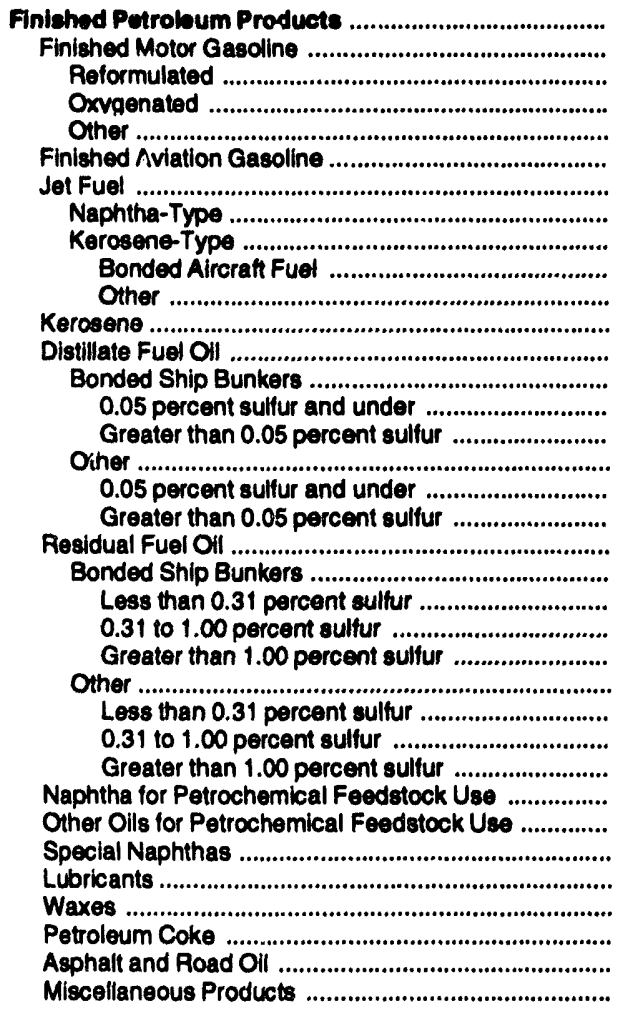 & $\begin{array}{r}300,550 \\
84,432 \\
0 \\
421 \\
84,011 \\
0 \\
29,937 \\
2,046 \\
27,891 \\
15,803 \\
12,088 \\
239 \\
59,237 \\
0 \\
0 \\
0 \\
59,237 \\
22,128 \\
37,109 \\
110,481 \\
0 \\
0 \\
0 \\
0 \\
0 \\
110,481 \\
16,871 \\
20,890 \\
72,720 \\
1,806 \\
0 \\
188 \\
2,156 \\
413 \\
0 \\
10,960 \\
701\end{array}$ & $\begin{array}{r}5,647 \\
908 \\
0 \\
0 \\
908 \\
32 \\
831 \\
792 \\
39 \\
0 \\
39 \\
0 \\
2,244 \\
0 \\
0 \\
0 \\
2,244 \\
1,051 \\
1,193 \\
257 \\
0 \\
0 \\
0 \\
0 \\
257 \\
0 \\
182 \\
75 \\
215 \\
0 \\
756 \\
161 \\
70 \\
0 \\
100 \\
73\end{array}$ & $\begin{array}{r}7,651 \\
1,363 \\
0 \\
0 \\
1,363 \\
0 \\
2,445 \\
584 \\
1,861 \\
0 \\
1,861 \\
47 \\
2,399 \\
0 \\
0 \\
0 \\
2,399 \\
2,399 \\
0 \\
18,195 \\
0 \\
0 \\
0 \\
0 \\
18,195 \\
2,128 \\
520 \\
15,547 \\
11,010 \\
40,343 \\
796 \\
148 \\
105 \\
512 \\
220 \\
68\end{array}$ & $\begin{array}{r}1,310 \\
120 \\
0 \\
0 \\
120 \\
0 \\
0 \\
0 \\
0 \\
0 \\
0 \\
0 \\
1,138 \\
19 \\
19 \\
0 \\
1,119 \\
456 \\
663 \\
18 \\
0 \\
0 \\
0 \\
0 \\
18 \\
5 \\
5 \\
8 \\
0 \\
0 \\
0 \\
0 \\
34 \\
0 \\
0 \\
0\end{array}$ & $\begin{array}{r}17,156 \\
3,419 \\
0 \\
0 \\
3,419 \\
46 \\
3,270 \\
268 \\
3,002 \\
300 \\
2,702 \\
21 \\
2,166 \\
639 \\
0 \\
639 \\
1,527 \\
1,004 \\
523 \\
7,200 \\
0 \\
0 \\
0 \\
0 \\
7,200 \\
1,412 \\
426 \\
5,362 \\
255 \\
0 \\
50 \\
31 \\
24 \\
177 \\
491 \\
6\end{array}$ & $\begin{array}{r}402,314 \\
80,242 \\
0 \\
421 \\
89,821 \\
78 \\
38,463 \\
3,690 \\
32,793 \\
16,103 \\
16,690 \\
307 \\
67,184 \\
658 \\
19 \\
639 \\
66,526 \\
27,038 \\
39,488 \\
136,151 \\
0 \\
0 \\
0 \\
0 \\
0 \\
136,151 \\
20,416 \\
22,023 \\
93,712 \\
13,286 \\
40,343 \\
1,790 \\
2,496 \\
646 \\
689 \\
11,771 \\
848\end{array}$ & $\begin{array}{r}1,102 \\
247 \\
0 \\
1 \\
246 \\
(8) \\
100 \\
10 \\
90 \\
44 \\
46 \\
1 \\
184 \\
2 \\
(8) \\
2 \\
182 \\
74 \\
108 \\
373 \\
0 \\
0 \\
0 \\
0 \\
373 \\
56 \\
60 \\
257 \\
36 \\
111 \\
5 \\
7 \\
2 \\
2 \\
32 \\
2\end{array}$ \\
\hline Total & 853,468 & 526,352 & $1,599,443$ & 39,381 & 127,810 & $3,146,454$ & 8,620 \\
\hline
\end{tabular}

a Crude oil and unfinished olls are reported by the PAD Distribt in which they are to be processed; all other products are reported by the PAD District of entry.

b Includes crude oll imported for storage in the Strategic Petroleum Reserve.

c Includes ethyl tertiany butyl ether (ETBE), tertiany amyl methyl ether (TAME), tertlary butyl alcohol (TBA), and other allphatic alcohols and ethers intended for motor gasoline blending (e.g., lsopropyl ether (IPE) or n-propanol).

(s) = Less than 500 barrels per day.

Note: Totals may not equal sum of components dus to independent rounding.

Sources: Energy Information Administration (EIA) Form EIA-814, "Monthly Imports Raport." 
Table 21. Imports of Crude Oll and Petroloum Products Into the United States by Country of Origin," 1993

(Thousand Barrels)

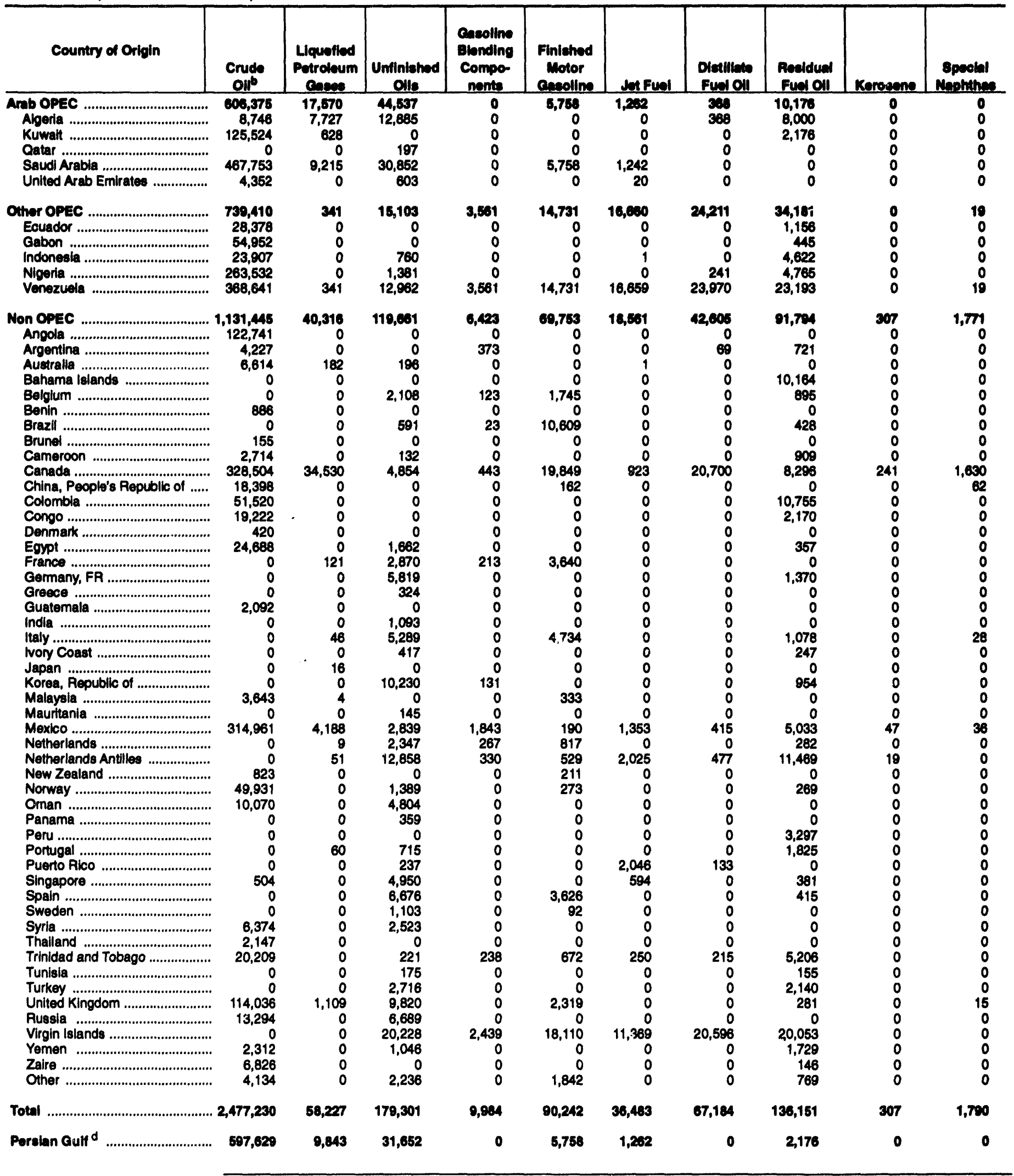

See footnotes at end of table. 
Table 21. Importe of Crude Oll and Petroloum Producte Into the United States by Country of Orlgin," 1993 (Continued)

(Thousand Barrels)

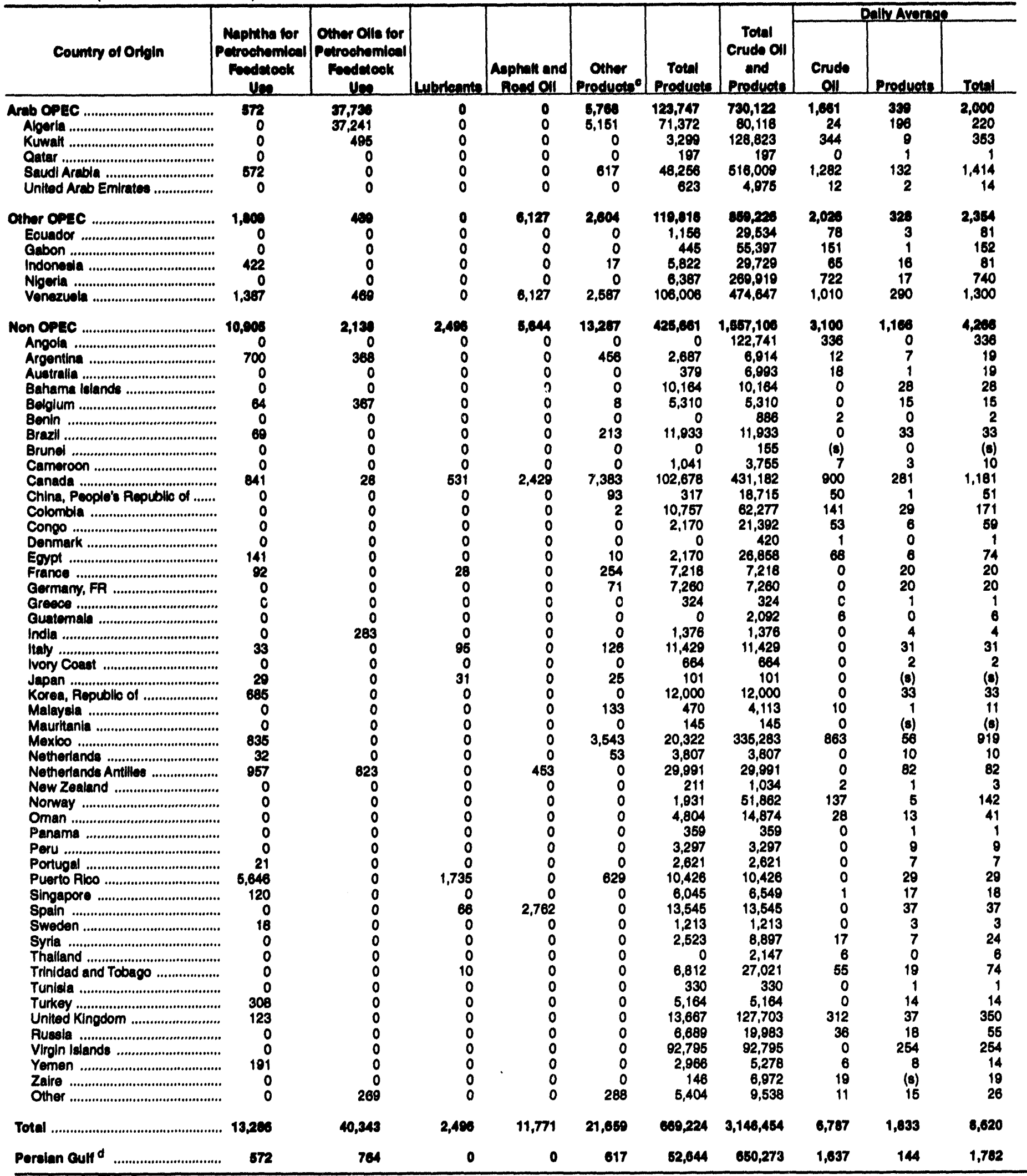

- Crude oll and uniniahed olls are reported by the PAD Dietrict in which they are to be processed; all other products are reported by the PAD Distriot of entry.

b includes crude oll imported for etorage in the Strategic Petroleum Reserve.

- includes avlation gasoline, avlation gasoline blending components, miscellaneous products, other hydrocarbons and oxygenates, pentanes plus, petroleum coke, and waxeg.

dincludes Bahrain, Iran, Ireq, Kuwail, Qatar, Saudi Arebla, and United Arab Emirates.

(a) = Less than 600 barrele per day.

Note: Totals may not equal sum of components due to independent rounding.

Source: Energy Information Administration (EIA) Form EIA-814, "Monthly Imports Report."

Enorgy Information Adminiatration/Potroloum Supply Annual 1993, Volume 1 
Table 22. PAD District $\vdash$ Imports of Cruds OII and Petroloum Products by Country of Origin," 1993

(Thousand Barrels)

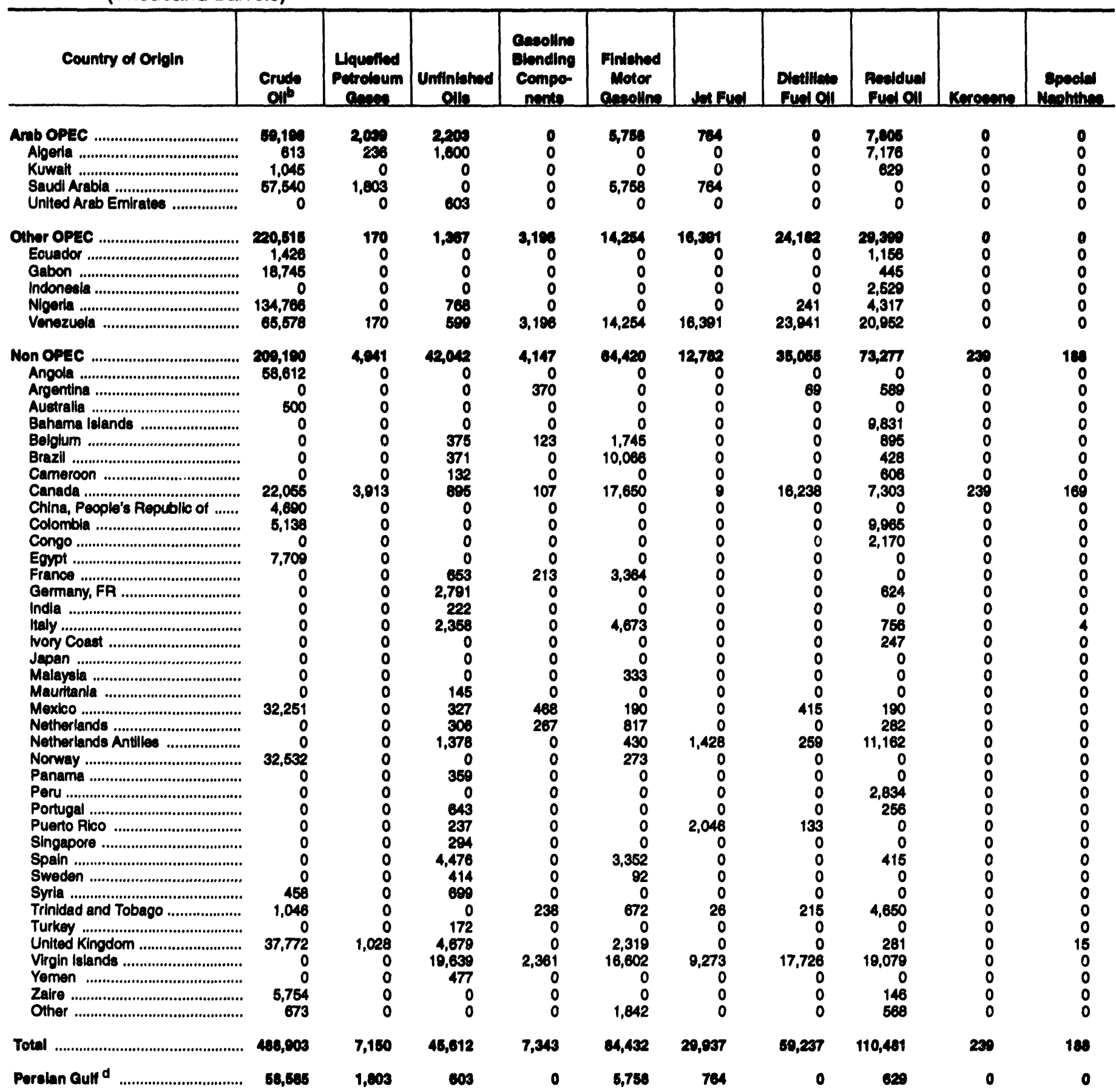

See footnotes at end of table. 
Table 22. PAD District I-Imports of Crude OII and Petroloum Products by Country of Origin, ${ }^{a} 1093$ (Continued) (Thousand Barrels)

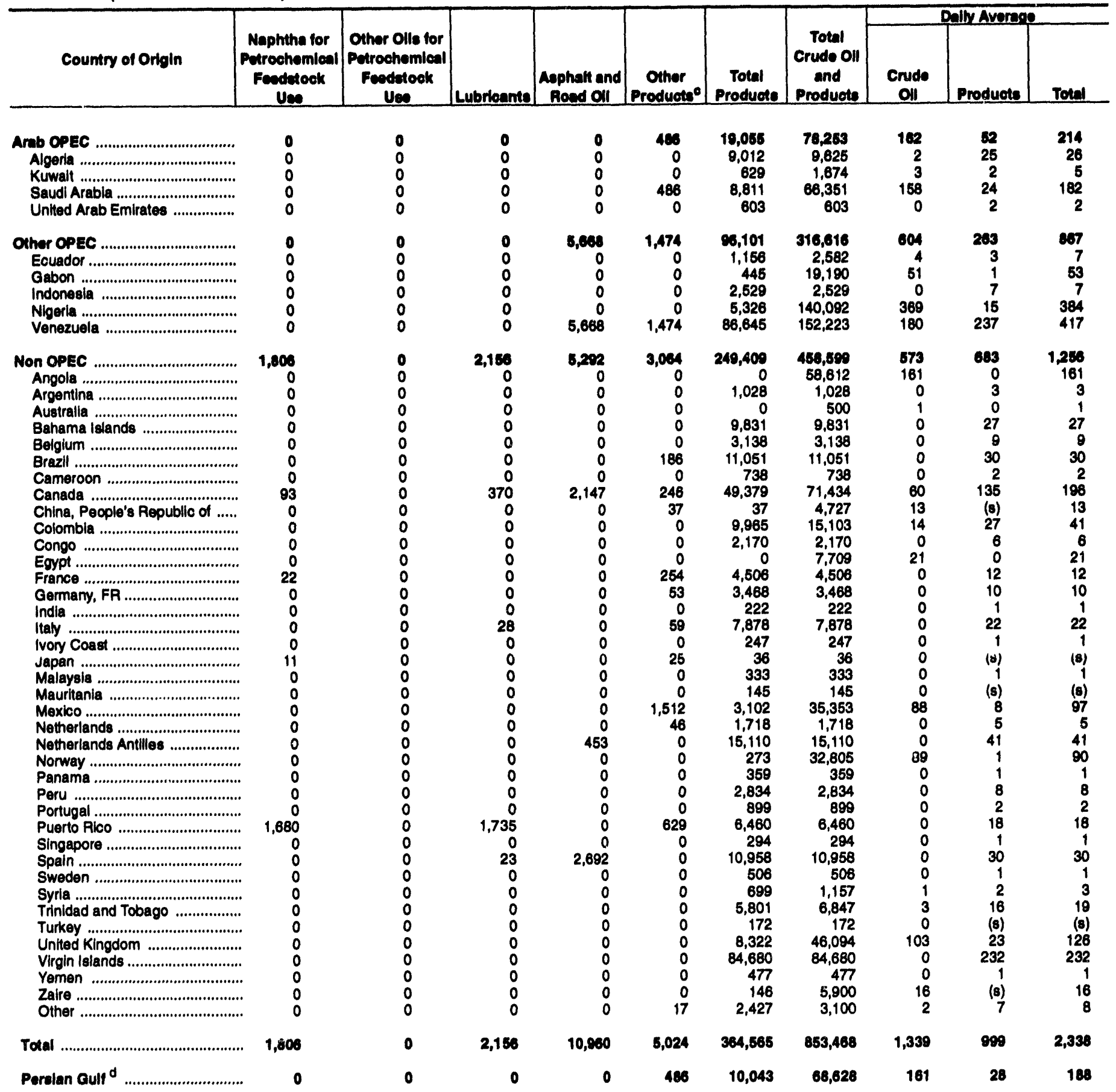

a Crude oll and unfinished oils are reported by the PAD District in which they are to be processed; all other products are reported by the PAD District of entry.

b Includes crude oll imported for storage in the Strategic Petroloum Reserve.

c includes aviation gasoline, aviation gasoline blending components, miscellaneous products, other hydrocarbons and oxygenates, pentanes plus, petroleum coke, and waxes.

Includes Bahrain, Iran, Iraq, Kuwalt, Oatar, Saudl Arabla, and United Arab Emirates.

$(s)=$ Less than 500 harrels per day.

Note: Totals may not equal sum of components due to independent rounding

Source: Energy information Administration (EIA) Form ElA-814, "Monthly Imports Report." 
Table 23. PAD District II-Importe of Crude Oll and Potroloum Products by Country of Origin," 1993

(Thousand Barrels)

\begin{tabular}{|c|c|c|c|c|c|c|c|c|c|c|}
\hline Country of Orlgin & $\begin{array}{l}\text { Crude } \\
\text { Oilb }\end{array}$ & $\begin{array}{l}\text { Llquefled } \\
\text { Petroloum } \\
\text { Oneses }\end{array}$ & $\begin{array}{c}\text { Untinished } \\
\text { Olls }\end{array}$ & $\begin{array}{l}\text { Casollne } \\
\text { Blending } \\
\text { Compo- } \\
\text { nents }\end{array}$ & $\begin{array}{l}\text { Finlahed } \\
\text { Motor } \\
\text { Cecolline }\end{array}$ & Jet Funel & $\begin{array}{l}\text { Disulliato } \\
\text { Fund OAl }\end{array}$ & $\begin{array}{l}\text { Realdual } \\
\text { Fuel OII }\end{array}$ & Keroeene & $\begin{array}{l}\text { Epoeldal } \\
\text { Nephthes }\end{array}$ \\
\hline 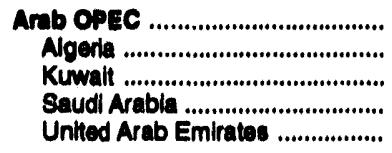 & $\begin{array}{r}16,497 \\
1,091 \\
13,379 \\
29,981 \\
1,046\end{array}$ & $\begin{array}{l}0 \\
0 \\
0 \\
0 \\
0\end{array}$ & $\begin{array}{l}0 \\
0 \\
0 \\
0 \\
0\end{array}$ & $\begin{array}{l}0 \\
0 \\
0 \\
0 \\
0\end{array}$ & $\begin{array}{l}0 \\
0 \\
0 \\
0 \\
0\end{array}$ & $\begin{array}{l}0 \\
0 \\
0 \\
0 \\
0\end{array}$ & $\begin{array}{l}0 \\
0 \\
0 \\
0 \\
0\end{array}$ & $\begin{array}{l}0 \\
0 \\
0 \\
0 \\
0\end{array}$ & $\begin{array}{l}0 \\
0 \\
0 \\
r .\end{array}$ & $\begin{array}{l}0 \\
0 \\
0 \\
0 \\
0\end{array}$ \\
\hline 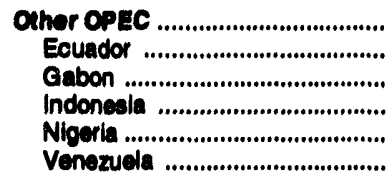 & $\begin{array}{r}98,941 \\
9,219 \\
1,053 \\
397 \\
36,646 \\
51,726\end{array}$ & $\begin{array}{l}0 \\
0 \\
0 \\
0 \\
0 \\
0\end{array}$ & $\begin{array}{l}0 \\
0 \\
0 \\
0 \\
0 \\
0\end{array}$ & $\begin{array}{l}0 \\
0 \\
0 \\
0 \\
0 \\
0\end{array}$ & $\begin{array}{l}0 \\
0 \\
0 \\
0 \\
0 \\
0\end{array}$ & $\begin{array}{l}0 \\
0 \\
0 \\
0 \\
0 \\
0\end{array}$ & $\begin{array}{l}0 \\
0 \\
0 \\
0 \\
0 \\
0\end{array}$ & $\begin{array}{l}0 \\
0 \\
0 \\
0 \\
0 \\
0\end{array}$ & $\begin{array}{l}0 \\
0 \\
0 \\
0 \\
0 \\
0\end{array}$ & $\begin{array}{l}0 \\
0 \\
0 \\
0 \\
0 \\
0\end{array}$ \\
\hline 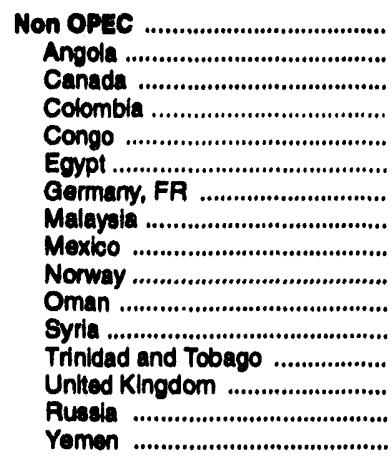 & $\begin{array}{r}347,133 \\
6,945 \\
243,211 \\
8,861 \\
4,012 \\
521 \\
0 \\
438 \\
48,652 \\
7,523 \\
1,067 \\
1,273 \\
1,767 \\
21,286 \\
1,030 \\
460\end{array}$ & $\begin{array}{r}27,923 \\
0 \\
27,923 \\
0 \\
0 \\
0 \\
0 \\
0 \\
0 \\
0 \\
0 \\
0 \\
0 \\
0 \\
0 \\
0\end{array}$ & $\begin{array}{r}370 \\
0 \\
370 \\
0 \\
0 \\
0 \\
0 \\
0 \\
0 \\
0 \\
0 \\
0 \\
0 \\
0 \\
0 \\
0\end{array}$ & $\begin{array}{r}335 \\
0 \\
335 \\
0 \\
0 \\
0 \\
0 \\
0 \\
0 \\
0 \\
0 \\
0 \\
0 \\
0 \\
0 \\
0\end{array}$ & $\begin{array}{r}208 \\
0 \\
908 \\
0 \\
0 \\
0 \\
0 \\
0 \\
0 \\
0 \\
0 \\
0 \\
0 \\
0 \\
0 \\
0\end{array}$ & $\begin{array}{r}831 \\
0 \\
831 \\
0 \\
0 \\
0 \\
0 \\
0 \\
0 \\
0 \\
0 \\
0 \\
0 \\
0 \\
0 \\
0\end{array}$ & $\begin{array}{r}2,24 \\
0 \\
2,244 \\
0 \\
0 \\
0 \\
0 \\
0 \\
0 \\
0 \\
0 \\
0 \\
0 \\
0 \\
0 \\
0\end{array}$ & $\begin{array}{r}257 \\
0 \\
257 \\
0 \\
0 \\
0 \\
0 \\
0 \\
0 \\
0 \\
0 \\
0 \\
0 \\
0 \\
0 \\
0\end{array}$ & $\begin{array}{l}0 \\
0 \\
0 \\
0 \\
0 \\
0 \\
0 \\
0 \\
0 \\
0 \\
0 \\
0 \\
0 \\
0 \\
0 \\
0\end{array}$ & $\begin{array}{r}760 \\
0 \\
768 \\
0 \\
0 \\
0 \\
0 \\
0 \\
0 \\
0 \\
0 \\
0 \\
0 \\
0 \\
0 \\
0\end{array}$ \\
\hline 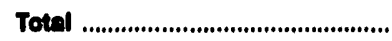 & 401,074 & 27,923 & 370 & 335 & 808 & 831 & 2,244 & 207 & 0 & 786 \\
\hline 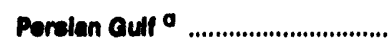 & 4,408 & 0 & 0 & 0 & $\mathbf{0}$ & 0 & 0 & $\mathbf{0}$ & 0 & 0 \\
\hline
\end{tabular}

See footnotes at end of table.

60 
Table 23. PAD Dietrict II-Imports of Cruds OII and Potroloum Products by Country of Origin," 1993 (Continued)

(Thousand Barrels)

\begin{tabular}{|c|c|c|c|c|c|c|c|c|c|c|}
\hline \multirow[b]{2}{*}{ Country of Orlgin } & \multirow[b]{2}{*}{$\begin{array}{c}\text { Nophtha for } \\
\text { Petrochemieal } \\
\text { Fecdetcok } \\
\text { Une }\end{array}$} & \multirow[b]{2}{*}{$\begin{array}{c}\text { Other Olle for } \\
\text { Petroohomieal } \\
\text { Prodetcok } \\
\text { Une }\end{array}$} & \multirow[b]{2}{*}{ Luprieante } & \multirow[b]{2}{*}{$\begin{array}{c}\text { Aephali and } \\
\text { Read ell }\end{array}$} & \multirow[b]{2}{*}{$\begin{array}{c}\text { Othor } \\
\text { Producte }\end{array}$} & \multirow[b]{2}{*}{$\begin{array}{c}\text { Total } \\
\text { Produete }\end{array}$} & \multirow[b]{2}{*}{$\begin{array}{c}\text { Total } \\
\text { Crude OII } \\
\text { and } \\
\text { Produete }\end{array}$} & \multicolumn{3}{|c|}{ Dally Averse } \\
\hline & & & & & & & & $\begin{array}{c}\text { Crude } \\
\text { ell }\end{array}$ & Producte & Total \\
\hline 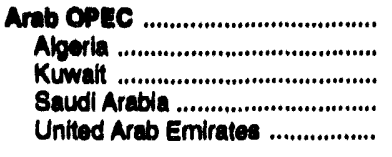 & $\begin{array}{l}0 \\
0 \\
0 \\
0 \\
0\end{array}$ & $\begin{array}{l}0 \\
0 \\
0 \\
0 \\
0\end{array}$ & $\begin{array}{l}0 \\
0 \\
0 \\
0 \\
0\end{array}$ & $\begin{array}{l}0 \\
0 \\
0 \\
0 \\
0\end{array}$ & $\begin{array}{l}0 \\
0 \\
0 \\
0 \\
0\end{array}$ & $\begin{array}{l}0 \\
0 \\
0 \\
0 \\
0\end{array}$ & $\begin{array}{r}48,497 \\
1,091 \\
13,379 \\
29,981 \\
1,046\end{array}$ & $\begin{array}{r}125 \\
3 \\
37 \\
82 \\
3\end{array}$ & $\begin{array}{l}0 \\
0 \\
0 \\
0 \\
0\end{array}$ & $\begin{array}{r}128 \\
3 \\
37 \\
82 \\
3\end{array}$ \\
\hline 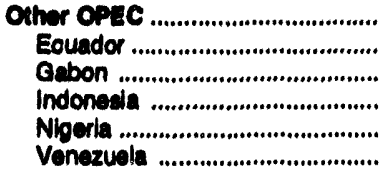 & $\begin{array}{l}0 \\
0 \\
0 \\
0 \\
0 \\
0\end{array}$ & $\begin{array}{l}0 \\
0 \\
0 \\
0 \\
0 \\
0\end{array}$ & $\begin{array}{l}0 \\
0 \\
0 \\
0 \\
0 \\
0\end{array}$ & $\begin{array}{l}0 \\
0 \\
0 \\
0 \\
0 \\
0\end{array}$ & $\begin{array}{l}0 \\
0 \\
0 \\
0 \\
0 \\
0\end{array}$ & $\begin{array}{l}0 \\
0 \\
0 \\
0 \\
0 \\
0\end{array}$ & $\begin{array}{r}98,941 \\
9,219 \\
1,053 \\
3897 \\
38,646 \\
51,726\end{array}$ & $\begin{array}{r}271 \\
25 \\
3 \\
1 \\
100 \\
142\end{array}$ & $\begin{array}{l}0 \\
0 \\
0 \\
0 \\
0 \\
0\end{array}$ & $\begin{array}{r}271 \\
25 \\
3 \\
1 \\
100 \\
142\end{array}$ \\
\hline 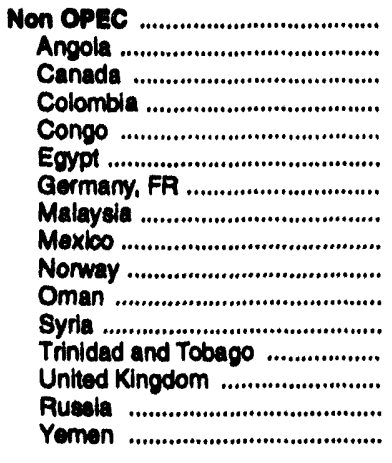 & $\begin{array}{r}215 \\
0 \\
215 \\
0 \\
0 \\
0 \\
0 \\
0 \\
0 \\
0 \\
0 \\
0 \\
0 \\
0 \\
0 \\
0\end{array}$ & $\begin{array}{l}0 \\
0 \\
0 \\
0 \\
0 \\
0 \\
0 \\
0 \\
0 \\
0 \\
0 \\
0 \\
0 \\
0 \\
0 \\
0\end{array}$ & $\begin{array}{r}181 \\
0 \\
161 \\
0 \\
0 \\
0 \\
0 \\
0 \\
0 \\
0 \\
0 \\
0 \\
0 \\
0 \\
0 \\
0\end{array}$ & $\begin{array}{r}100 \\
0 \\
100 \\
0 \\
0 \\
0 \\
0 \\
0 \\
0 \\
0 \\
0 \\
0 \\
0 \\
0 \\
0 \\
0\end{array}$ & $\begin{array}{r}679 \\
0 \\
667 \\
0 \\
0 \\
0 \\
11 \\
0 \\
0 \\
0 \\
0 \\
0 \\
0 \\
0 \\
0 \\
0\end{array}$ & $\begin{array}{r}34,77 \\
0 \\
34,767 \\
0 \\
0 \\
0 \\
11 \\
0 \\
0 \\
0 \\
0 \\
0 \\
0 \\
0 \\
0 \\
0\end{array}$ & $\begin{array}{r}301,914 \\
6,945 \\
277,978 \\
8,961 \\
4,012 \\
621 \\
11 \\
438 \\
48,652 \\
7,623 \\
1,067 \\
1,273 \\
1,757 \\
21,286 \\
1,030 \\
460\end{array}$ & $\begin{array}{r}981 \\
19 \\
666 \\
25 \\
11 \\
1 \\
0 \\
1 \\
133 \\
21 \\
3 \\
3 \\
5 \\
58 \\
3 \\
1\end{array}$ & $\begin{array}{r}98 \\
0 \\
96 \\
C \\
0 \\
0 \\
(8) \\
0 \\
0 \\
0 \\
0 \\
0 \\
0 \\
0 \\
0 \\
0\end{array}$ & $\begin{array}{r}1,046 \\
19 \\
762 \\
25 \\
11 \\
1 \\
(8) \\
1 \\
133 \\
21 \\
3 \\
3 \\
5 \\
58 \\
3 \\
1\end{array}$ \\
\hline 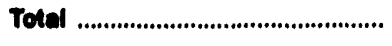 & 218 & 0 & 161 & 100 & 678 & 34,778 & $82.9,382$ & 1,347 & 98 & 1,442 \\
\hline Perelan Culf d ................................ & 0 & 0 & 0 & $\mathbf{0}$ & $\mathbf{0}$ & 0 & 4,408 & 122 & 0 & 122 \\
\hline
\end{tabular}

\footnotetext{
Crude oil and unfinished olls are reported by the PAD District in which they are to be processed; all other products are reported by the PAD District of entry.

b Includes crude oll imported lor slorage in the Strategic Petroleum Reserve.

c Includes avlation gasollne, aviation gasoline blending components, miscellaneous products, other hydrocarbons and oxygenates, pentanes plus, petroleum coke, and waxes.

includes Bahrain, Iran, Iraq, Kuwait, Qatar, Saudi Arabla, and United Arab Emirates.

(s) = Leas than 500 barrels per day.

Note: Totals may not equal sum ol components due to independent rounding.

Source: Energy Information Administration (EIA) Form EIA-814, "Monthly Imports Pepon."
} 
Table 24. PAD District III-Importe of Cruds OII and Potroleum Products by Country of Origin," 1908 (Thousand Barre/s)

\begin{tabular}{|c|c|c|c|c|c|c|c|c|c|c|}
\hline Country of Origln & & 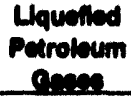 & $\begin{array}{c}\text { Unlinished } \\
\text { Qilh }\end{array}$ & $\begin{array}{l}\text { Dlending } \\
\text { Compo- } \\
\text { nent: }\end{array}$ & $\begin{array}{l}\text { Finlahed } \\
\text { Motor } \\
\text { enapline }\end{array}$ & Seft & $\begin{array}{l}\text { Dictilleto } \\
\text { Puel OAl }\end{array}$ & $\begin{array}{l}\text { Reddud } \\
\text { Fud ell }\end{array}$ & Kerosen & $\begin{array}{l}\text { Epolut } \\
\text { Nephth. }\end{array}$ \\
\hline 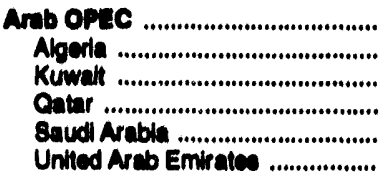 & $\begin{array}{r}7,042 \\
7,02,128 \\
0 \\
390,282 \\
3,300\end{array}$ & $\begin{array}{r}18,691 \\
7,491 \\
628 \\
0 \\
7,412 \\
0\end{array}$ & $\begin{array}{r}4,284 \\
11,286 \\
0 \\
197 \\
30,862 \\
0\end{array}$ & $\begin{array}{l}0 \\
0 \\
0 \\
0 \\
0 \\
0\end{array}$ & $\begin{array}{l}0 \\
0 \\
0 \\
0 \\
0 \\
0\end{array}$ & $\begin{array}{l}0 \\
0 \\
0 \\
0 \\
0 \\
0\end{array}$ & $\begin{array}{r}368 \\
0 \\
0 \\
0 \\
0\end{array}$ & $\begin{array}{r}1,873 \\
824 \\
1,040 \\
0 \\
0 \\
0\end{array}$ & $\begin{array}{l}0 \\
0 \\
0 \\
0 \\
0 \\
0\end{array}$ & $\begin{array}{l}0 \\
0 \\
0 \\
0 \\
0 \\
0\end{array}$ \\
\hline 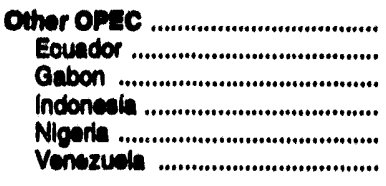 & $\begin{array}{r}37,011 \\
3,102 \\
36,164 \\
1,776 \\
02,220 \\
246,700\end{array}$ & $\begin{array}{r}171 \\
0 \\
0 \\
0 \\
0 \\
171\end{array}$ & $\begin{array}{r}12,171 \\
0 \\
0 \\
641 \\
613 \\
12,017\end{array}$ & $\begin{array}{r}138 \\
0 \\
0 \\
0 \\
0 \\
138\end{array}$ & $\begin{array}{l}0 \\
0 \\
0 \\
0 \\
0 \\
0\end{array}$ & $\begin{array}{l}0 \\
0 \\
0 \\
0 \\
0 \\
0\end{array}$ & $\begin{array}{l}0 \\
0 \\
0 \\
0 \\
0 \\
0\end{array}$ & $\begin{array}{r}3,043 \\
0 \\
0 \\
648 \\
448 \\
1,900\end{array}$ & $\begin{array}{l}0 \\
0 \\
0 \\
0 \\
0 \\
0\end{array}$ & $\begin{array}{r}10 \\
0 \\
0 \\
0 \\
0 \\
19\end{array}$ \\
\hline 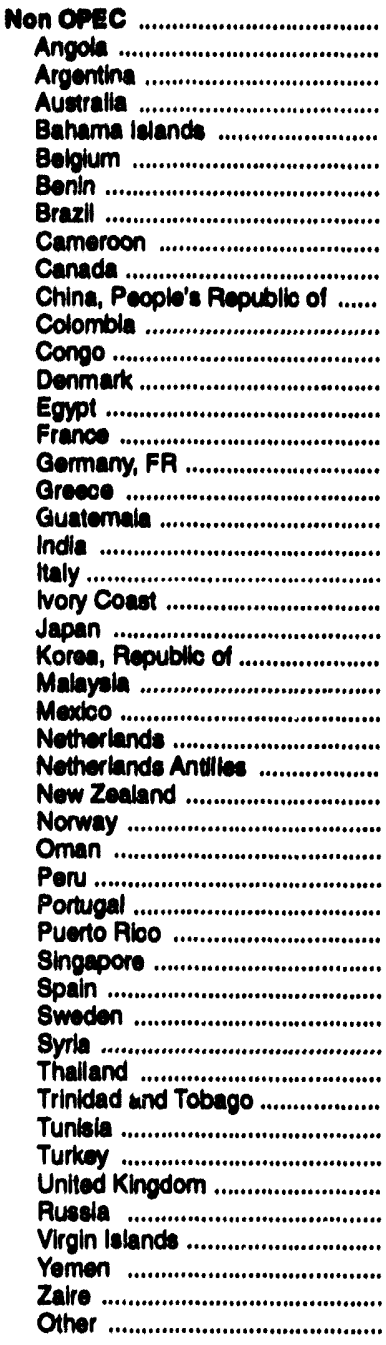 & $\begin{array}{r}41,091 \\
67,184 \\
4,227 \\
1,688 \\
0 \\
0 \\
888 \\
0 \\
2,714 \\
4,428 \\
6,647 \\
36,681 \\
16,210 \\
420 \\
16,468 \\
0 \\
0 \\
0 \\
2,092 \\
0 \\
0 \\
0 \\
0 \\
0 \\
1,288 \\
232,040 \\
0 \\
0 \\
280 \\
9,876 \\
3,682 \\
0 \\
12,264 \\
0 \\
1,862 \\
1,072 \\
2,468\end{array}$ & $\begin{array}{r}4,78 \\
0 \\
0 \\
182 \\
0 \\
0 \\
0 \\
0 \\
0 \\
0 \\
0 \\
0 \\
0 \\
0 \\
0 \\
121 \\
0 \\
0 \\
0 \\
0 \\
48 \\
0 \\
16 \\
0 \\
4 \\
4,188 \\
0 \\
51 \\
0 \\
0 \\
0 \\
0 \\
60 \\
0 \\
0 \\
0 \\
0 \\
0 \\
0 \\
0 \\
0 \\
0 \\
81 \\
0 \\
0 \\
0 \\
0 \\
0\end{array}$ & 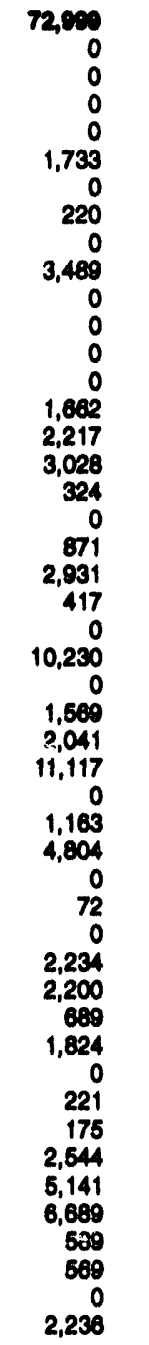 & $\begin{array}{r}1,732 \\
0 \\
3 \\
0 \\
0 \\
0 \\
0 \\
23 \\
0 \\
1 \\
0 \\
0 \\
0 \\
0 \\
0 \\
0 \\
0 \\
0 \\
0 \\
0 \\
0 \\
0 \\
0 \\
0 \\
0 \\
1,375 \\
0 \\
330 \\
0 \\
0 \\
0 \\
0 \\
0 \\
0 \\
0 \\
0 \\
0 \\
0 \\
0 \\
0 \\
0 \\
0 \\
0 \\
0 \\
0 \\
0 \\
0 \\
0\end{array}$ & $\begin{array}{r}1,333 \\
0 \\
0 \\
0 \\
0 \\
0 \\
0 \\
281 \\
0 \\
0 \\
0 \\
0 \\
0 \\
0 \\
0 \\
245 \\
0 \\
0 \\
0 \\
0 \\
0 \\
0 \\
0 \\
0 \\
0 \\
0 \\
0 \\
0 \\
0 \\
0 \\
0 \\
0 \\
0 \\
0 \\
0 \\
0 \\
0 \\
0 \\
0 \\
0 \\
0 \\
0 \\
0 \\
0 \\
837 \\
0 \\
0 \\
0\end{array}$ & $\begin{array}{r}2,48 \\
0 \\
0 \\
0 \\
0 \\
0 \\
0 \\
0 \\
0 \\
10 \\
0 \\
0 \\
0 \\
0 \\
0 \\
0 \\
0 \\
0 \\
0 \\
0 \\
0 \\
0 \\
0 \\
0 \\
0 \\
572 \\
0 \\
0 \\
0 \\
0 \\
0 \\
0 \\
0 \\
0 \\
0 \\
0 \\
0 \\
0 \\
0 \\
224 \\
0 \\
0 \\
0 \\
0 \\
0 \\
0 \\
0\end{array}$ & $\begin{array}{r}2,081 \\
0 \\
0 \\
0 \\
0 \\
0 \\
0 \\
0 \\
0 \\
0 \\
0 \\
0 \\
0 \\
0 \\
0 \\
0 \\
0 \\
0 \\
0 \\
0 \\
0 \\
0 \\
0 \\
0 \\
0 \\
0 \\
0 \\
0 \\
0 \\
0 \\
0 \\
0 \\
0 \\
0 \\
0 \\
0 \\
0 \\
0 \\
0 \\
0 \\
0 \\
0 \\
0 \\
0 \\
0 \\
0 \\
0\end{array}$ & $\begin{array}{r}13,293 \\
0 \\
132 \\
0 \\
333 \\
0 \\
0 \\
0 \\
303 \\
386 \\
0 \\
790 \\
0 \\
0 \\
367 \\
0 \\
746 \\
0 \\
0 \\
0 \\
322 \\
0 \\
0 \\
0 \\
0 \\
1,484 \\
0 \\
307 \\
0 \\
269 \\
0 \\
132 \\
1,589 \\
0 \\
381 \\
0 \\
0\end{array}$ & $\begin{array}{l}17 \\
0 \\
0 \\
0 \\
0 \\
0 \\
0 \\
0 \\
0 \\
0 \\
0 \\
0 \\
0 \\
0 \\
0 \\
0 \\
0 \\
0 \\
0 \\
0 \\
0 \\
0 \\
0 \\
0 \\
0 \\
47 \\
0 \\
0 \\
0 \\
0 \\
0 \\
0 \\
0 \\
0 \\
0 \\
0 \\
0 \\
0 \\
0 \\
0 \\
0 \\
0 \\
0 \\
0 \\
0 \\
0 \\
0 \\
0\end{array}$ & $\begin{array}{r}\pi \\
0 \\
0 \\
0 \\
0 \\
0 \\
0 \\
0 \\
0 \\
655 \\
62 \\
0 \\
0 \\
0 \\
0 \\
0 \\
0 \\
0 \\
0 \\
0 \\
24 \\
0 \\
0 \\
0 \\
0 \\
36 \\
0 \\
0 \\
0 \\
0 \\
0 \\
0 \\
0 \\
0 \\
0 \\
0 \\
0 \\
0 \\
0 \\
0 \\
0 \\
0 \\
0 \\
0 \\
0 \\
0 \\
0 \\
0\end{array}$ \\
\hline Total & $1,898,640$ & 20,400 & 128,604 & 1,037 & 1,383 & 2,445 & 2,300 & 18,108 & 47 & 79 \\
\hline Perclan Cuff ${ }^{d}$................................ & 498,698 & 8,040 & 31,040 & 0 & $\mathbf{0}$ & 0 & $\mathbf{0}$ & 1,049 & 0 & $\mathbf{0}$ \\
\hline
\end{tabular}

See footnotes at end of table. 
Table 24. PAD Dientet If-mports of Cruds Oll and Petroleum Producte by Country of Origin, "19es (Continued) (Thousand Barrola)

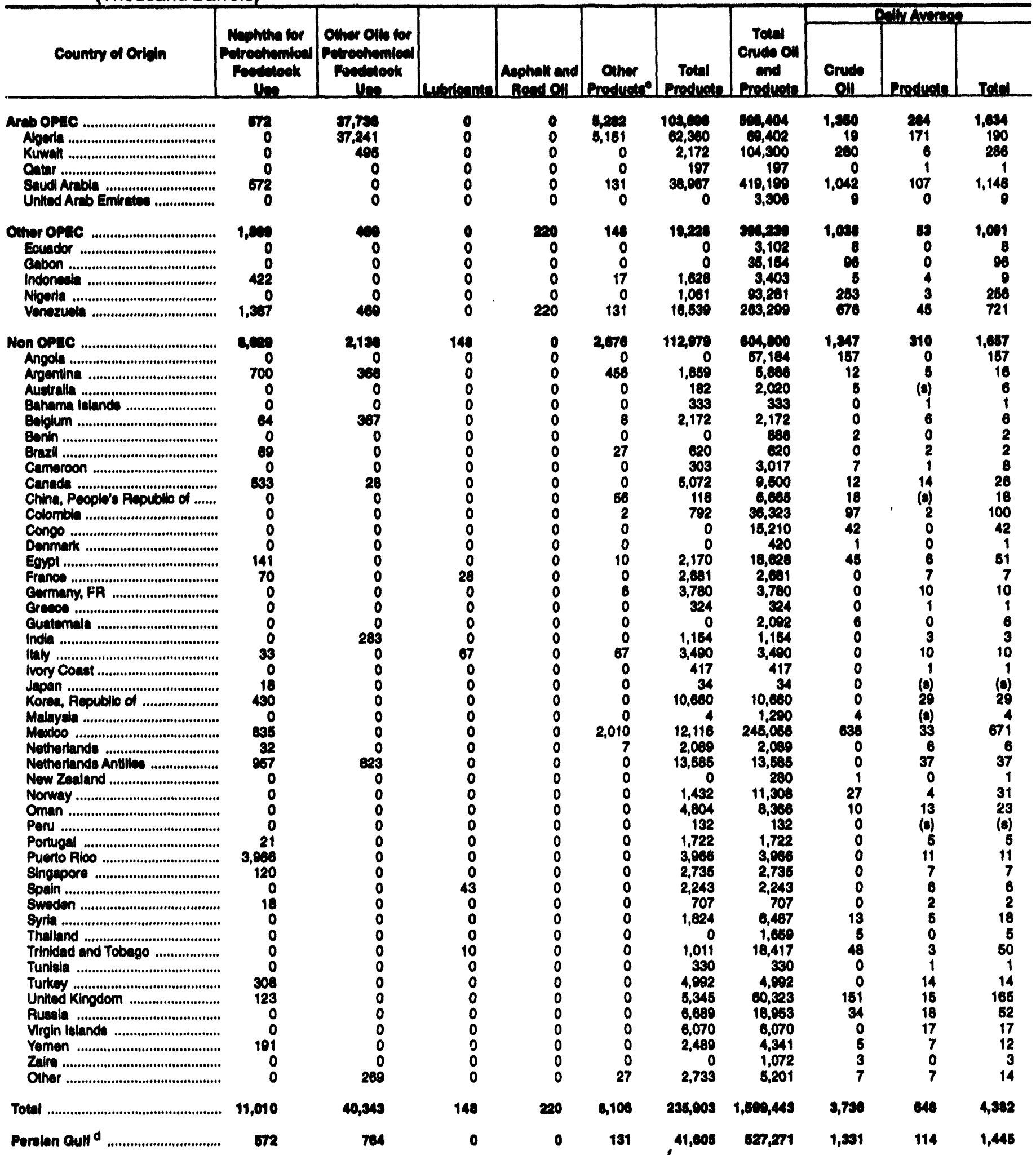

Crude oll and uniniluhed olls are reported by the PAD Dietrict in whioh they are to be processed; all other products are reported by the PAD Distrlot of entry

b Includes onde oll imported for storage in the strategio Potroleum Reserve.

- Includes aviation gasoline, eviation gacoline blending componente, miscellaneous products, other hydrocarbons and axygenatce, pentanes plus, petroleum coke, and waxes.

Includes Bahrain, Iran, Iraq, Kuwalt, Qatar, saudi Arabla, and Unlted Arab Emirates.

(s) = Lees than 800 barrole per day.

Note: Totals may not equal sum of componente dus to independent rounding.

Source: Energy Information Administration (EIA) Form ELA-814, "Monthly Imports Report. 
Table 25. PAD Distriots IV and V-Imports of Cruds Oll and Potroloum Producte by Country of Origin," 1993

(Thousand Barrels)

\begin{tabular}{|c|c|c|c|c|c|c|c|c|c|c|}
\hline Country of Origin & $\begin{array}{l}\text { Crude } \\
\text { Oal }\end{array}$ & $\begin{array}{l}\text { Wquetied } \\
\text { Petroloum } \\
\text { onen }\end{array}$ & $\begin{array}{c}\text { Uniliniahed } \\
\text { Oile }\end{array}$ & $\begin{array}{l}\text { Casoline } \\
\text { Elending } \\
\text { Compo- } \\
\text { nente }\end{array}$ & $\begin{array}{l}\text { Finlahod } \\
\text { Motor } \\
\text { Ceacolline }\end{array}$ & afred & $\begin{array}{l}\text { Diestinto } \\
\text { Prelloil }\end{array}$ & $\begin{array}{l}\text { Pealdual } \\
\text { Eund Oil }\end{array}$ & Konosen & $\begin{array}{l}\text { Epeolal } \\
\text { Nephth: }\end{array}$ \\
\hline & \multicolumn{10}{|c|}{ PAD Dietriot IV } \\
\hline 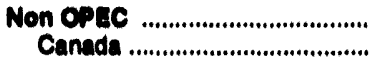 & $\begin{array}{l}32,941 \\
32,941\end{array}$ & $\begin{array}{l}2,462 \\
2,462\end{array}$ & $\begin{array}{l}0 \\
0\end{array}$ & $\stackrel{0}{0}$ & $\begin{array}{l}120 \\
120\end{array}$ & $\begin{array}{l}0 \\
0\end{array}$ & $\begin{array}{l}1,139 \\
1,136\end{array}$ & $\begin{array}{l}18 \\
18\end{array}$ & $\begin{array}{l}0 \\
0\end{array}$ & : \\
\hline Total & 32,041 & 2,400 & 0 & 0 & 120 & 0 & 1,133 & 10 & 0 & 0 \\
\hline
\end{tabular}

PAD Dietriet V

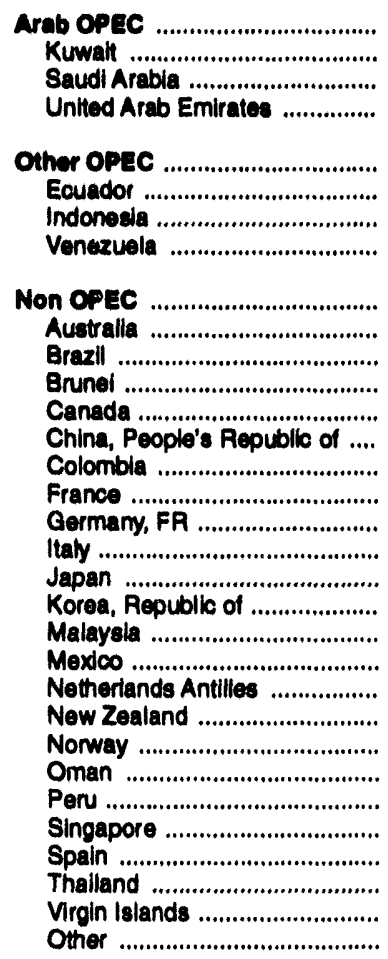

Total

Poraian Culf ${ }^{d}$

$\begin{array}{rrr}8,972 & 0 & 0 \\ 8,972 & 0 & 0 \\ 0 & 0 & 0 \\ 0 & 0 & 0 \\ 40,943 & 0 & 860 \\ 14,631 & 0 & 0 \\ 21,736 & 0 & 219 \\ 4,677 & 0 & 340 \\ 80,397 & 232 & 4,280 \\ 4,276 & 0 & 196 \\ 0 & 0 & 0 \\ 156 & 0 & 0 \\ 25,869 & 232 & 100 \\ 7,161 & 0 & 0 \\ 1,890 & 0 & 0 \\ 0 & 0 & 0 \\ 0 & 0 & 0 \\ 0 & 0 & 0 \\ 0 & 0 & 0 \\ 0 & 0 & 0 \\ 1,919 & 0 & 0 \\ 1,118 & 0 & 943 \\ 0 & 0 & 363 \\ 543 & 0 & 0 \\ 0 & 0 & 226 \\ 5,441 & 0 & 0 \\ 0 & 0 & 0 \\ 504 & 0 & 2,422 \\ 0 & 0 & 0 \\ 488 & 0 & 0 \\ 0 & 0 & 0 \\ 993 & 0 & 0 \\ 100,272 & 232 & 1,818 \\ 8,972 & 0 & 0 \\ & & \end{array}$

0
0
0
0

0
0
0
0

489
0
478
20

490
498
0
0

\section{0}

a

230
0
0

a77 200

0
477

200
0
1
268

1,006

1,606
0
1,445

200

2,040

2,042
0
262
0

2,600

2,600
1
0
0
73

1.171
182

0
73
0

$\begin{array}{rrr}0 & 182 & 0 \\ 0 & 0 & 0 \\ 0 & 31 & 0 \\ 0 & 0 & 0 \\ 0 & 61 & 0\end{array}$

73
0
0
0

$\begin{array}{rrr}0 & 01 & 0 \\ 0 & 0 & 0 \\ 131 & 0 & 0 \\ 0 & 0 & 0\end{array}$

$\begin{array}{rr}0 & 0 \\ 0 & 781 \\ 99 & 597 \\ 211 & 0 \\ 0 & 0 \\ 0 & 0 \\ 0 & 0 \\ 0 & 594 \\ 274 & 0 \\ 0 & 0 \\ 671 & 457 \\ 0 & 0 \\ & 0\end{array}$

$\begin{array}{rrr}430 & 3,410 & 3,270 \\ 0 & 0 & 490\end{array}$

$\begin{array}{rr}2,160 & 7,200 \\ 0 & 490\end{array}$

$\begin{array}{rr}2,197 & 8,000 \\ 0 & 0 \\ 0 & 0 \\ 0 & 0 \\ 1,080 & 362 \\ 0 & 0 \\ 0 & 0 \\ 0 & 0 \\ 0 & 0 \\ 0 & 0 \\ 0 & 0 \\ 0 & 954 \\ 0 & 0 \\ 0 & 3,369 \\ 218 & 0 \\ 0 & 0 \\ 0 & 0 \\ 0 & 0 \\ 0 & 331 \\ 0 & 0 \\ 0 & 0 \\ 0 & 0 \\ 839 & 0 \\ 0 & 0 \\ 2,160 & 7,200 \\ 0 & 490\end{array}$

$\begin{array}{cc}0 & 0 \\ 0 & 0 \\ 0 & 0 \\ 0 & 0 \\ 0 & 0 \\ 0 & 0 \\ 0 & 0 \\ 0 & 0 \\ 21 & 50 \\ 0 & 0 \\ 0 & 0 \\ 0 & 0 \\ 2 & 50 \\ 0 & 0 \\ 0 & 0 \\ 0 & 0 \\ 0 & 0 \\ 0 & 0 \\ 0 & 0 \\ 0 & 0 \\ 0 & 0 \\ 0 & 0 \\ 19 & 0 \\ 0 & 0 \\ 0 & 0 \\ 0 & 0 \\ 0 & 0 \\ 0 & 0 \\ 0 & 0 \\ 0 & 0 \\ 0 & 0 \\ 0 & 0 \\ 21 & 50 \\ 0 & 0\end{array}$

See footnotes at end of table. 
Table 25. PAD Districts IV and V-Imports of Cruds Oll and Petrobum Producte by Country of Origin," 1908 (Continued)

(Thousand Barrels)

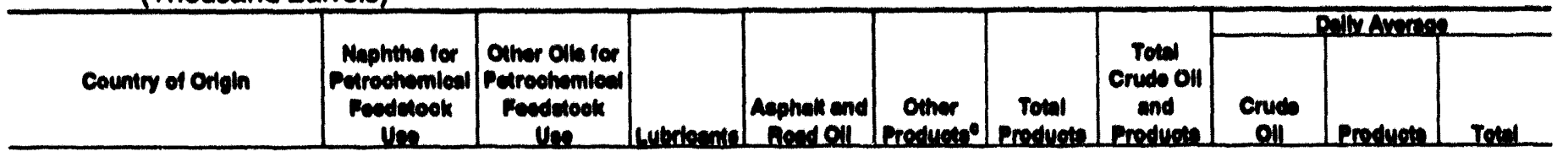

PAD Dietriot N

\begin{tabular}{|c|c|c|c|c|c|c|c|c|c|c|}
\hline 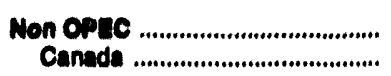 & $\begin{array}{l}0 \\
0\end{array}$ & $\begin{array}{l}0 \\
0\end{array}$ & 0 & $\begin{array}{l}0 \\
0\end{array}$ & $\begin{array}{l}2,700 \\
2,702\end{array}$ & $\begin{array}{r}6,400 \\
6,410\end{array}$ & 30,001 & $\infty$ & $\begin{array}{l}10 \\
18\end{array}$ & $\begin{array}{l}108 \\
108\end{array}$ \\
\hline clal ........... & 0 & 0 & 0 & 0 & 2,700 & 0,440 & $0,2 \times 1$ & $\omega$ & 10 & 10 \\
\hline
\end{tabular}

PAD Diatried $v$

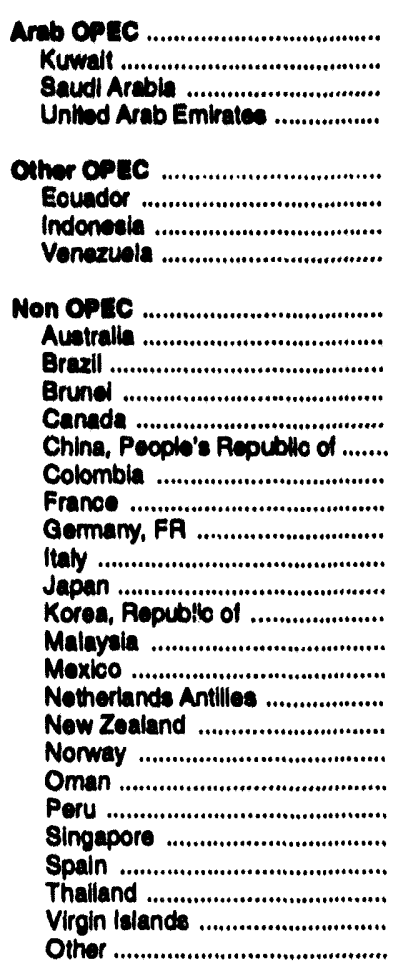

Total

Ponalan Qulf d

0
0
0
0
0
0
0
0

$\begin{array}{ll}0 & 0 \\ 0 & 0 \\ 0 & 0 \\ 0 & 0\end{array}$

0

0
0
0
0

$2 a 5$

0

0

0
0
0

0

0
258
0

0
0
0

0
0
0

0

0

0
0
0
0

285

o 
Tablo 26. Imports of Rosidual Fuel Oll by sulfur Content and by PAD Distrlot and 8tato of Entry, 1903 (Thousand Barrels)

\begin{tabular}{|c|c|c|c|c|}
\hline \multirow[b]{2}{*}{ PAD Dietriet and Eute of Eniry } & \multicolumn{4}{|c|}{ Roaldual Fuet Oll } \\
\hline & $\begin{array}{l}\text { Lexethan } \\
\text { o.sis } \\
\text { enlitu }\end{array}$ & $\begin{array}{l}0.110 \\
1.00 \% \\
\text { nilly }\end{array}$ & $\begin{array}{l}\text { Crrostor } \\
\text { than } 1.00 \% \\
\text { Orlity }\end{array}$ & ToA:I \\
\hline 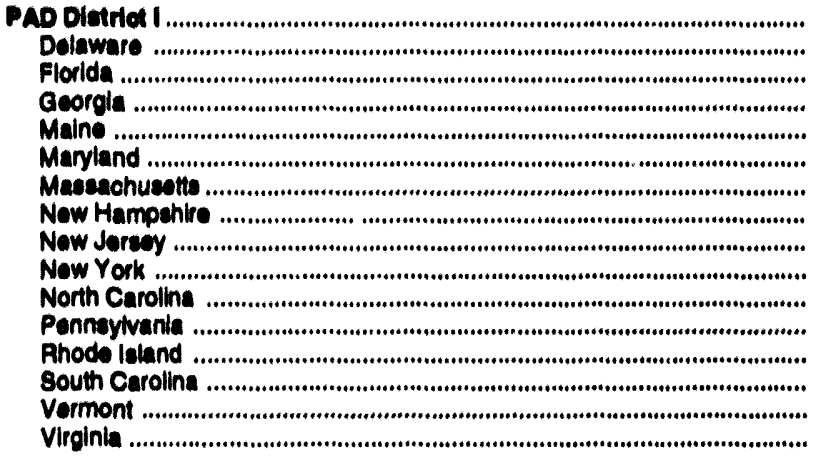 & $\begin{array}{r}16,671 \\
621 \\
0 \\
0 \\
608 \\
888 \\
1,028 \\
0 \\
2,118 \\
6,734 \\
0 \\
6,808 \\
0 \\
113 \\
0 \\
0\end{array}$ & $\begin{array}{r}20,090 \\
0 \\
2,062 \\
0 \\
683 \\
780 \\
3,216 \\
0 \\
2,178 \\
7,028 \\
10 \\
3,008 \\
0 \\
28 \\
0 \\
742\end{array}$ & $\begin{array}{r}72,720 \\
601 \\
14,140 \\
2,160 \\
4,937 \\
4,790 \\
7,179 \\
1,324 \\
12,040 \\
10,841 \\
5,080 \\
1,711 \\
372 \\
2,884 \\
21 \\
6,243\end{array}$ & $\begin{array}{r}110,461 \\
1,222 \\
16,702 \\
2,169 \\
6,326 \\
6,137 \\
11,422 \\
1,324 \\
18,338 \\
23,903 \\
8,106 \\
10,672 \\
372 \\
2,098 \\
21 \\
5,986\end{array}$ \\
\hline 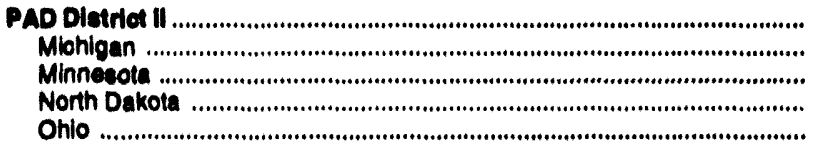 & $\begin{array}{l}0 \\
0 \\
0 \\
0 \\
0\end{array}$ & $\begin{array}{r}102 \\
68 \\
1 \\
113 \\
0\end{array}$ & $\begin{array}{r}78 \\
0 \\
4 \\
28 \\
43\end{array}$ & $\begin{array}{r}287 \\
68 \\
5 \\
141 \\
43\end{array}$ \\
\hline 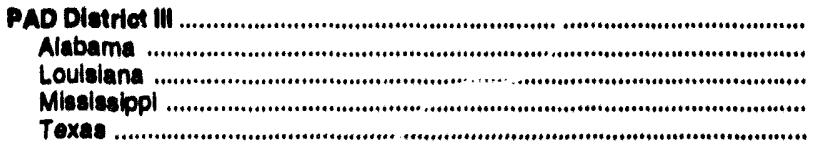 & $\begin{array}{r}2,120 \\
0 \\
1,089 \\
0 \\
1,089\end{array}$ & $\begin{array}{r}620 \\
0 \\
0 \\
0 \\
520\end{array}$ & $\begin{array}{r}18,047 \\
1,073 \\
3,162 \\
2,250 \\
0,053\end{array}$ & $\begin{array}{r}18,198 \\
1,073 \\
4,231 \\
2,259 \\
10,632\end{array}$ \\
\hline PAD Distriet N & 8 & 8 & 8 & $\begin{array}{l}18 \\
18\end{array}$ \\
\hline 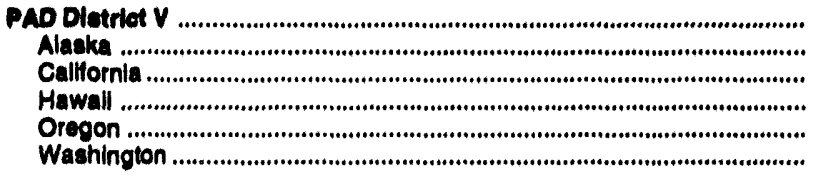 & $\begin{array}{r}1,412 \\
0 \\
849 \\
100 \\
463 \\
0\end{array}$ & $\begin{array}{r}420 \\
0 \\
380 \\
0 \\
0 \\
46\end{array}$ & $\begin{array}{r}5,802 \\
48 \\
4,845 \\
0 \\
0 \\
469\end{array}$ & $\begin{array}{r}7,200 \\
48 \\
6,074 \\
100 \\
463 \\
515\end{array}$ \\
\hline 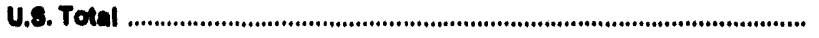 & 20,410 & 22,023 & 98,712 & 130,181 \\
\hline
\end{tabular}

Source: Energy intormation Administration (EIA) Form EIA-814, Monthiy Importa Rcport." 
Tablo 27. Exporte of Cruds Oll and Petroloum Products by PAD Dletrict, 1903 (Thousand Barrels)

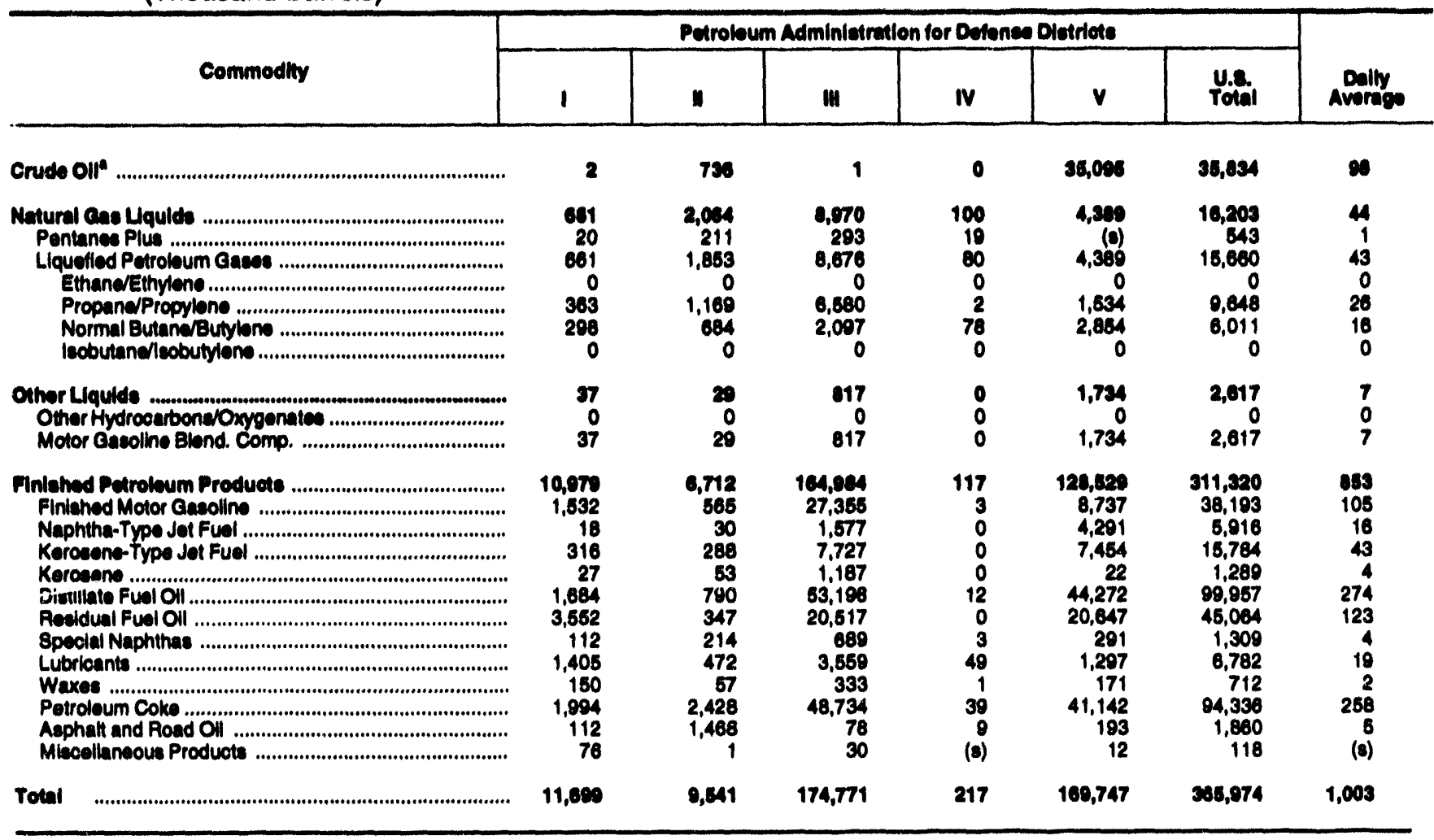

- Crude oll exports are reatricted to: (1) orude oll derived from flelds under the State waters of Alaka's Cook Inlet; (2) certuin domeatically produced crude oll deatined for Canada; and (3) shipments to U.8. terrtiories, and Calfornia orude oil to Peoifio Aim countries. On December 6, 1991, the U.S. Department of commercs approved a libense bo oxport 28,000 barrels per day of Calfornia heavy orude oll (lese than 20 degrees API gravity) to Pacifio Rim countries for one year. (s) w Leas than 500 barrels or lese than 500 barrele per day.

Nole: Totals may not cqual eum of components dus 10 independent rounding.

Sources: Energy Information Administration (ELA) Form EIA-810, "Monthly Refinery Report" and the U.S. Bureau of the Census. 
Table 28. Exports of Crude Oil and Petroleum Products by Destination, 1993 (Thousand Barrels)

\begin{tabular}{|c|c|c|c|c|c|c|c|c|}
\hline Destination & $\begin{array}{c}\text { Crude } \\
\text { elp }\end{array}$ & $\begin{array}{l}\text { Pemtanes } \\
\text { Plus }\end{array}$ & $\begin{array}{c}\text { Laquibd } \\
\text { Puroleum } \\
\text { coens }\end{array}$ & $\begin{array}{l}\text { Finishod } \\
\text { Motor } \\
\text { esselline }\end{array}$ & Let Ful & Keroesen & $\begin{array}{c}\text { Dietillato Fust } \\
\text { Oft }\end{array}$ & $\begin{array}{l}\text { Rosidual } \\
\text { Fuel oll }\end{array}$ \\
\hline 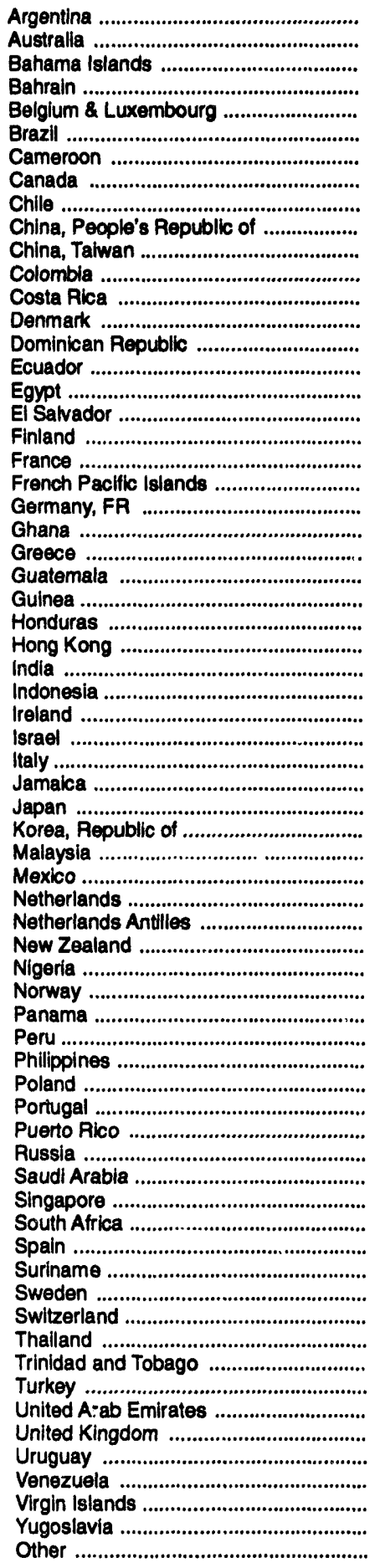 & $\begin{array}{r}0 \\
0 \\
0 \\
0 \\
0 \\
0 \\
0 \\
738 \\
0 \\
0 \\
0 \\
0 \\
0 \\
0 \\
0 \\
0 \\
0 \\
0 \\
0 \\
0 \\
0 \\
0 \\
0 \\
0 \\
0 \\
0 \\
0 \\
0 \\
0 \\
0 \\
0 \\
0 \\
0 \\
0 \\
0 \\
0 \\
0 \\
0 \\
0 \\
0 \\
0 \\
0 \\
0 \\
0 \\
0 \\
0 \\
0 \\
0 \\
0 \\
0 \\
0 \\
0 \\
0 \\
0 \\
0 \\
0 \\
0 \\
2 \\
0 \\
0 \\
0 \\
0 \\
0 \\
0 \\
0 \\
0\end{array}$ & 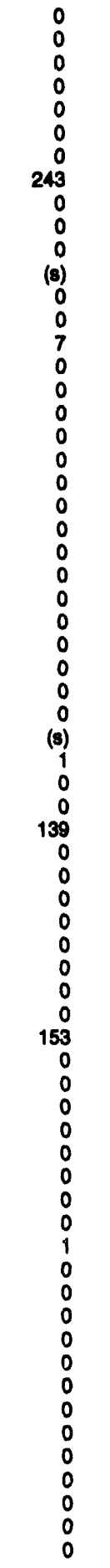 & 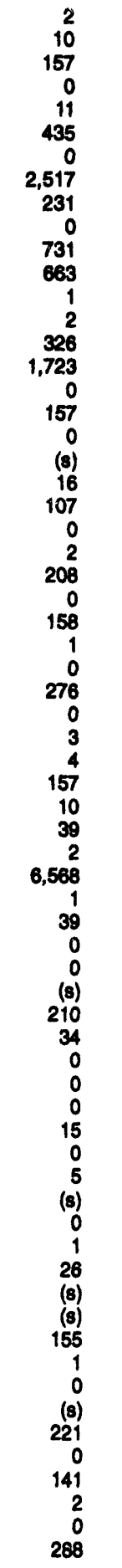 & $\begin{array}{r}769 \\
839 \\
915 \\
0 \\
2 \\
(8) \\
0 \\
2,986 \\
0 \\
700 \\
1,882 \\
0 \\
392 \\
0 \\
347 \\
0 \\
0 \\
174 \\
0 \\
15 \\
4 \\
1 \\
0 \\
0 \\
1,591 \\
0 \\
672 \\
0 \\
0 \\
0,190 \\
0 \\
0 \\
0 \\
0 \\
0 \\
0 \\
0 \\
0 \\
0 \\
0 \\
0 \\
0 \\
0 \\
14 \\
18 \\
121 \\
196 \\
272 \\
0 \\
0 \\
503\end{array}$ & $\begin{array}{r}48 \\
0 \\
113 \\
0 \\
0 \\
140 \\
0 \\
2,465 \\
6 \\
715 \\
0 \\
0 \\
31 \\
0 \\
6 \\
0 \\
0 \\
13 \\
0 \\
0 \\
19 \\
0 \\
0 \\
0 \\
67 \\
1 \\
128 \\
(8) \\
796 \\
0 \\
0 \\
1,407 \\
0 \\
30 \\
10,993 \\
2,493 \\
0 \\
55 \\
226 \\
0 \\
1 \\
0 \\
0 \\
0 \\
0 \\
0 \\
0 \\
0 \\
0 \\
0 \\
1 \\
0 \\
0 \\
30 \\
0 \\
11 \\
50 \\
0 \\
0 \\
0 \\
0\end{array}$ & 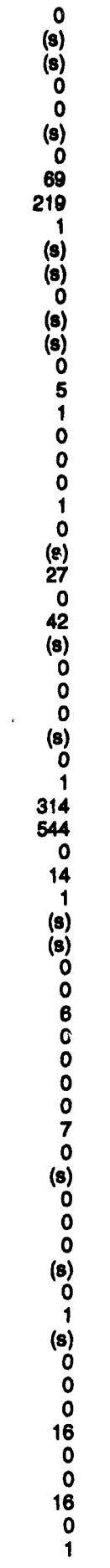 & 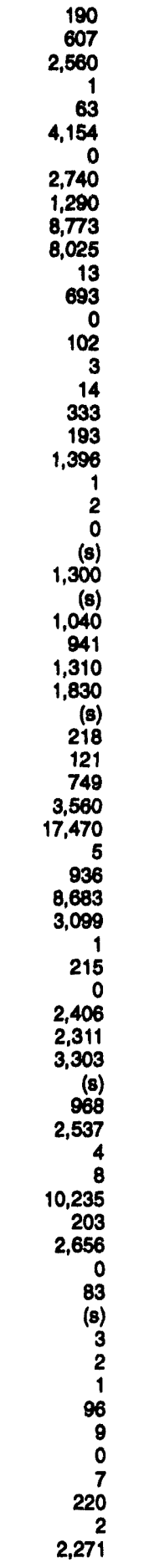 & $\begin{array}{r}0 \\
139 \\
2,067 \\
0 \\
0 \\
38 \\
0 \\
5,505 \\
0 \\
240 \\
2,406 \\
0 \\
102 \\
0 \\
255 \\
0 \\
0 \\
31 \\
(8) \\
0 \\
346 \\
0 \\
0 \\
0 \\
854 \\
0 \\
0 \\
303 \\
3,150 \\
0 \\
26 \\
57 \\
0 \\
0 \\
0 \\
0 \\
0 \\
294 \\
(8) \\
2,538 \\
6,272 \\
2,018 \\
3,745 \\
0 \\
8,432 \\
549 \\
428 \\
0 \\
1,50 \\
0 \\
0 \\
1,552 \\
298 \\
209 \\
0 \\
0 \\
0 \\
785\end{array}$ \\
\hline Total & 35,834 & 548 & 15,600 & 38,193 & 21,700 & 1,280 & 90,957 & 45,004 \\
\hline
\end{tabular}

See footnotes at end of table. 
Table 28. Exports of Crude Oll and Petroleum Products by Destination, 1993 (Continued) (Thousand Barrels)

\begin{tabular}{|c|c|c|c|c|c|c|c|c|}
\hline \multirow{2}{*}{ Deatination } & \multirow[b]{2}{*}{$\begin{array}{l}\text { Spocial } \\
\text { Naphthes }\end{array}$} & \multirow[b]{2}{*}{ Luprienta } & \multirow[b]{2}{*}{ Meres } & \multirow[b]{2}{*}{$\begin{array}{l}\text { Petroloum } \\
\text { Cole }\end{array}$} & \multirow{2}{*}{$\begin{array}{c}\text { Asphalt } \\
\text { and Road } \\
\text { Oll }\end{array}$} & \multirow[b]{2}{*}{$\begin{array}{c}\text { Other } \\
\text { Producteb }\end{array}$} & \multicolumn{2}{|c|}{ Crude Oll and Products } \\
\hline & & & & & & & Tothl & $\begin{array}{c}\text { Dally } \\
\text { Averese }\end{array}$ \\
\hline 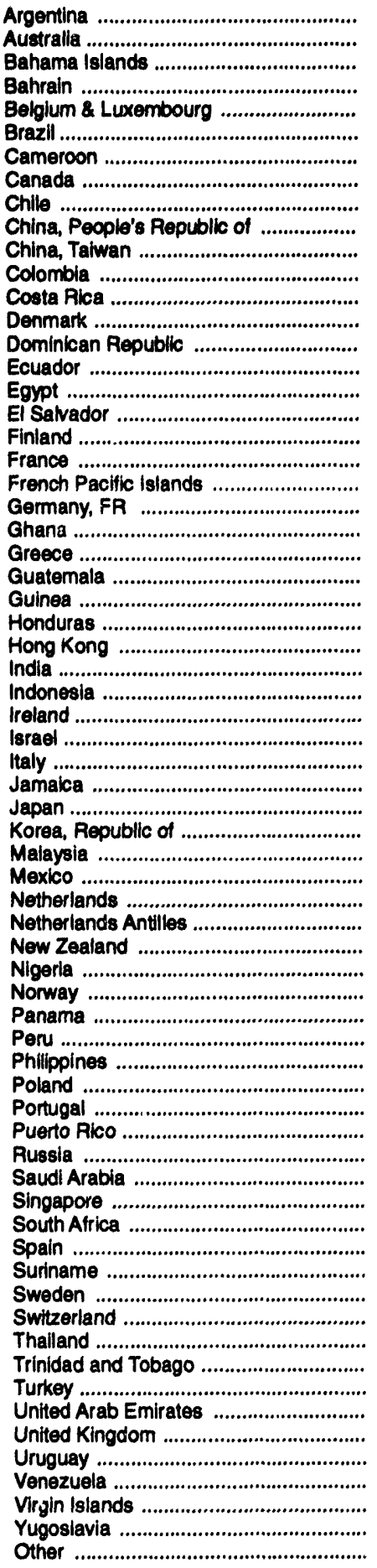 & 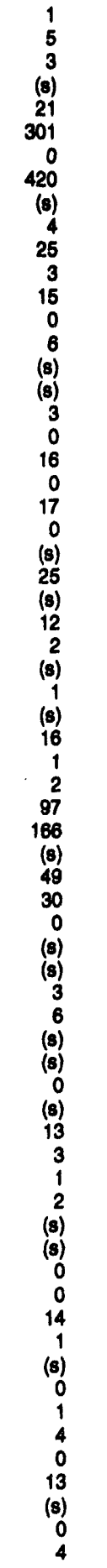 & 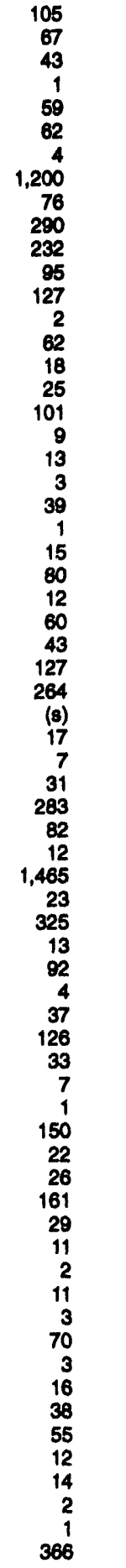 & 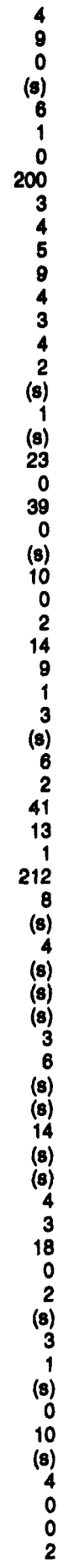 & $\begin{array}{r}3 \\
3,846 \\
0 \\
685 \\
7,396 \\
687 \\
143 \\
5,179 \\
6 \\
0 \\
5 \\
272 \\
0 \\
1,825 \\
100 \\
0 \\
2 \\
0 \\
526 \\
1,608 \\
0 \\
1,353 \\
0 \\
1,657 \\
0 \\
0 \\
0 \\
0 \\
0,299 \\
0 \\
4333 \\
58 \\
14,239 \\
347 \\
0 \\
0 \\
1,530 \\
0 \\
324 \\
165 \\
1,217 \\
307 \\
538 \\
9,597 \\
127 \\
20,924 \\
1,652 \\
(8) \\
890 \\
6,877 \\
0 \\
547 \\
0 \\
36 \\
30 \\
723 \\
140 \\
30 \\
0 \\
177 \\
0\end{array}$ & 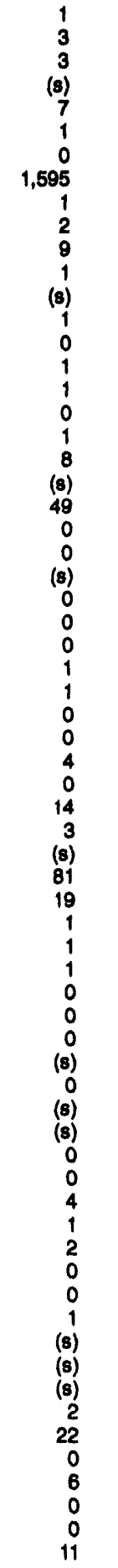 & 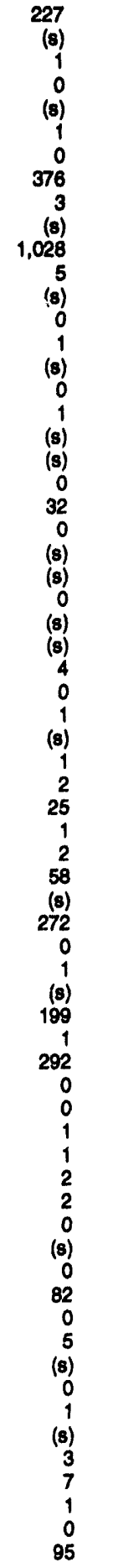 & 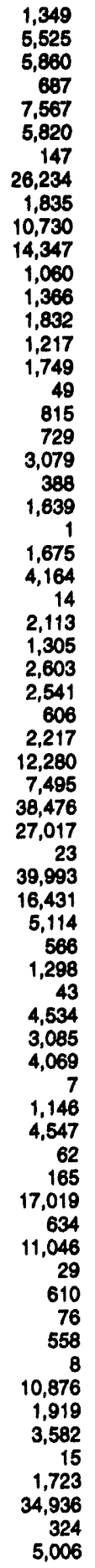 & 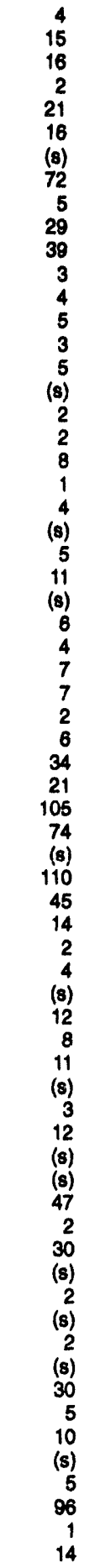 \\
\hline Total & 1,300 & 6,782 & 712 & 94,333 & 1,880 & 2,739 & 335,974 & 1,003 \\
\hline
\end{tabular}

a Crude oll exports are restricted to: (1) crude ofl derived from fields under the State waters of Alaska's Cook Inlet; (2) certain domestically produced crude oll destined for Canada; and (3) shipments to U.S. territories, and Califomia crude oil to Pacific Rim countries. On December 6, 1991, the U.S. Department of Commerce approved a license to export 25,000 barrels per day of California heavy crude oil (less than 20 degrees API gravity) to Pacific Rim countries for one year.

$\mathrm{b}$ includes miscellaneous products, motor gasoline blending components, and other hydrocarbons and oxygenates.

(s) = Less than 500 barrels or less than 500 barrels per day.

Note: Totais may not equal sum of components due to independent rounding.

Sources: Energy Information Administration (EIA) Form EIA-B10, "Monthly Refinery Report" and the U.S. Bureau of the Census. 
Table 29. Net Imports of Crude Oll and Petroleum Products into the United States by Country, 1993 (Thousand Barrels per Day)

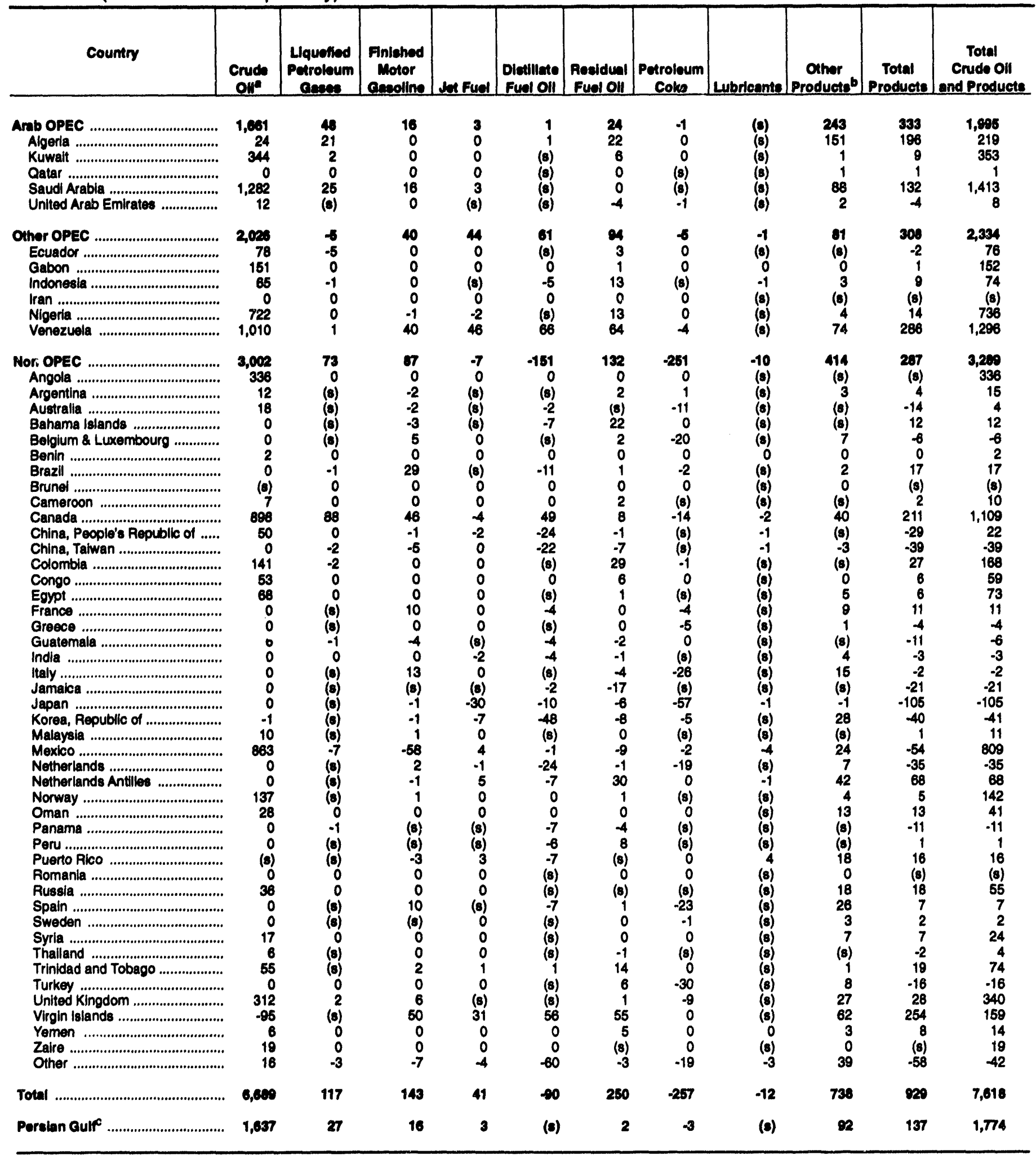

Includes crude oll imported for storage in the Strategic Petroleum Reserve.

b Includes asphalt and road oll, aviation gasoline, aviation gasoline blending components, kerosene, miscellaneous producte, motor gasoline blending components, naphtha for petrochernical feedstock use, other hydrocarbons and oxygenates, other olls for petrochemical feedstock use, pentanes plus, special naphthas, unfinished olls, and waxes.

C Includes Bahrain, Iran, Iraq, Kuyalt, Catar, Saudl Arabia, and United Arab Emirates.

(8) = Less than 500 barrels per day.

Note: Totals may not equal sum of components due to independent rounding.

Sources: Energy Iniormation Administration (ElA) Forms ElA-810, "Monthly Refinery Report," ElA-814, "Monthly Imports Report" and the U.S. Bureau of the Census. 
Table 30. Stocks of Crude Oll and Petroleum Products by PAD District, 1993 (Thousand Barrels)

\begin{tabular}{|c|c|c|c|c|c|c|}
\hline \multirow{2}{*}{ Commodity } & \multicolumn{5}{|c|}{ Potroleum Administration for Dofienes Dietriets } & \multirow[b]{2}{*}{ Y. 8.} \\
\hline & $\mathbf{1}$ & " & IIII & N & $v$ & \\
\hline 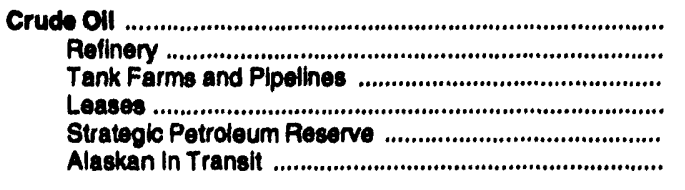 & $\begin{array}{r}14,320 \\
13,354 \\
959 \\
7 \\
0 \\
0\end{array}$ & $\begin{array}{r}76,006 \\
14,196 \\
61,439 \\
1,171 \\
0 \\
0\end{array}$ & $\begin{array}{r}762,228 \\
43,574 \\
108,371 \\
15,203 \\
587,080 \\
0\end{array}$ & $\begin{array}{r}11,133 \\
1,861 \\
8,330 \\
646 \\
0 \\
0\end{array}$ & $\begin{array}{r}67,976 \\
20,178 \\
32,376 \\
1,052 \\
0 \\
14,371\end{array}$ & $\begin{array}{r}922,465 \\
93,261 \\
209,475 \\
18,278 \\
587,080 \\
14,371\end{array}$ \\
\hline 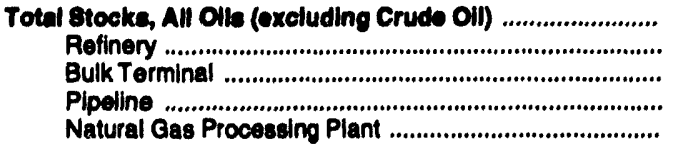 & $\begin{array}{r}179,216 \\
56,858 \\
93,073 \\
29,227 \\
68\end{array}$ & $\begin{array}{r}168,616 \\
58,984 \\
67,380 \\
36,878 \\
3,273\end{array}$ & $\begin{array}{r}283,001 \\
130,513 \\
88,923 \\
40,346 \\
3,220\end{array}$ & $\begin{array}{r}16,841 \\
11,690 \\
2,610 \\
2,298 \\
243\end{array}$ & $\begin{array}{r}90,161 \\
65,577 \\
28,316 \\
7,154 \\
135\end{array}$ & $\begin{array}{r}\mathbf{7 2 4 , 7 5 4} \\
323,622 \\
278,301 \\
115,902 \\
6,829\end{array}$ \\
\hline 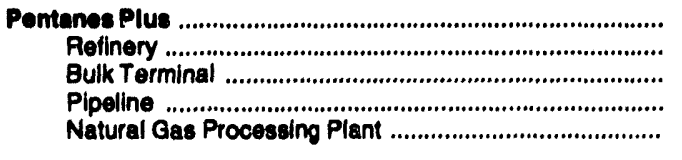 & $\begin{array}{r}288 \\
270 \\
11 \\
0 \\
7\end{array}$ & $\begin{array}{r}3,970 \\
708 \\
2,151 \\
668 \\
443\end{array}$ & $\begin{array}{r}6,074 \\
350 \\
3,936 \\
1,135 \\
653\end{array}$ & $\begin{array}{r}183 \\
6 \\
4 \\
75 \\
96\end{array}$ & $\begin{array}{r}86 \\
8 \\
47 \\
0 \\
11\end{array}$ & $\begin{array}{r}10,681 \\
1,342 \\
6,149 \\
1,878 \\
1,212\end{array}$ \\
\hline 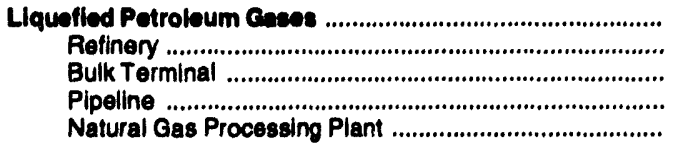 & $\begin{array}{r}5,204 \\
1,637 \\
1,775 \\
1,741 \\
61\end{array}$ & $\begin{array}{r}32,337 \\
3,775 \\
18,060 \\
7,682 \\
2,830\end{array}$ & $\begin{array}{r}64,218 \\
8,426 \\
45,988 \\
7,237 \\
2,567\end{array}$ & $\begin{array}{r}1,176 \\
435 \\
140 \\
456 \\
145\end{array}$ & $\begin{array}{r}3,819 \\
1,252 \\
2,173 \\
0 \\
124\end{array}$ & $\begin{array}{r}106,484 \\
15,525 \\
68,126 \\
17,116 \\
6,717\end{array}$ \\
\hline 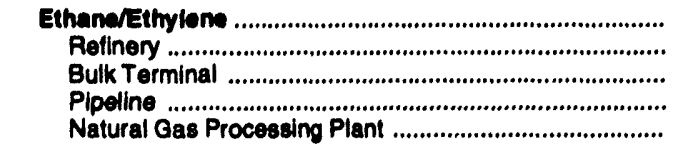 & $\begin{array}{l}0 \\
0 \\
0 \\
0 \\
0\end{array}$ & $\begin{array}{r}4,304 \\
3 \\
2,010 \\
1,621 \\
750\end{array}$ & $\begin{array}{r}15,670 \\
946 \\
11,477 \\
2,736 \\
511\end{array}$ & $\begin{array}{r}170 \\
0 \\
0 \\
174 \\
2\end{array}$ & $\begin{array}{l}0 \\
0 \\
0 \\
0 \\
0\end{array}$ & $\begin{array}{r}20,230 \\
949 \\
13,487 \\
4,531 \\
1,263\end{array}$ \\
\hline 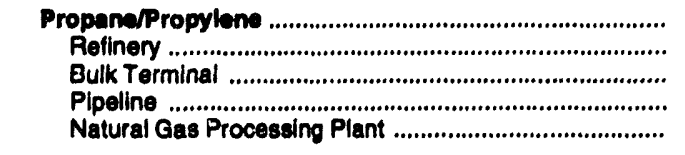 & $\begin{array}{r}3,650 \\
542 \\
1,503 \\
1,562 \\
43\end{array}$ & $\begin{array}{r}19,045 \\
1,863 \\
11,525 \\
4,355 \\
1,302\end{array}$ & $\begin{array}{r}28,930 \\
2,314 \\
20,352 \\
3,133 \\
1,151\end{array}$ & $\begin{array}{r}480 \\
83 \\
136 \\
166 \\
85\end{array}$ & $\begin{array}{r}1,000 \\
130 \\
889 \\
0 \\
61\end{array}$ & $\begin{array}{r}51,205 \\
4,942 \\
34,405 \\
9,216 \\
2,642\end{array}$ \\
\hline 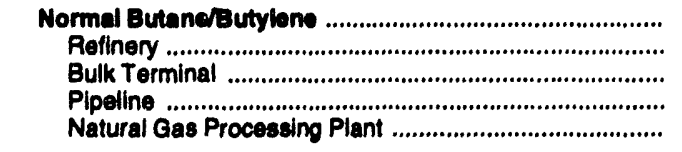 & $\begin{array}{r}1,444 \\
988 \\
272 \\
179 \\
5\end{array}$ & $\begin{array}{r}6,432 \\
1,387 \\
3,254 \\
1,215 \\
576\end{array}$ & $\begin{array}{r}14,000 \\
3,407 \\
9,920 \\
891 \\
590\end{array}$ & $\begin{array}{r}311 \\
216 \\
4 \\
53 \\
38\end{array}$ & $\begin{array}{r}1,817 \\
733 \\
1,077 \\
0 \\
7\end{array}$ & $\begin{array}{r}24,812 \\
6,731 \\
14,527 \\
2,338 \\
1,216\end{array}$ \\
\hline 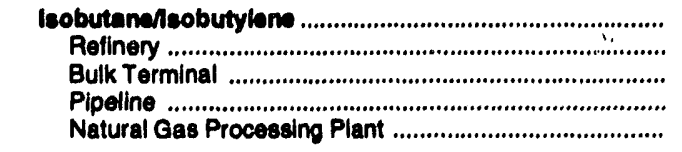 & $\begin{array}{r}110 \\
107 \\
0 \\
0 \\
3\end{array}$ & $\begin{array}{r}2,476 \\
522 \\
1,261 \\
491 \\
202\end{array}$ & $\begin{array}{r}6,700 \\
1,759 \\
4,239 \\
477 \\
315\end{array}$ & $\begin{array}{r}200 \\
126 \\
0 \\
63 \\
20\end{array}$ & $\begin{array}{r}882 \\
389 \\
207 \\
0 \\
56\end{array}$ & $\begin{array}{r}10,237 \\
2,903 \\
5,707 \\
1,031 \\
596\end{array}$ \\
\hline 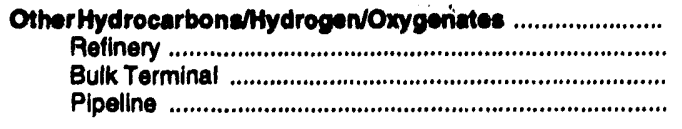 & $\begin{array}{r}1,680 \\
1,152 \\
408 \\
0\end{array}$ & $\begin{array}{r}1,025 \\
324 \\
701 \\
0\end{array}$ & $\begin{array}{r}6,013 \\
2,079 \\
3,557 \\
377\end{array}$ & $\begin{array}{r}248 \\
164 \\
61 \\
0\end{array}$ & $\begin{array}{r}3,830 \\
2,296 \\
738 \\
796\end{array}$ & $\begin{array}{r}12,673 \\
6,035 \\
5,465 \\
1,173\end{array}$ \\
\hline 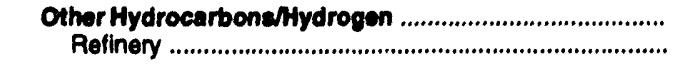 & $\begin{array}{l}5 \\
5\end{array}$ & $\begin{array}{l}27 \\
27\end{array}$ & 1 & $\begin{array}{l}0 \\
0\end{array}$ & $\begin{array}{l}7 \\
7\end{array}$ & $\begin{array}{l}40 \\
40\end{array}$ \\
\hline 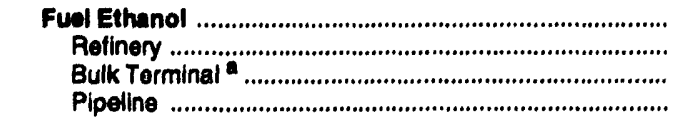 & $\begin{array}{l}162 \\
W \\
W \\
W\end{array}$ & $\begin{array}{r}779 \\
118 \\
W \\
W\end{array}$ & $\underset{w}{w} \underset{w}{w}$ & $\begin{array}{l}97 \\
w \\
w \\
w\end{array}$ & $\underset{w}{w} \underset{w}{w}$ & $\begin{array}{r}2,114 \\
337 \\
W \\
W\end{array}$ \\
\hline 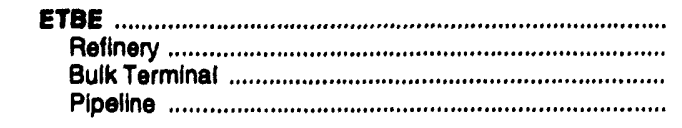 & $\begin{array}{l}\underset{w}{w} \\
\underset{w}{w} \\
w\end{array}$ & $\begin{array}{l}w \\
w \\
w \\
w \\
w\end{array}$ & $\begin{array}{l}\mathbf{w} \\
\mathbf{w} \\
\mathbf{w} \\
\mathbf{w}\end{array}$ & $\begin{array}{l}\underset{w}{w} \\
\underset{w}{w} \\
\mathbf{w}\end{array}$ & $\begin{array}{l}\mathbf{w} \\
\mathbf{w} \\
\mathbf{w} \\
\mathbf{w}\end{array}$ & $\begin{array}{l}\mathbf{w} \\
\mathbf{w} \\
\mathbf{w} \\
\mathbf{w}\end{array}$ \\
\hline 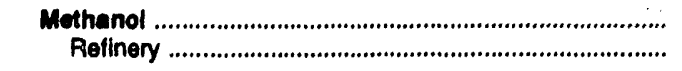 & $\underset{w}{w}$ & $\begin{array}{l}w \\
w\end{array}$ & $\begin{array}{l}\mathbf{w} \\
\mathbf{w}\end{array}$ & $\underset{W}{W}$ & $\begin{array}{l}w \\
w\end{array}$ & $\begin{array}{l}316 \\
316\end{array}$ \\
\hline
\end{tabular}

See footnotes at end of table. 
Table 30. Stocks of Crude OII and Petroleum Products by PAD District, 1993 (Continued) (Thousand Barrels)

\begin{tabular}{|c|c|c|c|c|c|c|}
\hline \multirow{2}{*}{ Commodity } & \multicolumn{5}{|c|}{ Potroleum Adminiatration for Dofenes Dietrlets } & \multirow{2}{*}{ U. 8 . } \\
\hline & $\mathbf{I}$ & $\boldsymbol{n}$ & III & $\mathbf{N}$ & $\mathbf{v}$ & \\
\hline 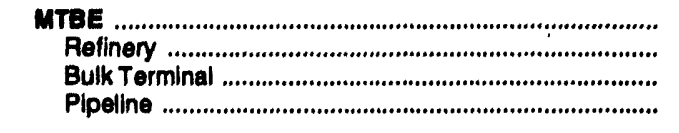 & $\begin{array}{r}1,348 \\
1,053 \\
W \\
W\end{array}$ & $\begin{array}{l}\mathbf{w} \\
\mathbf{w} \\
\mathbf{w}\end{array}$ & $\begin{array}{r}5,022 \\
1,740 \\
2,806 \\
377\end{array}$ & $\begin{array}{l}w \\
w \\
w \\
w\end{array}$ & $\begin{array}{r}3,301 \\
2,239 \\
275 \\
787\end{array}$ & $\begin{array}{r}10,085 \\
5,314 \\
3,557 \\
1,164\end{array}$ \\
\hline 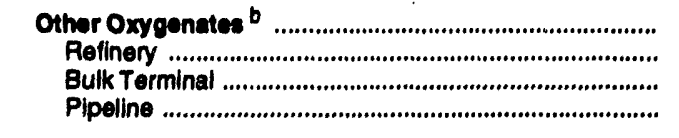 & $\begin{array}{l}\underset{w}{W} \\
w \\
w\end{array}$ & $\begin{array}{l}\underset{w}{w} \\
\underset{w}{W}\end{array}$ & $\begin{array}{l}w \\
w \\
w \\
w\end{array}$ & $\begin{array}{l}w \\
w \\
w \\
w\end{array}$ & $\begin{array}{l}\mathbf{w} \\
\mathbf{w} \\
\mathbf{w}\end{array}$ & $\begin{array}{l}w \\
w \\
w \\
w\end{array}$ \\
\hline 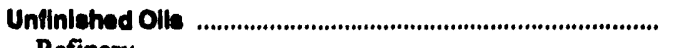 & 8,937 & 14,124 & 41,440 & 2,727 & 21,108 & $\mathbf{8 6 , 4 2 0}$ \\
\hline 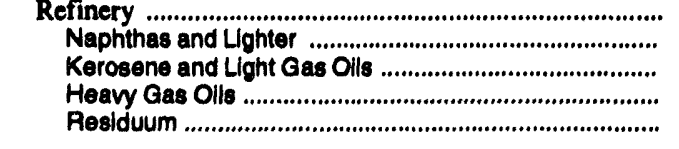 & $\begin{array}{r}2,048 \\
1,949 \\
4,040 \\
900\end{array}$ & $\begin{array}{l}3,553 \\
1,922 \\
5,199 \\
3,450\end{array}$ & $\begin{array}{r}10,530 \\
6,551 \\
16,308 \\
8,060\end{array}$ & $\begin{array}{r}447 \\
413 \\
1,614 \\
353\end{array}$ & $\begin{array}{r}3,732 \\
3,618 \\
10,649 \\
3,184\end{array}$ & $\begin{array}{l}20,310 \\
14,453 \\
37,710 \\
15,947\end{array}$ \\
\hline 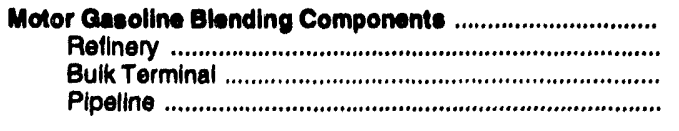 & $\begin{array}{r}6,676 \\
6,357 \\
218 \\
0\end{array}$ & $\begin{array}{r}7,202 \\
6,555 \\
338 \\
369\end{array}$ & $\begin{array}{r}15,703 \\
14,468 \\
933 \\
302\end{array}$ & $\begin{array}{r}2,082 \\
2,082 \\
0 \\
0\end{array}$ & $\begin{array}{r}8,784 \\
7,728 \\
23 \\
1,033\end{array}$ & $\begin{array}{r}39,406 \\
36,190 \\
1,512 \\
1,704\end{array}$ \\
\hline 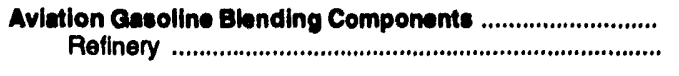 & 42 & $\begin{array}{l}0 \\
0\end{array}$ & $\begin{array}{l}27 \\
27\end{array}$ & $\begin{array}{l}0 \\
0\end{array}$ & 9 & $\begin{array}{l}78 \\
78\end{array}$ \\
\hline 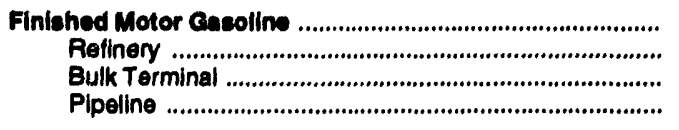 & $\begin{array}{l}69,370 \\
15,093 \\
30,189 \\
14,086\end{array}$ & $\begin{array}{l}48,170 \\
10,935 \\
22,415 \\
14,820\end{array}$ & $\begin{array}{l}49,481 \\
20,445 \\
10,558 \\
18,480\end{array}$ & $\begin{array}{l}4,532 \\
1,999 \\
1,501 \\
1,032\end{array}$ & $\begin{array}{r}25,480 \\
11,353 \\
11,649 \\
2,488\end{array}$ & $\begin{array}{r}187,051 \\
59,825 \\
76,320 \\
50,906\end{array}$ \\
\hline 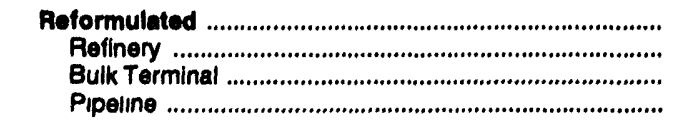 & $\begin{array}{l}0 \\
0 \\
0 \\
0\end{array}$ & $\begin{array}{l}0 \\
0 \\
0 \\
0\end{array}$ & $\begin{array}{l}0 \\
0 \\
0 \\
0\end{array}$ & $\begin{array}{l}0 \\
0 \\
0 \\
0\end{array}$ & $\begin{array}{l}0 \\
0 \\
0 \\
0\end{array}$ & $\begin{array}{l}0 \\
0 \\
0 \\
0\end{array}$ \\
\hline 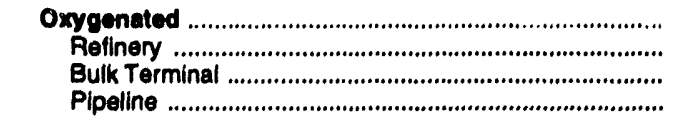 & $\begin{array}{r}16,014 \\
3,283 \\
6,835 \\
4,896\end{array}$ & $\begin{array}{r}1,113 \\
697 \\
289 \\
127\end{array}$ & $\begin{array}{r}6,642 \\
2,649 \\
683 \\
3,430\end{array}$ & $\begin{array}{r}855 \\
215 \\
341 \\
98\end{array}$ & $\begin{array}{r}10,124 \\
5,397 \\
4,102 \\
625\end{array}$ & $\begin{array}{r}33,648 \\
12,141 \\
12,230 \\
9,177\end{array}$ \\
\hline 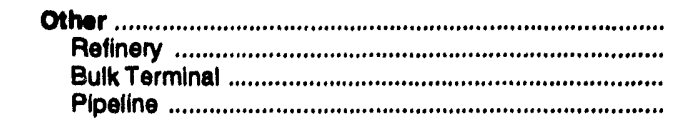 & $\begin{array}{r}\mathbf{4 4 , 3 8 4} \\
11,810 \\
23,364 \\
9,190\end{array}$ & $\begin{array}{l}47,057 \\
10,238 \\
22,126 \\
14,693\end{array}$ & $\begin{array}{r}42,890 \\
17,896 \\
9,893 \\
15,050\end{array}$ & $\begin{array}{r}3,877 \\
1,784 \\
1,160 \\
833\end{array}$ & $\begin{array}{r}15,368 \\
5,956 \\
7,547 \\
1,863\end{array}$ & $\begin{array}{r}153,503 \\
47,684 \\
64,090 \\
41,729\end{array}$ \\
\hline 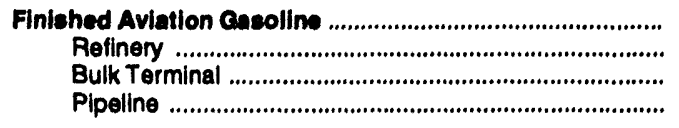 & $\begin{array}{r}613 \\
64 \\
547 \\
2\end{array}$ & $\begin{array}{r}433 \\
122 \\
263 \\
48\end{array}$ & $\begin{array}{r}323 \\
269 \\
46 \\
8\end{array}$ & $\begin{array}{r}47 \\
40 \\
7 \\
0\end{array}$ & $\begin{array}{r}395 \\
105 \\
290 \\
0\end{array}$ & $\begin{array}{r}1,811 \\
600 \\
1,163 \\
58\end{array}$ \\
\hline 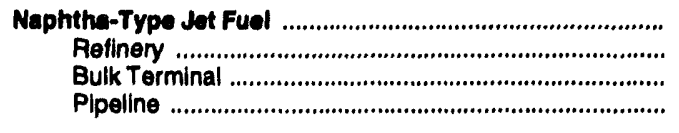 & $\begin{array}{r}271 \\
119 \\
60 \\
92\end{array}$ & $\begin{array}{l}773 \\
362 \\
203 \\
208\end{array}$ & $\begin{array}{r}756 \\
595 \\
0 \\
160\end{array}$ & $\begin{array}{r}281 \\
167 \\
0 \\
84\end{array}$ & $\begin{array}{r}298 \\
52 \\
1 \\
245\end{array}$ & $\begin{array}{r}2,348 \\
1,295 \\
264 \\
789\end{array}$ \\
\hline 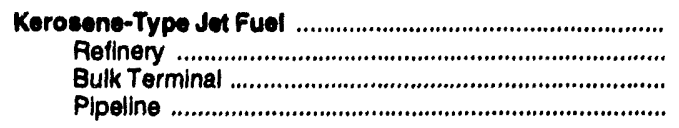 & $\begin{array}{l}8,651 \\
1,258 \\
3,256 \\
4,138\end{array}$ & $\begin{array}{l}7,190 \\
2,127 \\
1,999 \\
3,073\end{array}$ & $\begin{array}{r}13,083 \\
5,490 \\
2,882 \\
4,711\end{array}$ & $\begin{array}{l}462 \\
207 \\
125 \\
130\end{array}$ & $\begin{array}{l}8,649 \\
4,177 \\
3,411 \\
1,081\end{array}$ & $\begin{array}{l}38,044 \\
13,259 \\
11,672 \\
13,113\end{array}$ \\
\hline
\end{tabular}

See footnotes at end of table. 
Table 30. Stocks of Crude Oll and Petroleum Producte by PAD Dietrict, 1993 (Continued) (Thousand Barrels)

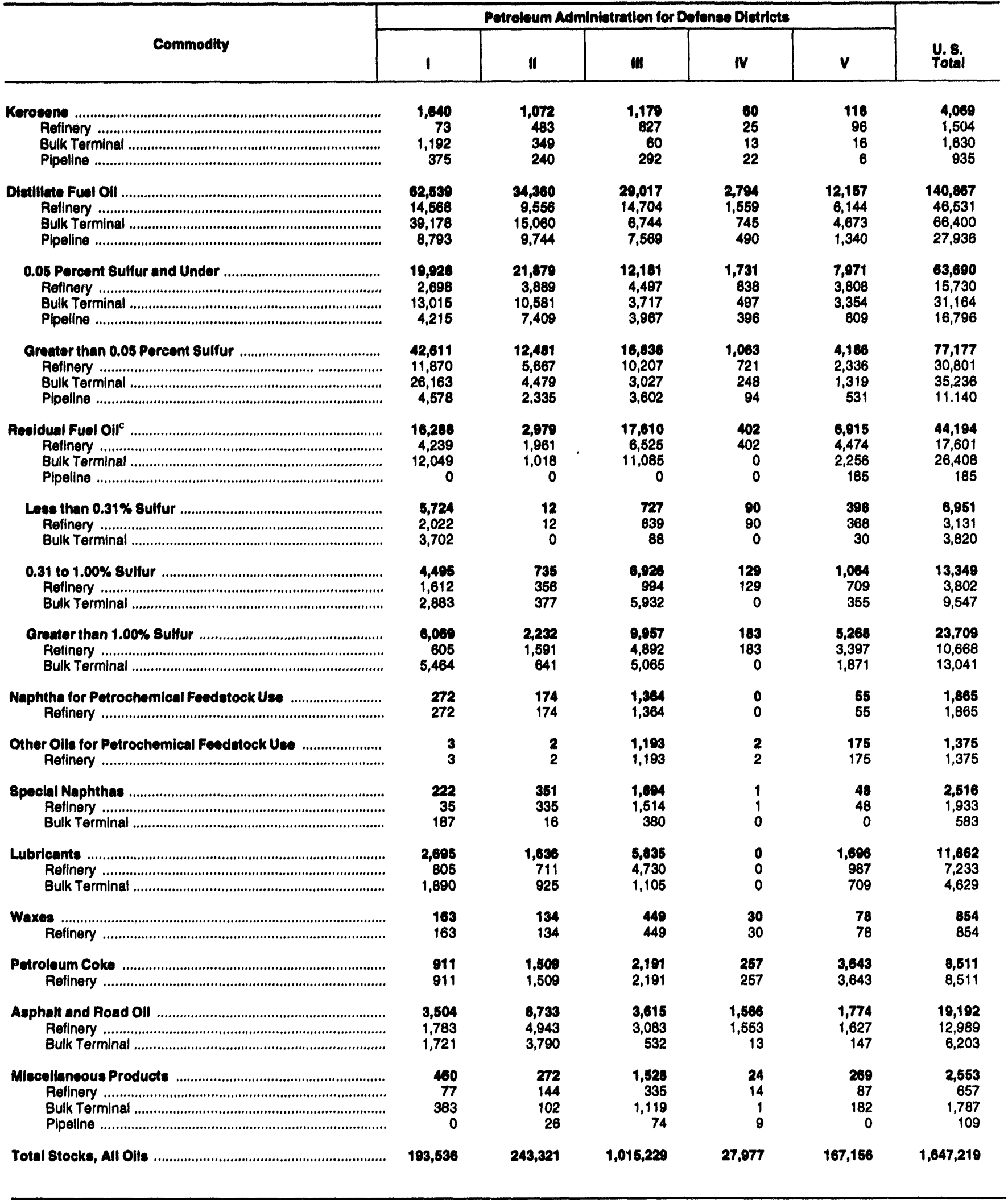

\footnotetext{
Includes stocks held by producers.
b Includes tertiany amyl methyl ether (TAME), terilary butyl alcohol (TBA), and other aliphatic alcohols and ethers Intended for motor gasoline blending (e.g., lsopropyl ether (IPE) or n-propanol).

c Sulfur content not available for stocks held by pipelines.

$W=$ Withheld to avoid disclosure of individual company data.

Note: Stocks are reported as of the end of December.

Sources: Energy Information Administration (EIA) Forms EIA-810, "Monthly Refinery Report," ElA-811, "Monthly Bulk Terminal Report," ElA-812, "Monthly Product Plpeline Report," ElA-813, "Monthly Crude Oll Report," and ElA-816, "Monthly Natural Gas Liquids Report."
} 
Table 31. Refinery, Bulk Torminal, and Natural Gas Plant Stooks of Solocted Petroloum Producte by PAD Dlstrict and State, 1993

(Thousand Barrels)

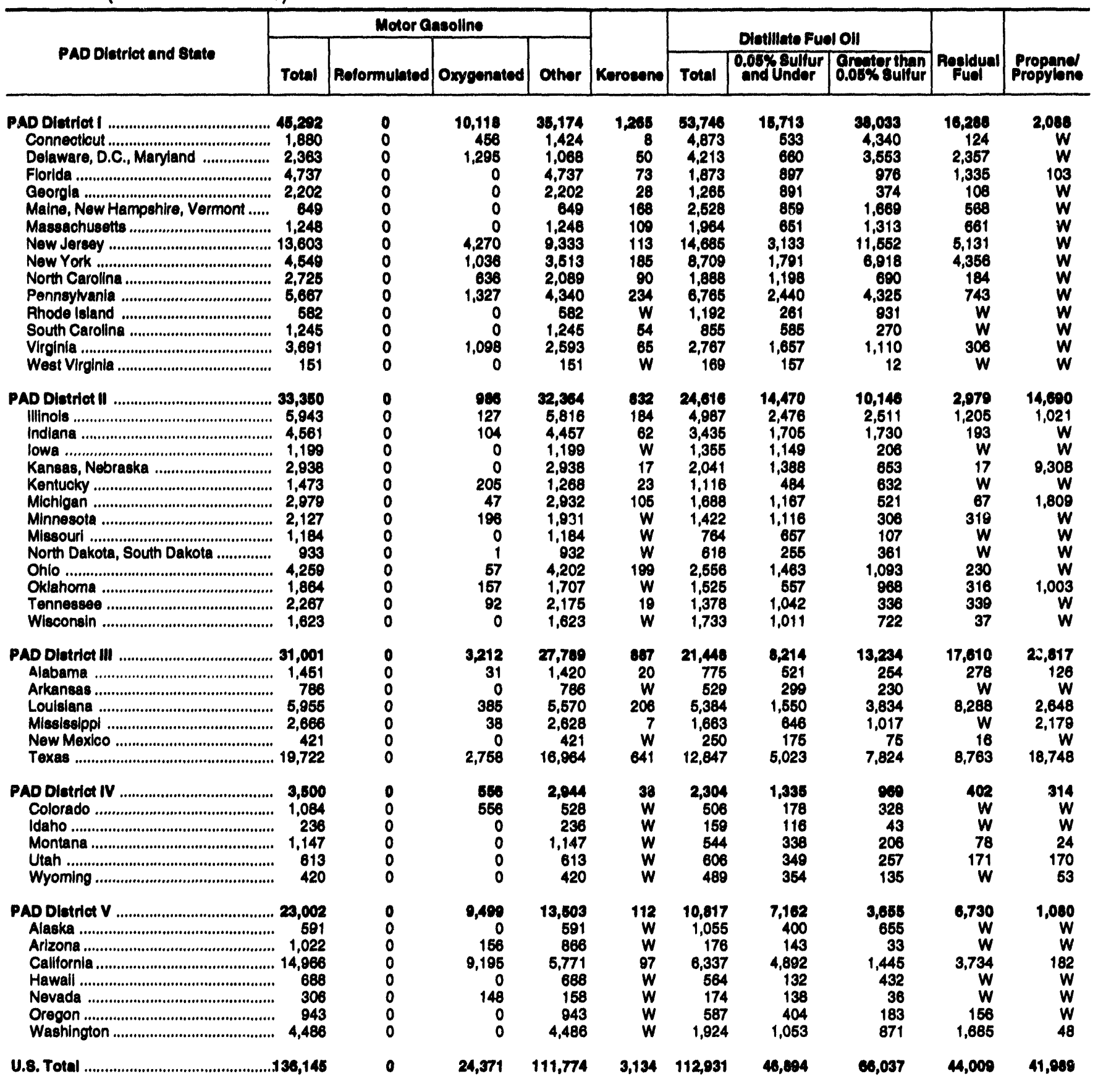

$W=$ Withheld to avoid disclosure of individual company data.

Notes: - Stocks are reported as of the end of December. - Totals may not equal sum of components due to independent rounding

Sources: Energy Intormation Administration (ElA) Forme EIA-810, "Monthly Refinery Report," ElA-811, "Monthly Bulk Terminal Report," and ElA-816, "Monthly Natural Gas Liquids Report." 
Table 32. Movements of Crude Oll and Petrobum Products by Pipeline, Tanker, and Barge Between PAD Districta, 1993

(Thousand Barrels)

\begin{tabular}{|c|c|c|c|c|c|c|c|c|c|}
\hline \multirow{2}{*}{ Commodity } & \multicolumn{3}{|c|}{ From I to } & \multicolumn{4}{|c|}{ From II to } & \multicolumn{2}{|c|}{ From III to } \\
\hline & $\boldsymbol{\|}$ & III & $\mathbf{V}$ & $\mathbf{I}$ & III & IV & $\mathbf{v}$ & 1 & \| \\
\hline 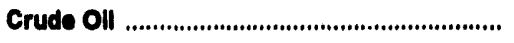 & 1,101 & 0 & 0 & 1,390 & 16,474 & 4,300 & 0 & $\mathbf{0}$ & 673,490 \\
\hline 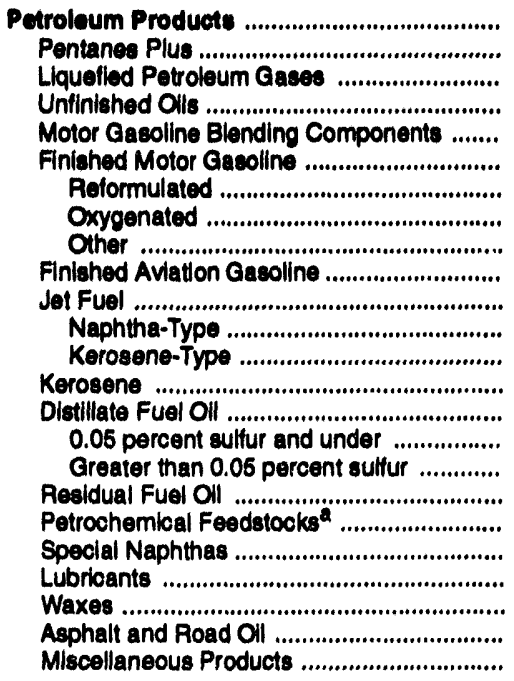 & $\begin{array}{r}04,629 \\
0 \\
0 \\
327 \\
192 \\
63,652 \\
0 \\
128 \\
63,424 \\
01 \\
3,661 \\
0 \\
3,661 \\
579 \\
25,531 \\
6,058 \\
19,473 \\
0 \\
538 \\
0 \\
57 \\
0 \\
0 \\
0\end{array}$ & $\begin{array}{r}1,934 \\
0 \\
0 \\
0 \\
388 \\
161 \\
0 \\
0 \\
161 \\
0 \\
0 \\
0 \\
0 \\
0 \\
983 \\
0 \\
983 \\
0 \\
0 \\
17 \\
465 \\
0 \\
0 \\
0\end{array}$ & $\begin{array}{l}0 \\
0 \\
0 \\
0 \\
0 \\
0 \\
0 \\
0 \\
0 \\
0 \\
0 \\
0 \\
0 \\
0 \\
0 \\
0 \\
0 \\
0 \\
0 \\
0 \\
0 \\
0 \\
0 \\
0\end{array}$ & $\begin{array}{r}45,240 \\
0 \\
12,785 \\
210 \\
224 \\
19,421 \\
0 \\
1,316 \\
18,108 \\
0 \\
2,031 \\
0 \\
2,031 \\
363 \\
7,441 \\
2,304 \\
5,137 \\
422 \\
0 \\
0 \\
938 \\
0 \\
1,435 \\
0\end{array}$ & $\begin{array}{r}r 8,180 \\
2,491 \\
36,001 \\
425 \\
223 \\
12,395 \\
0 \\
0 \\
12,395 \\
0 \\
3,492 \\
743 \\
2,749 \\
0 \\
3,203 \\
894 \\
2,309 \\
4,665 \\
0 \\
0 \\
230 \\
0 \\
36 \\
119\end{array}$ & $\begin{array}{r}36,160 \\
1 \\
1,094 \\
0 \\
0 \\
19,907 \\
0 \\
99 \\
19,808 \\
131 \\
8,802 \\
0 \\
8,802 \\
0 \\
6,216 \\
4,829 \\
1,386 \\
0 \\
0 \\
0 \\
0 \\
0 \\
0 \\
0\end{array}$ & $\begin{array}{r}0 \\
0 \\
0 \\
81 \\
0 \\
0 \\
0 \\
0 \\
0 \\
0 \\
0 \\
0 \\
0 \\
0 \\
0 \\
0 \\
0 \\
0 \\
0 \\
0 \\
0 \\
0 \\
0 \\
8\end{array}$ & $\begin{array}{r}886,847 \\
0 \\
26,957 \\
3,640 \\
2,008 \\
587,088 \\
0 \\
67,408 \\
519,680 \\
1,297 \\
121,953 \\
1,890 \\
119,863 \\
6,896 \\
226,618 \\
32,722 \\
193,896 \\
2,862 \\
10 \\
894 \\
5,584 \\
26 \\
2,263 \\
751\end{array}$ & $\begin{array}{r}332,684 \\
10,446 \\
61,757 \\
1,177 \\
9,693 \\
154,954 \\
0 \\
107 \\
154,847 \\
996 \\
37,751 \\
1,432 \\
36,319 \\
1,216 \\
46,531 \\
18,619 \\
27,912 \\
475 \\
20 \\
1,079 \\
3,383 \\
0 \\
2,424 \\
683\end{array}$ \\
\hline 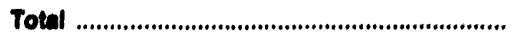 & 98,020 & 1,094 & $\mathbf{0}$ & 48,020 & 79,684 & 40,549 & 89 & 088,047 & $1,008,080$ \\
\hline
\end{tabular}

\begin{tabular}{|c|c|c|c|c|c|c|c|c|c|}
\hline \multirow[b]{2}{*}{ Commodity } & \multicolumn{2}{|c|}{ From ill to } & \multicolumn{3}{|c|}{ From IV to } & \multicolumn{4}{|c|}{ From V to } \\
\hline & IV & $\mathbf{v}$ & II & III & $\mathbf{v}$ & $\mathbf{I}$ & $\|$ & III & IV \\
\hline 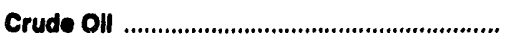 & 0 & 0 & 20,411 & 18,149 & 0 & 0 & 0 & 71,747 & $\mathbf{0}$ \\
\hline 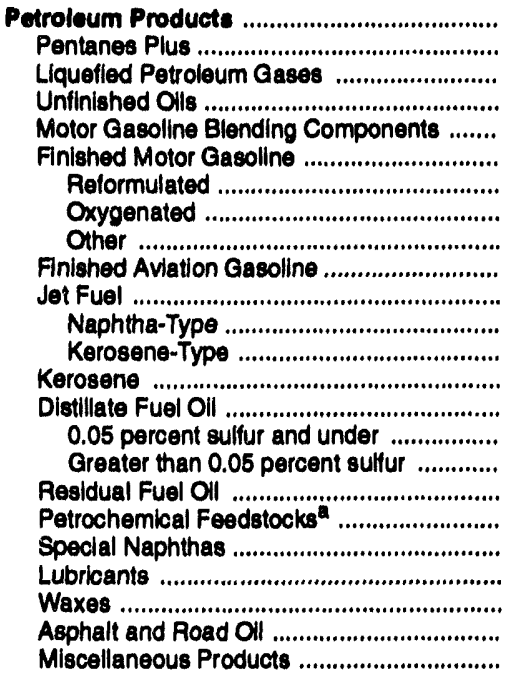 & $\begin{array}{l}0 \\
0 \\
0 \\
0 \\
0 \\
0 \\
0 \\
0 \\
0 \\
0 \\
0 \\
0 \\
0 \\
0 \\
0 \\
0 \\
0 \\
0 \\
0 \\
0 \\
0 \\
0 \\
0 \\
0\end{array}$ & $\begin{array}{r}21,407 \\
0 \\
0 \\
593 \\
1,049 \\
12,432 \\
0 \\
0 \\
12,432 \\
0 \\
2,798 \\
1,034 \\
1,764 \\
0 \\
3,633 \\
732 \\
2,901 \\
0 \\
0 \\
0 \\
902 \\
0 \\
0 \\
0\end{array}$ & $\begin{array}{r}21,300 \\
1,614 \\
9,454 \\
0 \\
0 \\
5,774 \\
0 \\
0 \\
5,774 \\
0 \\
957 \\
928 \\
29 \\
196 \\
3,403 \\
836 \\
2,567 \\
0 \\
0 \\
0 \\
0 \\
0 \\
0 \\
0\end{array}$ & $\begin{array}{r}18,817 \\
2,109 \\
16,708 \\
0 \\
0 \\
0 \\
0 \\
0 \\
0 \\
0 \\
0 \\
0 \\
0 \\
0 \\
0 \\
0 \\
0 \\
0 \\
0 \\
0 \\
0 \\
0 \\
0 \\
0\end{array}$ & $\begin{array}{r}12,916 \\
0 \\
0 \\
0 \\
0 \\
8,682 \\
0 \\
0 \\
8,582 \\
0 \\
1,885 \\
884 \\
1,101 \\
0 \\
2,349 \\
684 \\
1,685 \\
0 \\
0 \\
0 \\
0 \\
0 \\
0 \\
0\end{array}$ & $\begin{array}{r}395 \\
0 \\
0 \\
0 \\
0 \\
395 \\
0 \\
0 \\
395 \\
0 \\
0 \\
0 \\
0 \\
0 \\
0 \\
0 \\
0 \\
0 \\
0 \\
0 \\
0 \\
0 \\
0 \\
0\end{array}$ & $\begin{array}{l}0 \\
0 \\
0 \\
0 \\
0 \\
0 \\
0 \\
0 \\
0 \\
0 \\
0 \\
0 \\
0 \\
0 \\
0 \\
0 \\
0 \\
0 \\
0 \\
0 \\
0 \\
0 \\
0 \\
0\end{array}$ & $\begin{array}{r}420 \\
0 \\
0 \\
0 \\
0 \\
0 \\
0 \\
0 \\
0 \\
0 \\
0 \\
0 \\
0 \\
0 \\
0 \\
0 \\
0 \\
0 \\
0 \\
0 \\
392 \\
0 \\
0 \\
28\end{array}$ & $\begin{array}{l}0 \\
0 \\
0 \\
0 \\
0 \\
0 \\
0 \\
0 \\
0 \\
0 \\
0 \\
0 \\
0 \\
0 \\
0 \\
0 \\
0 \\
0 \\
0 \\
0 \\
0 \\
0 \\
0 \\
0\end{array}$ \\
\hline Total & $\mathbf{0}$ & 21,407 & 80,830 & 33,898 & 12,916 & 395 & 0 & 72,167 & $\mathbf{0}$ \\
\hline
\end{tabular}

Includes naphtha less than $401^{\circ} \mathrm{F}$ endpoint and other olls equal to or greater than $401^{\circ} \mathrm{F}$ endpoint.

Sources: Energy Intormation Administration (EIA) Forms EIA-812, "Monthly Product Pipeline Report," EIA-813, "Monthly Crude Oll Report," and EIA-817, "Monthly Tanker and Barge Movement Report." 
Table 33. Movements of Crude Oll and Potroleum Products by Plpeline Between PAD Distrlete, 1993 (Thousand Barrels)

\begin{tabular}{|c|c|c|c|c|c|c|c|}
\hline \multirow{2}{*}{ Commodity } & \multicolumn{2}{|c|}{ From 1 to } & \multicolumn{3}{|c|}{ From II to } & \multicolumn{2}{|c|}{ From ilf to } \\
\hline & $\|$ & III & 1 & III & $\mathbf{I V}$ & 1 & $\|$ \\
\hline 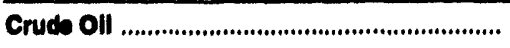 & 1,076 & 0 & 30 & 16,474 & 4,300 & $\mathbf{0}$ & 672,470 \\
\hline 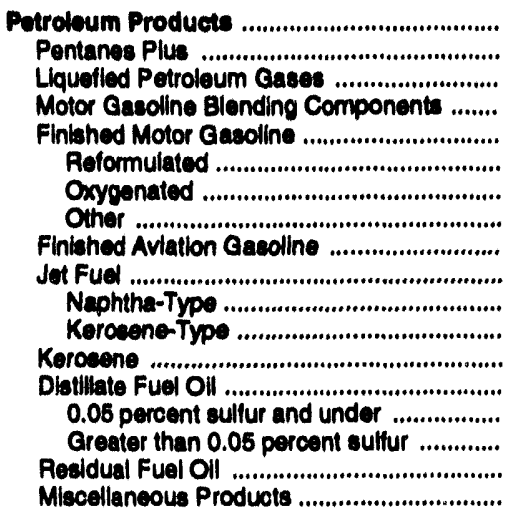 & $\begin{array}{r}93,168 \\
0 \\
0 \\
0 \\
63,403 \\
0 \\
0 \\
63,408 \\
91 \\
3,681 \\
0 \\
3,681 \\
572 \\
25,428 \\
6,058 \\
19,370 \\
0 \\
0\end{array}$ & $\begin{array}{l}0 \\
0 \\
0 \\
0 \\
0 \\
0 \\
0 \\
0 \\
0 \\
0 \\
0 \\
0 \\
0 \\
0 \\
0 \\
0 \\
0 \\
0\end{array}$ & $\begin{array}{r}28,788 \\
0 \\
12,756 \\
224 \\
9,431 \\
0 \\
0 \\
9,431 \\
0 \\
1,118 \\
0 \\
1,118 \\
168 \\
2,087 \\
887 \\
1,180 \\
0 \\
0\end{array}$ & $\begin{array}{r}6,344 \\
2,491 \\
36,001 \\
0 \\
11,763 \\
0 \\
0 \\
11,763 \\
0 \\
3,492 \\
743 \\
2,749 \\
0 \\
2,488 \\
688 \\
1,830 \\
0 \\
119\end{array}$ & $\begin{array}{r}39,180 \\
1 \\
1,004 \\
0 \\
19,807 \\
0 \\
00 \\
19,808 \\
131 \\
13,802 \\
0 \\
8,802 \\
0 \\
6,216 \\
4,829 \\
1,386 \\
0 \\
0\end{array}$ & $\begin{array}{r}797,410 \\
0 \\
24,446 \\
1,407 \\
468,924 \\
0 \\
59,299 \\
404,686 \\
01 \\
102,580 \\
1,444 \\
101,136 \\
6,780 \\
198,212 \\
28,015 \\
170,197 \\
0 \\
0\end{array}$ & $\begin{array}{r}29,045 \\
10,446 \\
61,675 \\
7,441 \\
137,242 \\
0 \\
0 \\
137,242 \\
886 \\
35,561 \\
1,432 \\
34,129 \\
1,010 \\
39,628 \\
17,240 \\
22,388 \\
0 \\
156\end{array}$ \\
\hline Total & 04,201 & $\mathbf{0}$ & 28,703 & 72,010 & 40,849 & 707,440 & Q98,624 \\
\hline
\end{tabular}

\begin{tabular}{|c|c|c|c|c|c|c|c|}
\hline \multirow[b]{2}{*}{ Commodity } & \multicolumn{2}{|c|}{ From ill to } & \multicolumn{3}{|c|}{ From IV to } & \multicolumn{2}{|c|}{ From V to } \\
\hline & $\mathbf{W}$ & $\mathbf{v}$ & u & III & $\mathbf{v}$ & mi & IV \\
\hline 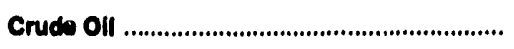 & $\mathbf{0}$ & $\mathbf{0}$ & 29,441 & 18,149 & 0 & 45,492 & $\mathbf{0}$ \\
\hline 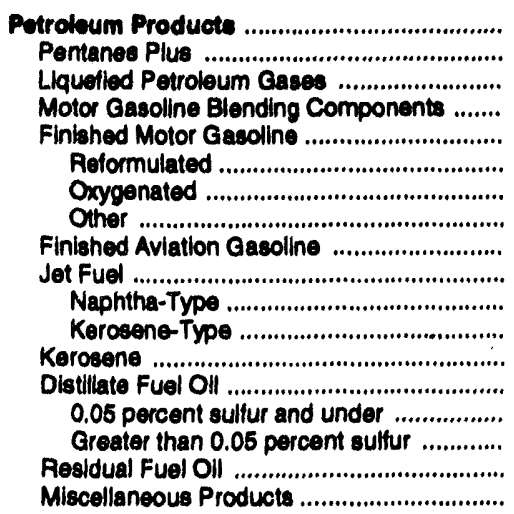 & $\begin{array}{l}0 \\
0 \\
0 \\
0 \\
0 \\
0 \\
0 \\
0 \\
0 \\
0 \\
0 \\
0 \\
0 \\
0 \\
0 \\
0 \\
0 \\
0\end{array}$ & $\begin{array}{r}18,681 \\
0 \\
0 \\
0 \\
12,160 \\
0 \\
0 \\
12,160 \\
0 \\
2,798 \\
1,034 \\
1,764 \\
0 \\
3,633 \\
732 \\
2,901 \\
0 \\
0\end{array}$ & $\begin{array}{r}21,398 \\
1,614 \\
9,454 \\
0 \\
6,774 \\
0 \\
0 \\
5,774 \\
0 \\
957 \\
928 \\
29 \\
198 \\
3,403 \\
836 \\
2,567 \\
0 \\
0\end{array}$ & $\begin{array}{r}18,017 \\
2,109 \\
16,708 \\
0 \\
0 \\
0 \\
0 \\
0 \\
0 \\
0 \\
0 \\
0 \\
0 \\
0 \\
0 \\
0 \\
0 \\
0\end{array}$ & $\begin{array}{r}12,916 \\
0 \\
0 \\
0 \\
0 \\
8,682 \\
0 \\
0 \\
8,682 \\
0 \\
1,885 \\
884 \\
1,101 \\
0 \\
2,349 \\
664 \\
1,685 \\
0 \\
0\end{array}$ & $\begin{array}{l}0 \\
0 \\
0 \\
0 \\
0 \\
0 \\
0 \\
0 \\
0 \\
0 \\
0 \\
0 \\
0 \\
0 \\
0 \\
0 \\
0 \\
0\end{array}$ & $\begin{array}{l}0 \\
0 \\
0 \\
0 \\
0 \\
0 \\
0 \\
0 \\
0 \\
0 \\
0 \\
0 \\
0 \\
0 \\
0 \\
0 \\
0 \\
0\end{array}$ \\
\hline Total & 0 & 18,601 & 80,839 & 33,930 & 12,916 & 48,402 & 0 \\
\hline
\end{tabular}

Sources: Energy Inlormation Administration (EIA) Forms ELA-812, "Monthly Product Pipeline Report," and EIA-813, Monthly Crude Oll Report." 
Table 34. Movemente of Crude Oll and Potroloum Producte by Tanker and Barge Between PAD Districte, 1993

(Thousand Barrels)

\begin{tabular}{|c|c|c|c|c|c|c|c|c|}
\hline \multirow[b]{2}{*}{ Commodity } & \multicolumn{3}{|c|}{ From 1 to } & \multicolumn{3}{|c|}{ From ll to } & \multicolumn{2}{|c|}{ From III to } \\
\hline & $n$ & HII & $\mathbf{V}$ & 1 & III & $\mathbf{v}$ & 1 & $\begin{array}{l}\text { Now } \\
\text { Enoland }\end{array}$ \\
\hline 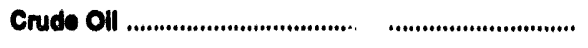 & 26 & 0 & 0 & 1,340 & 0 & 0 & $\mathbf{0}$ & $\mathbf{0}$ \\
\hline 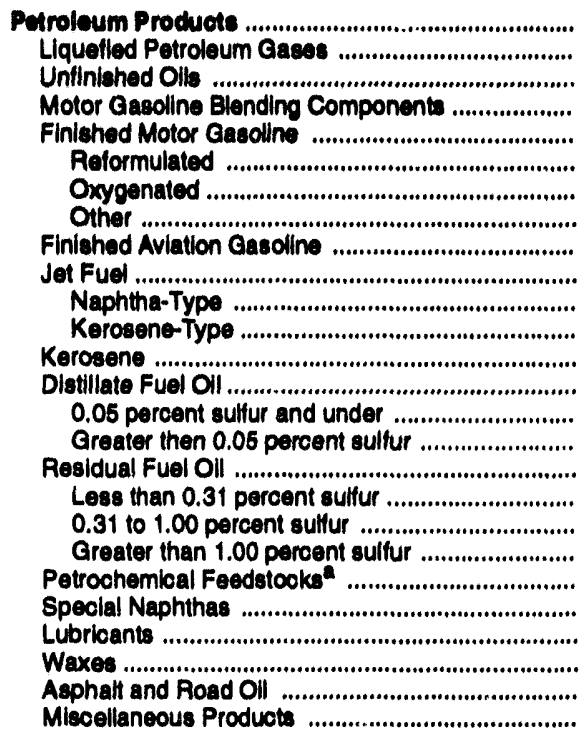 & $\begin{array}{r}1,373 \\
0 \\
327 \\
192 \\
149 \\
0 \\
128 \\
21 \\
0 \\
0 \\
0 \\
0 \\
7 \\
103 \\
0 \\
103 \\
0 \\
0 \\
0 \\
0 \\
538 \\
0 \\
57 \\
0 \\
0 \\
0\end{array}$ & $\begin{array}{r}1,94 \\
0 \\
0 \\
388 \\
161 \\
0 \\
0 \\
161 \\
0 \\
0 \\
0 \\
0 \\
0 \\
933 \\
0 \\
933 \\
0 \\
0 \\
0 \\
0 \\
0 \\
17 \\
465 \\
0 \\
0 \\
0\end{array}$ & $\begin{array}{l}0 \\
0 \\
0 \\
0 \\
0 \\
0 \\
0 \\
0 \\
0 \\
0 \\
0 \\
0 \\
0 \\
0 \\
0 \\
0 \\
0 \\
0 \\
0 \\
0 \\
0 \\
0 \\
0 \\
0 \\
0 \\
0\end{array}$ & $\begin{array}{r}19,407 \\
0 \\
210 \\
0 \\
9,990 \\
0 \\
1,316 \\
8,676 \\
0 \\
913 \\
0 \\
913 \\
206 \\
5,374 \\
1,417 \\
3,957 \\
422 \\
0 \\
0 \\
422 \\
0 \\
0 \\
938 \\
0 \\
1,435 \\
0\end{array}$ & $\begin{array}{r}6,830 \\
0 \\
425 \\
223 \\
642 \\
0 \\
0 \\
642 \\
0 \\
0 \\
0 \\
0 \\
0 \\
715 \\
236 \\
479 \\
4,565 \\
0 \\
0 \\
4,565 \\
0 \\
0 \\
230 \\
0 \\
36 \\
0\end{array}$ & $\begin{array}{r}80 \\
0 \\
81 \\
0 \\
0 \\
0 \\
0 \\
0 \\
0 \\
0 \\
0 \\
0 \\
0 \\
0 \\
0 \\
0 \\
0 \\
0 \\
0 \\
0 \\
0 \\
0 \\
0 \\
0 \\
0 \\
8\end{array}$ & $\begin{array}{r}191,207 \\
2,611 \\
3,640 \\
601 \\
123,164 \\
0 \\
0,169 \\
114,995 \\
1,206 \\
19,373 \\
546 \\
18,827 \\
116 \\
28,406 \\
4,707 \\
23,699 \\
2,662 \\
1,425 \\
0 \\
1,237 \\
10 \\
894 \\
5,584 \\
26 \\
2,263 \\
751\end{array}$ & $\begin{array}{r}8,433 \\
0 \\
0 \\
0 \\
1,863 \\
0 \\
765 \\
1,098 \\
74 \\
330 \\
0 \\
330 \\
68 \\
2,880 \\
0 \\
2,880 \\
228 \\
0 \\
0 \\
228 \\
0 \\
0 \\
0 \\
0 \\
0 \\
0\end{array}$ \\
\hline 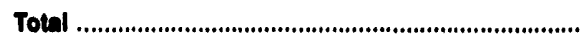 & 1,398 & 1,694 & 0 & 20,897 & 6,838 & 8 & 191,207 & 5,433 \\
\hline
\end{tabular}

\begin{tabular}{|c|c|c|c|c|c|c|c|}
\hline \multirow{2}{*}{ Commodity } & \multicolumn{4}{|c|}{ From III to } & \multicolumn{3}{|c|}{ From V to } \\
\hline & $\begin{array}{l}\text { Contral } \\
\text { Atlantle }\end{array}$ & $\begin{array}{l}\text { Lower } \\
\text { Atlantlo }\end{array}$ & $\|$ & $\mathbf{V}$ & 1 & $\|$ & IIII \\
\hline 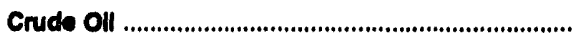 & 0 & 0 & 1,017 & $\mathbf{0}$ & 0 & $\mathbf{0}$ & $\mathbf{2 6 , 2 6 5}$ \\
\hline 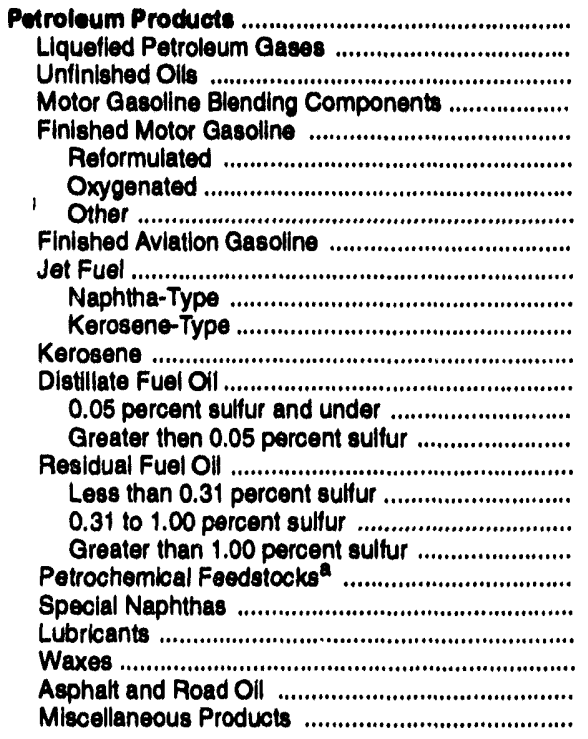 & $\begin{array}{r}11,500 \\
0 \\
570 \\
432 \\
3,028 \\
0 \\
572 \\
2,456 \\
227 \\
561 \\
372 \\
179 \\
0 \\
1,872 \\
509 \\
1,363 \\
185 \\
0 \\
0 \\
185 \\
0 \\
0 \\
4,391 \\
26 \\
30 \\
197\end{array}$ & $\begin{array}{r}174,205 \\
2,511 \\
3,070 \\
169 \\
118,283 \\
0 \\
6,842 \\
111,441 \\
905 \\
18,492 \\
174 \\
18,318 \\
48 \\
23,654 \\
4,198 \\
19,466 \\
2,249 \\
1,425 \\
0 \\
824 \\
10 \\
894 \\
1,193 \\
0 \\
2,233 \\
554\end{array}$ & $\begin{array}{r}38,580 \\
82 \\
1,177 \\
2,252 \\
17,712 \\
0 \\
107 \\
17,605 \\
110 \\
2,190 \\
0 \\
2,190 \\
205 \\
6,903 \\
1,379 \\
5,524 \\
475 \\
0 \\
32 \\
443 \\
20 \\
1,079 \\
3,383 \\
0 \\
2,424 \\
527\end{array}$ & $\begin{array}{r}2,828 \\
0 \\
593 \\
1,049 \\
282 \\
0 \\
0 \\
282 \\
0 \\
0 \\
0 \\
0 \\
0 \\
0 \\
0 \\
0 \\
0 \\
0 \\
0 \\
0 \\
0 \\
0 \\
902 \\
0 \\
0 \\
0\end{array}$ & $\begin{array}{r}395 \\
0 \\
0 \\
0 \\
395 \\
0 \\
0 \\
395 \\
0 \\
0 \\
0 \\
0 \\
0 \\
0 \\
0 \\
0 \\
0 \\
0 \\
0 \\
0 \\
0 \\
0 \\
0 \\
0 \\
0 \\
0\end{array}$ & $\begin{array}{l}0 \\
0 \\
0 \\
0 \\
0 \\
0 \\
0 \\
0 \\
0 \\
0 \\
0 \\
0 \\
0 \\
0 \\
0 \\
0 \\
0 \\
0 \\
0 \\
0 \\
0 \\
0 \\
0 \\
0 \\
0 \\
0\end{array}$ & $\begin{array}{r}420 \\
0 \\
0 \\
0 \\
0 \\
0 \\
0 \\
0 \\
0 \\
0 \\
0 \\
0 \\
0 \\
0 \\
0 \\
0 \\
0 \\
0 \\
0 \\
0 \\
0 \\
0 \\
392 \\
0 \\
0 \\
28\end{array}$ \\
\hline 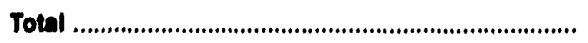 & 11,800 & 174,208 & 39,580 & 2,828 & 395 & 0 & 26,675 \\
\hline
\end{tabular}

a Includes naphtha less than $401^{\circ} F$ endpoint and other olls equal to or greater than $401^{\circ} \mathrm{F}$ endpoint.

Sources: Energy Information Administration (EIA) Forms ElA-812, "Monthly Product Pipeline Report" and ElA-817, "Monthly Tanker and Barge Movement Report." 
Table 35. Net Movements of Cruds Oll and Potrobum Produote by Pipolime, Tanker, and Barns Ectween PAD Dictriote, 1993

(Thousand Barrels)

\begin{tabular}{|c|c|c|c|c|c|c|}
\hline \multirow{2}{*}{ Comnodity } & \multicolumn{3}{|c|}{ PAD Dlotrital } & \multicolumn{3}{|c|}{ PAO Dientiot II } \\
\hline & Recolpte & Shlpmonts & Net Recolpte & Reoulpos & Chlpmente & Nex Rocolpts \\
\hline 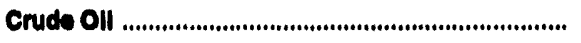 & 1,380 & 1,101 & 270 & 704,095 & 20,263 & 681,768 \\
\hline 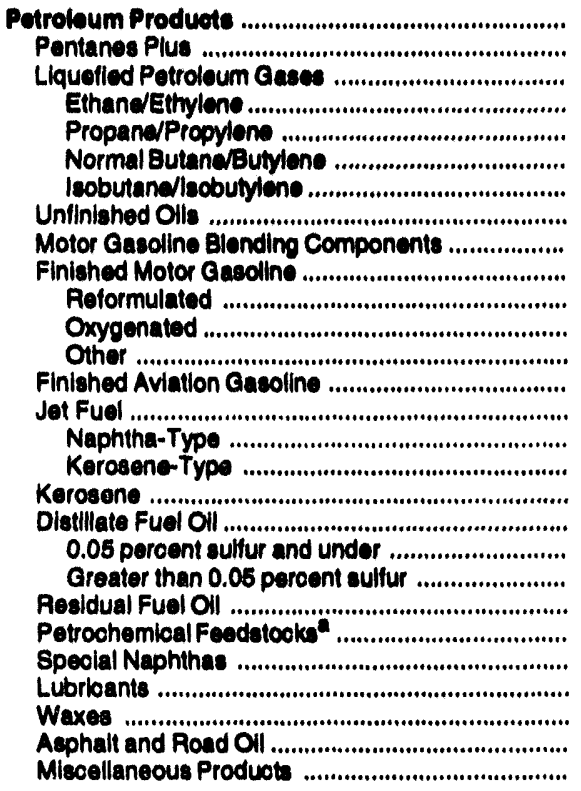 & $\begin{array}{r}1,034,282 \\
0 \\
39,712 \\
0 \\
37,679 \\
1,680 \\
383 \\
3,860 \\
2,232 \\
606,904 \\
0 \\
68,723 \\
638,181 \\
1,297 \\
123,984 \\
1,090 \\
121,984 \\
7,259 \\
234,059 \\
35,026 \\
109,033 \\
3,084 \\
10 \\
894 \\
6,522 \\
28 \\
3,698 \\
751\end{array}$ & $\begin{array}{r}89,492 \\
0 \\
0 \\
0 \\
0 \\
0 \\
0 \\
327 \\
680 \\
63,713 \\
0 \\
128 \\
63,696 \\
91 \\
3,661 \\
0 \\
3,681 \\
579 \\
26,464 \\
6,058 \\
20,406 \\
0 \\
538 \\
17 \\
522 \\
0 \\
0 \\
0\end{array}$ & 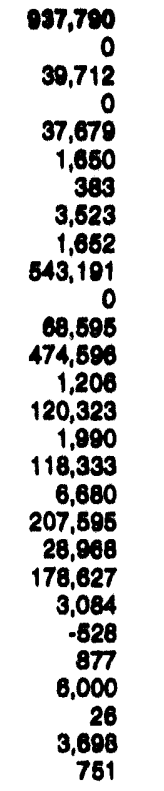 & $\begin{array}{r}44,610 \\
12,000 \\
71,211 \\
8,449 \\
46,767 \\
8,673 \\
8,322 \\
1,604 \\
9,885 \\
224,280 \\
0 \\
285 \\
224,045 \\
1,087 \\
42,389 \\
2,360 \\
40,000 \\
1,090 \\
76,465 \\
26,613 \\
40,062 \\
475 \\
568 \\
1,079 \\
3,440 \\
0 \\
2,424 \\
683\end{array}$ & $\begin{array}{r}14,090 \\
2,492 \\
49,860 \\
19,014 \\
22,168 \\
6,698 \\
2,088 \\
716 \\
447 \\
51,723 \\
0 \\
1,414 \\
50,300 \\
131 \\
14,325 \\
743 \\
13,882 \\
363 \\
16,860 \\
8,027 \\
8,832 \\
4,987 \\
0 \\
0 \\
1,188 \\
0 \\
1,471 \\
127\end{array}$ & $\begin{array}{r}303,081 \\
9,688 \\
21,381 \\
-10,686 \\
23,612 \\
2,077 \\
6,237 \\
788 \\
9,438 \\
172,657 \\
0 \\
-1,179 \\
173,736 \\
966 \\
28,044 \\
1,617 \\
26,427 \\
1,627 \\
68,606 \\
17,486 \\
41,120 \\
-4,612 \\
568 \\
1,079 \\
2,272 \\
0 \\
963 \\
568\end{array}$ \\
\hline Total & $1,088,682$ & 97,503 & 938,090 & $1,182,840$ & 168,912 & 988,638 \\
\hline
\end{tabular}

\begin{tabular}{|c|c|c|c|c|c|c|c|c|c|}
\hline \multirow[b]{2}{*}{ Commodity } & \multicolumn{3}{|c|}{ PAD Distriot ill } & \multicolumn{3}{|c|}{ PAD Distriet N } & \multicolumn{3}{|c|}{ PAD Dietriter V } \\
\hline & Rocolpte & Shipments & $\begin{array}{c}\text { Not } \\
\text { Recolpts }\end{array}$ & Recolpte & Shipmente & necolpte & Rocolpes & Shlpments & Noetpte \\
\hline Crude Oll & 103,370 & 673,498 & $-570,120$ & 4,309 & 4,800 & $-10,191$ & 0 & 71,747 & $-71,747$ \\
\hline 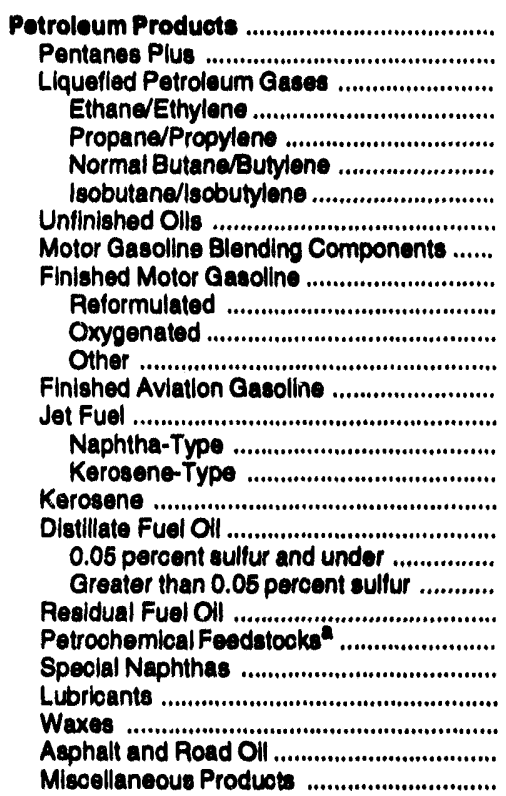 & $\begin{array}{r}4,391 \\
4,600 \\
52,709 \\
27,268 \\
15,135 \\
7,033 \\
3,273 \\
425 \\
611 \\
12,566 \\
0 \\
0 \\
12,556 \\
0 \\
3,492 \\
743 \\
2,749 \\
0 \\
4,136 \\
894 \\
3,242 \\
4,565 \\
0 \\
17 \\
1,087 \\
0 \\
36 \\
147\end{array}$ & $\begin{array}{r}1,342,638 \\
10,446 \\
88,714 \\
5,318 \\
68,931 \\
6,958 \\
7,607 \\
5,410 \\
12,750 \\
754,474 \\
0 \\
67,515 \\
686,959 \\
2,293 \\
162,502 \\
4,456 \\
158,046 \\
8,111 \\
276,782 \\
52,073 \\
224,709 \\
3,137 \\
30 \\
1,973 \\
9,869 \\
26 \\
4,687 \\
1,434\end{array}$ & $\begin{array}{r}-1,288,257 \\
-5,846 \\
-36,005 \\
21,960 \\
-53,796 \\
75 \\
-4,234 \\
-4,985 \\
-12,139 \\
-741,918 \\
0 \\
-67,515 \\
-674,403 \\
-2,293 \\
-159,010 \\
-3,713 \\
-155,297 \\
-8,111 \\
-272,646 \\
-51,179 \\
-221,467 \\
1,428 \\
-30 \\
-1,956 \\
-8,782 \\
-26 \\
-4,651 \\
-1,287\end{array}$ & $\begin{array}{r}30,150 \\
1 \\
1,094 \\
0 \\
1,075 \\
19 \\
0 \\
0 \\
0 \\
19,807 \\
0 \\
99 \\
19,808 \\
131 \\
8,802 \\
0 \\
8,802 \\
0 \\
6,215 \\
4,829 \\
1,386 \\
0 \\
0 \\
0 \\
0 \\
0 \\
0 \\
0\end{array}$ & $\begin{array}{r}83,131 \\
3,723 \\
26,162 \\
11,385 \\
8,570 \\
3,821 \\
2,386 \\
0 \\
0 \\
14,350 \\
0 \\
0 \\
14,366 \\
0 \\
2,942 \\
1,812 \\
1,130 \\
196 \\
5,752 \\
1,500 \\
4,262 \\
0 \\
0 \\
0 \\
0 \\
0 \\
0 \\
0\end{array}$ & $\begin{array}{r}-19,001 \\
-3,722 \\
-25,068 \\
-11,385 \\
-7,496 \\
-3,802 \\
-2,386 \\
0 \\
0 \\
5,861 \\
0 \\
99 \\
6,452 \\
131 \\
5,860 \\
-1,812 \\
7,672 \\
-198 \\
463 \\
3,329 \\
-2,866 \\
0 \\
0 \\
0 \\
0 \\
0 \\
0 \\
0\end{array}$ & $\begin{array}{r}34,412 \\
0 \\
0 \\
0 \\
0 \\
0 \\
0 \\
674 \\
1,049 \\
21,014 \\
0 \\
0 \\
21,014 \\
0 \\
4,783 \\
1,918 \\
2,865 \\
0 \\
5,982 \\
1,396 \\
4,686 \\
0 \\
0 \\
0 \\
902 \\
0 \\
0 \\
8\end{array}$ & $\begin{array}{r}818 \\
0 \\
0 \\
0 \\
0 \\
0 \\
0 \\
0 \\
0 \\
395 \\
0 \\
0 \\
395 \\
0 \\
0 \\
0 \\
0 \\
0 \\
0 \\
0 \\
0 \\
0 \\
0 \\
0 \\
392 \\
0 \\
0 \\
28\end{array}$ & $\begin{array}{r}33,697 \\
0 \\
0 \\
0 \\
0 \\
0 \\
0 \\
674 \\
1,049 \\
20,619 \\
0 \\
0 \\
20,619 \\
0 \\
4,783 \\
1,918 \\
2,865 \\
0 \\
5,982 \\
1,386 \\
4,586 \\
0 \\
0 \\
0 \\
510 \\
0 \\
0 \\
-20\end{array}$ \\
\hline Total & 187,751 & $2,016,134$ & $-1,020,383$ & 40,540 & 97,721 & $-8,172$ & 34,412 & $7, D_{2}$ & $-30,160$ \\
\hline
\end{tabular}

- Inoludes naphtha lose than $401^{\circ} \mathrm{F}$ endpoint and other olis equal to or greater than $401^{\circ} \mathrm{F}$ endpoint.

Sources: Enorgy Information Adminietration (EIA) Forme EIA-812, "Monthly Product Pipelline Report," ElA-813, "Monthly Crude Oll Report," and ElA-817, "Monthly Tanker and Bargo Movement Report."

78

Enorgy Information Administration/Potroloum Supply Annual 19es, Volume 1 


\section{Refinery Capacity}

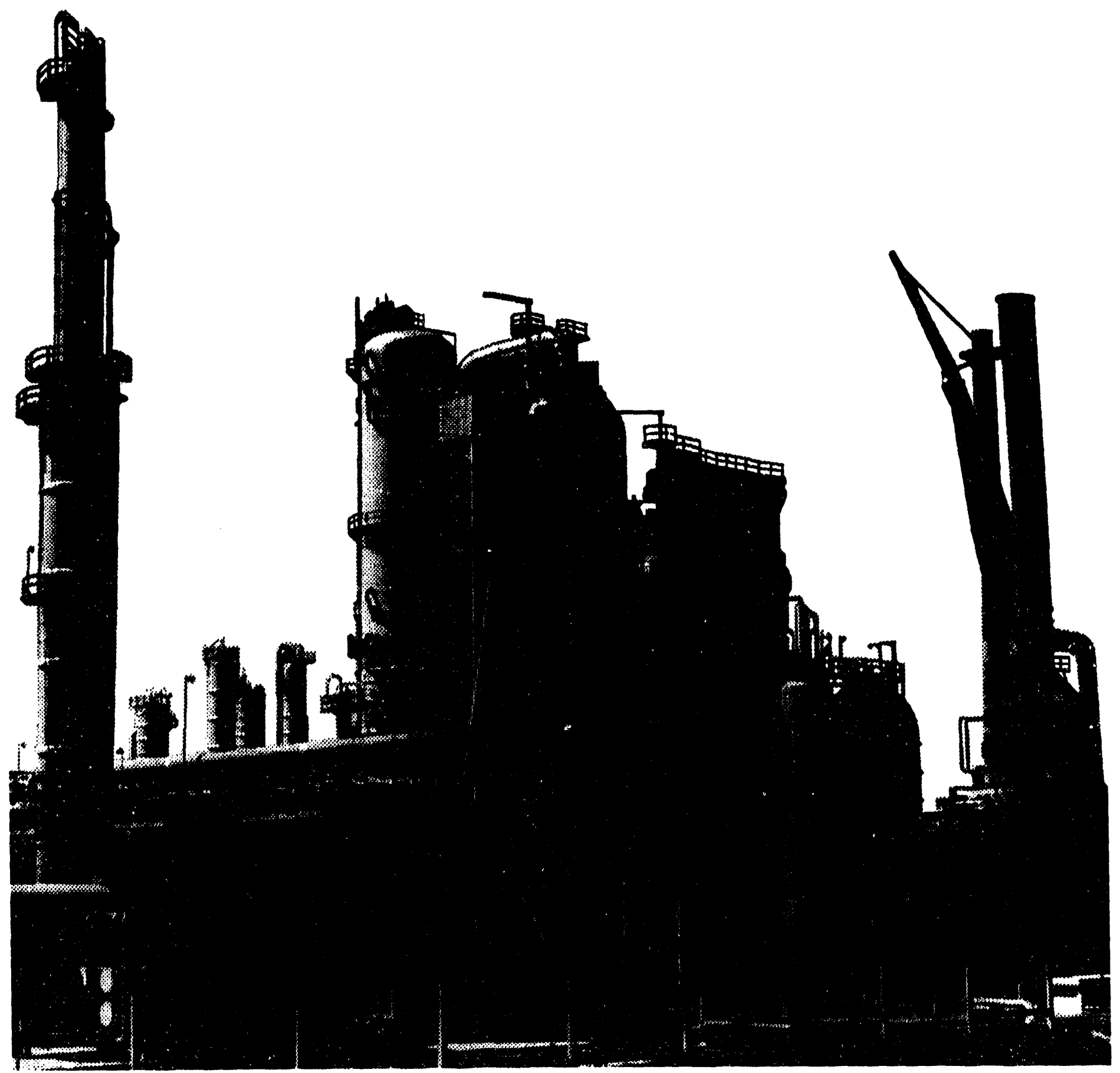

As part of the refining process, a catalytic cracking unit is used to increase the yield of gasoline from crude oil. 


\section{Changes to the 1994 Refinery Capacity Tables}

Several enhancements have been made to the 1994 Refinery Capacity tables to fulfill customer requests for additional data and to improve the usefulness of the tables. These enhancements focus primarily on eliminating excessive footnotes and displaying more detailed information on downstream processing units. Detailed changes are listed below.

Table $38 \quad$ (Capacity of Operable Petroleum Refineries by State)

A separate column for charge capacity for each type of downstream unit is now displayed. To accommodate this expansion, production capacity has been removed and a separate table created (Table 39).

Table 39 (Production Capacity of Operable Petroleum Refineries by State)

This information was formerly included in Table 38. To provide more detailed information, this data is now displayed as a separate table.

Table $40 \quad$ (Refiners' Operable Atmospheric Crude Oil Distillation Capacity)

Formerly Table 39.

Table 41 (Operable Crude and Downstream Charge Capacity of Petroleum Refineries)

Formerly Table 40.

Table 42 (Operable Production Capacity of Petroleum Refineries)

Formerly Table 41.

Table 4.3 (Working Storage Capacity at Refineries and Gasoline Blending Plants)

Formerly Table 42.

This table has been redesigned to display data on a PAD District basis. State information is no longer displayed.

Table 44 (Shell Storage Capacity at Refineries and Gasoline Blending Plants)

Formerly Table 43.

This table has been redesigned to display data on a PAD District basis. State information is no longer displayed.

Table 45

(Capacity, Fresh Feed Input and Utilization Rates of Selected Downstream Units at U.S. Refineries)

New table added. Data are displayed for the most recent eight years.

Table 46 (Refinery Receipts of Crude Oil by Method of Transportation)

Formerly Table 44.

Table $47 \quad$ (Fuels Consumed at Refineries by PAD District)

Formerly Table 45.

Table $48 \quad$ (Reactivated and Shutdown Refineries During 1993)

Formerly Table 46.

Table $49 \quad$ (Refinery Sales January 1993 through February 1994)

Formerly Table 47. 
Table 36. Number and Capacity of Operable Potroloum Rofineribs by PAD Dlotrlot and state as of January 1, 1994

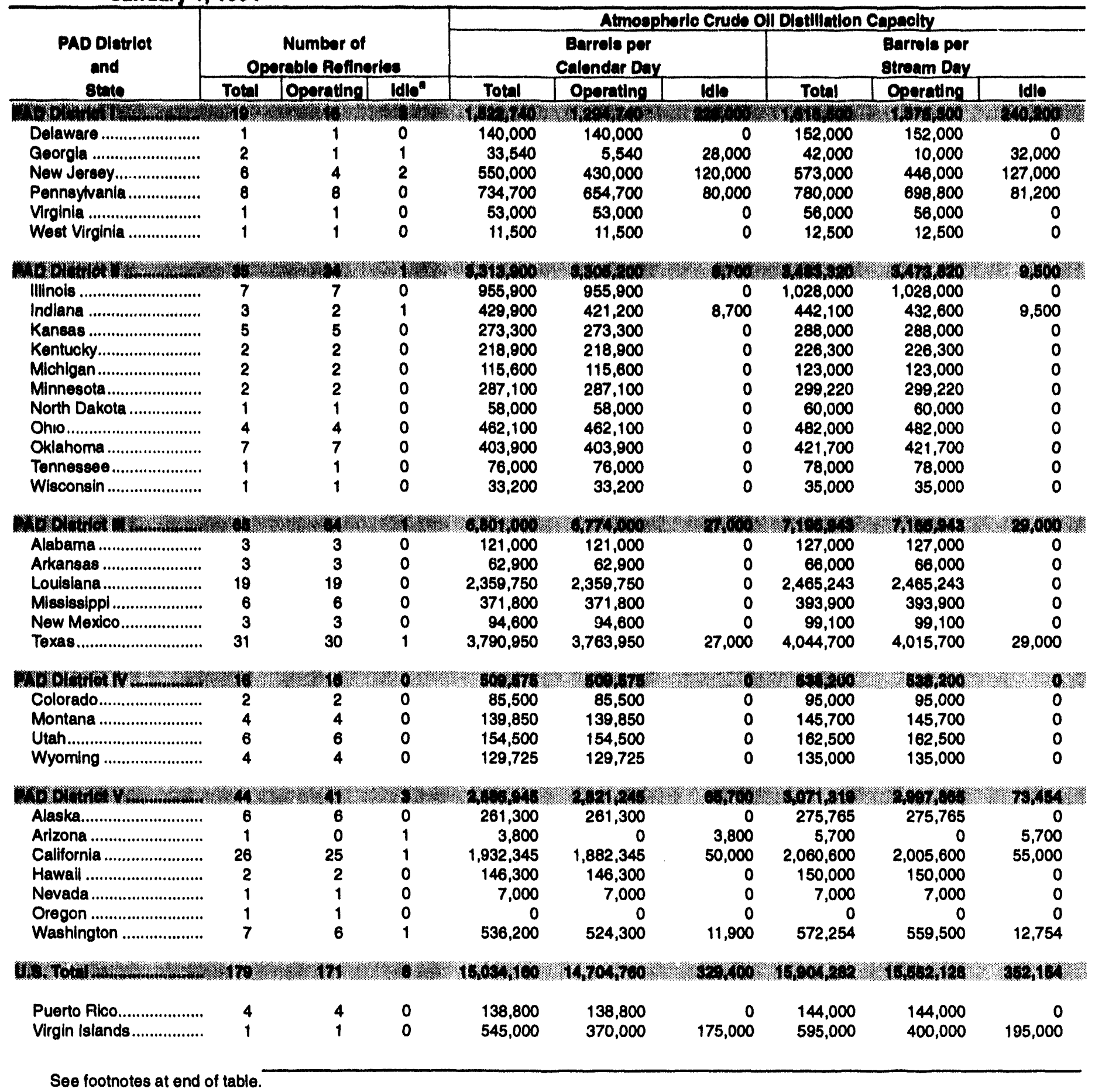



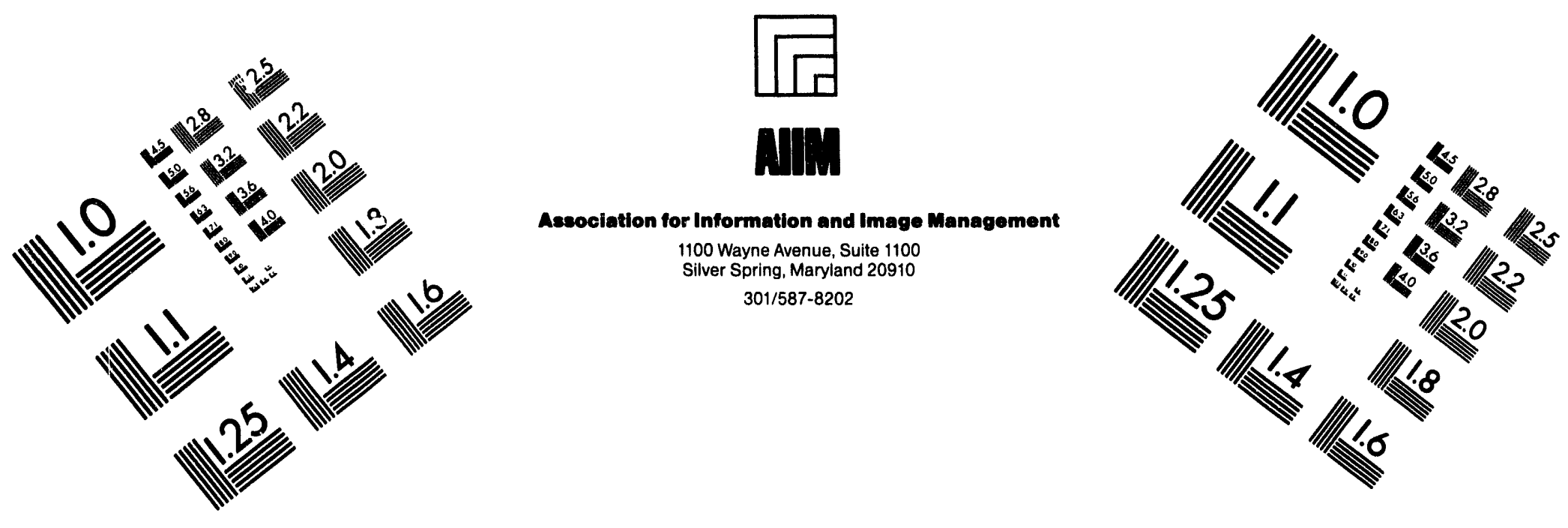

\section{Centimeter}

$\begin{array}{llllllllllllllll}1 & 2 & 3 & 4 & 5 & 6 & 7 & 8 & 9 & 10 & 11 & 12 & 13 & 14 & 15 & \mathrm{~mm}\end{array}$

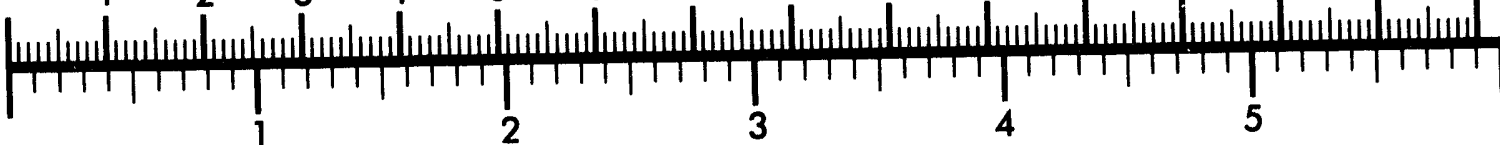
Inches

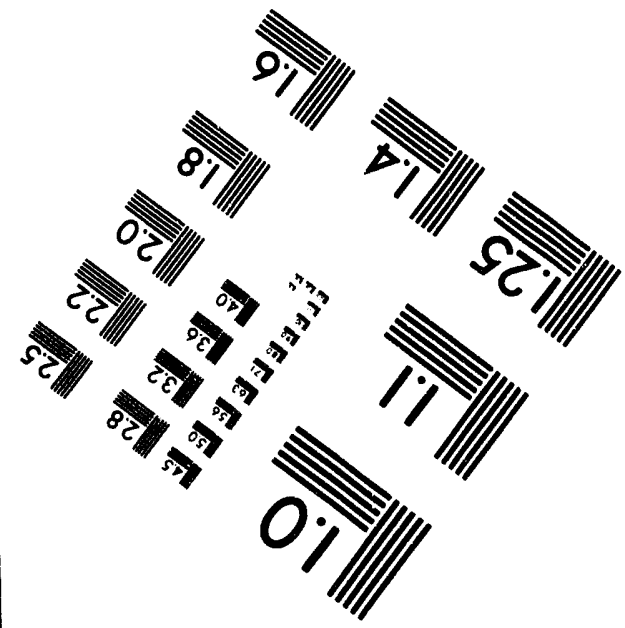

MANUFACTURED TO AIIM STANDARDS

BY APPLIED IMAGE. INC.

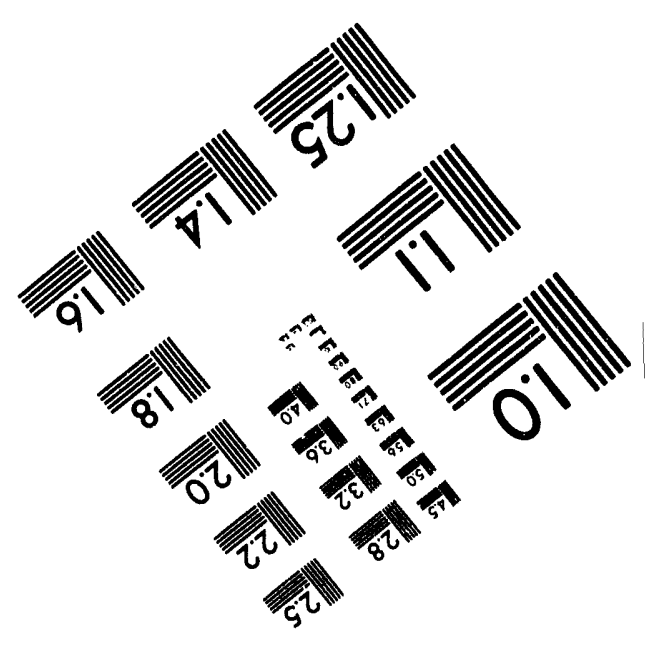



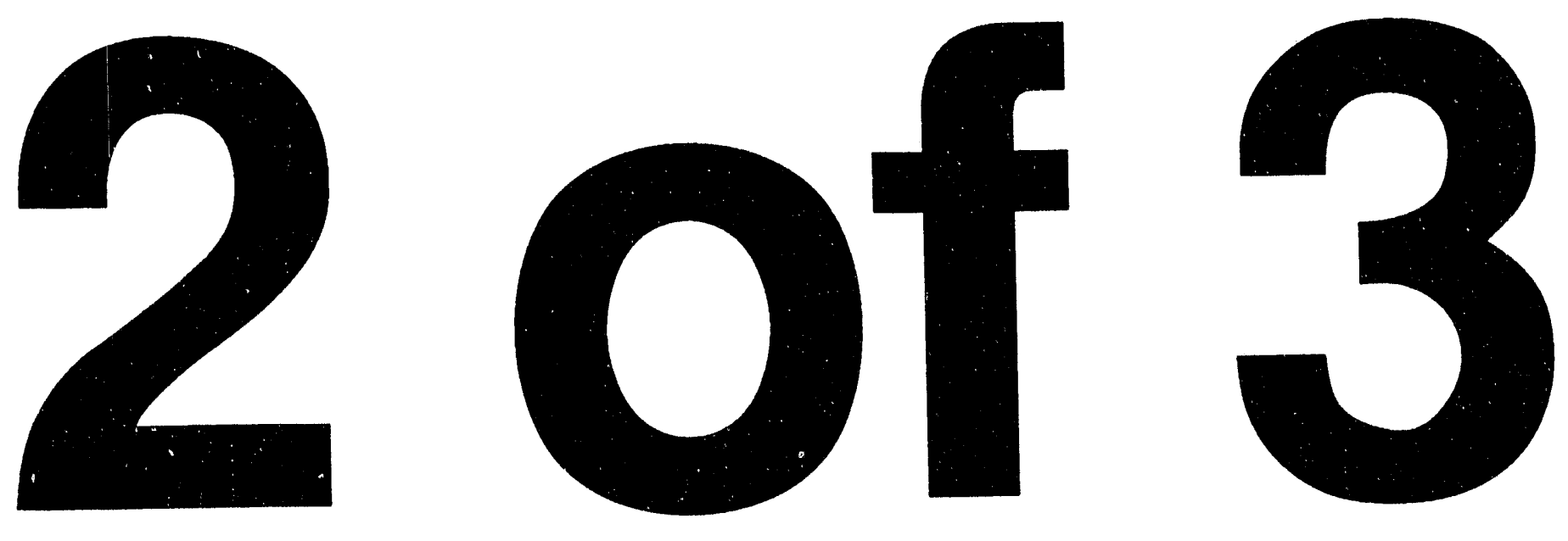
Table 36. Number and Capacity of Operable Petroleum Refinerles by PAD District and State as of January 1, 1994 (Continued)

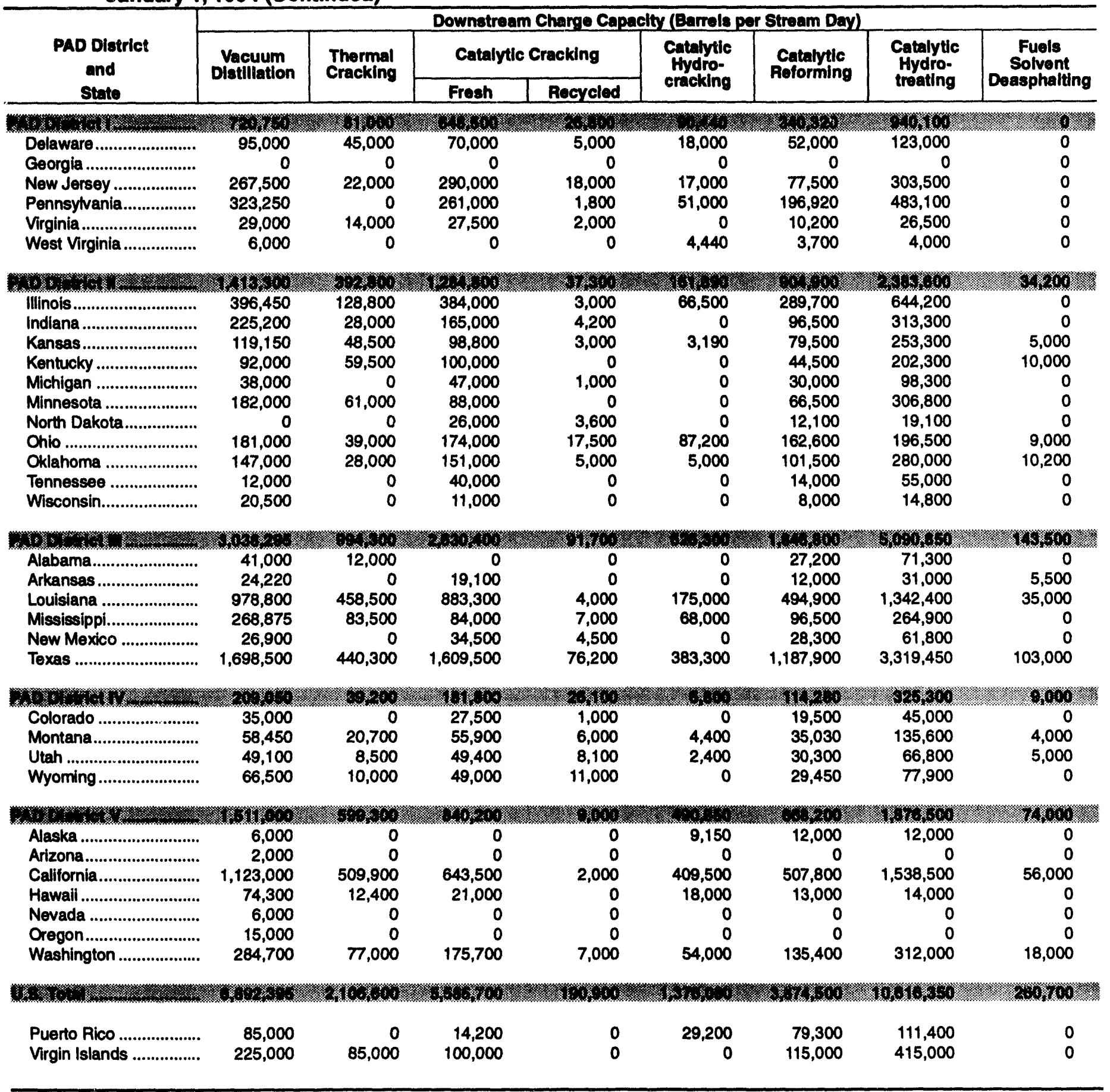

Refineries where distillation units were completely idle but not permanently shutdown on January 1, 1994.

Source: Energy Information Administration (EIA), Form EIA-820, "Annual Refinery Report." 
Table 37. Production Capacity of Operable Petroleum Refinerles by PAD District and State as of January 1, 1994 (Barrels per Stream Day, Except Where Noted)

\begin{tabular}{|c|c|c|c|c|c|c|c|c|}
\hline \multirow[b]{2}{*}{$\begin{array}{c}\text { PAD District } \\
\text { and } \\
\text { State } \\
\end{array}$} & \multicolumn{8}{|c|}{ Production Capacity } \\
\hline & Alkylates & Aromatics & $\begin{array}{c}\text { Asphalt and } \\
\text { Road OII }\end{array}$ & Isomers & Lubricants & $\begin{array}{c}\text { Marketable } \\
\text { Petroleum } \\
\text { Coke }\end{array}$ & $\begin{array}{l}\text { Hydrogen } \\
\text { (MMcid) }\end{array}$ & $\begin{array}{c}\text { Sulfur (short } \\
\text { tons/day) }\end{array}$ \\
\hline 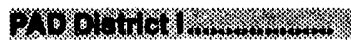 & or 63 & $14 x_{3}$ & \&oror & $\% 010$ & 00034 & 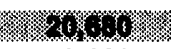 & (W6. & 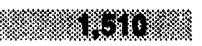 \\
\hline 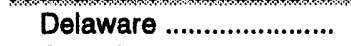 & 11,500 & 0 & 0 & 0 & 0 & 9,080 & 40 & 440 \\
\hline 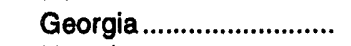 & 0 & 0 & 27,000 & 0 & 2,000 & 0 & 0 & 0 \\
\hline New Jersey...................... & 30,000 & 3,500 & 62,600 & 25,000 & 9,000 & 6,500 & 21 & 511 \\
\hline Pennsylvania .................... & 51,000 & 11,000 & 33,000 & 15,950 & 6,850 & 0 & 56 & 486 \\
\hline 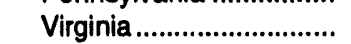 & 0 & 0 & 0 & 0 & 0 & 5,100 & 0 & 73 \\
\hline West Virginia...................... & 0 & 0 & 0 & 0 & 5,056 & 0 & 1 & 0 \\
\hline 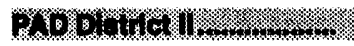 & $2+1 \times 13$ & $50 / 26$ & 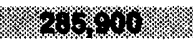 & \%ork, & 20,13 & 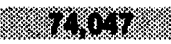 & 86 & 86 \\
\hline 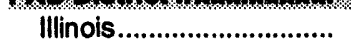 & 96,500 & 8,000 & 59,000 & 12,900 & 7,200 & 24,619 & 68 & 1,675 \\
\hline 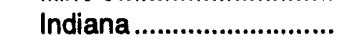 & 32,700 & 15,000 & 66,000 & 24,200 & 6,000 & 10,540 & 35 & 410 \\
\hline 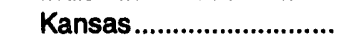 & 31,000 & 3,000 & 6,300 & 35,000 & 0 & 10,673 & 8 & 406 \\
\hline 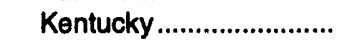 & 12,000 & 6,000 & 30,000 & 16,250 & 8,500 & 0 & 20 & 400 \\
\hline Michigan ........................... & 10,400 & 0 & 28,000 & 3,500 & 0 & 0 & 0 & 178 \\
\hline 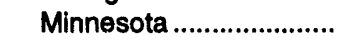 & 15,500 & 0 & 39,000 & 23,300 & 0 & 16,000 & 76 & 700 \\
\hline North Dakota .................. & 4,800 & 0 & 0 & 5,100 & 0 & 0 & 0 & 17 \\
\hline Ohio & 25,800 & 16,000 & 21,800 & 10,693 & 200 & 6,190 & 72 & 295 \\
\hline 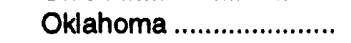 & 34,000 & 2,200 & 18,800 & 30,400 & 7,500 & 6,025 & 36 & 226 \\
\hline 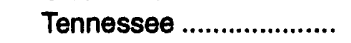 & 2,500 & 0 & 3,500 & 4,000 & 0 & 0 & 0 & 50 \\
\hline 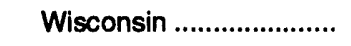 & 1,600 & 0 & 13,500 & 2,000 & 0 & 0 & 0 & 14 \\
\hline DOBWHAOL & 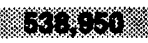 & 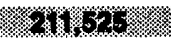 & $200 \times 16$ & 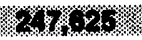 & $20 \% 10$ & (4) & 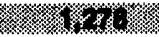 & 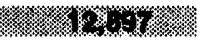 \\
\hline Alabama .............................. & 0 & 0 & 20,000 & 5,000 & 0 & 400 & 6 & 63 \\
\hline 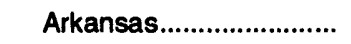 & 4,900 & 0 & 10,550 & 6,000 & 4,700 & 0 & 3 & 32 \\
\hline 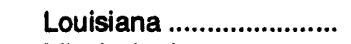 & 191,200 & 23,950 & 68,560 & 80,100 & 37,500 & 83,225 & 227 & 3,574 \\
\hline 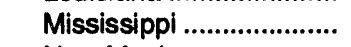 & 20,200 & 5,500 & 42,700 & 0 & 6,400 & 21,600 & 223 & 1,270 \\
\hline New Mexico ..................... & 11,200 & 0 & 6,400 & 4,000 & 0 & 0 & 0 & 24 \\
\hline 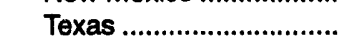 & 311,450 & 182,075 & 60,125 & 152,525 & 79,800 & 85,010 & 819 & 7,934 \\
\hline 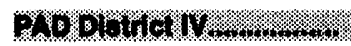 & $8 \mathrm{~s} / \mathrm{m}$ & (1) & 6.100 & 10000 & 0 & $8 \%$ & 16. & .416 \\
\hline 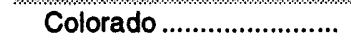 & 1,200 & 0 & 8,500 & 0 & 0 & 0 & 0 & 97 \\
\hline 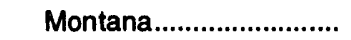 & 13,780 & 0 & 27,900 & 5,450 & 0 & 5,775 & 43 & 250 \\
\hline 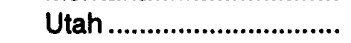 & 12,400 & 0 & 3,300 & 8,550 & 0 & 2,000 & 0 & 29 \\
\hline 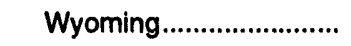 & 7,834 & 0 & 16,400 & 4,000 & 0 & 621 & 5 & 139 \\
\hline MVDWHO & $10 \%, 13$ & $\% 1 \mathrm{gr}$ & Wros & $2 \pi, 30$ & $\times 1031$ & mas & (WI & 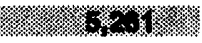 \\
\hline 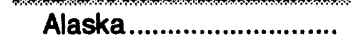 & 0 & 2,100 & 2,500 & 4,000 & 0 & 0 & 13 & 15 \\
\hline 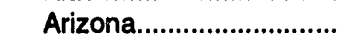 & 0 & 0 & 1,200 & 0 & 0 & 0 & 0 & 0 \\
\hline 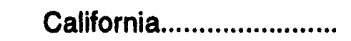 & 118,900 & 0 & 72,663 & 13,600 & 31,800 & 94,512 & 1,057 & 4,866 \\
\hline Hawaii............................ & 5,000 & 0 & 15,950 & 3,200 & 0 & 0 & 21 & 0 \\
\hline 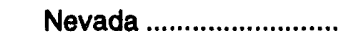 & 0 & 0 & 1,000 & 0 & 0 & 0 & 0 & 0 \\
\hline 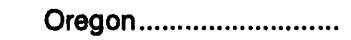 & 0 & 0 & 11,250 & 0 & 0 & 0 & 0 & 0 \\
\hline 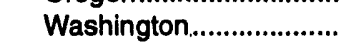 & 28,200 & 0 & 15,200 & 4,000 & 0 & 22,500 & 90 & 380 \\
\hline A & $100000 \%$ & 276,26 & $10 / 200$ & 20210 & $21,16 \%$ & nowo & 2.0 & $2 \%+6 \%$ \\
\hline 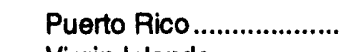 & 0 & 15,000 & 1,000 & 0 & 9,500 & 0 & 26 & 69 \\
\hline Virgin Islands .................. & 20,000 & 20,000 & 0 & 18,000 & 0 & 0 & 0 & 600 \\
\hline
\end{tabular}

MMcfd = Million cubic feet per day.

Source: Energy Information Administration (EIA), Form EIA-820, "Annual Refinery Report." 


\begin{tabular}{|c|c|}
\hline Refiner & Page(s) \\
\hline 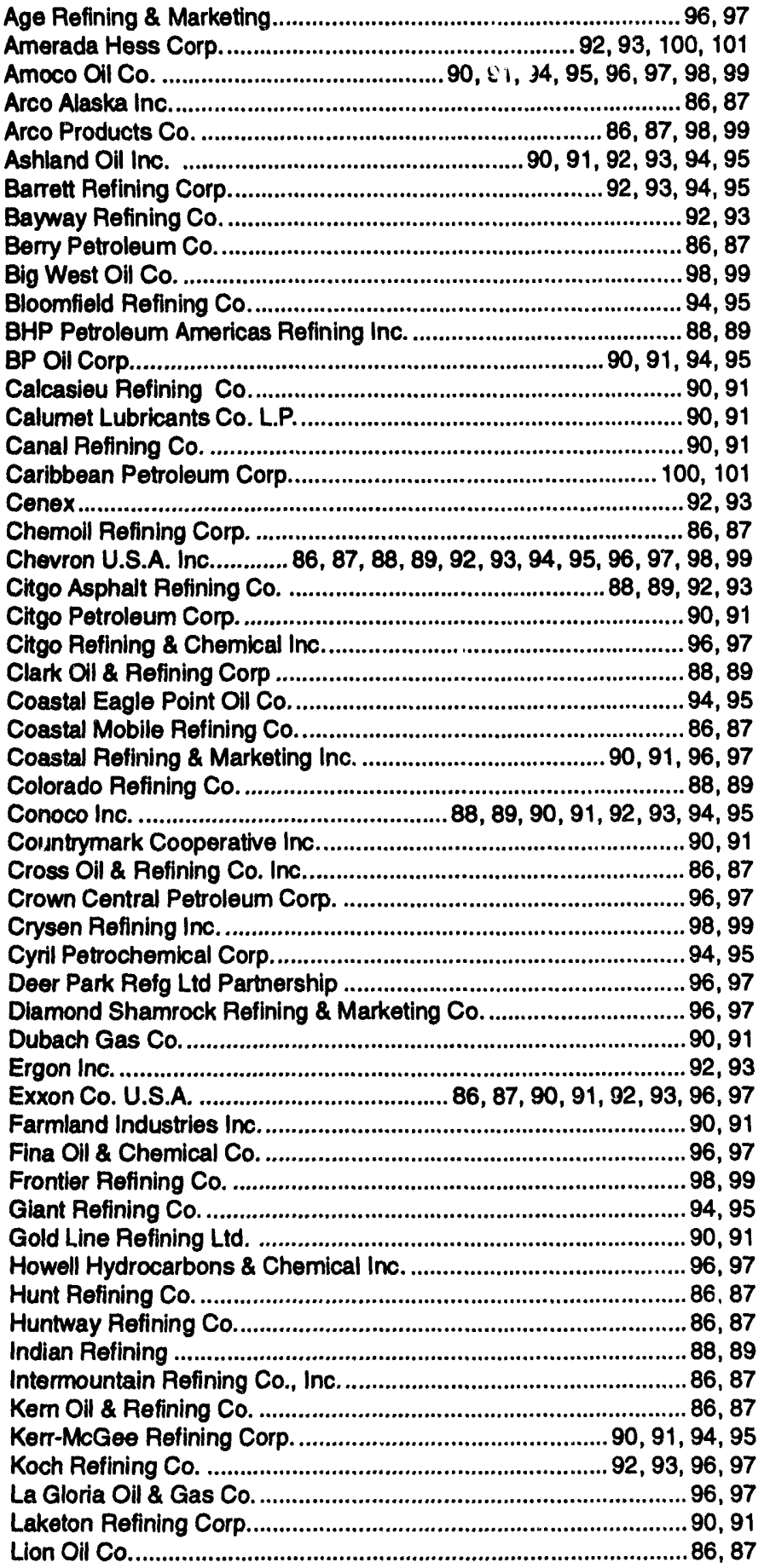 & 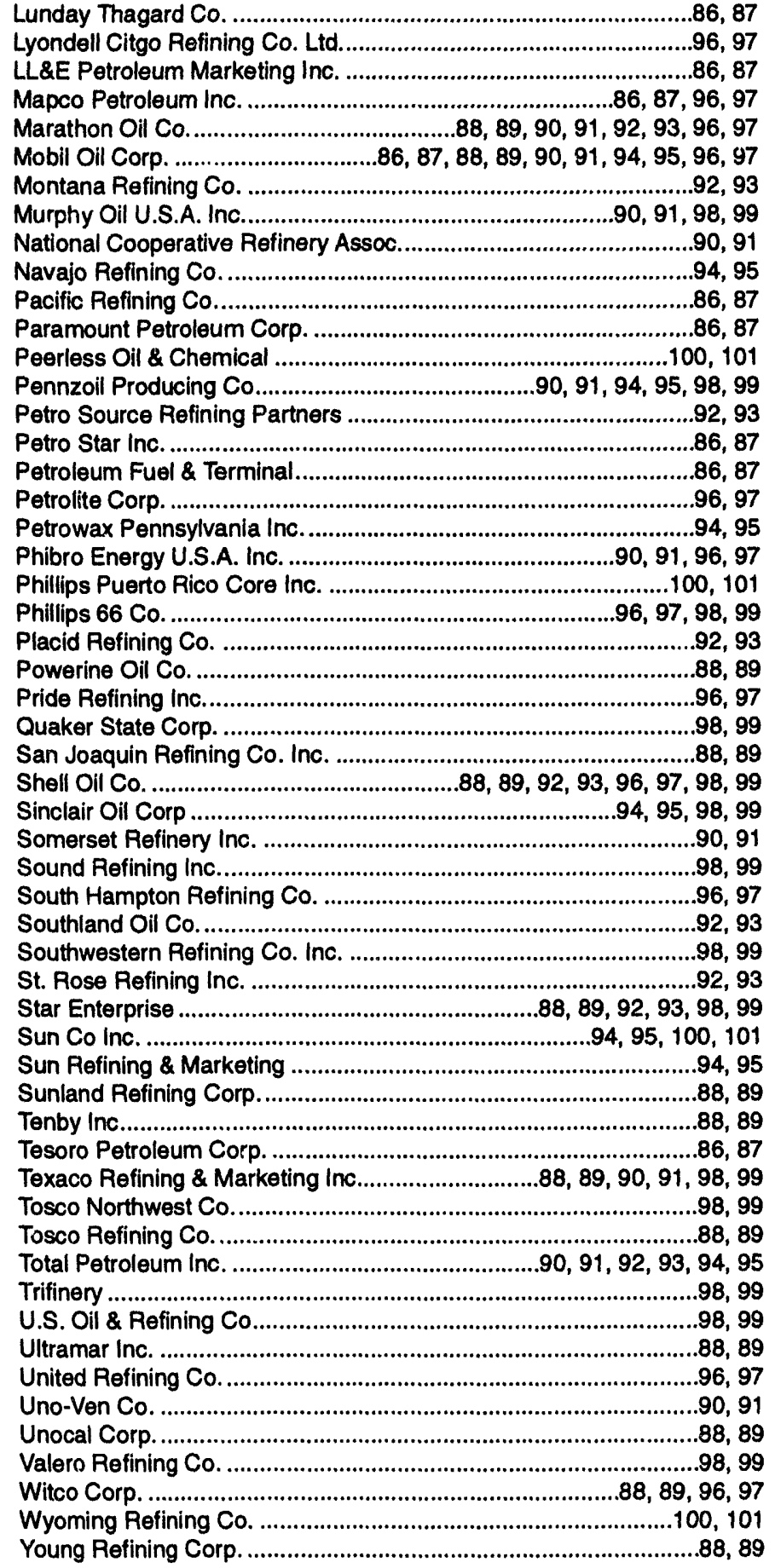 \\
\hline
\end{tabular}

Little America Refining Co.

$.98,99$ 
Table 38. Capacity of Operable Petroleum Refineries by State as of January 1, 1994 (Barrels per Stream Day, Except Where Noted)

\begin{tabular}{|c|c|c|c|c|c|c|c|c|c|}
\hline \multirow[b]{4}{*}{ StateRefiner/Rocation } & \multicolumn{4}{|c|}{ Atmospherle Crude Oll Distlllation Capacity } & \multicolumn{5}{|c|}{ Downstream Cherge Capecily } \\
\hline & \multirow{2}{*}{\multicolumn{2}{|c|}{$\begin{array}{l}\text { Barrels por } \\
\text { Calondar Day }\end{array}$}} & \multirow{2}{*}{\multicolumn{2}{|c|}{$\begin{array}{l}\text { Barrels per } \\
\text { Stream Day }\end{array}$}} & \multirow{3}{*}{$\begin{array}{l}\text { Vacuum } \\
\text { Distlliation }\end{array}$} & \multicolumn{4}{|c|}{ Thermal Cracking } \\
\hline & & & & & & \multirow{2}{*}{$\begin{array}{l}\text { Dolayed } \\
\text { Colkng }\end{array}$} & \multirow[b]{2}{*}{ Fluld Coking } & \multirow[b]{2}{*}{ Visbreaking } & \multirow{2}{*}{$\begin{array}{l}\text { Otherl } \\
\text { Cas Olf } \\
\end{array}$} \\
\hline & Operating & Idle & Operating & Idle & & & & & \\
\hline \multicolumn{5}{|c|}{ N. } & 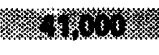 & (x): & 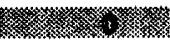 & (2) & \% \\
\hline \multirow{3}{*}{ 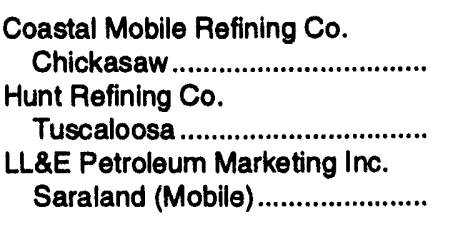 } & 16,500 & 0 & 18,000 & 0 & 14,000 & 0 & 0 & 0 & 0 \\
\hline & 33,500 & 0 & 35,000 & 0 & 15,000 & 12,000 & 0 & 0 & 0 \\
\hline & 71,000 & 0 & 74,000 & 0 & 12,000 & 0 & 0 & 0 & 0 \\
\hline 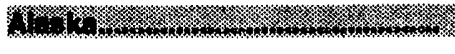 & 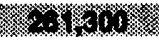 & sis & 然 & (3) & (2) & 策 & 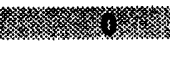 & 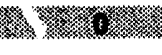 & 㭴 \\
\hline $\begin{array}{l}\text { Arco Alaska Inc. } \\
\text { Kuparuk.................................... } \\
\text { Prudhoe Bay ........................... } \\
\text { Mapco Petroleum Inc. }\end{array}$ & $\begin{array}{l}12,000 \\
15,000\end{array}$ & $\begin{array}{l}0 \\
0\end{array}$ & $\begin{array}{l}14,500 \\
16,000\end{array}$ & $\begin{array}{l}0 \\
0\end{array}$ & $\begin{array}{l}0 \\
0\end{array}$ & $\begin{array}{l}0 \\
0\end{array}$ & $\begin{array}{l}0 \\
0\end{array}$ & $\begin{array}{l}0 \\
0\end{array}$ & $\begin{array}{l}0 \\
0\end{array}$ \\
\hline $\begin{array}{l}\text { North Pole } \\
\text { Petro Star Inc. }\end{array}$ & 128,000 & 0 & 130,000 & 0 & 6,000 & 0 & 0 & 0 & 0 \\
\hline 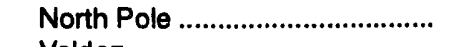 & 8,000 & 0 & 8,225 & 0 & 0 & 0 & 0 & 0 & 0 \\
\hline $\begin{array}{l}\text { Valdez } \\
\text { Tesoro Petroleum Corp. }\end{array}$ & 26,300 & 0 & 27,040 & 0 & 0 & 0 & 0 & 0 & 0 \\
\hline 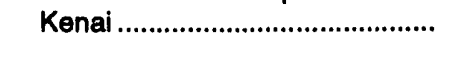 & 72,000 & 0 & 80,000 & 0 & 0 & 0 & 0 & 0 & 0 \\
\hline \multicolumn{3}{|c|}{ 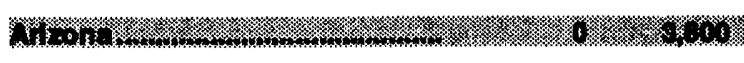 } & (2) & Whe & 样 & a. & 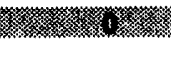 & 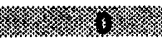 & 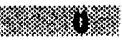 \\
\hline 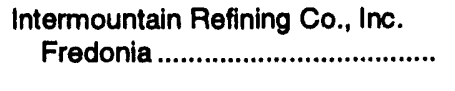 & 0 & 3,800 & 0 & 5,700 & 2,000 & 0 & 0 & 0 & 0 \\
\hline 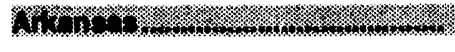 & 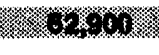 & 6. & 2014 & (6) & 28.43 & 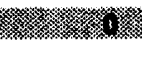 & (2) & 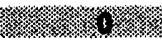 & (1) \\
\hline $\begin{array}{l}\text { Berry Petroleum Co. } \\
\text { Stephens...................................... } \\
\text { Cross Oil \& Refining Co. Inc. }\end{array}$ & 6,700 & 0 & 7,000 & 0 & 1,800 & 0 & 0 & 0 & 0 \\
\hline 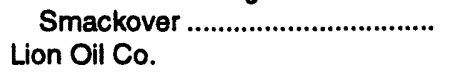 & 6,200 & 0 & 7,000 & 0 & 3,920 & 0 & 0 & 0 & 0 \\
\hline 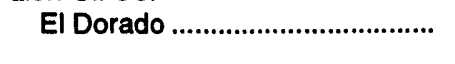 & 50,000 & 0 & 52,000 & 0 & 18,500 & 0 & 0 & 0 & 0 \\
\hline (m) & $10, m, 16$ & Q6m, & 20006 & \% & Whman & x.ming & 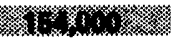 & 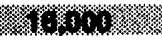 & (6) \\
\hline $\begin{array}{l}\text { Arco Products Co. } \\
\text { Los Angeles................................ } \\
\text { Chemoil Refining Corp. }\end{array}$ & 237,000 & 0 & 242,000 & 0 & 115,500 & 0 & 58,000 & 0 & 0 \\
\hline $\begin{array}{l}\text { Long Beach ........................................... } \\
\text { Chevron U.S.A. }\end{array}$ & 18,000 & 0 & 20,000 & 0 & 0 & 0 & 0 & 0 & 0 \\
\hline 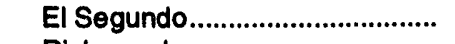 & 226,000 & 0 & 263,000 & 0 & 122,000 & 66,000 & 0 & 0 & 0 \\
\hline 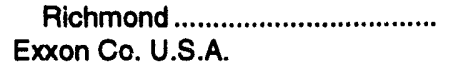 & 235,000 & 0 & 248,000 & 0 & 115,000 & 0 & 0 & 0 & 0 \\
\hline $\begin{array}{l}\text { Benicia .................................... } \\
\text { Huntway Refining Co. }\end{array}$ & 128,000 & 0 & 132,000 & 0 & 67,000 & 0 & 27,500 & 0 & 0 \\
\hline 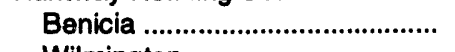 & 8,600 & 0 & 9,000 & 0 & 8,000 & 0 & 0 & 0 & 0 \\
\hline $\begin{array}{l}\text { Wilmington ................................. } \\
\text { Kern Oil \& Refining Co. }\end{array}$ & 5,500 & 0 & 6,000 & 0 & 5,500 & 0 & 0 & 0 & 0 \\
\hline 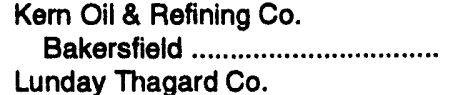 & 21,400 & 0 & 23,000 & 0 & 0 & 0 & 0 & 0 & 0 \\
\hline 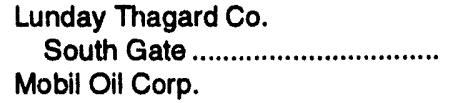 & 8,100 & 0 & 8,500 & 0 & 7,000 & 0 & 0 & 0 & 0 \\
\hline $\begin{array}{l}\text { Torrance .................................... } \\
\text { Pacific Refining Co. }\end{array}$ & 130,000 & 0 & 140,000 & 0 & 101,000 & 48,000 & 0 & 0 & 0 \\
\hline $\begin{array}{l}\text { Hercules .................................. } \\
\text { Paramount Petroleum Corp. }\end{array}$ & 0 & 50,000 & 0 & 55,000 & 25,000 & 0 & 0 & 11,000 & 0 \\
\hline $\begin{array}{l}\text { Paramount................................... } \\
\text { Petroleum Fuel \& Terminal" }\end{array}$ & 46,500 & 0 & 46,500 & 0 & 28,000 & 0 & 0 & 0 & 0 \\
\hline 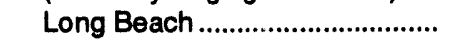 & 15,000 & 0 & 15,000 & 0 & 0 & 0 & 0 & 0 & 0 \\
\hline
\end{tabular}


Table 38. Capacity of Operable Petroleum Refineries by State as of January 1, 1994 (Continued) (Barrels per Stream Day, Except Where Noted)

\begin{tabular}{|c|c|c|c|c|c|c|c|c|c|c|}
\hline \multirow[b]{3}{*}{ Locution } & \multicolumn{10}{|c|}{ Downstream Charge Capacity (Continued) } \\
\hline & \multicolumn{2}{|c|}{ Catalytic Cracking } & \multirow[b]{2}{*}{$\begin{array}{c}\text { Catalytic } \\
\text { Hydrocracking }\end{array}$} & \multicolumn{2}{|c|}{ Catalytic Roforming } & \multicolumn{4}{|c|}{ Catalytic Hydrotreating } & \multirow{2}{*}{$\begin{array}{c}\text { Fuel } \\
\text { Solvents } \\
\text { Deasphalting }\end{array}$} \\
\hline & Fresh & Recyclod & & $\begin{array}{l}\text { Low } \\
\text { Pressure }\end{array}$ & $\begin{array}{c}\text { High } \\
\text { Pressure }\end{array}$ & $\begin{array}{l}\text { Heavy } \\
\text { Gas OII }\end{array}$ & $\begin{array}{l}\text { Naphtha } \\
\text { Relormer Feed }\end{array}$ & Distillate & $\begin{array}{l}\text { Otherl } \\
\text { Restlual }\end{array}$ & \\
\hline
\end{tabular}

Chickasaw.

$\begin{array}{ll}0 & 0 \\ 0 & 0\end{array}$

$$
0
$$

Tuscaloosa.

$\begin{array}{ll}0 & 0 \\ 0 & 0\end{array}$

$0 \quad 7,200$

Saraland (Mobile).

\title{
$\begin{array}{lr}0 & 0 \\ \omega & \end{array}$
}

\section{nh . .}

\begin{tabular}{|c|c|c|c|c|c|c|c|c|c|}
\hline Kuparuk & $\begin{array}{l}0 \\
0\end{array}$ & $\begin{array}{l}0 \\
0\end{array}$ & $\begin{array}{l}0 \\
0\end{array}$ & $\begin{array}{l}0 \\
0\end{array}$ & $\begin{array}{l}0 \\
0\end{array}$ & $\begin{array}{l}0 \\
0\end{array}$ & $\begin{array}{l}0 \\
0\end{array}$ & $\begin{array}{l}0 \\
0\end{array}$ & $\begin{array}{l}0 \\
0\end{array}$ \\
\hline 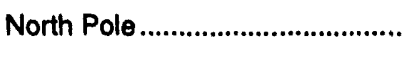 & 0 & 0 & 0 & 0 & 0 & 0 & 0 & 0 & 0 \\
\hline $\begin{array}{l}\text { North Pole } \\
\text { Valdez }\end{array}$ & $\begin{array}{l}0 \\
0\end{array}$ & $\begin{array}{l}0 \\
0\end{array}$ & $\begin{array}{l}0 \\
0\end{array}$ & $\begin{array}{l}0 \\
0\end{array}$ & $\begin{array}{l}0 \\
0\end{array}$ & $\begin{array}{l}0 \\
0\end{array}$ & $\begin{array}{l}0 \\
0\end{array}$ & $\begin{array}{l}0 \\
0\end{array}$ & $\begin{array}{l}0 \\
0\end{array}$ \\
\hline 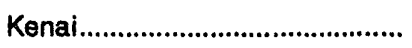 & 0 & 0 & 9,150 & 12,000 & 0 & 0 & 12,000 & 0 & 0 \\
\hline
\end{tabular}

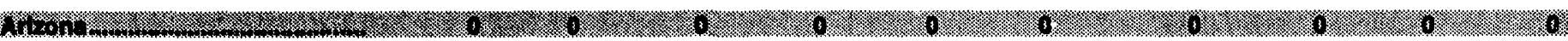

Fredonia.

$0 \quad 0$

$0 \quad 0$

\section{F⿻上丨า}

\begin{tabular}{|c|c|c|c|c|c|c|c|c|c|}
\hline Stephens & 0 & 0 & 0 & 0 & 0 & 0 & 0 & 0 & 0 \\
\hline 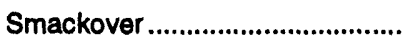 & 0 & 0 & 0 & 0 & 0 & 0 & 0 & 0 & 4,000 \\
\hline 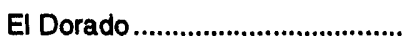 & 19,100 & 0 & 0 & 12,000 & 0 & 0 & 20,000 & 7,000 & 0 \\
\hline
\end{tabular}

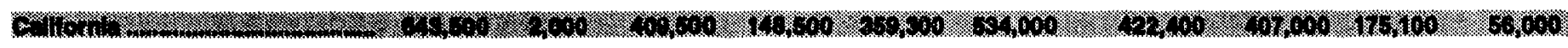

\begin{tabular}{|c|c|c|c|c|c|c|c|c|c|c|}
\hline Los Angeles .................................... & 93,000 & 0 & 38,000 & 0 & 50,000 & 85,000 & 39,000 & 17,000 & 15,700 & 0 \\
\hline 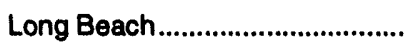 & 0 & 0 & 0 & 0 & 0 & 0 & 0 & 0 & 0 & 0 \\
\hline 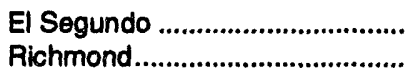 & $\begin{array}{l}65,000 \\
67,000\end{array}$ & $\begin{array}{l}0 \\
0\end{array}$ & $\begin{array}{l}45,000 \\
95,000\end{array}$ & $\begin{array}{r}27,000 \\
0\end{array}$ & $\begin{array}{l}24,000 \\
62,000\end{array}$ & $\begin{array}{r}65,000 \\
0\end{array}$ & $\begin{array}{l}56,000 \\
55,000\end{array}$ & $\begin{array}{r}74,000 \\
110,000\end{array}$ & $\begin{array}{r}0 \\
81,000\end{array}$ & $\begin{array}{r}0 \\
56,000\end{array}$ \\
\hline 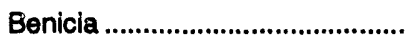 & 67,000 & 0 & 32,000 & 0 & 32,000 & 37,000 & 49,000 & 25,000 & 17,000 & 0 \\
\hline 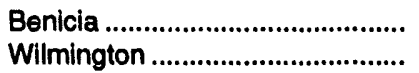 & $\begin{array}{l}0 \\
0\end{array}$ & 0 & $\begin{array}{l}0 \\
0\end{array}$ & $\begin{array}{l}0 \\
0\end{array}$ & $\begin{array}{l}0 \\
0\end{array}$ & $\begin{array}{l}0 \\
0\end{array}$ & $\begin{array}{l}0 \\
0\end{array}$ & $\begin{array}{l}0 \\
0\end{array}$ & $\begin{array}{l}0 \\
0\end{array}$ & $\begin{array}{l}0 \\
0\end{array}$ \\
\hline 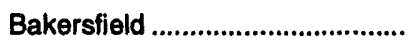 & 0 & 0 & 0 & 0 & 2,800 & 0 & 5,000 & 7,300 & 0 & \\
\hline 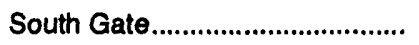 & 0 & 0 & 0 & 0 & 0 & 0 & 0 & 0 & 0 & \\
\hline 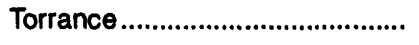 & 82,000 & 0 & 22,400 & 0 & 40,000 & 93,500 & 23,000 & 28,000 & 0 & \\
\hline 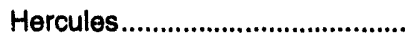 & 4,000 & 0 & 0 & 0 & 11,200 & 0 & 11,200 & 0 & 0 & \\
\hline Paramount & 0 & 0 & 0 & 0 & 11,500 & 0 & 0 & 7,000 & 21,500 & \\
\hline Beach....................................... & 0 & 0 & 0 & 0 & 0 & 0 & 0 & 0 & 0 & \\
\hline
\end{tabular}


Table 38. Capacity of Operable Petroleum Refineries by State as of January 1, 1994 (Continued) (Barrels per Stream Day, Except Where Noted)

\begin{tabular}{|c|c|c|c|c|c|c|c|c|c|}
\hline \multirow[b]{4}{*}{ State/Refiner/Location } & \multicolumn{4}{|c|}{ Atmospheric Crude Cll Distillation Capacity } & \multicolumn{5}{|c|}{ Downstream Charge Capacliy } \\
\hline & \multirow{2}{*}{\multicolumn{2}{|c|}{$\begin{array}{l}\text { Barrels per } \\
\text { Calendar Day }\end{array}$}} & \multirow{2}{*}{\multicolumn{2}{|c|}{$\begin{array}{l}\text { Barrels per } \\
\text { Stream Day }\end{array}$}} & \multirow{3}{*}{$\begin{array}{c}\text { Vacuum } \\
\text { Distillation }\end{array}$} & \multicolumn{4}{|c|}{ Thermal Crecking } \\
\hline & & & & & & \multirow{2}{*}{$\begin{array}{l}\text { Delayed } \\
\text { Cokhng }\end{array}$} & \multirow{2}{*}{ Fluid Coking } & \multirow{2}{*}{ Visbreaking } & \multirow{2}{*}{$\begin{array}{l}\text { Otherl } \\
\text { Gas OII }\end{array}$} \\
\hline & Operating & Idle & Operating & Idle & & & & & \\
\hline 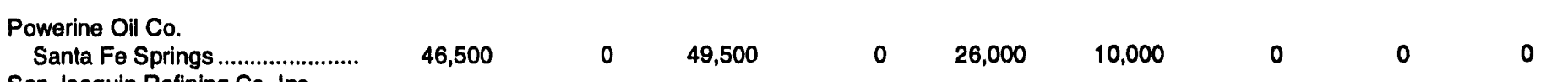 & 46,500 & 0 & 49,500 & 0 & 26,000 & 10,000 & 0 & 0 & 0 \\
\hline 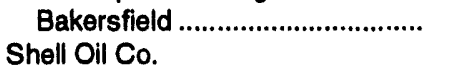 & 24,300 & 0 & 27,000 & 0 & 12,000 & 0 & 0 & 5,000 & 0 \\
\hline $\begin{array}{l}\text { Martinez } \\
\text { Sunland Refining Corp. }\end{array}$ & 147,100 & 0 & 150,100 & 0 & 101,900 & 0 & 22,500 & 0 & 0 \\
\hline 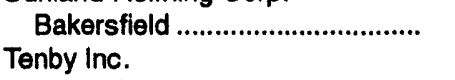 & 12,000 & 0 & 17,000 & 0 & 0 & 0 & 0 & 0 & 0 \\
\hline 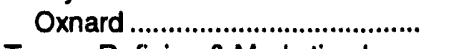 & 4,000 & 0 & 5,000 & 0 & 0 & 0 & 0 & 0 & 0 \\
\hline \multicolumn{10}{|l|}{ Texaco Refining \& Marketing Inc. } \\
\hline 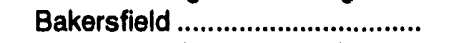 & 54,000 & 0 & 58,000 & 0 & 34,000 & 21,000 & 0 & 0 & 0 \\
\hline Wilmington (Los Angeles) .......... & 64,000 & 0 & 70,000 & 0 & 54,000 & 75,000 & 0 & 0 & 0 \\
\hline \multicolumn{10}{|l|}{ Tosco Refining Co. } \\
\hline $\begin{array}{l}\text { Martinez (Avon) } \\
\text { Uitramar Inc. }\end{array}$ & 148,000 & 0 & 160,000 & 0 & 102,000 & \multicolumn{3}{|c|}{ Uitramar Inc. } & 0 \\
\hline $\begin{array}{l}\text { Wilmington } \\
\text { Unocal Corp. }\end{array}$ & 68,000 & 0 & 70,000 & 0 & 40,000 & 23,000 & 0 & 0 & 0 \\
\hline Arroyo Grande (Santa Maria).... & 40,745 & 0 & 44,000 & 0 & 32,000 & 23,200 & 0 & 0 & 0 \\
\hline Rodeo (San Francisco) ............... & 73,100 & 0 & 77,000 & 0 & 42,100 & 23,700 & 0 & 0 & 0 \\
\hline Wilmington (Los Angeles) .......... & 121,500 & 0 & 125,000 & 0 & 75,000 & 50,000 & 0 & 0 & 0 \\
\hline \multicolumn{10}{|l|}{ Witco Corp. } \\
\hline 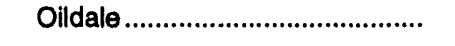 & 0 & 0 & 0 & 0 & 10,000 & 0 & 0 & 0 & 0 \\
\hline 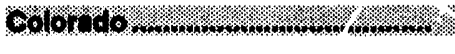 & 8.500 & 0 & 05,000 & $\%$ & 00060 & (1) & 6 & 10 & 0 \\
\hline 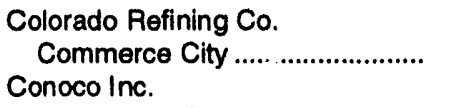 & 28,000 & 0 & 35,000 & 0 & 10,000 & 0 & 0 & 0 & 0 \\
\hline 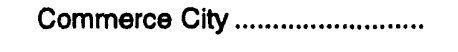 & 57,500 & 0 & 60,000 & 0 & 25,000 & 0 & 0 & 0 & 0 \\
\hline 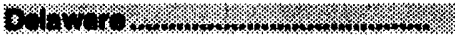 & 120000 & 0 & 152000 & 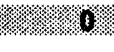 & 060000 & (1) & 20000 & 0 & 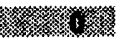 \\
\hline \multicolumn{10}{|l|}{ Star Enterprise } \\
\hline 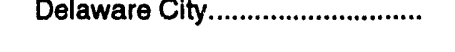 & 140,000 & 0 & 152,000 & 0 & 95,000 & 0 & 45,000 & 0 & 0 \\
\hline 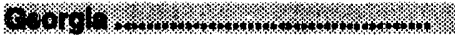 & 5,60 & 20000 & 10000 & 84000 & 0. & 行: & (3) & 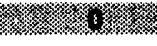 & 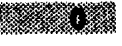 \\
\hline \multicolumn{10}{|l|}{$\begin{array}{l}\text { Citgo Asphalt Refining Co. } \\
\text { (Formerly Amoco Oil Co.) }\end{array}$} \\
\hline $\begin{array}{l}\text { Savannah................................ } \\
\text { Young Refining Corp. }\end{array}$ & 0 & 28,000 & 0 & 32,000 & 0 & 0 & 0 & 0 & 0 \\
\hline 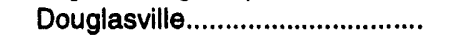 & 5,540 & 0 & 10,000 & 0 & 0 & 0 & 0 & 0 & 0 \\
\hline A & 16.303 & 10. & 16000 & ?. & $2 / 23 s$ & 6. & 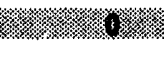 & Sos & 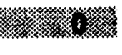 \\
\hline \multicolumn{10}{|c|}{$\begin{array}{l}\text { BHP Petroleum Americas Refining Inc. } \\
\text { (Formerly Hawailan Independent Refining Inc.) }\end{array}$} \\
\hline 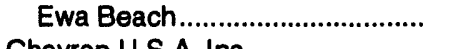 & 93,500 & 0 & 95,000 & 0 & 43,000 & 0 & 0 & 12,400 & 0 \\
\hline Chevron U.S.A. Inc. & & & & & & & & & \\
\hline 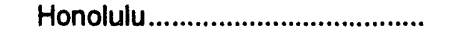 & 52,800 & 0 & 55,000 & 0 & 31,300 & 0 & 0 & 0 & 0 \\
\hline Maro - . & 06000 & 0 & 10028000 & 0 & (2) 6.460 & (1) & 10 & Xar & 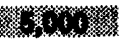 \\
\hline 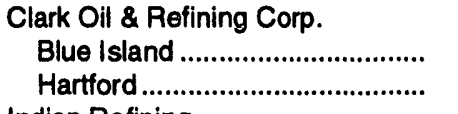 & $\begin{array}{l}71,900 \\
57,000\end{array}$ & $\begin{array}{l}0 \\
0\end{array}$ & $\begin{array}{l}77,000 \\
60,000\end{array}$ & $\begin{array}{l}0 \\
0\end{array}$ & $\begin{array}{l}35,950 \\
30,000\end{array}$ & $\begin{array}{r}0 \\
15,000\end{array}$ & $\begin{array}{l}0 \\
0\end{array}$ & $\begin{array}{l}0 \\
0\end{array}$ & $\begin{array}{l}0 \\
0\end{array}$ \\
\hline \multicolumn{10}{|l|}{ Indian Refining } \\
\hline $\begin{array}{l}\text { Lawrenceville } \\
\text { Marathon Oil Co. }\end{array}$ & 73,000 & 0 & 77,000 & 0 & 23,000 & 0 & 0 & 0 & 0 \\
\hline $\begin{array}{l}\text { Robinson...................................... } \\
\text { Mobil Oil Corp. }\end{array}$ & 175,000 & 0 & 180,000 & 0 & 50,000 & 21,700 & 0 & 0 & 5,000 \\
\hline 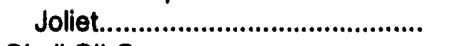 & 180,000 & 0 & 190,000 & 0 & 92,500 & 40,000 & 0 & 0 & 0 \\
\hline Shell Oil Co. & & & & & & & & & \\
\hline Wood River & 252,000 & 0 & 291,000 & 0 & 103,000 & 0 & 0 & 18,000 & 0 \\
\hline
\end{tabular}

See footnotes at end of table. 
Table 38. Capacity of Operable Petroleum Refineries by State as of January 1, 1994 (Continued) (Barrels per Stream Day, Except Where Noted)

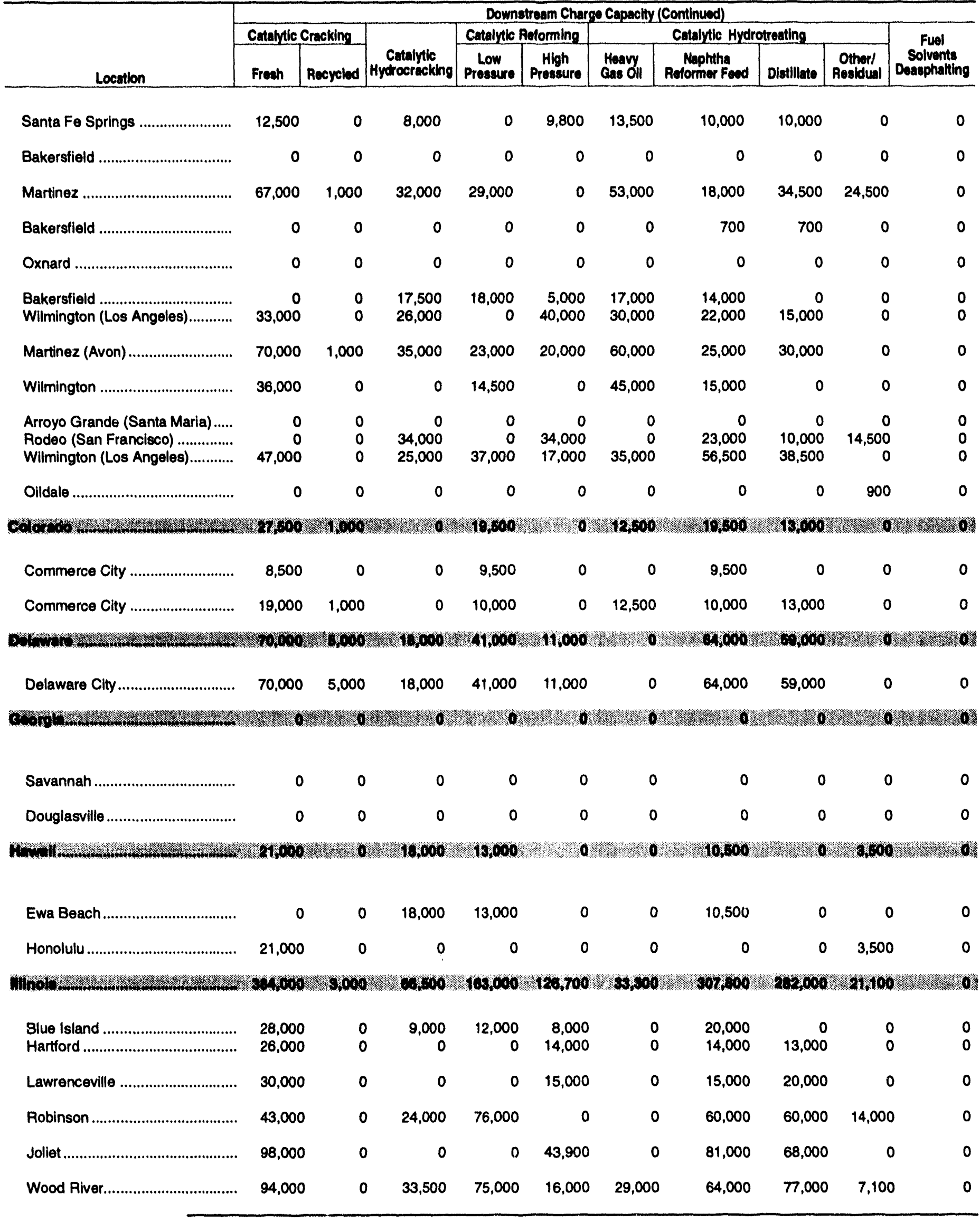

See footnotes at end of table. 
Table 38. Capacity of Operable Petroloum Refineries by State as of January 1, 1994 (Continued) (Barrels per Stream Day, Except Where Noted)

\begin{tabular}{|c|c|c|c|c|c|c|c|c|c|}
\hline \multirow[b]{4}{*}{ State/Roflher/Locatton } & \multicolumn{4}{|c|}{ Almospheric Cruds OII Dietllibtion Capacity } & \multicolumn{5}{|c|}{ Downstream Charge Cepacily } \\
\hline & \multirow{2}{*}{\multicolumn{2}{|c|}{$\begin{array}{l}\text { Berrels par } \\
\text { Calondar Day }\end{array}$}} & \multirow{2}{*}{\multicolumn{2}{|c|}{$\begin{array}{l}\text { Barrols por } \\
\text { Stream Day }\end{array}$}} & \multirow{3}{*}{$\begin{array}{c}\text { Vacuum } \\
\text { Distillation }\end{array}$} & \multicolumn{4}{|c|}{ Thermal Cracking } \\
\hline & & & & & & \multirow{2}{*}{$\begin{array}{l}\text { Dolayod } \\
\text { Colking }\end{array}$} & \multirow[b]{2}{*}{ Fluid Coking } & \multirow[b]{2}{*}{ Vlebreaking } & \multirow{2}{*}{$\begin{array}{l}\text { Othall } \\
\text { Gas OII }\end{array}$} \\
\hline & Operting & Idle & Oporathg & Idle & & & & & \\
\hline $\begin{array}{l}\text { Uno-Ven Co. } \\
\text { Lemont (Chicago) .......................... }\end{array}$ & 147,000 & 0 & 153,000 & 0 & 62,000 & 29,100 & 0 & 0 & 0 \\
\hline hatan & stonts & 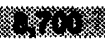 & (4) & 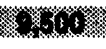 & 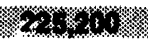 & $2014 s$ & 3 & 样 & 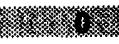 \\
\hline $\begin{array}{l}\text { Amoco Oil Co. } \\
\text { Whiting.................................... } \\
\text { Countrymark Cooperative Inc. }\end{array}$ & 400,000 & 0 & 410,000 & 0 & 212,000 & 28,000 & 0 & 0 & 0 \\
\hline $\begin{array}{l}\text { Mount Vernon ................................ } \\
\text { Laketon Refining Corp. }\end{array}$ & 21,200 & 0 & 22,600 & 0 & 7,200 & 0 & 0 & 0 & 0 \\
\hline 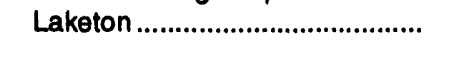 & 0 & 8,700 & 0 & 9,500 & 6,000 & 0 & 0 & 0 & 0 \\
\hline 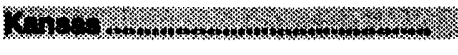 & 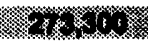 & 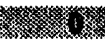 & 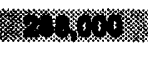 & (x) & st 1 & 然 & 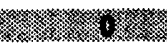 & 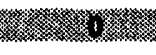 & 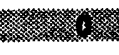 \\
\hline 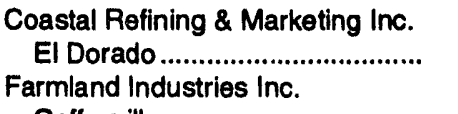 & 0 & 0 & 0 & 0 & 15,000 & 0 & 0 & 0 & 0 \\
\hline $\begin{array}{l}\text { Coffeyville .............................. } \\
\text { National Cooperative Refinery Assoc. }\end{array}$ & 61,700 & 0 & 63,000 & 0 & 28,000 & 14,000 & 0 & 0 & 0 \\
\hline $\begin{array}{l}\text { McPherson............................. } \\
\text { Texaco Refining \& Marketing Inc. }\end{array}$ & 75,600 & 0 & 80,000 & 0 & 27,000 & 22,000 & 0 & 0 & 0 \\
\hline 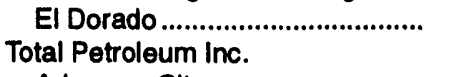 & 80,000 & 0 & 85,000 & 0 & 33,000 & 12,500 & 0 & 0 & 0 \\
\hline 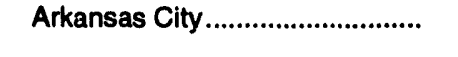 & 56,000 & 0 & 60,000 & 0 & 16,150 & 0 & 0 & 0 & 0 \\
\hline Rura & 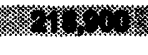 & & 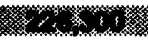 & , & oms & wa & we & & \\
\hline 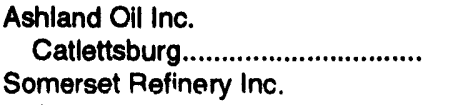 & 213,400 & 0 & 220,000 & 0 & 92,000 & 0 & 0 & 4,500 & 55,000 \\
\hline 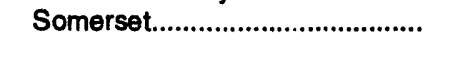 & 5,500 & 0 & 6,300 & 0 & 0 & 0 & 0 & 0 & 0 \\
\hline 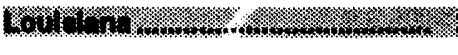 & $\left.x^{2}-1\right)$ & & $x$ & 10 & 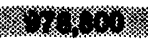 & 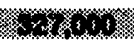 & r & 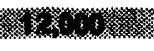 & XXrs \\
\hline $\begin{array}{l}\text { BP Oil Corp. } \\
\text { Belle Chasse (Alliance) .............. } \\
\text { Calcasieu Refining Co. }\end{array}$ & 223,300 & 0 & 230,000 & 0 & 89,000 & 24,000 & 0 & 0 & 0 \\
\hline $\begin{array}{l}\text { Lake Charles................................ } \\
\text { Calumet Lubricants Co. L.P. }\end{array}$ & 12,500 & 0 & 13,500 & 0 & 0 & 0 & 0 & 0 & 0 \\
\hline $\begin{array}{l}\text { Princeton ............................................ } \\
\text { Canal Refining Co. }\end{array}$ & 6,000 & 0 & 6,443 & 0 & 6,000 & 0 & 0 & 0 & 0 \\
\hline $\begin{array}{l}\text { Church Point .................................... } \\
\text { Citgo Petroleum Corp. }\end{array}$ & 9,500 & 0 & 10,000 & 0 & 0 & 0 & 0 & 0 & 0 \\
\hline 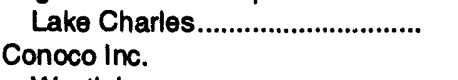 & 305,000 & 0 & 320,000 & 0 & 83,000 & 94,000 & 0 & 0 & 0 \\
\hline 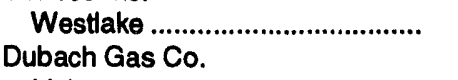 & 182,000 & 0 & 189,000 & 0 & 87,800 & 65,000 & 0 & 0 & 11,500 \\
\hline $\begin{array}{l}\text { Lisbon } \\
\text { Exxon Co. U.S.A. }\end{array}$ & 7,350 & 0 & 7,500 & 0 & 0 & 0 & 0 & 0 & 0 \\
\hline $\begin{array}{l}\text { Baton Rouge ................................. } \\
\text { Gold Line Refining Ltd. }\end{array}$ & 424,000 & 0 & 441,000 & 0 & 186,000 & 90,000 & 0 & 0 & 0 \\
\hline $\begin{array}{l}\text { Lake Charles............................... } \\
\text { Kerr-McGee Refining Corp. }\end{array}$ & 27,600 & 0 & 30,000 & 0 & 10,000 & 0 & 0 & 0 & 0 \\
\hline 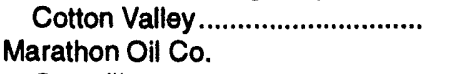 & 7,800 & 0 & 8,500 & 0 & 0 & 0 & 0 & 0 & 0 \\
\hline $\begin{array}{l}\text { Garyville ........................................... } \\
\text { Mobil Oil Corp. }\end{array}$ & 255,000 & 0 & 263,000 & 0 & 125,000 & 0 & 0 & 0 & 0 \\
\hline 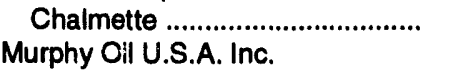 & 170,000 & 0 & 177,800 & 0 & 92,500 & 33,000 & 0 & 0 & 0 \\
\hline $\begin{array}{l}\text { Meraux................................... } \\
\text { Pennzoil Producing Co. }\end{array}$ & 95,000 & 0 & 100,000 & 0 & 40,000 & 0 & 0 & 0 & 0 \\
\hline $\begin{array}{l}\text { Shreveport ......................... } \\
\text { Phibro Energy U.S.A. Inc. }\end{array}$ & 46,200 & 0 & 50,000 & 0 & 23,000 & 0 & 0 & 0 & 0 \\
\hline 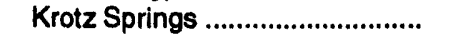 & 60,000 & 0 & 61,500 & 0 & 22,000 & 0 & 0 & 0 & 0 \\
\hline
\end{tabular}


Table 38. Capacity of Operable Petroleum Refineries by State as of January 1, 1994 (Continued) (Barrels per Stream Day, Except Where Noted)

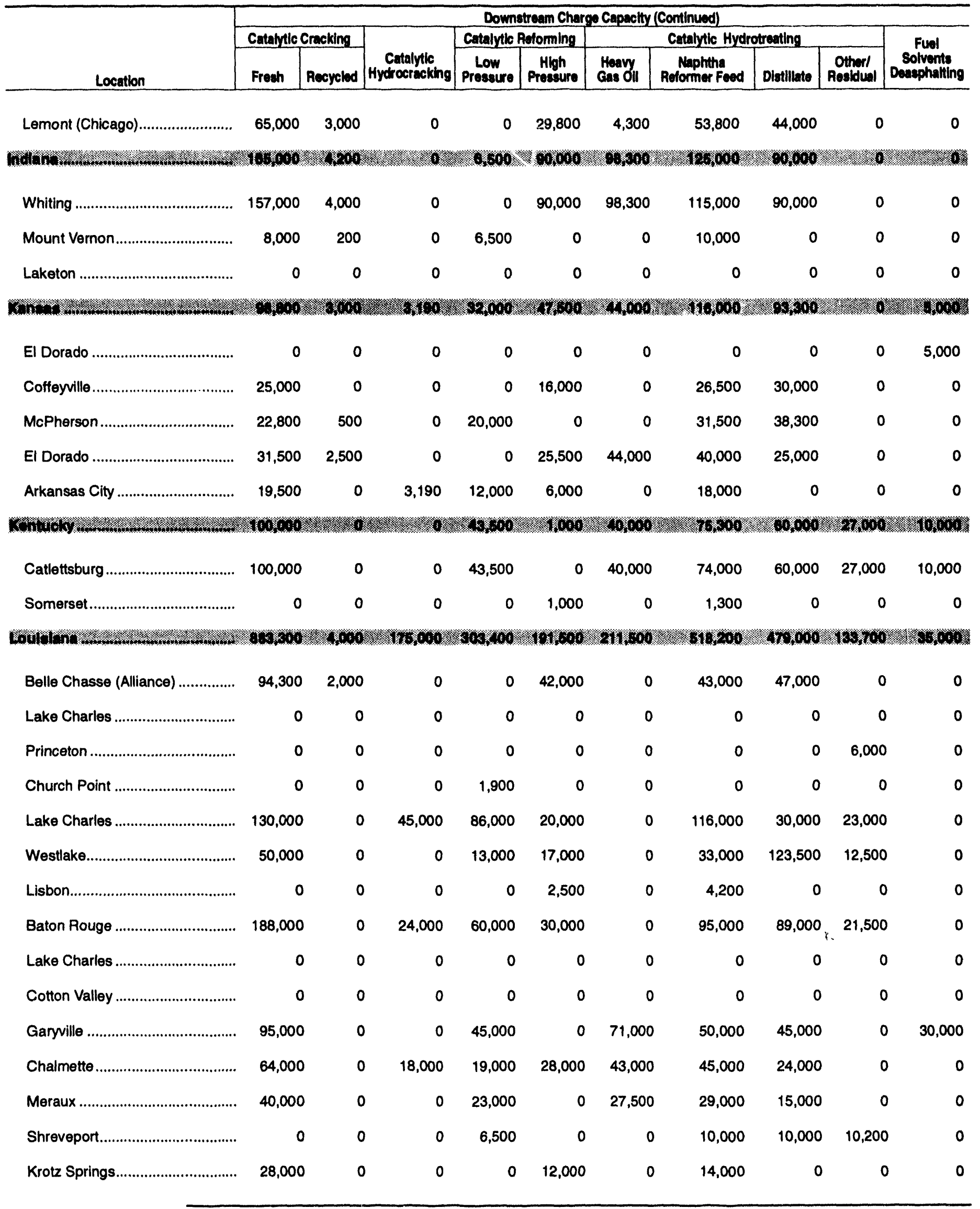


Table 38. Capacity of Operable Petroleum Refineries by State as of January 1, 1994 (Continued) (Barrels per Stream Day, Except Where Noted)

\begin{tabular}{|c|c|c|c|c|c|c|c|c|c|}
\hline \multirow[b]{4}{*}{ State/Rofiner/Location } & \multicolumn{4}{|c|}{ Amospheric Crude Oll Distillation Capacity } & \multicolumn{5}{|c|}{ Downstream Charge Capacliy } \\
\hline & \multirow{2}{*}{\multicolumn{2}{|c|}{$\begin{array}{l}\text { Barrols per } \\
\text { Calendar Day }\end{array}$}} & \multirow{2}{*}{\multicolumn{2}{|c|}{$\begin{array}{l}\text { Barrels per } \\
\text { Stream Day }\end{array}$}} & \multirow{3}{*}{$\begin{array}{l}\text { Vacuum } \\
\text { Distlllation }\end{array}$} & \multicolumn{4}{|c|}{ Thermal Cracking } \\
\hline & & & & & & \multirow{2}{*}{$\begin{array}{l}\text { Dolayed } \\
\text { Cokng }\end{array}$} & \multirow[b]{2}{*}{ Fuld Coking } & \multirow[b]{2}{*}{ Visbroakding } & \multirow{2}{*}{$\begin{array}{l}\text { Othorl } \\
\text { Cas OII }\end{array}$} \\
\hline & Operating & Idle & Operating & Idle & & & & & \\
\hline \multicolumn{10}{|c|}{$\begin{array}{l}\text { Placid Refining Co. } \\
\text { Port Allen.................................. } \\
\text { Shell Oil Co. } \\
\text { Norco }\end{array}$} \\
\hline $\begin{array}{l}\text { Norco } \\
\text { St. Rose Refining Inc. } \\
\text { (Formerly Phibro Energy U.S.A. Inc }\end{array}$ & $\begin{array}{l}215,000 \\
\text { c.) }\end{array}$ & 0 & 220,000 & 0 & 78,000 & 21,000 & 0 & 0 & 108,000 \\
\hline $\begin{array}{l}\text { St. Rose ...................................... } \\
\text { Star Enterprise }\end{array}$ & 40,000 & 0 & 45,000 & 0 & 18,000 & 0 & 0 & 0 & 0 \\
\hline 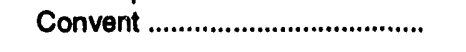 & 225,000 & 0 & 242,500 & 0 & 98,500 & 0 & 0 & 12,000 & 0 \\
\hline N⿴囗十) & (2000 & (1) & (28,100 & 0 & 2600 & 3. & 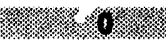 & (2) & 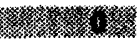 \\
\hline $\begin{array}{l}\text { Marathon Oil Co. } \\
\text { Detroit ......................................... } \\
\text { Total Petroleum Inc. }\end{array}$ & 70,000 & 0 & 72,000 & 0 & 38,000 & 0 & 0 & 0 & 0 \\
\hline Alma................................................ & 45,600 & 0 & 51,000 & 0 & 0 & 0 & 0 & 0 & 0 \\
\hline \multicolumn{10}{|c|}{ Mnnom } \\
\hline 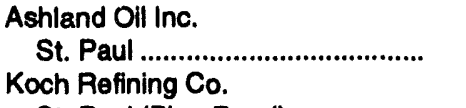 & 67,100 & 0 & 69,220 & 0 & 32,000 & 0 & 0 & 0 & 0 \\
\hline St. Paul (Pine Bend) .................. & 220,000 & 0 & 230,000 & 0 & 150,000 & 61,000 & 0 & 0 & 0 \\
\hline 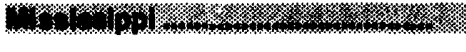 & 610 & 6 & xases: & 20 & 2.08210 & Trows & 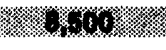 & 2) & 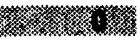 \\
\hline $\begin{array}{l}\text { Amerada Hess Corp. } \\
\text { Purvis...................................... } \\
\text { Barrett Refining Corp. }\end{array}$ & 30,000 & 0 & 32,000 & 0 & 0 & 0 & 8,500 & 0 & 0 \\
\hline $\begin{array}{l}\text { Vicksburg ..................................... } \\
\text { Chevion U.S. }\end{array}$ & 7,000 & 0 & 7,900 & 0 & 0 & 0 & 0 & 0 & 0 \\
\hline $\begin{array}{l}\text { Pascagoula } \\
\text { Ergon Inc. }\end{array}$ & 295,000 & 0 & 310,000 & 0 & 243,000 & 75,000 & 0 & 0 & 0 \\
\hline 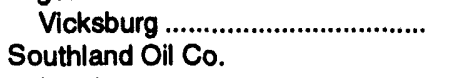 & 23,000 & 0 & 25,000 & 0 & 19,000 & 0 & 0 & 0 & 0 \\
\hline 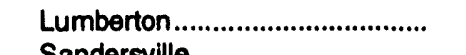 & 5,800 & 0 & 6,500 & 0 & 0 & 0 & 0 & 0 & 0 \\
\hline 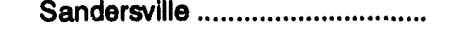 & 11,000 & 0 & 12,500 & 0 & 6,875 & 0 & 0 & 0 & 0 \\
\hline 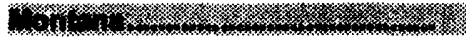 & 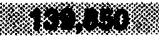 & (6) & Moros. & 10. & ontos & (ass & (1) $x \omega$ & 3. & 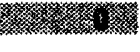 \\
\hline 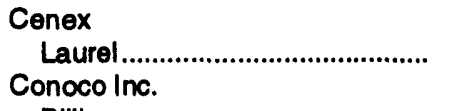 & 41,450 & 0 & 42,500 & 0 & 12,000 & 0 & 0 & 0 & 0 \\
\hline $\begin{array}{l}\text { Billings.................................. } \\
\text { Exxon Co. U.S.A. }\end{array}$ & 49,500 & 0 & 52,000 & 0 & 25,000 & 13,000 & 0 & 0 & 0 \\
\hline $\begin{array}{l}\text { Billings........................................ } \\
\text { Montana Refining Co. }\end{array}$ & 42,000 & 0 & 44,000 & 0 & 18,000 & 0 & 7,700 & 0 & 0 \\
\hline 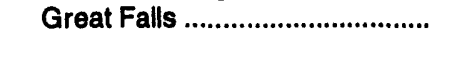 & 6,900 & 0 & 7,200 & 0 & 3,450 & 0 & 0 & 0 & 0 \\
\hline 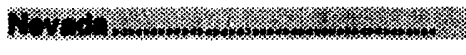 & row & 0 & 7000 & 0 & 63030 & 4 & (3) & 0 & 3. \\
\hline 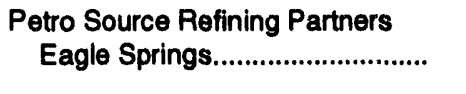 & 7,000 & 0 & 7,000 & 0 & 6,000 & 0 & 0 & 0 & 0 \\
\hline m. & 6000 & 120000 & 0.0000 & $12 \pi 000$ & 20.7.00s: & 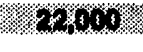 & 6. & (1) & (6) \\
\hline $\begin{array}{l}\text { Amerada Hess Corp. } \\
\text { Port Reading (Sewaren) ............ } \\
\text { Bayway Refining Co. } \\
\text { (Formerly Exxon Co. U.S.A.) }\end{array}$ & 0 & 0 & 0 & 0 & 0 & 0 & 0 & 0 & 0 \\
\hline $\begin{array}{l}\text { Bayway ........................................... } \\
\text { Chevron U.S. }\end{array}$ & 200,000 & 0 & 200,000 & 0 & 65,000 & 0 & 0 & 0 & 0 \\
\hline $\begin{array}{l}\text { Perth Amboy ................................. } \\
\text { Citgo Asphalt Refining Co. }\end{array}$ & 0 & 80,000 & 0 & 83,000 & 47,000 & 0 & 0 & 0 & 0 \\
\hline 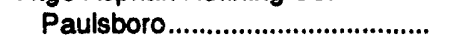 & 0 & 40,000 & 0 & 44,000 & 40,000 & 0 & 0 & 0 & 0 \\
\hline
\end{tabular}

See footnotes at end of table. 
Table 38. Capacity of Operable Petroleum Refineries by State as of January 1, 1994 (Continued) (Barrels per Stream Day, Except Where Noted)

\begin{tabular}{|c|c|c|c|c|c|c|c|c|c|c|}
\hline \multirow[b]{3}{*}{ Location } & \multicolumn{10}{|c|}{ Downstream Charge Capacity (Continued) } \\
\hline & \multicolumn{2}{|c|}{ Cratalytic Cracking } & \multirow[b]{2}{*}{$\begin{array}{c}\text { Cetalytic } \\
\text { Hydrocracking }\end{array}$} & \multicolumn{2}{|c|}{ Catalytic Roforming } & \multicolumn{4}{|c|}{ Catalytic Hydrotreating } & \multirow{2}{*}{$\begin{array}{c}\text { Fual } \\
\text { Solvents } \\
\text { Desesphalting }\end{array}$} \\
\hline & Freas & Rocyolod & & Low & $\begin{array}{c}\text { Hlgh } \\
\text { Pressure }\end{array}$ & $\begin{array}{l}\text { Hoavy } \\
\text { Cas ON }\end{array}$ & $\begin{array}{c}\text { Naphtha } \\
\text { Retormer Fead }\end{array}$ & Distillate & $\begin{array}{c}\text { Otherl } \\
\text { Residual } \\
\end{array}$ & \\
\hline Port Allen & 19,000 & 2,000 & 0 & 10,000 & 0 & 0 & 10,000 & 0 & 0 & 5,000 \\
\hline 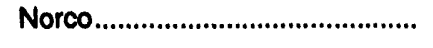 & 90,000 & 0 & 38,000 & 39,000 & 0 & 70,000 & 29,000 & 0 & 28,000 & 0 \\
\hline 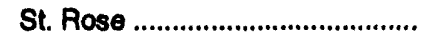 & 0 & 0 & 0 & 0 & 0 & 0 & 0 & 0 & 0 & 0 \\
\hline 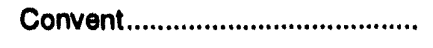 & 85,000 & 0 & 50,000 & 0 & 40,000 & 0 & 40,000 & 95,500 & 32,500 & 0 \\
\hline 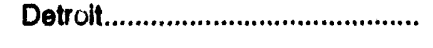 & 27,500 & 1,000 & 0 & 15,000 & 0 & 11,200 & 17,000 & 21,800 & 0 & 0 \\
\hline Alma & 19,500 & 0 & 0 & 15,000 & 0 & 3,800 & 23,000 & 19,500 & 2,000 & 0 \\
\hline 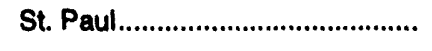 & 23,000 & 0 & 0 & 0 & 23,500 & 23,000 & 32,800 & 20,000 & 0 & 0 \\
\hline 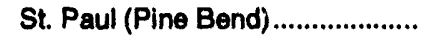 & 65,000 & 0 & 0 & 30,000 & 13,000 & 80,000 & 63,000 & 88,000 & 0 & 0 \\
\hline 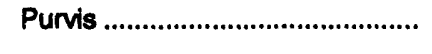 & 16,000 & 7,000 & 0 & 0 & 6,500 & 0 & 6,500 & 6,000 & 0 & 0 \\
\hline 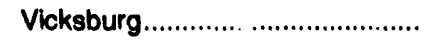 & 0 & 0 & 0 & 0 & 0 & 0 & 0 & 0 & 0 & 0 \\
\hline 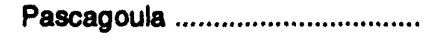 & 68,000 & 0 & 68,000 & 57,000 & 33,000 & 37,000 & 48,000 & 65,000 & 96,000 & the \\
\hline 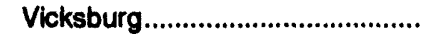 & 0 & 0 & 0 & 0 & 0 & 0 & 0 & 0 & 6,400 & 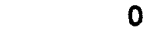 \\
\hline 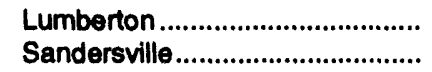 & $\begin{array}{l}0 \\
0\end{array}$ & $\begin{array}{l}0 \\
0\end{array}$ & $\begin{array}{l}0 \\
0\end{array}$ & $\begin{array}{l}0 \\
0\end{array}$ & $\begin{array}{l}0 \\
0\end{array}$ & $\begin{array}{l}0 \\
0\end{array}$ & $\begin{array}{l}0 \\
0\end{array}$ & $\begin{array}{l}0 \\
0\end{array}$ & $\begin{array}{l}0 \\
0\end{array}$ & 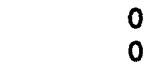 \\
\hline
\end{tabular}

46.

\begin{tabular}{|c|c|c|c|c|c|c|c|c|c|c|}
\hline 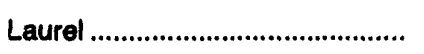 & 13,500 & 1,500 & 0 & 12,000 & 0 & 16,000 & 18,000 & 15,000 & 0 & 4,000 \\
\hline 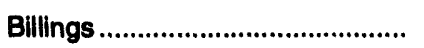 & 19,000 & 1,000 & 0 & 0 & 12,000 & 0 & 2,500 & 8,500 & 28,000 & 0 \\
\hline Billings & 21,000 & 3,500 & 4,400 & 0 & 10,000 & 0 & 15,500 & 20,000 & 6,000 & 0 \\
\hline 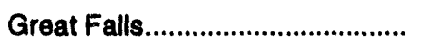 & 2,400 & 0 & 0 & 0 & 1,030 & 0 & 1,100 & 2,000 & 3,000 & 0 \\
\hline
\end{tabular}

Nata

Eagle Springs.............................. $00 \begin{array}{lllllllll} & 0 & 0 & 0 & 0 & 0 & 0 & 0 & 0\end{array}$

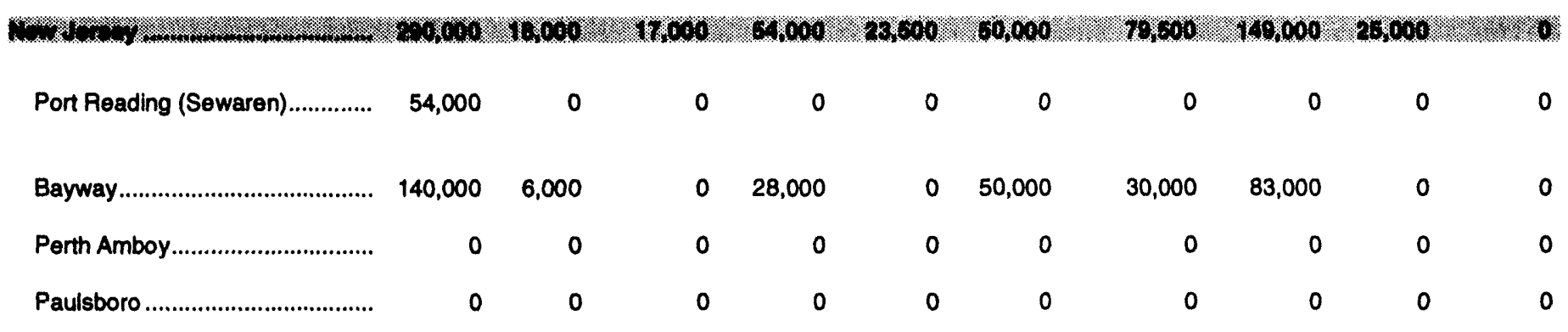

See footnotes at end of table. 
Table 38. Capacity of Operable Petroleum Refinerles by State as of January 1, 1994 (Continued) (Barrels per Stream Day, Except Where Noted)

\begin{tabular}{|c|c|c|c|c|c|c|c|c|c|}
\hline \multirow[b]{4}{*}{ Stateriefinemocition } & \multicolumn{4}{|c|}{ Almoepheric Cruds Oll Distilletion Cepecity } & \multicolumn{5}{|c|}{ Dounstrem Chargs Cepectly } \\
\hline & \multirow{2}{*}{\multicolumn{2}{|c|}{$\begin{array}{l}\text { Berrels per } \\
\text { Colender Day }\end{array}$}} & \multirow{2}{*}{\multicolumn{2}{|c|}{$\begin{array}{l}\text { Berrels par } \\
\text { Streem Day }\end{array}$}} & \multirow{3}{*}{$\begin{array}{l}\text { Koutum } \\
\text { Dictillation }\end{array}$} & \multicolumn{4}{|c|}{ Thermal Crecking } \\
\hline & & & & & & \multirow{2}{*}{$\begin{array}{l}\text { Dolayed } \\
\text { Cokng }\end{array}$} & \multirow[b]{2}{*}{ Fludd Coluting } & \multirow[b]{2}{*}{ Vobrualing } & \multirow{2}{*}{$\begin{array}{l}\text { Otherl } \\
\text { Gas OII }\end{array}$} \\
\hline & Opereting & late & Operathg & lolle & & & & & \\
\hline \multirow{2}{*}{ 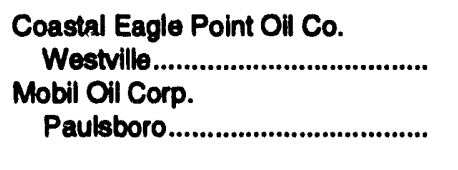 } & 117,000 & 0 & 128,000 & 0 & 50,000 & 0 & 0 & 0 & 0 \\
\hline & 113,000 & 0 & 118,000 & 0 & 65,500 & 22,000 & 0 & 0 & 0 \\
\hline \multirow{4}{*}{ 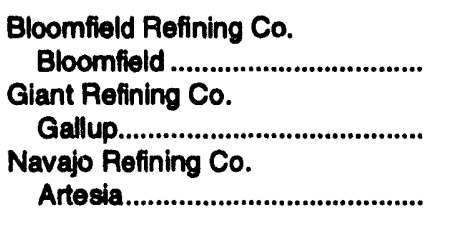 } & & & & & & & & & \\
\hline & 16,800 & 0 & 18,100 & 0 & 0 & 0 & 0 & 0 & 0 \\
\hline & 20,800 & 0 & 21,000 & 0 & 7,900 & 0 & 0 & 0 & 0 \\
\hline & 57,000 & 0 & 60,000 & 0 & 19,000 & 0 & 0 & 0 & 0 \\
\hline \multicolumn{10}{|c|}{ Whim $;$; } \\
\hline $\begin{array}{l}\text { Amoco Oll Co. } \\
\text { Mandan }\end{array}$ & 58,000 & 0 & 60,000 & 0 & 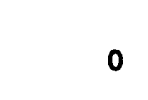 & 0 & 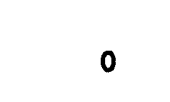 & 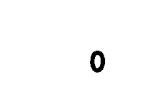 & 0 \\
\hline \multicolumn{10}{|c|}{ 1. } \\
\hline 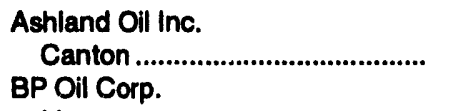 & 66,000 & 0 & 68,000 & 0 & 33,000 & 0 & 0 & 0 & 0 \\
\hline 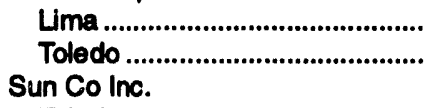 & $\begin{array}{l}145,000 \\
126,100\end{array}$ & $\begin{array}{l}0 \\
0\end{array}$ & $\begin{array}{l}155,000 \\
130,000\end{array}$ & $\begin{array}{l}0 \\
0\end{array}$ & $\begin{array}{l}50,000 \\
68,000\end{array}$ & $\begin{array}{l}20,000 \\
19,000\end{array}$ & $\begin{array}{l}0 \\
0\end{array}$ & $\begin{array}{l}0 \\
0\end{array}$ & $\begin{array}{l}0 \\
0\end{array}$ \\
\hline Toledo ............................................. & 125,000 & 0 & 129,000 & 0 & 30,000 & 0 & 0 & 0 & 0 \\
\hline \multicolumn{10}{|l|}{ Barrett Refining Corp. } \\
\hline $\begin{array}{l}\text { Thomas (Custer) ............................ } \\
\text { Conoco Inc. }\end{array}$ & 10,500 & 0 & 11,200 & 0 & 0 & 0 & 0 & 0 & 0 \\
\hline $\begin{array}{l}\text { Ponca City.................................. } \\
\text { Cyril Petrochemical Corp." }\end{array}$ & 140,000 & 0 & 145,000 & 0 & 45,000 & 21,500 & 0 & 0 & 0 \\
\hline Kerr-MoGee Refining Corp. & 7,400 & 0 & 7,500 & 0 & 0 & 0 & 0 & 0 & 0 \\
\hline $\begin{array}{l}\text { Wynnewood } \\
\text { Sinclair Oil Corp. }\end{array}$ & 43,000 & 0 & 45,000 & 0 & 14,000 & 0 & 0 & 0 & 0 \\
\hline 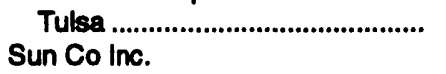 & 50,000 & 0 & 53,000 & 0 & 27,000 & 0 & 0 & 0 & 0 \\
\hline $\begin{array}{l}\text { Tulsa ..................................... } \\
\text { Total Petroleum Inc. }\end{array}$ & 85,000 & 0 & 90,000 & 0 & 29,000 & 6,500 & 0 & 0 & 0 \\
\hline 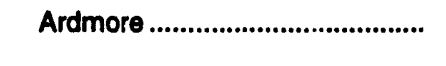 & 68,000 & 0 & 70,000 & 0 & 32,000 & 0 & 0 & 0 & 0 \\
\hline \multicolumn{10}{|c|}{ fer } \\
\hline $\begin{array}{l}\text { Chevron U.S.A. Inc. } \\
\text { Portland (Willbridge) }\end{array}$ & 0 & 0 & 0 & 0 & 15,000 & 0 & 0 & 0 & 0 \\
\hline \multicolumn{10}{|c|}{ 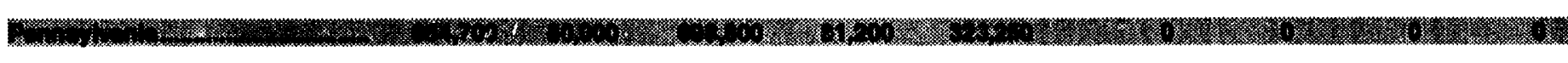 } \\
\hline $\begin{array}{l}\text { BP Oil Corp. } \\
\text { Marcus Hook............................... } \\
\text { Chevron U.S.A. Inc. }\end{array}$ & 172,000 & 0 & 186,000 & 0 & 78,000 & 0 & 0 & 0 & 0 \\
\hline $\begin{array}{l}\text { Philadelphia .............................. } \\
\text { Pennzoil Producing Co. }\end{array}$ & 172,000 & 0 & 185,000 & 0 & 80,000 & 0 & 0 & 0 & 0 \\
\hline $\begin{array}{l}\text { Rouseville .................................. } \\
\text { Petrowax Pennsyivania Inc. }\end{array}$ & 15,700 & 0 & 16,500 & 0 & 6,500 & 0 & 0 & 0 & 0 \\
\hline $\begin{array}{l}\text { Farmer's Valley (Smethport) } \\
\text { Sun Co... Inc. }\end{array}$ & 0 & 0 & 0 & 0 & 3,750 & 0 & 0 & 0 & 0 \\
\hline $\begin{array}{l}\text { Marcus Hook........................... } \\
\text { Sun Refining \& Marketing }\end{array}$ & 175,000 & 0 & 185,000 & 0 & 46,000 & 0 & 0 & 0 & 0 \\
\hline 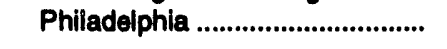 & 50,000 & 80,000 & 50,800 & 81,200 & 83,000 & 0 & 0 & 0 & 0 \\
\hline
\end{tabular}

See footnotes at end of table. 
Table 38. Capacity of Operable Petroleum Refineries by State as of January 1, 1994 (Continued) (Barrels per Stream Day, Except Where Noted)

\begin{tabular}{|c|c|c|c|c|c|c|c|c|c|c|}
\hline \multirow[b]{3}{*}{ Location } & \multicolumn{10}{|c|}{ Downstreem Cherge Cepecily (Continued) } \\
\hline & \multicolumn{2}{|c|}{ Cetalytic Crecking } & \multirow[b]{2}{*}{$\begin{array}{c}\text { Cetalytile } \\
\text { Hydrecrecking }\end{array}$} & \multicolumn{2}{|c|}{ Cataly tic Roforming } & \multicolumn{4}{|c|}{ Cestantic Hydroteding } & \multirow{2}{*}{$\begin{array}{c}\text { Fual } \\
\text { Solvonts } \\
\text { Desecphalting }\end{array}$} \\
\hline & Frech & Recyeled & & Low & $\begin{array}{c}\text { High } \\
\text { Preacure }\end{array}$ & $\begin{array}{l}\text { Haany } \\
\text { cas oll }\end{array}$ & $\begin{array}{c}\text { Maphtha } \\
\text { Roformer Fead }\end{array}$ & Dientilinte & \begin{tabular}{c|c} 
Otherl \\
Recidiual
\end{tabular} & \\
\hline 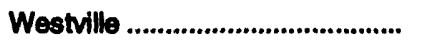 & 52,000 & 12,000 & 17,000 & 26,000 & 0 & 0 & 26,000 & 15,000 & 10,000 & 0 \\
\hline 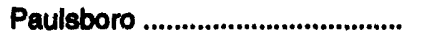 & 44,000 & 0 & 0 & 0 & 23,500 & 0 & 23,500 & 51,000 & 15,000 & 0 \\
\hline 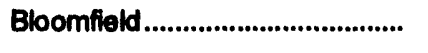 & 6,000 & 500 & 0 & 0 & 4,000 & 0 & 4,000 & 3,000 & 0 & 0 \\
\hline 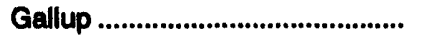 & 8,500 & 3,000 & 0 & 0 & 6,800 & 0 & 6,800 & 3,000 & 0 & 0 \\
\hline 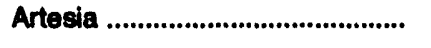 & 20,000 & 1,000 & 0 & 12,500 & 5,000 & 0 & 20,000 & 25,000 & 0 & 0 \\
\hline 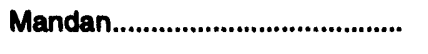 & 26,000 & 3,600 & 0 & 0 & 12,100 & 0 & 19,100 & 0 & 0 & 0 \\
\hline 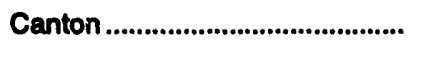 & 25,000 & 0 & 0 & 20,000 & 0 & 23,000 & 26,500 & 7,000 & 0 & 0 \\
\hline Uma & $\begin{array}{l}34,000 \\
55,000\end{array}$ & $\begin{array}{r}0 \\
16,500\end{array}$ & $\begin{array}{l}24,000 \\
35,000\end{array}$ & $\begin{array}{l}0 \\
0\end{array}$ & $\begin{array}{l}55,000 \\
42,000\end{array}$ & $\begin{array}{l}0 \\
0\end{array}$ & $\begin{array}{l}60,000 \\
40,000\end{array}$ & $\begin{array}{l}0 \\
0\end{array}$ & $\begin{array}{l}0 \\
0\end{array}$ & $\begin{array}{l}0 \\
0\end{array}$ \\
\hline 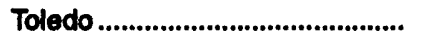 & 60,000 & 1,000 & 28,200 & 0 & 45,600 & 0 & 40,000 & 0 & 0 & 9,000 \\
\hline Thomas (Custer)............................ & 0 & 0 & 0 & 0 & 0 & 0 & 0 & 0 & 0 & 0 \\
\hline 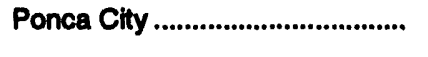 & 53,000 & 0 & 0 & 0 & 36,000 & 0 & 36,000 & 53,500 & 0 & 0 \\
\hline Cyril & 0 & 0 & 0 & 0 & 0 & 0 & 0 & 0 & 0 & 0 \\
\hline 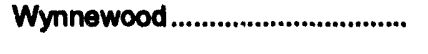 & 18,000 & 0 & 5,000 & 12,500 & 0 & 0 & 11,000 & 0 & 0 & 4,400 \\
\hline 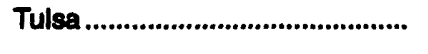 & 19,000 & 5,000 & 0 & 0 & 12,000 & 0 & 20,000 & 17,500 & 0 & 0 \\
\hline 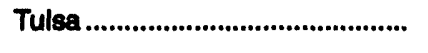 & 35,000 & 0 & 0 & 0 & 24,000 & 0 & 24,000 & 0 & 10,000 & 5,800 \\
\hline 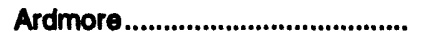 & 26,000 & 0 & 0 & 17,000 & 0 & 28,000 & 24,000 & 28,000 & 28,000 & 0 \\
\hline Portland (Willbridge) .................... & 0 & 0 & 0 & 0 & 0 & 0 & 0 & 0 & 0 & 0 \\
\hline Marcus Hook ................................. & 51,000 & 1,600 & 21,000 & 48,000 & 0 & 42,000 & 56,000 & 44,000 & 0 & 0 \\
\hline 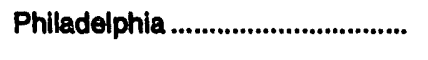 & 64,000 & 0 & 0 & 0 & 34,000 & 0 & 34,000 & 31,000 & 0 & 0 \\
\hline 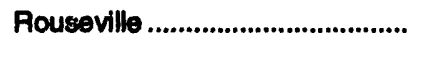 & 0 & 0 & 0 & 5,820 & 0 & 0 & 6,500 & 0 & 8,000 & 0 \\
\hline Farmer's Valley (Smethport)...... & 0 & 0 & 0 & 0 & 0 & 0 & 0 & 0 & 0 & 0 \\
\hline 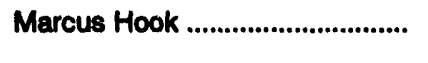 & 86,000 & 0 & 0 & 0 & 41,300 & 0 & 57,500 & 28,800 & 6,000 & 0 \\
\hline 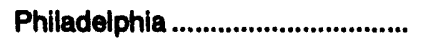 & 40,000 & 0 & 30,000 & 0 & 50,000 & 24,000 & 54,000 & 50,000 & 0 & 0 \\
\hline
\end{tabular}

See footnotes at end of table. 
Table 38. Capacity of Operable Petroleum Refineries by Stete as of January 1, 1994 (Continued) (Barrels per Stream Day, Except Where Noted)

\begin{tabular}{|c|c|c|c|c|c|c|c|c|c|}
\hline \multirow[b]{4}{*}{ State/Refiner/Location } & \multicolumn{4}{|c|}{ 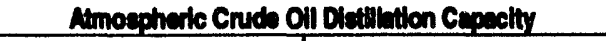 } & \multicolumn{5}{|c|}{ Downetreem Chargs Capecily } \\
\hline & \multirow{2}{*}{\multicolumn{2}{|c|}{$\begin{array}{l}\text { Barrels por } \\
\text { Calondar Day }\end{array}$}} & \multirow{2}{*}{\multicolumn{2}{|c|}{$\begin{array}{l}\text { Barels por } \\
\text { Streem Doy }\end{array}$}} & \multirow{3}{*}{$\begin{array}{c}\text { Vecuum } \\
\text { Distllition }\end{array}$} & \multicolumn{4}{|c|}{ Thermal Crecking } \\
\hline & & & & & & \multirow{2}{*}{$\begin{array}{l}\text { Delayed } \\
\text { Coling }\end{array}$} & \multirow[b]{2}{*}{ Fuid Coking } & \multirow{2}{*}{ Visbreaking } & \multirow{2}{*}{$\begin{array}{l}\text { Otharl } \\
\text { Cas OII }\end{array}$} \\
\hline & Operating & Vollo & Operating & Ite & & & & & \\
\hline \multicolumn{10}{|l|}{ 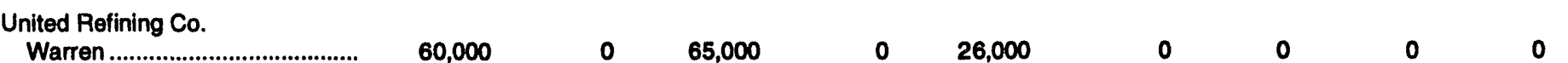 } \\
\hline 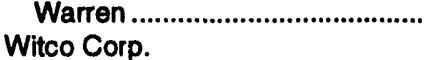 & 60,000 & 0 & 65,000 & 0 & 26,000 & 0 & 0 & 0 & 0 \\
\hline 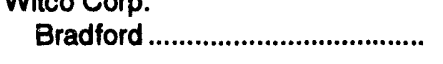 & 10,000 & 0 & 10,500 & 0 & 0 & 0 & 0 & 0 & 0 \\
\hline \multicolumn{10}{|c|}{ 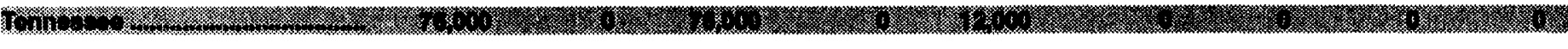 } \\
\hline \multicolumn{10}{|l|}{ 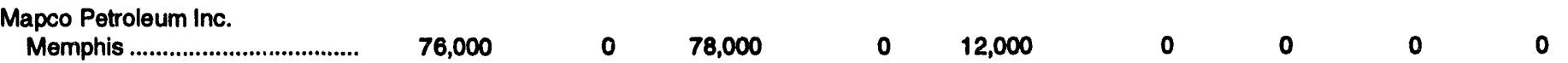 } \\
\hline \multicolumn{10}{|c|}{ H. } \\
\hline \multicolumn{10}{|l|}{ Age Refining \& Marketing } \\
\hline $\begin{array}{l}\text { San Antonio.................................. } \\
\text { Amoco Oil Co. }\end{array}$ & 4,000 & 0 & 5,000 & 0 & 0 & 0 & 0 & 0 & 0 \\
\hline $\begin{array}{l}\text { Texas City ..................................... } \\
\text { Chevron U.S.A. Inc. }\end{array}$ & 433,000 & 0 & 460,000 & 0 & 240,000 & 36,000 & 0 & 0 & 0 \\
\hline 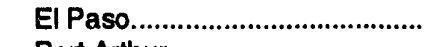 & 81,900 & 0 & 97,500 & 0 & 40,000 & 3,000 & 0 & 0 & 0 \\
\hline $\begin{array}{l}\text { Port Arthur ............................. } \\
\text { Citgo Refining \& Chemical Inc. }\end{array}$ & 185,000 & 0 & 195,000 & 0 & 85,000 & 35,000 & 0 & 0 & 0 \\
\hline 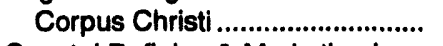 & 130,000 & 0 & 142,000 & 0 & 67,000 & 34,000 & 0 & 0 & 0 \\
\hline Coastal Refining \& Marketing Inc. & & & & & & & & & \\
\hline $\begin{array}{l}\text { Corpus Christi ............................ } \\
\text { Crown Central Petroleum Corp. }\end{array}$ & 85,000 & 0 & 95,000 & 0 & 45,000 & 14,500 & 0 & 10,000 & 0 \\
\hline 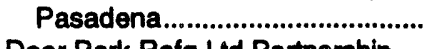 & 100,000 & 0 & $1 \cup 3,000$ & 0 & 38,000 & 12,500 & 0 & 0 & 0 \\
\hline $\begin{array}{l}\text { Deer Park Refg Ltd Partnership } \\
\text { (Formerly Shell Oil Co.) }\end{array}$ & & & & & & & & & \\
\hline Deer Park & 215,900 & 0 & 227,000 & 0 & 90,500 & 0 & 0 & 17,000 & 55,000 \\
\hline \multicolumn{10}{|c|}{ Diamond Shamrock Refining \& Marketing Co. } \\
\hline Sunray (McKe日) ........................... & 125,000 & 0 & 128,000 & 0 & 47,000 & 0 & 0 & 0 & 0 \\
\hline 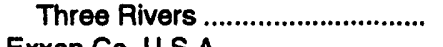 & 70,000 & 0 & 73,000 & 0 & 28,000 & 0 & 0 & 0 & 0 \\
\hline $\begin{array}{l}\text { Exxon Co. U.S.A. } \\
\text { Baytown ....................................... }\end{array}$ & & & & & & & & & \\
\hline Fina Oil \& Chemical Co. & 396,000 & 0 & 418,000 & 0 & 230,000 & 0 & 32,000 & 0 & 0 \\
\hline 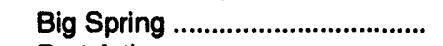 & 55,000 & 0 & 60,000 & 0 & 24,000 & 0 & 0 & 0 & 0 \\
\hline 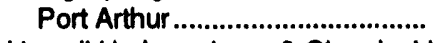 & 144,000 & 0 & 150,000 & 0 & 53,000 & 0 & 0 & 0 & 0 \\
\hline \multicolumn{10}{|l|}{ Howell Hydrocarbons \& Chemical Inc. } \\
\hline $\begin{array}{l}\text { Channelview .......................................... } \\
\text { Koch Refining Co. }\end{array}$ & 1,400 & 0 & 1,700 & 0 & 0 & 0 & 0 & 0 & 0 \\
\hline $\begin{array}{l}\text { Corpus Christi ................................ } \\
\text { La Gloria Oil \& Gas Co. }\end{array}$ & 125,000 & 0 & 130,000 & 0 & 42,600 & 12,800 & 0 & 0 & 0 \\
\hline Tyler ..................................... & 52,000 & 0 & 55,000 & 0 & 14,000 & 6,500 & 0 & 0 & 0 \\
\hline \multicolumn{10}{|c|}{$\begin{array}{l}\text { Lyondell Citgo Refining Co. Ltd. } \\
\text { (Formerly Lyondell Petrochemical Co.) }\end{array}$} \\
\hline $\begin{array}{l}\text { Houston ....................................... } \\
\text { Marathon Oil Co. }\end{array}$ & 265,000 & 0 & 286,000 & 0 & 127,000 & 45,000 & 0 & 0 & 0 \\
\hline $\begin{array}{l}\text { Texas City ............................................ } \\
\text { Mobil Oil Corp. }\end{array}$ & 70,000 & 0 & 74,000 & 0 & 0 & 0 & 0 & 0 & 0 \\
\hline $\begin{array}{l}\text { Beaumont ............................................ } \\
\text { Petrolite Corp. }\end{array}$ & 310,000 & 0 & 325,000 & 0 & 135,000 & 40,000 & 0 & 0 & 0 \\
\hline $\begin{array}{l}\text { Kilgore................................... } \\
\text { Phibro Energy U.S.A. In. }\end{array}$ & 1,000 & 0 & 1,000 & 0 & 400 & 0 & 0 & 0 & 0 \\
\hline 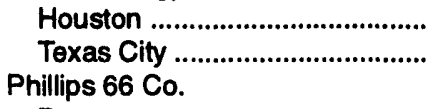 & $\begin{array}{r}71,000 \\
123,500\end{array}$ & $\begin{array}{l}0 \\
0\end{array}$ & $\begin{array}{r}75,000 \\
130,700\end{array}$ & $\begin{array}{l}0 \\
0\end{array}$ & $\begin{array}{l}39,000 \\
64,000\end{array}$ & $\begin{array}{l}0 \\
0\end{array}$ & $\begin{array}{l}0 \\
0\end{array}$ & $\begin{array}{r}0 \\
21,000\end{array}$ & $\begin{array}{l}0 \\
0\end{array}$ \\
\hline 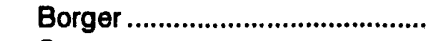 & 105,000 & 0 & 110,000 & 0 & 0 & 0 & 0 & 0 & 0 \\
\hline 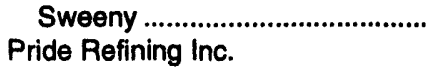 & 175,000 & 0 & 195,000 & 0 & 83,000 & 0 & 0 & 0 & 0 \\
\hline $\begin{array}{l}\text { Abilene ............................................. } \\
\text { Shell Oil Co. }\end{array}$ & 42,750 & 0 & 45,000 & 0 & 13,500 & 0 & 0 & 0 & 0 \\
\hline South Hampton Refining Co. & 28,600 & 0 & 29,000 & 0 & 0 & 0 & 0 & 0 & 0 \\
\hline 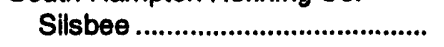 & 0 & 0 & 0 & 0 & 0 & 0 & 0 & 0 & 0 \\
\hline
\end{tabular}


Table 38. Capacity of Operable Petroleum Refineries by State as of January 1, 1994 (Continued) (Barrels per Stream Day, Except Where Noted)

\begin{tabular}{|c|c|c|c|c|c|c|c|c|c|c|}
\hline \multirow[b]{3}{*}{ Location } & \multicolumn{10}{|c|}{ Downetrean Cherp Cepaclity (Continued) } \\
\hline & \multicolumn{2}{|c|}{ Centalytic Cracking } & \multirow[b]{2}{*}{$\begin{array}{c}\text { Coitalytic } \\
\text { Hyctoctecing }\end{array}$} & \multicolumn{2}{|c|}{ Cotalytic Relorming } & \multicolumn{4}{|c|}{ Cotabytic Hydrotreeting } & \multirow{2}{*}{$\begin{array}{c}\text { Furl } \\
\text { Solvents } \\
\text { Desaphating }\end{array}$} \\
\hline & Freah & Recyoled I & & $\begin{array}{c}\text { Low } \\
\text { Preasure }\end{array}$ & $\begin{array}{c}\text { Hogh } \\
\text { Proseure }\end{array}$ & $\begin{array}{l}\text { Heayy } \\
\text { cas on }\end{array}$ & $\begin{array}{l}\text { Nuphtitu } \\
\text { Reforiner Feed }\end{array}$ & Dtotillato & \begin{tabular}{c|c} 
Otherl \\
Reaidual
\end{tabular} & \\
\hline Warren & 20,000 & 200 & 0 & 0 & 16,000 & 0 & 20,000 & 18,000 & 0 & 0 \\
\hline 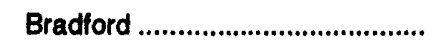 & 0 & 0 & 0 & 0 & 1,800 & 0 & 3,300 & 0 & 0 & 0 \\
\hline 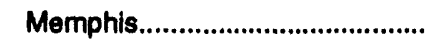 & 40,000 & 0 & 0 & 14,000 & 0 & 0 & 19,000 & 36,000 & 0 & 0 \\
\hline 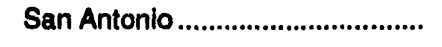 & 0 & 0 & 0 & 0 & 1,200 & 0 & 0 & 0 & 0 & 0 \\
\hline 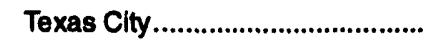 & 204,000 & 43,000 & 120,000 & 60,000 & 78,000 & 85,000 & 146,000 & 130,000 & 0 & 0 \\
\hline 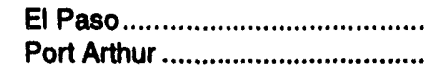 & $\begin{array}{l}25,000 \\
65,000\end{array}$ & $\begin{array}{l}0 \\
0\end{array}$ & $\begin{array}{l}0 \\
0\end{array}$ & $\begin{array}{l}25,000 \\
48,000\end{array}$ & $\begin{array}{l}0 \\
0\end{array}$ & $\begin{array}{r}0 \\
68,000\end{array}$ & $\begin{array}{l}25,000 \\
50,000\end{array}$ & $\begin{array}{l}18,000 \\
82,000\end{array}$ & $\begin{array}{l}0 \\
0\end{array}$ & $\begin{array}{l}0 \\
0\end{array}$ \\
\hline 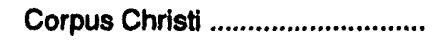 & 78,000 & 0 & 0 & 52,000 & 0 & 65,000 & 60,000 & 41,700 & 0 & 0 \\
\hline 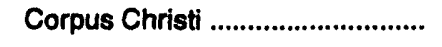 & 20,000 & 0 & 11,000 & 0 & 30,500 & 22,500 & 35,000 & 24,000 & 0 & 0 \\
\hline 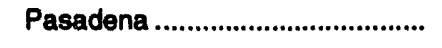 & 56,000 & 0 & 0 & 23,000 & 13,000 & 0 & 28,000 & 7,000 & 16,000 & 0 \\
\hline Deer Park & 65,000 & 5,000 & 65,000 & 43,000 & 20,000 & 45,000 & 65,000 & 70,000 & 55,500 & 0 \\
\hline 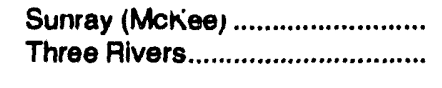 & $\begin{array}{l}47,000 \\
21,000\end{array}$ & $\begin{array}{l}0 \\
0\end{array}$ & $\begin{array}{l}25,000 \\
26,000\end{array}$ & $\begin{array}{l}42,000 \\
30,000\end{array}$ & $\begin{array}{l}0 \\
0\end{array}$ & $\begin{array}{l}0 \\
0\end{array}$ & $\begin{array}{l}35,000 \\
20,000\end{array}$ & $\begin{array}{r}31,500 \\
4,000\end{array}$ & $\begin{array}{l}0 \\
0\end{array}$ & $\begin{array}{r}10,000 \\
7,000\end{array}$ \\
\hline 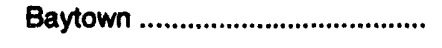 & 195,000 & 5,000 & 24,000 & 123,000 & 0 & 110,000 & 148,000 & 212,000 & 44,100 & 35,000 \\
\hline 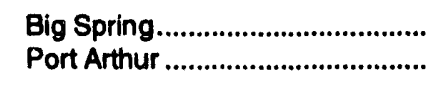 & $\begin{array}{l}23,500 \\
59,000\end{array}$ & $\begin{array}{l}0 \\
0\end{array}$ & $\begin{array}{l}0 \\
0\end{array}$ & $\begin{array}{l}21,000 \\
35,000\end{array}$ & $\begin{array}{l}0 \\
0\end{array}$ & $\begin{array}{r}6,000 \\
0\end{array}$ & $\begin{array}{l}25,500 \\
40,000\end{array}$ & $\begin{array}{l}22,750 \\
48,000\end{array}$ & $\begin{array}{r}2,500 \\
23,000\end{array}$ & $\begin{array}{l}10,000 \\
18,000\end{array}$ \\
\hline 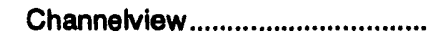 & 0 & 0 & 0 & 0 & 0 & 0 & 0 & 0 & 0 & 0 \\
\hline 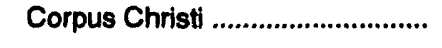 & 50,000 & 0 & 12,500 & 48,000 & 0 & 0 & 54,000 & 9,000 & 0 & 0 \\
\hline Tyler & 18,000 & 800 & 0 & 13,000 & 4,600 & 0 & 22,000 & 12,000 & 0 & 0 \\
\hline Houston & 92,000 & 0 & 0 & 25,000 & 83,000 & 82,000 & 132,000 & 93,000 & 6,600 & 0 \\
\hline 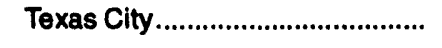 & 34,000 & 0 & 0 & 0 & 10,000 & 0 & 0 & 0 & 0 & 0 \\
\hline 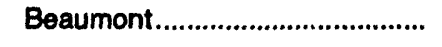 & 102,000 & 0 & 45,000 & 132,000 & 0 & 0 & 109,000 & 95,000 & 0 & 0 \\
\hline 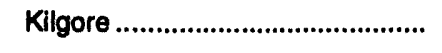 & 0 & 0 & 0 & 0 & 0 & 0 & 0 & 0 & 0 & 0 \\
\hline Houston & $\begin{array}{l}61,000 \\
42,000\end{array}$ & $\begin{array}{l}0 \\
0\end{array}$ & $\begin{array}{l}0 \\
0\end{array}$ & $\begin{array}{r}0 \\
12,100\end{array}$ & $\begin{array}{l}13,500 \\
11,100\end{array}$ & $\begin{array}{l}0 \\
0\end{array}$ & $\begin{array}{l}14,000 \\
29,200\end{array}$ & $\begin{array}{l}31,500 \\
59,000\end{array}$ & $\begin{array}{l}0 \\
0\end{array}$ & $\begin{array}{r}17,000 \\
0\end{array}$ \\
\hline Borger & $\begin{array}{l}60,000 \\
87,000\end{array}$ & $\begin{array}{l}10,400 \\
12,000\end{array}$ & $\begin{array}{l}0 \\
0\end{array}$ & $\begin{array}{l}0 \\
0\end{array}$ & $\begin{array}{l}26,000 \\
36,000\end{array}$ & $\begin{array}{l}0 \\
0\end{array}$ & $\begin{array}{l}26,500 \\
53,000\end{array}$ & $\begin{array}{l}40,000 \\
50,000\end{array}$ & $\begin{array}{l}50,000 \\
75,000\end{array}$ & $\begin{array}{l}0 \\
0\end{array}$ \\
\hline Abilene & 0 & 0 & 0 & 7,500 & 0 & 0 & 7,500 & 12,000 & 0 & 0 \\
\hline 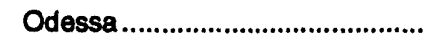 & 10,000 & 0 & 0 & 0 & 10,000 & 0 & 10,700 & 0 & 0 & 0 \\
\hline Silsbee & 0 & 0 & 0 & 1,400 & 0 & 0 & 3,500 & 500 & 500 & 0 \\
\hline
\end{tabular}

See footnotes at end of table. 
Table 38. Capacity of Operable Petroleum Rofinerlos by State as of January 1, 1994 (Continued) (Barrels per Stream Day, Except Where Noted)

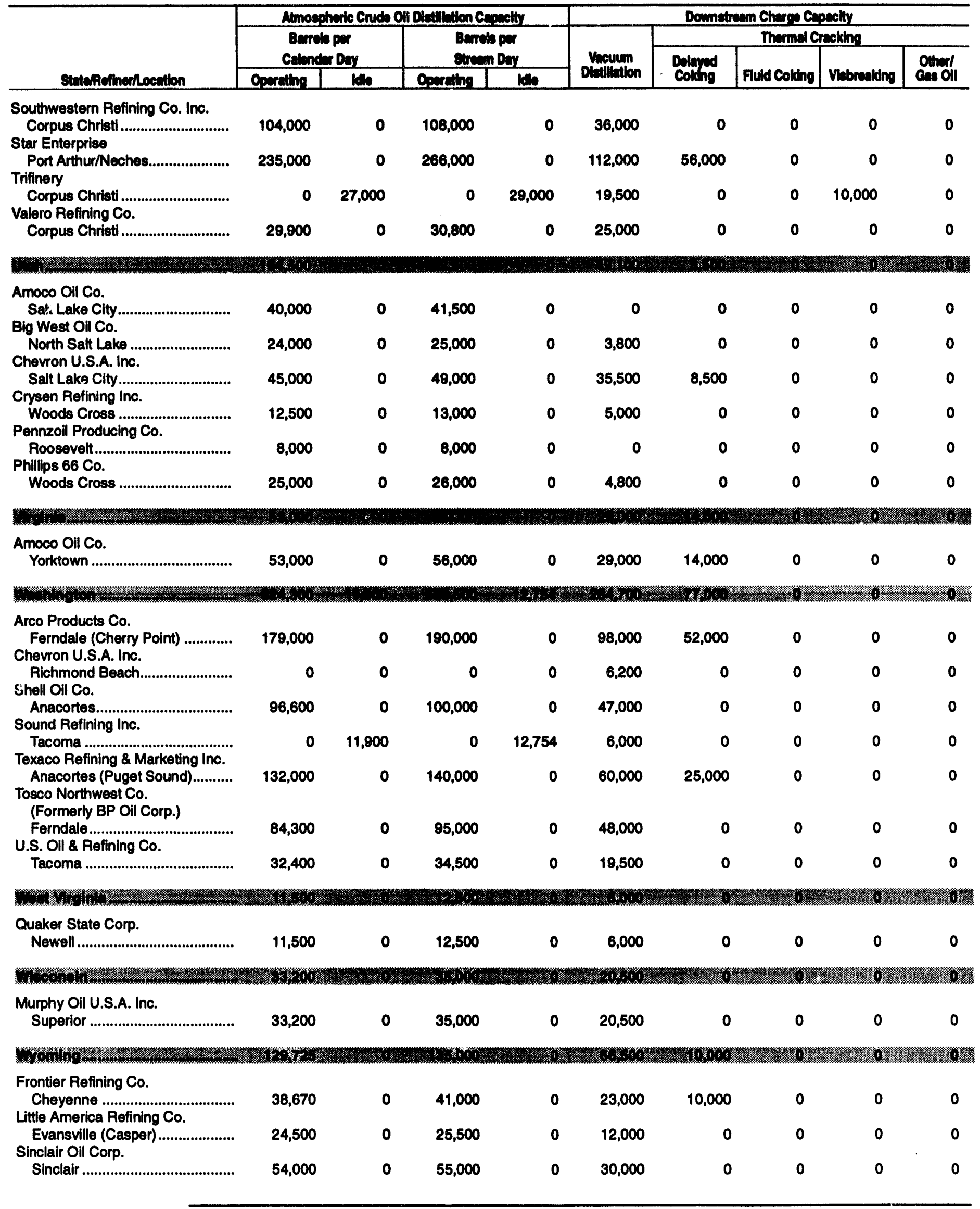

See footnotes at end of table. 
Table 38. Capacity of Operable Petroleum Rofinories by State as of January 1, 1994 (Continued) (Barrels per Stream Day, Except Where Noted)

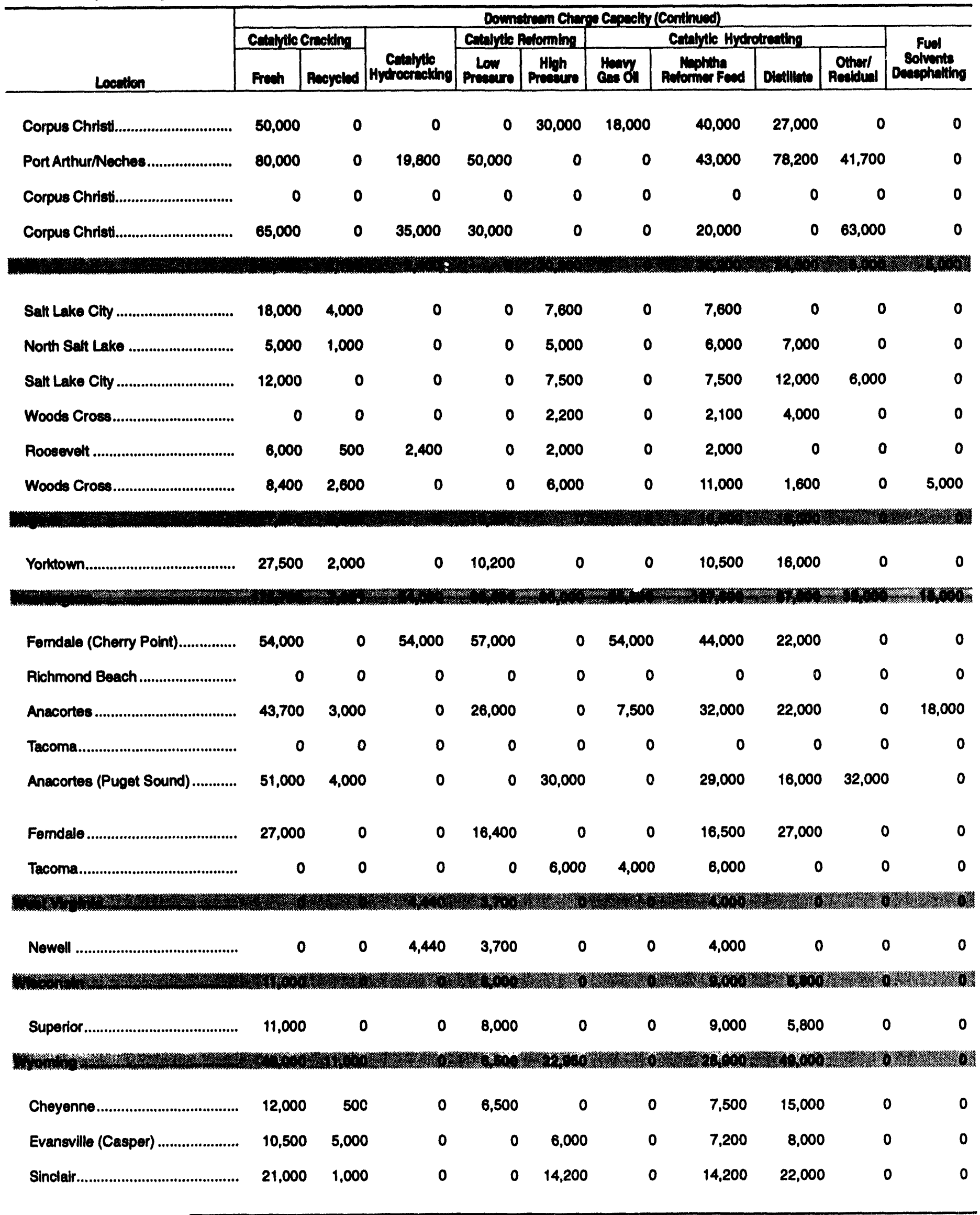


Table 38. Capacity of Operable Potroleum Refineries by State as of January 1, 1994 (Continued) (Barrels per Stream Day, Except Where Noted)

\begin{tabular}{|c|c|c|c|c|c|c|c|c|c|}
\hline \multirow[b]{4}{*}{ Staterietinorhocation } & \multicolumn{4}{|c|}{ Atmospheric Crude Oll Distllition Ceppeity } & \multicolumn{5}{|c|}{ Downatroam Charge Cepecliy } \\
\hline & \multirow{2}{*}{\multicolumn{2}{|c|}{$\begin{array}{l}\text { Berrots per } \\
\text { Calender Day }\end{array}$}} & \multirow{2}{*}{\multicolumn{2}{|c|}{$\begin{array}{l}\text { Barrebs por } \\
\text { Stream Day }\end{array}$}} & \multirow{3}{*}{$\begin{array}{l}\text { Vhoulum } \\
\text { Distlilation }\end{array}$} & \multicolumn{4}{|c|}{ Thermal Crecking } \\
\hline & & & & & & \multirow{2}{*}{$\begin{array}{l}\text { Delayed } \\
\text { Colkng }\end{array}$} & \multirow{2}{*}{ Fluid Coking } & \multirow{2}{*}{ Vhabreaking } & \multirow{2}{*}{$\begin{array}{l}\text { Otheyl } \\
\text { Gas OII }\end{array}$} \\
\hline & Operating & Iolle & Operating & Idile & & & & & \\
\hline 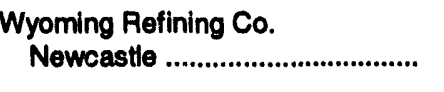 & 12,555 & 0 & 13,500 & 0 & 1,500 & 0 & 0 & 0 & 0 \\
\hline \multicolumn{10}{|c|}{ 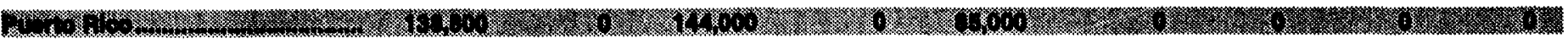 } \\
\hline $\begin{array}{l}\text { Caribbean Petroleum Corp. } \\
\text { San Juan (Bayamon) } \\
\text { Peerle................ }\end{array}$ & 45,000 & 0 & 48,000 & 0 & 22,000 & 0 & $\mathbf{0}$ & 0 & 0 \\
\hline $\begin{array}{l}\text { Ponce................................ } \\
\text { Phillips Puerto Rico Core Inc. }\end{array}$ & 8,800 & 0 & 9,000 & 0 & 18,000 & 0 & 0 & 0 & 0 \\
\hline $\begin{array}{l}\text { Guayama } \\
\text { Sun Co Inc. }\end{array}$ & 0 & 0 & 0 & 0 & 0 & 0 & 0 & 0 & 0 \\
\hline Yabucoa & 85,000 & 0 & 87,000 & 0 & 45,000 & c & 0 & 0 & $\mathbf{0}$ \\
\hline $\begin{array}{l}\text { Amerada Hess Corp. } \\
\text { St. Croix }\end{array}$ & 370,000 & 175,000 & 400,000 & 195,000 & 225,000 & 0 & 0 & 85,000 & 0 \\
\hline
\end{tabular}


Table 38. Capacity of Operable Petroleum Rofinerles by State as of January 1, 1994 (Continued) (Barrels per Stream Day, Except Where Noted)

\begin{tabular}{|c|c|c|c|c|c|c|c|c|c|c|}
\hline \multirow[b]{3}{*}{ Loetion } & \multicolumn{10}{|c|}{ Downetrenen Charge Cepelty (Conthued) } \\
\hline & \multicolumn{2}{|c|}{ Cetaintic Creolin? } & \multirow[b]{2}{*}{$\begin{array}{l}\text { Cetabyte } \\
\text { Hydrearcoling }\end{array}$} & \multicolumn{2}{|c|}{ Conjtis Rifoming } & \multicolumn{4}{|c|}{ Catalytic Hydrotrating } & \multirow{2}{*}{$\begin{array}{c}\text { Fual } \\
\text { Solvints } \\
\text { Danephaling }\end{array}$} \\
\hline & Freah & Resyoled & & $\begin{array}{l}\text { Low } \\
\text { Prenewo }\end{array}$ & $\begin{array}{l}\text { High } \\
\text { Prencurs }\end{array}$ & Hay of & $\begin{array}{l}\text { Maphtha } \\
\text { Retormer Feed }\end{array}$ & Detetllate & $\begin{array}{c}\text { Othorl } \\
\text { Recldual }\end{array}$ & \\
\hline
\end{tabular}

Newcastle

$5,500 \quad 4,500$

0

o 2,750

0

$0 \quad 4,000$

0

0

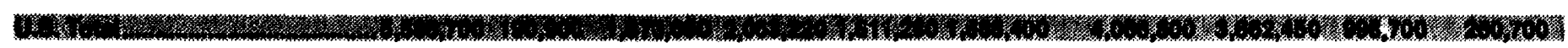
15.

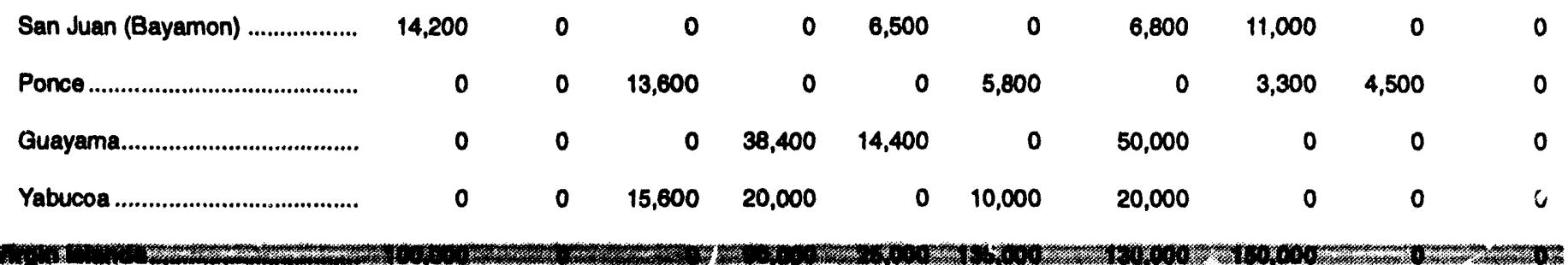

St. Croix

100,000

$\mathbf{0}$

$\begin{array}{llll}0 & 80,000 & 25,000 & 135,000\end{array}$

$130,000 \quad 150,000$

0

0

- Refinery was reactivated during 1993.

Source: Energy Information Administration (EIA), Form EIA-820, "Annual Refinery Report." 
Table 39. Production Capacity of Operable Petroleum Refinerles by State as of January 1, 1994 (Barrels per Stream Day, Except Where Noted)

\begin{tabular}{|c|c|c|c|c|c|c|c|c|c|}
\hline \multirow[b]{2}{*}{ State/Refiner/Rocation } & \multirow[b]{2}{*}{ Allyylates } & \multirow[b]{2}{*}{ Aromatles } & \multirow[b]{2}{*}{$\begin{array}{c}\text { Aephalt } \\
\text { and } \\
\text { Road OII }\end{array}$} & \multicolumn{2}{|c|}{ bomere } & \multirow[b]{2}{*}{ Lubricants } & \multirow[b]{2}{*}{$\begin{array}{l}\text { Markoteble } \\
\text { Petroleum } \\
\text { Coke }\end{array}$} & \multirow[b]{2}{*}{$\begin{array}{l}\text { Hydrogen } \\
\text { (Imedd) }\end{array}$} & \multirow[b]{2}{*}{$\begin{array}{l}\text { sulturur } \\
\text { (short tons } \\
\text { por day) }\end{array}$} \\
\hline & & & & Lolutane & $\begin{array}{l}\text { leopentane } \\
\text { and } \\
\text { leohexane }\end{array}$ & & & & \\
\hline \multicolumn{10}{|c|}{ 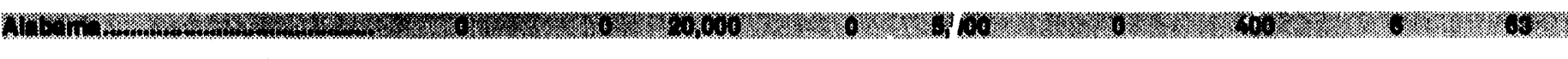 } \\
\hline $\begin{array}{l}\text { Coastal Mobile Refining Co. } \\
\text { Chickasaw ...................................... } \\
\text { Hunt Refining Co. }\end{array}$ & 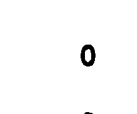 & 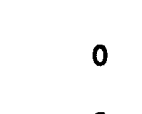 & 10,500 & 0 & 0 & 0 & 0 & 0 & 0 \\
\hline $\begin{array}{l}\text { Tuscaloosa...................................... } \\
\text { LL\&E Petroleum Marketing Inc. }\end{array}$ & 0 & 0 & 9,500 & 0 & 0 & 0 & 400 & 6 & 8 \\
\hline 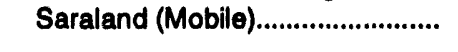 & 0 & 0 & 0 & 0 & 5,000 & 0 & 0 & 0 & 55 \\
\hline \multicolumn{10}{|c|}{ 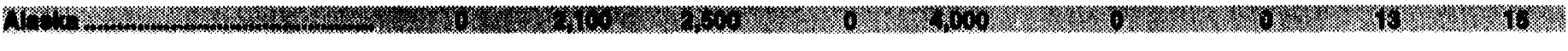 } \\
\hline $\begin{array}{l}\text { Mapco Petroleum Inc. } \\
\text { North Pole...................................... } \\
\text { Tesoro Petroleum Corp. }\end{array}$ & 0 & 2,100 & 2,500 & 0 & 0 & 0 & 0 & 0 & 0 \\
\hline Kenail................................................. & 0 & 0 & 0 & 0 & 4,000 & 0 & 0 & 13 & 15 \\
\hline \multicolumn{10}{|c|}{ 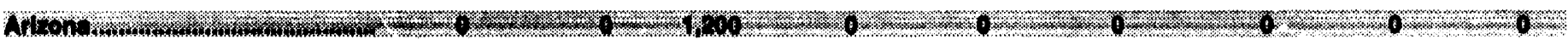 } \\
\hline 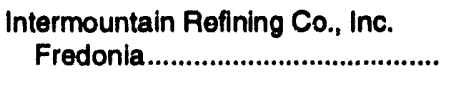 & 0 & 0 & 1,200 & 0 & 0 & 0 & 0 & 0 & 0 \\
\hline \multicolumn{10}{|c|}{ Min . . } \\
\hline $\begin{array}{l}\text { Berry Petroleum Co. } \\
\text { Stephens...................................... } \\
\text { Cross Oil \& Refining Co. Inc. }\end{array}$ & 0 & 0 & 800 & 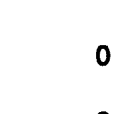 & 0 & 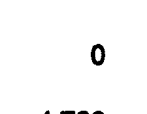 & 0 & 0 & 0 \\
\hline 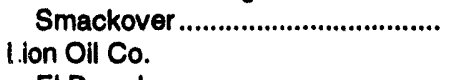 & 0 & 0 & 2,050 & 0 & 0 & 4,700 & 0 & 3 & 0 \\
\hline El Dorado ........................................... & 4,900 & 0 & 7.700 & 0 & 6,000 & 0 & 0 & 0 & 32 \\
\hline \multicolumn{10}{|c|}{ - mhnL } \\
\hline 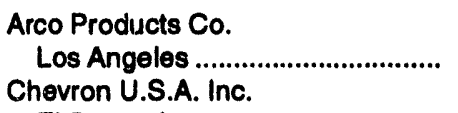 & 13,000 & 0 & 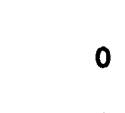 & 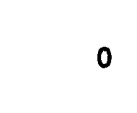 & 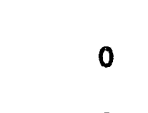 & 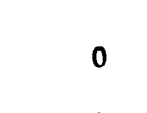 & 10,064 & 75 & 290 \\
\hline El Segundo & $\begin{array}{l}8,400 \\
8,000\end{array}$ & $\begin{array}{l}0 \\
0\end{array}$ & $\begin{array}{r}0 \\
2,000\end{array}$ & $\begin{array}{l}0 \\
0\end{array}$ & $\begin{array}{l}0 \\
0\end{array}$ & $\begin{array}{r}0 \\
13,000\end{array}$ & $\begin{array}{r}16,500 \\
0\end{array}$ & $\begin{array}{l}130 \\
185\end{array}$ & $\begin{array}{l}840 \\
672\end{array}$ \\
\hline $\begin{array}{l}\text { Exxon Co. U.S.A. } \\
\text { Benicia ................................................ } \\
\text { Huntway Refining Co. }\end{array}$ & 14,000 & 0 & 0 & 0 & 0 & 0 & 5,500 & 104 & 304 \\
\hline 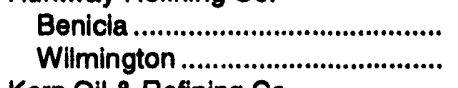 & $\begin{array}{l}0 \\
0\end{array}$ & $\begin{array}{l}0 \\
0\end{array}$ & $\begin{array}{l}4,500 \\
3,500\end{array}$ & $\begin{array}{l}0 \\
0\end{array}$ & $\begin{array}{l}0 \\
0\end{array}$ & $\begin{array}{l}0 \\
0\end{array}$ & $\begin{array}{l}0 \\
0\end{array}$ & $\begin{array}{l}0 \\
0\end{array}$ & $\begin{array}{l}0 \\
0\end{array}$ \\
\hline 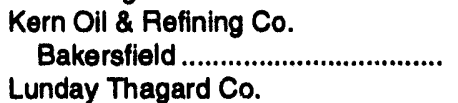 & 0 & 0 & 0 & 0 & 0 & 0 & 0 & 0 & 4 \\
\hline $\begin{array}{l}\text { South Gate................................................. } \\
\text { Mobil Oil Corp. }\end{array}$ & 0 & 0 & 5,833 & 0 & 0 & 0 & 0 & 0 & 0 \\
\hline $\begin{array}{l}\text { Torrance } \\
\text { Pacific Refining Co. }\end{array}$ & 20,000 & 0 & 0 & 0 & 0 & 0 & 14,500 & 122 & 441 \\
\hline $\begin{array}{l}\text { Hercules.................................. } \\
\text { Paramount Petroleum Corp. }\end{array}$ & 0 & 0 & 6,000 & 0 & 0 & 0 & 0 & 0 & 0 \\
\hline $\begin{array}{l}\text { Paramount ................................. } \\
\text { Petroleum Fuel \& Terminal" } \\
\text { (Formerly Edgington Oil Co.) }\end{array}$ & 0 & 0 & 15,000 & 0 & 0 & 0 & 0 & 0 & 0 \\
\hline $\begin{array}{l}\text { Long Beach............................................ } \\
\text { Powerine Oil Co. }\end{array}$ & 0 & 0 & 6,630 & 0 & 0 & 0 & 0 & 0 & 0 \\
\hline $\begin{array}{l}\text { Santa Fe Springs .............................. } \\
\text { San Joaquin Refining Co. Inc. }\end{array}$ & 2,700 & 0 & 0 & 1,500 & 0 & 0 & 2,500 & 19 & 45 \\
\hline $\begin{array}{l}\text { Bakersfield ................................................. } \\
\text { Shell Oil Co. }\end{array}$ & 0 & 0 & 8,000 & 0 & 0 & 4,000 & 0 & 0 & 0 \\
\hline 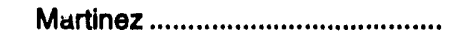 & 9,500 & 0 & 15,000 & 0 & 0 & 4,100 & 700 & 107 & 259 \\
\hline
\end{tabular}

See footnotes at end of table. 
Table 39. Production Capacity of Operable Petroleum Refineries by State as of January 1, 1994 (Continued) (Barrels per Stream Day, Except Where Noted)

\begin{tabular}{|c|c|c|c|c|c|c|c|c|c|}
\hline \multirow[b]{2}{*}{ StatedRefhernocation } & \multirow[b]{2}{*}{ Alkylates } & \multirow[b]{2}{*}{ Aromatics } & \multirow[b]{2}{*}{$\begin{array}{l}\text { Asphal } \\
\text { and } \\
\text { Road OII }\end{array}$} & \multicolumn{2}{|c|}{ bomers } & \multirow[b]{2}{*}{ Lubricants } & \multirow[b]{2}{*}{$\begin{array}{l}\text { Nortortable } \\
\text { Petrolvum } \\
\text { Colve }\end{array}$} & \multirow[b]{2}{*}{ Hydrogen } & \multirow[b]{2}{*}{$\begin{array}{l}\text { Sulfur } \\
\text { (chort tons } \\
\text { per day) }\end{array}$} \\
\hline & & & & bobutan & $\begin{array}{l}\text { leopentione } \\
\text { end } \\
\text { bohoxene }\end{array}$ & & & & \\
\hline $\begin{array}{l}\text { Tenby Inc. } \\
\text { Oxnard....................................... } \\
\text { Texaco Refining \& Marketing Inc. }\end{array}$ & 0 & 0 & 1,200 & 0 & 0 & 0 & 0 & 0 & 0 \\
\hline $\begin{array}{l}\text { Wilmington (Los Angeles)............. } \\
\text { Tosco Refining Co. }\end{array}$ & 6,300 & 0 & 0 & 0 & 0 & 0 & 9,850 & 70 & 392 \\
\hline $\begin{array}{l}\text { Martinez (Avon) } \\
\text { Ultramar Inc............................ }\end{array}$ & 16,000 & 0 & 0 & 0 & 0 & 0 & 1,500 & 80 & 200 \\
\hline 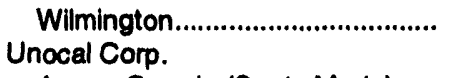 & 10,500 & 0 & 0 & 4,000 & 0 & 0 & 1,350 & 0 & 200 \\
\hline Arroyo Grande (Santa Maria) ...... & 0 & 0 & 0 & 0 & 0 & 0 & 7,000 & 0 & 107 \\
\hline Colorado Refining Co. & & & & & & & & & \\
\hline 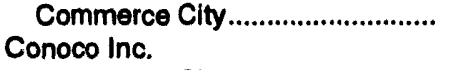 & 1,200 & 0 & 0 & 0 & 0 & 0 & 0 & 0 & 2 \\
\hline 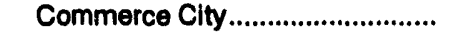 & 0 & 0 & 8,500 & 0 & 0 & 0 & 0 & 0 & 95 \\
\hline \multicolumn{10}{|c|}{ 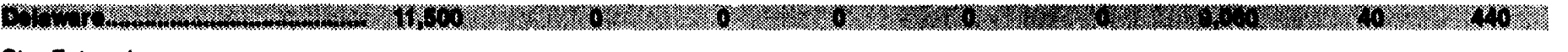 } \\
\hline 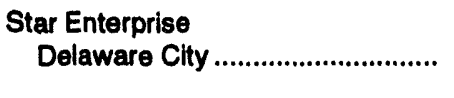 & 11,500 & 0 & 0 & 0 & 0 & 0 & 9,080 & 40 & 440 \\
\hline \multicolumn{10}{|c|}{ coun } \\
\hline \multicolumn{10}{|c|}{$\begin{array}{l}\text { Citgo Asphalt Refining Co. } \\
\text { (Formerly Amoco Oil Co.) }\end{array}$} \\
\hline Savannah ............................................. & 0 & 0 & 24,000 & 0 & 0 & 0 & 0 & 0 & 0 \\
\hline 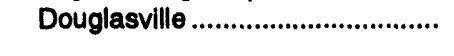 & 0 & 0 & 3,000 & 0 & 0 & 2,000 & 0 & 0 & 0 \\
\hline \multicolumn{10}{|c|}{ W.111. } \\
\hline \multicolumn{10}{|c|}{$\begin{array}{l}\text { BHP Petroleum Americas Refining Inc. } \\
\text { (Formerly Hawailan Independent Refining Inc.) }\end{array}$} \\
\hline $\begin{array}{l}\text { Ewa Beach ................................... } \\
\text { Chevron U.S.A. Inc. }\end{array}$ & 0 & 0 & 950 & 0 & 0 & 0 & 0 & 18 & 0 \\
\hline 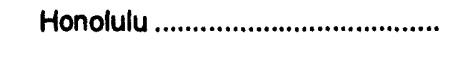 & 5,000 & 0 & 15,000 & 3,200 & 0 & 0 & 0 & 3 & 0 \\
\hline \multicolumn{10}{|l|}{ MHab $-2.3 \%$} \\
\hline 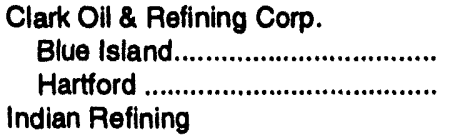 & $\begin{array}{l}5,000 \\
8,000\end{array}$ & $\begin{array}{l}0 \\
0\end{array}$ & $\begin{array}{l}7,000 \\
2,500\end{array}$ & $\begin{array}{r}1,000 \\
0\end{array}$ & $\begin{array}{l}3,000 \\
4,000\end{array}$ & $\begin{array}{l}0 \\
0\end{array}$ & $\begin{array}{r}0 \\
4,500\end{array}$ & $\begin{array}{r}19 \\
3\end{array}$ & $\begin{array}{r}22 \\
110\end{array}$ \\
\hline $\begin{array}{l}\text { Lawrenceville........................................ } \\
\text { Marathon Oil Co. }\end{array}$ & 6,000 & 0 & 4,500 & 0 & 0 & 0 & 0 & 0 & 70 \\
\hline $\begin{array}{l}\text { Robinson } \\
\text { Mobil Oil Corp. }\end{array}$ & 12,500 & 0 & 0 & 0 & 4,900 & 0 & 6,400 & 0 & 180 \\
\hline Joliet ................................................. & 25,000 & 0 & 0 & 0 & 0 & 0 & 11,750 & 0 & 395 \\
\hline $\begin{array}{l}\text { Wood Awer....................................... } \\
\text { Uno-Ven Co. }\end{array}$ & 22,000 & 4,500 & 45,000 & 0 & 0 & 7,200 & 0 & 35 & 450 \\
\hline 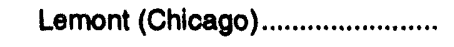 & 18,000 & 3,500 & 0 & 0 & 0 & 0 & 1,969 & 11 & 448 \\
\hline
\end{tabular}


Table 39. Production Capacity of Operable Petroleum Rofinerles by State as of January 1, 1994 (Continued) (Barrels per Stream Day, Except Where Noted)

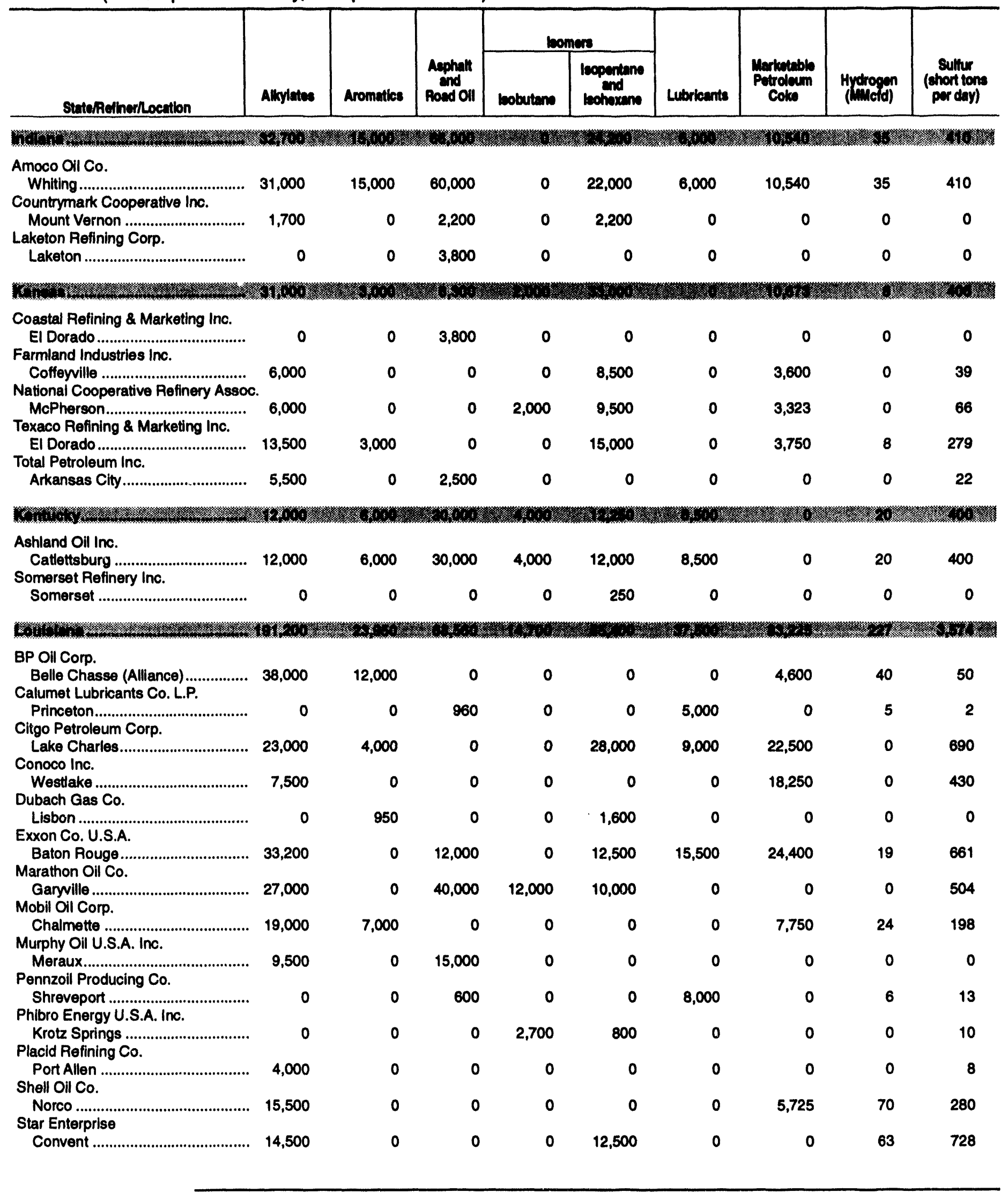


Table 39. Production Capacity of Operable Potroleum Refineries by State as of January 1, 1994 (Continued) (Barrels per Stream Day, Except Where Noted)

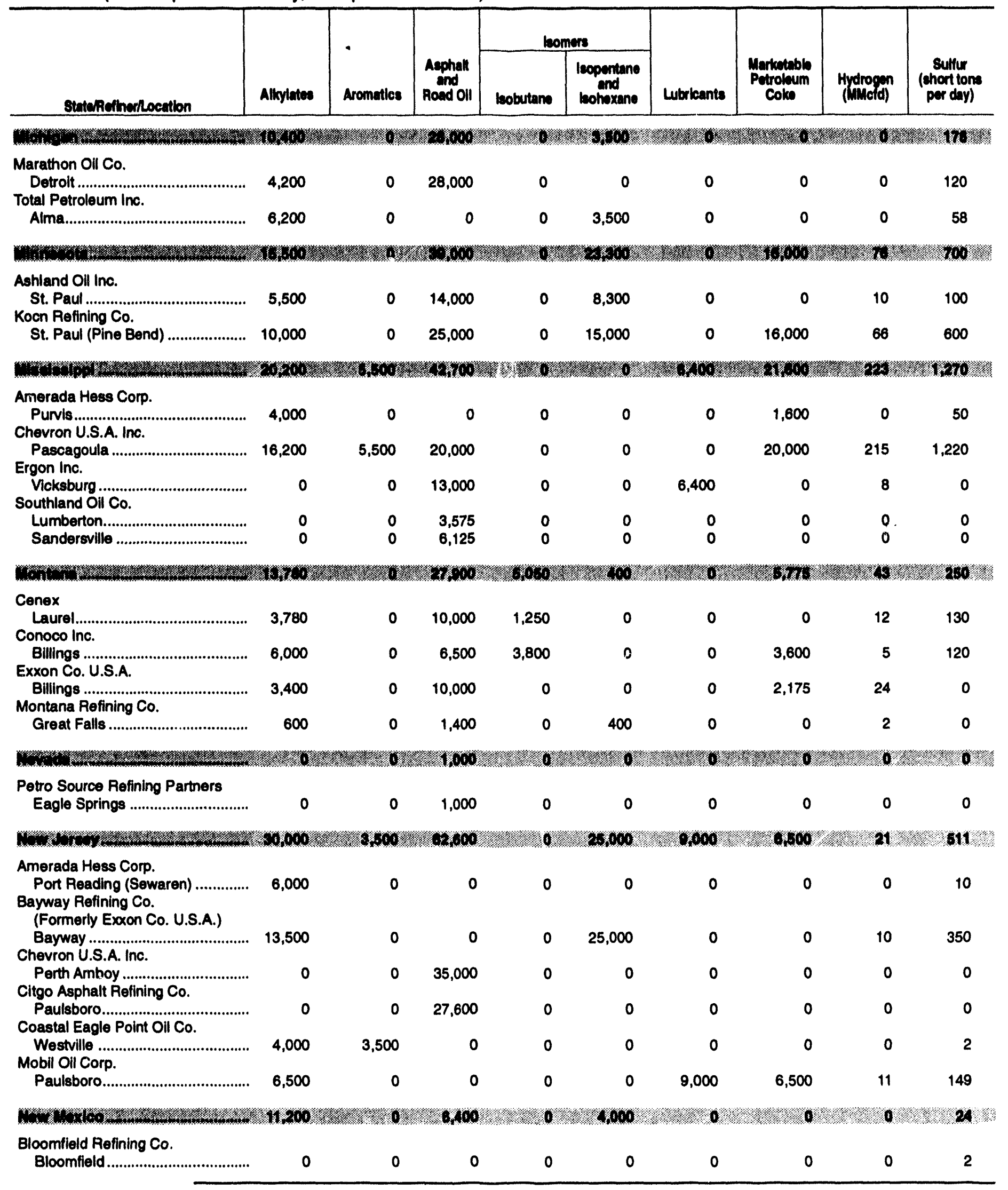


Table 39. Production Capacity of Operable Petroleum Refinerles by State as of January 1, 1994 (Continued) (Barrels per Stream Day, Except Where Noted)

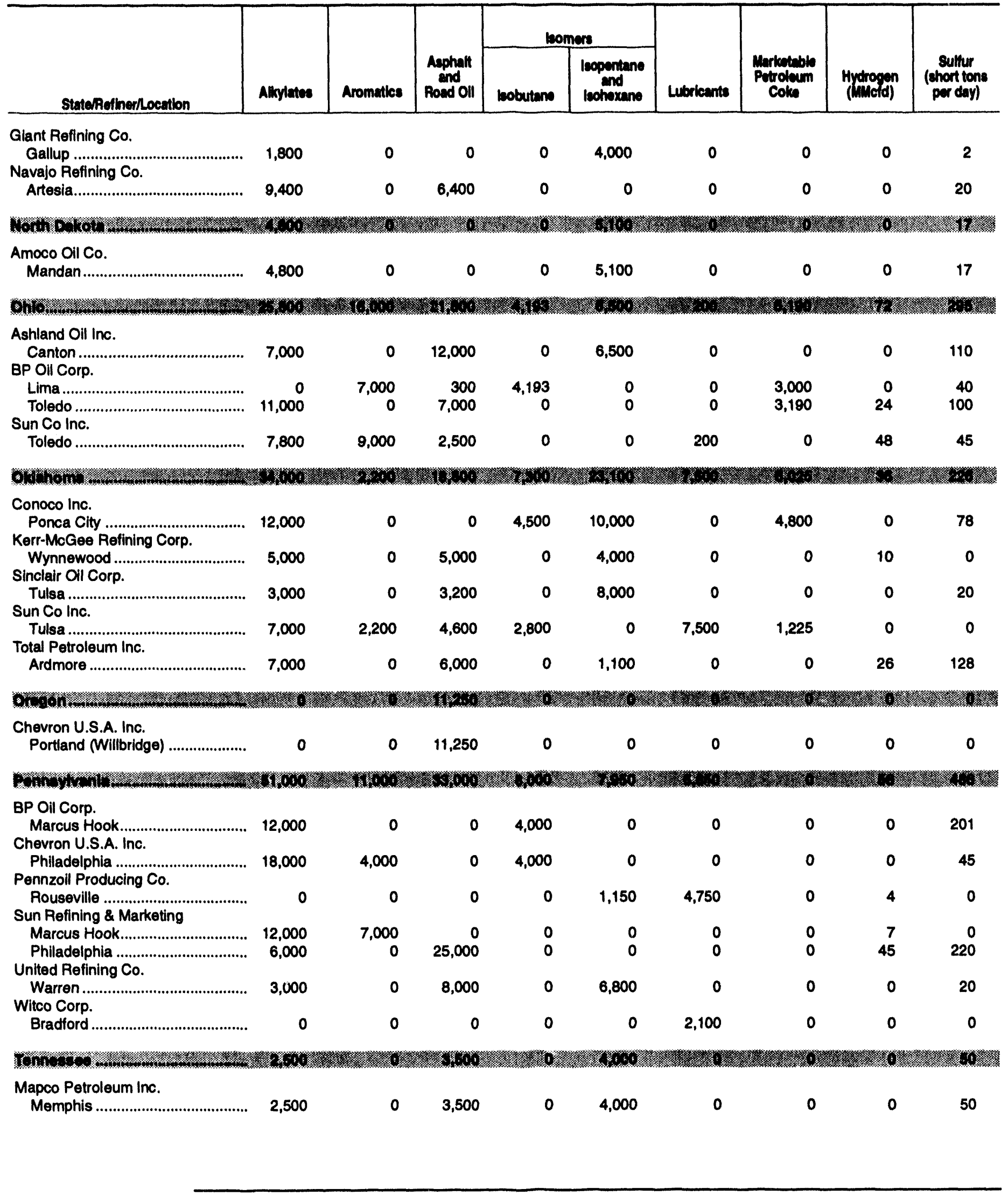

See footnotes at end of table. 
Table 39. Production Capacity of Operable Petroleum Rofinorles by State as of January 1, 1994 (Continued) (Barrels per Stream Day, Except Where Noted)

\begin{tabular}{|c|c|c|c|c|c|c|c|c|c|}
\hline \multirow[b]{2}{*}{ State/Rellner/Location } & \multirow[b]{2}{*}{ Alkylates } & \multirow[b]{2}{*}{ Aromatics } & \multirow[b]{2}{*}{$\begin{array}{l}\text { Acphall } \\
\text { and } \\
\text { Roand oll }\end{array}$} & \multicolumn{2}{|c|}{ comere } & \multirow[b]{2}{*}{ Lubricants } & \multirow[b]{2}{*}{$\begin{array}{l}\text { Marketabls } \\
\text { Petroloum } \\
\text { Coke }\end{array}$} & \multirow[b]{2}{*}{$\begin{array}{l}\text { Hydrogen } \\
\text { (mimold) }\end{array}$} & \multirow[b]{2}{*}{$\begin{array}{l}\text { sulth } \\
\text { (short tons } \\
\text { per day) }\end{array}$} \\
\hline & & & & nobutane & $\begin{array}{l}\text { leopention } \\
\text { ind } \\
\text { bohoxene }\end{array}$ & & & & \\
\hline \multicolumn{10}{|c|}{ nu } \\
\hline $\begin{array}{l}\text { Age Refining \& Marketing } \\
\text { San Antonio ................................................ } \\
\text { Amoco Oll Co. }\end{array}$ & 0 & 1,200 & 0 & 0 & 0 & 0 & 0 & 0 & 0 \\
\hline $\begin{array}{l}\text { Texas City...................................... } \\
\text { Chevron U.S.A. Inc. }\end{array}$ & 62,000 & 45,000 & 0 & 0 & 28,000 & 0 & 13,800 & 204 & 1,400 \\
\hline 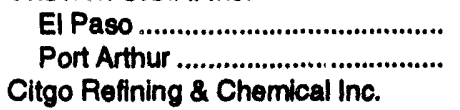 & $\begin{array}{r}9,000 \\
16,900\end{array}$ & $\begin{array}{r}0 \\
15,800\end{array}$ & $\begin{array}{r}5,600 \\
0\end{array}$ & $\begin{array}{l}0 \\
0\end{array}$ & $\begin{array}{l}0 \\
0\end{array}$ & $\begin{array}{l}0 \\
0\end{array}$ & $\begin{array}{r}675 \\
9,750\end{array}$ & $\begin{array}{l}0 \\
0\end{array}$ & $\begin{array}{r}64 \\
580\end{array}$ \\
\hline $\begin{array}{l}\text { Corpus Christi... } \\
\text { Coastal Refining \& Marketing Inc. }\end{array}$ & 19,200 & 6,500 & 0 & 0 & 0 & 0 & 11,500 & 0 & 783 \\
\hline $\begin{array}{l}\text { Corpus Christi............................ } \\
\text { Deer Park Refg Lid Partnership } \\
\text { (Formerly Shell Oll Co.) }\end{array}$ & 3,000 & 19,100 & 5,000 & 0 & 5,200 & 0 & 3,250 & 24 & 85 \\
\hline $\begin{array}{l}\text { Deor Park } \\
\text { Diamond Shamrock Refining \& Mark }\end{array}$ & $\begin{array}{l}8,100 \\
\text { keting Co. }\end{array}$ & 11,900 & 5,400 & 0 & 0 & 10,000 & 0 & 60 & 577 \\
\hline 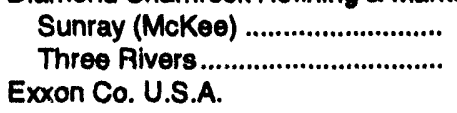 & $\begin{array}{l}9,150 \\
6,000\end{array}$ & $\begin{array}{l}0 \\
0\end{array}$ & $\begin{array}{r}7,500 \\
500\end{array}$ & $\begin{array}{l}0 \\
0\end{array}$ & $\begin{array}{l}0 \\
0\end{array}$ & $\begin{array}{r}0 \\
1,500\end{array}$ & $\begin{array}{l}0 \\
0\end{array}$ & $\begin{array}{l}0 \\
0\end{array}$ & $\begin{array}{r}45 \\
0\end{array}$ \\
\hline $\begin{array}{l}\text { Baytown.................... } \\
\text { Fina Oll \& Chemical Co. }\end{array}$ & 29,000 & 0 & 7,000 & 0 & 0 & 31,200 & 1,250 & 147 & 1,030 \\
\hline 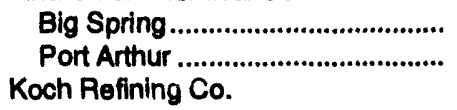 & $\begin{array}{l}5,000 \\
5,500\end{array}$ & $\begin{array}{l}1,000 \\
9,500\end{array}$ & $\begin{array}{l}7,600 \\
2,000\end{array}$ & $\begin{array}{l}0 \\
0\end{array}$ & 8,000 & $\begin{array}{l}0 \\
0\end{array}$ & $\begin{array}{l}0 \\
0\end{array}$ & $\begin{array}{r}0 \\
34\end{array}$ & $\begin{array}{l}129 \\
253\end{array}$ \\
\hline $\begin{array}{l}\text { Corpus Christi............................... } \\
\text { La Gloria Oil \& Gas Co. }\end{array}$ & 9,900 & 18,500 & 0 & 0 & 2,000 & 0 & 2,350 & 0 & 50 \\
\hline 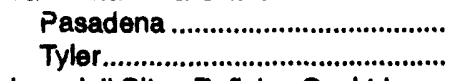 & $\begin{array}{r}10,000 \\
4,700\end{array}$ & $\begin{array}{l}0 \\
0\end{array}$ & $\begin{array}{l}0 \\
0\end{array}$ & $\begin{array}{r}0 \\
500\end{array}$ & $\begin{array}{l}5,000 \\
4,000\end{array}$ & $\begin{array}{l}0 \\
0\end{array}$ & $\begin{array}{l}1,500 \\
1,500\end{array}$ & $\begin{array}{l}0 \\
0\end{array}$ & $\begin{array}{l}20 \\
11\end{array}$ \\
\hline $\begin{array}{l}\text { Lyondell Citgo Refining Co. Litd. } \\
\text { (Formerly Lyondell Petrochemical }\end{array}$ & (Co.) & & & & & & & & \\
\hline $\begin{array}{l}\text { Houston ............................. } \\
\text { Marathon Oil Co. }\end{array}$ & 0 & 36,000 & 0 & 0 & 0 & 6,600 & 10,900 & 0 & 400 \\
\hline $\begin{array}{l}\text { Texas City................................. } \\
\text { Mobil Oil Corp. }\end{array}$ & 10,000 & 2,500 & 0 & 0 & 0 & 0 & 0 & 0 & 0 \\
\hline $\begin{array}{l}\text { Beaumont ............................................ } \\
\text { Petrolite Corp. }\end{array}$ & 13,500 & 0 & 0 & 0 & 20,000 & 11,000 & 12,000 & 60 & 560 \\
\hline $\begin{array}{l}\text { Kilgore .................................. } \\
\text { Phibro Energy U.S. }\end{array}$ & 0 & 0 & 125 & 0 & 0 & 0 & 0 & 0 & 0 \\
\hline Mouston & $\begin{array}{l}8,500 \\
6,000\end{array}$ & $\begin{array}{r}2,600 \\
0\end{array}$ & $\begin{array}{r}8,000 \\
0\end{array}$ & $\begin{array}{l}0 \\
0\end{array}$ & $\begin{array}{r}0 \\
6,000\end{array}$ & $\begin{array}{l}0 \\
0\end{array}$ & $\begin{array}{l}0 \\
0\end{array}$ & $\begin{array}{l}0 \\
0\end{array}$ & $\begin{array}{l}100 \\
145\end{array}$ \\
\hline Phillips 66 Co. & & & & & & & & & \\
\hline 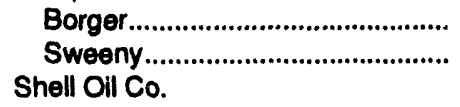 & $\begin{array}{l}14,000 \\
20,000\end{array}$ & $\begin{array}{r}0 \\
5,575\end{array}$ & $\begin{array}{l}0 \\
0\end{array}$ & $\begin{array}{r}11,000 \\
0\end{array}$ & $\begin{array}{l}24,600 \\
16,900\end{array}$ & $\begin{array}{l}0 \\
0\end{array}$ & $\begin{array}{l}0 \\
0\end{array}$ & $\begin{array}{r}50 \\
120\end{array}$ & $\begin{array}{l}340 \\
385\end{array}$ \\
\hline Odessa & 3,300 & 0 & 0 & 0 & 0 & 0 & 0 & 0 & 0 \\
\hline $\begin{array}{l}\text { Silsbee............... } \\
\text { Southwestern Refining Co. Inc. }\end{array}$ & 0 & 400 & 0 & 0 & 825 & 0 & 0 & 2 & 0 \\
\hline $\begin{array}{l}\text { Corpus Christi............................................ } \\
\text { Star Enterprise }\end{array}$ & 7,500 & 6,500 & 0 & 0 & 7,500 & 0 & 0 & 0 & 123 \\
\hline $\begin{array}{l}\text { Port Arthur/Neches.......................... } \\
\text { Trifinery }\end{array}$ & 20,000 & 0 & 0 & 0 & 0 & 19,500 & 16,535 & 0 & 484 \\
\hline $\begin{array}{l}\text { Corpus Christl................................. } \\
\text { Valero Refining Co. }\end{array}$ & 0 & 0 & 11,400 & 0 & 0 & 0 & 0 & 0 & 0 \\
\hline 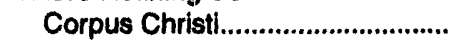 & . 11,200 & 0 & 0 & 13,000 & 0 & 0 & 0 & 118 & 370 \\
\hline
\end{tabular}

See footnotes at end of table. 
Table 39. Production Capacity of Operable Petroleum Refineries by State as of January 1, 1994 (Continued) (Barrels per Stream Day, Excr.pt Where Noted)

\begin{tabular}{|c|c|c|c|c|c|c|c|c|c|}
\hline \multirow[b]{2}{*}{ Staterietherhocution } & \multirow[b]{2}{*}{ Alloylates } & \multirow[b]{2}{*}{ Arometies } & \multirow[b]{2}{*}{$\begin{array}{l}\text { Aephall } \\
\text { end } \\
\text { Roed OII }\end{array}$} & \multicolumn{2}{|c|}{ bomers } & \multirow[b]{2}{*}{ Lubricants } & \multirow[b]{2}{*}{$\begin{array}{l}\text { Marketable } \\
\text { Petroleum } \\
\text { Colw }\end{array}$} & \multirow[b]{2}{*}{ Hydrogen } & \multirow[b]{2}{*}{$\begin{array}{l}\text { Suliur } \\
\text { (ehort tons } \\
\text { per day) }\end{array}$} \\
\hline & & & & bobutane & $\begin{array}{l}\text { locpentime } \\
\text { and } \\
\text { bohoxene }\end{array}$ & & & & \\
\hline \multicolumn{10}{|c|}{ W } \\
\hline 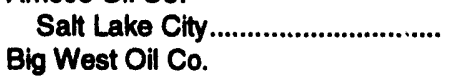 & 4,000 & 0 & 0 & 3,000 & 0 & 0 & 0 & 0 & 0 \\
\hline $\begin{array}{l}\text { North Salt Lake.............................. } \\
\text { Chevron U.S.A. Inc. }\end{array}$ & 1,300 & 0 & 0 & 200 & 1,650 & 0 & 0 & 0 & 0 \\
\hline $\begin{array}{l}\text { Salt Lake City............................... } \\
\text { Crysen Refining Inc. }\end{array}$ & 5,000 & 0 & 0 & 1,100 & 0 & 0 & 2,000 & 0 & 18 \\
\hline $\begin{array}{l}\text { Woods Cross } \\
\text { Phillips } 66 \text { Co............................. }\end{array}$ & 0 & 0 & 1,600 & $\mathbf{0}$ & 0 & 0 & 0 & 0 & 0 \\
\hline Woods Cross & 2,100 & 0 & 1,700 & 0 & 2,600 & 0 & 0 & 0 & 11 \\
\hline \multicolumn{10}{|c|}{ win } \\
\hline 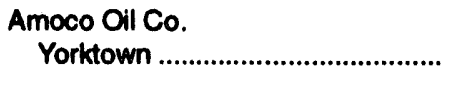 & 0 & 0 & 0 & 0 & 0 & 0 & 5,100 & 0 & 73 \\
\hline \multicolumn{10}{|c|}{ 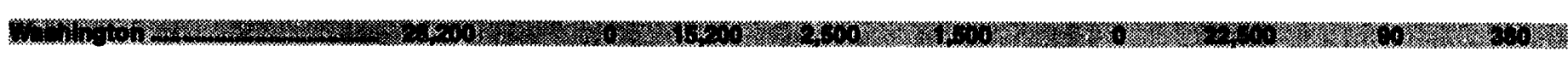 } \\
\hline $\begin{array}{l}\text { Arco Products Co. } \\
\text { Ferndale (Cherry Point) ............... } \\
\text { Chevron U.S.A. Inc. }\end{array}$ & 0 & 0 & 0 & 0 & 0 & 0 & 15,000 & 90 & 225 \\
\hline $\begin{array}{l}\text { Richmond Beach ............................ } \\
\text { Shell Oil Co. }\end{array}$ & 0 & 0 & 4,200 & 0 & 0 & 0 & 0 & 0 & 0 \\
\hline $\begin{array}{l}\text { Anacortes....................................... } \\
\text { Sound Refining Inc. }\end{array}$ & 11,500 & 0 & 0 & 2,500 & 0 & 0 & 0 & 0 & 0 \\
\hline $\begin{array}{l}\text { Tacoma ....................................... } \\
\text { Texaco Refining \& Marketing Inc. }\end{array}$ & 0 & 0 & 3,000 & 0 & 0 & 0 & 0 & 0 & 0 \\
\hline $\begin{array}{l}\text { Anacortes (Puget Sound)............. } \\
\text { Tosco Northwest Co. } \\
\text { (Formerly BP Oil Corp.) }\end{array}$ & 10,500 & 0 & 0 & 0 & 0 & 0 & 7,500 & 0 & 108 \\
\hline $\begin{array}{l}\text { Ferndale......................... } \\
\text { U.S. Oil \& Refining Co. }\end{array}$ & 6,200 & 0 & 0 & 0 & 0 & 0 & 0 & 0 & 40 \\
\hline Tacoma & 0 & 0 & 8,000 & 0 & 1,500 & 0 & 0 & 0 & 7 \\
\hline \multicolumn{10}{|l|}{ Quaker State Corp. } \\
\hline 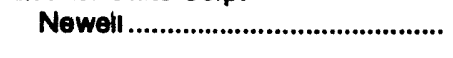 & 0 & 0 & 0 & 0 & 0 & 5,056 & 0 & 1 & 0 \\
\hline \multicolumn{10}{|c|}{ 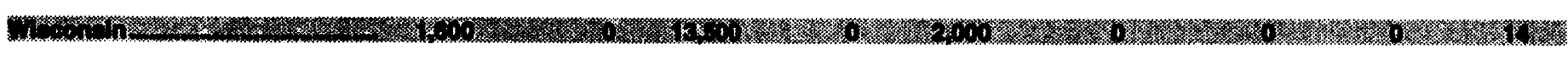 } \\
\hline 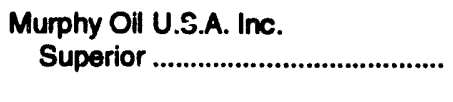 & 1,600 & 0 & 13,500 & 0 & 2,000 & 0 & 0 & 0 & 14 \\
\hline 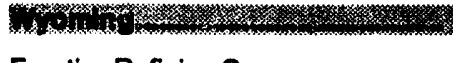 & ments & 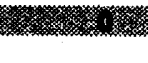 & mysis & 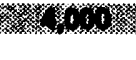 & & 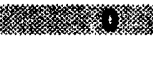 & & 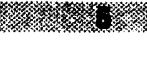 & \\
\hline $\begin{array}{l}\text { Frontier Refining Co. } \\
\text { Cheyenne ....................................... } \\
\text { Sinclair Oil Corp. }\end{array}$ & 3,000 & 0 & 7,000 & 2,000 & 0 & 0 & 621 & 5 & 97 \\
\hline 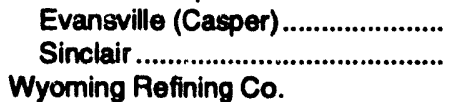 & $\begin{array}{r}0 \\
4,000\end{array}$ & $\begin{array}{l}0 \\
0\end{array}$ & $\begin{array}{l}4,400 \\
5,000\end{array}$ & $\begin{array}{r}0 \\
2,000\end{array}$ & $\begin{array}{l}0 \\
0\end{array}$ & $\begin{array}{l}0 \\
0\end{array}$ & $\begin{array}{l}0 \\
0\end{array}$ & $\begin{array}{l}0 \\
0\end{array}$ & $\begin{array}{r}0 \\
40\end{array}$ \\
\hline 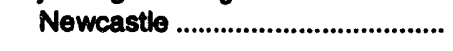 & 834 & 0 & 0 & 0 & 0 & 0 & 0 & 0 & 2 \\
\hline
\end{tabular}

See footnotes at end of table. 
Table 39. Production Capactly of Operable Petroloum Rofinerles by State as of January 1, 1994 (Continued) (Barrels per Stream Day, Except Where Noted)

\begin{tabular}{|c|c|c|c|c|c|c|c|c|c|}
\hline \multirow[b]{2}{*}{ Stuterintinerlocention } & \multirow[b]{2}{*}{ Alloyletes } & \multirow[b]{2}{*}{ Aromatios } & \multirow[b]{2}{*}{$\begin{array}{l}\text { Aephall } \\
\text { and } \\
\text { Roend OII }\end{array}$} & \multicolumn{2}{|c|}{ bomere } & \multirow[b]{2}{*}{ Lubriants } & \multirow[b]{2}{*}{ 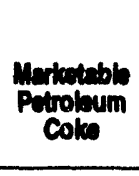 } & \multirow[b]{2}{*}{$\begin{array}{l}\text { Hydrogen } \\
\text { (Mincid) }\end{array}$} & \multirow[b]{2}{*}{$\begin{array}{l}\text { Sultur } \\
\text { (short fons } \\
\text { per day) }\end{array}$} \\
\hline & & & & Dobuten & $\begin{array}{l}\text { bopentane } \\
\text { and } \\
\text { bohoxine }\end{array}$ & & & & \\
\hline \multirow{4}{*}{$\begin{array}{l}\text { Caribbean Petroleum Corp. } \\
\text { San Juan (Bayamon) .................... } \\
\text { Peerless Oll \& Chemical } \\
\text { Ponce.......................................... } \\
\text { Phillips Puento Rico Core Inc. } \\
\text { Guayama ........................................ } \\
\text { Sun Co Inc. } \\
\text { Yabucoa................................... }\end{array}$} & 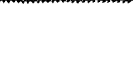 & 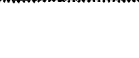 & & & tom & 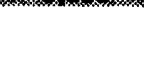 & 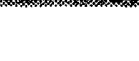 & & $m$ \\
\hline & 0 & 0 & 0 & 0 & 0 & 0 & 0 & 7 & 0 \\
\hline & 0 & 15,000 & 0 & 0 & 0 & 0 & 0 & 0 & 0 \\
\hline & 0 & 0 & 0 & 0 & 0 & 9,500 & 0 & 19 & 50 \\
\hline
\end{tabular}




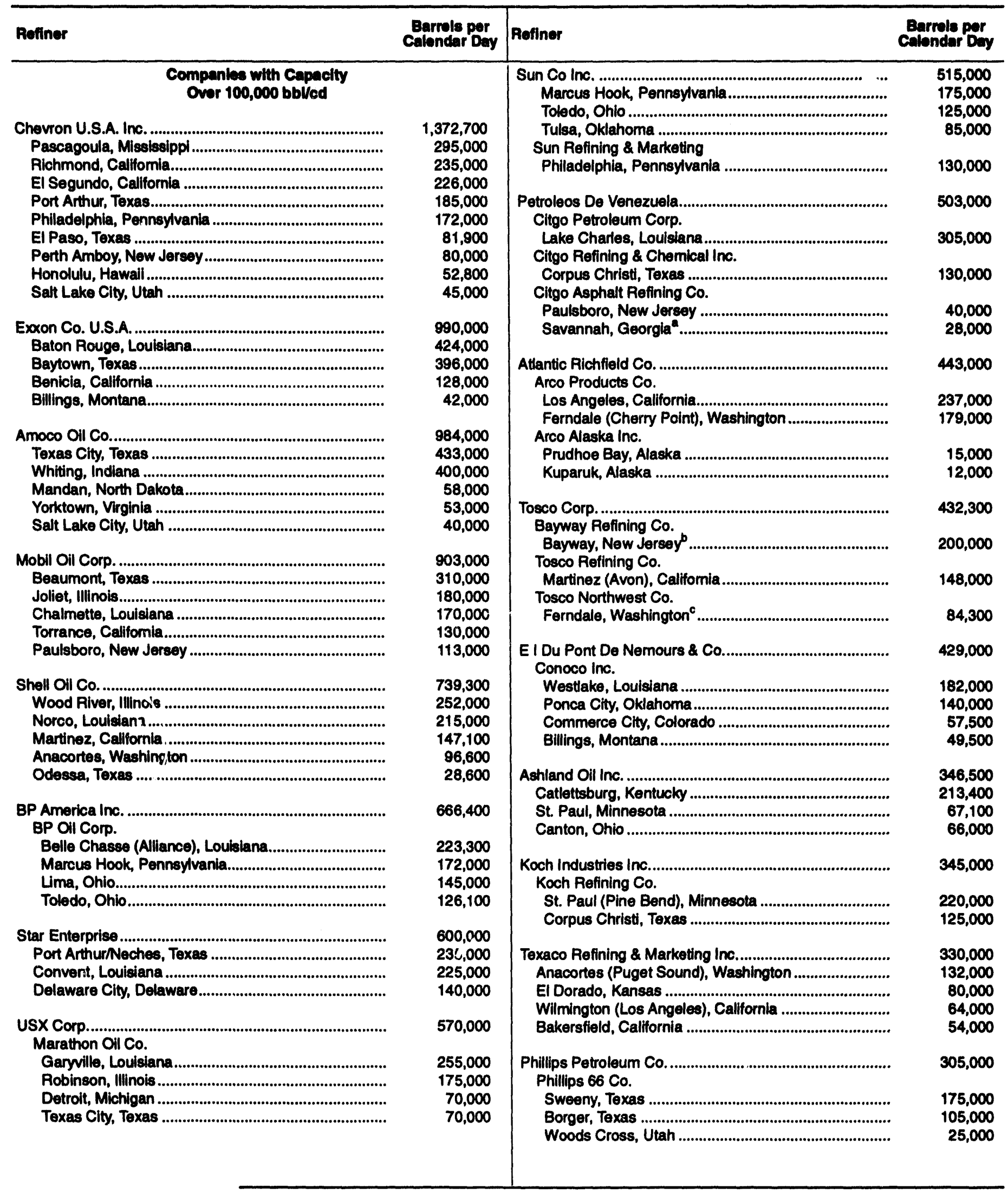

See footnotes at end of table. 
Tablo 40. Rofiners' Operable Atmospheric Crude Oll Distillation Capacity as of January 1, 1994 (Continued)

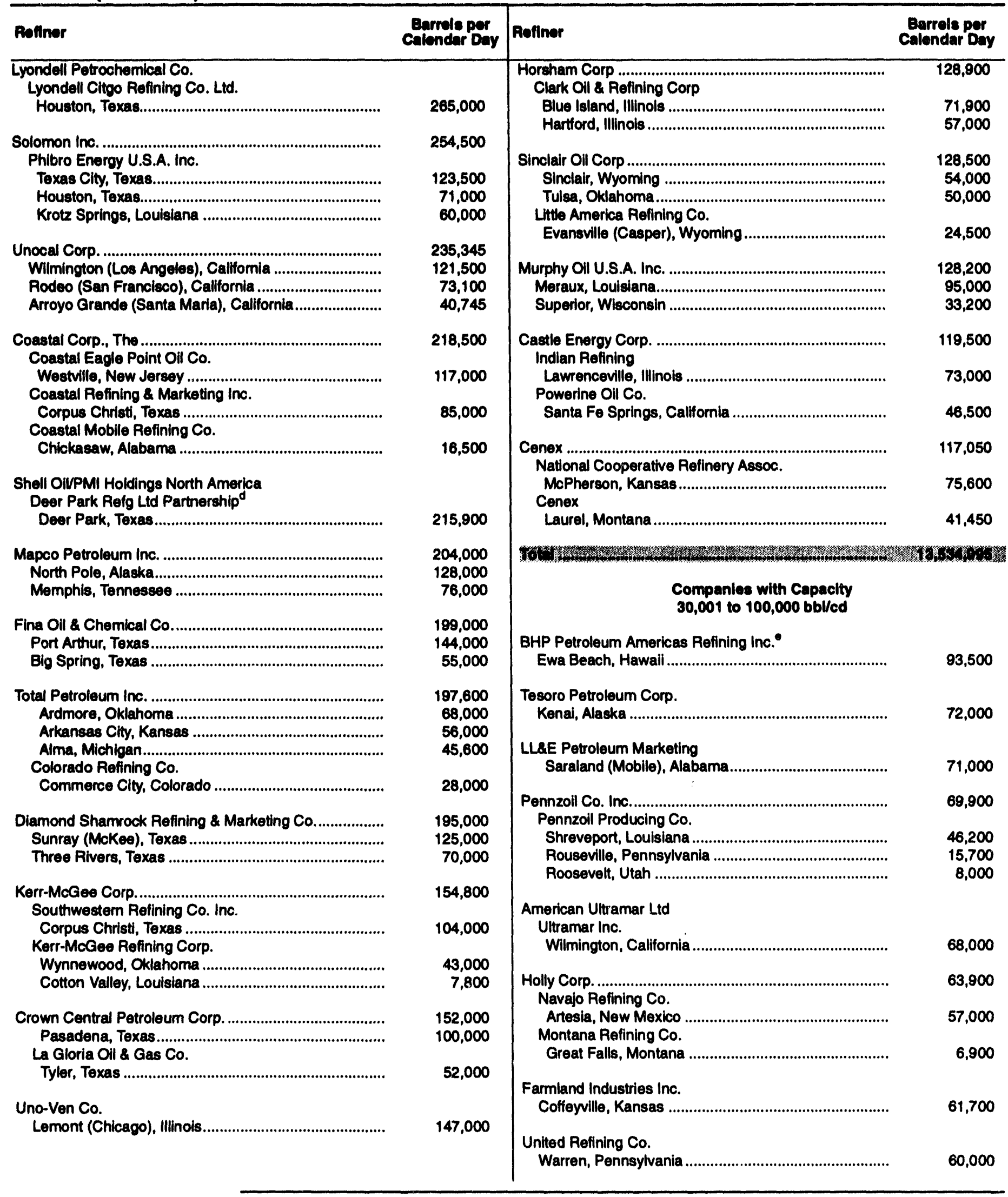

See footnotes at end of table. 
Table 40. Refiners' Operable Atmospheric Crude Oll Distillation Capacity as of January 1, 1994 (Continued)

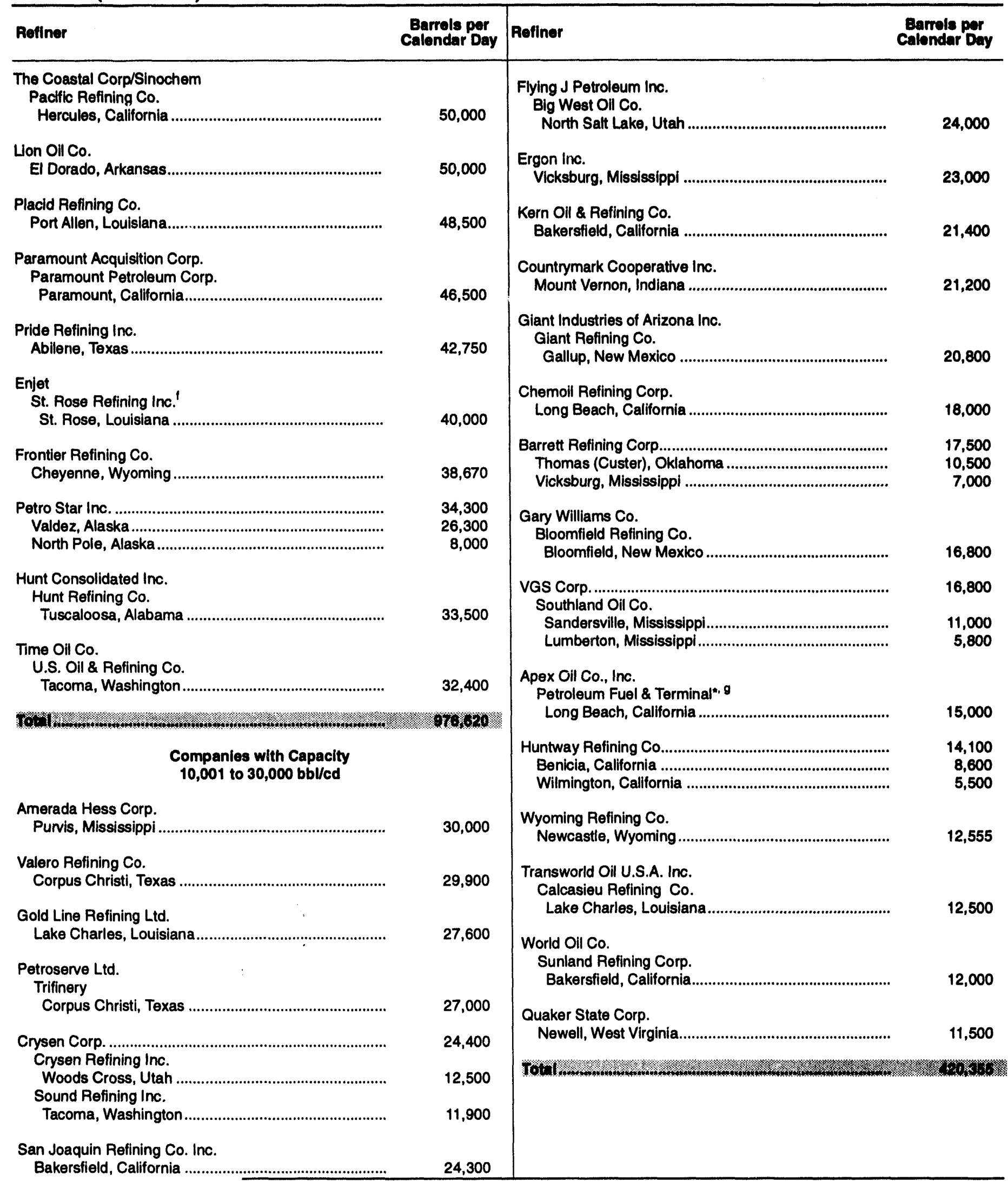

See footnotes at end of table. 
Table 40. Refiners' Operable Atmosphoric Crude Oll Distillation Capacity as of January 1, 1994 (Continued)

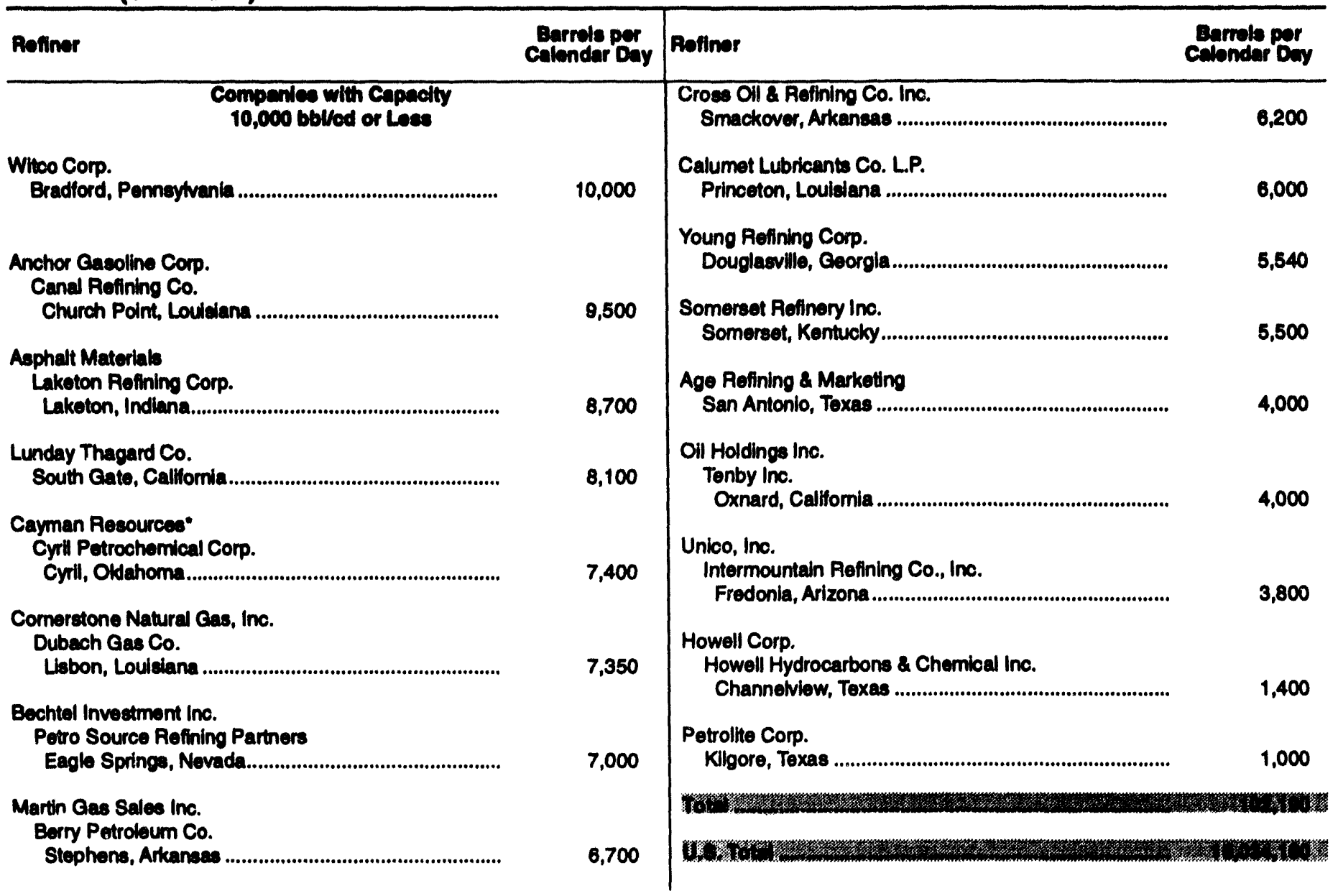

- Formerly Amoco On Co.

b Formerly Exoxon Co. U.S.A

c Formerly BP Amerion Inc.

d Formerly Shell OH Co.

- Formerly Peclitic Resources inc.

I Formerty Phibro Enaroy U.S.A. Inc.

- Formerly Edgington Oll Co.

boved = Barrels per Calender Doy.

- Refinery becamo operable during 1993.

Source: Enorgy information Administration (EIA), Form EIA-820, "Annual Refinery Report." 
Table 41. Operable Crude and Downatream Charge Capaclty of Petroleum Rofinerles, January 1, 1981 to January 1, 1995

(Thousand Barrels per Stream Day, Except Where Noted)

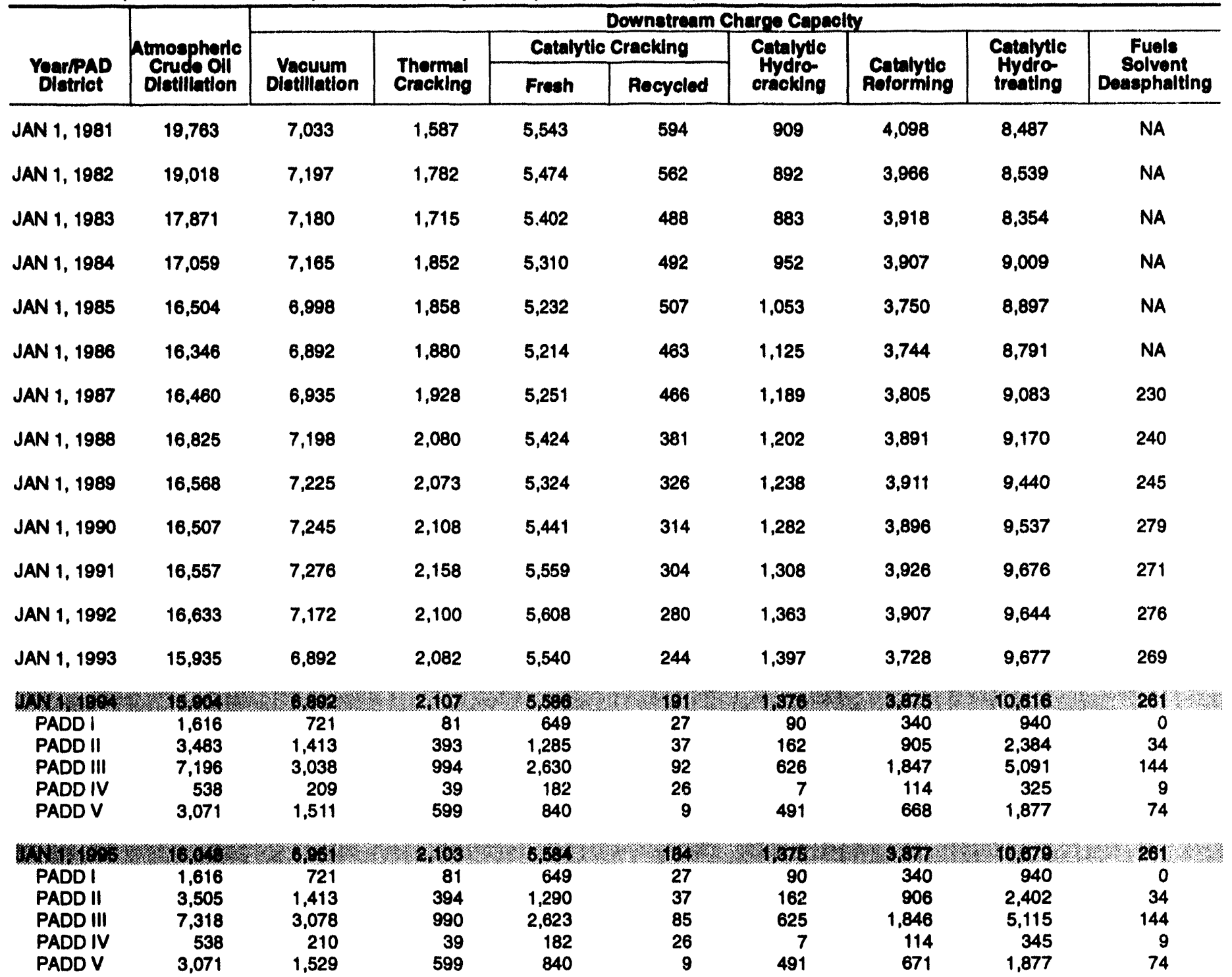

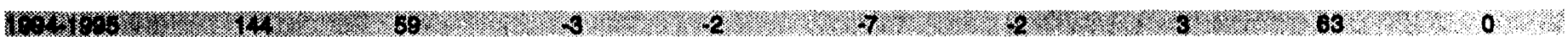
(Not Change)

$\begin{array}{lrrrrrrrrr}\text { PADD I } & 0 & 0 & 0 & 0 & 0 & 0 & 0 & 0 & 0 \\ \text { PADD II } & 22 & 0 & 1 & 5 & 0 & 0 & 0 \\ \text { PADD III } & 122 & 39 & -4 & -7 & -7 & -2 & 0 \\ \text { PADD IV } & 0 & 1 & 0 & 0 & 0 & 0 & 0 \\ \text { PADD V } & 0 & 18 & 0 & 0 & 0 & 0 & 0 \\ \end{array}$

NA $=$ Not available.

Note: Totals may not equal sum of components due to independent rounding.

Source: Energy Information Administration (EIA), Form ElA-820, "Annual Refinery Report." 
Tcble 42. Operabls Production Capaclity of Petroloum Aefineribe, January 1, 1981 to January 1, 1998 (Thousand Barrels per Straam Day, Except Where Noted)

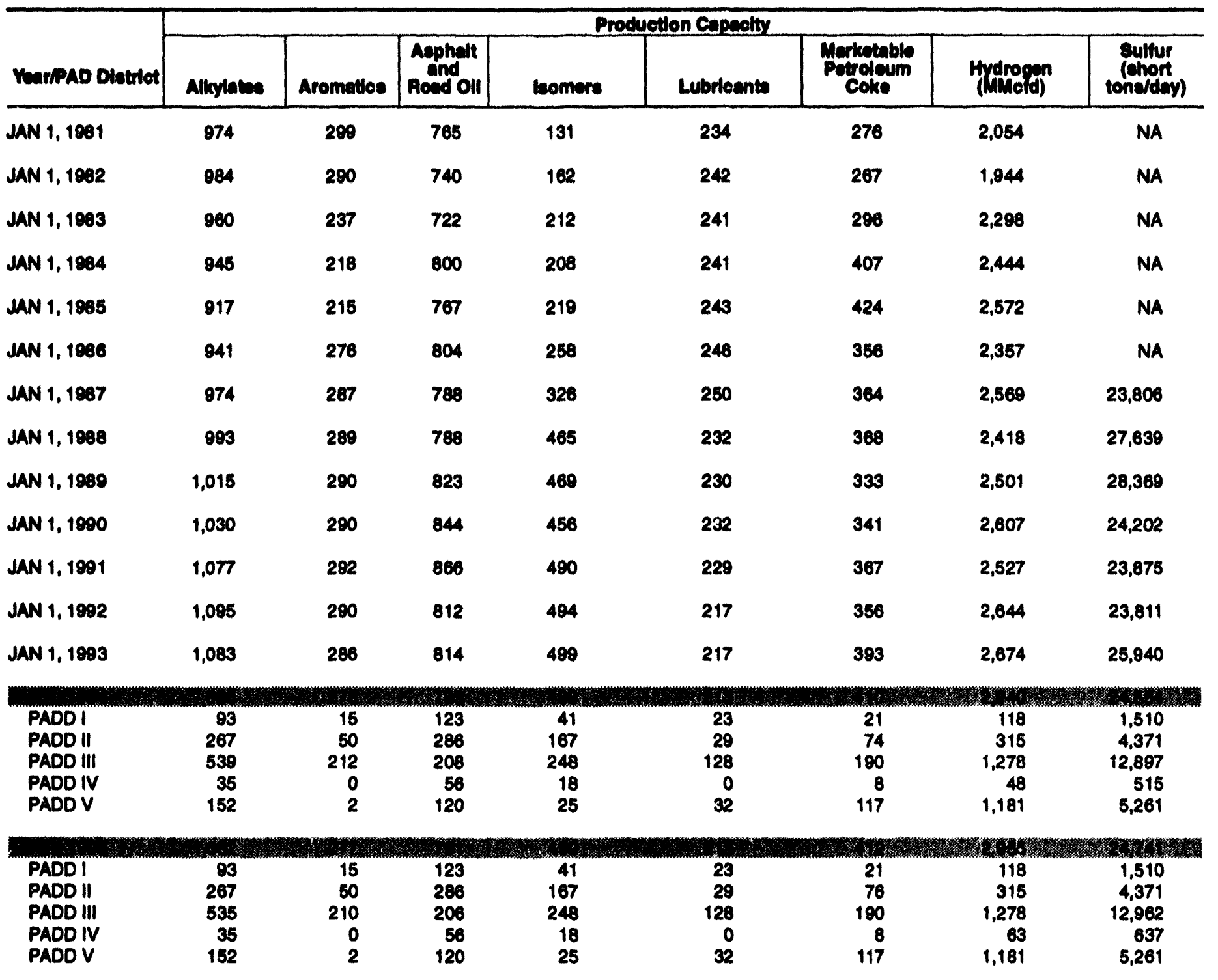

(Net Change)

\begin{tabular}{lrrrrrrrr} 
PADD I & 0 & 0 & 0 & 0 & 0 & 0 & 0 \\
PADD II & 0 & 0 & 0 & 0 & 0 & 0 & 0 \\
PADD III & -4 & -2 & -2 & 0 & 0 & 0 & 0 \\
PADD IV & 0 & 0 & 0 & 0 & 0 & 0 & 15 \\
PADD V & 0 & 0 & 0 & 0 & 0 & 0 & 122 \\
\hline
\end{tabular}

NA $=$ Not avallable. MiMotd $=$ Million cuble foet per day.

(s) = Less than 500 barrels per stream day.

Note: Totals may not equal sum of components dus to independent rounding.

Source: Energy Information Adminiatration (EIA), Form EIA-820, "Annual Refinery Report." 
Table 43. Working Storage Capaclty" at Rofineries and Gasollne Blending Plants by PAD Dlatrict as of January 1, 1994

(Thousand Barrels)

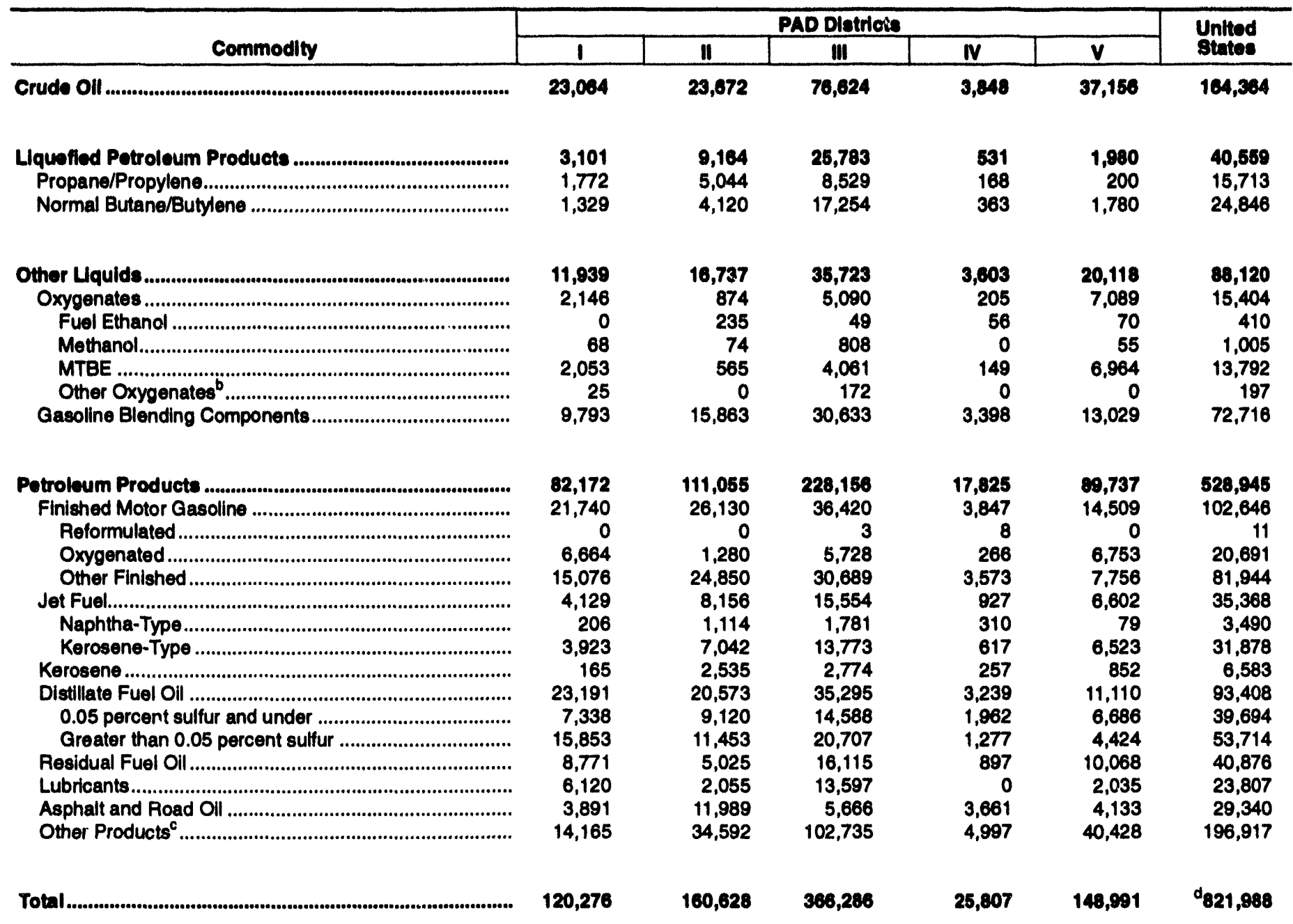

\footnotetext{
- The difference in volume between the maximum safe fill capacity and tank bottoms.

- Includes ethyl tertiary butyl ether (ETBE), tertiary amyl methyl ether (TAME), tertiary butyl alcohol(TBA), and other aliphatic alcohols and ethers intended for motor gasoline blending (e.g., lsopropyl ether (IPE) or n-propanol).

'includes ethane/ethylene, isobutane/sobutylene, pentanes plus, other hydrocarbons/hydrogen, unfinished olls, finished aviation gasoline, special naphthas, wax, petroleum coke, still gas, petrochemical feedstocks and miscellaneous products.

Includes 5,297 thousand barrels of storage capacity at shuidown refineries and shutcown gasoline blending plants, and 44,892 thousand barrels storage capacity at operating gasoline blending plants.

Source: Energy Information Administration (EIA), Form EIA-820, "Annual Refinery Report."
} 
Table 44. 8holl Storage Capacity" at Refineries and Gasoline Blonding Plants by PAD Dietrict as of January 1, 1994

(Thousand Barrels)

\begin{tabular}{|c|c|c|c|c|c|c|}
\hline \multirow[b]{2}{*}{ Commodity } & \multicolumn{5}{|c|}{ PAD Districte } & \multirow{2}{*}{$\begin{array}{l}\text { Unitiod } \\
\text { Statios } \\
\end{array}$} \\
\hline & 1 & II & III & $\mathbf{N}$ & $\mathbf{v}$ & \\
\hline 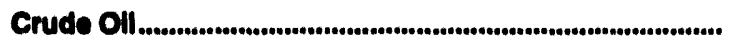 & 25,712 & 26,697 & 91,450 & 4,306 & 42,354 & 190,519 \\
\hline 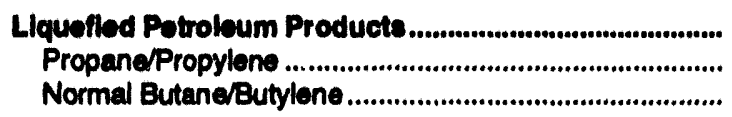 & $\begin{array}{l}3,420 \\
1,978 \\
1,442\end{array}$ & $\begin{array}{l}9,690 \\
5,299 \\
4,391\end{array}$ & $\begin{array}{l}29,341 \\
10,198 \\
19,143\end{array}$ & $\begin{array}{l}588 \\
181 \\
387\end{array}$ & $\begin{array}{r}2,102 \\
214 \\
1,888\end{array}$ & $\begin{array}{l}45,121 \\
17,870 \\
27,251\end{array}$ \\
\hline 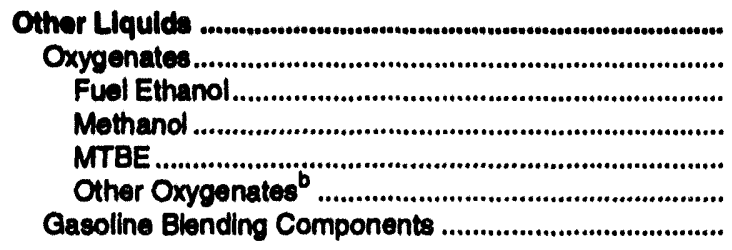 & $\begin{array}{r}13,127 \\
2,339 \\
0 \\
90 \\
2,221 \\
28 \\
10,788\end{array}$ & $\begin{array}{r}18,903 \\
952 \\
255 \\
80 \\
617 \\
0 \\
17,951\end{array}$ & $\begin{array}{r}41,333 \\
5,744 \\
62 \\
916 \\
4,574 \\
192 \\
35,589\end{array}$ & $\begin{array}{r}4,025 \\
220 \\
62 \\
0 \\
158 \\
0 \\
3,805\end{array}$ & $\begin{array}{r}22,399 \\
7,671 \\
85 \\
62 \\
7,524 \\
0 \\
14,728\end{array}$ & $\begin{array}{r}99,787 \\
16,926 \\
464 \\
1,148 \\
15,094 \\
220 \\
82,861\end{array}$ \\
\hline 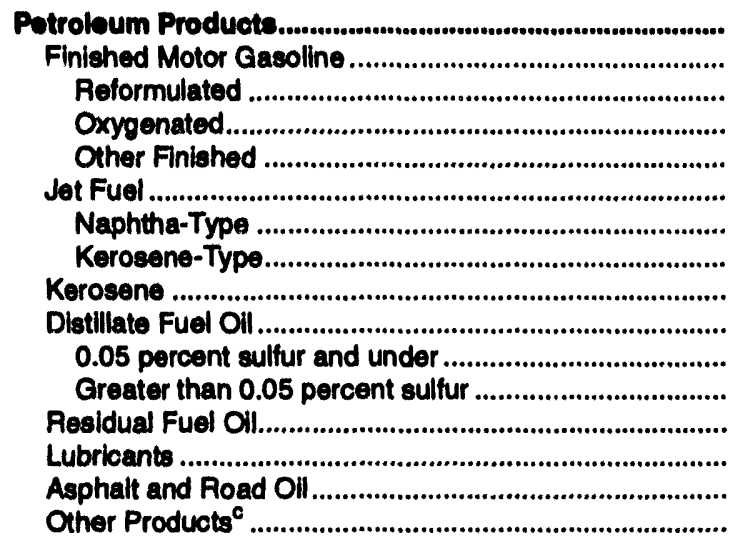 & $\begin{array}{r}88,611 \\
23,560 \\
0 \\
7,196 \\
16,364 \\
4,369 \\
236 \\
4,133 \\
182 \\
24,937 \\
7,763 \\
17,174 \\
9,554 \\
6,280 \\
4,219 \\
15,510\end{array}$ & $\begin{array}{r}121,251 \\
28,757 \\
0 \\
1,378 \\
27,379 \\
8,856 \\
1,234 \\
7,622 \\
2,765 \\
22,048 \\
9,815 \\
12,233 \\
5,484 \\
2,210 \\
12,966 \\
38,165\end{array}$ & $\begin{array}{r}262,884 \\
42,034 \\
3 \\
6,425 \\
35,606 \\
17,335 \\
1,951 \\
15,384 \\
3,141 \\
39,531 \\
16,190 \\
23,341 \\
18,228 \\
15,018 \\
6,616 \\
120,981\end{array}$ & $\begin{array}{r}21,376 \\
4,282 \\
10 \\
313 \\
3,959 \\
1,023 \\
362 \\
661 \\
282 \\
3,480 \\
2,109 \\
1,371 \\
968 \\
0 \\
3,884 \\
7,457\end{array}$ & $\begin{array}{r}99,738 \\
16,598 \\
0 \\
7,837 \\
8,761 \\
7,255 \\
84 \\
7,171 \\
971 \\
12,462 \\
7,486 \\
4,976 \\
10,938 \\
2,161 \\
4,459 \\
44,894\end{array}$ & $\begin{array}{r}593,860 \\
115,231 \\
13 \\
23,149 \\
92,069 \\
38,838 \\
3,867 \\
34,971 \\
7,341 \\
102,458 \\
43,363 \\
59,095 \\
45,172 \\
25,669 \\
32,144 \\
227,007\end{array}$ \\
\hline ........ & 130,870 & 176,541 & 425,008 & 30,275 & 168,593 & $\$ 29,287$ \\
\hline
\end{tabular}

\footnotetext{
- The design capacily of the tank.

- Includes ethyl tertiary butyl ether (ETBE), tertiary amyl melhyl ether (TAME), tertiany butyl alcohol (TBA), and other aliphatic alcohols and ethers intended for motor gasoline blending (e.g., lsopropyl ether (IPE) or n-propanol).

"Includes ethane/ethylene, loobutane/lsobutylene, pentanes plus, other hydrocarbons/hydrogen, unfinished olls, finished aviation gasoline, special naphthas, wax, petroleum coke, still gas, petrochemical feedstocks and miscellaneous products.

Includes 6,000 thousand barrels of storage capacily at shuidown refineries and shutdown gasoline blending plants, and 48,115 thousand barrels storage capacily at operating gasoline blending plants.

Source: Energy Information Administration (ELA), Form ElA-820, "Annual Refinery Report."
} 
Table 45. Cepacity, Freeh Fced Input and Utillzation Rates of Solected Downstream Unite at U.S. Rofinorise, 1987 to Present (Barrels per Calendar Day, Expect Where Noted)

\begin{tabular}{|c|c|c|c|c|c|c|c|c|}
\hline PAD Distrlot/hom & 1997 & 1988 & 1880 & 1000 & 1901 & 1992 & 1803 & 1904 \\
\hline & \multicolumn{8}{|c|}{ PAD DISTIOTI } \\
\hline \multicolumn{9}{|l|}{ Cokere } \\
\hline 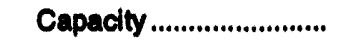 & 72,680 & 74,520 & 74,520 & 74,520 & 73,600 & 73,600 & 74,520 & 79,400 \\
\hline 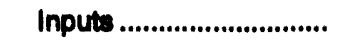 & 62,737 & 69,918 & 67,027 & 70,842 & 69,814 & 75,605 & 78,683 & \\
\hline Utilization (percent) ....... & 85.2 & 93.8 & 89.9 & 95.8 & 94.9 & 102.0 & 102.1 & \\
\hline \multicolumn{9}{|l|}{ Catalytie Crackers } \\
\hline 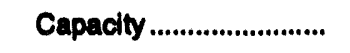 & 578,360 & 572,185 & 552,235 & 557,175 & 567,625 & 582,826 & 580,425 & 595,625 \\
\hline 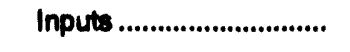 & 503,110 & 543,120 & 539,595 & 498,830 & 531,167 & 579,344 & 601,852 & \\
\hline Utilization (percent) ....... & $\because \quad 87.5$ & 86.6 & 97.3 & 88.7 & 82.3 & $\mathbf{8 8 . 8}$ & 101.5 & \\
\hline \multicolumn{9}{|l|}{ Hydrocrackers } \\
\hline 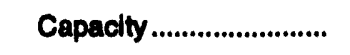 & 66,986 & 66,996 & 78,300 & 82,260 & 81,396 & 81,390 & 81,398 & 76,400 \\
\hline 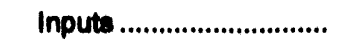 & 50,827 & 52,923 & 61,644 & 62,710 & 62,945 & 64,817 & 52,123 & \\
\hline \multirow[t]{2}{*}{ Utilization (percent) ....... } & 75.9 & 72.8 & 76.8 & 76.6 & 77.3 & 79.6 & 68.1 & \\
\hline & \multicolumn{8}{|c|}{ PAD DETARICT II } \\
\hline \multicolumn{9}{|l|}{ Cokere } \\
\hline 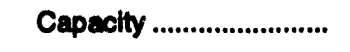 & 260,268 & 268,088 & 271,492 & 276,736 & 277,840 & 279,220 & 284,096 & 282,750 \\
\hline 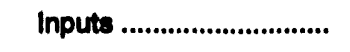 & 233,074 & 261,536 & 249,323 & 258,811 & 263,551 & 267,527 & 275,162 & \\
\hline Utllization (percent) ....... & 88.2 & 86.9 & 91.0 & 93.3 & 94.6 & 85.0 & 85.4 & \\
\hline \multicolumn{9}{|l|}{ Catalytic Crackere } \\
\hline 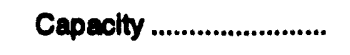 & $1,128,890$ & $1,165,080$ & $1,172,870$ & $1,180,870$ & $1,230,060$ & $1,247,160$ & $1,247,160$ & $1,204,350$ \\
\hline 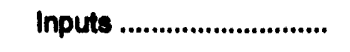 & 988,693 & 987,669 & 894,378 & $1,073,205$ & $1,100,378$ & $1,130,388$ & $1,110,277$ & \\
\hline Utilization (percent) ....... & 87.1 & 84.5 & 84.5 & 89.0 & 88.8 & 90.6 & 80.6 & \\
\hline \multicolumn{9}{|l|}{ Hydrocrackers } \\
\hline 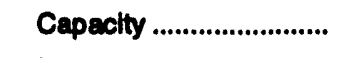 & 142,821 & 145,521 & 146,151 & 146,601 & 146,601 & 146,601 & 147,501 & 134,080 \\
\hline 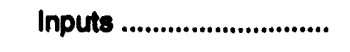 & 111,855 & 119,025 & 109,784 & 122,077 & 113,433 & 127,314 & 120,573 & \\
\hline \multirow[t]{2}{*}{ Utilization (percent) ...... } & 77.6 & 81.6 & 75.0 & 83.3 & $\pi .4$ & 86.6 & 85.6 & \\
\hline & \multicolumn{8}{|c|}{ PAD DIBTAICT WI } \\
\hline \multicolumn{9}{|l|}{ Cokere } \\
\hline 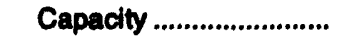 & 535,072 & 556,048 & 541,696 & 565,800 & 611,616 & 611,800 & 638,756 & 698,300 \\
\hline 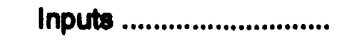 & 509,482 & 551,776 & 563,011 & 551,953 & 588,458 & 622,557 & 652,674 & \\
\hline Utilization (percent) ...... & 93.4 & 100.5 & 101.7 & 93.8 & 96.2 & 99.6 & 97.6 & \\
\hline \multicolumn{9}{|l|}{ Catahytic Crackere } \\
\hline 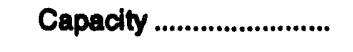 & $2,391,150$ & $2,511,705$ & $2,420,505$ & $2,494,225$ & $2,537,355$ & $2,601,955$ & $2,524,625$ & $2,499,800$ \\
\hline Inputs & $2,075,416$ & $2,171,074$ & $2,219,296$ & $2,271,704$ & $2,265,729$ & $2,265,992$ & $2,359,879$ & \\
\hline Utillization (percent) ....... & 84.7 & 88.0 & 90.3 & 90.3 & 88.2 & 88.4 & 93.9 & \\
\hline \multicolumn{9}{|l|}{ Hydrocrackers } \\
\hline 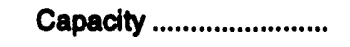 & 435,600 & 443,700 & 461,700 & 484,650 & 499,050 & 529,650 & 552,680 & 571,040 \\
\hline Inputs & 384,852 & 355,719 & 381,622 & 378,986 & 399,967 & 397,451 & 433,666 & \\
\hline Utilization (percent) ....... & 87.5 & 78.6 & 80.7 & $\pi 7.1$ & 77.8 & 73.4 & 77.2 & \\
\hline
\end{tabular}

See footnotes at end of table. 
Table 45. Capaolty, Frech Feed Input and Utilization Rates of Solectod Downatream Unite at U.8. Rofinerios, 1987 to Present (Continued) (Barrels per Calendar Day, Expect Where Noted)

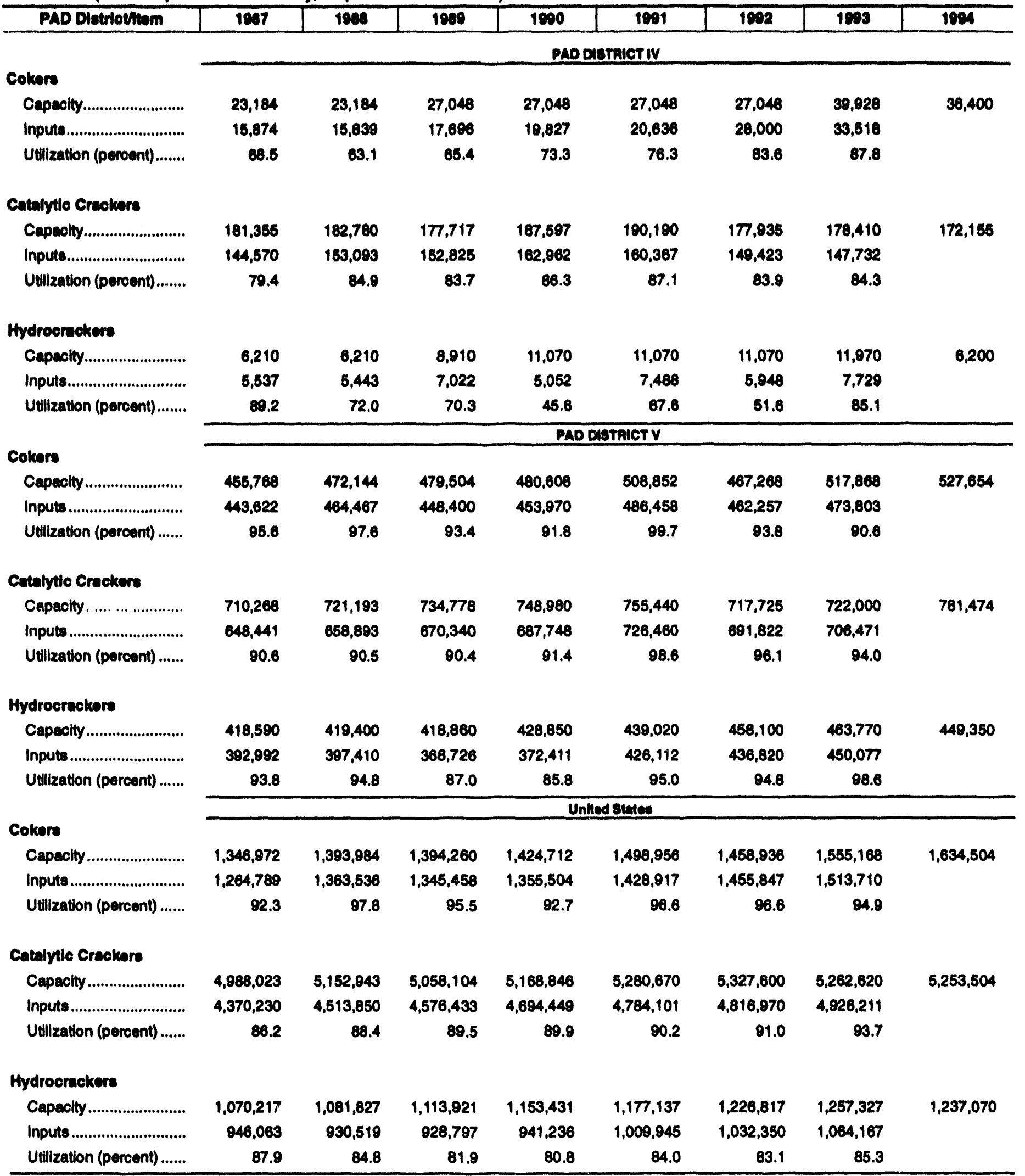

Note: - Capacities are as of January 1 of the indicated year. For 1987 through 1993, stream day capacilles were adjusted to calendar day capecities by calculating calendar day capecilies at 92 percent, 85 percent, and 90 percemt of stream day capacilies for delayed and fhild cokers, celtalytic crackers, and hyctocrackers, respectively. For 1984, calendar day capaciles for theee units were collected for the first time on the Form ELA-820, "Annual Refinery Report." Utilization rates repizsem fresh feed inputs divided by the average annual capacity. Average annual capacities were obtained by averaging the January 1 capacity for the indicated year and the January 1 capacity for the following year.

Sources: Capactibs are trom the Energy Intormation Administration Form EIA-820, "Annual Refinery Report." Inputs are from the Energy Information Administration Form ElA-810, "Monthly Refinery Report." 
Table 46. Rofinery Recelpts of Crude Oil by Mothod of Transportation by PAD District, 1993 (Thousand Barrels)

\begin{tabular}{|c|c|c|c|c|c|c|}
\hline \multirow[b]{2}{*}{ Mothod } & \multicolumn{5}{|c|}{ PAD Diatriots } & \multirow{2}{*}{$\begin{array}{l}\text { Unitod } \\
\text { 8tatios }\end{array}$} \\
\hline & 1 & $\|$ & III & IV & $\mathbf{v}$ & \\
\hline \multicolumn{7}{|l|}{ Plpollin } \\
\hline Domestio & $\begin{array}{r}1,765 \\
22,854\end{array}$ & $\begin{array}{l}623,068 \\
504,711\end{array}$ & $\begin{array}{l}782,651 \\
331,933\end{array}$ & $\begin{array}{r}114,851 \\
30,502\end{array}$ & $\begin{array}{r}333,778 \\
17,152\end{array}$ & $\begin{array}{r}1,858,111 \\
807,152\end{array}$ \\
\hline \multicolumn{7}{|l|}{ Tanker } \\
\hline Domestic & $\begin{array}{r}229 \\
435,969\end{array}$ & $\begin{array}{l}0 \\
0\end{array}$ & $\begin{array}{r}7,254 \\
1,081,027\end{array}$ & $\begin{array}{l}0 \\
0\end{array}$ & $\begin{array}{r}514,527 \\
66,779\end{array}$ & $\begin{array}{r}522,010 \\
1,583,775\end{array}$ \\
\hline \multicolumn{7}{|l|}{ Barge } \\
\hline Domestic & $\begin{array}{r}1,411 \\
21,981\end{array}$ & $\begin{array}{r}1,180 \\
0\end{array}$ & $\begin{array}{r}109,574 \\
15,977\end{array}$ & $\begin{array}{l}0 \\
0\end{array}$ & $\begin{array}{r}5,781 \\
10,805\end{array}$ & $\begin{array}{r}117,966 \\
48,763\end{array}$ \\
\hline \multicolumn{7}{|l|}{ Tank Care } \\
\hline 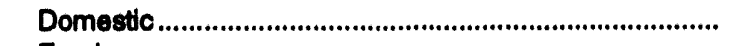 & 5,449 & 0 & 713 & 863 & 1,827 & 8,852 \\
\hline 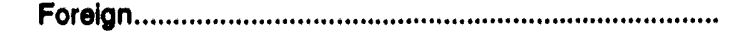 & 0 & 0 & 0 & 59 & 0 & 59 \\
\hline \multicolumn{7}{|l|}{ Trucks } \\
\hline 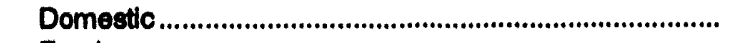 & 4,385 & 18,847 & 27,711 & 17,398 & 8,509 & 76,850 \\
\hline 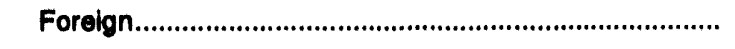 & 0 & 0 & 0 & 0 & 0 & 0 \\
\hline \multicolumn{7}{|l|}{ Total } \\
\hline Domestic & $\begin{array}{r}13,239 \\
480,804\end{array}$ & $\begin{array}{l}643,103 \\
504,711\end{array}$ & $\begin{array}{r}927,903 \\
1,428,937\end{array}$ & $\begin{array}{r}133,112 \\
30,561\end{array}$ & $\begin{array}{r}864,422 \\
94,736\end{array}$ & $\begin{array}{l}2,581,779 \\
2,539,749\end{array}$ \\
\hline
\end{tabular}

Source: Energy Information Administration (EIA), Form ElA-820, "Annual Refinery Report."

Table 47. Fuels Consumed at Refineries by PAD District, 1993 (Thousand Barrels, Except Where Noted)

\begin{tabular}{|c|c|c|c|c|c|c|}
\hline \multirow[b]{2}{*}{ Commodity } & \multicolumn{5}{|c|}{ PAD Districts } & \multirow{2}{*}{$\begin{array}{l}\text { United } \\
\text { States }\end{array}$} \\
\hline & 1 & II & III & $\mathbf{N}$ & $v$ & \\
\hline 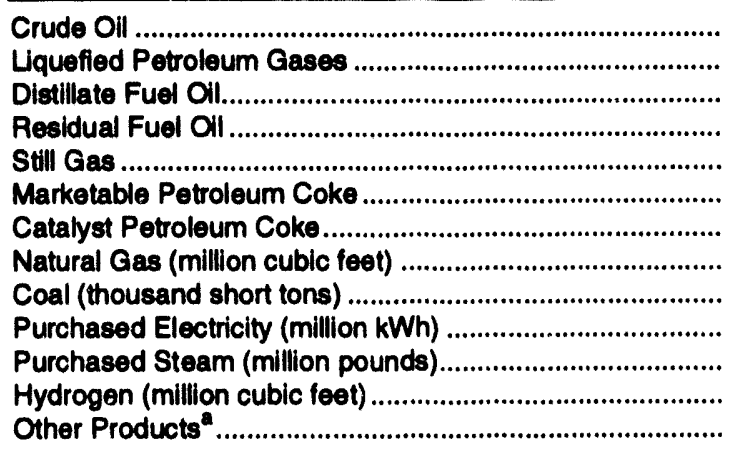 & $\begin{array}{r}0 \\
340 \\
126 \\
3,445 \\
19,642 \\
1,405 \\
10,892 \\
32,691 \\
123 \\
3,057 \\
2,339 \\
W \\
169\end{array}$ & $\begin{array}{r}0 \\
1,273 \\
106 \\
3,577 \\
47,676 \\
0 \\
18,964 \\
99,677 \\
19 \\
8,569 \\
3,260 \\
W \\
92\end{array}$ & $\begin{array}{r}0 \\
618 \\
47 \\
1,354 \\
106,480 \\
795 \\
39,971 \\
499,028 \\
0 \\
16,054 \\
13,216 \\
W \\
206\end{array}$ & $\begin{array}{r}0 \\
180 \\
1 \\
757 \\
7,224 \\
144 \\
2,139 \\
17,483 \\
0 \\
1,271 \\
0 \\
W \\
0\end{array}$ & $\begin{array}{r}0 \\
6,103 \\
235 \\
1,327 \\
49,738 \\
1,121 \\
12,782 \\
87,060 \\
0 \\
5,962 \\
13,110 \\
W \\
597\end{array}$ & $\begin{array}{r}0 \\
8,514 \\
515 \\
10,460 \\
230,760 \\
3,465 \\
84,748 \\
735,939 \\
142 \\
34,913 \\
31,925 \\
W \\
1,064\end{array}$ \\
\hline
\end{tabular}

"Includee naphtha-type jet fuel, miscellaneous products, unfinished olls, motor gasoline blending components, asphalt and road oll, speclal naphthas and wax.

$W=$ Whihheld to avold disclosure of individual company data.

Source: Energy Information Administration (EIA), Form ElA-820, "Annual Retinery Report." 
Table 48. Reactivated and Shutdown Rofinerles During 1993

\begin{tabular}{|c|c|c|c|c|c|c|}
\hline PAD Distrlet / Roflinery & Location & $\begin{array}{l}\text { Total Atmospheric } \\
\text { Crude oll } \\
\text { Diatiliation } \\
\text { Capacity (bbled) }\end{array}$ & $\begin{array}{c}\text { Total Downatream } \\
\text { Charge Capacity } \\
\text { (bbled) }\end{array}$ & $\begin{array}{c}\text { Date } \\
\text { Operable }\end{array}$ & $\begin{array}{l}\text { Dats of Last } \\
\text { Oporation }\end{array}$ & $\begin{array}{l}\text { Date } \\
\text { shutdown }\end{array}$ \\
\hline
\end{tabular}

\section{REACTIVATIONS}

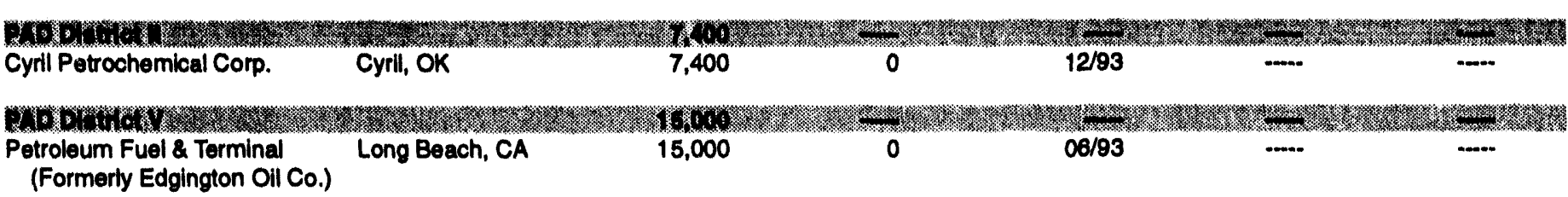

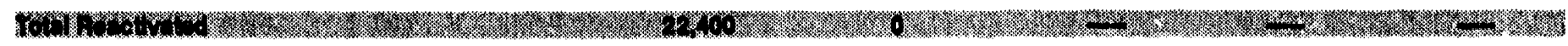

\section{SHUTDOWNS}

\begin{tabular}{|c|c|c|c|c|c|c|}
\hline $\begin{array}{l}\text { Cibro Refining } \\
\text { Saint Mary's Refg Co. }\end{array}$ & $\begin{array}{l}\text { Albany, NY } \\
\text { Saint Mary's, WV }\end{array}$ & $\begin{array}{r}41,850 \\
4,000\end{array}$ & $\begin{array}{r}27,000 \\
4,480\end{array}$ & $\begin{array}{r}10 / 78 \\
\text { (a) }\end{array}$ & $\begin{array}{l}07 / 93 \\
02 / 93\end{array}$ & $\begin{array}{l}09 / 93 \\
03 / 93\end{array}$ \\
\hline \multicolumn{7}{|c|}{ 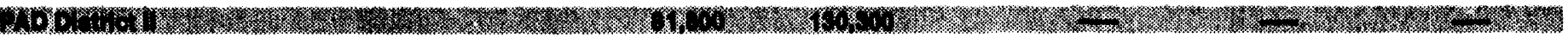 } \\
\hline $\begin{array}{l}\text { Coastal Refg \& Mktg } \\
\text { Coastal Refg \& Mktg } \\
\text { Crystal Refining } \\
\text { Marathon }\end{array}$ & $\begin{array}{l}\text { Augusta, KS } \\
\text { Wichita, KS } \\
\text { Carson City, MI } \\
\text { Indianapolls, IN }\end{array}$ & $\begin{array}{r}0 \\
28,800 \\
3,000 \\
50,000\end{array}$ & $\begin{array}{r}21,000 \\
41,300 \\
0 \\
68,000\end{array}$ & $\begin{array}{r}05 / 86 \\
\text { (a) } \\
\text { (a) } \\
\text { (a) }\end{array}$ & $\begin{array}{l}06 / 93 \\
05 / 93 \\
10 / 92 \\
09 / 93\end{array}$ & $\begin{array}{l}06 / 93 \\
06 / 93 \\
09 / 93 \\
10 / 93\end{array}$ \\
\hline \multicolumn{7}{|c|}{ 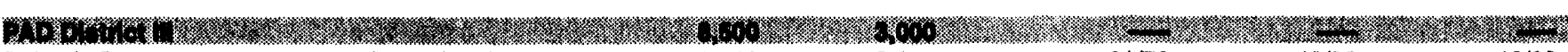 } \\
\hline Dubach Gas & Dubach, LA & 8,500 & 3,000 & $01 / 73$ & $12 / 93$ & $12 / 93$ \\
\hline Landmark Refining & Fruita, CO & 10,000 & 25,900 & $01 / 89$ & $01 / 92$ & $11 / 93$ \\
\hline \multicolumn{7}{|c|}{ 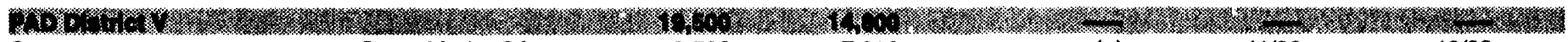 } \\
\hline $\begin{array}{l}\text { Conoco } \\
\text { Sunbelt Refining }\end{array}$ & $\begin{array}{l}\text { Santa Maria, CA } \\
\text { Coolidge, AZ }\end{array}$ & $\begin{array}{r}9,500 \\
10,000\end{array}$ & $\begin{array}{l}7,800 \\
7,000\end{array}$ & $\begin{array}{r}(a) \\
11 / 89\end{array}$ & $\begin{array}{l}11 / 92 \\
08 / 93\end{array}$ & $\begin{array}{l}12 / 93 \\
09 / 93\end{array}$ \\
\hline
\end{tabular}

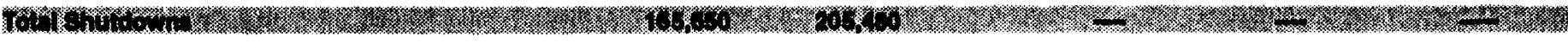

"Refinery was operable prior to 1948.

bbVod = Barrels per calendar day.

bbVsd = Barrels per stream day.

Source: Energy Information Administration (EIA) Form EIA-820, "Annual Refinery Report." 
Table 49. Refinery Sales, January 1993 through Fobruary 1994

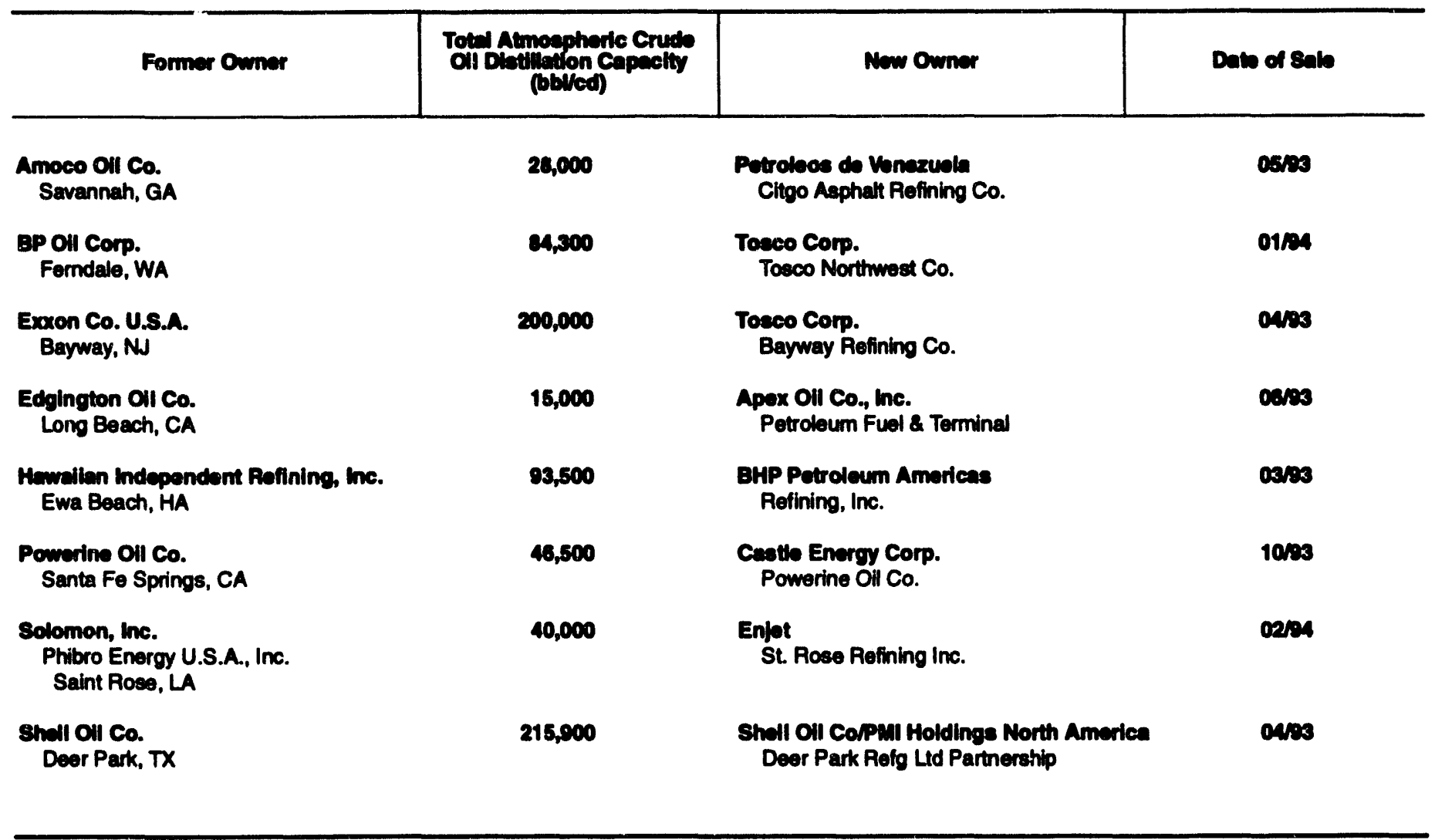

bovcd = Barrets per calendar day.

Source: Energy Intormation Administration (ELA) Form EA-820, "Annual Refinery Report" 


\section{Oxygenate Capacity}

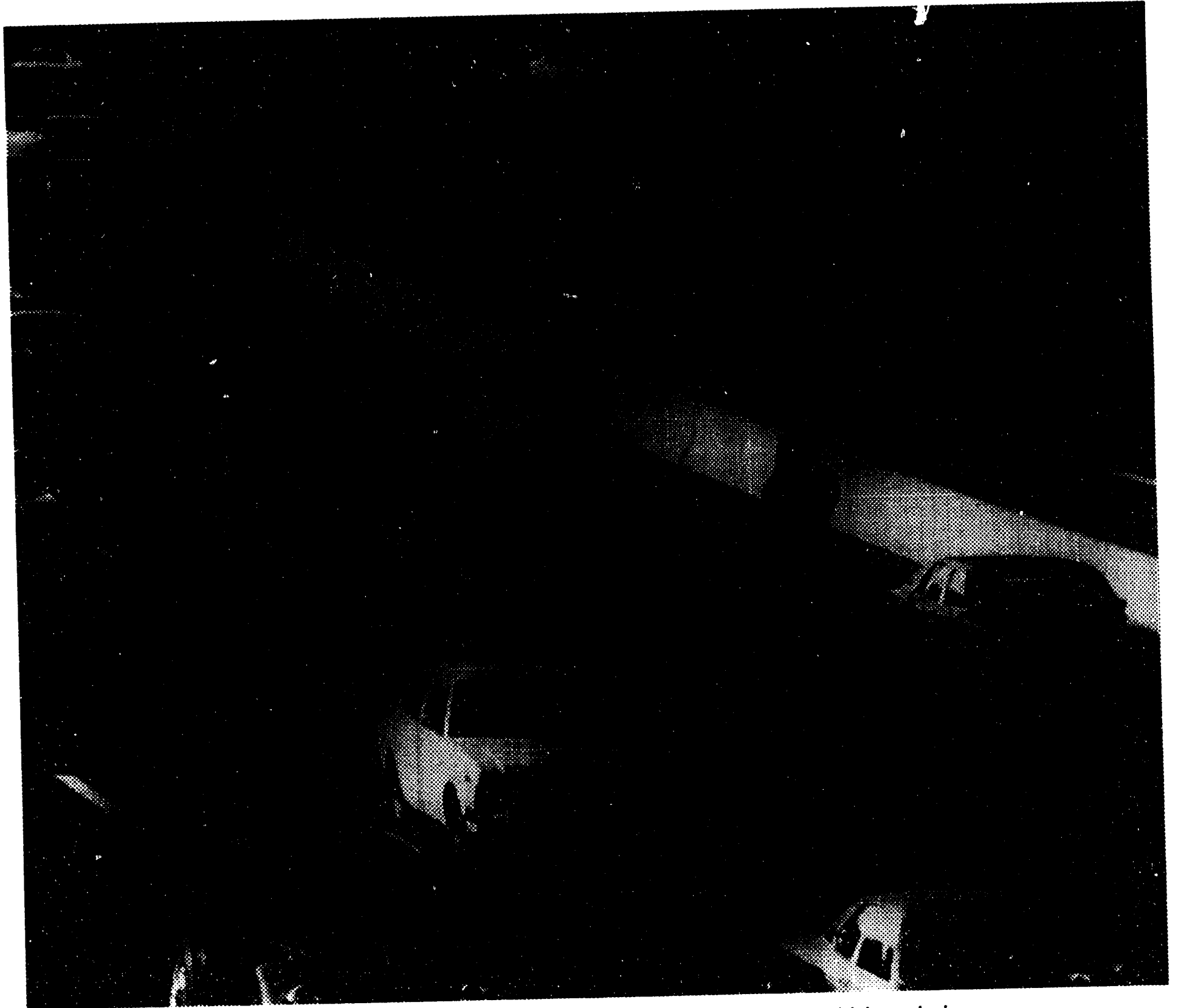


Table 50. Number and Production Capaclty of Operable Oxygenate Plants by PAD District and State as of January 1, 1994

\begin{tabular}{|c|c|c|c|c|c|c|c|c|c|c|}
\hline \multirow{3}{*}{$\begin{array}{l}\text { PAD Dletrict } \\
\text { and } \\
\text { 8ute }\end{array}$} & \multirow{2}{*}{\multicolumn{3}{|c|}{$\begin{array}{c}\text { Number of } \\
\text { Oporable Plants }\end{array}$}} & \multicolumn{7}{|c|}{ Barrels per Day } \\
\hline & & & & \multirow{2}{*}{$\begin{array}{c}\text { Fud } \\
\text { Ethanol }\end{array}$} & \multirow[b]{2}{*}{ ETBE } & \multirow[b]{2}{*}{ Mothanol } & \multirow[b]{2}{*}{ MTBE } & \multirow[b]{2}{*}{ TAME } & \multirow[b]{2}{*}{ TBA } & \multirow{2}{*}{$\begin{array}{c}\text { Other } \\
\text { Omyenates" }\end{array}$} \\
\hline & Totel & Operating & Idilo" & & & & & & & \\
\hline & & & & 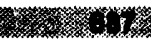 & 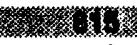 & (x) & and & 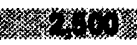 & 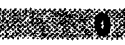 & \multirow[b]{2}{*}{0} \\
\hline \multirow{3}{*}{ 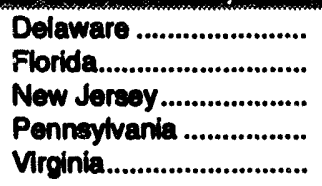 } & 2 & 1 & 1 & 0 & \multirow{3}{*}{$\begin{array}{r}0 \\
0 \\
0 \\
0 \\
815\end{array}$} & \multirow{3}{*}{$\begin{array}{r}7,000 \\
4,150 \\
0 \\
0 \\
0\end{array}$} & 2,500 & 2,500 & \multirow{3}{*}{$\begin{array}{l}0 \\
0 \\
0 \\
0 \\
0\end{array}$} & \\
\hline & 2 & 1 & 1 & 361 & & & 0 & \multirow{2}{*}{$\begin{array}{l}0 \\
0 \\
0 \\
0\end{array}$} & & \multirow{2}{*}{$\begin{array}{l}0 \\
0 \\
0 \\
0\end{array}$} \\
\hline & 1 & 1 & 0 & 0 & & & 2,000 & & & \\
\hline \multirow{10}{*}{ 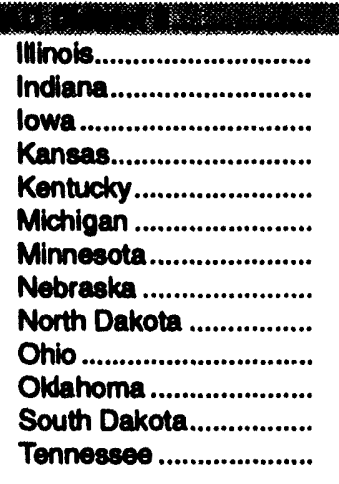 } & & & & & \multicolumn{3}{|c|}{ Hork } & & \multirow{2}{*}{$\begin{array}{c}0 \\
0\end{array}$} \\
\hline & 6 & 6 & 0 & ${ }^{0} 58,409$ & \multicolumn{2}{|c|}{0} & 1,450 & 250 & o & \\
\hline & 2 & 1 & 1 & 5,000 & 0 & 0 & 3,897 & 0 & 0 & 0 \\
\hline & $\overline{7}$ & 7 & 0 & ' 5,387 & 0 & 0 & 0 & 0 & 0 & 0 \\
\hline & 4 & 4 & 0 & 2,386 & 0 & 0 & 0 & 0 & 0 & 0 \\
\hline & 1 & 1 & 0 & 0 & 0 & 0 & 3,200 & 0 & 0 & 0 \\
\hline & 1 & 1 & 0 & 3,914 & 0 & 0 & 0 & 0 & 0 & 0 \\
\hline & 1 & 1 & 0 & 0 & 0 & 0 & 1,200 & 0 & 0 & 0 \\
\hline & 2 & 2 & 0 & 535 & 0 & 0 & 0 & 0 & 0 & 0 \\
\hline & 2 & 2 & 0 & 3,095 & 0 & 3,590 & 0 & 0 & 0 & 0 \\
\hline & & & & & & & & 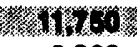 & 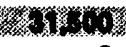 & \\
\hline Lovisiana .......................... & 10 & 9 & 1 & 0 & 0 & 38,458 & 25,975 & 6,800 & 0 & 0 \\
\hline Miseissippi ....................... & 1 & 1 & 0 & 0 & 0 & 0 & 2,500 & 0 & 0 & 0 \\
\hline 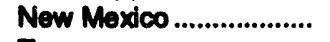 & 2 & 1 & 1 & 1,220 & 0 & 0 & 0 & 0 & 0 & 0 \\
\hline Texas & 27 & 24 & 3 & 0 & ${ }^{d} 0$ & 69,470 & 163,480 & 4,950 & 31,500 & 5,950 \\
\hline & & & & & & & & & & \\
\hline 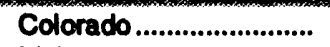 & 2 & 2 & 0 & 103 & 0 & 1,794 & 0 & 0 & 0 & 0 \\
\hline 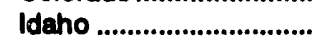 & 2 & 2 & 0 & 392 & 0 & 0 & 0 & 0 & 0 & 0 \\
\hline
\end{tabular}

\footnotetext{
2 Plants where units are completely ldle but not permanently shutdown on January 1, 1994.

- Other aliphatic alcohols and ethers intended for motor gasoline blending (e.g., Isopropyl ether (IPE) or n-propanol).

- Capacity for Ilinois includes plants operated by Archer Daniels Midland Co. located in Decatur and Peoria, illinois; Cedar Rapids and Clinton, lowa; and Wahalla, North Dakota.

Arco Chemical Co. Corpus Chriati, Texas plant has the capabilly of producing either 9,500 barrels per day of ETBE or 12,000 barrels per day of MTBE. As of January 1, 1994, the plant was producing MTBE and therefore is included in the MTBE production capacity data. Their ETBE production capacity of 9,500 barrels per day is not included in the ETBE production capacity data.

Source: Energy Information Administration (EIA) Form EIA-819A, "Annual Oxygenate Capacity Report."
} 
Table 51. Fuel Ethanol Production Capacity by PAD District (Barrels per Day)

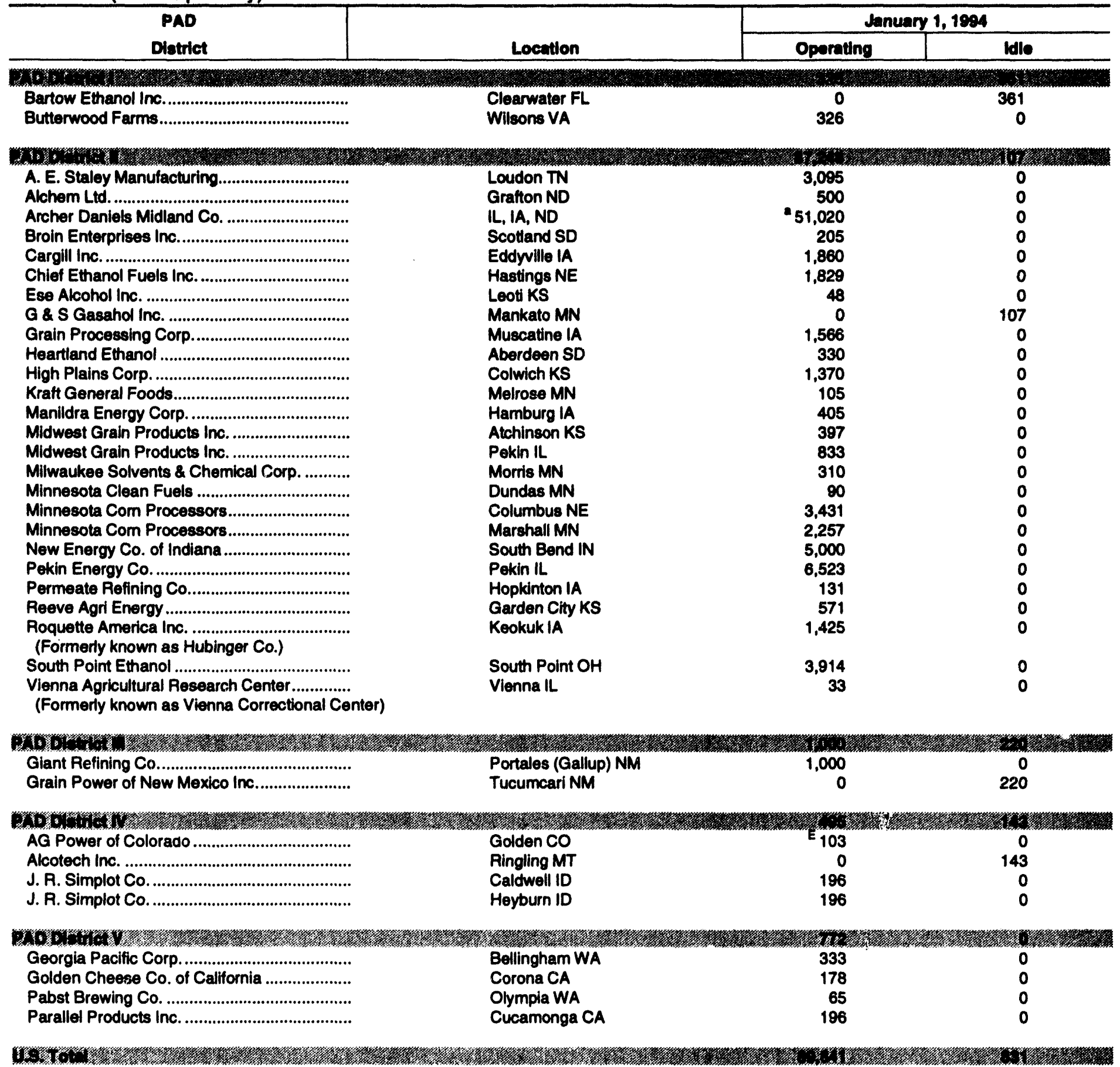

\footnotetext{
- Reported a combined capacity for plants located in Decatur and Peoria, Ililnois; Cedar Raplds and Cinton, lowa; and Wahalla, North Dakota. $E=$ Estimated.

Sounce: Energy Information Administration (EIA) Form EIA-819A, "Annual Oxygenate Capacity Report."
} 
Table 52. Ethyl Tortiary Butyl Ether (ETBE) Production Capacity by PAD District (Barrels per Day)

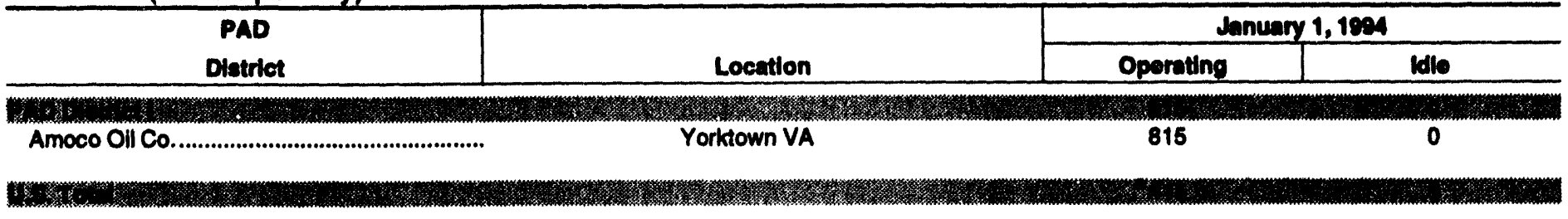

Arco Chemical Co., Corpus Christ, Texas plant has the capability of producing elther 9,500 barrels per day of ETBE or 12,000 barrels per day of MTBE. As of January 1 , 1994, the plant was producing MTBE and therefore is included in the MTBE production capactly data. Their ETBE production capecily of 8,500 barrele per day is not inoluded in the ETBE production capacity data.

Source: Energy Intormation Adminietration (EIA) Form ElA-819A, "Annual Oxygenate Capacity Report."

Table 53. Mothanol Production Capacity by PAD District (Barrels per Day)

\begin{tabular}{|c|c|c|c|}
\hline \multirow{2}{*}{$\begin{array}{c}\text { PAD } \\
\text { Diatrict }\end{array}$} & \multirow[b]{2}{*}{ Location } & \multicolumn{2}{|c|}{ danuary 1, 1994} \\
\hline & & Operating & Idle \\
\hline \multicolumn{4}{|c|}{ 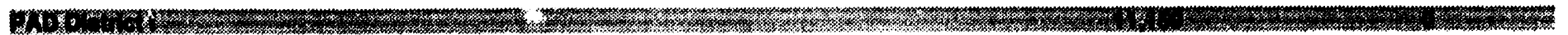 } \\
\hline 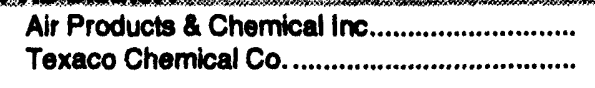 & $\begin{array}{l}\text { Pensacola FL } \\
\text { Delaware City DE }\end{array}$ & $\begin{array}{l}4,150 \\
7,000\end{array}$ & $\begin{array}{l}0 \\
0\end{array}$ \\
\hline $\begin{array}{l}\text { Tennessee Eastman Div........................... } \\
\text { (Formerly known as Eastman Chemical Co) }\end{array}$ & Kingsport TN & 3,580 & 0 \\
\hline 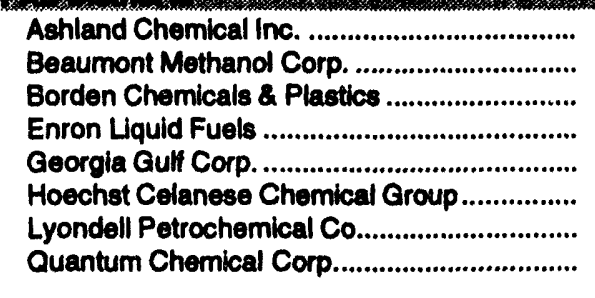 & $\begin{array}{l}\text { Plaquemine LA } \\
\text { Beaumont TX } \\
\text { Geismar LA } \\
\text { Pasadena TX } \\
\text { Plaquemine LA } \\
\text { Bishop TX } \\
\text { Channelview TX } \\
\text { Deer Park TX }\end{array}$ & $\begin{array}{r}10,476 \\
18,270 \\
18,850 \\
10,000 \\
9,132 \\
13,000 \\
15,200 \\
E_{13,000}\end{array}$ & $\begin{array}{l}0 \\
0 \\
0 \\
0 \\
0 \\
0 \\
0 \\
0\end{array}$ \\
\hline 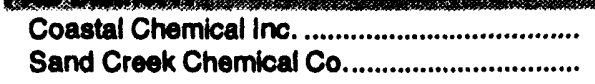 & $\begin{array}{l}\text { Cheyenne WY } \\
\text { Commerce City co }\end{array}$ & $\begin{array}{l}1,475 \\
1,794\end{array}$ & $\begin{array}{l}0 \\
0\end{array}$ \\
\hline
\end{tabular}

Emestimated.

Source: Energy Information Administration (EIA) Form EIA-819A, "Annual Oxygenate Capacily Report."

Table 54. Tertiary Amyl Mothyl Ether (TAME) Production Capacity by PAD District (Barrels per Day)

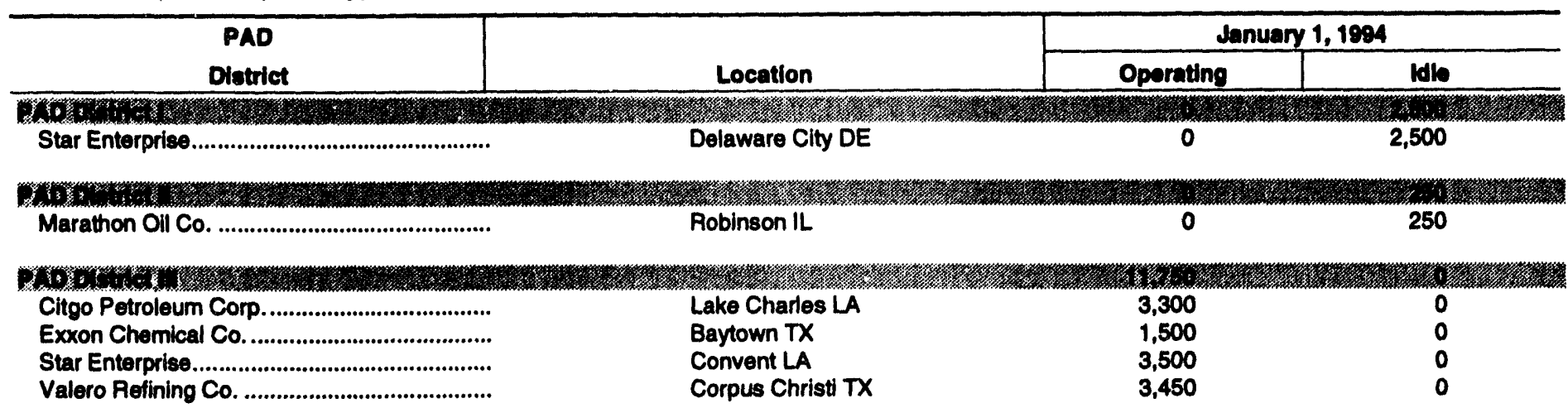

1 IIn

Source: Energy Information Administration (EIA) Form EIA-819A, "Annual Oxygenate Capacity Report." 
Table 55. Methyl Tertlary Butyl Ether (MTBE) Production Capacity by PAD District (Barrels per Day)

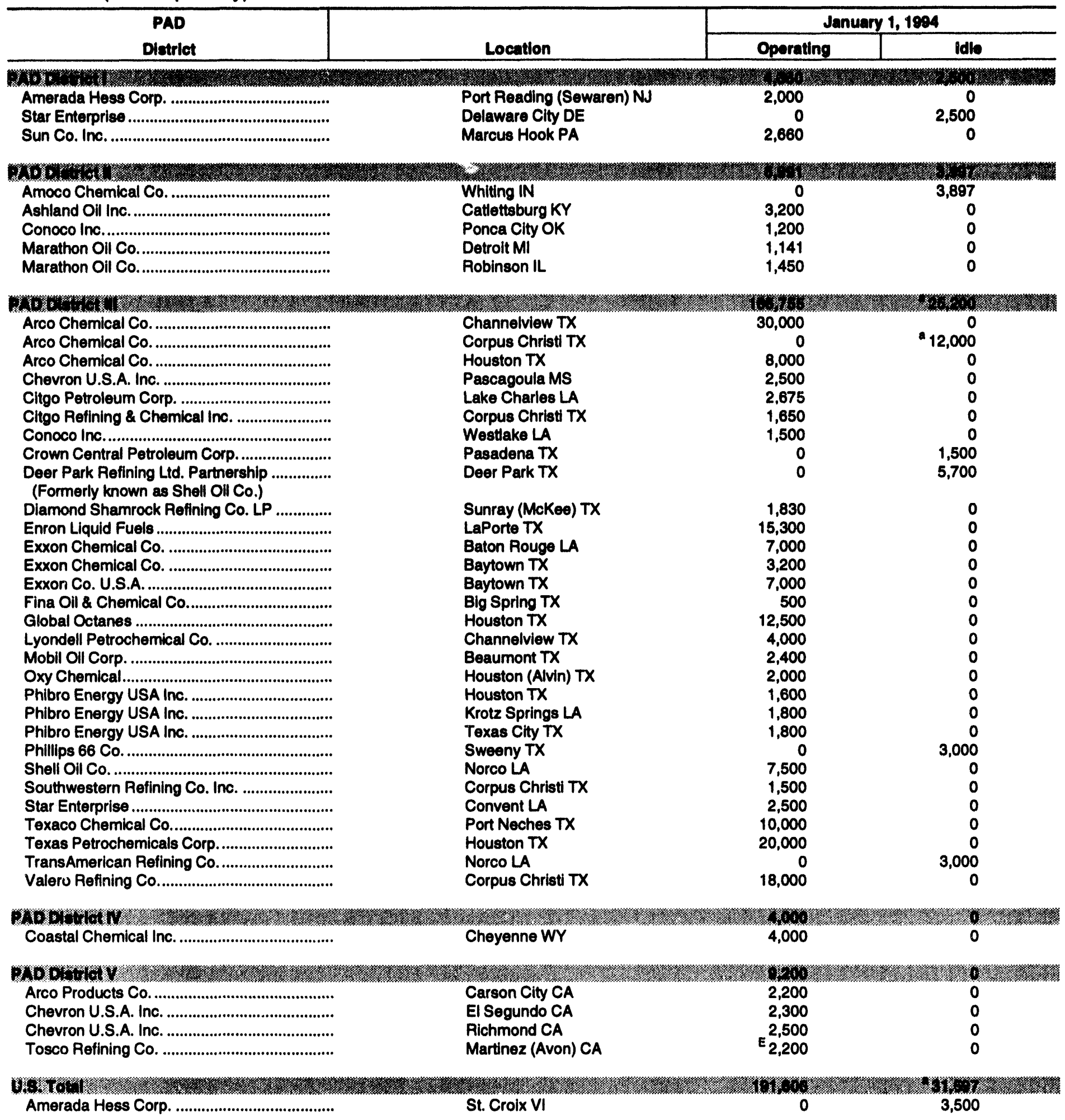

- Arco Chemical Co., Corpus Christi, Texas plant has the capabilly of producing elther 9,500 barrels per day of ETBE or 12,000 barrels per day of MTBE. As of January 1, 1994, the plant was producing MTBE and therefore is included in the MTBE production capacity data. Their ETBE production capacity of 9,500 barrels per day is not included in the ETBE production capacity data.

Ex=Estimated.

Source: Energy Information Administration (EIA) Form ElA-819A, "Annual Oxygenate Capacity Report." 
Table 56.Tertiary Butyl Alcohol (TBA) Production Capacity by PAD District

(Barrels per Day)

\begin{tabular}{|c|c|c|c|}
\hline \multirow{2}{*}{$\begin{array}{c}\text { PAD } \\
\text { Distrlat } \\
\end{array}$} & \multirow[b]{2}{*}{ Loeation } & \multicolumn{2}{|c|}{ January 1,1994} \\
\hline & & Oporating & Ide \\
\hline 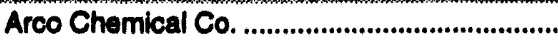 & ayport TX & 31,500 & 0 \\
\hline
\end{tabular}

Source: Energy Information Administration (EIA) Form EIA-819A, 'Annual Oxygenate Capacity Report."

Table 57. Other Oxygenates" Production Capacity by PAD District

(Barrels per Day)

\begin{tabular}{|c|c|c|c|}
\hline PAD & & & \\
\hline Distrlet & Location & Operating & Idile \\
\hline 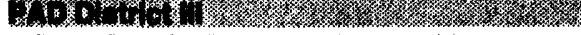 & & & \\
\hline $\begin{array}{l}\text { Deer Park Refining Ltd. Partnership ............... } \\
\text { (Formerly known as Shell Oil Co.) }\end{array}$ & Deer Park TX & 5,950 & 0 \\
\hline
\end{tabular}

"Other aliphatic alcohols and ethers intended for motor gasoline blending (e.g., lsopropyl ether (IPE) or n-propanol).

Source: Enargy Information Administration (EIA) Form EIA-819A, "Annugl Oxygenate Capacity Report." 
Table 58. Production Capacity of Operable Oxycenate Plants by PAD District January 1, 1993 - January 1, 1996

(Barrels per Day)

\begin{tabular}{|c|c|c|c|c|c|c|c|}
\hline $\begin{array}{c}\text { YearipaD } \\
\text { Diotriet }\end{array}$ & $\begin{array}{c}\text { Fuel } \\
\text { Ethenol }\end{array}$ & ETEE & Emenel & MTEE & TAME & TEA & $\begin{array}{c}\text { Othor } \\
\text { Oxyenntes" }\end{array}$ \\
\hline $\begin{array}{l}\text { PADD I } \\
\text { PADD II } \\
\text { PADD III } \\
\text { PADD IV } \\
\text { PADD V }\end{array}$ & $\begin{array}{r}687 \\
87,355 \\
1,220 \\
638 \\
772\end{array}$ & $\begin{array}{r}815 \\
0 \\
0 \\
0 \\
0\end{array}$ & $\begin{array}{r}11,150 \\
3,590 \\
107,828 \\
3,269 \\
0\end{array}$ & $\begin{array}{r}7,160 \\
10,888 \\
181,955 \\
4,000 \\
9,200\end{array}$ & $\begin{array}{r}2,500 \\
250 \\
11,750 \\
0 \\
0\end{array}$ & $\begin{array}{r}0 \\
0 \\
31,500 \\
0 \\
0\end{array}$ & $\begin{array}{r}0 \\
0 \\
5,950 \\
0 \\
0\end{array}$ \\
\hline $\begin{array}{l}\text { PADD I } \\
\text { PADD II } \\
\text { PADD III } \\
\text { PADD IV } \\
\text { PADD V }\end{array}$ & $\begin{array}{l}\text { NA } \\
\text { NA } \\
\text { NA } \\
\text { NA } \\
\text { NA }\end{array}$ & $\begin{array}{r}815 \\
0 \\
0 \\
0 \\
0\end{array}$ & $\begin{array}{r}11,160 \\
6,490 \\
109,471 \\
3,269 \\
0\end{array}$ & $\begin{array}{r}7,160 \\
10,888 \\
235,805 \\
4,000 \\
11,700\end{array}$ & $\begin{array}{r}2,500 \\
250 \\
13,890 \\
0 \\
4,000\end{array}$ & $\begin{array}{r}0 \\
0 \\
31,500 \\
0 \\
0\end{array}$ & $\begin{array}{r}0 \\
0 \\
5,950 \\
0 \\
0\end{array}$ \\
\hline $\begin{array}{l}\text { (Net Change) } \\
\text { PADD I } \\
\text { PADD II } \\
\text { PADD III } \\
\text { PADD IV } \\
\text { PADD V }\end{array}$ & $\begin{array}{l}\text { NA } \\
\text { NA } \\
\text { NA } \\
\text { NA } \\
\text { NA }\end{array}$ & $\begin{array}{l}0 \\
0 \\
0 \\
0 \\
0\end{array}$ & $\begin{array}{r}0 \\
2,900 \\
1,543 \\
0 \\
0\end{array}$ & $\begin{array}{r}0 \\
0 \\
43,850 \\
0 \\
2,500\end{array}$ & $\begin{array}{r}0 \\
0 \\
2,140 \\
0 \\
4,000\end{array}$ & $\begin{array}{l}0 \\
0 \\
0 \\
0 \\
0\end{array}$ & $\begin{array}{l}0 \\
0 \\
0 \\
0 \\
0\end{array}$ \\
\hline
\end{tabular}

Arco Chemical Co., Corpus Christi, Texas plant has the capabilly of producing elther 9,500 barrels per day of ETBE of 12,000 barrels per day of MTBE. Reler to Tables 52 and 55 for current plant status.

Other aliphatic alcohols and ethers intended for motor gasoline blending (e.g., bopropyl ether (IPE) or n-propanol).

NA $=$ Not available.

Note: Totals may not equal sum of components due to independent rounding.

Source: Energy Information Administration (EIA), Form EIA-819A, "Annual Oxygenate Capacity Report." 


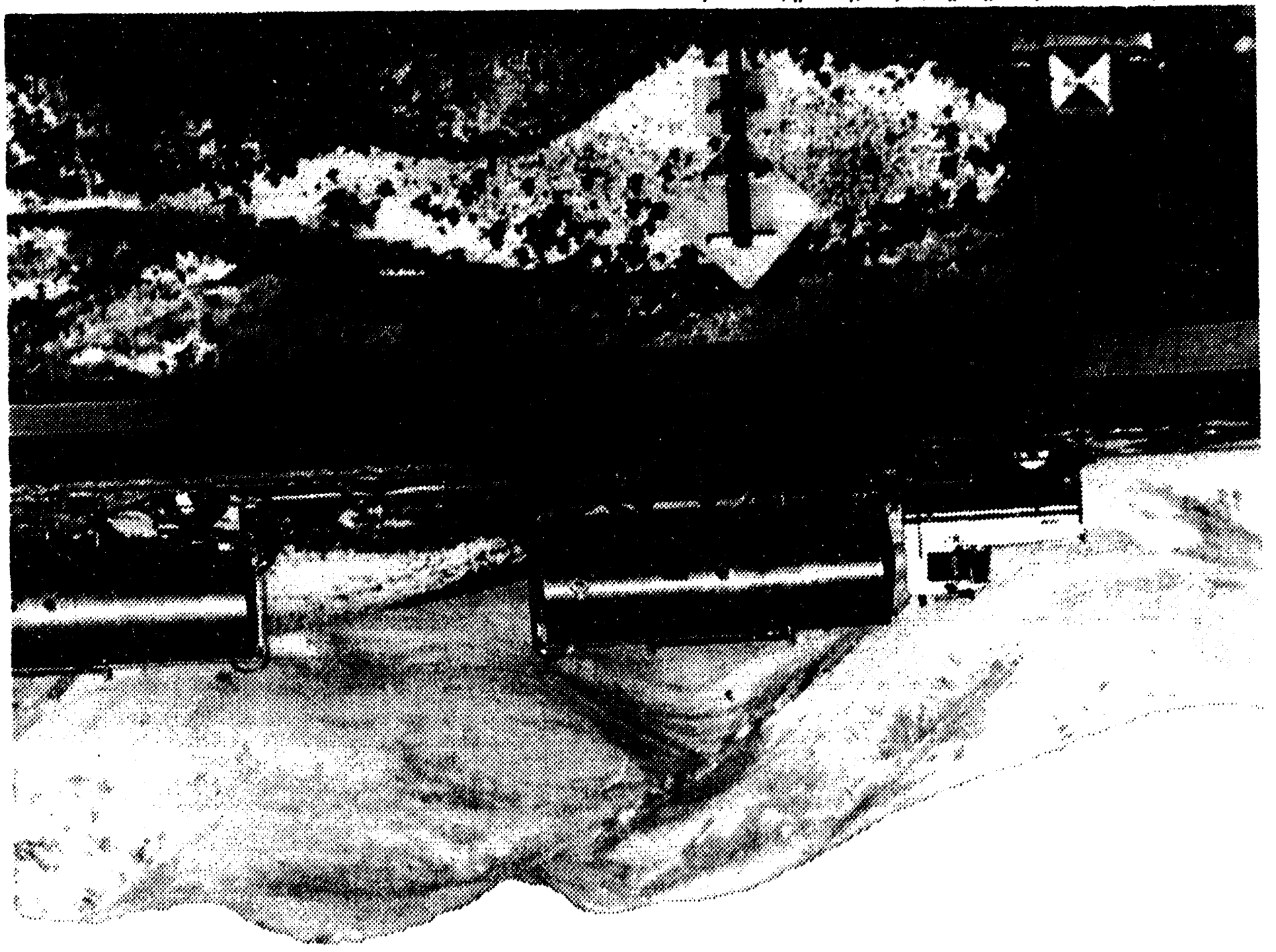

\section{sdew pue suo!̣d!ıseg เอ!גม!}

\section{$\forall x \mid p u e d d \forall$}




\section{District Descriptions and Maps}

The following are the Refining Districts which make up the Petroleum Administration for Defense (PAD) Districts.

\section{PAD District I}

East Coast: District of Columbia and the States of Maine, New Hampshire, Vermont, Massachusetts, Rhode Island, Connecticut, New Jersey, Delaware, Maryland, Virginia, North Carolina, South Carolina, Georgia, Florida, and the following counties of the State of New York: Cayuga, Tompkins, Chemung, and all counties east and north thereof. Also the following counties in the State of Pennsylvania: Bradford, Sullivan, Columbia, Montour, Northumberland, Dauphin, York, and all counties east thereof.

Appalachian No. 1: The State of West Virginia and those parts of the States of Pennsylvania and New York not included in the East Coast District.

\section{Sub-PAD District I}

New England: The States of Connecticut, Maine, Massachusetts, New Hampshire, Rhode Island and Vermont.

Central Atlantic: The District of Columbia and the States of Delaware, Maryland, New Jersey, New York, and Pennsylvania.

Lower Atlantic: The States of Florida, Georgia, North Carolina, South Carolina, Virginia and West Virginia.

\section{PAD District II}

Indiana-Illinois-Kentucky: The States of Indiana, Illinois, Kentucky, Tennessee, Michigan, and Ohio.

Minnesota-Wisconsin-North and South Dakota: The States of Minnesota, Wisconsin, North Dakota, and South Dakota.

Oklahoma-Kansas-Missouri: The States of Oklahoma, Kansas, Missouri, Nebraska, and lowa.

\section{PAD District III}

Texas Inland: The State of Texas except the Texas Gulf Coast District.

Texas Gulf Coast: The following counties of the State of Texas: Newton, Orange, Jefferson, Jasper, Tyler, Hardin, Liberty, Chambers, Polk, San Jacinto, Montgomery, Harris, Galveston, Waller, Fort Bend, Brazcria, Wharton, Matagorda, Jackson, Victoria, Calhoun, Refugio, Aransas, San Patricio, Nueces, Kleberg, Kenedy, Willacy, and Cameron.

Louisiana Gulf Coast: The following Parishes of the State of Louisiana: Vernon, Rapides, Avoyelles, Pointe Coupee, West Feliciana, East Feliciana, Saint Helena, Tangipahoa, Washington, and all Parishes south thereof. Also the following counties of the State of Mississippi: Pearl River, Stone, George, Hancock, Harrison, and Jackson. Also the following counties of the State of Alabama: Mobile and Baldwin.

North Louisiana-Arkansas: The State of Arkansas and those parts of the States of Louisiana, Mississippi, and Alabama not included in the Louisiana Gulf Coast District.

New Mexico: The State of New Mexico.

\section{PAD District IV}

Rocky Mountain: The States of Montana, Idaho, Wyoming, Utah, and Colorado.

\section{PAD District V}

West Coast: The States of Washington, Oregon, California, Nevada, Arizona, Alaska, and Hawaii. 

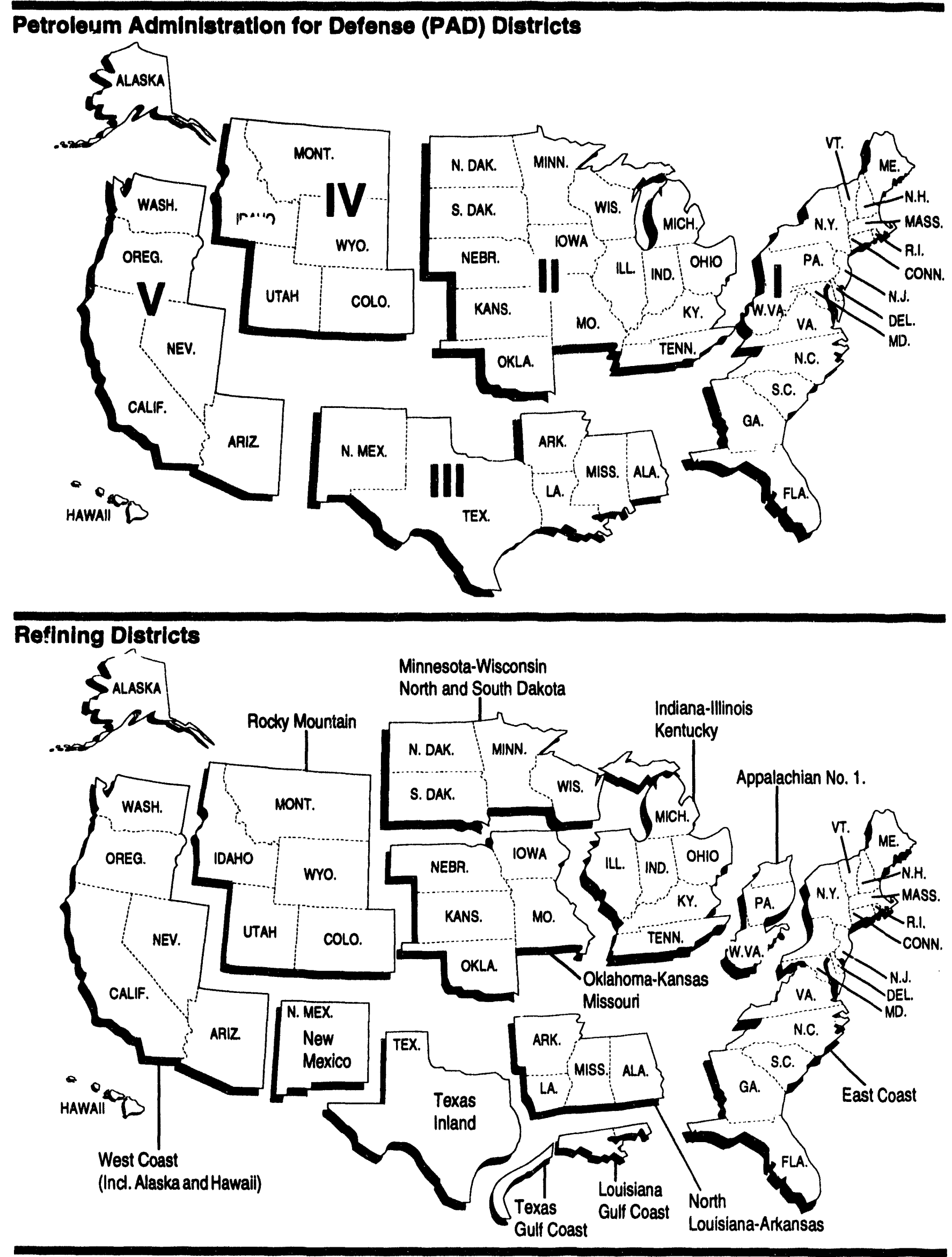


\section{Appendix B}

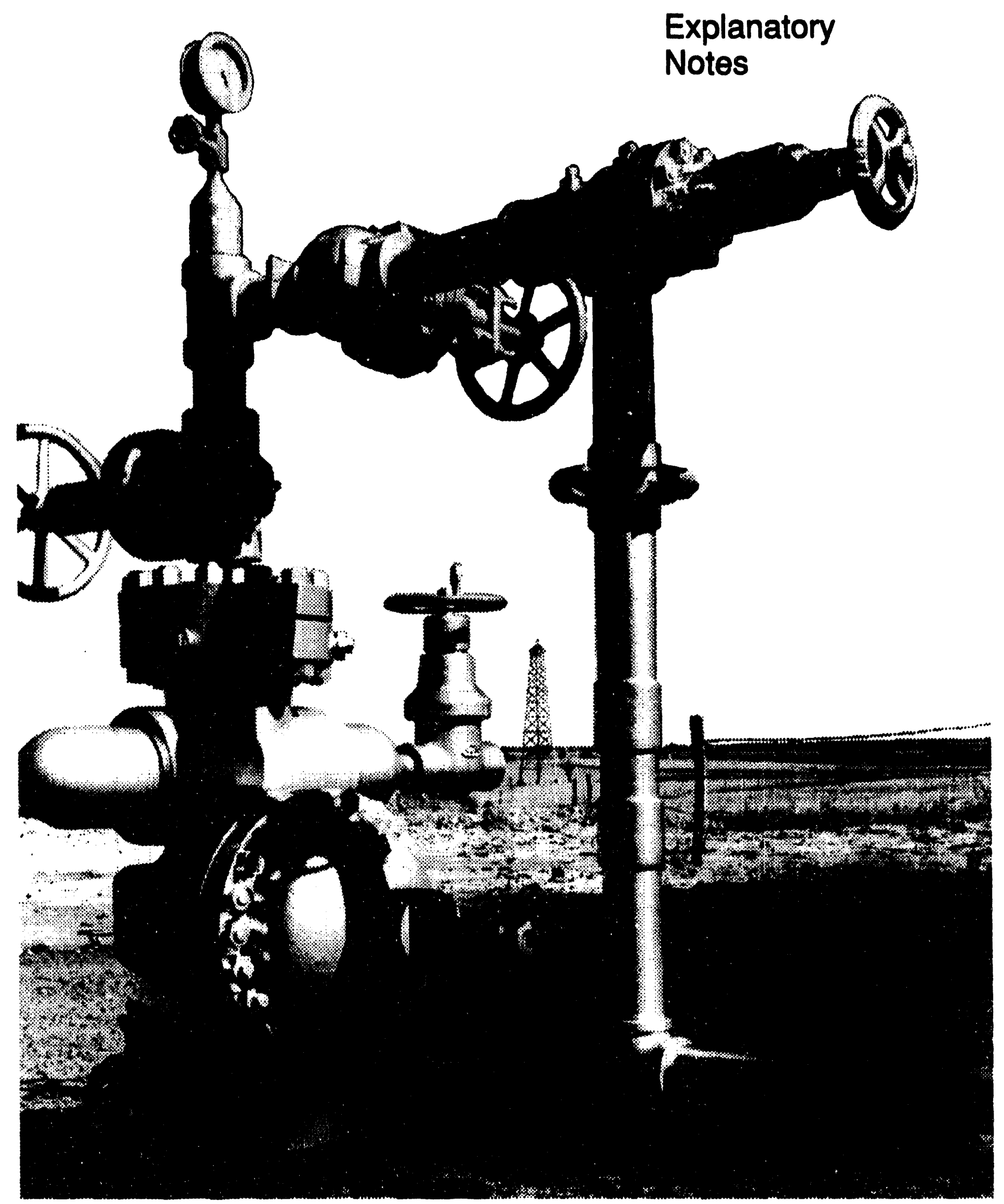

The cluster of pipes and valves that control the flow of oil at the mouth of an oil well is what oilmen call a "Christmas Tree." 


\section{Explanatory Notes}

The following Explanatory Notes are provided to assist in understanding and interpreting the data presented in this publication.

- Note 1. Petroleum Supply Reporting System

- Note 2. Monthly Petroleum Supply Reporting System

- Note 3. Form EIA-820: Annual Refinery Report

- Note 4. Form EIA-819A: Annual Oxygenate Capacity Report

- Note 5. Technical Notes for Detailed Statistics Tables

- Note 6. Domestic Crude Oil Production

- Note 7. Export Data

- Note 8. Quality Control and Data Revision

- Note 9. Frames Maintenance

- Note 10. Descriptive Monthly Statistics

- Note 11. Practical Limitations of Data Collection Efforts

- Note 12. 1981 Changes in the Petroleum Supply Reporting System

- Note 13. 1983 Changes in the Petroleum Supply Reporting System

- Note 14. 1984 Changes in the Petroleum Supply Reporting System

- Note 15. 1985 Changes in the Petroleum Supply Reporting System

- Note 16. 1986 Changes in the Petroleum Supply Reporting System

- Note 17. 1987 Changes in the Petroleum Supply Reporting System

- Note 18. 1989 Changes in the Petroleum Supply Reporting System

- Note 19. 1990 Changes in the Petroleum Supply Reporting System

- Note 201993 Changes in the Petroleum Supply Reporting System

\section{Note 1. Petroleum Supply Reporting System}

The Petroleum Supply Reporting System (PSRS) represents a family of data collection survey forms, data processing systems, and publication systems that have been consolidated to achieve comparability and consis- tency throughout. The survey forms that comprise the PSRS are:

\begin{tabular}{ll|}
\hline Form & \\
Number & Name \\
ElA-800 & "Weekly Refinery Report" \\
ElA-801 & "Weekly Bulk Terminal Report" \\
EIA-802 & "Weekly Product Pipeline Report" \\
EIA-803 & "Weekly Crude Oil Stocks Report" \\
ElA-804 & "Weekly Imports Report" \\
EIA-807 & "Propane Telephone Survey" \\
EIA-810 & "Monthly Refinery Report" \\
EIA-811 & "Monthly Bulk Terminal Report" \\
EIA-812 & "Monthly Product Pipeline Report" \\
EIA-813 & "Monthly Crude Oil Report" \\
EIA-814 & "Monthly Imports Report" \\
EIA-816 & "Monthly Natural Gas Liquids Report" \\
EIA-817 & "Monthly Tanker and Barge Movement \\
& Report" \\
EIA -819M & "Monthly Oxygenate Telephone Report" \\
EIA-819A & "Annual Oxygenate Report" \\
EIA-820 & "Annual Refinery Report"
\end{tabular}

Forms EIA-800 through 804 comprise the Weekly Petroleum Supply Reporting System (WPSRS). A sample of all petroleum companies report weekly data to the Energy Information Administration (EIA) on crude oil and petroleum product stocks, refinery inputs and production, and crude oil and petroleum product imports. The sample of companies that report weekly is selected from the universe of companies that report on the comparable monthly surveys. Data collected from the WPSRS are used to develop estimates of the most current monthly quantities in the Summary Statistics section of the Petroleum Supply Monthly (PSM) and which appear in the Weekly Petroleum Status Report(WPSR).

The Form EIA-807, "Propane Telephone Survey," is used to collect data on production, stocks, and imports of propane. These data are used to monitor the supply of propane and to report to the Congress and others on supplies when requested. Data are collected from a sample of respondents reporting on the Monthly Petroleum Supply Reporting System (MPSRS) surveys. Data are collected 
on a weekly basis during the heating season (October through March) and published in the Winter Fuels Report. During the non-heating season (April through September) data are collected on end-of-month stocks only. These data are published in the WPSR.

Forms ElA-810 through 814,816 , and 817 comprise the MPSRS. These surveys are used to collect detailed refinery, natural gas plant and oxygenate plant operations data; refinery, bulk terminal, oxygenate plant, natural gas plant and pipeline stocks data; crude oil and petroleum product imports data; and data on movements of petroleum products and crude oil between Petroleum Administration for Defense (PAD) Districts. A description of the MPSRS forms follows in Explanatory Note 2.

Data from these surveys are published in preliminary form in the PSM. They are published in final form in the Petroleum Supply Annual (PSA), Volumes 1 and 2.

Summary information on the revision error between preliminary and final data is published once a year in the PSM feature article entitled, "Timeliness and Accuracy of Petroleum Supply Data." The last article was published in the August 1993 issue and evaluated the accuracy of the data for 1992 compared with previous years.

The Form EIA-819M, "Monthly Oxygenate Telephone Report," is used to collect preliminary data on production, imports, and stocks of oxygenates by PAD District. These data are used to monitor the supply of oxygenates. Data are collected from a sample of respondents reporting on the MPSRS surveys and from the universe of oxygenate producers reporting on Form EIA-819A, "Annual Oxygenate Capacity Report." Data are published in Appendix D of the PSM and also in the WPSR.

The Form EIA-819A, "Annual Oxygenate Capacity Report," is used to collect data on current and projected production capacity of oxygenates and annual production and end-of-year inventories of fuel ethanol. The results of this survey are published in the Oxygenate Capacity section of the PSA, Volume 1 and is described in more detail in Explanatory Note 4.

The Form EIA-820, "Annual Refinery Report," is used to collect data on refinery fuel use and consumption of steam and electricity, refinery receipts of crude oil by method of transportation, operable capacity for atmospheric crude oil distillation units and downstream units, as well as production capacity and storage capacity for petroleum products. This survey is the primary source of data in the Refinery Capacity section of the PSA, Volume 1 and is described in more detail in Explanatory Note 3.
Note 2.

\section{Monthly Petroleum Supply Reporting System}

The Monthly Petroleum Supply Reporting System (MPSRS) was implemented in January 1983 as the result of an extensive effort by the Energy Information Administration (EIA) to integrate the collection and processing of petroleum supply data that had been collected on other survey forms for many years. The collection of monthly petroleum supply statistics began as early as 1918 when the U.S. Bureau of Mines began collecting data on refinery operations and crude oil stocks and movements. The collection systems were further expanded in 1925 to include natural gas plant liquids production and storage, imports of crude oil and petroleum products and storage and movement of petroleum products in 1959, and tanker and barge movements of crude oil and petroleum products in 1964. Since their inception, each survey has undergone numerous changes, but the MPSRS was the first effort to make them all consistent and comparable. The forms that comprise the MPSRS are:

\begin{tabular}{ll}
\hline $\begin{array}{l}\text { Form } \\
\text { Number }\end{array}$ & Name \\
EIA-810 & "Monthly Refinery Report" \\
EIA-811 & "Monthly Bulk Terminal Report" \\
EIA-812 & "Monthly Product Pipeline Report" \\
EIA-813 & "Monthly Crude Oil Report" \\
EIA-814 & "Monthly Imports Report" \\
EIA-816 & "Monthly Natural Gas Liquids Report" \\
EIA-817 & "Monthly Tanker and Barge Movement \\
& Report" \\
EIA-819M & "Monthly Oxygenate Telephone Report"
\end{tabular}

\section{Respondont Frame}

Form EIA-810, "Monthly Refinery Report" - Operators of all operating and idle petroleum refineries and blending plants located in the 50 States, the District of Columbia, Puerto Rico, the Virgin Islands, Guam and other U.S. possessions. Approximately 240 respondents report on the Form EIA-810.

Form EIA-811, "Monthly Bulk Terminal Report" - Every bulk terminal operating company located in the 50 States, the District of Columbia, Puerto Rico, the Virgin Islands, and other U.S. possessions. A bulk terminal is primarily used for storage and/or marketing of petroleum products and has a total bulk storage capacity of 50,000 barrels or more, and/or receives petroleum products by tanker, barge, or pipeline. Bulk terminal facilities associated with a product pipeline are included. Approximately 330 respondents report on the Form EIA-811. 
Form EIA-812, "Monthly Product Pipeline Report" - All product pipeline companies that carry petroleum products (including interstate, intrastate, and intracompany pipelines) in the 50 States and the District of Columbia. Approximately 80 respondents report on the Form EIA812.

Form EIA-813, "Monthly Crude Oil Report" - All companies which carry or store 1,000 barrels or more of crude oil. Included in this survey are gathering and trunk pipeline companies (including interstate, intrastate, and intracompany pipelines), crude oil producers, terminal operators, storers of crude oil (except refineries), and companies transporting Alaskan crude oil by water in the 50 States and the District of Columbia. Approximately 160 respondents report on the Form EIA-813.

Form EIA-8 14, "Monthly Imports Report" - All companies, including subsidiary or affiliated companies, that import crude oil or petroleum products (1) into the 50 States and the District of Columbia, (2) into Puerto Rico, the Virgin Islands and other U.S. possessions (Guam, Midway Islands, Wake Island, American Samoa, and Northern Mariana Islands), and (3) from Puerto Rico, the Virgin Islands and other U.S. possessions into the $\mathbf{5 0}$ States and the District of Columbia. Imports into Foreign Trade Zones located in the 50 States and the District of Columbia are considered imports into the 50 States and the District of Columbia and must be reported. A report is required only if there has been an import during the month unless the importer has been selected as part of a sample to report every month regardless of activity. Approximately 220 respondents report on the Form EIA-814.

Form EIA-816, "Monthly Natural Gas Liquids Report" Operators of all facilities that extract liquid hydrocarbons from a natural gas stream (natural gas processing plant) and/or separate a liquid hydrocarbon stream into its component products (fractionator). Approximately 720 respundents report on the Form EIA-816.

Form EIA-817, "Monthly Tanker and Barge Movement Report" - All companies that have custody of crude oil or petroleum products transported by tanker or barge between Petroleum Administration for Defense (PAD) Districts or between the Panama Canal and the United States. For purposes of this report, custody is defined as physical possession of crude oil or petroleum products on a company-owned tanker or barge. Also, companies which lease vessels or contract for the movement of crude oil or petroleum products on a tanker or barge between PAD Districts or between the Panama Canal and the United States are considered to have custody. Approximately $\mathbf{4 0}$ respondents report on the Form EIA-817.

Form EIA-819M, "Monthly Oxygenate Telephone Report" - The sample of companies that report on the
EIA-819M are selected from the universe of companies that report on the MPSRS surveys and from the universe of oxygenate producers reporting on the Form EIA-819A, "Annual Oxygenate Capacity Report." The universe consists of (1) operators of facilities that produce (manufacture or distill) oxygenates (including MTBE plants, petrochemical plants, and refineries that produce oxygenates as part of their operations); (2) operators of petroleum refineries; (3) operators of bulk terminals, bulk stations, blending plants, and other nonrefinery facilities that store and/or blend oxygenates; and (4) importers of oxygenates (importer of record) located in or importing oxygenates into the 50 States and the District of Columbia. Approximately 100 respondents report on the Form BIA-819M.

\section{Sampling}

The sampling procedure used for the survey Form EIA$819 \mathrm{M}$ is the cut-off method and is performed using software developed by EIA's Office of Statistical Standards. In the cut-off method, companies are ranked from largest to smallest on the basis of quantities reported (oxygenate production, oxygenate stocks, and oxygenate imports) during 1993. Companies are chosen for the sample beginning with the largest and adding companies until the total sample covers approximately 90 percent of the total for each oxygenate item and supply type by geographic region (PAD Districts I through V) for which data may be published.

\section{Description of Sunvey Forms}

The Form EIA-810, "Monthly Refinery Report," is used to collect data on refinery input and capacity, sulfur content and API gravity of crude oil, and data on supply (beginning stocks, receipts, and production) and disposition (inputs, shipments, fuel use and losses, and ending stocks) of crude oil and refined products.

The Form EIA-811, "Monthly Bulk Terminal Report," is used to collect data on end-of-month stock levels of finished petroleum products by State in the custody of the bulk terminal company regardless of ownership. Leased tankage at other facilities is excluded. All domestic and foreign stocks held at bulk terminals and in-transit thereto, except those in-transit by pipeline are included. Petroleum products in-transit by pipeline are reported by pipeline operators on Form EIA-812, "Monthly Product Pipeline Report."

The Form EIA-812, "Monthly Product Pipeline Report," is used to collect data on end-of-month stock levels and movements of petroleum products transported by pipeline. Intermediate movements for pipeline systems operating in more than two PAD Districts are included. 
The Form EIA-813, "Monthly Crude Oil Report," is used to collect data on end-of-month stocks of crude oil held at pipeline and tank farms (associated with the pipelines) and terminals operated by the reporting company. Also, crude oil consumed by pipelines and on leases as pump fuel, boiler fuel, etc., is reported. Data are reported on a PAD District basis.

Total Alaskan crude oil stocks in-transit by water (including stocks held at transshipment terminals between Alaska and the continental United States) to the 50 States, the District of Columbia, Puerto Rico, and the Virgin Islands are also reported by the transporting company having custody of the stocks.

Inter-PAD District movements of crude oil by pipeline are collected by the shipping and receiving PAD District. Intermediate movements for pipeline systems operating in more than two PAD Districts are not included.

The Form EIA-814, "Monthly Imports Report," is used to collect data on imports of crude oil and petroleum products (1) into the 50 States and the District of Columbia, (2) into Puerto Rico, the Virgin Islands, and other U.S. possessions (Guam, Midway Islands, Wake Island, American Samoa, and Northern Mariana Islands), and (3) from Puerto Rico, the Virgin Islands, and other U.S. possessions into the 50 States and the District of Columbia. imports into Foreign Trade Zones located in the 50 Itates and the District of Columbia are considered imports into the 50 States and the District of Columbia.

The type of commodity, port of entry, country of origin, quantity (thousand barrels), sulfur percent by weight, API gravity, and name and location of the processing or storage facility are reported. Sulfur percent by weight is requested for crude oil, crude oil bumed as fuel, and residual fuel oil only. API gravity is requested for crude oil only. The name and location of the processing or storage facility is requested for crude oil, unfinished oils, other hydrocarbons/hydrogen/oxygenates, and blending components only.

The Form EIA-816, "Monthly Natural Gas Liquids Report," is used to collect data on the operations of natural gas processing plants and fractionators. Beginning and end-of-month stocks, receipts, inputs, production, shipments, and plant fuel use and losses during the month are collected from operators of natural gas processing plants. End-of-month stocks are collected from fractionators.

The Form EIA-817, "Monthly Tanker and Barge Movement Report," is used to collect data on the movements of crude oil and petroleum products between PAD Districts. Data are reported by shipping and receiving PAD District and sub-PAD District. Shipments to and from the Panama
Canal are also included if the shipment was delivered to the Canal.

The Form EIA-819M, "Monthly Oxygenate Telephone Report," is used to collect data on production, stocks, and imports of oxygenates. Data on end-of-month stocks are reported on a custody basis regardless of ownership. Data are reported on a PAD District basis.

\section{Collection Mothods}

Except for the EIA- 819M, survey forms for the MPSRS can be submitted by mail, facsimile, or electronic transmission. Completed forms are required to be postmarked by the 20th calendar day following the end of the report month. Data collection for the EIA-819M begins on the seventh working day of each month. Data are solicited by telephone or transmitted to the EIA by facsimile. Receipt of the reports are monitored using an automated respondent mailing list. Telephone follow-up calls are made to nonrespondents prior to the publication deadline.

\section{Response Rate}

The response rate is generally 98 to 100 percent. Chronic nonrespondents and late filing respondents are contacted in writing and reminded of their requirement to report. Companies that file late or fail to file are subject to criminal fines, civil penalties, and other sanctions as provided by Section 13(i) of the Federal Energy Administration (FEA) Act.

\section{Data Imputation}

Imputation is performed for companies that fail to file Forms EIA-810 through 813, 816, and 819M. For such companies, previous monthly values are used for current values. On the EIA-819M, data are aggregated for each geographic region. Estimation factors, which are derived from the previous year's data, are then applied to each cell to generate published estimates. Data for nonrespondents on the Forms EIA-814 and 817 are not imputed because these data series, by respondent, are highly variable.

\section{Confidentiality}

The Office of Legal Counsel of the Department of Justice concluded on March 20,1991, that the Federal Energy Administration Act requires the Energy Information Administration to provide company-specific data to the Department of Justice, or to any Federal agency when requested for official use, which may include enforcement of Federal law. The information contained on this form may also be made available, upon request, to another component of the Department of Energy (DOE), to any Committee of Congress, the General Accounting Office, or other Congressional agencies authorized by law to 
receive such information. A court of competent jurisdiction may obtain this information in response to an order.

The information contained on Forms EIA-810 through $813,816,817$, and $819 \mathrm{M}$ are kept confidential and not disclosed to the public to the extent that they satisfy the criteria for exemption under the Freedom of Information Act (FOIA), 5 U.S.C. 552, the Department of Energy (DOE) regulations, 10 C.F.R. 1004.11, implementing the FOIA, and the Trade Secrets Act, 18 U.S.C. 1905. The information contained on Form EIA-814 are not considered confidential and historically has not been treated as such.

Upon receipt of a request for this information under the FOIA, the DOE shall make a final determination whether the information is exempt from disclosure in accordance with the procedures and criteria provided in the regulations. To assist us in this determination, respondents should demonstrate to the DOE that, for example, their information contains trade secrets or commercial or financial information whose release would be likely to cause substantial harm to their company's competitive position. A letter accompanying the submission that explains (on an element-by-element basis) the reasons why the information would be likely to cause the respondent substantial competitive harm if released to the public would aid in this determination. A new justification does not need to be provided each time information is submitted on the form, if the company has previously submitted a justification for that information and the justification has not changed. Company specific data are also provided to other DOE offices for the purpose of examining operations in the context of emergency response planning and actual emergencies.

The data collected on Forms EIA-810 through 814, 816, and 817 appear in EIA publications such as Petroleum Supply Monthly (PSM), Monthly Energy Review, Petroleum Supply Annual (PSA), and the Annual Energy Review.

Data on the breakdown between liquefied refinery gases and olefins and lubricants is suppressed on Table 16, "Refinery Net Production of Finished Petroleum Products by PAD and Refining Districts" to avoid disclosure of company identifiable data.

Statistics representing data aggregated from less than three companies or aggregated data representing 60 percent or more of a single company's data are suppressed on the PSA tables listed below. In addition, complementary suppression is performed to avoid any residual disclosure.
- Table 16, "Refinery Input of Crude Oil and Petroleum Products by PAD and Refining Districts," (inputs of oxygenates)

- Table 18, "Refinery Stocks of Crude Oil and Petroleum Products by PAD and Refining Districts," (stocks of oxygenates)

- Table 30, "Stocks of Crude Oil and Petroleum Products by PAD District," (stocks of oxygenates)

- Table 31, "Refinery, Bulk Terminal, and Natural Gas Plant Stocks of Selected Petroleum Products," (all products)

With the exception of the tables listed above, the tables in the PSA are not subject to statistical : 1ondisclosure procedures. Thus, there may be some tuble cells which are based on data from only one or two risspondents, or which are dominated by data from one or tv'o large respondents. In these cases, it may be possible for a knowledgeable user of the data to make inferences a sout the data reported by a specific respondent.

\section{Note 3. Form ElA-820: Annual Refinery Report}

Refinery capacity data collection was begun in 1918 by the Bureau of Mines, then in the Department of Commerce, and was operated on a voluntary basis until 1980 . In 1980, the mandatory Energy Information Administration (EIA) Form EIA-177, Capacity of Petroleum Refineries, was implemented. Information on refining capacity was expanded to include not only current year operations, two-year projections, and refinery input/production data. Working storage capacity data was also added to the form and product categories were added for total coverage. Information on refinery downstream facilities was expanded to include a breakdown of thermal operations and to add vacuum distillation, catalytic hydrorefining and hydrotreating. Production capacity was also added to include information on isomerization, alkylation, aromatics, asphal/road oil, coking, lubricants and hydrogen.

In 1983, the form was revised to improve the consistency and quality of the data collected by the EIA and redesignated as Form EIA-820, "Annual Refinery Report." Two sections for data previously reported monthly were added: (1) refinery receipts of crude oil by method of transportation, and (2) fuels consumed for all purposes at refineries. Also, the second year projections on refining capacity were eliminated. As a result of a study conducted by the EIA evaluating motor gasoline data collected by the Federal Highway Administration (FHWA) and by the EIA, motor gasoline blending plants were included for the first time to the respondent frame in order to produce more accurate statistics on the production of motor gasoline. 
In 1987, the form was revised to reduce respondent burden and to better reflect current refinery operations through updated terminology. Information on projected input/production of refinery processing facilities was deleted. Several categories under catalytic hydrotreating were combined: naphtha and reformer feeds were combined into a single category as well as residual fuel oil and other. Thermal cracking types, gas oil and "other" were also combined into a single category. Catalytic reforming types, conventional and bi-metallic were replaced with low and high pressure processing units. Two new categories were added: fuels solvent deasphalting was added to downstream charge capacity and sulfur recovery was added to production capacity.

In 1994, the form was revised to enable EIA to calculate utilization rates for certain downstream processing units and to reflect storage capacity of fuels mandated by the Clean Air Act Amendments of 1990. Additions to the form included calendar day downstream charge capacity for fluid and delayed coking, catalytic cracking, and catalytic hydrocracking. Also storage capacity categories for reformulated, oxygenated, and other finished motor gasoline were added, as well as oxygenate storage capacity and separate categories for high and low sulfur distillate fuel oil.

\section{Respondent Frame}

The respondent frame consists of all operating and idle petroleum refineries (including new refineries under construction), motor gasoline blending plants, shutdown refineries and shutdown motor gasoline blending plants with usable storage capacity, located in the 50 States, the District of Columbia, Puerto Rico, the Virgin Islands, Guam and other U.S. possessions. As of January 1, 1994, there were 179 refineries and 32 motor gasoline blending plants operating in the 50 States. The following is a list of motor gasoline blending plants and their locations that operated during 1993.

Citgo Petroleum Corp. Linden, NJ

Phillips 66 Co. Commerce City, CO

Citgo Petroleum Corp. East Chicago, IN

Santa Fe Pacific Pipeline Phoenix, AZ

Citgo Petroleum Corp. East Braintree, MA

Citgo Petroleum Corp. Richmond, VA

Santa Fe Pacific Pipeline Reno, NV

Shell Oil Co.

Carson, CA

Citgo Petroleum Corp. Bryan, TX

Sinclair Oil Corp. Denver, CO

Citgo Petroleum Corp. Waco, TX
GATX Terminal Corp.

Pasadena, TX

GATX Terminal Corp.

Carteret, NJ

Golden West Refg. Co.

Santa Fe Springs, CA

Hartford/Woodriver Term.

Hartford, IL

International Matex Tank

Terminals

Bayonne, NJ

Northville Industries

Linden, NJ

Oiltanking Houston Inc.

Houston, TX

Phillips 66 Co.

East St. Louis, IL

Phillips 66 Co.

East Chicago, IN

Phillips 66 Co.

Decatur, IL
Texaco, Inc.

Phoenix, AZ

Texaco, Inc. Tucson, AZ

Thriftway Refg. Co. Bloomfield, NM

Unocal

Beaumont, TX

Westec Petroleum Inc. Grand Junction, CO

Westec Petroleum Inc. Denver, CO

Westec Petroleum Inc. Woods Cross, UT

Wickland Pipeline Co. Crockett, CA

Williams Pipeline Co. Des Moines, IA

Williams Pipeline Co. lowa City, IA

The respondent frame is maintained by monitoring the monthly Form EIA-810, "Monthly Refinery Report," and industry publications for changes and developments in the . petroleum industry such as refinery sales, mergers and new operations.

\section{Description of Survey Form}

The Form EIA-820 is used to collect data on fuels consumed for all purposes at the refinery during the preceding year; refinery receipts of crude oil by method of transportation during the preceding year; current and next year projections for operable atmospheric crude oil distillation capacity, downstream charge capacity and production capacity; and current year working and shell storage capacity for crude oil and petroleum products at the refinery.

\section{Collection Methods}

The Form EIA-820 is sent to respondents in December. Survey forms can be submitted by mail or facsimile. Completed forms are required to be postmarked by the 15th day of February of the current report year. Receipt of the reports is monitored using an automated respondent mailing list. Telephone follow-up calls are made to secure 
responses from those companies failing to report by February 15 th.

\section{Responce Rab}

The response rate for the Form EIA-820 is normally very high. Data are estimated and non-compliance procedures are implemented for those companies still not reporting data by close-out for the report year. For the January 1, 1994 survey, there were no nonrespondents.

\section{Data Imputation}

Imputation is performed for companies that fail to file prior to the publication deadline. For the January 1, 1994 survey, there were no nonrespondents; therefore, imputation was not necessary. However, when nonresponse does occur, values for these companies are imputed from data reported on the previous year's Form EIA-820 and/or from data reported on Form EIA-810, "Monthly Refinery Report," for that company. For most surveyed items, the value imputed for nonrespondents is the value that company reported on the Form EIA-820 for the previous year. For three categories of information however, the imputed value is also based on their data from the Form EIA-810 as follows:

\section{Section 1: Fud, Electricity, and Steam Consumed for all Purposes at Refinerios}

Data are imputed for crude oil, distillate and residual fuel oil, liquefied petroleum gases, still gas, and marketable and catalyst petroleum coke based upon data reported on the monthly Form EIA-810. A ratio of the fuel use reported on the previous year's annual report to the fuel use and losses reported on the aggregated monthly data for the previous year is derived for these product categories. This ratio is then applied to the monthly aggregated data for the current year for similar product categories.

Estimates for natural gas, coal, electricity and steam are taken directly from data reported on the previous year's annual Form EIA-820.

\section{Section 2: Refinery Recelpts of Crude OII by Mothod of Transportation}

The imputation methodology for this section is based on data reported on both the monthly Form EIA-810 and the annual Form EIA-820. Annual refinery receipts of domestic and foreign crude oil for a nonrespondent are imputed by aggregating the values for the refinery on the monthly survey. These values are allocated to the method of transportation by using the percentages reported for the refinery in the previous year. The difference between the values reported on the two surveys by all respondents in 1994 is 0.6 percent.

\section{Section 3: Operable and Storage Capacity as of January 1}

Operable atmospheric crude oil distillation capacity in barrels per calendar day is collected on the monthly Form ElA-810 as of the first day of each month and on the annual Form EIA-820 as of January 1. As part of the editing process for the Form EIA-820, these two values are compared. Companies are contacted and any discrepancies are resolved by the time of publication. Imputed values for operable atmospheric crude oil distillation capacity in barrels per calendar day are taken directly from the January Form EIA-810. A barrels per stream day capacity is then derived by dividing the reported barrels per calendar day capacity by .95 .

Current year and projected year data for downstream charge capacity, production capacity, and data for working and shell storage capacity are taken directly from the previous year's annual report.

\section{Confidentiality}

The Office of Legal Counsel of the Department of Justice concluded on March 20, 1991, that the Federal Energy Administration Act requires the Energy Information Administration to provide company-specific data to the Department of Justice, or to any other Federal agency when requested for official use, which may include enforcement of Federal law. The information contained on this form may also be made available, upon request, to another component of the Department of Energy (DOE), to any Committee of Congress, the General Accounting Office, or other Congressional agencies authorized by law to receive such information. A court of competent jurisdiction may obtain this information in response to an order.

Information on operable atmospheric crude oil distillation capacity, downstream charge capacity, and production capacity on Form ElA-820 are not considered as confidential, and historically have not been treated as such. Company identifiable data are published in the Petroleum Supply Annual (PSA), Volume 1, Tables 38 and 39.

Other data on the Form EIA-820 are kept confidential and not disclosed to the public to the extent that it satisfies the criteria for exemption under the Freedom of Information Act (FOIA), 5 U.S.C.552, Department of Energy (DOE) regulations, 10 C.F.R.1004.11, implementing the FOIA, and the Trade Secrets Act, 18 U.S.C.1905.

Upon receipt of a request for this information under the FOIA, the DOE shall make a final determination whether the information is exempt from disclosure in accordance with the procedures and criteria provided in the regula- 
tions. To assist us in this determination, respondents should demonstrate to the DOE that, for example, their information contains trade secrets or commercial or financial information whose release would be likely to cause substantial harm to their company's competitive position. A letter accompanying the submission that explains (on an element-by-element basis) the reasons why the information would be likely to cause the respondent substantial competitive harm if released to the public would aid in this determination. A new justification does not need to be provided each time information is submitted on the form, if the company has previously submitted a justification for that information and the justification has not changed.

The data collected on Form EIA-820, "Annual Refinery Report," is used to report aggregate statistics on and conduct analyses of the operation of U.S. petroleum refineries and blending plants. The data appear in EIA publications such as PSA, and the Annual Energy Review. Company specific data are also provided to other DOE offices for the purpose of examining specific refinery operations in the context of emergency response planning and actual emergencies.

The tables pertaining to refinery receipts of crude oil by method of transportation and fuels consumed at the refinery published in the PSA are not subject to statistical nondisclosure procedures. Thus, there may be some table cells which are based on data from only one or two respondents, or which are dominated by data from one or two large respondents. In these cases, it may be possible for a knowledgeable user of the data to make inferences about the data reported by a specific respondent.

\section{Quality Control}

There are two types of errors usually associated with data produced from a survey - sampling errors and nonsampling errors. Because estimates from the Form EIA-820 survey are based on a complete census of the frame of petroleum refineries and motor gasoline blending plants, there is no sampling error in the data presented in this report. The data, however, are subject to nonsampling errors. Nonsampling errors are those which can arise from: (1) the inability to obtain data from all companies in the frame or sample (nonresponse) and the method used to account for nonresponses; (2) definitional difficulties and/or improperly worded questions which lead to different interpretations; (3) mistakes in recording or coding the data obtained from respondents; and (4) other errors of collection, response, coverage, and estimation. Quality control procedures are employed in the collection and editing operations to minimize misrepresentation and misreporting. Nonresponse follow-up procedures are employed to reduce the number of nonrespondents, and procedures employed to impute missing data, introduce a minimal amount of error, given the relatively small volume of imputed data.

\section{Resubmisolons}

Resubmissions are required whenever an error greater than 5 percent of the true value is discovered. In the event of a reporting error, company reports are updated after contact with the company and are followed up by corrected report resubmissions. Late submissions or resubmissions received after the publication date are entered into a "working" file. This file contains the most up-todate data for the Form EIA-820 and is used to edit next year's data.

\section{Note 4. Form ElA-819A: Annual Oxygenate Capacity Report}

The Clean Air Act Amendments of 1990, mandated several changes to the composition of motor gasoline. Among these is the requirement for the addition of liquid components containing oxygen to motor gasoline starting in the Fall of 1992. Because of their importance to gasoline supply, the Energy Information Administration (EIA) began collecting oxygenate production capacity as of January 1993 in order to monitor the availability of oxygenates in the United States.

\section{Respondent Frame}

The respondent frame consists of all operating and idle facilities that produce or distill oxygenates (including fuel-grade ethanol producers, methyl tertiary butyl ether (MTBE) plants, petrochemical plants and refineries that produce oxygenates as part of their operations), and new plants under construction located in the 50 States, the District of Columbia, Puerto Rico, the Virgin Islands, Guam and other U.S. possessions. As of January 1, 1994, there were 98 oxygenate producers.

The respondent frame is maintained by monitoring industry periodicals that report changes in status (births, deaths, sales, and acquisitions) of oxygenate facilities, producing, transporting, importing, and/or storing oxygenates.

\section{Description of Survey Form}

The Form EIA-819A is used to collect data on current and projected production capacities. The following oxygenates are collected: fuel ethanol, ethyl tertiary butyl ether (ETBE), methanol, MTBE, tertiary amyl methyl ether (TAME), tertiary butyl alcohol (TBA), and other oxygenates (i.e., includes other aliphatic alcohols and ethers intended for motor gasoline blending (e.g., isopropyl ether (IPE) or n-propanol)). 


\section{Collection Methode}

The Form EIA-819A is sent to respondents in December. Survey forms can be submitted by mail or facsimile. Completed forms are required to be postmarked by the 15 th day of February of the current report year. Receipt of the reports is monitored using an automated respondent mailing list. Telephone follow-up calls are made to secure responses from those companies failing to report by February 15 th.

\section{Reoponse Rate}

Data are imputed and non-compliance procedures are implemented for those companies still not reporting data by close-out for the report year. The response rate for the January 1, 1994 survey, was very high with only two nonrespondents.

\section{Data Imputation}

Imputation is performed for companies that fail to file prior to the publication deadline. For the January 1, 1994 survey, one fuel ethanol and one MTBE U.S. producer did not respond. Their total operable capacity for each oxygenate is estimated to be less than 0.01 percent of the U.S. total. Data for these respondents were imputed using previous year's data.

\section{Confidentiality}

The Office of Legal Counsel of the Department of Justice concluded on March 20, 1991, that the Federal Energy Administration Act requires the EIA to provide companyspecific data to the Department of Justice, or to any other Federal agency when requested for official use, which may include enforcement of Federal law. The information contained on this form may also be made available, upon request, to another component of the Department of Energy (DOE), to any Committee of Congress, the General Accounting Office, or other Congressional agencies authorized by law to receive such information. A court of competent jurisdiction may obtain this information in response to an order.

The information on current year "production capacity" is not considered as confidential. Company identifiable data are published in the Petroleum Supply Annual, Volume 1, Tables 51 through 57.

Projected year production capacity is kept confidential and not disclosed to the public to the extent that it satisfies the criteria for exemption under the Freedom of Information Act (FOIA), 5 U.S.C. section 552, the DOE regulations, 10 C.F.R. section 1004.11, implementing the FOIA, and the Trade Secrets Act, 18 U.S.C. section 1905.
Upon receipt of a request for this information under the FOIA, the DOE shall make a final determination whether the information is exempt from disclosure in accordance with the procedures and criteria provided in the regulations. To assist us in this determination, respondents should demonstrate to the DOE that, for example, their information contains trade secrets or commercial or financial information whose release would be likely to cause substantial harm to their company's competitive position. A letter accompanying the submission that explains (on an element-by-element basis) the reasons why the information would be likely to cause the respondent substantial competitive harm if released to the public would aid in this determination. A new justification does not need to be provided each time information is submitted on the form, if the company has previously submitted a justification for that information and the justification has not changed.

\section{Quality Control}

There are two types of errors usually associated with data produced from a survey - sampling errors and nonsampling errors. Because estimates from the Form EIA-819A survey are based on a complete census of the frame of oxygenate producers, there is no sampling error in the data presented in this report. The data, however, are subject to nonsampling errors Nonsampling errors are those which can arise from: (1) the inability to obtain data from all companies in the frame or sample (nonresponse) and the method used to account for nonresponses; (2) definitional difficulties and/or improperly worded questions which lead to different interpretations; (3) mistakes in recording or coding the data obtained from respondents; and (4) other errors of collection, response, coverage, and estimation. Quality control procedures are employed in the collection and editing operations to minimize misrepresentation and misreporting. Nonresponse follow-up procedures are employed to reduce the number of nonrespondents, and procedures employed to impute missing data, introduce a minimal amount of error, given the relatively small volume of imputed data.

For the January 1, 1994 survey, several reporting issues were identified when generating publication table. These issues were resolved as follows:

(1) The Arco Chemical Co., Corpus Christi, Texas plant reported the capability of producing either 9,500 barrels per day of ETBE or 12,000 barrels per day of MTBE. ETBE or MTBE can be produced at this site but not simultaneously; the unit must be designated for production of only one product. As of January 1, 1994, the plant was producing MTBE and therefore was included in the MTBE production capacity data. Their ETBE production capacity of 9,500 barrels per day was idle on January 1, 1994 and was not included 
in the ETBE production capacity data to avoid double counting of the oxygenate production capacity of this specific unit.

(2) Archer Daniels Midland (ADM) Co. reported a combined fuel ethanol production capacity for their five locations: Decatur and Peoria, Illinois; Cedar Rapids and Clinton, lowa; and Cenex Agri-Fuels located in Wahalla, North Dakota. The combined fuel ethanol production capacity was included in the total fuel ethanol production capacity for the State of Illinois. However, the number of operable plants located in each state reflects the correct location of the five plants.

In addition, ADM did not provide a projected year fuel ethanol production capacity siting an insufficient basis with which to project its future fuel ethanol production capacity including the period covering calendar year 1995 until regulations are adopted defining ethanol's role in the oxygenates program in the Clean Air Act. Because ADM accounts for such a large portion of the fuel ethanol production capacity, publication of projected production capacity and net change capacity for fuel ethanol was not feasible.

\section{Resubmissions}

Resubmissions are required whenever an error greater than five percent of the true value is discovered. In the event of a reporting error, company reports are updated after contact with the company and are followed up by corrected report submissions. Late submissions or resubmissions received after the publication date are entered into a "working" file. This file contains the most up-todate data for the Form EIA-819A and is used to edit next year's data.

\section{Note 5. Technical Notes for Detailed Statistics Tables}

The detailed statistics tables in the Petroleum Supply Annual provide complete supply and demand information for the previous year. The tables are organized to locate $\mathrm{Na}$ tional and Petroleum Administration for Defense (PAD) District summary data at the front followed by tables on crude oil and petroleum product production, import/export data, stocks information, and lastly, data on crude oil and petroleum product movements. To assist in the interpretation of these tables, the following technical notes are provided. Column and row headings are defined in the Glossary.

\section{Supply}

Field Production - Field production is the sum of crude oil production, natural gas plant liquids production, other liquids production, and finished petroleum products production.

Crude oil production is an estimate based on data received from State conservation agencies and the Mineral Management Service of the U.S. Department of the Interior. Refer to Explanatory Note 6 for further details.

Field production of natural gas plant liquids is reported on Form ElA-816 and published on a net basis (i.e., production minus inputs) in this column.

Other liquids field production is calculated by forcing the product supplied to be zero: thereby backing into field production.

Field production of finished petroleum products is calculated by (1) adding the amount of fuel ethanol that has been blended into finished motor gasoline, and (2) plus $(+)$ or minus (-) the field production of motor gasoline blending components. Refer to Explanatory Note 11 for a further discussion of this calculation.

Negative field production of motor gasoline blending components represents an understatement for finished motor gasoline.

Negative field production of other finished motor gasoline represents an overstatement of other finished motor gasoline and an understatement of oxygenated motor gasoline.

Refinery Production - Published production of these products equal refinery production minus refinery input. Refinery production of other hydrocarbons, hydrogen and alcohol, unfinished oils, and motor and aviation gasoline blending components appear on a net basis under refinery input. Negative refinery production will occur when the amount of a product produced during the month is less than the amount of that same product that is reprocessed (input) or reclassified to become another product during the same month.

Unaccounted for Crude Oil - This column is a balancing item for crude oil. This data element represents the difference between crude oil supply and disposition. Crude oil supply is the sum of field production and imports. Crude oil disposition is the sum of stock change, losses, refinery inputs, exports, and products supplied. A positive result indicates that refiners and exporters reported use of more crude oil than was reported to have been available to them. (This occurs, for example, when imports are undercounted due to late reporting or other problems.) A nega- 
tive result indicates that more crude oil was reported to have been supplied to refiners and exporters than they reported to have used.

\section{Disposition}

Stock Change - This column is calculated as the difference between the Ending Stocks column of this table and the Ending Stocks column of the prior year's publication. A negative number indicates a decrease in stocks and a positive number indicates an increase in stocks.

Crude Losses - The volume of crude oil reported by petroleum refineries as being lost in their operations. These losses are due to spills, contamination, fires, etc., as opposed to refining processing losses or gains.

Refinery Inputs - Refinery inputs of crude oil and intermediate materials (unfinished oils, gasoline blending components, other hydrocarbons and oxygenates, liquefied petroleum gases, and pentanes plus) that are processed at refineries to produce finished petroleum products.

Crude oil inputs represents total crude oil (domestic and foreign) input to atmospheric crude oil distiliation units and other refinery processing units (i.e., catalytic cracking units, cokers).

Inputs of natural gas liquids are natural gas liquids received from natural gas plants for blending and processing. Published inputs of natural gas liquids are reported on a gross basis.

Inputs of unfinished oils, motor and aviation gasoline blending components, and other hydrocarbons and oxygenates are published on a net basis (i.e., refinery input minus refinery production).

Inputs of finished petroleum products are published on a net basis (i.e., refinery production minus refinery inputs) and displayed under the refinery production column.

Exports - Exports include crude oil shipments from the 50 States to Puerto Rico, and the Virgin Islands.

Products Supplied - Products supplied is equal to field production, plus refinery production, plus imports. plus unaccounted for crude oil, (plus net receipts on a PAD District basis), minus stock change, minus crude losses, minus refinery inputs, minus exports.

Products supplied indicates those quantities of petroleum products supplied for domestic consumption. Occasionally, the result for a product is negative because total disposition of the product exceeds total supply. Negative products supplied may occur for a number of reasons: (1) product reclassification has not been reported; (2) data were misreported or reported late; (3) in the case of calculations on a PAD District basis, the figure for net receipts was inaccurate because the coverage of interdistrict movements was incomplete; and (4) products such as gasoline blending components and unfinished oils have entered the primary supply channels with their production not having been reported, e.g., streams returned to refineries from petrochemical plants.

Product supplied for crude oil is the sum of crude oil burned on leases and by pipelines as fuel. Prior to January 1983, crude oil burned on leases and by pipelines as fuel were reported as either distillate or residual fuel oil and were included in product supplied for these products.

\section{Molds}

The refinery yield of finished motor gasoline is calculated by subtracting the inputs of pentanes plus, liquefied petroleum gases, other hydrocarbons/alcohol and motor gasoline blending components from the production of finished motor gasoline before dividing by the sum of crude oil input and unfinished oils input (net).

The refinery yield of finished aviation gasoline is calculated by subtracting the inputs of aviation gasoline blending components from the production of finished aviation gasoline before dividing by the sum of crude oil input and unfinished oils input (net).

Refinery yields for all products (except finished motor gasoline and finished aviation gasoline) are calculated by dividing the production for each product by the sum of crude oil input and unfinished oils input (net) reported in the U.S. total.

\section{Stocks}

Primary stocks of petroleum products do not include either secondary stocks held by dealers and jobbers or tertiary stocks held by consumers.

\section{Movements}

Movements of crude oil by pipeline between PAD Districts include trunk pipeline companies (interstate, intrastate, and intracompany pipelines). Intermediate movements for crude oil pipeline systems operating in more than two PAD Districts are not included.

Movements of petroleum products by pipeline between PAD Districts include trunk pipeline companies (interstate, intrastate and intracompany pipelines). Intermediate movements for product pipeline systems operating in more than two PAD Districts are included. For example, a shipment originating in PAD District 3, 
passing through PAD District 2 to PAD District 1, is reported as a movement from PAD District 3 to PAD District 2 and also from PAD District 2 to PAD District 1.

Waterborne movements of crude oil and petroleum products between PAD Districts include all shipments of crude oil or petroleum products for which the transporter has custody at the time of shipment. Custody is defined as physical possession of crude oil or petroleum products on a company-owned tanker and barge.

\section{Note 6. Domestic Crude Oll Production}

The Energy Information Administration (EIA) collects monthly crude oil production data on an ongoing basis. Data on crude oil production for States are reported to the EIA by State government agencies. Data on crude oil production for Federal offshore areas are reported to the EIA by the Minerals Management Service of the U.S. Department of the Interior and the Conservation Committee of California Oil and Gas Producers. Currently, all except four crude oil producing States (Michigan, New York, Ohio, and Pennsylvania) report production on a monthly basis. These four States report crude oil production on an annual basis. Estimates of monthly crude oil production for these four States are made by the EIA using data reported on Form EIA-182, "Domestic Crude Oil First Purchase Report."

After the end of each calendar year, the monthly crude oil production estimates are updated using annual reports from various State agencies, the Mineral Management Service, and the Conservation Committee of California Oil and Gas Producers. The EIA incorporates production data into its Crude Oil Production System (COPS) as the data are received from the reporting agencies. EIA publications show portions of this database at specific points in time. Table 14 of this publication presents the 1993 crude oil production data received by the EIA as of April 1994. Crude oil production data for 1993 received after April 1994 will be published later as an appendix in the following year's Petroleum Supply Annual (PSA). Table C1 of this publication presents the 1992 crude oil production a year after it was published in the PSA 1992.

\section{Note 7. Export Data}

Each month the Energy Information Administration (EIA) receives magnetic tapes of aggregated export statistics from the U.S. Bureau of the Census (EM-522 and EM594).

Census export statistics used in the Petroleum Supply Annual reflect both government and nongovernment exports of domestic and foreign merchandise from the
United States (the 50 States and the District of Columbia) to foreign countries and U.S. possessions, without regard to whether or not the exportation involves a commercial transaction. The following types of transactions are excluded from the statistics:

(1) Merchandise shipped in transit through the United States from one foreign country to another, when documented as such with U.S. Customs.

(2) Bunker fuels and other supplies and equipment for use on departing vessels, planes, or other carriers engaged in foreign trade.

\section{Source of Export information}

The official U.S. export statistics are compiled by the U.S. Bureau of the Census. Exporters are required to file export documents with U.S. Customs officials (Customs Form 7525).

\section{Country and Area of Destination}

The country of destination is defined as the country of ultimate destination or the country where the goods are to be consumed, further processed, or manufactured, as known to the shipper at the time of exportation. If the shipper does not know the country of ultimate destination, the shipment is credited to the last country to which the shipper knows that the merchandise will be shipped in the same form as it was when exported.

\section{Note 8. Quality Control and Data Revision}

\section{Quality Control}

The Energy Information Administration (EIA) monitors the supply and disposition of crude oil, petroleum products, and natural gas liquids in the United States. Through a tracking system, the EIA provides insight into the activities of primary operators and distributors in the petroleum industry. The tracking system, known as the Petroleum Supply Reporting System (PSRS), consists of production, inputs, imports, inventories, movements, and other petroleum-related data collected on weekly, month$l y$, and annual surveys.

Survey forms are periodically reviewed for completeness, meaningfulness, and clarity. Modifications are made, when needed, to maintain efficient measure of the intended data items and to track product movement accurately throughout the industry. Through this process, the EIA can maintain consistency among forms, minimize respondent burden, and eliminate ambiguity. 


\section{Sampling and Nonaampling Errora}

There are two types of errors usually associated with data produced from a survey -- nonsampling errors and sampling errors. Because the estimates for the monthly surveys 810 through 813,816 , and 817 are based on a complete census of the frame, there is no sampling error in the data presented. The data, however, are subject to nonsampling errors. Nonsampling errors, sometimes referred to as biases, are those which can arise from a number of sources: (1) the inability to obtain data from all companies in the frame or sample (nonresponse and the method used to account for nonresponses, (2) definitional difficulties and/or improperly worded questions which lead to different interpretations. (3) mistakes in recording or coding the data obtained from respondents, and (4) other errors of collection, response, coverage, and estimation.

Response rates on the monthly surveys are very high. In general, response rates average above 95 percent for the weekly survey and above 98 percent for monthly surveys. Whenever survey responses are not received in time to be included in published statistics, the data are imputed. Although imputing for missing data may not eliminate the total error associated with nonresponse, it can serve to reduce the error. The data reported in the previous month are used as imputed values for missing data for all surveys except the Forms EIA-814, "Monthly Imports Report," and EIA-817, "Monthly Tanker and Barge Movement Report." There is no imputation procedure for these surveys because these data series, by respondent, are highly variable.

Response error is the major factor affecting the accuracy of PSRS data. Response, or reporting error, is the difference between the true value and the value reported on a survey form. Response error can occur for any number of reasons. For example, figures may be entered incorrectly when written on forms by the respondent, or errors may result from the misunderstanding of survey form instructions or definitions. Response error can also occur from the use of preliminary data when final data are not available. This can result in differences between published preliminary and final data. To help detect and minimize probable reporting errors, automated editing procedures are used to check current data for consistency with past data, as well as for internal consistency (e.g., totals equal to the sums of the parts), and to flag those data elements that fail edit criteria.

Errors can also be introduced during data processing. For example, while creating computer data files, key errors can occur in transcribing or coding the data; or information can be entered into the wrong cell. Using well designed edit criteria which examine orders of magnitude, cell position, and historical reporting patterns, many of these errors can be identified and corrected.

Monthly data are compared to weekly data on a regular basis. Discrepancies betweenly weekly and monthly data are documented and respondents are called when discrepancies are either large (usually over 300 thousand barrels) or consistent (e.g., weekly data are always lower than monthly data). In addition, a comparison of the data collected on the PSRS with other similar data series from sources outside of the Petroleum Supply Division is performed each year. The results of this data comparison are published once a year in the Petroleum Supply Monthly (PSM) feature article, "Comparison of Independent Statistics on Petroleum Supply."

Sampling errors are those errors that occur when survey estimates are based on a sample rather than being derived from a complete census of the frame. The $819 \mathrm{M}$ data, which are based on sample estimates, serve as leading indicators of the PSRS monthly data for oxygenates. To assess the accuracy of the $819 \mathrm{M}$ statistics, data are compared with the monthly aggregate data for the EIA-810, 811 , and 812 surveys. Although monthly data are still subject to error, they have been thoroughly reviewed and edited, and are considered to be the most accurate data available.

\section{Data Rovision}

Resubmissions are any changes to the originally submitted data that were either requested by the EIA or initiated by the respondent. Resubmissions are compared with the original submission and processed at the time of receipt. For Forms EIA-810 through 813, 816, and 817 the Resubmission Tracking System (RTS) is run after resubmissions have been processed for the month. The RTS enables the user to study major products and data series to see how company resubmissions impact published data on a month by month basis. During the processing year, a summary of the effect of these resubmissions to major series is provided in Appendix C of the PSM.

For the EIA-819M data, a determination is made on whether to process the resubmissions based on the magnitude of the revision. Cell entries on publication tables are marked with an "R" for revised.

\section{Lato Response}

Respondents who fail to respond within the prescribed time limit (25th day following the end of the report month) become nonrespondents for that particular report period and are contacted by phone to obtain the current month's data. Respondents who are chronically late (i.e., 3 consecutive months) are notified by EIA either by letter or telephone. 
Follow-up action is taken when a company fails to respond adequately to data requests from the EIA. Preliminary attempts to gather delinquent reports are made by phone. Noncompliance form letters are sent to those companies that have not submitted reports and have not responded to data requests by phone.

\section{Note 9. Frames Maintenance}

The Petroleum Supply Division (PSD) maintains complete lists of respondents to its monthly surveys. Each survey has a list of companies and facilities required to submit petroloum activity data. This list is known as the survey frame. Frame maintenance procedures are used to monitor the status of petroleum companies and facilities currently contained in each survey frame as well as to identify new members to be added to the frame. As a result, all known petroleum supply organizations falling within the definition of "Who Must Submit" participate in the survey.

The activities for frames maintenance are conducted on a monthly and annual basis. Monthly frames maintenance procedures focus on examining several frequently published industry periodicals that report changes in status (births, deaths, sales, and acquisitions) of petroleum facilities producing, transporting, importing, and/or storing crude oil and petroleum products. These sources are augmented by articles in newspapers, letters from respondents indicating changes in status, and information received from survey systems operated by other offices. Survey managers review these sources to monitor changes in company operations and to develop lists of potential respondents. These activities assure coverage of the reporting universe and maintain accurate facility information on addresses and ownership.

Annual frames maintenance focuses on re-evaluating the "must submit" companies filing the Form EIA-814 and reviewing the sample frame for the Form EIA-819M, "Monthly Oxygenate Telephone Report."

To supplement the monthly and annual frames maintenance activities and to provide more comprehensive coverage, the PSD periodically conducts a comprehensive frames investigation. These investigations result in the reassessment and recompilation of the complete frame for each survey. The effort also impact of potential frame changes on the historical time serves data published from these respondents. The results of this frame study are usually implemented in January to provide a full year under the same frame.
Beginning in January 1981, the Energy Information Administration (EIA) expanded its universe to include nonrefinery blenders; redefined motor gasoline into two categories (finished leaded and finished unleaded); and separated blending components from finished motor gasoline as a reporting category. Refer to Explanatory Note 12 for future discussion.

In January 1981, 1983, and 1984 numerous respondents were added to bulk terminal and pipeline surveys affecting subsequent stocks reported and stock change calculations. Table B1 displays the end-of-year stocks, in million barrels using the expanded coverage (new basis).

Tablo B1. Now Basls Stocks' (Million Barrols)

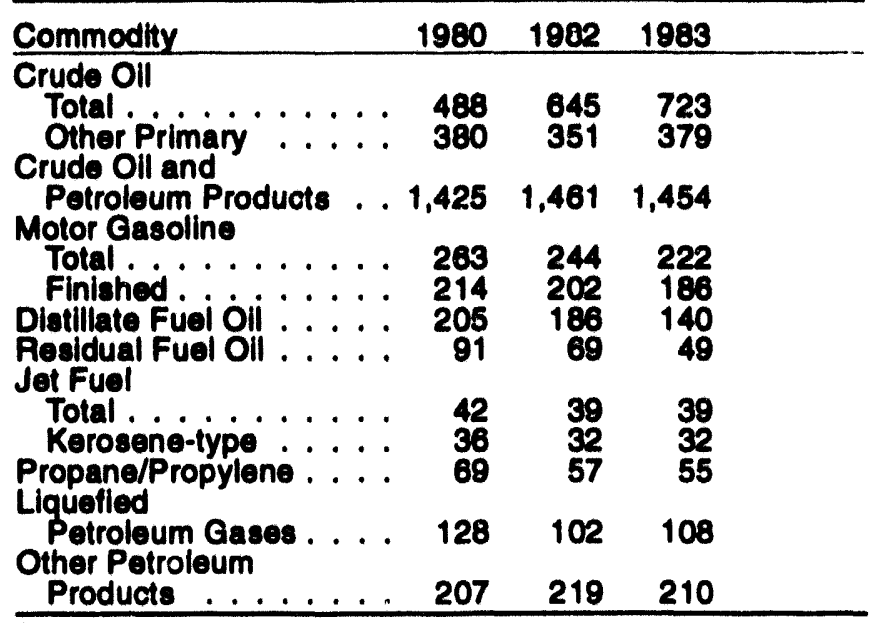

'stocke as of December 31.

Beginning in January 1986, as a result of frames maintenance activities, 39 respondents were added to the monthly survey frames: $\mathbf{2}$ motor gasoline blenders, 30 bulk terminal operators, 3 pipeline operators, 3 crude oil stock holders, and 1 tanker and barge operator. Table B2 shows the impact of the data reported by the new respondents on published data for production and stocks of major petroleum products.

Also, beginning in January 1986, a major petroleum company consolidated production and stocks reporting for some of its facilities. Data previously reported separately on Form EIA-811, "Monthly Bulk Terminal Report," and on Form EIA-816, "Monthly Natural Gas Liquids Report" for two facilities were combined with data reported for two refineries on Form EIA-810, "Monthly Refinery Report." The primary impact of this reporting change is on Table 24, "Stocks of Crude Oil and Petroleum Products by PAD District," which showed a decrease in natural gas 


\begin{tabular}{|c|c|c|c|c|}
\hline \multirow[b]{2}{*}{ Product } & \multicolumn{2}{|c|}{$\frac{\text { Refinery Production }}{\text { (thousand barrele per day) }}$} & \multicolumn{2}{|c|}{$\frac{\text { Stocks" }}{\text { (thouseandbarrols) }}$} \\
\hline & $\begin{array}{c}\text { Reported } \\
\text { by Now } \\
\text { Respondente } \\
\end{array}$ & $\begin{array}{l}\text { Publlahed } \\
\text { U.S. Total }\end{array}$ & $\begin{array}{c}\text { Reported } \\
\text { by Now } \\
\text { Respondents }\end{array}$ & $\begin{array}{l}\text { Published } \\
\text { U.S. Total } \\
\end{array}$ \\
\hline $\begin{array}{l}\text { Leaded Gasoline } \\
\text { Unleaded Gasoline } \\
\text { Distillate Fuel OII } \\
\text { Realdual Fuel OII } \\
\text { NGLs \& LRGs } \\
\text { Other Producte } \\
\text { Crude OII (excl. SPR) }\end{array}$ & $\begin{array}{r}1.3 \\
0.6 \\
0 \\
0 \\
0 \\
0 \\
-\end{array}$ & $\begin{array}{r}2,326 \\
4,323 \\
3,174 \\
1,055 \\
393 \\
3,302 \\
-\end{array}$ & $\begin{array}{r}224 \\
276 \\
1,217 \\
1,747 \\
409 \\
1,413 \\
2,314\end{array}$ & $\begin{array}{r}81,379 \\
108,422 \\
143,911 \\
50,671 \\
80,898 \\
239,158 \\
318,695\end{array}$ \\
\hline
\end{tabular}

- Stocks as of December 31, 1995.

liquids (NGL) stocks at bulk terminals and natural gas processing plants, and an increase in NGL stocks at refineries.

\section{Note 10. Descriptive Monthly Statistics}

The universe of each of the Petroleum Supply surveys (refinery, bulk terminal, pipeline, crude oil stock, import, etc.) is relatively small and ever-changing due to company births, deaths, mergers and splits. The frequency distributions of the petroleum supply variables are non-normal, highly variable, positive skewed and leptokurtic; that is, there are many small units and few large ones. Zeros often dominate the responses; that is, not all of the sampling units produce and/or store all products.

The statistics described in Table B3 were calculated from the monthly surveys over a 12-month period and display the following petroleum supply variables:

(1) The number of active sampling units (respondents).

(2) The number of sampling units reporting nonzero values (nonzero respondents).

(3) The average of nonzero values reported in thousand barrels (average).

(4) The standard deviation of nonzero values reported in thousand barrels (standard deviation).

\section{Note 11. Practlcal Limitations of Data Collectlon Efforts}

\section{Cruds OII Lease Stock Adjustment}

End-of-month crude oil stocks held on leases are reported on the EIA-813, "Monthly Crude Oil Report." However. only those companies that store 1,000 barrels or more of crude oil are required to submit a report. Previous frames analysis has shown that crude oil stocks held on leases reported to the EIA are consistently lower than the lease stocks reported to individual states.

Up until 1983, monthly state government data on lease stocks were substituted for ElA data wherever possible in order to rectify the understatement of lease crude oil stocks. State data were available from three states -Texas, New Mexico, and Montana. To calculate the "lease adjustment," a comparison between EIA reported data and the state government data was made and the difference added to the EIA data for the respective states.

In 1983, the EIA modified the Form EIA-813 to eliminate state data on crude oil stocks and began collecting crude oil stock data by Petroleum Administration for Defense (PAD) District. With this change, the "lease adjustment" could no longer be calculated on a state basis and was changed to a PAD District level.

\section{Trans Alaskan Pipeline System Adjustment}

Beginning with the January 1989 data, adjustments are made to refinery inputs and product supplied of natural gas liquids (NGLs) and refinery inputs of crude oil to account for refiner misreporting. Substantial volumes of 
Tabio B3. Descriptive 8tatiaties for Selectod Potroleum Supply Voriables

Petinary Crese Input to Atmoesphorie Cruds Oll Dietlliation Uniti

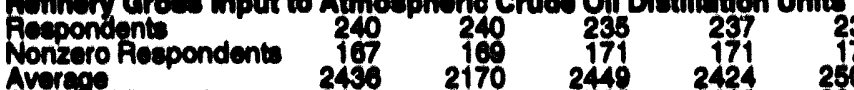

Btandifd Doviation 2681

$\begin{array}{ll}2449 & 2424 \\ 2726 & 2613\end{array}$ 174
2505
2600 Jun Jul Aug

Sop

Oot

Nov

Deo

Polinery Crude Oll input

Nonzoro Respondents 17

Averece Respondents 17

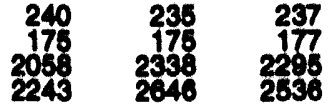

237
240

238
174
2485

238
174
2576
2783

238
173
2516

235
171
2464
2618

237
171
2580
2681

23

289

2814

288
168
2628
2735

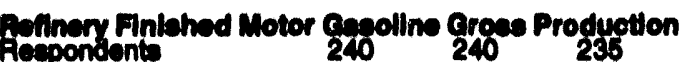

Nonzero Reapondent 240

$172 \quad 168 \quad 168$

1316

$\begin{array}{ll}1197 & 1313 \\ 1292 & 1377\end{array}$

237
161
1332

236
180
2434
2663

238

238

235
179
2320

237
175
2430

234

17517

2590

2633

25582690

Folinary Dietlliws Ful OU Croses Production

$\begin{array}{lll} & \text { Clloes Production } \\ 240 & 240 & 235 \\ 165 & 165 & 164 \\ 568 & 495 & 571 \\ 591 & 522 & 643\end{array}$

237

$\begin{array}{r}238 \\ 161 \quad 238 \\ \hline\end{array}$

1379

238
100
1414

235
165
1360

237
169
$139 \%$
1408

$\begin{array}{rr}234 & 235 \\ 1304 & 1445 \\ 1510 & 1500\end{array}$

Nonzero Reepondent

Standifd Doviation

501 520

$\begin{array}{ll}237 & 237 \\ 167 & 167 \\ 566 & 571 \\ 615 & 595\end{array}$

$\begin{array}{ll}238 & 238 \\ 170 & 168 \\ 561 & 600\end{array}$

238
168
612

236
160
605
603

237
167
680

$\begin{array}{ll}234 & 238 \\ 168 & 16 \\ 653 & 600\end{array}$

Aofinery Roddual Full Oll Croses Production

Recoondent

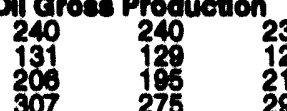

235
128
212
298

237
127
221
314

237
130
227
327

238
128
200
260

238
127
199
276

238
124
204
281

235
126
207
299

237
127
214
280

234
126
224
329

238
127
219
318

Rofinery Finibhed Motor Cesolino stocke

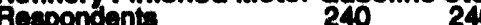

Averege

240

235
170
346

237

171
325
378

$237 \quad 238$

170
317

236

293

238

279

235

309

237
166
317
362

234
165
335
378

235

350

Eulk Terminal Finished Motor Cesoline stocks

$\begin{array}{llll}\text { Reepondents } & 342 & 341 & 339 \\ \text { Nonzero Respondents } & 152 & 149 & 150 \\ & 565 & 563 & 514\end{array}$

335

335
151
522
1070

330

527

330
151
403

493

331
149

485

331

479

391
148
486

333
140
515

332
148
516

Plpelline Finiahed Motor Cgeolline 8tgeke

Reepondent:

$88 \quad 88$

86
56
94

86
58
95
2050

86
55
885

86
56
915
2016

85
56
946

85
55
881
1849

85
55
898
1991

85
56
911
2195

85
54
945
2145

85
54
943
2143

Refinery Dlatilats Fuel Oll stocke

Rofinepondents
Nonzero Reepondents 240
Avereae
205

240
193
182
239

235237

$192 \quad 191$

237
190
181

$\begin{array}{ll}238 \\ 102 & 236 \\ 191 & \end{array}$

192
184

191
218
285

238
190

190
217
307

189

189
220
327

237

192

234
255

235

Bulk Torminal Dietillato Fupl Oll stocks

$\begin{array}{lll}\text { Respondents } & 342 & 341 \\ \text { Nonzero Respondents } 223 & 223\end{array}$

$\begin{array}{lll}\text { Average } & 225 & 233 \\ \text { Standard Deviation } & \mathbf{7 2 7} & 534\end{array}$

$\begin{array}{ll}339 & 335 \\ 223 & 221\end{array}$

187

335

219

330
221
234

330

219

258
607

331

283

331

302

331

327

223

329

220

300

Pipelline Distlilate Fuel Oll Stocke

Respondents

Nonzero Respondents

Average

$\begin{array}{rr}86 & 86 \\ 57 & 57 \\ 449 & 409 \\ 1341 & 1055\end{array}$

$\begin{array}{rr}86 & 86 \\ 57 & 56 \\ 432 & 412 \\ 1217 & 1028\end{array}$

86
56
378

86
55
418
1006

85
54
415
1120

85
53
461
1214

85
56
429
1113

85
55
490
1236

$\begin{array}{r}85 \\ 56 \\ 482 \\ \hline 204\end{array}$

85
56
499

Rofinery Recldual Fuel Oll Stocks

Aespondents 240 240

Nonzoro Respondents 149

Averege

Standard Doviation

$\begin{array}{ll}128 & 133 \\ 207 & 207\end{array}$

$235 \quad 237$

123

172

237

144
134
188

238

143

236
142

142
133
202

236
142
134

235

140

237

139

$\begin{array}{ll}234 & 235 \\ 139 & 138\end{array}$

143128

Bulk Torminal Pealdual Fupl Oll Stocks

Respondents

Nonzero Respondents

Average

$\begin{array}{rr}34 & 341 \\ 65 & 64 \\ 379 & 347 \\ 706 & 658\end{array}$

$339 \quad 335$

$\begin{array}{rr}339 & 335 \\ 63 & 65 \\ 350 & 339\end{array}$

$335 \quad 330$

330
65
395
851

330
65
358
736

331
64
398
797

331

331

67
387

33
613
86

332

767

$\begin{array}{ll}238 & 236 \\ 176 & 176\end{array}$

236
176
572
661

236

176
539

235

532

237

174

557

234

566

566
646

172

$\begin{array}{lll}\text { Average } & \\ \text { Standard Doviation } & \mathbf{7 6 7} & 556\end{array}$

Pipoline/Tank Farm Cruds Oll stocks

Respondents

Aospondents

Average

Standard Deviation

3011

162
122
1657

161

162
121
1732
17372

163
124
1727
3436

162
122
1725
3270

162
123
1762
3509

163
124
1663

163
124
160

163
124
1623

163
124
165

1653

163
124
1689
3456

3176 
NGLs are produced at natural gas processing plants in Alaska and injected into the crude oil moving in the Trans Alaska Pipeline System (TAPS). Refiners receiving any crude oil commingled with NGLs are instructed to report the NGL portion of that stream separately from the crude oil portion. This has not been done for Alaskan crude oil because refiners are unable to identify these volumes for accounting purposes. As a result, the NGL production in Alaska has been credited directly toward product supplied and also toward product supplied from refinery production when the refiner processes the crude oil-NGL mixture. In addition, the reporting of the commingled stream as crude oil by the refiner has overstated crude oil inputs and resulted in an increase in unaccounted for crude oil equal to the volume of NGL in the crude oil.

To offset this reporting error, an adjustment is made to refinery input in all PAD Districts receiving Alaskan crude oil. The adjustment reduces the crude oil inputs and increases the NGL inputs by an equal amount. Each PAD District adjustment is a portion of the known Alaskan-
NGL production that is proportional to the PAD District's share of Alaskan crude oil received at all refineries in the United States. The greatest impact occurs in PAD District $\mathrm{V}$ for butane and pentanes plus.

The reporting problem which began in 1987 grew as injections on NGLs into the TAPS increased. Data for 1988 was revised in the Petroleum Supply Annual to account for the adjustment.

\section{Finished Motor Gasoline Product Supplied Adjustment}

Beginning with the reporting of January 1993 data, adjustments were made to the product supplied series for finished motor gasoline. It was recognized that motor gasoline statistics published by the EIA through 1992 were underreported because the reporting system was not collecting all fuel ethanol and motor gasoline blending components being blended downstream from the refinery. The EIA was able to quantify these volumes and make corrective adjustments for 1992 in 1993 (refer to Table B4).

Table B4. Finished Motor Gasoline Product Supplied Adjustment, 1992 and 1993 (Thousand Barrels per Day)

\begin{tabular}{|c|c|c|c|c|c|}
\hline Year/Month & $\begin{array}{l}\text { Product Supplied } \\
1992 \text { Basis }\end{array}$ & $\begin{array}{l}\text { Fuel Ethanol } \\
\text { Adjustment }\end{array}$ & $\begin{array}{l}\text { Motor Gasoline Blending } \\
\text { Component Adjustment }\end{array}$ & $\begin{array}{c}\text { Product Supplied } \\
1993 \text { Basis }\end{array}$ & Difference \\
\hline \multicolumn{6}{|l|}{1992} \\
\hline January & 6,869 & 68 & -8 & 6,929 & 60 \\
\hline February ...... & 6,963 & 68 & -6 & 7,025 & 62 \\
\hline March ........ & 7.137 & 62 & 59 & 7,258 & 121 \\
\hline April ......... & 7,238 & 68 & 49 & 7,355 & 117 \\
\hline May ......... & 7,328 & 55 & 36 & 7,419 & 91 \\
\hline June $\ldots \ldots \ldots$ & 7,460 & 64 & 11 & 7,535 & 75 \\
\hline July . . . . . . & 7,639 & 52 & 75 & 7,766 & 127 \\
\hline August $\ldots \ldots \ldots$ & 7,380 & 66 & 91 & 7,537 & 157 \\
\hline September ..... & 7,344 & 54 & 43 & 7,441 & 97 \\
\hline October ....... & 7,338 & 76 & -14 & 7,400 & 62 \\
\hline November . . . . . & 7,102 & 91 & 68 & 7,261 & 159 \\
\hline December ..... & 7,396 & 100 & 66 & 7,562 & 166 \\
\hline Average . . . . & 7,268 & 69 & 39 & 7,376 & 108 \\
\hline \multicolumn{6}{|l|}{1993} \\
\hline January . . . . . . & -. & 61 & -59 & 6,639 & -. \\
\hline February ..... & - & 67 & -61 & 7,112 & -- \\
\hline March ........ & -- & 70 & 15 & 7,389 & -- \\
\hline April ......... & - & 61 & -32 & 7,435 & -- \\
\hline May . . . . . . . & $\cdots$ & 58 & -3 & 7,585 & - \\
\hline June $\ldots \ldots \ldots$ &.- & 63 & -5 & 7,700 & -- \\
\hline July . . . . . . . . & - & 62 & -19 & 7,785 & -. \\
\hline August $\ldots \ldots$ & -- & 48 & 54 & 7,864 & -. \\
\hline September ..... & -- & 68 & 79 & 7,607 & -- \\
\hline October ....... & - & 69 & .72 & 7,382 & -- \\
\hline November ..... & -- & 84 & .72 & 7,533 & -- \\
\hline December ..... & -- & 81 & 48 & 7,661 & -- \\
\hline Average ..... & -- & 66 & -10 & 7,476 & $\because$ \\
\hline
\end{tabular}

Note: Totals may not equal sum of components due to independent rounding.

Source: - Fuel Ethanol Adjustment - 1992, Energy Information Administration (ElA), Petroleum Supply Monthly , Appendix D. • Motor Gasoline Blending Component Adjustment - 1992 and 1993, EIA, Petroleum Supply Annual, Volumes I and II. 


\section{Fuel Ethanol Adjustment}

Prior to 1993, an estimated 60 to 70 thousand barrels per day of fuel ethanol were added to motor gasoline to produce gasohol but were not included in the EIA finished motor gasoline production data. In 1992, the EIA attempted to collect these data from downstream fuel ethanol motor gasoline blenders but found that this effort was impractical and the results were inaccurate.

Beginning in January 1993, an estimate for the missing fuel ethanol blended into motor gasoline was calculated. This estimate was calculated as production (from the EIA819M, "Monthly Oxygenate Telephone Report"), plus imports (from the EIA-814, "Monthly Imports Report"), minus inputs at refineries (from the EIA-810, "Monthly Refinery Report"), plus or minus stock change (from the EIA-819M survey). This estimate for the amount of fuel ethanol blended into motor gasoline was added to Table 1 for Natural Gas Liquids Field Production (line 14) and in the Field Production column for finished motor gasoline in Tables 2 through 13 published in the PSA.

An estimate for the total amount of gasohol produced with the ethanol is given as 10 times the estimated fuel ethanol blended (this assumes a 10 percent ethanol blend). This amount is added to the column labeled field production of "oxygenated gasoline" and subtracted from the field production of "other" finished gasoline. The PAD District level detail was obtained by allocating the national level estimates according to the percent of gasohol sales from the U.S. Department of Transportation, Federal Highway Administration, Monthly Motor Fuel Reported by States, 1991.

\section{Motor Gasoline Blending Component Adjustment}

Prior to 1993, the EIA published a "product supplied" for motor gasoline blending components. Since these components are to be blended into finished motor gasoline, there is no actual demand for this intermediate product. The EIA corrected this series by including the quantity of "product supplied" for motor gasoline blending components with "other" finished motor gasoline. This change was accomplished in Tables 2 through 13 by adding product supplied for motor gasoline blending components to the column labeled field production of "other" motor gasoline, and subtracting it from the field production column for "motor gasoline blending components."

\section{Fuol Ethanol Stock Adjustment}

Total end-of-month stocks of fuel ethanol are underreported in the PSRS because of the inability to collect data from downstream fuel ethanol motor gasoline blenders. Total stocks of fuel ethanol are assumed to be those reported by ethanol producers on the Form EIA-
819M, "Monthly Oxygenate Telephone Report." The difference between the stocks reported on the EIA-819M and the stocks reported in the PSRS (from refiners, bulk terminal and pipeline operators) is added to the stocks shown for bulk terminals. If the stocks for the PSRS are higher than those reported on the EIA-819M, no adjustment is made.

\section{Note 12. 1981 Changes in the Petroleum Supply Reporting System}

Petroleum statistics contained in this report for all years through 1980 were developed using definitions, concepts, reporting procedures, and aggregation methods that are consistent with those developed by the U.S. Bureau of Mines. Research conducted by the Energy Information Administration (EIA) in 1979 and 1980 indicated that changes had occurred in the petroleum industry that were not being adequately reflected in EIA's reporting system.

The EIA reporting forms, definitions, and procedures were modified beginning in January 1981 to describe industry operations more accurately. Unfortunately, empirical information is not available to precisely measure the data shortcomings through 1980 . Estimates of the magnitudes of differences in the major data series are described below to form a basis for comparing 1979, 1980 , and 1981 data.

\section{Motor Gasoline}

Prior to 1979, the EIA product-supplied series for motor gasoline was consistently about 2 percent lower than the Federal Highway Administration (FHWA) gasoline sales data series, which is derived from State tax receipts. The difference increased to about 3 percent in 1979 and 1980. There were two primary causes for this growing difference. First, refinery operations, particularly the flows of unfinished oils and the redesignation of some finished products, were not being accurately described on the EIA survey forms. Second, a large amount of gasoline was being produced away from refineries at "downstream blending stations" to take advantage of provisions in regulations governing the amount of lead that could be added. These blending stations were not reporting gasoline production to the EIA until the data system was changed in January 1981.

Quantitative estimates of the magnitude of the difference in EIA's gasoline product supplied data in 1979 and 1980 have been made by the EIA and the American Petroleum Institute (API). Table B5 provides 1979 and 1980 data as published in the Petroleum Statement, Annual, as well as EIA and API estimates of "recast" motor gasoline product supplied. 
The EIA recast estimates were based upon preliminary monthly information in the Monthly Petroleum Statement. The ranges displayed in the EIA column reflect uncertainty in the estimates. Also shown are the FHWA motor gasoline sales statistics for those years.

Table 85. Finished Motor Gasoline Product Supplied (Thousand Barrels per Day)

\begin{tabular}{|c|c|c|c|c|}
\hline & $\begin{array}{c}\text { EIA } \\
\text { Reported }\end{array}$ & $\begin{array}{c}\text { APT } \\
\text { Recast }\end{array}$ & $\begin{array}{l}\text { EIA } \\
\text { Recast }\end{array}$ & FHWA \\
\hline $\begin{array}{l}1979 \\
1980\end{array}$ & $\begin{array}{l}7,034 \\
6,579\end{array}$ & $\begin{array}{l}7,302 \\
6,882\end{array}$ & $\begin{array}{l}7,183-7,347 \\
6,806-6,889\end{array}$ & $\begin{array}{l}7,258 \\
6,792\end{array}$ \\
\hline
\end{tabular}

FHWA gasoline statistics based on data from Federal Highway Administration, Estimate of Total Gasoline Use, Table MF-21A published October 1980 and September 1981. Aviation gasoline (Table MF-24) has been subtracted from FHWA product supplied quantities to make data comparable.

\section{Distillate and Residual Fuel OII}

Distillate and residual fuel oil refinery production statistics through 1980 were adjusted to account for an imbalance between unfinished oil supply and disposition. The reported quantities of refinery inputs of unfinished oils typically exceed the available supply of unfinished oils. It has been assumed that this occurs when distillate and residual fuel oils produced by a refinery are shipped to another refinery, where it is treated as unfinished oil. This oil is then reprocessed rather than used or sold as distillate or residual fuel oil.

For many years (including 1980), the difference between unfinished oil disposition and supply was subtracted from distillate and residual fuel oil production to adjust for this discrepancy. Two-thirds of the difference was applied to distillate fuel oil, and one-third to residual fuel oil.

Table B6. Distillate and Residual Fuel OII Production and Product Supplied (Thousand Barrels per Day)

\begin{tabular}{|c|c|c|c|c|}
\hline & $\begin{array}{c}\text { Adjusted } \\
\text { Refinery } \\
\text { Production }\end{array}$ & $\begin{array}{l}\text { Unadjusted } \\
\text { Refinery } \\
\text { Production }\end{array}$ & Difference & $\begin{array}{l}\text { Unadjusted } \\
\text { Product } \\
\text { Supplied }\end{array}$ \\
\hline \multicolumn{5}{|l|}{$\begin{array}{l}\text { Distillate } \\
\text { Fuel Oil }\end{array}$} \\
\hline $1979 \ldots \ldots$ & 3,152 & 3,169 & 16 & 3,327 \\
\hline $1980 \ldots \ldots$ & 2,661 & 2,764 & 103 & 2,969 \\
\hline \multicolumn{5}{|l|}{$\begin{array}{l}\text { Residual } \\
\text { Fuel Oil }\end{array}$} \\
\hline $1979 \ldots \ldots$ & 1,687 & 1,695 & 8 & 2,834 \\
\hline $1980 \ldots \ldots$ & 1,580 & 1,634 & 54 & 2,562 \\
\hline
\end{tabular}

Beginning in January 1981, this adjustment was discontinued because there was not sufficient empirical evidence to support it. Table B6 presents distillate and residual fuel oil refinery production in 1979 and 1980 as published (adjusted) and on the same basis as 1981 statistics (unadjusted) to permit comparison.

Adjusted distillate and residual fuel oil product supplied volumes differ from the unadjusted volumes by the same amounts as the adjusted and unadjusted production volumes.

\section{Total Potroleum Products}

The imbalance between the supply and disposition of unfinished oils and gasoline blending components is included with other products (line 35 ) in Table 1 . These imbalances are reported as negative product supplied in Table 2 . Since these changes only involve redistribution of the volumes of finished motor gasoline, distillate and residual fuel oil, gasoline blending components, and unfinished oils, the total volume of petroleum products supplied remains unaffected by them.

\section{Alaskan In Tranait Stocks}

Stocks of Alaskan crude oil in-transit were included for the first time in January 1981. The major impact of this change is on the reporting of stock change calculations. Using the expanded coverage (new basis), 1980 end-ofyear crude oil stocks would have been $\mathbf{4 8 8}$ million barrels (Total) and 380 million barrels (Other Primary).

\section{Note 13. 1983 Changes in the Petroleum Supply Reporting System}

January 1983 marked the implementation of recent changes in the collection, processing and availability of the Energy Information Administration's (EIA) petroleum supply data. Survey forms and definitions were made consistent; frames for bulk terminals, petroleum product pipelines and crude oil stock holders were updated, and the survey processing system was redesigned and incorporated into the new Petroleum Supply Reporting System (PSRS).

\section{Changes in Data Collection}

Changes in data collection can be grouped into five categories. Some were made to improve consistency, others to classify activity more precisely, and others to combine or eliminate information elements or to reduce the frequency of reporting in recognition of the trade-off between data value and reporting burden. The changes are itemized below. 
- Motor gasoline was divided into three standard categories (finished leaded motor gasoline, finished unleaded motor gasoline and motor gasoline blending components).

- Aviation gasoline blending components were added to Form EIA-817.

- Crude oil burned as fuel on leases and by pipelines is reported as a single item on Form EIA-813. Previously it was reported as distillate or residual fuel oil consumption.

- Number 4 Fuel Oil is now included with distillate fuel oil.

- Gasohol was eliminated as a separate category and is now reported as either "finished leaded motor gasoline" or "finished unleaded motor gasoline."

- Waterborne movements of petrochemical feedstocks are now divided into naphtha-less than 401 degrees end-point and other-oils equal to or greater than 401 degrees end-point on Form EIA-817.

- Data aggregation for Petroleum Administration for Defense District (PADD) I was divided into three subdistricts on Forms EIA-812 and 817.

- Detailed categories of Gross Input to Crude Oil Distillation Units were eliminated, and only Total Gross Inputs are collected on Form EIA-810.

- Waterborne movements of crude oil and petroleum products between PADDs, on Form EIA-817, no longer reflect shipping and receiving States.

- Reporting of production and stocks of Number 4 Fuel Oil by sulfur levels were eliminated from Forms EIA$810,811,812$, and 817 .

- Crude oil stocks are collected at PADD levels rather than State levels on Form EIA-813.

- Shipments from natural gas processing plants no longer reflect destination by facility type on Form EIA-816.

- The four categories for unfinished oils were reduced to two on Form EIA-810.

- The five categories for sulfur content of residual fuel oil were reduced to three on Forms EIA-810, 811, and 817.

- Normal Butane and Other Butanes were combined into a single category on Forms EIA-810, 811, and 816.
- Three subcategories of lubricating oils (bright stock, neutral, and other) were combined into a single category on the Form EIA-810.

- Three subcategories of waxes (microcrystalline, crystalline-fully refined, and crystalline-other) were combined into a single category on the Form EIA-810.

- Asphalt and Road Oil were combined into a single category on Forms EIA-810 and 811.

- Plant fuel use and Losses were combined on Form EIA-816.

- Natural Gasoline and Isopentane were combined on Form EIA-816.

\section{Change in Crude Oil Lease Stocks}

The end-of-month crude oil stocks held on leases are reported on the Form EIA-813, "Monthly Crude Oil Report." However, only those companies that store 1,000 barrels or more of crude oil are required to submit a report. Previous frames analysis has shown that crude oil stocks held on leases reported to the Energy Information Administration (EIA) are consistently lower than the lease stocks reported to individual states.

Up until 1983, monthly state government data on lease stocks were substituted for EIA data wherever possible in order to rectify the understatement of lease crude oil stocks. State data were available from three states -Texas, New Mexico, and Montana. To calculate the "lease adjustment", a comparison between the EIA reported data and the state government data was made and the difference added to the EIA data for respective states.

In 1983, the EIA modified the Form EIA-813 to eliminate state data on crude oil stocks and began collecting crude oil stock data by PAD District. With this change, the "lease adjustment" could no longer be calculated on a state basis and was changed to a PAD District level.

\section{Note 14. 1984 Changes in the Petroleum Supply Reporting System}

iii Ieniuary 1984, a number of changes in the reporting of natural gas liquids (NGL) were implemented. The modified system reflects supply and disposition of NGL on a component, rather than a product, basis.

From 1979 to 1983, the Energy Information Administration (EIA) collected and reported information on the supply and disposition of nine NGL products. Beginning with January 1984, NGL supply and disposition data were 
reported for 5 components to be consistent with record keeping practices used by the industry. Table B7 shows the product category under the new and old basis. Four Petroleum Supply Reporting System surveys were modified beginning in January 1984 . They were:

$\begin{array}{ll}\text { EIA-810 } & \text { "Monthly Refinery Report" } \\ \text { EIA-811 } & \text { "Monthly Bulk Terminal Report" } \\ \text { EIA-812 } & \text { "Monthly Product Pipeline Report" } \\ \text { EIA-816 } & \text { "Monthly Natural Gas Liquids Report" }\end{array}$

Tablo B7. Product Basis vs. Component Basls Reporting

\begin{tabular}{|c|c|c|c|c|c|}
\hline \multirow[b]{2}{*}{$\begin{array}{l}1979-1983 \\
\text { Product Basis }\end{array}$} & \multicolumn{5}{|c|}{$\begin{array}{c}1984 \text { Component } \\
\text { Basis }\end{array}$} \\
\hline & $\begin{array}{l}\stackrel{\Phi}{\Phi} \\
\text { 总 }\end{array}$ & 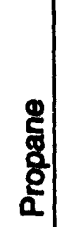 & 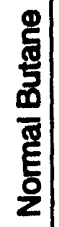 & 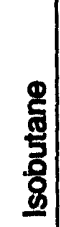 & 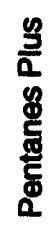 \\
\hline Ethane & $\bullet$ & & & & \\
\hline Ethane-Propane Mixtures & $\bullet$ & $\bullet$ & & & \\
\hline Propane & & $\bullet$ & & & \\
\hline Butane-Propane Mixtures & & $\bullet$ & - & & \\
\hline Butane & & & $\bullet$ & & \\
\hline Isobutane & & & & $\cdot$ & \\
\hline Unfractionated Stream & $\bullet$ & $\bullet$ & $\bullet$ & $\bullet$ & $\bullet$ \\
\hline Natural Gasoline and Isopentane & & & & & $\bullet$ \\
\hline Plant Condensate & & & & & $\cdot$ \\
\hline
\end{tabular}

This change affected stocks reported and stock change calculations. Under the new basis, end-of-year 1983 stocks would have been 108 million barrels (Liquefied Petroleum Gases) and 210 million barrels (Other Petroleum Products).

A fifth survey, Form EIA-814, "Monthly Imports Report" (formerly Form ERA-60), was not modified. Therefore, in order to allocate imports and exports of mixed NGL streams to individual component parts, the EIA developed a statistical algorithm.

\section{Imports}

The imports algorithm was based on information gathered from the larger importers of NGL, who were asked to provide component analysis of the products they imported during the first 6 months of 1983 . The percentages shown in Table B8 are derived from the weighted averages of the data provided by the importers.

\section{Exports}

The exports algorithm was based on information gathered from the larger exporters of NGL, who were asked to provide component analysis of the products they exported during 1983. The percentages shown in Table B8 are derived from the weighted averages of the data provided by the exporters. It was necessary to derive percentages by Petroleum Administration for Defense Districts of exportation, due to the wide variation of components included in the mixed streams.

\section{Note 15. 1985 Changes in the Petroleum Supply Reporting System}

Beginning in January 1985, inter-Petroleum Administration for Defense (PAD) District pipeline movements of crude oil were included in the crude oil supply balance at the PAD District level but did not affect National level statistics. As a result of including these movements, Net Receipts of crude oil and Unaccounted for Crude Oil at the PAD District level changed significantly. Also affected were crude oil imports and unfinished oil imports at the PAD District level which are provided by PAD District of Entry (Tables 4-8) and by PAD District of Processing (Table 14).

The tables in the Petroleum Supply Annual that were changed due to the inclusion of inter-PAD District pipeline movements of crude oil are listed below:

- Tables 4 through 8, "PAD Districts I to V, Supply and Disposition of Crude Oil and Petroleum Products."

- Effective January 1985, crude oil imports and unfinished oil imports in Tables 4 through 8 were reported at the PAD District of Entry rather than at the PAD District of Processing. Net Receipts now include movements by pipeline as well as by tanker and barge.

- Table 20, "Movements of Crude Oil and Petroleum Products by Pipeline, Tanker, and Barge Between PAD Districts."

- The crude oil line includes movements by pipeline as well as by tanker and barge.

- Table 21, "Movements of Crude Oil and Petroleum Products by Pipeline Between PAD Districts."

- A line was added to report crude oil movements. 


\begin{tabular}{|c|c|c|c|c|c|}
\hline \multirow[b]{2}{*}{ Product } & \multicolumn{5}{|c|}{ EIA Component Slate } \\
\hline & Ethane & Propane & Normal Butane & Isobutane & Pentanes Plus \\
\hline \multicolumn{6}{|l|}{ Import Product } \\
\hline Natural Gasoline and lsopentane (EIA-814) ... & - & - & - & - & 100 \\
\hline Plant Condensate (EIA-814) . . . . . . . . . . . & - & - & - & - & 100 \\
\hline Ethane $(\mathrm{IM}-145) \ldots \ldots \ldots \ldots \ldots \ldots \ldots$ & 100 & - & - & - & - \\
\hline Propane $(\mathrm{IM}-145) \ldots \ldots \ldots \ldots \ldots \ldots \ldots$ & - & 100 & - & - & - \\
\hline Butane $(I M-145) \ldots \ldots \ldots \ldots \ldots \ldots \ldots$ & - & - & 65 & 35 & - \\
\hline Butane-Propane Mixtures (IM-145) ........ & - & 40 & 35 & 20 & 5 \\
\hline Ethane-Propane Mixtures (IM-145) $\ldots \ldots \ldots$ & 60 & 40 & - & - & - \\
\hline \multicolumn{6}{|l|}{ Export Product } \\
\hline Ethane (All PAD Districts) & 100 & - & - & - & - \\
\hline Propane (All PAD Districts) $\ldots \ldots \ldots \ldots \ldots \ldots$ & - & 100 & - & - & - \\
\hline Butane (All PAD Districts) . . . . . . . . . & - & - & 100 & - & - \\
\hline 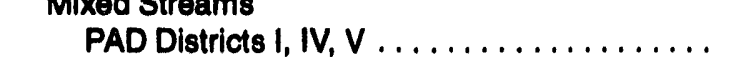 & - & 40 & 60 & - & - \\
\hline PAD Dlstrict $\| \ldots \ldots \ldots \ldots \ldots \ldots \ldots$ & 30 & 25 & 15 & 15 & 15 \\
\hline PAD Dlstrict III $\ldots \ldots \ldots \ldots \ldots \ldots \ldots$ & - & 80 & 20 & - & - \\
\hline
\end{tabular}

- Table 23, "Net Movements of Crude Oil and Petroleum Products by Pipeline, Tanker, and Barge Between PAD Districts."

- The crude oil line includes movements by pipeline as well as by tanker and barge.

\section{Note 16. 1986 Changes in the Petroleum Supply Reporting System}

Beginning in January 1986, several changes to the Petroleum Supply Reporting System (PSRS) went into effect. These changes affected the frame of operators of petroleum facilities required to complete the monthly surveys in the PSRS and resulted in some changes to the tables presented in the Petroleum Supply Monthly and were subsequently published in the Petroleum Supply Annual (PSA). Refer to Explanatory Note 9 for a detailed description of frames maintenance and updates.

\section{Changes in Data Collection}

- The unit of measure used on Form EIA-814, "Monthly Imports Report," has been changed from barrels to thousands of barrels.
- Unfinished oil imports data, previously reported as one product on the Form EIA-814, are now reported separately under four classifications. These classifications are:

- Naphthas and lighter

- Kerosene and light gas oils

- Heavy gas oils

- Residuum

- The number of categories for reporting natural gas liquids and liquefied petroleum gases data on Form EIA814 was reduced from 19 to 5 by eliminating the requirement to separately identify categories for further processing, petrochemical use, and fuel use.

- The requirements to report the type of processing facility and the applicable section of the oil import regulations were eliminated for the Form EIA-814.

- The requirement to report data for imports of crude oil, unfinished oils, and finished products on separate schedules of the Form EIA-814 was eliminated.

- The requirement to report two end-use categories, petrochemical use and other use, for still gas and liquefied refinery gases, was eliminated on Form EIA810, "Monthly Refinery Report." 
- Form EIA-815, "Monthly Shipments from Puerto Rico to the United States Report," was discontinued. The data previously reported on this form are now reported on Form-814.

\section{Changes in Publication Tables}

Several changes were also made to tables in the PSA either as a direct result of changes in reporting requirements or to improve the usefulness of the publication. These changes were:

- Table 11, "Refinery Input of Crude Oil and Petroleum Products by PAD District."

- Alaskan crude oil receipts were shown separately.

- Table 12, "Refinery Production of Petroleum Products by PAD District."

- The breakout between "petrochemical feedstock use" and "other use" were no longer shown separately for still gas or for liquefied refinery gases.

- Table 14, "Imports of Crude Oil and Petroleum Products by PAD District."

- Imports of anfinished oils were separated into four categories: naphthas and lighter, kerosene and light gas oils, heavy gas oils, and residuum.

- Table 15, "Imports of Crude Oil and Petroleum Products by Source."

- Countries formerly included in the categories "Other Western Hemisphere" and "Other Eastern Hemisphere" were shown individually.

- Table 18, "Stocks of Crude Oil and Petroleum Products by PAD District."

- The breakout between "petrochemical feedstock use" and "other use" for each liquefied petroleum gas was eliminated.

\section{Note 17. 1987 Changes in the Petroleum Supply Reporting System}

Several changes to the Petroleum Supply Reporting System went irto effect at the beginning of January 1987. These changes were made as part of the Energy Information Administration's (EIA's) continuing effort to provide pertinent, timely, and consistent energy information. These changes were subsequently reflected in the Petroleum Supply Annual(PSA).

\section{Changes in Data Collection}

Fresh feed input to catalytic cracking units, hydrocracking units, and cokers were added to the Form EIA-810, "Monthly Refinery Report."

\section{Changes in Publication Tables}

- The "Appalachian No. 2" Refining District was combined with the "Indiana, Illinois, Kentucky," .lefining District. This affected PSA Tables 10 through 13, 18, 24 , and 25.

- Fresh feed inputs to catalytic cracking units, hydrocracking units, and cokers were added to Table 11, "Refinery Input of Crude Oil and Petroleum Products by PAD District."

\section{Clarification}

In 1986, several refineries and terminals in the United States applied for Foreign Trade Zone (FTZ) status and applications from three refineries were approved. Consequently, during 1986, some refineries with FTZ status were treated as if they were within the United States while the Hawaiian FTZ was considered outside.

Effective with the January 1987 data, all FTZ facilities located within the 50 United States are considered domestic entities and are included in PSA statistics. The principal differences in the PSA data series as a result of adding the Hawaiian FTZ was an approximate 1 percent increase in crude imports and a 3 percent decrease in product imports.

\section{Note 18. 1989 Changes in the Petroleum Supply Reporting System}

Several changes to the Petroleum Supply Reporting System (PSRS) went into effect at the beginning of January 1989. These changes were made to reduce respondent burden, to fulfill user requests for additional data, and to improve accuracy and consistency in reporting. To reflect these changes and to improve the usefulness of the Petroleum Supply Monthly (PSM) publication, the following changes were made in January 1989 and are subsequently reflected in the Petroleum Supply Annual (PSA) publication.

\section{Changes in Data Collection}

- Data on inputs and production of naphthenic and paraffinic lubricants were added to the Form EIA-810, "Monthly Refinery Report." 
- Separate lines for the collection of inputs and production of olefins (ethylene, propylene, and butylene)were adcied to Form EIA-810, "Monthly Refinery Report."

- The collection of data on the movement of Liquefied Petroleum Gases (LPGs) and Liquefied Refinery Gases (LRGs) on a component basis were added to the Forms EIA-812, "Monthly Product Pipeline Report," and the EIA-817, "Monthly Tanker and Barge Movement Report."

- Bonded imports of jet fuel and fuel oils and imports of LPGs previously published from data provided by the U.S. Bureau of the Census were discontinued. Data are now published from the data reported on the Form EIA-814, "Monthly Imports Report."

- Exports of butane/propane and ethane/propane mixtures were split in a ratio of 60 percent for the butane and ethane portions and 40 percent for the propane portion.

- The reporting of products other than Natural Gas Liquids (NGLs) by natural gas processing plants was eliminated on the Form EIA-816, "Monthly Natural Gas Liquids Report."

- Fractionators were required to report only end-ofmonth stocks of NGLs on the Form EIA-816. "Monthly Natural Gas Liquids Report."

\section{Changes in Natural Gas Llquids and Crude Oll Statistics}

Beginning with the January 1989 issue of the PSM, adjustments were made to refinery inputs and product supplied of NGLs and refinery inputs of crude oil to account for refiner misreporting. Substantial volumes of NGLs are produced at natural gas processiris plants in Alaska and injected into the crude oil moving in the Trans Alaska Pipeline System (TAPS). Refiners receiving any crude oil commingled with NGLs are instructed to report the NGL portion of that stream separately from the crude oil portion. This has not been done for Alaskan crude oil because refiners are unable to identify these volumes for accounting purposes. As a result, the NGL production in Alaska has been credited directly toward product supplied and also toward product supplied from refinery production when the refiner processes the crude oil-NGL mixture. In addition, the reporting of the commingled stream as crude oil by the refiner has overstated crude oil inputs and resulted in an increase in unaccounted for crude oil equal to the volume of NGL in the crude oil.

To offset this reporting error, an adjustment was made to refinery input in all Petroleum Administration for Defense (PAD) Districts receiving Alaskan crude oil. The adjustment reduces the crude oil inputs and increases the NGL inputs by an equal amount. Each PAD District adjustment is a portion of the known Alaskan NGL production that is proportional to the PAD District's share of Alaskan crude oil received at all refineries in the United States. The greatest impact occurs in PAD District $V$ for butane and pentanes plus.

The reporting problem began in 1987 and has grown as injections of NGLs into the TAPS have increased. Data for 1988 was revised to account for the adjustment in the PSA. Revisions for 1987 data are not planned.

\section{Changes in Publication Tables}

- "Stock Withdrawal" was renamed "Stock Change" and was moved from Supply to Disposition in Tables 2 through 13. A negative number indicates a decrease in stocks and a positive number indicates an increase in stocks.

- A jet fuel total line was added to Tables $2-13,17,18$, $20,32-35$.

- PAD District Supply and Disposition tables (Tables 4 through 13) now display liquefied petroleum gases on a component basis.

- A table showing net imports by country for the current month (Table 29) was added.

- Table numbers were changed as a result of data additions and table reorganization. Table $B 9$ is provided to show the new to old table numbers for the detailed statistics tables.

- Table 15, "Natural Gas Plant Net Production and Stocks of Petroleum Products by PAD and Refining District."

- Stocks at natural gas processing plants by Refining District previously published on Table 10 was included with net production of petroleum products at natural gas plants.

- The reporting of products other than natural gas liquids by natural gas processing plants was eliminated.

- Table 17, "Net Refinery Production of Finished Petroleum Products by PAD and Refining District."

- Net production of olefins (ethylene, propylene, and butylene) was added.

- Net production of naphthenic and paraffinic lubricants was added. 


\begin{tabular}{|c|c|c|c|c|c|c|c|c|c|}
\hline \multicolumn{10}{|c|}{ Table Numbers } \\
\hline Old & Now & Old & Now & Old & Now & Old & Now & Old & Now \\
\hline $\begin{array}{c}1 \\
2 \\
3 \\
4 \\
N A \\
5 \\
N A \\
6\end{array}$ & $\begin{array}{l}1 \\
2 \\
3 \\
4 \\
5 \\
6 \\
7 \\
8\end{array}$ & $\begin{array}{c}\text { NA } \\
7 \\
\text { NA } \\
8 \\
\text { NA } \\
9 \\
10 \\
11\end{array}$ & $\begin{array}{c}9 \\
10 \\
11 \\
12 \\
13 \\
14 \\
15 \\
16\end{array}$ & $\begin{array}{c}12,24 \\
18,25 \\
13 \\
14,27 \\
15 \\
15 \\
15 \\
15\end{array}$ & $\begin{array}{l}17 \\
18 \\
19 \\
20 \\
21 \\
22 \\
23 \\
24\end{array}$ & $\begin{array}{c}15 \\
27 \\
16 \\
17 \\
N A \\
18,25 \\
19 \\
20\end{array}$ & $\begin{array}{l}25 \\
26 \\
27 \\
28 \\
29 \\
30 \\
31 \\
32\end{array}$ & $\begin{array}{c}21 \\
22,26 \\
23\end{array}$ & $\begin{array}{l}33 \\
34 \\
35\end{array}$ \\
\hline
\end{tabular}

NA = Not Applicable

- Net production of residual fuel oil by percent sulfur, previously published as Table 24, was added.

- Table 18, "Refinery Stocks of Crude Oil and Petroleum Products by PAD and Refining District."

- Stocks at refineries by Refining District were added from Table 18.

- Stocks of residual fuel oil by percent sulfur content, previously published as Table 25 , were added.

- Tables 21 through 25, "Imports of Crude Oil and Petroleum Products by Country of Origin."

- Data previously included in the "Other Products" category were displayed separately for naphthas for petrochemical feedstock use, other oils for petrochemical feedstock use, lubricants, and asphalt and road oil.

- Table 20,"Imports of Crude Oil and Petroleum Products by PAD District."

- Sulfur content categories for residual fuel oil, previously published as Table 27 , were added.

- Table 28, "Exports of Crude Oil and Petroleum Products by Destination."

- Data for exports by destination previously included in the Other Products category were displayed separately for pentanes plus, kerosene, naphthas for petrochemical feedstock use, and other oils for petrochemical feedstock use.

- Table 30, "Stocks of Crude Oil and Petroleum Products by PAD District."
- Refining District data were eliminated. Refinerv stocks and natural gas processing plant stocks by Refining District were added to Table 18.

- Sulfur content categories for residual fuel oil, previously published as Table 25 , were added.

\section{Note 19. 1990 Changes in the Petroleum Supply Reporting System}

Beginning with the May 1990 issue of the Petroleum Supply Monthly (PSM), stocks of propane/propylene were added to Table 42, "Refinery, Bulk Terminal, and Natural Gas Plant Stocks of Selected Petroleum Products by State." This change is also reflected in the corresponding table in the Petroleum Supply Annual (PSA).

Beginning with the 1991 March issue of the PSM, several changes were made to the Petroleum Supply Reporting System to provide additional data and to improve the usefulness of the publication. Although these changes were made in 1991, these changes have been incorporated into the 1990 PSA to provide consistent energy information.

\section{Changes in Publication Tables}

\section{Summary Statistics Tables}

- A new table (Table S7) has been added to display jet fuel supply and disposition.

- Table S8, "Other Petroleum Products Supply and Disposition" has been redesignated as Table S9. Jet fuel data are no longer included. Historical data have been revised to exclude jet fuel. 
- Table S3, "Crude Oil and Petroleum Product Imports" has been expanded to display all Organization of Petroleum Exporting Countries (OPEC) and additional Non-OPEC countries. A separate column for crude oil imports has also been added for each country.

- Time periods have been included in table titles.

\section{Flgures}

- Time periods have been included in figure titles.

- Sources have been provided for each figure.

- Bar graphs used to display end-of-month stocks have been replaced with line graphs.

\section{Sources}

The sources and explanatory notes for this section have been updated and are now located at the end of the Summary Statistics section.

\section{Dotailed Statistice Tables}

- Table 1, "U.S. Petroleum Balance"

- A line has been added to display jet fuel as a separate category for Total Products Supplied and Total Stocks (Lines 34 and 44, respectively).

- Imports of Crude Oil and Petroleum Products by PAD District

- Residual fuel oil sulfur categories have been added.

- Imports of Crude Oil and Petroleum Products by Country of Origin

- Residual fuel oil sulfur categories by country of origin have been eliminated. These categories are now reported on a PAD District basis.

- Separate daily average columns have been added for crude oil and petroleum products.

\section{Note 20. 1993 Changes in the Petroleum Supply Reporting System}

In keeping with the Department of Energy's (DOE's) mandated responsibilities, the Energy Information Administration (EIA) made several changes to the Petroleum Supply Reporting System (PSRS) effective in January 1993. These changes were designed to accommodate the revisions to the Clean Air Act of 1990, and to reflect current and upcoming changes in the petroleum industry. These changes are subsequently reflected in the 1993 Petroleum Supply Annual.

\section{Changes in Data Collection}

- Motor gasoline categories have been revised to reflect the change in the type of fuels produced. The new categories are: reformulated gasoline, oxygenated gasoline, and other finished gasoline. These changes were made to Forms EIA-810, "Monthly Refinery Report," EIA-811, "Monthly Bulk Terminal Report," EIA-812, "Monthly Product Pipeline Report," EIA814, "Monthly Imports Report," and EIA-817, "Monthly Tanker and Barge Movement Report."

- Distillate Fuel Oil has been split into two sulfur categories to meet Environmental Protection Agency requirements effective in October 1993. The new categories for inputs, production, end-of-month stocks and movements are: $0.05 \%$ sulfur and under, and greater than $0.05 \%$ sulfur. These changes were made to Forms EIA-810, "Monthly Refinery Report," EIA-811, "Monthly Bulk Terminal Report," EIA-812, "Monthly Product Pipeline Report," EIA-814, "Monthly Imports Report," and EIA-817, "Monthly Tanker and Barge Movement Report."

- Other hydrocarbons, hydrogen, and alcohol (Code 090) has been renamed "Other hydrocarbons, hydrogen, and oxygenates" on Form EIA-810, "Monthly Refinery Report." A new line has also been added to report Other hydrocarbons and hydrogen separately.

- Data on inputs and end-of-month stocks of oxygenates (i.e., fuel ethanol, ethyl tertiary butyl ether (ETBE), methanol, methyl tertiary butyl ether (MTBE), tertiary amyl methyl ether (TAME), tertiary butyl alcohol (TBA), and other oxygenates) has been added to Form EIA-810, "Monthly Refinery Report."

- Inputs and production of Isobutylene (Code 634) has been added as sub-categories to Isobutane (Code 615) on Form EIA-810, "Monthly Refinery Report."

- Data on inputs and production of military kerosenetype jet fuel and commercial kerosene-type jet fuel has been added to Form ElA-810, "Monthly Refinery Report."

- Liquefied Petroleum and Refinery Gases column headings for Ethane, Propane, Normal Butane, and Isobutane have been revised to include olefins (e.g., Ethane/Ethylene etc.) on Form EIA-811, "Monthly Bulk Terminal Report." 
- Data on end-of-month stocks of oxygenates (i.e., fuel othanol, ethyl tertiary butyl ether (ETBE), methyl tertiary butyl ether (MTBE), tertiary amyl methyl ether (TAME), tertiary butyl alcohol (TBA), and other oxygenates) have been added to Forms EIA-811, "Monthly Bulk Terminal Report," and EIA-812, "Monthly Product Pipeline Report." Data for methanol are not collected at this time but has been included on the form for future use.

- Imports of oxygenates (i.e., fuel ethanol, ethyl tertiary butyl ether (ETBE), methyl tertiary butyl ether (MTBE), tertiary amyl methyl ether (TAME), tertiary butyl alcohol (TBA), and other oxygenates) have been added to Form EIA-814, "Monthly Imports Report." Data for methanol are not requested at this time.

- Imports of olefins are collected separately from liquefied petroleum gases (i.e., ethylene, propylene, butylene, and isobutylene) on Form EIA-814, "Monthly Imports Report."

- Data on blended into motor gasoline has been eliminated on the Form EIA-819M, "Monthly Oxygenate Telephone Report."

- Data on methanol is no longer required on the Form EIA-819M, "Monthly Oxygenate Telephone Report" but remains on the form for future use.

\section{Changes in Summary Statistice Tables}

- Table S1. Crude and Petroleum Products Overview

- History data for 1973 through 1980 has been dropped. The table title has been changed to reflect the change in time series.

- Table S2. Crude Oil Supply and Disposition

- History data for 1973 through 1980 has been dropped. The table title has been changed to reflect the change in time series.

- The Crude Used Directly column has been eliminated. This column is no longer applicable since the years 1973 through 1980 have been eliminated. The data for 1981 and 1982 are provided in a footnote.

- Table S3. Crude Oil and Petroleum Product Imports

- History data for 1973 through 1980 has been dropped. The table title has been changed to reflect the change in time series.
- The Former USSR has been renamed Russia. The remaining states that comprised the Former USSR have been included in the Other Non-OPEC column.

- Table S4. Finished Motor Gasoline Supply and Disposition

- History data for 1973 through 1980 has been dropped. The table title has been changed to reflect the change in time series.

- Product supplied-unleaded and product supplied-unleaded (percent of Total) columns have been eliminated. A new column has been added to display end-of-month stocks of oxygenates. These stocks are not included in the Total Motor Gasoline endof-month stocks.

- Table S5. Distillate Fuel Oil Supply and Disposition

- History data for 1973 through 1980 has been dropped. The table title has been changed to reflect the change in time series.

- Distillate fuel oil stocks have been separated into two sulfur categories $(0.05 \%$ sulfur and under and greater than $0.05 \%$ sulfur).

- The Crude Used Directly column has been eliminated. This column is no longer applicable since the years 1973 through 1980 have been eliminated. The data for 1981 and 1982 are provided in a footnote.

- Table S6. Residual Fuel Oil Supply and Disposition

- History data for 1973 through 1980 has been dropped. The table title has been changed to reflect the change in time series.

- The Crude Used Directly column has been eliminated. This column is no longer applicable since the years 1973 through 1980 have been eliminated. The data for 1981 and 1982 are provided in a footnote.

- Table S7. Jet Fuel Supply and Disposition

- History data for 1973 through 1980 has been dropped. The table title has been changed to reflect the change in time series.

- Table S8. Propane/Propylene Supply and Disposition

- A new summary table has been addéd to display supply and disposition data for propane/propylene. 
This information will continue to be included in the Liquefied Petroleum Gases Supply and Disposition table (renumbered as Table S9).

- Table S9. Liquefied Petroleum Gases Supply and Disposition

- Formerly numbered as Table S8.

- History data for 1973 through 1980 has been dropped. The table title has been changed to reflect the change in time series.

- Table S10. Other Petroleum Products Supply and Disposition

- Formerly numbered as Table S9.

- History data for 1973 through 1980 has been dropped. The table title has been changed to reflect the change in time series.

\section{Changes in Detalled Statistics Tables}

- Table 1. U.S. Petroleum Balance

- Line 14 includes fuel ethanol blended into finished motor gasoline. This quantity is comparable to the sum of field production of finished motor gasoline and natural gas liquids and LRGs on Table 2.

- Line 20 has been modified to read: Other Liquids New Supply (Field Production) to accommodate motor gasoline blending components field production.

- Tables 2 through 13. Supply and Disposition

- Isobutane has been renamed Isobutane/Isobutylene under Liquefied Petroleum Gases for clarification.

- Other Hydrocarbons/Hydrogen/Alcohol has been renamed Qther Hydrocarbons/Hydrogen/ Oxygenates for clarification.

- Unleaded and leaded motor gasoline categories have been replaced with the new types of gasolines produced: reformulated, oxygenated, and other.

- Distillate fuel oil sulfur categories $(0.05 \%$ sulfur and under and greater than $0.05 \%$ sulfur) have been added.

- Table 16. Refinery Input

- Other Hydrocarbons/Hydrogen/Alcohol has been renamed Qther Hydrocarbons/Hydrogen/
Oxygenater for clarification. Sub-categories are displayed for Other_Hydrocarbons/Hydresen and for Oxygenates.

- Oxygenates are displayed separately for fuel ethanol, methanol, MTBE, and other oxygenates. Other oxygenates includes ethyl tertiary butyl ether (ETBE), tertiary amyl methyl alcohol (TAME), tertiary butyl alcohol (TBA), and other aliphatic alcohols and ethers intended for motor gasoline blending (e.g., isopropyl ether (IPE) or n-propanol).

\section{- Table 17. Refinery Net Production}

- Isobutane has been renamed Isobutane/lsobutylene under Liquefied Petroleum Gases for clarification. Isobutylene is displayed as a sub-category to be consistent with the other liquefied gases.

- Unleaded and leaded motor gasoline categories have been replaced with the new types of gasolines produced: reformulated, oxygenated, and other.

- Military and commercial kerosene-type jet fuel has been added.

- Distillate fuel oil sulfur categories $(0.05 \%$ sulfur and under and greate, than $0.05 \%$ sulfur) have been added.

- Table 18. Refinery Stocks

- Isobutane has been renamed Isobutane/Isobutylene under Liquefied Petroleum Gases for clarification.

- Other Hydrocarbons/Hydrogen/Alcohol has been renamed Other Hydrocarbons/Hydrogen/ exygenates for clarification. Sub-categories are displayed for Other Hydrocarbons/Hydrogen and for Oxygenates.

- Oxygenates are displayed separately for fuel ethanol, methanol, MTBE, and other oxygenates. Other oxygenates includes ethyl tertiary butyl ether (ETBE), tertiary amyl methyl alcohol (TAME), tertiary butyl alcohol (TBA), and other aliphatic alcohols and ethers intended for motor gasoline blending (e.g., isopropyl ether (IPE) or n-propanol).

- Unleaded and leaded motor gasoline categories have been replaced with the new types of gasolines produced: reformulated, oxygenated, and other.

- Distillate fuel oil sulfur categories $(0.05 \%$ sulfur and under and greater than $0.05 \%$ sulfur) have been added. 
- Table 20. Imports by PAD District

- Data on olefins are displayed separately from liquefied petroleum gases.

- Other Hydrocarbons/Hydrogen/Alcohol has been renamed Qther Hydrocarbons/Hydrosen/ Exygenates for clarification. Sub-categories are displayed for Other Hydrocarbons/Hydrogen and for Oxygenates.

- Oxygenates are displayed separately for fuel ethanol, MTBE, and other oxygenates. Other oxygenates includes ethyl tertiary butyl ether (ETBE), tertiary amyl methyl alcohol (TAME), tertiary butyl alcohol (TBA), and other aliphatic alcohols and ethers intended for motor gasoline blending (e.g., isopropyl ether (IPE) or n-propanol).

- Unleaded and leaded motor gasoline categories have been replaced with the new types of gasolines produced: reformulated, oxygenated, and other.

- Distillate fuel oil sulfur categories $(0.05 \%$ sulfur and under and greater than $0.05 \%$ sulfur) have been added to both bonded ship bunkers and other.

- Tables 21-25. Imports by Country of Origin

- A new line has been added to appear below the Total line to show the sum of the Persian Gulf countries.

- Former USSR has been changed to read Russia. States formerly included in USSR are now included in the Other countries category under Non-OPEC.

- Table 27. Exports

- Isobutane has been renamed Isobutane/lsobutylene under Liquefied Petroleum Gases for clarification.

- Other Hydrocarbons/Oxygenates and Motor Gasoline Blending Components have been added as export products under the Other Liquids category.

- Table 28. Exports by Destination

- Miscellaneous products category has been renamed Other Products to accommodate exports of other hydrocarbons/ oxygenates and motor gasoline blending components.

- Table 29. Net Imports

- A new line has been added to appear below the Total line to show the sum of the Persian Gulf countries.
- Former USSR has been changed to read Russia. States formerly included in USSR are now included in the Other countries category under Non-OPEC.

- Table 30. Stocks

- Other Hydrocarbons/Hydrogen/Alcohol has been renamed Other Hydrocarbons/Hydrogen/ exygenates for clarification. Sub-categories are displayed for Other hydrocarbons/hydrogen fuel ethanol, ETBE, methanol, MTBE, and other oxygenates.

- Other oxygenates includes tertiary amyl methyl alcohol (TAME), tertiary butyl alcohol (TBA), and other aliphatic alcohols and ethers intended for motor gasoline blending (e.g., isopropyl ether (IPE) or n-propanol.

- Unleaded and leaded motor gasoline categories have been replaced with the new types of gasolines produced: reformulated, oxygenated, and other.

- Distillate fuel oil sulfur categories $(0.05 \%$ sulfur and under and greater than $0.05 \%$ sulfur) have been added.

- Table 31. Refinery, Bulk Terminal, and Natural Gas Plant Stocks

- Unleaded and leaded motor gasoline categories have been replaced with the new types of gasolines produced: reformulated, oxygenated, and other.

- Distillate fuel oil sulfur categories $(0.05 \%$ sulfur and under and greater than $0.05 \%$ sulfur) have been added.

- Table 32. Movements by Pipeline, Tanker, and Barge

- Unleaded and leaded motor gasoline categories have been replaced with the new types of gasolines produced: reformulated, oxygenated, and other.

- Distillate fuel oil sulfur categories $(0.05 \%$ sulfur and under and greater than $0.05 \%$ sulfur) have been added.

- Table 33. Movements by Pipeline

- Unleaded and leaded motor gasoline categories have been replaced with the new types of gasolines produced: reformulated, oxygenated, and other.

- Distillate fuel oil sulfur categories $(0.05 \%$ sulfur and und or and greater than $0.05 \%$ sulfur) have been added. 
- Table 34. Movements by Tanker and Barge

- Unleaded and leaded motor gasoline categories have been replaced with the new types of gasolines produced: reformulated, oxygenated, and other.

- Distillate fuel oil sulfur categories $(0.05 \%$ sulfur and under and greater than $0.05 \%$ sulfur) have been added.

- Table 35. Net Movements

- Isobutane has been renamed Isobutane/lsobutylene under Liquefied Petroleum Gases for clarification.

- Unleaded and leaded motor gasoline categories have been replaced with the new types of gasolines produced: reformulated, oxygenated, and other.

- Distillate fuel oil sulfur categories $(0.05 \%$ sulfur and under and greater than $0.05 \%$ sulfur) have been added.

\section{Changes in Appendix C (PSM)}

- Inputs

- Other hydrocarbons has been renamed Other Hydrocarbons/ Oxygenates for clarification.

- Production

- Isobutane has been renamed Isobutane/lsobutylene under Liquefied Petroleum Gases for clarification.

- Unleaded and leaded motor gasoline categories have been replaced with the new types of gasolines produced: reformulated, oxygenated, and other.

- A new line has been added to display field production of motor gasoline blending components.

- Imports

- Isobutane has been renamed Isobutane/lsobutylene under Liquefied Petroleum Gases for clarification.

- Unleaded and leaded motor gasoline categories have been replaced with the new types of gasolines produced: reformulated, oxygenated, and other.

- Stocks

- Other hydrocarbons has been renamed Other Hydrucarbons/Oxygenates for clarification.
- Isobutane has been renamed Isobutane//sobutulene under Liquefied Petroleum Oases for clarification.

- Unleaded and leaded motor gasoline categories have been replaced with the new types of gasolines produced: reformulated, oxygenated, and other.

- Product Supplied

- Isobutane has been renamed Isobutane/lsobutylene under Liquefied Petroleum Gases for clarification.

- Unleaded and leaded motor gasoline categories have been replaced with the new types of gasolines produced: reformulated, oxygenated, and other.

\section{Changes in Appendix D}

- Table D1. U.S. Summary Table

- Data on blended into motor gasoline has been eliminated. This information is no longer collected on the survey EIA-819M, "Monthly Oxygenate Telephone Report."

- Table D2. Monthly Fuel Ethanol Production and Ending Stocks

- Data for the previous year as well as current year are displayed.

- Data on blended into motor gasoline has been eliminated. This information is no longer collected on the survey EIA-819M, "Monthly Oxygenate Telephone Report."

- Data for fuel ethanol imports has been dropped due to small volumes reported by respondents.

- Table D3. Monthly MTBE Production and Ending Stocks

- Data for the previous year as well as current year are displayed.

- Data on blended into motor gasoline has been eliminated. This information is no longer collected on the survey EIA-819M, "Monthly Oxygenate Telephone Report."

- Data on MTBE imports has been dropped from the table due to small volumes reported by respondents. 


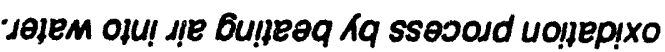

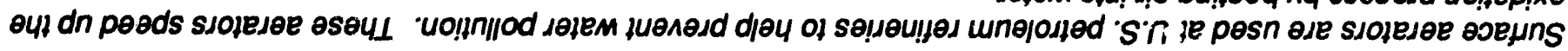

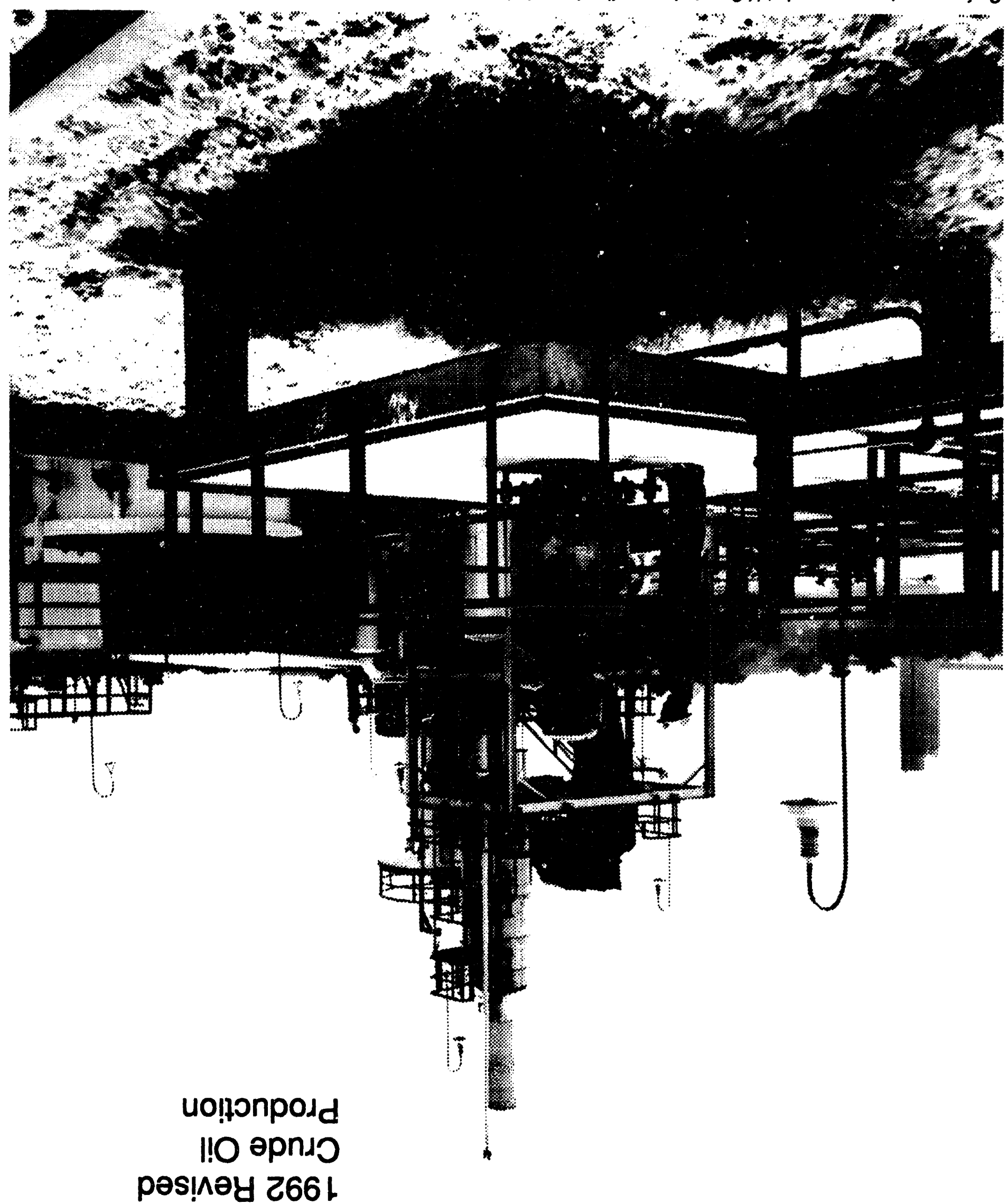

O x!puedd $\forall$ 
Table C1. Revised Crude Oll Production by PAD District and State, 1992 (Thousand Barrels)

\begin{tabular}{|c|c|c|c|c|c|c|c|}
\hline PAD District and State & January & February & March & April & May & June & July \\
\hline 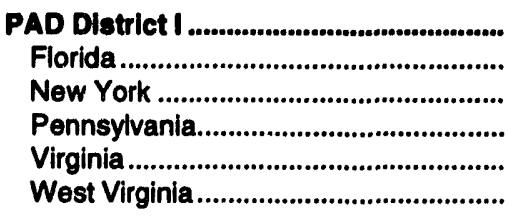 & $\begin{array}{r}881 \\
483 \\
33 \\
195 \\
1 \\
168\end{array}$ & $\begin{array}{r}783 \\
444 \\
21 \\
148 \\
1 \\
168\end{array}$ & $\begin{array}{r}835 \\
431 \\
34 \\
189 \\
1 \\
179\end{array}$ & $\begin{array}{r}844 \\
429 \\
33 \\
198 \\
1 \\
183\end{array}$ & $\begin{array}{r}828 \\
454 \\
34 \\
180 \\
1 \\
158\end{array}$ & $\begin{array}{r}859 \\
457 \\
37 \\
203 \\
1 \\
160\end{array}$ & $\begin{array}{r}826 \\
407 \\
38 \\
194 \\
1 \\
185\end{array}$ \\
\hline 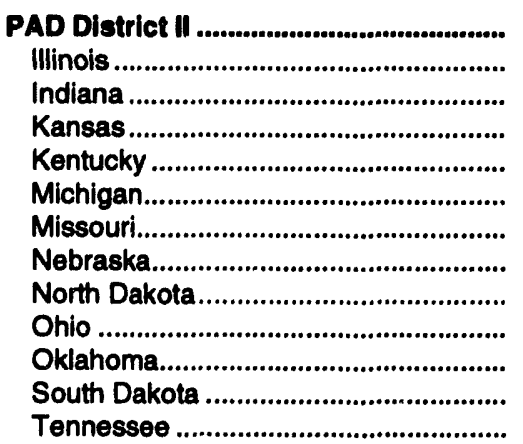 & $\begin{array}{r}21,642 \\
1,604 \\
250 \\
4,844 \\
446 \\
1,408 \\
11 \\
487 \\
2,879 \\
770 \\
8,761 \\
138 \\
44\end{array}$ & $\begin{array}{r}20,154 \\
1,527 \\
234 \\
4,322 \\
432 \\
1,321 \\
13 \\
454 \\
2,707 \\
737 \\
8,233 \\
128 \\
45\end{array}$ & $\begin{array}{r}21,563 \\
1,618 \\
265 \\
4,671 \\
346 \\
1,365 \\
13 \\
478 \\
2,862 \\
805 \\
8,968 \\
131 \\
41\end{array}$ & $\begin{array}{r}21,218 \\
1,592 \\
248 \\
4,592 \\
567 \\
1,300 \\
13 \\
466 \\
2,745 \\
813 \\
8,708 \\
127 \\
49\end{array}$ & $\begin{array}{r}20,875 \\
1,592 \\
241 \\
4,514 \\
438 \\
1,344 \\
12 \\
476 \\
2,807 \\
748 \\
8,524 \\
132 \\
46\end{array}$ & $\begin{array}{r}20,578 \\
1,619 \\
261 \\
4,462 \\
443 \\
1,291 \\
13 \\
450 \\
2,685 \\
787 \\
8,392 \\
130 \\
47\end{array}$ & $\begin{array}{r}21,022 \\
1,652 \\
253 \\
4,536 \\
459 \\
1,342 \\
12 \\
458 \\
2,755 \\
757 \\
8,631 \\
129 \\
38\end{array}$ \\
\hline 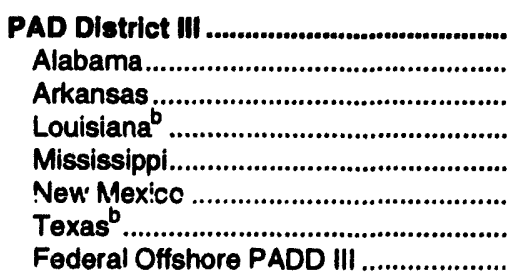 & $\begin{array}{r}106,316 \\
1,691 \\
920 \\
12,445 \\
2,192 \\
6,002 \\
56,323 \\
26,744\end{array}$ & $\begin{array}{r}99,307 \\
1,586 \\
854 \\
11,644 \\
2,021 \\
5,757 \\
52,552 \\
24,892\end{array}$ & $\begin{array}{r}105,965 \\
1,678 \\
899 \\
12,339 \\
2,112 \\
6,124 \\
56,220 \\
26,593\end{array}$ & $\begin{array}{r}101,609 \\
1,648 \\
870 \\
11,926 \\
2,072 \\
5,924 \\
54,054 \\
25,114\end{array}$ & $\begin{array}{r}103,969 \\
1,607 \\
858 \\
12,225 \\
2,152 \\
6,017 \\
54,953 \\
26,157\end{array}$ & $\begin{array}{r}100,235 \\
1,587 \\
846 \\
11,858 \\
2,118 \\
5,826 \\
52,661 \\
25,339\end{array}$ & $\begin{array}{r}103,639 \\
1,634 \\
868 \\
12,254 \\
2,153 \\
5,931 \\
54,505 \\
26,294\end{array}$ \\
\hline 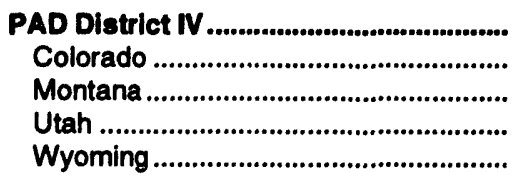 & $\begin{array}{r}14,797 \\
2,581 \\
1,599 \\
2,121 \\
8,495\end{array}$ & $\begin{array}{r}13,833 \\
2,472 \\
1,487 \\
1,996 \\
7,877\end{array}$ & $\begin{array}{r}14,675 \\
2,607 \\
1,603 \\
2,119 \\
8,346\end{array}$ & $\begin{array}{r}14,194 \\
2,629 \\
1,541 \\
1,979 \\
8,044\end{array}$ & $\begin{array}{r}14,589 \\
2,634 \\
1,583 \\
2,066 \\
8,306\end{array}$ & $\begin{array}{r}13,959 \\
2,538 \\
1,505 \\
1,973 \\
7,943\end{array}$ & $\begin{array}{r}14,391 \\
2,550 \\
1,545 \\
2,037 \\
8,259\end{array}$ \\
\hline 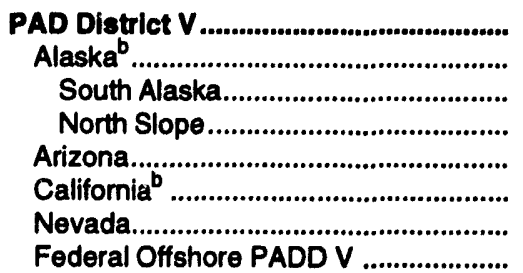 & $\begin{array}{r}85,142 \\
55,457 \\
1,322 \\
54,135 \\
9 \\
26,293 \\
250 \\
3,132\end{array}$ & $\begin{array}{r}80,418 \\
52,428 \\
1,237 \\
51,191 \\
8 \\
24,476 \\
238 \\
3,269\end{array}$ & $\begin{array}{r}85,091 \\
55,345 \\
1,335 \\
54,010 \\
8 \\
8 \\
26,170 \\
252 \\
3,317\end{array}$ & $\begin{array}{r}81,279 \\
52,233 \\
1,256 \\
50,976 \\
8 \\
8 \\
25,271 \\
271 \\
3,496\end{array}$ & $\begin{array}{r}82,281 \\
52,152 \\
1,212 \\
50,940 \\
8 \\
26,143 \\
316 \\
3,661\end{array}$ & $\begin{array}{r}79,785 \\
51,094 \\
1,251 \\
49,843 \\
8 \\
25,172 \\
305 \\
3,205\end{array}$ & $\begin{array}{r}81,627 \\
51,291 \\
1,242 \\
50,049 \\
8 \\
25,932 \\
325 \\
4,071\end{array}$ \\
\hline 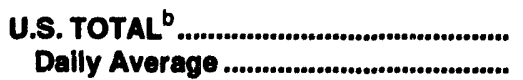 & $\begin{array}{r}228,778 \\
7,380\end{array}$ & $\begin{array}{r}214,494 \\
7,396\end{array}$ & $\begin{array}{r}228,129 \\
7,359\end{array}$ & $\begin{array}{r}219,144 \\
7,305\end{array}$ & $\begin{array}{r}222,542 \\
7,179\end{array}$ & $\begin{array}{r}215,416 \\
7,181\end{array}$ & $\begin{array}{r}221,505 \\
7,145\end{array}$ \\
\hline
\end{tabular}

This table contains updates on 1992 crude oil production statistics published in the Petroleum Supply Annual (PSA), 1992.

Statistics on crude oil production for States and for Federal offshore areas are reported to the Energy Information Administration (EIA) by State government agencie? and by the Minerals Management Service, U.S. Department of the interior. These data are updated periodically by the reporting agencies and are received by the EIA on an ongoing basis. At the time of publication of the 1992 PSA, the EIA had not received complete and/or updated statistics on crude oil production for several States. This table is provided to inform the user of updated monthly and annual crude oil production statistics for 1892 , and are not subject to further revision by the EIA. 
Table C1. Revised" Crude OII Production by PAD District and State, 1992 (Continued) (Thousand Barrels)

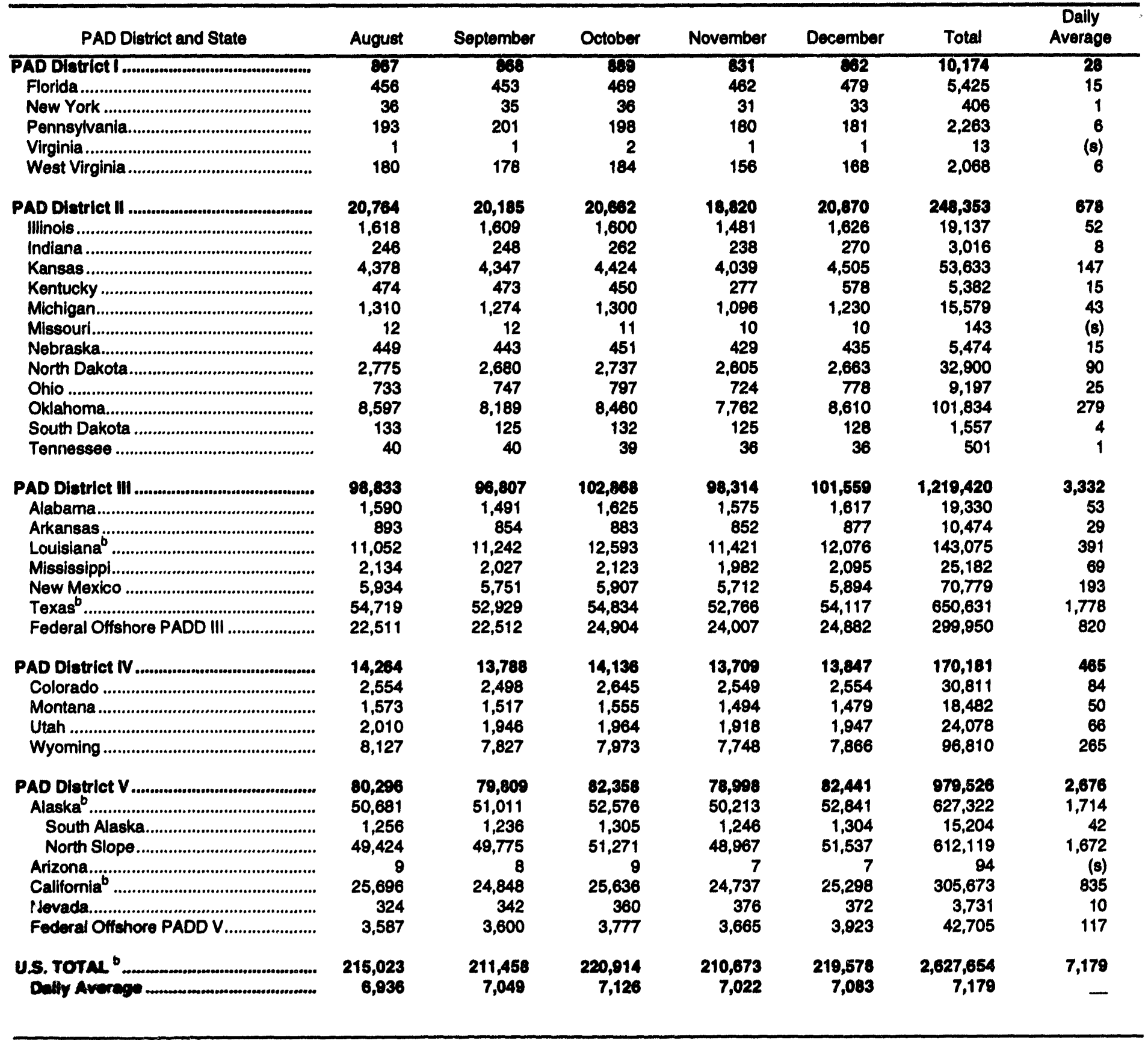

- Data are based upon revtions received as of April 1994.

b Includes the following ofthore production (thousand barrels): Alaska: State - 54,863; Calfornia: State - 21,944; Louisiana: State - 23,490; Texas: State - 2,138; U.S. Total, including Federal Offahore - 445,090.

$(8)=$ Less than 500 barrels por day.

Note: - Production data are revieed from those published in the Petroloum Supply Annual 1992 for the following States: Alabama, Arkansas, Callfornia, Colorado, Federal Offshore PAD District III, Federal Offshore PAD District V, Illinois, Kansas, Kentucky, Missouri, Montana, Nevada, New Mexico, New York, North Dakota, Oklahoma, Pennsyivania, Tennessee, Texas, Utah, and Virginia. - Annual crude oll production for Michigan, New York, Ohio, and Pennsylvania was prorated by month based on first purchaser monthly crude oil volumes collected on Form ElA-182, "Domestic Crude Oil First Purchase Report." - Totals may not equal sum of components due to independent rounding.

Source: State Conservation agencles, U.S. Department of the Interior, Minerals Management Service and the Conservation Committee of California Oil and Gas Producers. 


\section{Glossary}

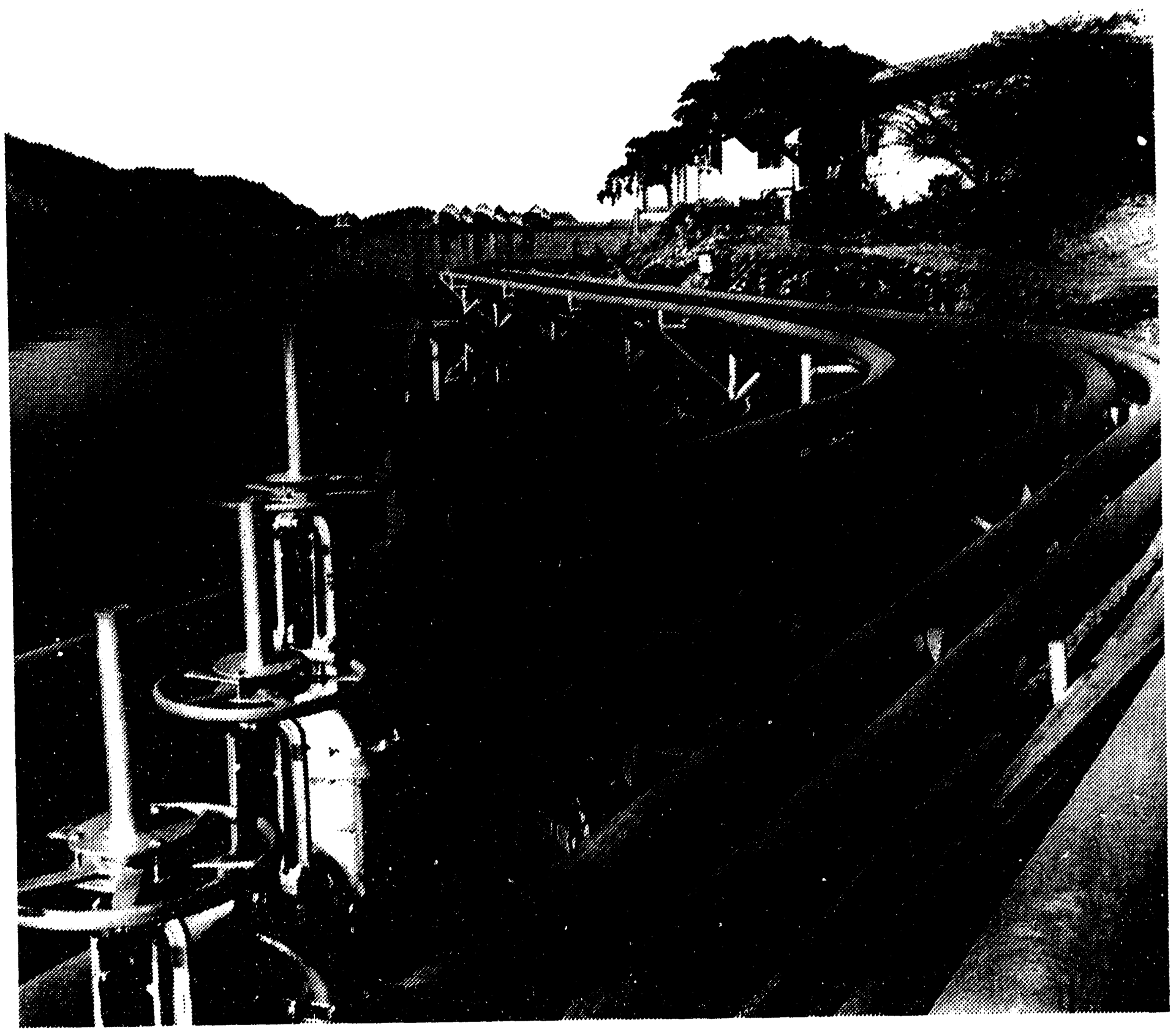

Pipelines carry natural gas across geographic regions. 


\section{Definitions of Petroleum Products and Other Terms}

Alcohol. The family name of a group of organic chemical compounds composed of carbon, hydrogen, and oxygen. The series of molecules vary in chain length and are composed of a hydrocarbon plus a hydroxyl group; $\mathrm{CH}_{3}-\left(\mathrm{CH}_{2}\right)$ n-OH (e.g., methanol, ethanol, and tertiary butyl alcohol).

Alkylate. The product of an alkylation reaction. It usually refers to the high octane product from alkylation units. This alkylate is used in blending high octane gasoline.

Alkylation. A refining process for chemically combining isobutane with olefin hydrocarbons (e.g., propylene, butylene) through the control of temperature and pressure in the presence of an acid catalyst, usually sulfuric acid or hydrofluoric acid. The product, alkylate, an isoparaffin, has high octane value and is blended with motor and aviation gasoline to improve the antiknock value of the fuel.

API Gravity. An arbitrary scale expressing the gravity or density of liquid petroleum products. The measuring scale is calibrated in terms of degrees API; it is calculated as follows:

$$
\text { Degrees API }=\frac{141.5}{s p .8 r .60^{\circ} \mathrm{F} / 60^{\circ} \mathrm{F}}-131.5
$$

Aromatics. Hydrocarbons characterized by unsaturated ring structures of carbon atoms. Commercial petroleum aromatics are benzene, toluene, and xylene (BTX).

Asphalt. A dark-brown-to-black cement-like material containing bitumens as the predominant constituent obtained by petroleum processing. The definition includes crude asphalt as well as the following finished products: cements, fluxes, the asphalt content of emulsions (exclusive of water), and petroleum distillates blended with asphalt to make cutback asphalts. The conversion factor for asphalt is $\mathbf{5 . 5}$ barrels per short ton.

ASTM. The acronym for the American Society for Testing and Materials.

Atmospheric Crude Oil Distillation. The refining process of separating crude oil components at atmospheric pressure by heating to temperatures of about $600^{\circ}$ to $750^{\circ} \mathrm{F}$ (depending on the nature of the crude oil and desired products) and subsequent condensing of the fractions by cooling.
Aviation Gasoline (Finished). All special grades of gasoline for use in aviation reciprocating engines, as given in ASTM Specification D910 and Military Specification MIL-G-5572. Excludes blending components which will be used in blending or compounding into finished aviation gasoline.

Aviation Gasoline Blending Components. Naphthas which will be used for blending or compounding into finished aviation gasoline (e.g., straight-run gasoline, alkylate, reformate, benzene, toluene, and xylene). Excludes oxygenates (alcohols, ethers), butane, and pentanes plus. Oxygenates are reported as other hydrocarbons, hydrogen, and oxygenates.

Barrel. A volumetric unit of measure for crude oil and petroleum products equivalent to 42 U.S. gallons. This measure is used in most statistical reports. Factors for converting petroleum coke, asphalt, still gas and wax to barrels are given in the definitions of these products.

Barrels Per Calendar Day. The maximum number of barrels of input that can be processed during a 24-hour period after making allowances for the following limitations:

the capability of downstream facilities to absorb the output of crude oil processing facilities of a given refinery. No reduction is made when a planned distribution of intermediate streams through other than downstream facilities is part of a refinery's normal operation;

the types and grades of inputs to be processed;

the types and grades of products expected to be manufactured;

the environmental constraints associated with refinery operations;

the reduction of capacity for scheduled downtime such as routine inspection, mechanical problems, maintenance, repairs, and turnaround; and

the reduction of capacity for unscheduled downtime such as mechanical problems, repairs, and slowdowns.

Barrels Per Stream Day. The amount a unit can process running at full capacity under optimal cıude oil and product slate conditions. 
Benzene $\left(\mathrm{C}_{6} \mathrm{H}_{6}\right)$. An aromatic hydrocarbon present in small proportion in some crude oils and made commercially from petroleum by the catalytic reforming of naphthenes in petroleum naphtha. Also made from coal in the manufacture of coke. Used as a solvent, in manufacturing detergents, synthetic fibers, and petrochemicals and as a component of high-octane gasoline.

Blending Components. See Motor or Aviation Gasoline Blending Components.

Blending Plant. A facility which has no refining capability but is either capable of producing finished motor gasoline through mechanical blending or blends oxygenates with motor gasoline.

Bonded Petroleum Imports. Petroleum imported and entered into Customs bonded storage. These imports are not included in the import statistics until they are: (1) withdrawn from storage free of duty for use as fuel for vessels and aircraft engaged in international trade; or (2) withdrawn from storage with duty paid for domestic use.

BTX. The acronym for the commercial petroleum aromatics benzene, toluene, and xylene. See individual categories for definitions.

Bulk Station. A facilitv used primarily for the storage and/or marketing of petroleum products which has a total bulk storage capacity of less than 50,000 barrels and receives its petroleum products by tank car or truck.

Bulk Terminal. A facility used primarily for the storage and/or marketing of petroleum products which has a total bulk storage capacity of 50,000 barrels or more and/or receives petroleum products by tanker, barge, or pipeline.

Butane $\left(\mathrm{C}_{4} \mathrm{H}_{10}\right)$. A normally gaseous straight-chain or branch-chain hydrocarbon extracted from natural gas or refinery gas streams. It includes isobutane and normal butane and is designated in ASTM Specification D1835 and Gas Processors Association Specifications for commercial butane.

Isobutane (CAH10). A normally gaseous branch-chain hydrocarbon. It is a colorless paraffinic gas that boils at a temperature of $10.9^{\circ} \mathrm{F}$. It is extracted from natural gas or refinery gas streams.

Normal Butane (CAH10). A normally gaseous straight-chain hydrocarbon. It is a colorless paraffinic gas that boils at a temperature of $31.1^{\circ} \mathrm{F}$. It is extracted from natural gas or refinery gas streams.

Butylene $\left(\mathrm{C}_{4} \mathrm{H}_{8}\right)$. An olefinic hydrocarbon recovered from refinery processes.
Captive Refinery Oxygenate Plants. Oxygenate production facilities located within or adjacent to a refinery complex.

Catalytic Cracking. The refining process of breaking down the larger, heavier, and more complex hydrocarbon molecules into simpler and lighter molecules. Catalytic cracking is accomplished by the use of a catalytic agent and is an effective process for increasing the yield of gasoline from crude oil. Catalytic cracking processes fresh feeds and recycled feeds.

Fresh Feeds. Crude oil or petroleum distillates which are being fed to processing units for the first time.

Recycled Feeds. Feeds that are continuously fed back for additional processing.

Catalytic Hydrocracking. A refining process that uses hydrogen and catalysts with relatively low temperatures and high pressures for converting middle boiling or residual material to high-octane gasoline, reformer charge stock, jet fuel, and/or high grade fuel oil. The process uses one or more catalysts, depending upon product output, and can handle high sulfur feedstocks without prior desulfurization.

Catalytic Hydrotreating. A refining procese for tresting petroleum fractions from atmospheric or vacuum distillation units (e.g., naphthas, middle distillates, reformer feeds, residual fuel oil, and heavy gas oil) and other petroleum (e.g., cat cracked naphtha, coker naphtha, gas oil, etc.) in the presence of catalysts and substantial quantities of hydrogen. Hydrotreating includes desulfurization, removal of substances (e.g., nitrogen compounds) that deactivate catalysts, conversion of olefins to paraffins to reduce gum formation in gasoline, and other processes to upgrade the quality of the fractions.

Catalytic Reforming. A refining process using controlled heat and pressure with catalysts to rearrange certain hydrocarbon molecules, thereby converting paraffinic and naphthenic type hydrocarbons (e.g., low-octane gasoline boiling range fractions) into petrochemical feedstocks and higher octane stocks suitable for blending into finished gasoline. Catalytic reforming is reported in two categories. They are:

Low Pressure. A processing unit operating at less than 225 pounds per square inch gauge (PSIG) measured at the outlet separator.

High Pressure. A processing unit operating at either equal to or greater than 225 pounds per square inch gauge (PSIG) measured at the outlet separator. 
Charge Capacity. The input (feed) capacity of the refinery processing facilities.

Coal. A black or brownish-black solid combustible substance formed by the partial decomposition of vegetable matter without access to air. The rank of coal, which includes anthracite, bituminous coal, subbituminous coal, and lignite, is based on fixed carbon, volatile matter, and heating value. Coal rank indicates the progressive alteration, or coalification, from lignite to anthracite. Lignite contains approximately 9 to 17 million BTU per ton. The heat contents of subbituminous and bituminous coal range from 16 to 24 million BTU per ton, and from 19 to 30 million BTU per ton, respectively. Anthracite contains approximately 22 to 28 million BTU per ton.

Commercial Kerosene-Type Jet Fuel. See Kerosene-Type Jet Fuel.

Crude Oil (Including Lease Condensate). A mixture of hydrocarbons that exists in liquid phase in underground reservoirs and remains liquid at atmospheric pressure after passing through surface-separating facilities. Included are lease condensate and liquid hydrocarbons produced from tar sands, gilsonite, and oil shale. Drip gases are also included, but topped crude oil (residual oil) and other unfinished oils are excluded. Liquids produced at natural gas processing plants and mixed with crude oil are likewise excluded where identifiable. Crude oil is considered as either domestic or foreign, according to the following:

Domestic. Crude oil produced in the United States or from its "outer continental shelf" as defined in 43 USC 1331 .

Foreign. Crude oil produced outside the United States. Imported Athabasca hydrocarbons are included.

Crude Oil, Refinery Receipts. Receipts of domestic and foreign crude oil at a refinery. Includes all crude oil in transit except crude oil in transit by pipeline. Foreign crude oil is reported as a receipt only after entry through customs. Crude oil of foreign origin held in bonded storage is excluded.

Crude Oil Losses. Represents the volume of crude oil reported by petroleum refineries as being lost in their operations. These losses are due to spills, contamination, fires, etc. as opposed to rizfinery processing losses.

Crude Oil Production. The volume of crude oil produced from oil reservoirs during given periods of time. The amount of such production for a given period is measured as volumes delivered from lease storage tanks (i.e., the point of custody transfer) to pipelines, trucks, or other media for transport to refineries or terminals with adjustments for (1) net differences between opening and closing lease inventories, and (2) basic sediment and water (BS\&W).

Crude Oil Qualities. Refers to two properties of crude oil, the sulfur content and API gravity, which affect processing complexity and product characteristics.

Delayed Coking. A process by which heavier crude oil fractions can be thermally decomposed under conditions of elevated temperatures and pressure to produce a mixture of lighter oils and petroleum coke. The light oils can be processed further in other refinery units to meet product specifications. The coke can be used either as a fuel or in other applications such as the manufacturing of steel or aluminum.

Disposition. The components of petroleum disposition are stock change, crude oil losses, refinery inputs, exports, and products supplied for domestic consumption.

Distillate Fuel Oil. A general classification for one of the petroleum fractions produced in conventional distillation operations. It is used primarily for space heating, on-and-off-highway diesel engine fuel (including railroad engine fuel and fuel for agricultural machinery), and electric power generation. Included are products known as No. 1, No. 2, and No. 4 fuel oils: No. 1. No. 2. and No. 4 diesel fuels. Distillate fuel oil is reported in the following sulfur categories: $0.05 \%$ sulfur and under and Greater than $0.05 \%$ sulfur.

No. 1 Distillate. A petroleum distillate which meets the specifications for No. 1 heating or fuel oil as defined in ASTM D 396 and/or the specifications for No. 1 diesel fuel as defined in ASTM Specification D 975 with distillation temperatures of $420^{\circ} \mathrm{F}$ at the 10-percent rec very point and $550^{\circ} \mathrm{F}$ at the 90-percent recovery point, and kinematic viscosities between 1.4 and 2.2 centistokes at $100^{\circ} \mathrm{F}$.

No. 2 Distillate. A petroleum distillate which meets the specifications for No. 2 heating or fuel oil as defined in ASTM D 396 and/or the specifications for No. 2 diesel fuel as defined in ASTM Specification D 975 with distillation temperatures of $540^{\circ}$ and $640^{\circ} \mathrm{F}$ at the 90-percent recovery point, and kinematic viscosities between 2.0 and 4.3 centistokes at $100^{\circ} \mathrm{F}$.

No. 4 Fuel Oil. A fuel oil for commercial burner installations not equipped with preheating facilities. It is used extensively in industrial plants. This grade is a blend of distillate fuel oil and residual fuel cil stocks that conforms to ASTM Specification D396 or Federal Specification VV-F-815C; with minimum and maximum kinematic viscosities between 5.8 and 26.4 centistokes at 
$100^{\circ}$ F. Also included is No. 4-D, a fuel oil for low and medium-speed diesel engines that conforms to ASTM Specification D975.

Electricity (Purchased). Electricity purchased for refinery operations that is not produced within the refinery complex.

Ending Stocks. Primary stocks of crude oil and petroleum products held in storage as of 12 midnight on the last day of the month. Primary stocks include crude oil or petroleum products held in storage at (or in) leases, refineries, natural gas processing plants, pipelines, tank farms, and bulk terminals that can store at least 50,000 barrels of petroleum products or that can receive petroleum products by tanker, barge, or pipeline. Crude oil that is in-transit by water from Alaska, or that is stored on Federal leases or in the Strategic Petroleum Reserve is included. Primary Stocks exclude stocks of foreign origin that are held in bonded warehouse storage.

ETBE (Ethyl tertiary butyl ether) ( $\left.\mathrm{CH}_{3}\right)_{3} \mathrm{COC}_{2} \mathrm{H}_{5}$. An oxygenate blend stock form: $d$ by the catalytic etherification of isobutylene with ethanol.

Ethane $\left(\mathrm{C}_{2} \mathrm{H}_{6}\right)$. A normally gaseous straight-chain hydrocarbon. It is a colorless paraffinic gas that boils at a temperature of $-127.48^{\circ} \mathrm{F}$. It is extracted from natural gas and refinery gas streams.

Ether. A generic term applied to a group of organic chemical compounds composed of carbon, hydrogen, and oxygen, characterized by an oxygen atom attached to two carbon atoms (e.g., methyl tertiary butyl ether).

Ethylene $\left(\mathrm{C}_{2} \mathrm{H}_{4}\right)$. An olefinic hydrocarbon recovered from refinery processes or petrochemical processes.

Exports. Shipments of goods from the 50 States and the District of Columbia to foreign countries, Puerto Rico, the Virgin Islands, and other U.S. possessions and territories.

Field Production. Represents crude oil production on leases, natural gas liquids production at natural gas processing plants, new supply of other hydrocarbons/ oxygenates and motor gasoline blending components, and fuel ethanol blended into finished motor gasoline.

Flexicoking. A thermal cracking process which converts heavy hydrocarbons such as crude oil, tar sands bitumen, and distillation residues into light hydrocarbons. Feedstocks can be any pumpable hydrocarbons including those containing high concentrations of sulfur and metals.

Fluid Coking. A thermal cracking process utilizing the fluidized-solids technique to remove carbon (coke) for continuous conversion of heavy, low-grade oils into lighter products.

Fresh Feed Input. Represents input of material (crude oil, unfinished oils, natural gas liquids, other hydrocarbons and oxygenates or finished products) to processing units at a refinery that is being processed (input) into a particular unit for the first time.

Examples:

(1) Unfinished oils coming out of a crude oil distillation unit which are input into a catalytic cracking unit are considered fresh feed to the catalytic cracking unit.

(2) Unfinished oils coming out of a catalytic cracking unit being looped back into the same catalytic cracking unit to be reprocessed are not considered fresh feed.

Fuel Ethanol ( $\left.\mathrm{C}_{2} \mathrm{H}_{5} \mathrm{OH}\right)$. An anhydrous denatured aliphatic alcohol intended for gasoline blending as described in Oxygenates definition.

Fuels Solvent Deasphalting. A refining process for removing asphalt compounds from petroleum fractions, such as reduced crude oil. The recovered stream from this process is used to produce fuel products.

Gas Oil. A liquid petroleum distillate having a viscosity intermediate between that of kerosene and lubricating oil. It derives its name from having originally been used in the manufacture of illuminating gas. It is now used to produce distillate fuel oils and gasoline.

Gasohol. A blend of finished motor gasoline and alcohol (generally ethanol but sometimes methanol), limited to 10 percent by volume of alcohol.

Gasoline Blending Components. Naphthas which will be used for blending or compounding into finished aviation or motor gasoline (e.g., straight-run gasoline, alkylate, reformate, benzene, toluene, and xylene). Excludes oxygenates (alcohols, ethers), butane, and pentanes plus.

Gross Input to Atmospheric Crude Oil Distillation Units. Total input to atmospheric crude oil distillation units. Includes all crude oil, lease condensate, natural gas plant liquids, unfinished oils, liquefied refinery gases, slop oils, and other liquid hydrocarbons produced from tar sands, gilsonite, and oil shale.

Heavy Gas Oil. Petroleum distillates with an approximate boiling range from $651^{\circ}$ to $1000^{\circ} \mathrm{F}$. 
Hydrogen. The lightest of all gases, occurring chiefly in combination with oxygen in water; exists also in acids, bases, alcohols, petroleum, and other hydrocarbons.

Idle Capacity. The component of operable capacity that is not in operation and not under active repair, but capable of being placed in operation within 30 days; and capacity not in operation but under active repair that can be completed within 90 days.

Imported Crude Oil Burned As Fuel. The amount of foreign crude oil burned as a fuel oil, usually as residual fuel oil, without being processed as such. Imported crude oil burned as fuel includes lease condensate and liquid hydrocarbons produced from tar sands, gilsonite, and oil shale.

Imports. Receipts of goods into the 50 States and the District of Columbia from foreign countries, Puerto Rico, the Virgin Islands, and other U.S. possessions and territories.

\section{Isobutane. See Butane.}

Isobutylene $\left(\mathrm{C}_{4} \mathrm{H}_{8}\right)$. An olefinic hydrocarbon recovered from refinery processes or petrochemical processes.

Isohexane ( $\left.\mathrm{C}_{6} \mathrm{H}_{14}\right)$. A saturated branch-chain hydrocarbon. It is a colorless liquid that boils at a temperature of $156.2^{\circ} \mathrm{F}$.

Isomerization. A refining process which alters the fundamental arrangement of atoms in the molecule without adding or removing anything from the original material. Used to convert normal butane into isobutane $\left(\mathrm{C}_{4}\right)$, an alkylation process feedstock, and normal pentane and hexane into isopentane $\left(\mathrm{C}_{5}\right)$ and isohexane $\left(\mathrm{C}_{6}\right)$, high-octane gasoline components.

\section{Isopentane. See Natural Gasoline and Isopentane.}

Kerosene. A petroleum distillate that has a maximum distillation temperature of $401^{\circ} \mathrm{F}$ at the 10-percent recovery point, a final boiling point of $572^{\circ} \mathrm{F}$, and a mini num flash point of $100^{\circ} \mathrm{F}$. Included are the two grades designated in ASTM D3699: No. 1-K and No. $2-\mathrm{K}$, and all grades of kerosene called range or stove oil. Kerosene is used in space heaters, cook stoves, and water heaters and is suitable for use as an illuminant when burned in wick lamps.

Kerosene-Type Jet Fuel. A quality kerosene product with a maximum distillation temperature of $400^{\circ} \mathrm{F}$ at the 10-percent recovery point and a final maximum boiling point of $572^{\circ} \mathrm{F}$. The fuel is designated in ASTM Specification D1655 and Military Specification MIL-T-5624L (Grades JP-5 and JP-8). A relatively low-freezing point distillate of the kerosene type used primarily for turbojet and turboprop aircraft engines.

Commercial. Kerosene-type jet fuel intended for commercial use.

Mulitary. Kerosene-type jet fuel intended for military use.

Lease Condensate. A natural gas liquid recovered from gas well gas (associated and non-associated) in lease separators or natural gas field facilities. Lease condensate consists primarily of pentanes and heavier hydrocarbons.

Light Gas Oils. Liquid petroleum distillates heavier than naphtha, with an approximate boiling range from $401^{\circ} \mathrm{F}$ to $650^{\circ} \mathrm{F}$.

Liquefied Petroleum Gases (LPG). Ethane, ethylene, propane, propylene, normal butane, butylene, isobutane, and isobutylene produced at refineries or natural gas processing plants, including plants that fractionate raw natural gas plant liquids.

Liquefied Refinery Gases (LRG). Liquefied petroleum gases fractionated from refinery or still gases. Through compression and/or refrigeration, they are retained in the liquid state. The reported categories are ethane/ethylene, propane/propylene, normal butane/butylene, and isobutane/isobutylene. Excludes still gas.

Lubricants. A substance used to reduce friction between bearing surfaces or as process materials either incorporated into other materials used as processing aids in the manufacturing of cther products, or as carriers of other materials. Petroleum lubricants may be produced either from distillates or residues. Other substances may be added to impart or improve certain required properties. Do not include byproducts of lubricating oil refining such as aromatic extracts derived from solvent extraction or tars derived from deasphalting. "Lubricants" includes all grades of lubricating oils from spindle oil to cylinder oil and those used in greases. Reporting categories include:

Paraffinic. Includes all grades of bright stock and neutrals with a Viscosity Index $>75$.

Naphthenic. Includes all lubricating oil base stocks with a Viscosity Index $<75$.

Note: The criterion for categorizing the lubricants is based solely on the Viscosity Index of the stocks and is independent of crude sources and type of processing used to produce the oils. 
Exceptions: Lubricating oil base stocks that have been historically classified as naphthenic or paraffinic by a refiner may continue to be so categorized irrespective of the Viscosity Index criterion.

\section{Example:}

(1) Unextracted paraffinic oils that would not meet the Viscosity Index test.

Merchant Oxygenate Plants. Oxygenate production facilities that are not associated with a petroleum refinery. Production from these facilities is sold under contract or on the spot market to refiners or other gasoline blenders.

Methanol $\left(\mathrm{CH}_{3} \mathrm{OH}\right)$. A light, volatile alcohol eligible for gasoline blending as described in Oxygenate definition.

Middle Distillates. A general classification of refined petroleum products that includes distillate fuel oil and kerosene.

Military Kerosene-Type Jet Fuel. See Kerosene-Type Jet Fuel.

Miscellaneous Products. Includes all finished products not classified elsewhere (e.g., petrolatum, lube refining byproducts (aromatic extracts and tars), absorption oils, ram-jet fuel. petroleum rocket fuels, synthetic natural gas feedstocks, and specialty oils).

Motor Gasoline (Finished). A complex mixture of relatively volatile hydrocarbons, with or without small quantities of additives, that has been blended to form a fuel suitable for use in spark-ignition engines. Motor gasoline, as given in ASTM Specification D439 or Federal Specification VV-G-1690B, includes a range in distillation temperatures from $122^{\circ}$ to $158^{\circ} \mathrm{F}$ at the 10 -percent recovery point and from $365^{\circ}$ to $374^{\circ} \mathrm{F}$ at the 90-percent recovery point. "Motor gasoline" includes reformulated gasoline, oxygenated gasoline, and other finished gasoline. Blendstock is excluded until blending has been completed.

Reformulated Gasoline. Gasoline formulated for use in motor vehicles, the composition and properties of which meet the requirements of the reformulated gasoline regulations promulgated by the U.S. Environmental Protection Agency under Section $211 \mathrm{~K}$ of the Clean Air Act.

Oxygenated Gasoline. Gasoline formulated for use in motor vehicles that has an oxygen content of 1.8 percent or higher, by weight. Includes gasohol.

Other Finished. Motor gasoline not included in the oxygenated or reformulated gasoline categories.
Motor Gasoline Blending. Mechanical mixing of motor gasoline blending components and oxygenates to produce finished motor gasoline (see definition of Motor Gasoline).

Motor Gasoline Blending Components. Naphthas which will be used for blending or compounding into finished motor gasoline (e.g. straight-run gasoline, alkylate, reformate, benzene, toluene, and xylene). Excludes oxygenates (alcohols, ethers), butane, and pentanes plus. Oxygenates are reported as individual components and included in the total for other hydrocarbons, hydrogens, and oxygenates.

MTBE (Methyl tertiary butyl ether) $\left(\mathrm{CH}_{3}\right)_{3} \mathrm{COCH}_{3}$. An ether intended for gasoline blending as described in Oxygenate definition.

Nameplate Capacity. The maximum design production capacity specified by the manufacturer of a processing unit or the maximum amount of a product that can be produced running the manufacturing unit at full capacity.

Naphtha. A generic term applied to a petroleum fraction with an approximate boiling range between $122^{\circ}$ and $400^{\circ}$ F.

\section{Naphtha Less Than $401^{\circ} \mathrm{F}$. See Petrochemical Feedstocks.}

Naphtha-Type Jet Fuel. A fuel in the heavy naphtha boiling range. ASTM Specification D1655 specifies for this fuel maximum distillation temperatures of $290^{\circ} \mathrm{F}$ at the 20-percent recovery point and $470^{\circ} \mathrm{F}$ at the 90 -percent point, meeting Military Specification MIL-T-5624L (Grade JP-4). JP-4 is used for turbojet and turboprop aircraft engines, primarily by the military. Excludes ram-jet and petroleum rocket fuels.

Natural Gas. A mixture of hydrocarbons and small quantities of various nonhydrocarbons existing in the gaseous phase or in solution with crude oil in underground reservoirs.

Natural Gas Field Facility. A field facility designed to process natural gas produced from more than one lease for the purpose of recovering condensate from a stream of natural gas; however, some field facilities are designed to recover propane, normal butane, pentane.: plus, etc., and to control the quality of natural gas to be marketed.

Natural Gas Plant Liquids. Natural gas liquids recovered from natural gas in gas processing plants, and in some situations, from natural gas field facilities. Natural gas liquids extracted by fractionators are also included. These liquids are defined according to the published specifications of the Gas Processors 
Association and the American Society for Testing and Materials and are classified as follows: ethane, propane, normal butane, isobutane, and pentanes plus.

Natural Gas Processing Plant. A facility designed (1) to achieve the recovery of natural gas liquids from the stream of natural gas which may or may not have been processed through lease separators and field facilities, and (2) to control the quality of the natural gas to be marketed. Cycling plants are classified as gas processing plants.

Natural Gasoline and Isopentane. A mixture of hydrocarbons, mostly pentanes and heavier, extracted from natural gas, that meets vapor pressure, end-point, and other specifications for natural gasoline set by the Gas Processors Association. Includes isopentane which is a saturated branch-chain hydrocarbon, $\left(\mathrm{C}_{5} \mathrm{H}_{12}\right)$, obtained by fractionation of natural gasoline or isomerization of normal pentane.

Net Receipts. The difference between total movements into and total movements out of each PAD District by pipeline, tanker, and barge.

\section{Normal Butane. See Butane.}

OPEC. The acronym for the Organization of Petroleum Exporting Countries, that have organized for the purpose of negotiating with oil companies on matters of oil production, prices and future concession rights. Current members are Algeria, Ecuador, Gabon, Indonesia, Iran, Iraq, Kuwait, Libya, Nigeria, Qatar, Saudi Arabia, United Arab Emirates, and Venezuela. The Neutral Zone between Kuwait and Saudi Arabia is considered part of OPEC.

Operable Capacity. The amount of capacity that, at the beginning of the period, is in operation; not in operation and not under active repair, but capable of being placed in operation within $\mathbf{3 0}$ days; or not in operation but under active repair that can be completed within 90 days. Operable capacity is the sum of the operating and idle capacity and is measured in barrels per calendar day or barrels per stream day.

Operating Capacity. The component of operable capacity that is in operation at the beginning of the period.

Operable Utilization Rate. Represents the utilization of the atmospheric crude oil distillation units. The rate is calculated by dividing the gross input to these units by the operable refining capacity of the units.

Operating Utilization Rate. Represents the utilization of the atmospheric crude oil distillation units. The rate is calculated by dividing the gross input to these units by the operating refining capacity of the units.
Other Finished. See Motor Gasoline (Finished).

Other Hydrocarbons. Materials received by a refinery and consumed as a raw material. Includes hydrogen, coal tar derivatives, gilsonite, and natural gas received by the refinery for reforming into hydrogen. Natural gas to be used as fuel is excluded.

Other Oils Equal To or Greater Than $401^{\circ} F$. See Petrochemical Feedstocks.

Other Oxygenates. Other aliphatic alcohols and aliphatic ethers intended for motor gasoline blending (e.g., isopropyl ether (IPE) or n-propanol).

Oxygenated Gasoline. See Motor Gasoline (Finished).

Oxygenates. Any substance which, when added to gasoline, increases the amount of oxygen in that gasoline blend. Through a series of waivers and interpretive rules, the Environmental Protection Agency (EPA) has determined the allowable limits for oxygenates in unleaded gasoline. The "Substantially Similar" Interpretive Rules (56 FR (February 11, 1991)) allows blends of aliphatic alcohols other than methanol and aliphatic ethers, provided the oxygen content does not exceed 2.7 percent by weight. The "Substantially Similar" Interpretive Rules also provides for blends of methanol up to 0.3 percent by volume exclusive of other oxygenates. and butanol or alcohols of a higher molecular weight up to 2.75 percent by weight. Individual waivers pertaining to the use of oxygenates in unleaded gasoline have been issued by the EPA. They include:

Fuel Ethanol. Blends of up to 10 percent by volume anhydrous ethanol (200 proof) (commonly referred to as the "gasohol waiver").

Methanol. Blends of methanol and gasoline-grade tertiary butyl alcohol (GTBA) such that the total oxygen content does not exceed 3.5 percent by weight and the ratio of methanol to GTBA is less than or equal to 1 . It is also specified that this blended fuel must meet ASTM volatility specifications (commonly referred to as the "ARCO" waiver).

Blends of up to 5.0 percent by volume methanol with a minimum of 2.5 percent by volume cosolvent alcohols having a carbon number of 4 or less (i.e., ethanol, propanol, butanol, and/or GTBA). The total oxygen must not exceed 3.7 percent by weight, and the blend must meet ASTM volatility specifications as well as phase separation and alcohol purity specifications (commonly referred to as the "DuPont" waiver).

MTBE (Methyl tertiary butyl ether). Blends up to 15.0 percent by volume MTBE which must meet the ASTM 
D4814 specifications. Blenders must take precautions that the blends are not used as base gasolines for other oxygenated blends (commonly referred to as the "Sun" waiver).

Pentanes Plus. A mixture of hydrocarbons, mostly pentanes and heavier, extracted from natural gas. Includes isopentane, natural gasoline, and plant condensate.

Persian Gulf. The countries that comprise the Persian Gulf are: Bahrain, Iran, Iraq, Kuwait, Qatar, Saudi Arabia, and the United Arab Emirates.

Petrochemical Feedstocks. Chemical feedstocks derived from petroleum principally for the manufacture of chemicals, synthetic rubber, and a variety of plastics. The categories reported are "Naphtha Less Than $401^{\circ} \mathrm{F}^{\prime}$ and "Other Oils Equal To or Greater Than $401^{\circ}$ F."

Naphtha Less Than $401^{\circ} \mathrm{F}$. A naphtha with a boiling range of less than $401^{\circ} \mathrm{F}$ that is intended for use as a petrochemical feedstock.

Other Oils Equal To or Greater Than $401^{\circ} F$. Oils with a boiling range equal to or greater than $401^{\circ} \mathrm{F}$ that are intended for use as a petrochemical feedstock.

Petroleum Administration for Defense (PAD) Districts. Geographic aggregations of the 50 States and the District of Columbia into five districts by the Petroleum Administration for Defense in 1950. These districts were originally defined during World War II for purposes of administering oil allocation.

Petroleum Coke. A residue, the final product of the condensation process in cracking. This product is reported as marketable coke or catalyst coke. The conversion factor is $\mathbf{5}$ barrels per short ton.

Marketable Coke. Those grades of coke produced in delayed or fluid cokers which may be recovered as relatively pure carbon. This "green" coke may be sold as is or further purified by calcining.

Catalyst Coke. In many catalytic operations (e.g., catalytic cracking) carbon is deposited on the catalyst, thus deactivating the catalyst. The catalyst is reactivated by burning off the carbon, which is used as a fuel in the refining process. This carbon or coke is not recoverable in a concentrated form.

Petroleum Products. Petroleum products are obtained from the processing of crude oil (including lease condensate), natural gas, and other hydrocarbon compounds. Petroleum products include unfinished oils, liquefied petroleum gases, pentanes plus, aviation gasoline, motor gasoline, naphtha-type jet fuel, kerosene-type jet fuel, kerosene, distillate fuel oil, residual fuel oil, petrochemical feedstocks, special naphthas, lubricants, waxes, petroleum coke, asphalt, road oil, still gas, and miscellaneous products.

Pipeline (Petroleum). Crude oil and product pipelines used to transport crude oil and petroleum products respectively, (including interstate, intrastate, and intracompany pipelines) within the 50 States and the District of Columbia.

Plant Condensate. One of the natural gas liquids, mostly pentanes and heavier hydrocarbons, recovered and separated as liquids at gas inlet separators or scrubbers in processing plants.

Processing Gain. The volumetric amount by which total output is greater than input for a given period of time. This difference is due to the processing of crude oil into products which, in total, have a lower specific gravity than the crude oil processed.

Processing Loss. The volumetric amount by which total refinery output is less than input for a given period of time. This difference is due to the processing of crude oil into products which, in total, have a higher specific gravity than the crude oil processed.

Product Supplied, Crude Oll. Crude oil burned on leases and by pipelines as fuel.

Production Capacity. The amount of product that can be produced from processing facilities.

Products Supplied. Approximately represents consumption of petroleum products because it measures the disappearance of these products from primary sources, i.e., refineries, natural gas processing plants, blending plants, pipelines, and bulk terminals. In general, product supplied of each product in any given period is computed as follows: field production, plus refinery production, plus imports, plus unaccounted for crude oil, (plus net receipts when calculated on a PAD District basis), minus stock change, minus crude oil losses, minus refinery inputs, minus exports.

Propane $\left(\mathrm{C}_{3} \mathrm{H}_{8}\right)$. A normally gaseous straight-chain hydrocarbon. It is a colorless paraffinic gas that boils at a temperature of $-43.67^{\circ} \mathrm{F}$. It is extracted from natural gas or refinery gas streams. It includes all products designated in ASTM Specification D1835 and Gas Processors Association Specifications for commercial propane and HD-5 propane.

Propylene $\left(\mathrm{C}_{3} \mathrm{H}_{6}\right)$. An olefinic hydrocarbon recovered from refinery processes or petrochemical processes. 
Refinery. An installation that manufactures finished petroleum products from crude oil, unfinished oils, natural gas liquids, other hydrocarbons, and oxygenates.

Reflicery Input, Crude Oil. Total crude oil (domestic plus foreign) input to crude oil distillation units and other refinery processing units (cokers, etc.).

Refinery Input, Total. The raw materials and intermediate materials processed at refineries to produce finished petroleum products. They include crude oil, products of natural gas processing plants, unfinished oils, other hydrocarbons and oxygenates, motor gasoline and aviation gasoline blending components and finished petroleum products.

Refinery Production. Petroleum products produced at a refinery or blending plant. Published production of these products equals refinery production minus refinery input. Negative production will occur when the amount of a product produced during the month is less than the amount of that same product that is reprocessed (input) or reclassified to become another product during the same month. Refinery production of unfinished oils, and motor and aviation gasoline blending components appear on a net basis under refinery input.

Refinery Yield. Refinery yield (expressed as a percentage) represents the percent of finished product produced from input of crude oil and net input of unfinished oils. It is calculated by dividing the sum of crude oil and net unfinished input into the individual net production of finished products. Before calculating the yield for finished motor gasoline, the input of natural gas liquids, other hydrocarbons and oxygenates, and net input of motor gasoline blending components must be subtracted from the net production of finished motor gasoline. Before calculating the yield for finished aviation gasoline, input of aviation gasoline blending components must be subtracted from the net production of finished aviation gasoline.

\section{Reformulated Gasoline. See Motor Gasoline} (Finished).

Residual Fuel Oil. The heavier oils that remain after the distillate fuel oils and lighter hydrocarbons are distilled away in refinery operations and that conform to ASTM Specifications D396 and 975 . Included are No. 5, a residual fuel oil of medium viscosity; Navy Special, for use in steam-powered vessels in government service and in shore power plants; No. 6, which includes Bunker C fuel oil, and is used for commercial and industrial heating, electricity generation and to power ships. Imports of residual fuel oil include imported crude oil burned as fuel.
Residuum. Residue from crude oil after distilling off all but the heaviest components, with a boiling range greater than $1000^{\circ} \mathrm{F}$.

Road Oll. Any heavy petroleum oil, including residual asphaltic oil used as a dust pallative and surface treatment on roads and highways. It is generally produced in six grades from 0 , the most liquid, to 5 , the most viscous.

Shell Storage Capacity. The design capacity of a petroleum storage tank which is always greater than or equal to working storage capacity.

Special Naphthas. All finished products within the naphtha boiling range that are used as paint thinners, cleaners, or solvents. These products are refined to a specified flash point. Special naphthas include all commercial hexane and cleaning solvents conforming to ASTM Specification D1836 and D484, respectively. Naphthas to be blended or marketed as motor gasoline or aviation gasoline, or that are to be used as petrochemical and synthetic natural gas (SNG) feedstocks are excluded.

Steam (Purchased). Steam, purchased for use by a refinery, that was not generated from within the refinery complex.

Still Gas (Refinery Gas). Any form or mixture of gascs produced in refineries by distillation, cracking, reforming, and other processes. The principal constituents are methane, ethane, ethylene, normal butane, butylene, propane, propylene, etc. Still gas is used as a refinery fuel and a petrochemical feedstock. The conversion factor is 6 million BTU's per fuel oil equivalent barrel.

Stock Change. The difference between stocks at the beginning of the month and stocks at the end of the month. A negative number indicates a decrease in stocks and a positive number indicates an increase in stocks.

Strategic Petroleum Reserve (SPR). Petroleum stocks maintained by the Federal Government for use during periods of major supply interruption.

Sulfur. A nonmetallic element of lemon-yellow color, sometimes known as "brimstone".

Supply. The components of petroleum supply are field production, refinery production, imports, and net receipts when calculated on a PAD District basis.

TAME (Tertiary amyl methyl ether) $\left(\mathrm{CH}_{3}\right)_{2}\left(\mathrm{C}_{2} \mathrm{H}_{5}\right) \mathrm{COCH}_{3}$. An oxygenate blend stock formed by the catalytic etherification of isoamylene with methanol. 
Tank Farm. An installation used by gathering and trunk pipeline companies, crude oil producers, and terminal operators (except refineries) to store crude oil.

Tanker and Barge. Vessels that transport crude oil or petroleum products. Data are reported for movements betweer. PAD Districts; from a PAD District to the Fanama Canal; or from the Panama Canal to a PAD District.

TBA (Tertiary butyl alcohol) $\left(\mathrm{CH}_{3}\right)_{3} \mathrm{COH}$. An alcohol primarily used as a chemical feedstock, a solvent or feedstock for isobutylene production for MTBE; produced as a co-product of propylene oxide production or by direct hydration of isobutylene.

Thermal Cracking. A refining process in which heat and pressure are used to break down, rearrange, or combine hydrocarbon molecules. Thermal cracking includes gas oil, visbreaking, fluid coking, delayed coking, and other thermal cracking processes (e.g., flexicoking). See individual categories for definition.

Toluene ( $\left.\mathrm{C}_{6} \mathrm{H}_{5} \mathrm{CH}_{3}\right)$. Colorless liquid of the aromatic group of petroleum hydrocarbons, made by the catalytic reforming of petroleum naphthas containing methyl cyclohexane. A high-octane gasoline-blending agent, solvent, and chemical intermediate, base for TNT.

Unaccounted for Crude Oil. Represents the arithmetic difference between the calculated supply and the calculated disposition of crude oil. The calculated supply is the sum of crude oil production plus imports minus changes in crude oil stocks. The calculated disposition of crude oil is the sum of crude oil input to refineries, crude oil exports, crude oil burned as fuel, and crude oil losses.

Unfinished Oils. Includes all oils requiring further processing, except those requiring only mechanical blending. Includes naphthas and lighter oils, kerosene and light gas oils, heavy gas oils, and residuum. See individual categories for definition.

Unfractionated Streams. Mixtures of unsegregated natural gas liquid components excluding those in plant condensate. This product is extracted from natural gas.

United States. The United States is defined as the $\mathbf{5 0}$ States and the District of Columbia.

Vacuum Distillation. Distillation under reduced pressure (less the atmospheric) which lowers the boiling temperature of the liquid being distilled. This technique with its relatively low temperatures prevents cracking or decomposition of the charge stock.

Visbreaking. A thermal cracking process in which heavy atmospheric or vacuum-still bottoms are cracked at moderate temperatures to increase production of distillate products and reduce viscosity of the distillation residues.

Wax. A solid or semi-solid material derived from petroleum distillates or residues by such treatments as chilling, precipitating with a solvent, or de-oiling. It is light-colored, more-or-less translucent crystalline mass, slightly greasy to the touch, consisting of a mixture of solid hydrocarbons in which the paraffin series predominates. Includes all marketable wax whether crude scale or fully refined. The three grades included are microcrystalline, crystalline-fully refined, and crystalline-other. The conversion factor is 280 pounds per 42 U.S. gallons per barrel.

Microcrystalline Wax. Wax extracted from certain petroleum residues having a finer and less apparent crystalline structure than paraffin wax and having the following physical characteristics: penetration at $77^{\circ} \mathrm{F}$ (D1321)-60 maximum; viscosity at $210^{\circ} \mathrm{F}$ in Saybolt Universal Seconds (SUS); (D88)-60 SUS (10.22 centistokes) minimum to 150 SUS (31.8 centistokes) maximum; oil content (D721)-5 percent minimum.

Crystalline-Fully Refined Wax. A light-colored paraffin wax having the following characteristics: viscosity at $210^{\circ}$ F (D88)-59.9 SUS (10.18 centistokes) maximum; oil content (D721)-0.5 percent maximum; other +20 color, Saybolt minimum.

Crystalline-Other Wax. A paraffin wax having the following characteristics: viscosity at $210^{\circ} \mathrm{F}$ (D88)-59.9 SUS (10.18 centistokes) maximum; oil content (D721)-0.51 percent minimum to 15 percent maximum.

Working Storage Capacity. The difference in volume between the maximum safe fill capacity and the quantity below which pump suction is ineffertive (bottoms).

Xylene $\left(\mathrm{C}_{6} \mathrm{H}_{4}\left(\mathrm{CH}_{3}\right)_{2}\right)$. Colorless liquid of the aromatic group of hydrocarbons made the catalytic reforming of certain naphthenic petroleum fractions. Used as high-octane motor and aviation gasoline blending agents, solvents, chemical intermediates. Isomers are metaxylene, orthoxylene, paraxylene. 

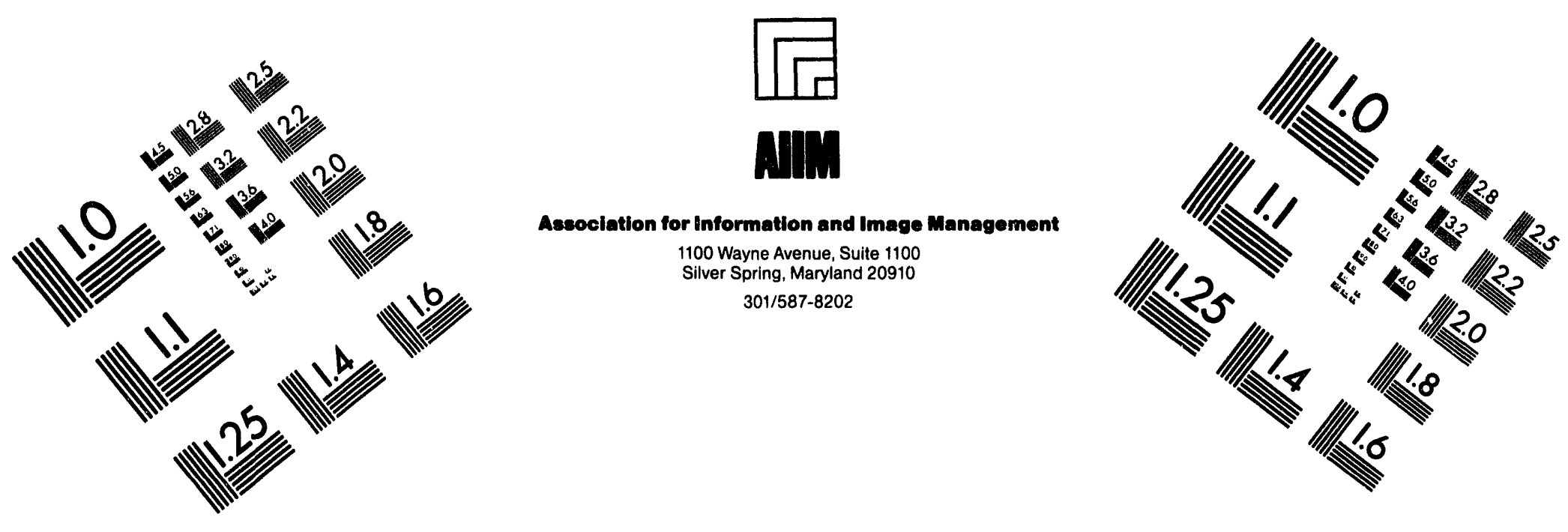

\section{Centimeter}

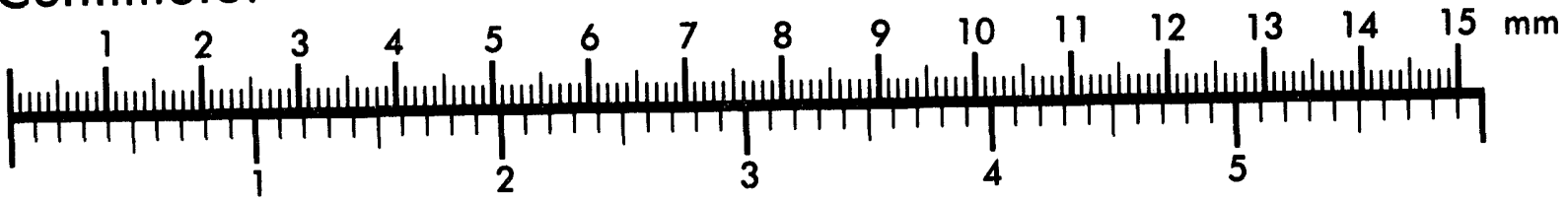

Inches
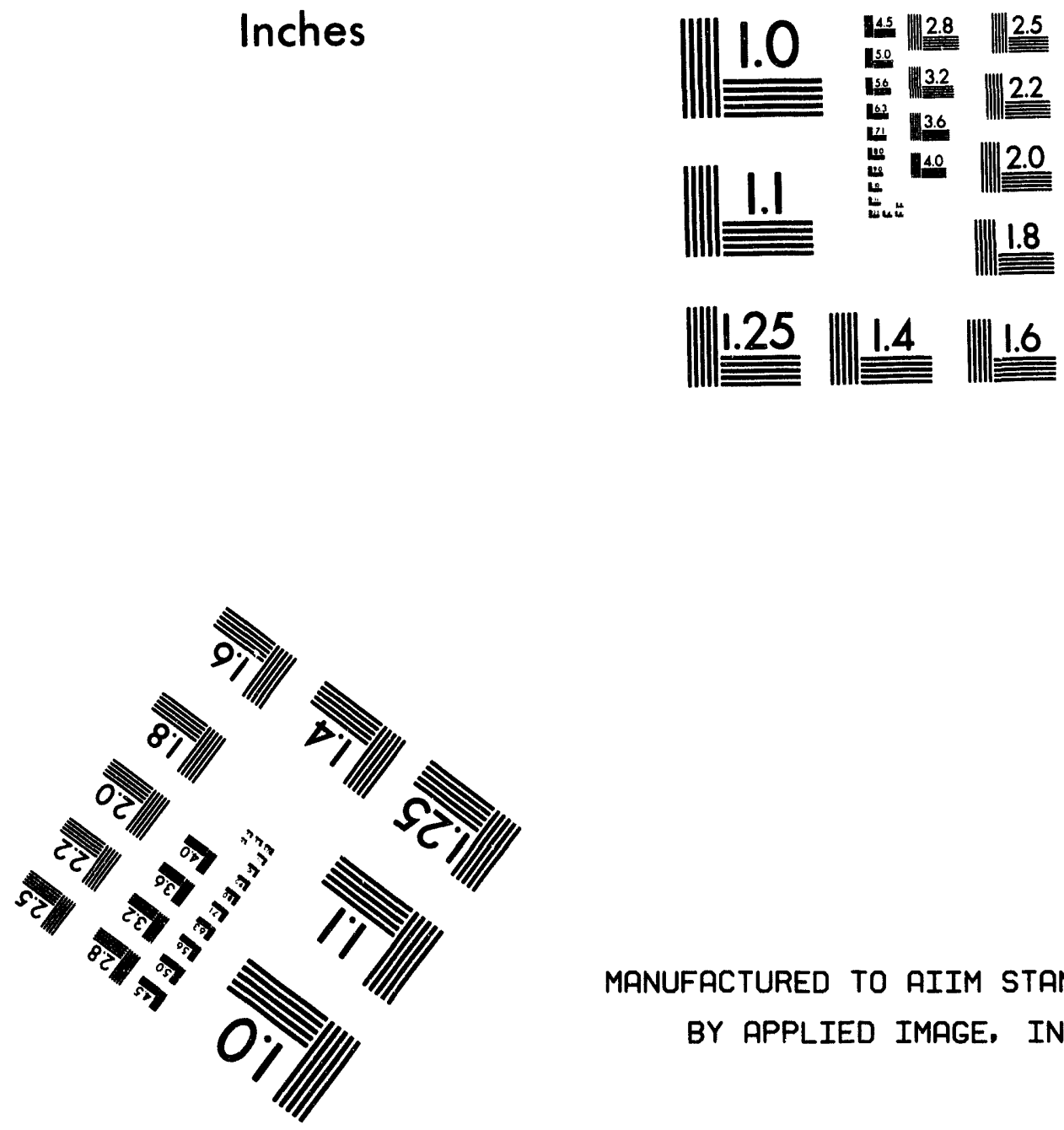

MANUFACTURED TO AIIM STANDARDS

BY APPLIED IMAGE, INC.

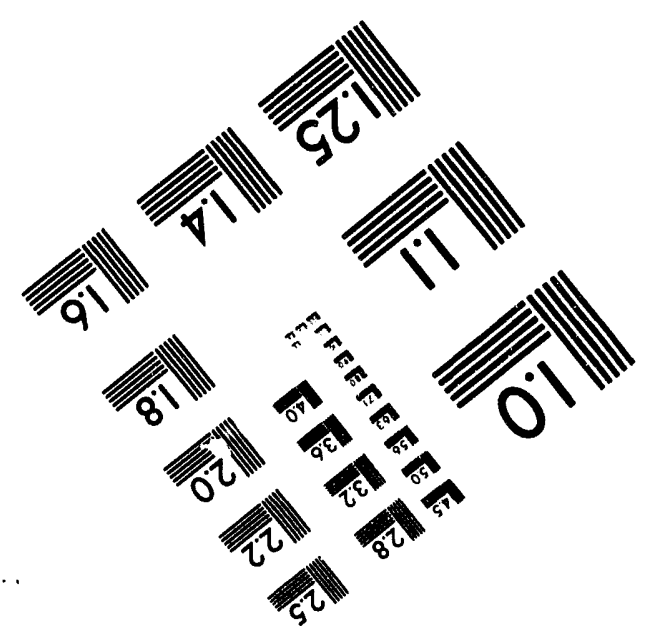




$$
\begin{aligned}
& \omega \\
& \stackrel{\rho}{\rho} \\
& \omega
\end{aligned}
$$



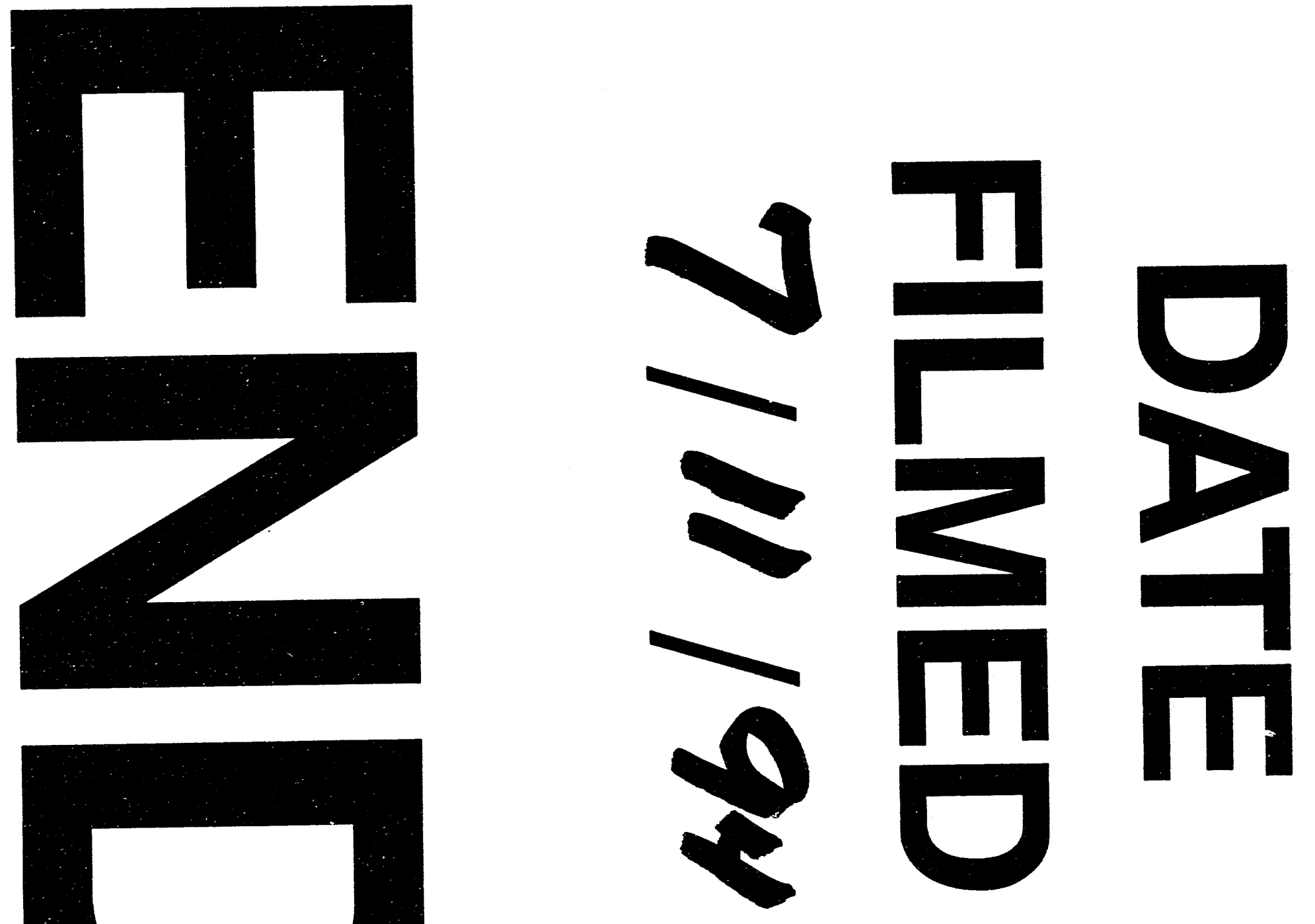

2

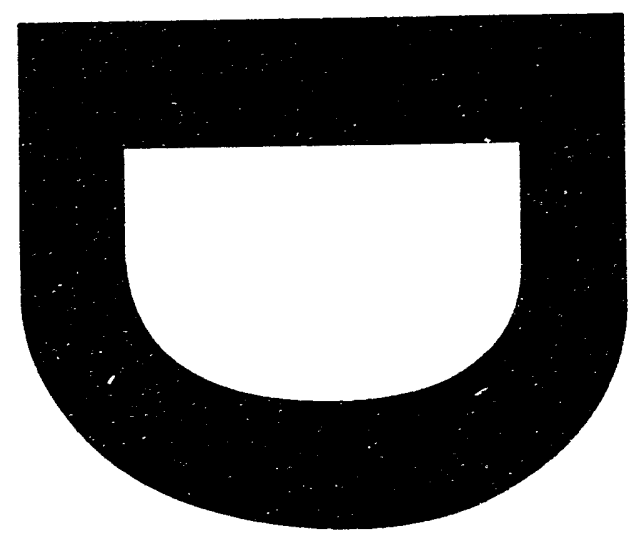


